
Biodiversity and the functioning of tropical forests

Masha T. van der Sande 


\section{Thesis committee}

\section{Promotor}

Prof. Dr Ir L. Poorter

Personal chair at the Forest Ecology and Forest Management Group

Wageningen University

\section{Co-promotors}

Dr M. Peña-Claros

Associate professor, Forest Ecology and Forest Management Group

Wageningen University

Dr E.J.M.M. Arets

Researcher at Vegetation, Forest and Landscape Ecology

Alterra Wageningen UR

\section{Other members}

Prof. Dr Ir N.P.R. Anten, Wageningen University

Prof. Dr Y. Malhi, University of Oxford, UK

Dr W.D. Gosling, University of Amsterdam

Dr J. van Ruijven, Wageningen University

This research was conducted under the auspices of the Wit Graduate School for Production Ecology and Resource Conservation 


\title{
Biodiversity and the functioning of tropical forests
}

\author{
Masha T. van der Sande
}

\section{Thesis}

submitted in fulfilment of the requirements for the degree of doctor

at Wageningen University

by the authority of the Rector Magnificus

Prof. Dr A.P.J. Mol,

in the presence of the

Thesis Committee appointed by the Academic Board

to be defended in public

on Wednesday 6 July 2016

at 4 p.m. in the Aula. 
Masha T. van der Sande

Biodiversity and the functioning of tropical forests, 284 pages.

PhD thesis, Wageningen University, Wageningen, NL (2016)

With references, with summary in English

ISBN 978-94-6257-802-9

DOI $10.18174 / 380256$ 


\section{Table of contents}

Chapter 1 General introduction 6

Chapter 2 Explaining biomass growth of tropical canopy trees: the 24 importance of sapwood

Oecologia (2015) 117: 1145-1155

Chapter 3 Soil fertility and species traits, but not species diversity, drive productivity and biomass stocks in a tropical rainforest In revision

Chapter 4 Drivers of biomass change in a Neotropical forest: testing for niche, mass-ratio, and environmental effects In revision

Chapter 5 Biodiversity, climate, and soil determine functioning of 104 Neotropical forests Submitted for publication

Chapter 6 Old-growth Neotropical forests are shifting in species and trait composition

Ecological Monographs (2016) 86: 228-243

Chapter 7 Biodiversity enhances climate change mitigation by tropical forests

In revision

Chapter 8 General discussion

References

Summary

Samenvatting

Resumen

Acknowledgements

Short biography

Publications 


$$
\text { Af }
$$

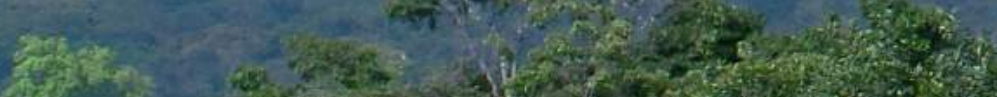

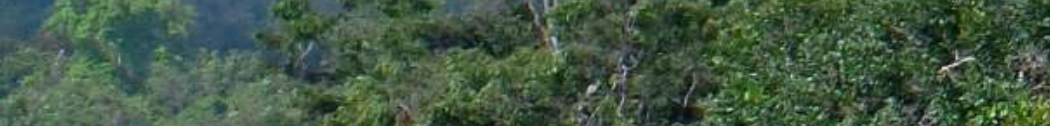

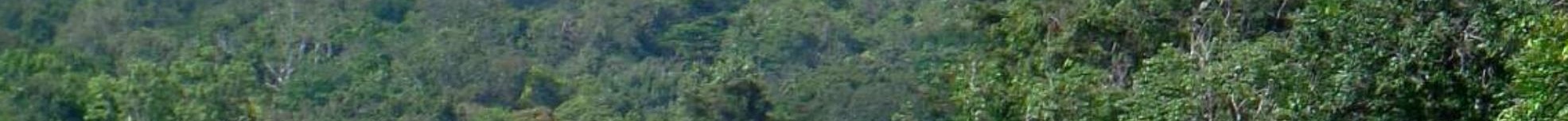

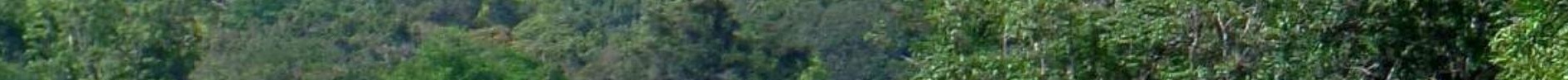

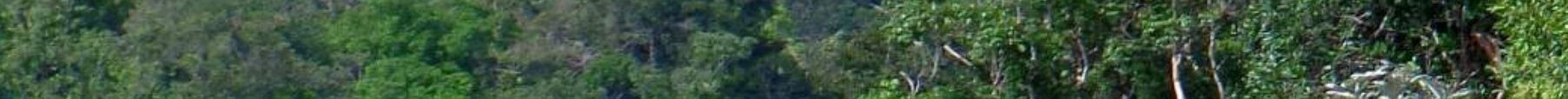

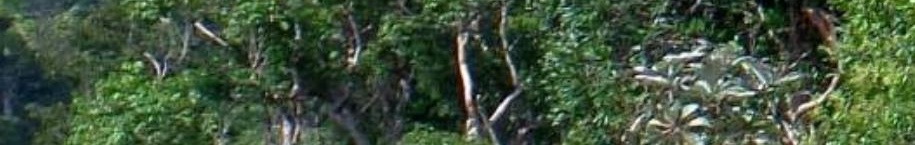
(1) (25) 3. 7.

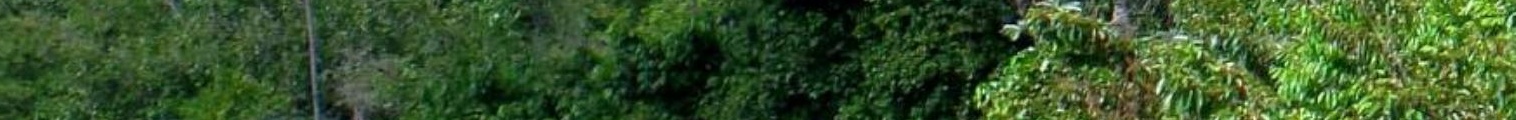
154 2.5. Fis

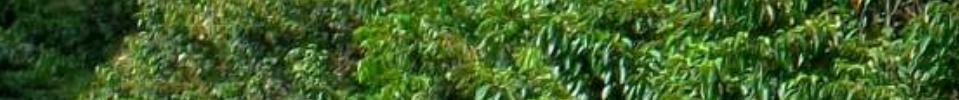

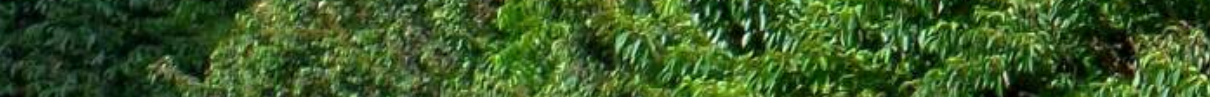

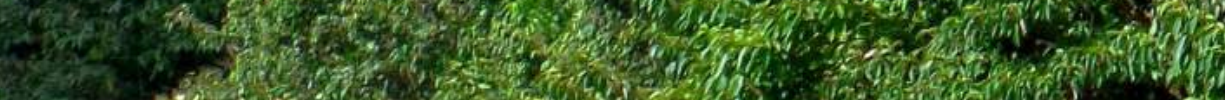

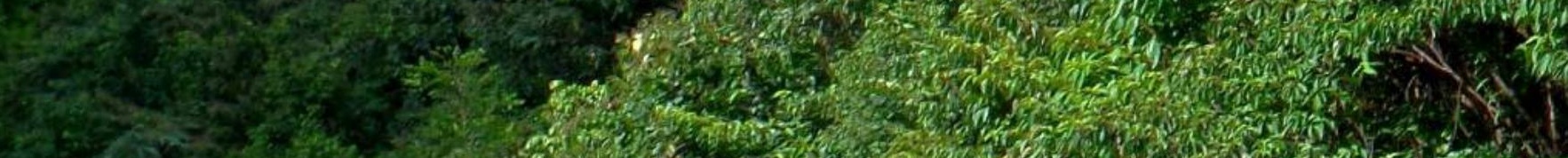

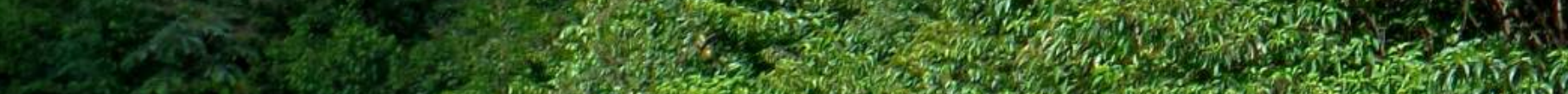

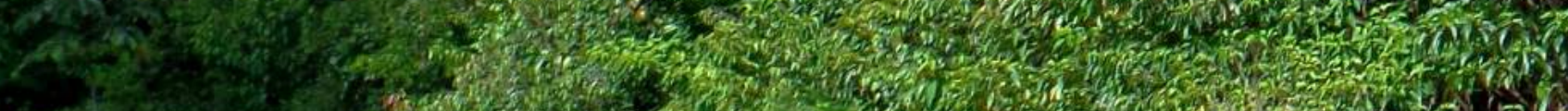

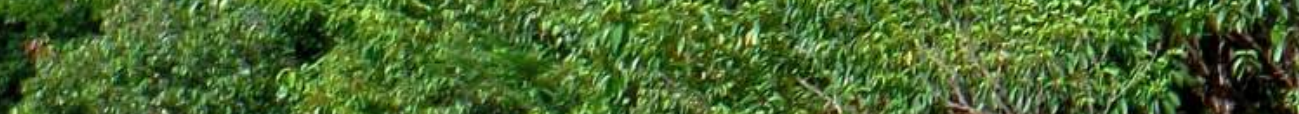
a

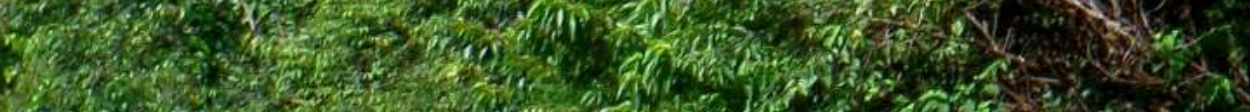

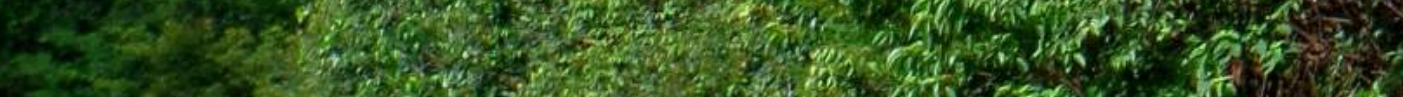

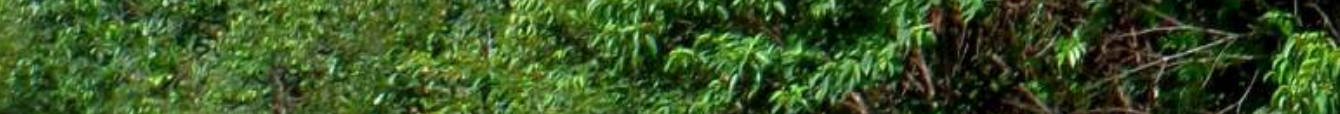




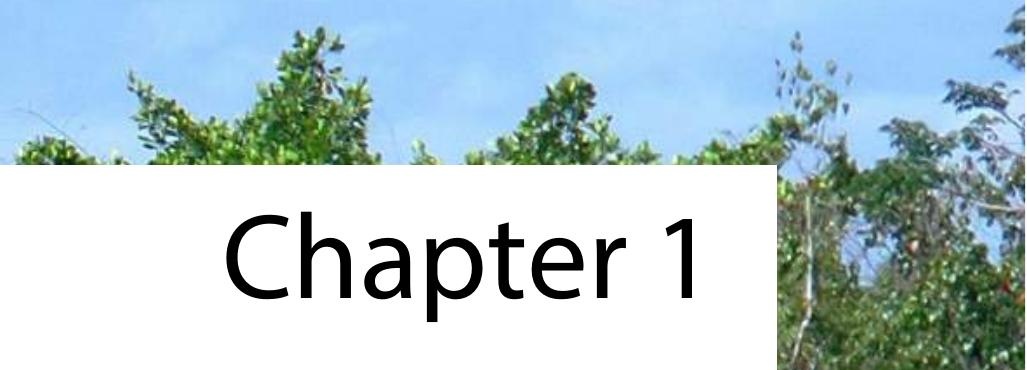
General introduction

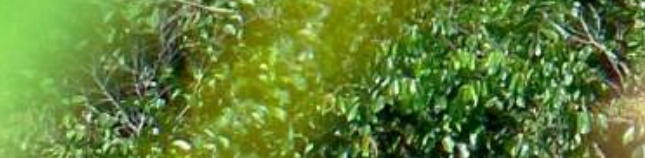

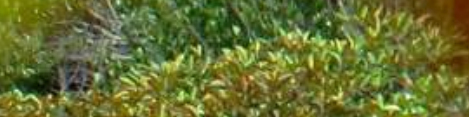

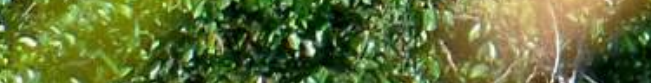

(1)

(2)

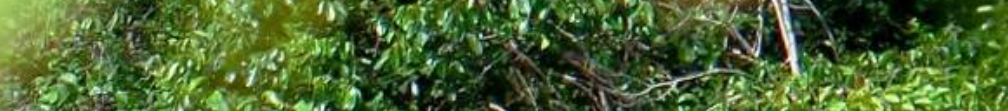
7.8.

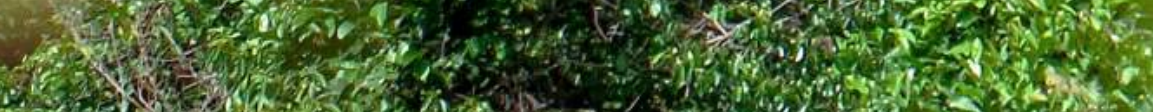

2.

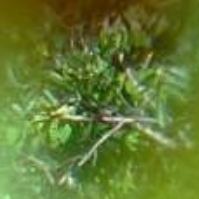

(2)

. 1.5. 27. 17. Th

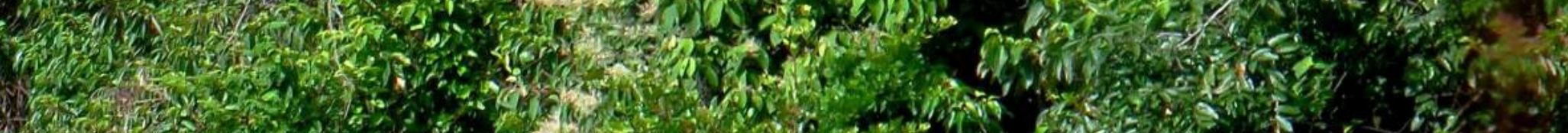

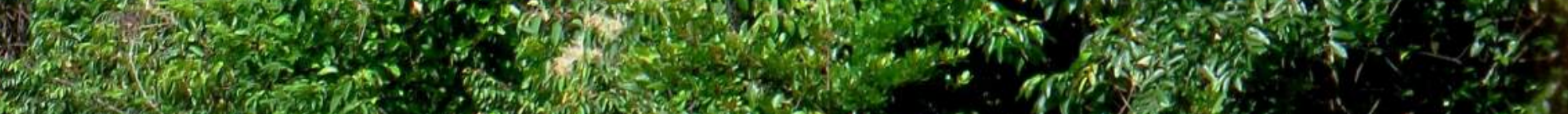

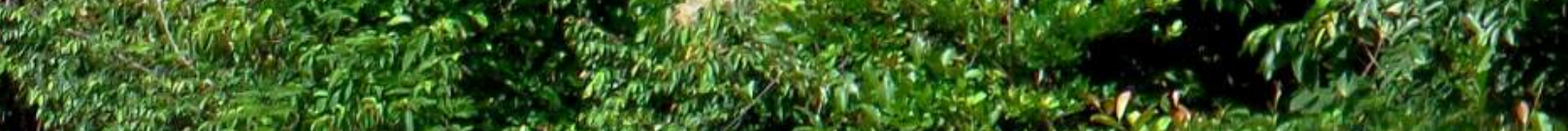

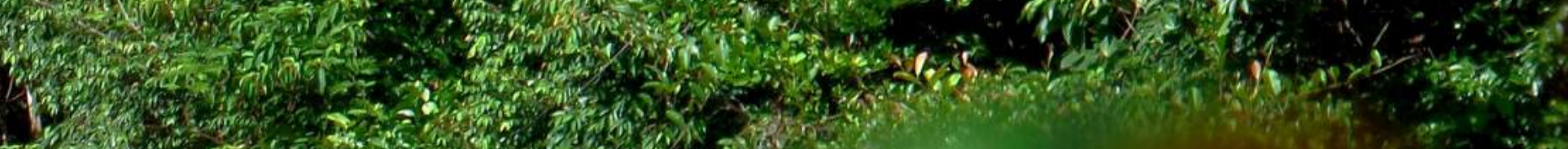

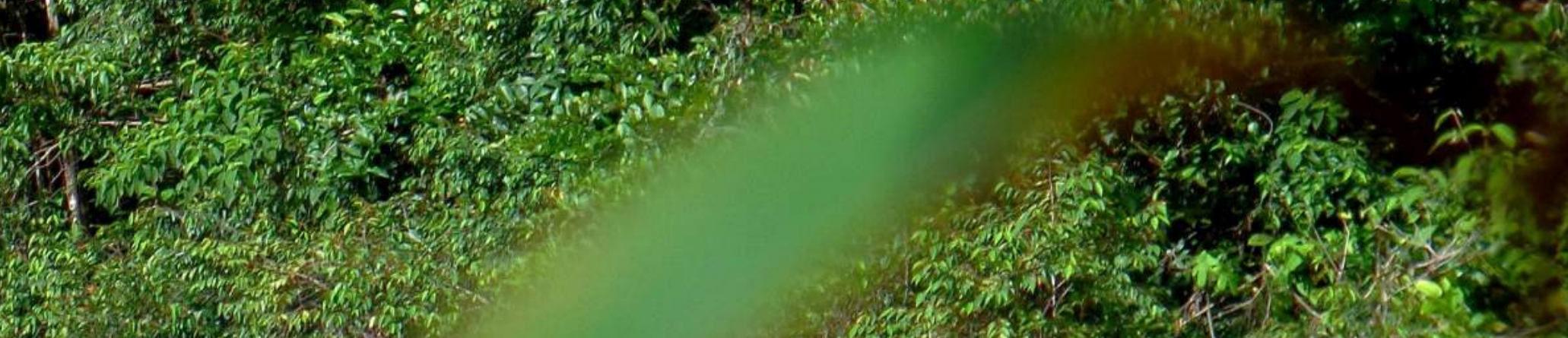




\section{Forest functioning in a changing world}

The human influence on planet Earth is increasing rapidly, in terms of both scale and intensity (Crutzen 2002, Steffen et al. 2011, Malhi et al. 2014). One of the major human-induced effects is global climate change. To keep climate change within safe boundaries (Rockström et al. 2009), international leaders have been discussing alternatives to mitigate and adapt. An important step was made during the Conference of the Parties (COP) of the United Nations Framework Convention on Climate Change (UNFCCC), in December 2015 in Paris. Here, 196 countries reached the agreement to reduce greenhouse gas emissions and increase $\mathrm{CO}_{2}$ removals from the atmosphere, with the ultimate goal to prevent global temperatures from rising more than $2{ }^{\circ} \mathrm{C}$ (United Nations 2015). $\mathrm{CO}_{2}$ removals from the atmosphere are naturally done by growing vegetation, through the process of photosynthesis. Vegetation types that store and remove large amounts of $\mathrm{CO}_{2}$, such as tropical forests, are therefore highly relevant for climate change mitigation.

Tropical forests contribute to climate change mitigation in three ways. First, biomass in tropical forests contains about $25 \%$ of all carbon on only about $12 \%$ of the area in the terrestrial biosphere (Bonan 2008), which means that preventing deforestation and forest degradation can reduce $\mathrm{CO}_{2}$ emissions. Second, tropical forests are $\mathrm{CO}_{2}$ 'sinks', meaning that they remove net $\mathrm{CO}_{2}$ from the atmosphere, and use this in photosynthesis to produce additional aboveground and belowground biomass (Brienen et al. 2015, Poorter et al. 2016). During the early $20^{\text {th }}$ century, standing old-growth tropical forests removed 1-1.2 Pg carbon $\mathrm{y}^{-1}$ and regrowing (or secondary) forests another 1.2-1.7 $\mathrm{Pg}^{-1}$, which was about $24 \%$ of the global annual anthropogenic carbon emissions (Canadell and Schulze 2014, Goodman and Herold 2014). Third, tropical forests reduce global temperatures due to high evapotranspiration rates. High evapotranspiration has a direct effect on temperatures through evaporative cooling, and an indirect effect through increasing cloud and rain formation and sunlight reflection which, in turn, reduce global temperatures (Bonan 2008, Canadell and Raupach 2008, Alkama and Cescatti 2016).

Besides their climate mitigation capacity, tropical forests are crucially important for various other functions that are relevant at local and global scales, such as timber and non-timber forest products and pollination (Laurance 1999, Malhi et al. 2008, Alkama and Cescatti 2016). Globally, the livelihood of more than a billion people depends directly on forests (FAO 2016), with most of them living in the tropics. Forest functioning ultimately depends on ecosystem processes, which are fluxes of carbon, water and nutrients at the ecosystem level (Box 1.1). To guarantee forest functioning, we thus need to understand what mechanisms determine ecosystem processes. In this thesis I focus on the biomass stocks and 
biomass dynamics of the tropical forest vegetation as measures of ecosystem processes, given that the vegetation is most directly related to climate change mitigation and other globally important functions. Biomass stocks refer to the amount of (above- and/or belowground) biomass per unit area, and biomass dynamics to fluxes in biomass per unit time per unit area (see Box 1.1). Biomass stock is a state variable rather than a process, but for simplicity I consider it as part of 'ecosystem processes' in the general introduction (chapter 1) and general discussion (chapter 8).

Tropical forests are thus important for climate change mitigation, but climate change in turn also affects the temporal dynamics of tropical forests and thus their mitigation capacity (Cox et al. 2000). Spatial variation in abiotic conditions such as soil fertility and annual rainfall strongly determines spatial variation in biomass (Malhi 2012, Poorter et al. 2015), and therefore temporal changes in abiotic conditions can lead to temporal changes in biomass stocks and other ecosystem processes (Box 1.1). In addition, biotic conditions, which are properties of the vegetation itself such as species diversity and community-weighted mean traits (Box 1.1), can determine ecosystem processes and in this way the climate mitigation potential of tropical forests. Consequently, the main aim of this thesis is to understand how underlying abiotic and biotic conditions determine the biomass stocks and dynamics of tropical forests (Fig. 1.1) across spatial scales (Fig. 1.2) and temporal scales.

\section{Biotic conditions}

Each tropical forest is unique in its species composition, species diversity, vegetation structure and ecological functioning. Depending on the context, throughout this thesis I interchangeably refer to such vegetation properties as 'biotic conditions', 'biotic factors' or 'biodiversity attributes' (see Box 1.1 and Table 7.2). Some evidence is emerging for effects of biotic conditions on biomass stocks and dynamics in tropical forests (e.g. Baker et al. 2009, Chisholm et al. 2013). The simultaneous effects of multiple biotic conditions have, however, rarely been evaluated, although this is important to identify their relative effects and thus their relevance for biomass stocks and dynamics. In this thesis I distinguish attributes that indicate vegetation 'quality', such as species diversity, trait mean and trait diversity, from other attributes that indicate vegetation 'quantity', such as plot basal area or tree density (cf. Lohbeck 2014). Vegetation quality can be important for ecosystem processes because the average and diversity in species' properties can influence the efficiency of resource acquisition and use of the plant community. Vegetation quantity can be important because a dense forest has greater biomass 
that can positively contribute to ecosystem process rates, or it can decrease ecosystem process rates due to lower light availability.

\section{Vegetation quality - species diversity}

Tropical forests host about 47000 different tree species (Slik et al. 2015), which makes them the most species-diverse terrestrial ecosystem (Dirzo and Raven 2003). Climate change, habitat loss and hunting, however, are causing unprecedented rates of species extinction (De Vos et al. 2015). This species loss is expected to be one of the main drivers of changes in ecosystem processes (Balvanera et al. 2006, Midgley 2012). Several theories have been proposed to understand the role of species diversity on ecosystem processes. The niche complementarity theory predicts that species diversity is crucially important for ecosystem processes (Tilman 1999), because high species diversity would increase the resource use efficiency of the community and as a result lead to higher community-level biomass stocks and carbon uptake. Comparably, the insurance theory (or temporal niche complementarity theory, Loreau 2000) predicts that high diversity increases the asynchrony in species' responses to environmental conditions and changes, which would increase the long-term stability of ecosystem processes (Yachi and Loreau 1999).

Box 1.1: Glossary with concepts and their explanation used in the general introduction (chapter 1) and general discussion (chapter 8), and synonyms used throughout this thesis.

Abiotic conditions (or factors or drivers): environmental variables such as climate, soil conditions, and light availability.

Biodiversity attributes: synonym for 'biotic conditions'

Biomass dynamics: the fluxes in biomass per unit area per unit time. Examples of positive fluxes (i.e. biomass increase) are: aboveground biomass increase, tree growth, seedling recruitment, or litter production. An example of a negative flux is tree mortality.

Biomass stocks: the amount of biomass per unit area. This can be based on aboveground living biomass, (fine) root biomass, or soil organic matter.

Biotic conditions (or factors or drivers): attributes of the vegetation, such as taxonomic diversity, trait diversity, community-mean trait values, and/or structural attributes (see also Table 7.2).

Carbon dynamics: fluxes in carbon (in vegetation or soil) per unit area per unit time. Carbon dynamics are sometimes used to replace biomass dynamics because they are very strongly related (biomass is about twice the mass of carbon).

Carbon stocks: the amount of carbon (in vegetation or soil) per unit area. Carbon stocks is sometimes used to replace biomass stocks because they are very strongly related.

Community-weighted mean (CWM) traits (or community-mean traits): community average trait values, such as specific leaf area, wood density and leaf nitrogen concentration, weighted by species' basal area or abundance. 
Ecosystem functioning: the combined effect of all ecosystem processes that are needed to sustain an ecosystem (Reiss et al. 2009).

Ecosystem functions: often used as a synonym for 'ecosystem processes'. In this thesis 'ecosystem functions' mainly refer to processes that provide benefits to the planet and humans, such as carbon sequestration.

Ecosystem processes: ecosystem-level fluxes or stocks of carbon, water and nutrients, such as biomass stocks or productivity of the whole community. For simplicity, in the general introduction (chapter 1) and general discussion (chapter 8) I also include biomass stock under 'ecosystem processes', even though this is a state variable rather than a process.

Ecosystem resilience: the rate at which an ecosystem returns to the pre-disturbance state following a perturbation, including maintaining its essential characteristics in taxonomic composition, structure, ecosystem functions, and process rates (Holling 1973).

Ecosystem stability: the capacity of an ecosystem to maintain similar ecosystem functioning during disturbances or changing environmental conditions, often calculated as the temporal mean of a process divided by its temporal standard deviation.

Environmental conditions: synonym for 'abiotic conditions'

Forest structure: synonym for 'vegetation quantity'

Functional (trait) diversity: synonym for 'trait diversity'

Functional trait: any measurable plant characteristic that affects the plant's resource acquisition and use, and thus determines its growth, reproduction and/or survival (Violle et al. 2007).

Insurance theory (or hypothesis): species respond differently to environmental changes and in this way insure long-term ecosystem functioning under environmental change (Yachi and Loreau 1999).

Mass-ratio theory (or hypothesis): the most dominant species and their traits mostly determine ecosystem processes (Grime 1998). That is, the community-weighted mean trait values more strongly determine ecosystem processes than diversity (in species or trait values) in the community.

Niche complementarity theory (or hypothesis): species are complementary in their resource acquisition and use. Therefore, high diversity (of species or traits) results in efficient acquisition and use at the community level, and thus in high biomass stocks and dynamics (Tilman 1999).

Species diversity: variation in species (e.g. the number or diversity) within a community

Structural attributes: synonym for 'vegetation quantity'

Taxonomic diversity: synonym for 'species diversity'.

Trait composition: synonym for 'community-weighted mean traits' (chapters 3 and 4). I also use 'trait composition' to refer to the multivariate community-weighted mean trait space (chapter 6).

Trait diversity: variation in trait values within a community. This can be based both on multivariate trait diversity as well as on the variation in single traits (Table 7.2).

Vegetation quantity: community-average or community-total values of structural components of the community, such as plot basal area and average stem diameter. 


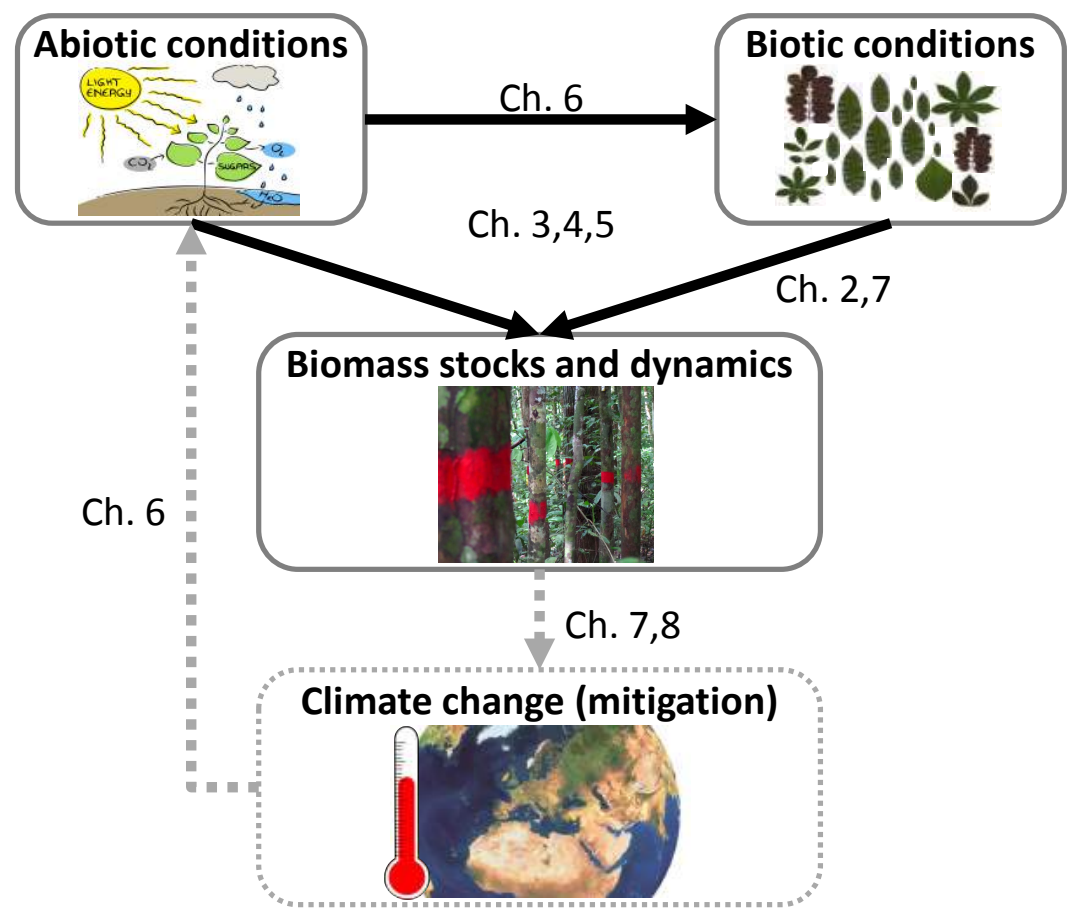

Figure 1.1: Conceptual framework linking abiotic conditions (e.g. light, water and nutrient availability) and biotic conditions (also referred to as 'biodiversity attributes' in this thesis, e.g. species diversity and community-weighted mean traits) with biomass stocks and dynamics. The chapters in which these relationships are addressed are provided. The black arrows represent directly analysed relationships, whereas the grey dashed arrows represent relationships that are discussed or hypothesized but not measured in this thesis. The boxes with a grey, solid border represent measured variables, whereas the box with a grey, dashed border represents nonmeasured variables. Chapters ("Ch.") 2 and 7 focus on effects of biotic conditions on biomass stocks and dynamics; chapters 3, 4 and 5 on the relationships between abiotic conditions, biotic conditions and biomass stocks and dynamics; and chapter 6 focuses on the effect of abiotic conditions on biotic conditions. Chapters 7 and 8 elaborately discuss the importance of biomass stocks and dynamics for climate change mitigation, and chapter 6 uses temporal changes in climate as cause for variation in abiotic conditions.

The niche complementarity and insurance theories have received ample support for relatively less complex ecosystems such as grasslands (Anten and Hirose 1999, Tilman et al. 2001, van Ruijven and Berendse 2005, Isbell et al. 2015) and temperate forests (Morin et al. 2011, Jucker et al. 2014). For tropical forests, however, evidence has only recently started to emerge, but does not yet provide a consistent understanding of species diversity effects on ecosystem processes (e.g. a negative effect by Potvin et al. 2011 and a positive by Chisholm et al. 2013). The relationship between species diversity and ecosystem processes may be difficult to understand because of the highly diverse and structurally complex nature of tropical forests. Nevertheless, it is important to obtain further insights into this relationship because of the potentially strong effects of diversity on ecosystem processes and functioning, as predicted by theories and demonstrated in grasslands and temperate forests. 


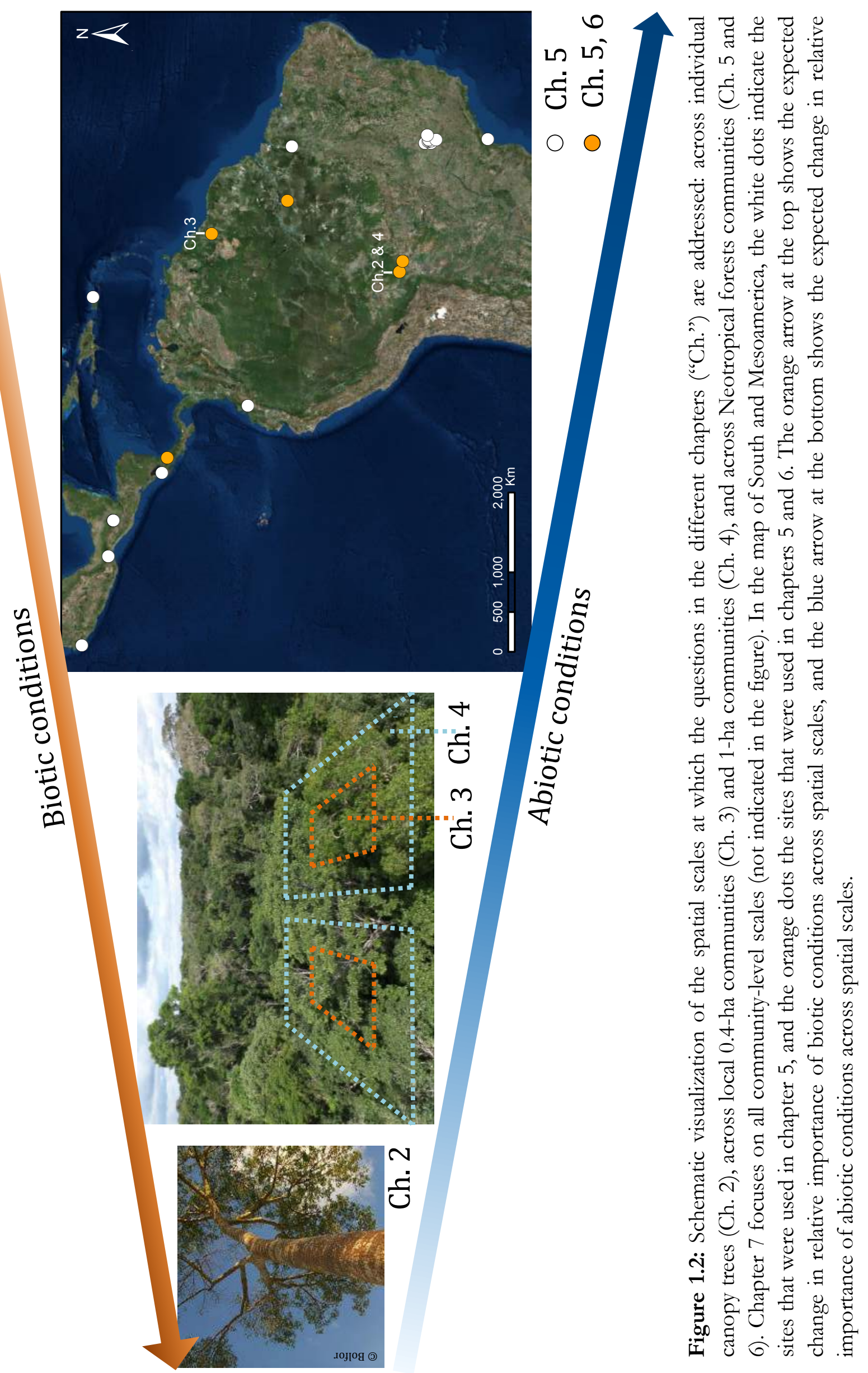


Species diversity is mostly defined as species richness. Species richness is based on the number of species per plot or calculated as rarefied species richness (i.e. number of species in a random draw of a fixed number of individuals). Besides species richness, various other measures for species diversity have been developed that to some degree take the species abundance into account, such as the ShannonWiener index, the Simpson index, and the evenness index (Peet 1974). Throughout this thesis, I make mainly use of (rarefied) species richness because this measure is most often used by other studies and thus allows comparison, but I also include the Shannon-Wiener index (chapter 5).

\section{Vegetation quality - trait mean and trait diversity}

Species diversity measures assume differences among species. Indeed, in a forest with hundreds of species, not even two are functionally equal. Nevertheless, the degree of niche complementarity may depend more strongly on the functional differences among species than on the number of species. In this thesis I use a trait-based approach to determine functional differences among individuals, species and communities, for example in terms of leaf and stem traits.

Functional differences among species or individuals can be measured by morphological, physiological or phenological traits that are important for their survival, growth and reproduction (Violle et al. 2007). For example, light and water acquisition are important for tree growth, and drought-tolerance is important for survival (Engelbrecht and Kursar 2003, Poorter and Markesteijn 2008). These trait examples are closely related to performance but are difficult to measure and are therefore called 'hard' traits (Hodgson et al. 1999). For that reason, most often 'soft' traits are used, which are more easily measurable traits that are a good proxy for a 'hard' trait or a process. For example, leaf traits such as specific leaf area (leaf area divided by leaf dry mass) and chlorophyll concentration are important for light acquisition and photosynthesis (Wright et al. 2004, Reich 2014), and a wood trait such as wood density is important for mechanic stability and drought-tolerance (Markesteijn et al. 2011a). Species' (soft) traits may therefore be good indicators of species' functioning (Pérez-Harguindeguy et al. 2013).

Information on species' traits can be scaled to the community level, to obtain a mechanistic understanding of ecosystem processes. Trait diversity measures can provide information on the niche complementarity theory. Contrary to the niche complementarity theory, however, is the mass-ratio theory (Grime 1998), which predicts that the functioning of the most dominant species in a community determines ecosystem processes, and that the diversity of species or their functioning matters less. In other words, the diversity of trait values is an indicator for niche complementarity, whereas the average trait value of the community, which is mainly determined by the dominant species, is an indicator for mass ratio. 
The trait sampling design depends on the ultimate research goal. Measuring intra-individual or intra-specific trait variation obviously trades off with the total number of species that can be measured. In this thesis I am mainly interested in community-level processes (except for chapter 2 where we look at intra-specific variation in biomass growth), for which trait values of at least $\sim 80 \%$ of the basal area or abundance in the community are needed to accurately determine community-average trait values (Pakeman and Quested 2007). This percentage should ideally be even higher for trait diversity measures (Pakeman 2014). At the community level, generally most variation in trait values is explained by interspecific differences (78\%; Rozendaal, Hurtado \& Poorter 2006). Therefore, I use locally collected average trait values (based on 1-10 individuals per species and 3-5 leaves per individual) for the species that represent $\sim 80 \%$ of the basal area abundance in the site, and I thus do not include intra-specific trait variation.

As an indicator for the mass-ratio theory, I use community-weighted mean (CWM) traits, which are based on species' average trait values and species' relative basal area (chapters 3-7) or abundance (chapter 6). Throughout my thesis, I also refer to CWM traits as 'trait composition' (chapters 3, 4 and 6) or 'communitymean traits' (chapters 4-7). As an indicator for niche complementarity, besides the species diversity indices, I use indices of multivariate trait diversity (also called functional diversity or variety, Mason et al. 2005), such as trait richness1 (chapter 4, Mason et al. 2005) and trait dispersion2 ('functional dispersion' in chapter 3, Pakeman 2014).

\section{Vegetation quantity}

Besides quality (represented by e.g. species diversity, CWM traits, and trait diversity), the quantity of the vegetation may also be important. For example, the total basal area or tree density in a community may strongly determine the potential of the forest to grow, although in opposite ways. On one hand, a dense forest has more individuals and biomass that can contribute to growth, but on the other hand a dense forest has low availability of resources (particularly light), which may decrease biomass stocks and dynamics. Also quantitative measures at the individual-tree level, for example tree biomass or total leaf area, may strongly determine tree growth (Stephenson et al. 2014). As measures of vegetation quantity, I mainly use plot basal area, because this represents the density and thus the competition within the forest (chapters 4 and 5).

\footnotetext{
${ }^{1}$ The amount of multivariate trait space occupied by species in the plot (Mason et al. 2005, Mouillot et al. 2005).

${ }^{2}$ Based on the mean distance in the multidimensional trait space of all individual species to the centroid of all species (Pakeman 2014).
} 


\section{Abiotic conditions}

Biotic conditions may thus strongly determine ecosystem processes, but they are not the only actor on stage. Abiotic conditions are another important group of variables to potentially influence ecosystem processes directly, and indirectly via their effects on biotic conditions (e.g. Figs. 1.1, 3.1, 4.1 and 5.1). The main three groups of abiotic conditions that I evaluate in this thesis are: 1) climatic wetness, such as rainfall, 2) soil conditions, such as nutrient concentrations and water availability, and 3) light availability, because these abiotic conditions are important for plant growth and may have different importance across spatial scales.

\section{Climate, soil, and light}

Climatic wetness (most often measured by annual rainfall) generally increases ecosystem process rates (Slik et al. 2013, Poorter et al. 2015), although at very high rainfall, strong nutrient leaching from the soil takes place which reduces ecosystem process rates (e.g. Hall and Swaine 1976). Differences in climate, however, may only be relevant at regional or continental scales and at longer temporal scales because it varies little at short-term local scales (Fig. 1.2).

High soil nutrient and soil water availability generally increase ecosystem processes (Telles et al. 2003, Malhi et al. 2004). Soil conditions can be very heterogeneous and therefore play an important role especially at local spatial scales (Roy and Singh 1994).

Light availability can vary locally because of natural or anthropogenic disturbances. It can also vary at larger spatial scales due to differences in vegetation structure, such as high light availability in dry forest with low total biomass. Disturbances reduce biomass and increase light availability. Hence, light availability may be strongly related with vegetation quantity, indicating that abiotic conditions may also have an effect on biotic conditions.

\section{How do abiotic conditions affect biotic conditions?}

Abiotic conditions may thus also determine biotic conditions and in this way indirectly affect ecosystem processes (Fig. 1.1). For example, sandy soils have more drought-tolerant species than clayey soils (Fayolle et al. 2012), and disturbance increases the community-weighted mean towards more acquisitive trait values (i.e, with high efficiency of resource use and capture, such as high specific leaf area) to benefit from high light availability (Carreño-Rocabado et al. 2012). In both examples, the changes in biotic conditions caused by abiotic conditions may in turn affect ecosystem processes. 


\section{The need for a comprehensive framework}

The interconnectedness of abiotic and biotic conditions and ecosystem processes in tropical forests indicates that it is difficult to separate the effects of all these variables. Additionally, replicates (often plots) vary in many abiotic and biotic conditions. These variables should be explicitly included in a comprehensive framework if one aims to evaluate their independent and direct vs. indirect effects. For these reasons, in various chapters of my thesis (3-5) I make use of a comprehensive framework including abiotic and biotic conditions that can possibly affect ecosystem processes (Figs. 1.1, 3.1, 4.1 and 5.1). This framework can be analysed using structural equation modelling, which allows for causal testing of multiple levels of variables (i.e, including direct and indirect effects on ecosystem processes, Shipley 2004, Grace 2006).

\section{At what spatial scale?}

The relative importance of all these abiotic and biotic conditions on biomass stocks and dynamics may depend on the spatial scale used in the study (Chisholm et al. 2013, Poorter et al. 2015). For example, as described earlier the importance of climate may be greater at large (e.g. continental) compared to small (e.g. within one forest type) spatial scales because of stronger spatial variation in climate at the larger scale. Throughout my thesis, I use 'large' spatial scale to refer to large geographic areas such as the Amazon or Neotropics (i.e. South and Mesoamerica), and 'small' spatial scale to refer to small geographic areas, such as those used in local studies (e.g. 10-50 km²). Also other abiotic conditions may vary more strongly at large spatial scales than at small spatial scales, and therefore strongly determine variation in biomass stocks and dynamics (Fig. 1.2). This means that across large spatial scales, we may find strong effects of environmental filtering, i.e. abiotic conditions strongly determine and limit the type of species present (e.g. ter Steege and Hammond 2001). Instead, at smaller spatial scales variation in abiotic conditions is smaller, but at this scale biotic interactions within and among species take place (Kunstler et al. 2016), which may result in strong niche complementarity effects. Other biotic effects, such mass-ratio, may also strongly determine biomass stocks and dynamics at small spatial scales (Fig. 1.2).

The expected scale-dependence of mechanisms underlying ecosystem processes asks for an explicit test of these processes at various spatial scales. In this thesis I evaluate the role of abiotic and biotic conditions on biomass stocks and dynamics at various spatial scales: across individual trees (chapter 2), across 0.4-ha communities (chapter 3), across 1-ha communities (chapter 4), across Neotropical forests (chapter 5), and at various spatial scales (chapter 7). 


\section{Climate-vegetation feedbacks}

Besides the climate change mitigation potential of tropical forests, tropical forests are also importantly affected by global climate. Increasing atmospheric $\mathrm{CO}_{2}$ concentrations, increasing temperature, and changing rainfall patterns will pose a challenge to the functioning of forests (Brienen et al. 2015). Whether ecosystem functioning will be maintained depends on whether species can adapt or acclimate to new abiotic conditions, and/or whether species composition can change so that better adapted species become more dominant. The questions are, therefore, how tropical forests respond to changes in abiotic conditions, and how biotic conditions (e.g. the type and diversity of species) contribute to this response capacity.

Evidence is increasing that old-growth tropical forests are not in a stable state but are accumulating biomass (Lewis et al. 2004, Brienen et al. 2015) and are changing in species composition (e.g. Enquist and Enquist 2011, Feeley et al. 2011). Various hypotheses have been proposed to explain these changes, such as $\mathrm{CO}_{2}$ fertilization or nitrogen deposition (Lewis et al. 2004, Wright 2005), but a general consensus is still lacking. A better understanding of temporal changes in forest composition and dynamics and their underlying drivers may be obtained by looking at changes in the CWM traits over time. Changing abiotic conditions should favour some species with specific trait values more than other species, leading to temporal changes in CWM trait values.

Changes in species composition and dynamics have been observed over relatively short timescales (10-30 y) compared to the much longer timescale of turnover of adult trees (200-400 y, Brienen \& Zuidema 2006) and at which climate change takes place. Hence, we have yet no idea of the response of tropical forests to long-term fluctuations and directional changes in climatic and other abiotic conditions. In line with the insurance theory (Yachi and Loreau 1999), several studies in grasslands and temperate forests find that biotic conditions, particularly species and trait diversity, are important for increasing the long-term stability of ecosystem processes (e.g. Hector et al. 2010, Morin et al. 2014). This phenomenon, however, has yet not been demonstrated for tropical forests because, due to their high diversity, high structural complexity, and the long turnover time of most tropical tree species, it is difficult to assess this relationship empirically. Global dynamic vegetation models that include realistic levels of diversity (e.g. Sakschewski et al. 2015) may provide an opportunity to evaluate effects of diversity on the longterm stability of tropical forests. This knowledge is crucial because tropical forests are important for global climate now, and should be so too in the future. 


\section{Questions \& hypotheses}

This thesis is embedded in the EU FP7 (7th Framework Programme for Research of the European Union) project on the 'Role Of Biodiversity In climate change mitigatioN' (ROBIN), which focuses on ecological, socio-economic, and policy aspects of climate change mitigation by tropical forests (www.robinproject.info). This thesis mainly focuses on ecological aspects, and aims to understand how abiotic and biotic conditions determine the biomass stocks and dynamics in tropical forests (Fig. 1.1) across spatial scales (Fig. 1.2) and across temporal scales. The specific questions are:

1. What are the independent relationships between abiotic conditions, biotic conditions, and biomass stocks and dynamics in tropical forests (chapters 2-5 and 7)?

2. How does spatial scale influence these relationships (chapters 2-5 and 7)?

3. How does temporal scale influence these relationships?;

a. How do biotic conditions respond to short-term temporal changes in abiotic conditions (chapter 6)?

b. How do biotic conditions determine the long-term stability of biomass stocks and dynamics (chapters 7 and 8 )?

The hypotheses corresponding to these questions are:

1. In forests that are limited by one or some abiotic conditions, such as low soil fertility and low rainfall, I expect that abiotic conditions will be important for biomass stocks and dynamics (e.g. Laurance et al. 1999). In such forests, strong environmental filtering due to low fertility and/or rainfall restricts the type of species that can perform well (ter Steege and Hammond 2001, Gourlet-Fleury et al. 2011), and therefore under such conditions the trait values of the dominant species (i.e. the community-weighted mean traits) would strongly drive biomass stocks and dynamics. For the effect of species and trait diversity on biomass stocks and dynamics, I have two alternative hypotheses. First, I expect that in forests where water, soil nutrients and/or light are limiting, high species or trait diversity will increase facilitation among species and lead to higher ecosystem process rates. Alternatively, I expect that strong nutrient and/or water limitation can lead to only a small set of species that is well adapted and strongly contributes to ecosystem processes (i.e. strong environmental filtering), which will thus result in a negative effect of species or trait diversity on biomass stocks and dynamics. Vegetation quantity can affect biomass stocks and dynamics in two ways: it can have a positive effect because a dense forest has more biomass that can contribute to growth, or a negative effect because of low light availability in the understorey. 
2. At large spatial scales (e.g. continental scales), I expect that abiotic conditions become more important for biomass stocks and dynamics compared to smaller spatial scales (e.g. within one study site) because stronger gradients in abiotic conditions will increase the effect size and make abiotic effects statistically easier to detect. At smaller spatial scales, I expect that biotic conditions become more important, as at these scale biotic interactions take place.

3. a. At relatively short time scales (10-30 y), I expect that tropical forests are experiencing changes in abiotic conditions, and as a result show compositional changes in community-weighted mean trait values and species composition reflecting the major underlying driver of change (cf. Fauset et al. 2012).

b. At longer time scales, I expect that the response to inter-annual climatic fluctuations (i.e. the stability) depends on the trait diversity within the forest, such that higher trait diversity would lead to more stable biomass productivity, and hence, biomass stocks (cf. Hector et al. 2010).

\section{General research approach}

\section{Methods and analyses}

To answer the main research questions, I combine different research approaches, using empirical data, statistical modelling, and a literature review. I have a strong focus on plant traits to understand growth of individual trees (chapter 2) and ecosystem processes (chapters 3-7). Chapters 3-6 are based on plot dynamics and species composition data from permanent sample plots in four sites (two in Bolivia, one in Brazil, and one in Guyana) managed by three local ROBIN-partners: Instituto Boliviano de Investigación Forestal in Bolivia, Empresa Brasileira de Pesquisa Agropecuária in Brazil, and the Guyana Forestry Commission in Guyana. In collaboration with the local partner institutions, I collected data on leaf and wood traits for the $\sim 80 \%$ most abundant species in each site: 98 species in the dry forest site (INPA) and 158 in the moist forest site (La Chonta) in Bolivia, 68 in the moist evergreen site (Tapajós) in Brazil, and 33 in the moist evergreen site (Pibiri) in Guyana. In chapters 5 and 6, plot and trait data for additional sites were obtained from collaborating researchers. These trait data were scaled to the community level by calculating community-weighted mean trait values and functional diversity indices. To tease apart the various underlying causal drivers of biomass stocks and dynamics, I used structural equation modelling (Shipley 2004, Grace 2006) (chapters 2-5). This is important, because one may overlook or find spurious relationships when not correcting for multiple possible explanatory variables. 
Finally, to assess the generality of relationships between biotic conditions and biomass stocks and dynamics, I perform a quantitative literature review based on studies using empirical, remote sensing, and numerical ecosystem modelling approaches (chapter 7).

\section{Study areas}

All chapters focus on Neotropical forests, mainly the Amazon, because this is the largest remaining tropical forest area that stores a substantial part of the global terrestrial carbon and hosts the majority of tropical tree species (Malhi et al. 2008). The specific chapters, however, are based on different sites or combinations of sites. To address the questions of this thesis, I focus on sites that together cover large abiotic gradients, ranging from nutrient poor to fertile soils, and that cover many tropical forest types across the Amazon, ranging from dry deciduous to wet evergreen (Table 1.1).

Table 1.1: Details of the five forest sites used in most of the chapters in this thesis: INPA, La Chonta, Tapajós, Pibiri, and Corinto. Additional sites used for chapter 5 can be found in Appendix 5.1.

\begin{tabular}{|c|c|c|c|c|c|}
\hline & INPA & La Chonta & Tapajós & Pibiri & Corinto \\
\hline Site used in chapters & 5,6 & $2,4,5,6$ & 5,6 & $3,5,6$ & 5,6 \\
\hline Coordinates & $\begin{array}{l}16^{\circ} 07^{\prime} \mathrm{S}, \\
61^{\circ} 43^{\prime} \mathrm{W}\end{array}$ & $\begin{array}{l}15^{\circ} 47^{\prime} \mathrm{S}, \\
62^{\circ} 55^{\prime} \mathrm{W}\end{array}$ & $\begin{array}{l}3^{\circ} 19^{\prime} \mathrm{S}, \\
54^{\circ} 57^{\prime} \mathrm{W}\end{array}$ & $\begin{array}{l}5^{\circ} 13^{\prime} \mathrm{N}, \\
58^{\circ} 38^{\prime} \mathrm{W}\end{array}$ & $\begin{array}{l}10^{\circ} 12^{\prime} \mathrm{N}, \\
83^{\circ} 52^{\prime} \mathrm{W}\end{array}$ \\
\hline Country & Bolivia & Bolivia & Brazil & Guyana & Costa Rica \\
\hline Forest type & $\begin{array}{l}\text { Dry } \\
\text { deciduous }\end{array}$ & $\begin{array}{l}\text { Moist semi- } \\
\text { deciduous }\end{array}$ & $\begin{array}{l}\text { Moist } \\
\text { evergreen }\end{array}$ & $\begin{array}{l}\text { Rainforest } \\
\text { (ch. 3), moist } \\
\text { evergreen (ch. 6) }\end{array}$ & $\begin{array}{l}\text { Wet } \\
\text { evergreen }\end{array}$ \\
\hline Rainfall $\left(\mathrm{mm} \mathrm{y}^{-1}\right)$ & 1160 & 1580 & 2110 & 2772 & 3900 \\
\hline $\begin{array}{l}\text { Number of dry } \\
\text { months }<100 \mathrm{~mm} \\
\text { rainfall }\end{array}$ & 7 & 6 & 3 & 0 & 0 \\
\hline $\begin{array}{l}\text { Average annual } \\
\text { temperature }\left({ }^{\circ} \mathrm{C}\right)\end{array}$ & 24.3 & 24.3 & 25 & 25.9 & 23.7 \\
\hline Soil type & Oxisols & Ultisols & Oxisols & Ferralsols & Inceptisols \\
\hline $\begin{array}{l}\text { Soil fertility from } \\
\text { highest (1) to lowest } \\
\text { (5), based on Fig. } 2 \text { of } \\
\text { Quesada et al. (2010). }\end{array}$ & $\begin{array}{l}2 \text { (middle- } \\
\text { high) }\end{array}$ & 1 (high) & $\begin{array}{l}3 \text { (middle- } \\
\text { low) }\end{array}$ & 4 (low) & n.a. \\
\hline
\end{tabular}

For chapters 2 and 4, we $^{3}$ use data from a moist semi-deciduous forest (1580 $\mathrm{mm}$ annual rainfall) with fertile soils in Bolivia (La Chonta). Chapter 3, on the other hand, is based on data of a wet forest $(2772 \mathrm{~mm})$ with very poor soils in Guyana

\footnotetext{
3 "we" is used when referring to research chapters in which co-authors are involved, and "I" for general thesis information in the general introduction and discussion (chapters 1 and 8).
} 
(Pibiri). In chapter 5, we use 26 forests across the Neotropics, with annual rainfall ranging between 784 - $3991 \mathrm{~mm}$ and covering many different soil types (see map in Fig. 1.2). Chapter 6 is based on five Neotropical forests (Inpa, La Chonta, Tapajós, Pibiri and Corinto), with annual rainfall ranging between $1160-3900 \mathrm{~mm}$ and with strong differences in soil fertility (see Table 1.1). Most of the sites are in forest management units and received logging treatments, which are used in the analyses of some of the chapters (3,4 and 5). More information of the five sites used in chapters 2, 3, 4, 6 and 7 can be found in Table 1.1, and of additional sites used in chapter 5 in Appendix 5.1.

\section{Thesis outline}

This thesis consists of eight chapters: the general introduction (this chapter), six research chapters (chapters 2-7) ordered from small to large spatial scale (2-5) and from short to long temporal scale (6-7), and the general discussion (chapter 8). We first focus on the individual tree-scale (chapter 2), then we scale up individual tree responses to the community level at the local scale within a forest (chapters 3 and 4 ), and finally assess site differences at the continental scale (chapter 5). We then look at short-term temporal dynamics of these communities (10-30 y, chapter 6). Finally, we review the generality of the relationships between biotic conditions and biomass stocks and dynamics (chapter 7), including the long-term ( $>200 \mathrm{y})$ temporal dynamics of these communities (see also the general discussion in chapter 8).

Biomass dynamics in tropical forests are most strongly determined by canopy trees that store most of the biomass (Slik et al. 2013) and have highest absolute biomass growth rates (Stephenson et al. 2014). However, factors driving differences in biomass growth among such large trees remain largely unknown. In chapter 2 we use a set of traits at the individual-tree level to explain absolute biomass growth of large trees.

At the community level, different variables may explain biomass stocks and dynamics than at the individual-tree level. We focus on two forests at the extremes in the Amazon basin, to evaluate whether similar mechanisms apply: Guyana and Bolivia (see the map in Fig. 1.2). In chapter 3 we evaluate how abiotic and biotic conditions drive biomass stocks (aboveground, fine root, and soil organic matter) and productivity of 0.4-ha plots in a wet forest in Guyana that grows on the very nutrient-poor Guiana shield.

In chapter 4 we assess how abiotic and biotic conditions drive three demographic processes that underlie net biomass change: biomass growth by trees that recruit, biomass growth by trees that survive, and biomass loss due to mortality 
in a moist semi-deciduous forest in Bolivia that grows on very fertile soils. Although net biomass change may be the most relevant variable for globally important ecosystem functions such as $\mathrm{CO}_{2}$ sequestration, it may be hard to predict because it is the final product of demographic processes (recruitment, growth and mortality). It is, therefore, important to tease apart net biomass change into its underlying demographic processes. In both chapters (3 and 4), we use structural equation modelling to answer our questions.

In chapters 3 and 4 we focus on the local scale. But how are biomass stocks and dynamics predicted by abiotic and biotic conditions at the continental scale? In chapter 5 we test the effects of abiotic and biotic conditions on the demographic processes that underlie net biomass change across 26 Neotropical forests that cover a large biogeographical range and climatic range (780-3990 $\mathrm{mm}$ annual rainfall), using a similar approach as in chapters 3 and 4.

The first four research chapters (2-5) are based on current ecosystem processes in tropical forests. Global change, however, is putting pressure on ecosystems, and it is yet not understood how ecosystems will change and what the main underlying global change drivers are. In chapter 6 we evaluate how five oldgrowth Neotropical forests are changing over 10-30 y in species and trait composition, and what is most likely the major underlying driver: increasing resource availability, increasing drought-stress, or recovery from disturbances.

Chapters 2-6 disentangle abiotic and biotic effects on ecosystem processes at various spatial and temporal scales. Yet, these chapters and other studies differ in many aspects, such as study site, and variables and analytical framework used. It remains therefore difficult to obtain a general idea of the abiotic and biotic effects on ecosystem processes. In chapter 7 we review the relationships between biotic conditions (called 'biodiversity attributes' in chapter 7) and biomass stocks and dynamics (called 'carbon stocks and dynamics'), focusing on results obtained from empirical, remote sensing, and ecosystem modelling studies.

Finally, I use the general discussion, chapter $\boldsymbol{8}$, to provide answers to the main research questions by synthesizing the results of the individual research chapters and presenting some additional analyses. Furthermore, I discuss the main scientific knowledge gaps and challenges regarding the understanding of the functioning of tropical forests. Last, I discuss the societal and political challenges to get towards long-term resilient and viable tropical forests that play a crucial role in important functions such as global climate change mitigation, water cycling and wood provisioning. 

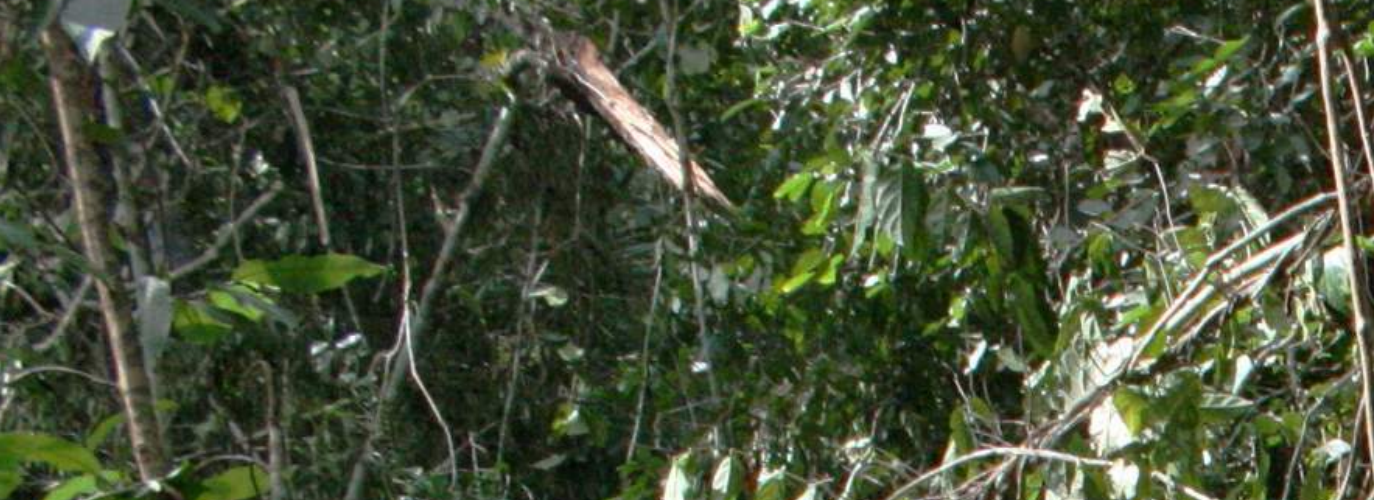

1.2.

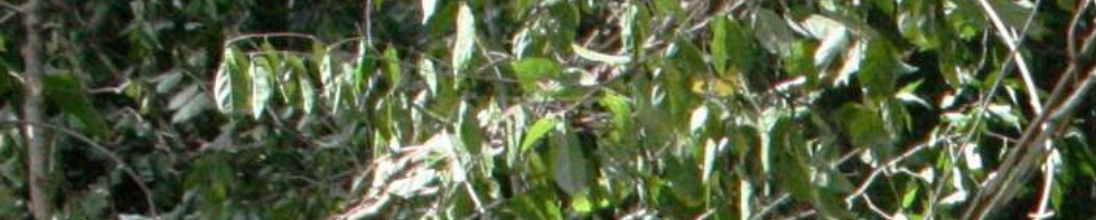

2. in

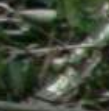

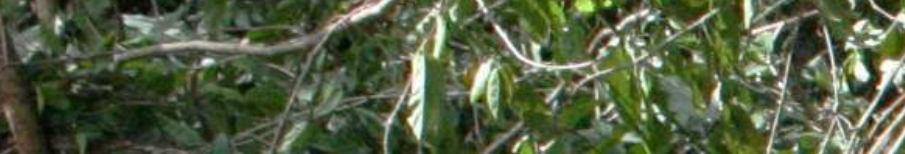

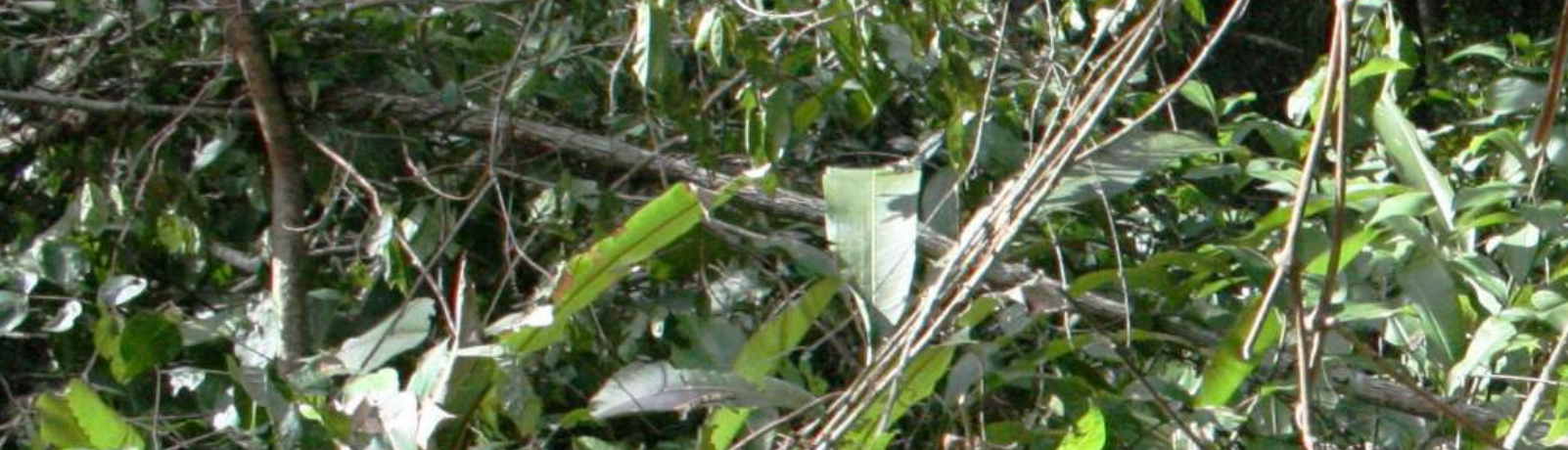

and 20 35120 ?

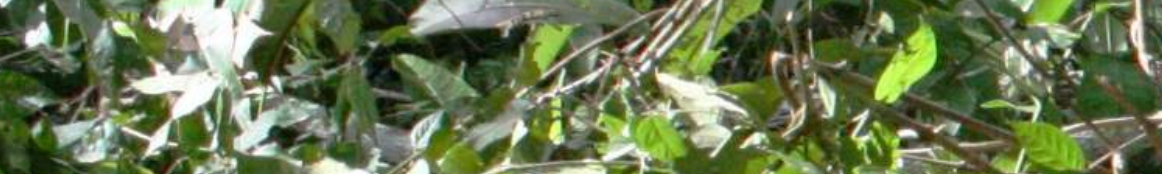
2.

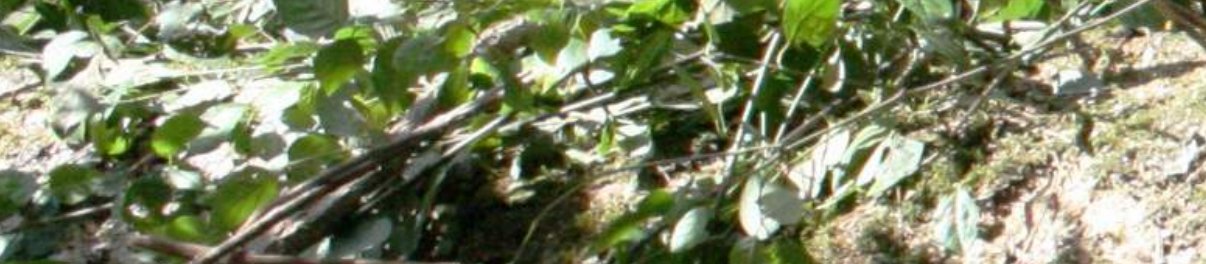
(a)

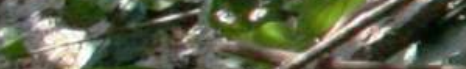
T. $+510$ (f) . at

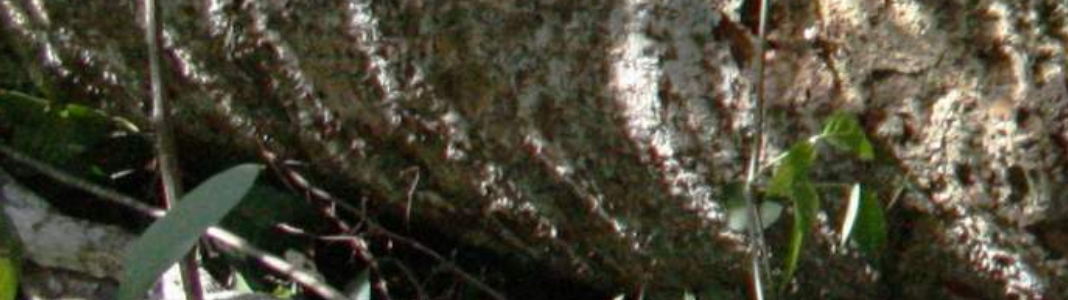

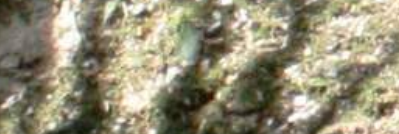

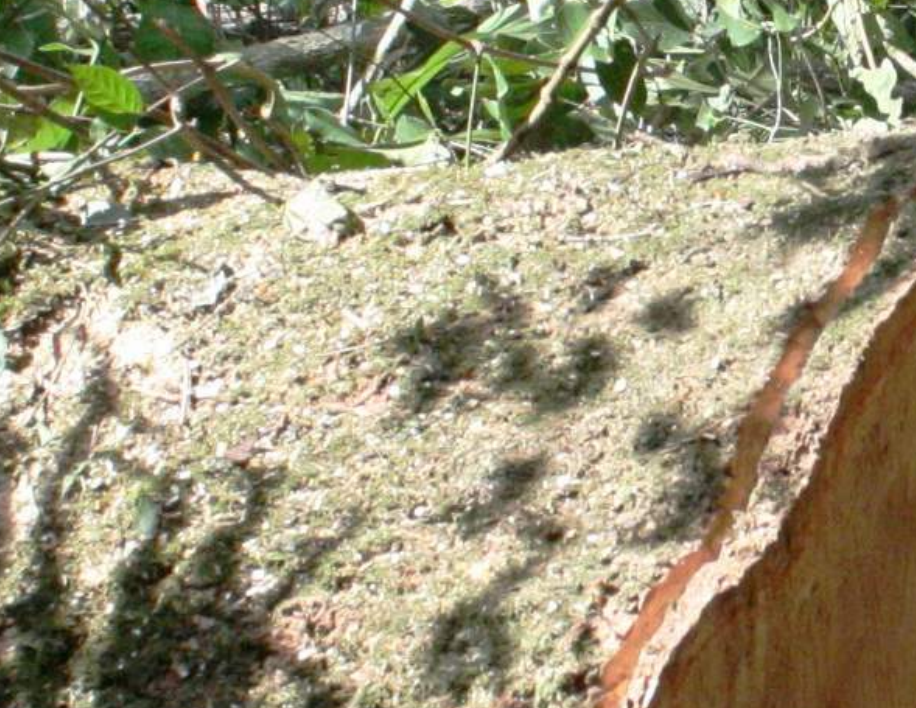

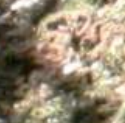

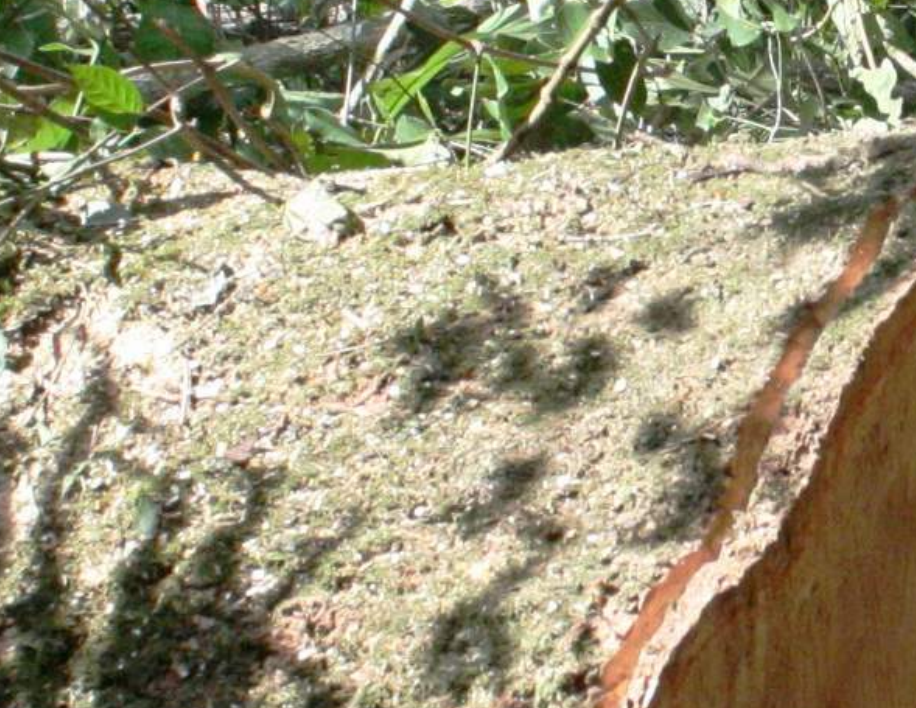

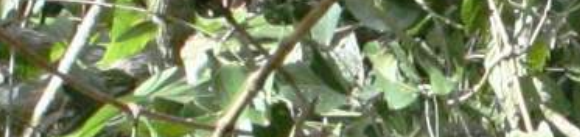

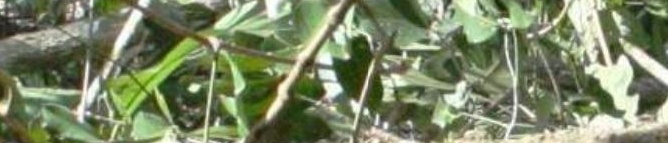
$\frac{1}{7-5}=$

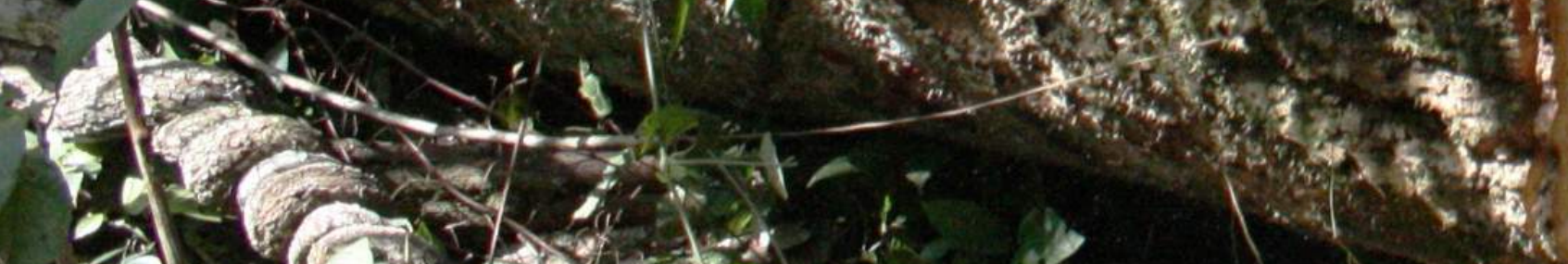

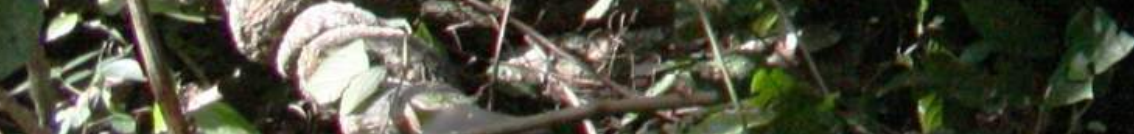

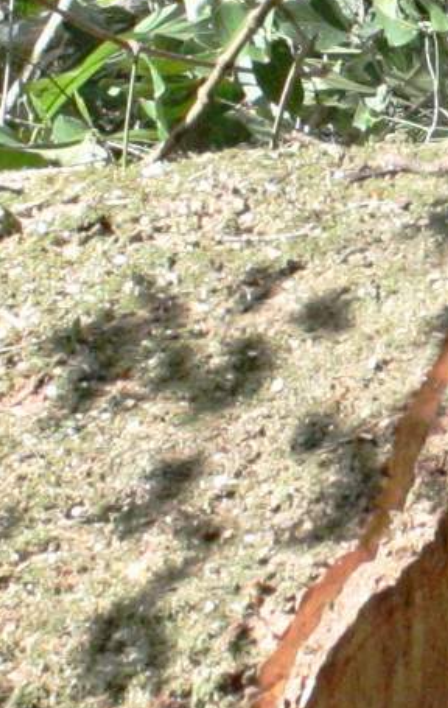

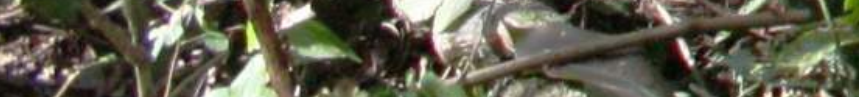

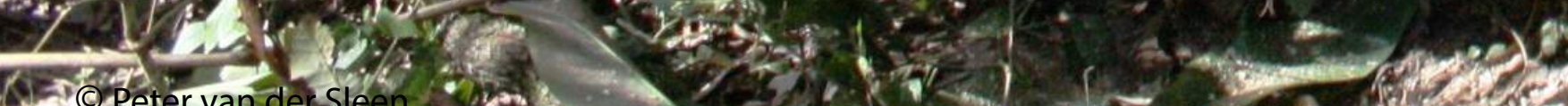
a. 3. $202 \sin 4$ ${ }^{2} y_{2}$ (

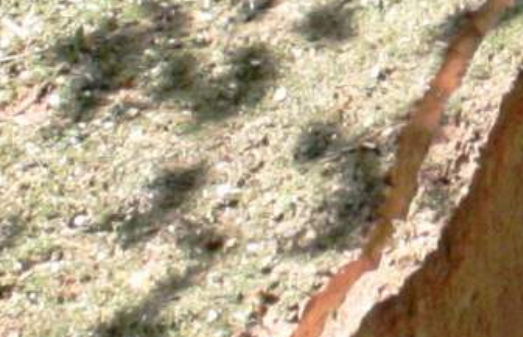
5.

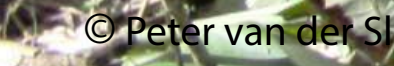




\section{Abstract}

Tropical forests are important in worldwide carbon (C) storage and sequestration. $\mathrm{C}$ sequestration of these forests may especially be determined by the growth of canopy trees. However, the factors driving variation in growth among such large individuals remain largely unclear. We evaluate how crown traits (total leaf area, specific leaf area and leaf nitrogen $(\mathrm{N})$ concentration) and stem traits (sapwood area (SA) and sapwood $\mathrm{N}$ concentration) measured for individual trees affect absolute biomass growth for 43 tropical canopy trees belonging to four species, in a moist forest in Bolivia. Biomass growth varied strongly among trees, between 17.3 and $367.3 \mathrm{~kg}$ year $^{-1}$, with an average of $105.4 \mathrm{~kg} \mathrm{year}^{-1}$. We found that variation in biomass growth was chiefly explained by a positive effect of SA, and not by tree size or other traits examined. SA itself was positively associated with sapwood growth, sapwood lifespan and basal area. We speculate that SA positively affects the growth of individual trees mainly by increasing water storage, thus securing water supply to the crown. These positive roles of sapwood on growth apparently offset the increased respiration costs incurred by more sapwood. This is one of the first individual-based studies to show that variation in sapwood traits and not crown traits - explains variation in growth among tropical canopy trees. Accurate predictions of $\mathrm{C}$ dynamics in tropical forests require similar studies on biomass growth of individual trees as well as studies evaluating the dual effect of sapwood (water provision vs. respiratory costs) on tropical tree growth.

Keywords: Bolivia, carbon economy, functional traits, sapwood area, sapwood turnover, stem growth, total leaf area, tropical forest, water relations 


\section{Introduction}

Tropical forests cover about $10 \%$ of the Earth surface, but store $25 \%$ of global terrestrial carbon and account for $34 \%$ of terrestrial gross primary productivity (Bonan 2008, Lewis et al. 2009, Malhi 2012). They therefore feature prominently in climate change mitigation policies, such as Reduced Emissions from Deforestation and forest Degradation (REDD+) (Houghton 2005, Bonan 2008). In these forests, the $2 \%$ largest stems account for at least $27 \%$ of the aboveground biomass (Clark and Clark 1996, Lindenmayer et al. 2012, Slik et al. 2013). Since absolute biomass growth often increases with tree size (Clark and Clark 1999, Stephenson et al. 2014), the growth of large canopy individuals may largely determine the total aboveground carbon sequestration per ground area. Although several studies have evaluated the effect of environmental conditions and functional traits on diameter growth rates (Hérault et al. 2011), or on growth for small trees and saplings (Poorter 1999, Sterck et al. 2003), the understanding of what drives the biomass growth of individual canopy trees is still very poor.

The growth of a tree is affected by its ontogenetic stage, biotic and abiotic environment, and functional traits. Most studies, however, do not consider the direct relation between biomass growth and factors driving this at the individualtree level, but rather focus on average species performance and average species traits (e.g. Poorter and Bongers 2006, Wright et al. 2010). Yet, as Clark et al. (2011) pointed out, "individuals are the objects responding to environmental gradients, not species". Species-specific performance of canopy trees may be partly driven by species-specific life-history traits that allow them to endure in the understory and eventually reach the canopy. Still, variation among individuals may be substantial (Paine et al. 2011, Thomas et al. 2013) and important for their ecological performance (Violle et al. 2007) and contributions to population growth (Zuidema et al. 2009). Hence, individual-tree level analyses may yield important insights into the drivers of tree growth (Binkley et al. 2010, Clark et al. 2011, Sterck and Schieving 2011).

Functional traits are expected to link environmental conditions to growth, and may therefore assist in developing a mechanistic understanding of factors that drive tree growth (McGill et al. 2006, Ordoñez et al. 2009). Many studies have highlighted the importance of leaf traits such as the positive effect of specific leaf area (SLA) and leaf nitrogen $\left(\mathrm{N}_{\text {leaf }}\right)$ on growth of saplings and small trees (Wright et al. 2005, Poorter and Bongers 2006, Sterck et al. 2006). However, these relationships are generally weak for large trees, possibly because size-related traits such as total leaf area (TLA) may determine absolute tree growth more strongly 
than leaf traits (Poorter et al. 2008, Wright et al. 2010). In addition, stem traits also potentially affect whole-tree growth (Chave et al. 2009). An important stem-related trait is the sapwood area of a tree, which may indirectly increase photosynthesis rates by sustaining water transport to the leaves (Meinzer et al. 2008). However, extra sapwood area may also incur additional maintenance respiration costs (see Meir and Grace (2002) for positive effect of stem diameter on respiration), counterbalancing the positive water-related effect on growth (Wullschleger et al. 1998). So far, the contributions of size- and tissue-related stem and crown traits on individual growth of tropical canopy trees is poorly understood.

In this study we evaluated the relative effect of various size- and tissue-related stem and crown traits on biomass growth of 43 tropical canopy trees belonging to four species. Specifically, we ask the question to what extent variation in biomass growth across individual canopy trees can be explained by crown and stem traits. We expected a positive relation between biomass growth and crown traits: TLA increases total light capture, a higher SLA increases the leaf area per unit biomass investment, and a higher $\mathrm{N}_{\text {leaf }}$ may increase the photosynthetic capacity (Poorter and Bongers 2006, Reich 2012). Furthermore, we expected that the sapwood nitrogen concentration $\left(\mathrm{N}_{\text {sapw }}\right)$ would negatively affect growth, because high levels of nitrogen in wood would increase respiration. We did not have an a priori hypothesis about the relation between sapwood area (SA) and tree growth, since the possible positive effects by augmenting water transport and storage might be offset by the negative effects of greater respiration loads.

\section{Methods}

\section{Research site}

This study was conducted in the moist, semi-deciduous forest of La Chonta, Bolivia $\left(15^{\circ} 47^{\prime} \mathrm{S}, 62^{\circ} 55^{\prime} \mathrm{W}\right)$. This is a 100,000 ha forestry concession that was established in 1974, with an average density of 367 trees per ha $(>10 \mathrm{~cm} \mathrm{DBH})$ and a species richness of about 59 per ha (Peña-Claros et al. 2008). The average canopy height is $25 \mathrm{~m}$, and most canopy trees have an estimated age of at least 150 years (Poorter and Bongers 2006, Rozendaal and Zuidema 2011). Average annual temperature is $24.3^{\circ} \mathrm{C}$ and annual precipitation is $1520 \mathrm{~mm}$, with a dry season from April until September. 


\section{Tree selection}

From early April until early June 2012, 43 emergent canopy trees were measured from four species representing different families and ecological growing strategies (Table 2.1): 15 individuals of Hura crepitans, 11 of Schizolobium parabyba, 9 of Cariniana ianeirensis and 8 of Sweetia fruticosa. Hereafter, these species will be referred to by their genus name. Moreover, these species were selected because they were known to produce well distinguishable annual growth rings (Lopez et al. 2012). We selected trees with undamaged and fully exposed crowns and no or little liana cover. This ensured that growth differences among study trees were not strongly determined by differences in light availability. All measurements were conducted within hours after the selected trees were felled.

Table 2.1: The four species used in this study with family, guild, maximum tree height, average crown exposure index as juvenile ( $\mathrm{CE}_{\text {juv }}$; value between 1-5 indicating increasing access to direct light), and average wood density $\left(\mathrm{g} \mathrm{cm}^{-3}\right)$ at breast height. Long lived pioneers (LLP) are long lived species that need high irradiance to establish, and partial shade tolerant trees (PST) are species that can establish under low irradiance. Wood density data are obtained from this study, but Guild, Maximum height and $\mathrm{CE}_{\text {juv }}$ are obtained from Poorter et al. (2006).

\begin{tabular}{llllll}
\hline & Family & Gaximum & & Wood \\
Species & height & CE $_{\text {juv }}$ & density \\
\hline Schizolobium parahyba & Fabaceae/ Caesalpiniaceae & LLP & 35 & 2.39 & 0.45 \\
Sweetia fruticosa & Fabaceae/ Papillionaceae & LLP & 30 & 1.91 & 0.82 \\
Hura crepitans & Euphorbiaceae & PST & 44 & 1.62 & 0.37 \\
Cariniana ianeirensis & Lecythidaceae & PST & 45 & 1.74 & 0.36 \\
\hline
\end{tabular}

\section{Biomass growth}

Directly following felling, we cut two stem discs using a chain saw. One disc was obtained at about $1 \mathrm{~m}$ from the stem base and one just below the first major branch (between 6-17 $\mathrm{m}$ from the stem base). Bark thickness of the discs was measured in four directions, and the distance from the soil to the first disc and from the soil to the second disc were measured using a measuring tape. The discs were brought to the laboratory where they were polished to identify ring boundaries. On these discs, the radial length of the heartwood, sapwood and pith diameter were measured at the longest radius, the shortest radius, and one intermediate radius, using a caliper and a ruler. In all species except Cariniana, the distinction between sapwood and heartwood was clear, with abrupt switches in contrasting colours. For Cariniana sapwood area could therefore not be measured. 
Per disc, ring width of the last five years was measured at the longest and shortest radius (using the pith as center) and at one intermediate radius between the longest and shortest, since the discs were never fully a circle with the pith exactly in the center. We measured ring width using the TSAP-Win 0.53 software. The measurements of the three radii and of the five years were averaged to obtain one value for annual ring width per tree. We based our growth estimates on an average of the last five years, to minimize the effect of climatic variability on the growth estimates. Based on this average annual ring width and the diameter of the disc, the annual basal area growth was calculated.

At the same two heights per tree, $3-4 \mathrm{~cm}$ wide sections were cut in radial direction, from the bark to the pith. The bark was removed and the section was cut in radial direction in sections of $6 \mathrm{~cm}$, starting from the youngest sapwood until the pith was included. For each sample, fresh volume was determined using the water displacement method, and dry mass was measured after oven drying at $70{ }^{\circ} \mathrm{C}$ until dry mass was stabilized. Wood density (WD; $\mathrm{g} \mathrm{cm}^{-3}$ ) was calculated per wood sample by dividing the dry mass by the fresh volume.

In Appendix 2.1 we show that, for our trees, taper only occurred between breast height and the first branch (i.e. along the main stem). We therefore calculated biomass growth separately for the stem (until the first branch) and crown. First, WD of the youngest sapwood was multiplied with the annual basal area growth of the same disc to get a measure for the annual biomass growth per unit tree height $\left(\mathrm{kg} \mathrm{m}^{-1} \mathrm{yr}^{-1}\right)$, which could later be multiplied with height (separately for the stem and crown, as explained below) to obtain total biomass growth. To determine stem biomass growth, we assumed that the averaged biomass growth of the two disc samples was a good representation of the average biomass growth along the whole length of the stem. Averaged biomass growth of the disc samples was subsequently multiplied with stem height to obtain an estimate of absolute stem biomass growth $\left(\mathrm{kg} \mathrm{yr}^{-1}\right)$. To determine growth of woody biomass in the crown, we assumed that the biomass growth of the disc below the first branch was a good representation of the biomass growth of the whole crown. This biomass growth was multiplied with the length of the crown (maximum tree height minus stem height), measured with a laser rangefinder (Nikon Forestry 550), to obtain crown biomass growth $\left(\mathrm{kg} \mathrm{yr}^{-1}\right)$. Note that we did not include leaf mass, as this strongly correlates with the total leaf area, which we used as one of the explanatory variables. Stem and crown biomass growth were subsequently summed to obtain an estimate of absolute aboveground biomass growth rate (AGR; $\mathrm{kg} \mathrm{yr}^{-1}$; Table 2.2). We chose this approach to calculate biomass rather than the more generally used allometric biomass equations, because it accounts for possible species-specific 
tapering within trunk and crown. As such, it likely provides a more direct and more reliable estimate of biomass than one based on generic biomass equations that are commonly used. We do acknowledge, though, that this is still an estimate of biomass (growth), which could be further refined, for example by using more detailed information on trunk tapering or wood density variation along the stem.

Table 2.2: List of variables with abbreviation, units, mean, minimum (Min), maximum (Max), standard deviation (Stdev) and coefficient of variation (CV).

\begin{tabular}{|c|c|c|c|c|c|c|c|}
\hline Abbreviation & Variable description & Unit & Mean & Min & $\operatorname{Max}$ & Stdev & $\mathrm{CV}$ \\
\hline AGR & $\begin{array}{l}\text { Absolute biomass } \\
\text { growth rate }\end{array}$ & $\mathrm{kg} \mathrm{yr}^{-1}$ & 105.43 & 17.32 & 367.3 & 80.68 & 0.77 \\
\hline Height & $\begin{array}{l}\text { Tree height until top of } \\
\text { crown }\end{array}$ & $\mathrm{m}$ & 26.22 & 21.6 & 32.4 & 3.03 & 0.12 \\
\hline TLA & $\begin{array}{l}\text { Total leaf area of the } \\
\text { crown }\end{array}$ & $\mathrm{m}^{2}$ & 1339.73 & 293.96 & 3641 & 759.23 & 0.57 \\
\hline SA & Sapwood area & $\mathrm{m}^{2}$ & 0.172 & 0.029 & 0.577 & 0.119 & 0.69 \\
\hline SLA & Specific leaf area & $\mathrm{cm}^{2} \mathrm{~g}^{-1}$ & 105.65 & 72.6 & 149.7 & 17.76 & 0.17 \\
\hline $\mathrm{N}_{\text {leaf }}$ & $\begin{array}{l}\text { Leaf nitrogen } \\
\text { concentration }\end{array}$ & $\%$ & 2.56 & 1.82 & 3.42 & 0.43 & 0.17 \\
\hline $\mathrm{N}_{\text {sapw }}$ & $\begin{array}{l}\text { Sapwood nitrogen } \\
\text { concentration }\end{array}$ & $\%$ & 0.25 & 0.11 & 0.47 & 0.09 & 0.36 \\
\hline $\mathrm{BA}$ & Stem basal area & $\mathrm{m}^{2}$ & 0.331 & 0.096 & 0.838 & 0.183 & 0.55 \\
\hline $\begin{array}{l}\text { Sapwood } \\
\text { lifespan }\end{array}$ & Age of the sapwood & $\mathrm{yr}$ & 29.78 & 5.75 & 88.64 & 21.77 & 0.73 \\
\hline $\begin{array}{l}\text { Sapwood } \\
\text { growth }\end{array}$ & $\begin{array}{l}\text { Basal area growth of } \\
\text { one year }\end{array}$ & $\mathrm{cm}^{2} \mathrm{yr}^{-1}$ & 101.37 & 12.05 & 332 & 76.54 & 0.76 \\
\hline
\end{tabular}

\section{Total leaf area}

Per tree, we selected four to five undamaged branches that had a stem diameter of 4-8 cm and were growing in different parts of the crown. For each branch, all the apices with leaf-bearing shoots were counted. Then, for five randomly selected apices, the number of leaves was counted and one leaf was randomly selected and harvested. We thus obtained 20-25 leaves per tree. We pooled these leaves to measure the average leaf area (without petioles), using a desktop scanner. At the lower end of each branch, a disc was cut from which BA excluding bark was determined.

Per branch, the TLA was calculated by multiplying the number of shoots, the average number of leaves per shoot, and the average leaf area (obtained at the tree 
level). The ratio of cross-sectional BA to leaf area was determined per branch and averaged over four to five branches to obtain one value per tree.

To estimate TLA $\left(\mathrm{m}^{2}\right)$, we assumed that the stem BA just below the first branch is proportional to its supporting leaf area. We tested this assumption by comparing the ratio of leaf area to BA at four sampling heights in the tree (see Appendix 2.1 for details): breast height (1), just below the first branch (2), and at two heights in the crown below the lowest leaves (3 and 4). BA just below the first branch did not differ from the two upper sampling heights, supporting our assumption of a constant ratio between BA and leaf area just below the first branch and in the crown (see Appendix 2.1). Therefore, we calculated TLA by dividing BA just below the first branch by the ratio BA : leaf area calculated from the branches of the same individual.

\section{Other traits}

Per tree, the leaf area of the $20-25$ pooled leaves was divided by their pooled dry mass (oven-dried at $70{ }^{\circ} \mathrm{C}$ until their mass was stabilized) to determine specific leaf area (SLA; $\left.\mathrm{cm}^{2} \mathrm{~g}^{-1}\right)$, and leaf samples per tree were analyzed for nitrogen concentration $\left(\mathrm{N}_{\text {leaf }} ; \%\right.$, Table 2.2). The youngest wood samples at the two heights along the stem were pooled per tree and analyzed for nitrogen concentration $\left(\mathrm{N}_{\text {sapw }}\right.$; $\%)$. Sapwood area $\left(\mathrm{SA} ; \mathrm{m}^{2}\right)$ per disc was determined by subtracting the heartwood and pith area from the total stem basal area. SA per tree was calculated as the average SA of the discs taken at the two heights. Sapwood growth was defined as the annual basal area growth (see Biomass growth), and sapwood lifespan was based on the number of annual rings in the sapwood. We estimated the number of annual rings in the sapwood by dividing the width of the sapwood by the average ring width of the last 15 years.

\section{Statistical analyses}

For Cariniana we could not distinguish sapwood from heartwood on the disc samples, so sapwood area (SA) could not be measured. We carried out two sets of statistical analyses: one without Cariniana and one that included Cariniana, in which SA for Cariniana was predicted based on a regression analysis of SA versus all traits and basal area of the other three species. These two approaches yielded similar results in terms of strength, direction and significance of coefficients of variables included in tests explaining variation in absolute biomass growth (Appendix 2.2). As including estimated SA values for Cariniana did not affect results, we present results of tests including Cariniana in the main text. 
Our main aim was to evaluate how traits of individual trees could explain variation in their growth, and not the mean effect of species per se. To account for variation in growth that is explained by species differences, we included species as a fixed factor in the analyses. Growth, basal area (BA) and SA were log-transformed and TLA was sqrt-transformed to meet the assumptions of equal variances and a normal distribution of the residuals. Possible interactions between species and each of the traits were first checked and included in further analyses if significant. Possible outlying observations were analyzed by applying the Cook's Distance to the linear models.

The model including all traits, species, and interactions was reduced using 'all subsets regression analysis', which evaluates all possible combinations of predictor variables (Burnham and Anderson 2002). We used this technique because various combinations of variables in multiple regression models can give comparable good fits (Burnham and Anderson 2002, Johnson and Omland 2004). We therefore selected and averaged the models that differed less than 2 AIC units from the model that was selected as 'best'. In this way, we obtained rather conservative but more robust model coefficients compared to what we would have obtained by selecting only the best model.

All analyses were performed using R 2.15.2. We used the following functions: $\mathrm{lm}$ for linear models, dredge for all subsets regression analysis, and model.avg for averaging regression models (the latter two from the MuMIn package; Barton 2015).

\section{Results}

The aboveground absolute growth rate (hereafter referred to as 'growth') ranged widely, between 17.32-367.25 $\mathrm{kg} \mathrm{yr}^{-1}$ with an average of $105.43 \mathrm{~kg} \mathrm{yr}^{-1}$ (Table 2.2). Many variables differed strongly among individuals and species, which can be seen from their high coefficient of variation. The averaged model, which included all variables, shows that only sapwood area (SA) had a significant positive effect on growth (standardized coefficient $=0.73$ ) and species differed in their intercept (Table 2.3, Fig. 2.1). The relative importance of SA and species on growth was both 1 and there were no significant interaction effects (species * traits). After SA, TLA had the strongest standardized coefficient, followed by SLA, $\mathrm{N}_{\text {sapw }}, \mathrm{N}_{\text {leaf }}$ and height $(0.17,-0.16,0.13,-0.12$, and 0.11 , respectively). The presented averaged model reflects the average of the five best-fitting models that differed less than 2 AIC units from the single best model. 
We evaluated the robustness of our results by adding a number of analyses, of which results are included in the appendices (2, 4, 5 and 6). First of all, we evaluated the results with different proxies for tree size, i.e. tree height or basal area. Results of statistical analyses showed that sapwood area and species were the most significant predictor variables, irrespective of the tree size proxy used (Appendix 2.2a vs. Table 2.3). We continued using tree height as size proxy, since this correlated more weakly than basal area with most of the other predictor variables of growth $(\mathrm{r}<0.6$ for tree height, Appendix 2.3, and $\mathrm{r}<0.86$ for basal area), suggesting that the effects of tree height on growth were independent of impacts by other crown or stem trait. Second, for sake of comparison, we present the analysis of the effect of traits on basal area growth in Appendix 2.4 (vs. analysis for absolute biomass growth in Table 2.3), which showed that traits similarly affect both growth measures. We further focused on biomass growth and not basal area growth or stem diameter growth, as biomass growth is most relevant for carbon sequestration. Third, in addition to our all subset regression analysis and model averaging, we added an analysis for biomass growth using the standard stepwise exclusion of variables, and showed that sapwood area and species were the most significant predictor variables in both analyses (Appendix 2.5 vs. Table 2.3). Last, we performed an analysis using a reduced model, in order to evaluate results for a pre-selected limited set of variables. The model in which only tree height, total leaf area and sapwood area were included as explanatory variables again confirmed that sapwood area and species were the only variables explaining variation in biomass growth (Appendix 2.6 vs. Table 2.3). The results of the analysis presented in Table 2.3 are thus in line with a number of alternative analyses presented in appendices $(2,4,5$, and 6$)$.

Because SA was the most important explanatory variable for growth, we elaborated further on factors that may explain variation in SA. We evaluated how SA depends on sapwood area growth, sapwood lifespan and stem basal area. In this analysis, sapwood area growth, i.e. newly formed sapwood area per year, ranged between 12.05-332.00 $\mathrm{cm}^{2} \mathrm{yr}^{-1}$ with an average of $101.37 \mathrm{~cm}^{2} \mathrm{yr}^{-1}$, sapwood lifespan ranged between 5.7-88.6 $\mathrm{yr}$ with an average of $29.78 \mathrm{yr}$, and basal area ranged between $0.10-0.83 \mathrm{~m}^{2}$ with an average of $0.33 \mathrm{~m}^{2}$ (Table 2.2). We included species as fixed factor (species did not interact with other predictor variables), and scaled all numeric variables by subtracting the mean and dividing by the standard deviation, to obtain standardized coefficients. The results showed that sapwood growth, sapwood lifespan, and stem basal area all positively affected SA, with standardized coefficients of $0.45,0.18$, and 0.22 , respectively (Table 2.3, Fig. 2.2). 


\section{Discussion}

Our aim was to explain variation in absolute biomass growth (referred to as 'growth') among individual tropical canopy trees by stem and crown traits. From all traits, sapwood area (SA) turned out to be the only variable that significantly increased with growth (Table 2.3, Fig. 2.1). Growth was not affected by tree height or basal area, indicating that size does not drive differences in growth among canopy trees. Further evaluation of factors explaining variation in SA across trees showed a positive effect of sapwood growth, sapwood lifespan and tree basal area on SA (Table 2.3, Fig. 2.2).

Table 2.3: Results from the two linear models with absolute growth rate (Growth) and SA as response variables. The standardized coefficient $(\beta)$, adjusted SE (SEadj), t-value, P-value, and relative variable importance (by summing the Akaike weights for all models where the specific variable was included (Barton 2015)) are given for each predictor variable. The effects on growth were evaluated by all subset regression analyses and subsequent averaging of the five models with Akaike information criteria values that differed by less than 2 units, therefore relative variable importance values could be obtained. The statistics of SA, however, were based on the full model (hence, no model averaging was applied and thus no relative variable importance values were calculated), based on variables scaled by subtracting the mean and dividing by the SD. Note that Cariniana was excluded from the analysis for SA.

\begin{tabular}{lllllll}
\hline $\begin{array}{l}\text { Response } \\
\text { variable }\end{array}$ & Predictor variable & $\boldsymbol{\beta}$ & SEadj & t-value & P-value & $\begin{array}{l}\text { Relative } \\
\text { importance }\end{array}$ \\
\hline Growth & $\log (\mathrm{SA})$ & 0.73 & 0.15 & 4.68 & $<0.001$ & 1 \\
& Intercept Sweetia & 0 & 0 & & & 1 \\
& Intercept Hura & -0.28 & 0.19 & 1.41 & 0.158 & \\
& Intercept Schirolobium & 0.56 & 0.14 & 3.93 & $<0.001$ & \\
& Intercept Cariniana & 0.07 & 0.12 & 0.56 & 0.574 & \\
& SLA & -0.16 & 0.10 & 1.60 & 0.111 & 0.56 \\
& $\mathrm{~N}_{\text {sapw }}$ & 0.13 & 0.08 & 1.47 & 0.142 & 0.33 \\
& sqrt(TLA) & 0.17 & 0.11 & 1.46 & 0.146 & 0.14 \\
& H & 0.11 & 0.10 & 1.03 & 0.304 & 0.08 \\
& $\mathrm{~N}_{\text {max }}$ f & -0.12 & 0.14 & 0.84 & 0.401 & 0.07 \\
\hline BA & BA & 0.22 & 0.07 & 3.32 & 0.002 & \\
& Sapwood growth & 0.45 & 0.08 & 5.99 & $<0.001$ & \\
& Sapwood lifespan & 0.18 & 0.07 & 2.50 & 0.019 & \\
& Intercept Sweetia & -0.77 & 0.12 & -6.55 & $<0.001$ & \\
& Intercept Hura & 1.42 & 0.16 & 8.67 & $<0.001$ & \\
& Intercept Schizolobium & 0.50 & 0.20 & 2.52 & 0.018 & \\
\hline
\end{tabular}



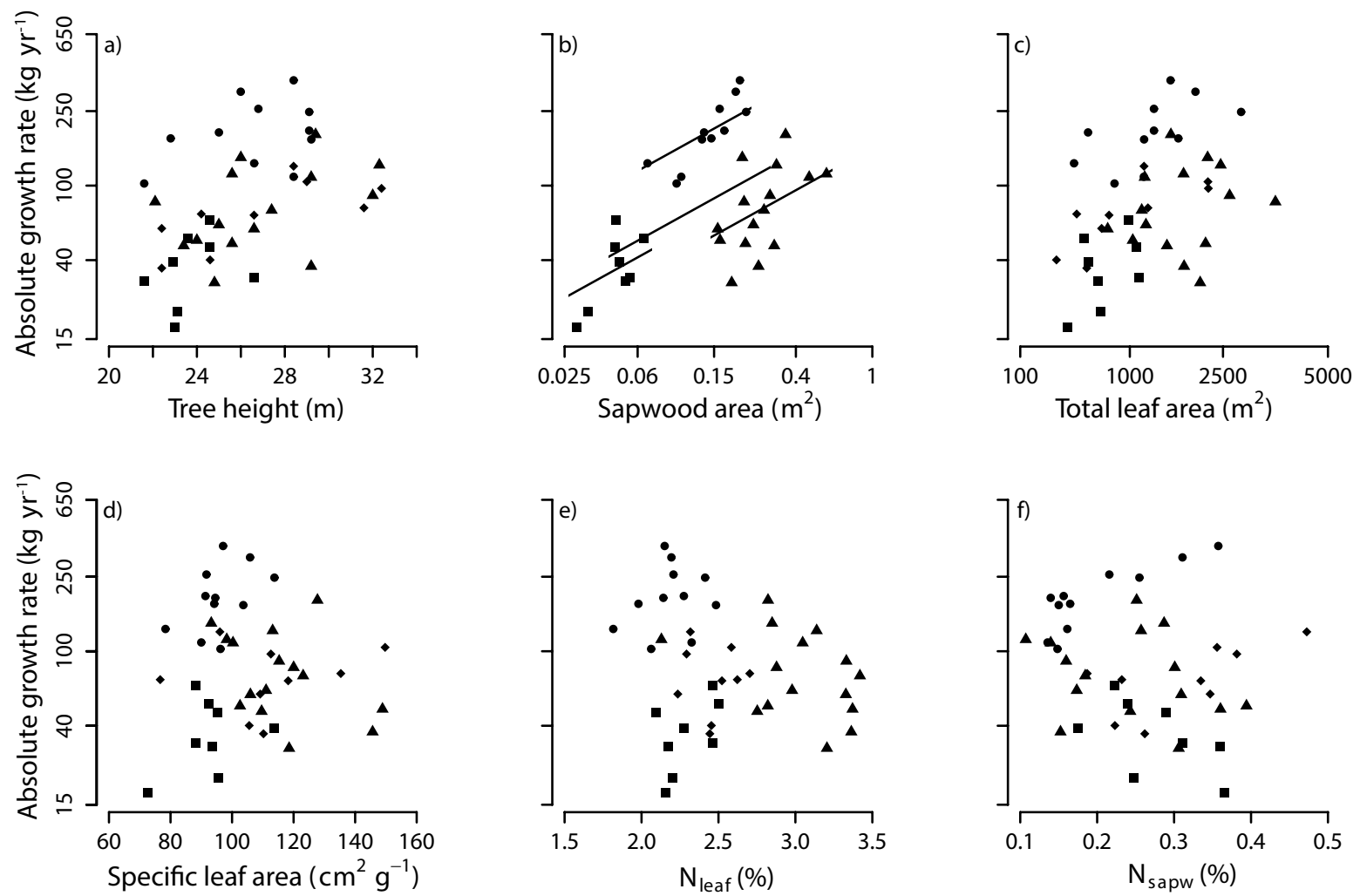

Figure 2.1: The relation of absolute biomass growth with a) tree height, b) sapwood area (SA), c) total leaf area (TLA), d) specific leaf area, e) leaf nitrogen concentration $\left(\mathrm{N}_{\text {leaf }}\right)$, and f) sapwood $\mathrm{N}$ concentration $\left(\mathrm{N}_{\text {sapw }}\right)$. Regression lines are based on the multiple regression analysis (by keeping the other predictor variables at their mean), but are only shown when the predictor variable contributed significantly in explaining absolute biomass growth (Table 2.3). Symbols represent four species: Sweetia (squares), Hura (triangles), Schizolobium (circles), and Cariniana (diamonds). Note that the axes for absolute biomass growth and SA have a log scale, and the axis for TLA a square root scale.

\section{An individual-based approach}

We used an individual approach to evaluate the factors driving variation in growth among tropical forest canopy trees. By combining individual traits and species in one statistical model, we were able to separate the effect that individual traits have on individual growth, from the variation caused by evolutionary differences among species (Clark et al. 2011). Our focus is on individuals because they are the units that grow and respond to their environment (Clark et al. 2011), rather than species. While other studies show that differences in growth and other traits among individuals of the same species even exceed the differences in average growth or traits among species (Bolnick et al. 2003, Clark 2010, Messier et al. 2010), this was not the case in our study. Possible explanations are that we used four species from different ecological growing strategies, and selected fully exposed canopy trees with 
reduced environmental variation among individuals. Nevertheless, we observed fully consistent trait impacts on growth among individuals, suggesting that similar functional relationships drive the growth variation amongst individuals for different species.
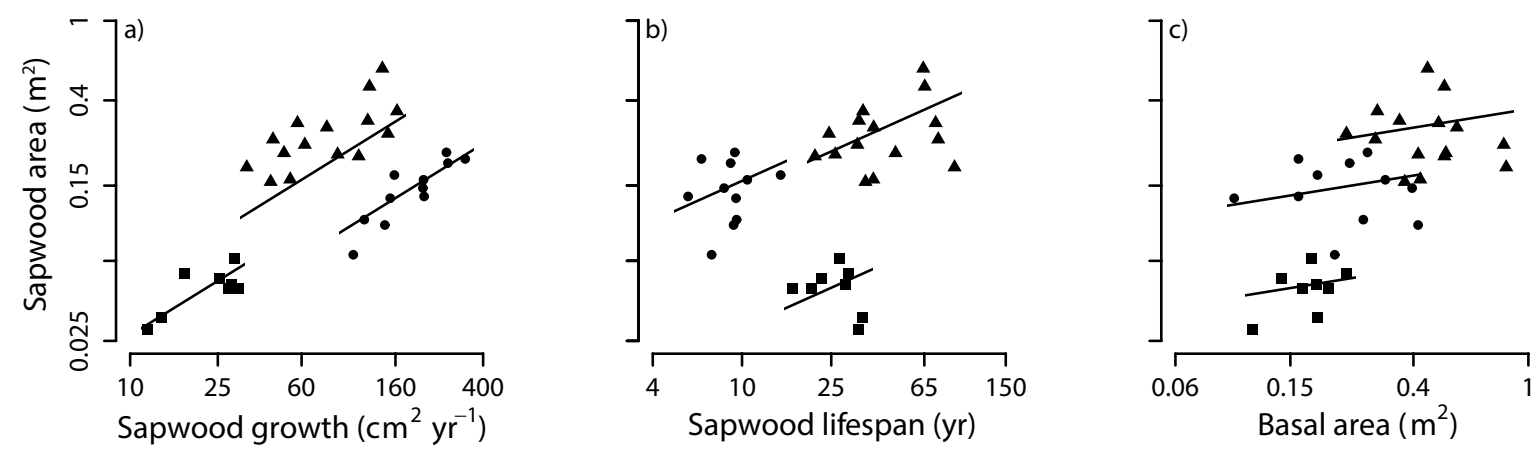

Figure 2.2: The relation of sapwood area (SA) with a sapwood growth, b sapwood lifespan, and c stem basal area (BA). Regression lines are based on the multiple regression analysis (by keeping the other predictor variables at their mean), but are only shown when the predictor variable contributed significantly in explaining absolute biomass growth (Table 2.3). Symbols represent four species: Sweetia (squares), Hura (triangles) and Schizolobium (circles). Cariniana was excluded because no SA could be distinguished. Note that the axes for SA and stem BA have a log scale.

\section{Sapwood is the major driver of growth, not crown traits}

Contrary to expectations, we found that none of the traits, except for SA, explained variation in growth of individual canopy trees. Many studies have found an important positive role of leaf traits such as TLA, SLA and $\mathrm{N}_{\text {leaf }}$ for species performance (Sterck et al. 2006, 2014), especially for saplings and small trees (Poorter 1999, Poorter and Bongers 2006). These traits indeed vary strongly among species and partially explain species-level growth responses of smaller trees, where a high TLA, SLA and $\mathrm{N}_{\text {leaf }}$ may strongly increase the light interception and photosynthesis per unit plant mass and therefore drive growth. The importance of such crown traits may be different for canopy trees that have full access to light and better developed crowns, with optimally distributed leaves that compensate for possible effects of leaf traits such as SLA and $\mathrm{N}_{\text {leaf }}$ on the light capture and carbon gain (McMurtrie et al. 2008, Sterck and Schieving 2011). Similar to our results, Staudhammer et al. (2013) found no effect of TLA on basal area growth of adult trees (although TLA did increase reproductive output). Thus, crown traits cannot explain the variation in stem growth among emergent tropical canopy trees.

Sapwood area was clearly the most important variable explaining aboveground biomass growth of individual trees in our study. A high amount of living wood may 
increase respiration costs (Ryan et al. 1994), especially when air temperature is high, and pose a negative effect on growth. Interestingly, a positive effect of large SA was superior to its high respiration costs (Table 2.3, Fig. 2.1), probably because tall trees can be water limited and SA improves the water supply to the crown. This relation could not be explained by larger trees that have both a high biomass growth and large sapwood area, since growth rate was not related to tree height (Table 2.3, Fig. 2.2) and neither to basal area (Appendix 2.2a). We added a structural equation model (Fig. 2.3) to summarize the relative effects of SA, TLA and tree height on growth when taking correlations among predictors into account. Even though the effect of TLA on growth was marginally significant as compared to the linear model (Table 2.3), the analysis confirmed that sapwood area is superior to any other effect on growth.

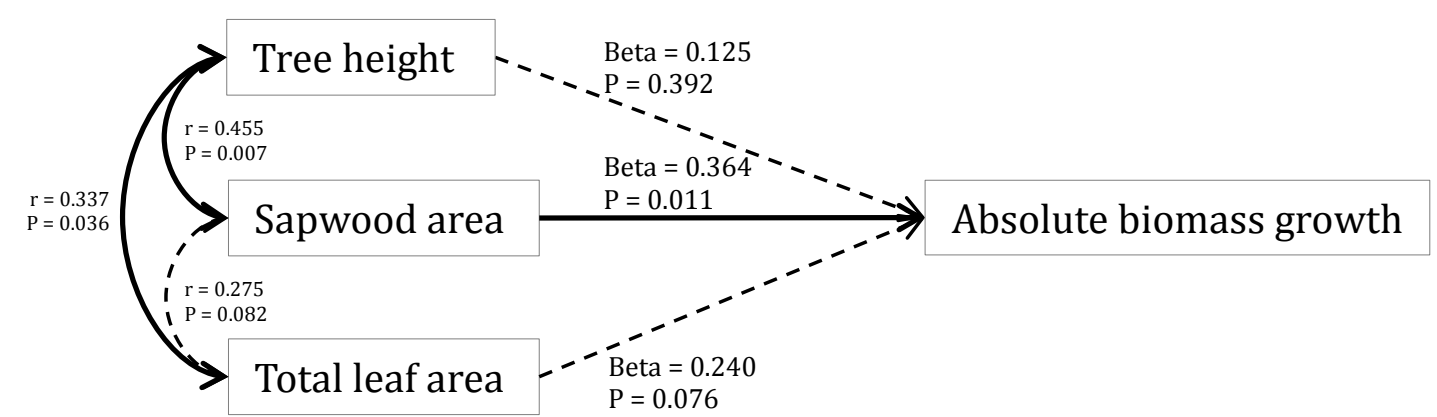

Figure 2.3: Structural equation model for the effects of tree height, sapwood area (SA) and total leaf area (TLA) on absolute biomass growth. For each variable, the species mean was subtracted from the individual measurements in order to exclude differences in intercept among species, as were found in previous analysis (Table 2.3). The one-headed arrows show regressions between variables, whereas the two-headed arrows between the predictor variables show correlations between variables. Black arrows show significant effects and dashed arrows show non-significant effects. For each relation, the coefficient $(\beta$ or $\mathrm{r}$ ) and significance $(\mathrm{P})$ are given, based on an $\mathrm{n}$ of 43. Note that the model is saturated (i.e. all possible arrows between boxes are drawn), therefore we cannot test the fit of the overall model. We nevertheless present this model in order to evaluate the relative strengths of size variables on growth while correcting for interrelatedness among predictor variables. The model was evaluated using the sem function of the lavaan package in R (Rosseel 2012).

\section{Growth and sapwood: chicken and egg?}

A question that arises from the positive relation between sapwood area and growth, is whether sapwood has a positive functional effect on growth, or is merely a passive consequence of growth (Galván et al. 2012). In other words, does large sapwood area increase growth, or does fast growth increase sapwood area? To better understand these relations, we evaluated some factors that may explain variation in sapwood area. A tree can have a lot of sapwood because of fast 
sapwood growth, long sapwood lifespan, and/or because the tree has a large basal area and consequently a large sapwood area. We found that all these three factors positively affect sapwood area (Table 2.3, Fig. 2.2). The positive effect of basal area on sapwood area indicates that larger trees have more sapwood area, but basal area did not affect growth (see Appendix 2.2a). The positive effect of sapwood growth and sapwood lifespan on sapwood area (Table 2.3, Fig. 2.2) suggests that trees can achieve a larger sapwood area by increasing sapwood growth and/or sapwood lifespan. However, the negative correlation between sapwood growth and sapwood lifespan (Appendix 2.3) suggests that trees with fast sapwood growth, which increases sapwood area, also have short sapwood lifespan, which decreases sapwood area. Hence, the sapwood area should not necessarily increase as a result of tree growth. Moreover, since the average sapwood lifespan is 30 years, average annual sapwood growth should at least be an order of magnitude smaller than the total sapwood area of the tree. Hence, it is unlikely that this small part of the sapwood area that is directly related to annual growth causes the strong positive relation between sapwood area and growth. These results imply that sapwood area is not only a passive consequence of growth, but that the positive effect of sapwood area on growth may be attributed to a functional role of sapwood underlying growth.

\section{Why does sapwood area increase growth?}

The functional role of sapwood is to supply water with nutrients to the crown, and this is likely how sapwood area increases biomass growth in our study trees. Sapwood assures water supply in two ways: by water transport from the roots to the leaves (Goldstein et al. 1998, Meinzer et al. 2001), and by water storage to buffer the use of soil water and allow more persistent water supply to the crown during the course of the day (e.g. during hot afternoons) or the dry season (Wullschleger et al. 1998). Our canopy trees were all emergent and thus most likely not primarily limited by light, but their high stature (on average $26.2 \mathrm{~m}$ ) may have caused hydraulic limitation for the supply of water to the crown. We found a positive effect of sapwood area on total leaf area (Fig. 2.4), without differences in slope and intercept between species. This suggests that a large sapwood area indeed supports a large total leaf area, and that, independent of species, a certain sapwood area is associated with a certain total leaf area. A positive relation between sapwood area and total leaf area was also found for two mountain ash species in south-east Australia (Vertessy et al. 1995), and a strong relation between sapwood area and water flow rate was found for five tropical canopy trees in Panama (Goldstein et al. 
1998). These studies and our results thus suggest that the water supply to the crown may limit the total leaf area and growth of these tropical forest trees.

The sapwood age (i.e. sapwood lifespan) of our trees ranged between 5.7 and 88.6 years with an average of 29.8 years (Table 2.2). We did not find other studies with data on sapwood lifespan for tropical trees, but Spicer and Holbrook (2007) found ages between 7.6 and 50 years for three temperate tree species, and Sterck et al. (2008) found ages between 25 and 50 years for Pinus sylvestris (a coniferous species) in an alpine valley. Compared to these studies, trees in our study varied strongly in sapwood lifespan, with some having remarkably old sapwood. Since water transport efficiency decreases with sapwood age (Spicer and Gartner 2001), it is unlikely that all 30 years of the sapwood have an equally important contribution to water transport. Instead, the oldest sapwood rings may be used to store water and nutrients in living cells and extracellular spaces (Goldstein et al. 1998), rather than to transport water. Goldstein et al. (1998) found that the majority of the stored water in large trees was used in the morning to supplement water that had been lost through transpiration during the previous day, before the soil water could reach these depleted sites. The stored water may act as a buffer to complement water supply to the upper leaves, which reduces the risk on drought-induced cavitation of the vessels, and simultaneously increases photosynthesis by allowing more water to be withdrawn for transpiration (Scholz et al. 2007).

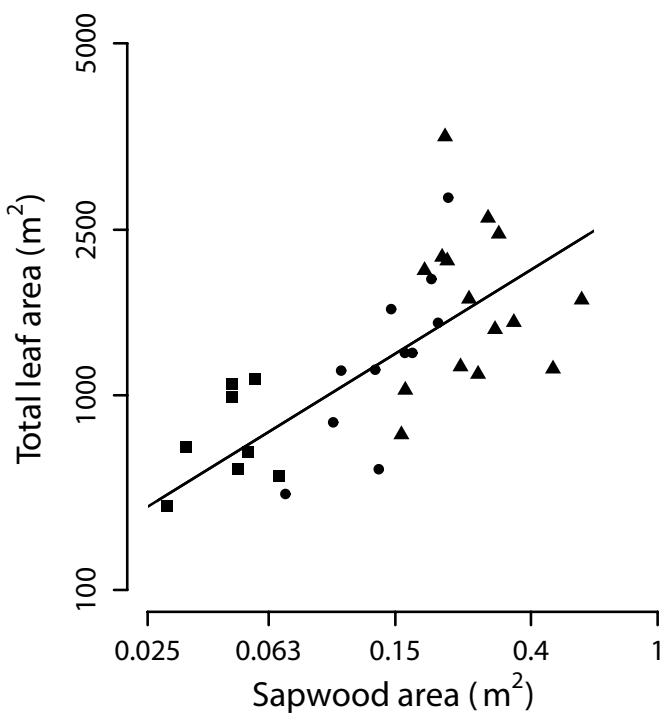

Figure 2.4: The relation of sapwood area (SA) with total leaf area (TLA), based on a regression analysis. Symbols represent four species: Sweetia (squares), Hura (triangles), Schirolobium (circles), and Cariniana (diamonds). Note that the axis for SA has a log scale and the axis for TLA a square root scale. 
The whole-tree hydraulic conductance can be evaluated by using the ratio between total leaf area and sapwood area. This ratio determines the water supply per unit leaf area and, hence, may affect actual rates of photosynthesis and growth (Whitehead et al. 1984, McDowell et al. 2002). For our trees, however, the ratio between sapwood area and total leaf area did not relate to growth (linear model with species as fixed factor; $\mathrm{t}=-1.33, \mathrm{P}=0.891)$. Probably, the sapwood area available per leaf is not a good indicator of water reaching the leaves for large trees, because of the reduced transport activity of the old sapwood. McDowell et al. (2002) showed that the ratio between leaf area and sapwood area decreases with tree height, indicating that for large trees the hydraulic conductance becomes relatively less important than their capacity to store water (Phillips et al. 2003). Given the old age of the sapwood in our trees (5.7-88.6 yr), the lack of effect of hydraulic conductance (the ratio between total leaf area and sapwood area) on growth, and the expected hydraulic limitations during periods of low water availability, we speculate that an increased sapwood area positively affects growth by improving water storage, rather than water transport.

We show that sapwood area may be one of the most important traits affecting growth of tropical canopy trees. Few studies have focussed on the role of sapwood for biomass growth (but see Galván et al. 2012), and no studies have done so for tropical trees. Our results suggest that the positive functional effects of sapwood area on growth largely offsets possible negative impacts of increasing respiration costs. We speculate that this is attributable to an increasing capacity for water storage that sustains water supply to the leaves, even in times of high evaporative demand and/or drought.

\section{Acknowledgements}

This project was financially supported by an ERC grant (\#242955) to P.A.Z. Furthermore, the research leading to these results has received partial funding from the European Union Seventh Framework Programme (FP7/2007-2013) under grant agreement $n^{\circ} 283093$ - The Role Of Biodiversity In climate change mitigatioN (ROBIN). We are grateful to Insituto Boliviano de Investigación Forestal (IBIF) and the logging company La Chonta for the use of facilities and logistical support. The fieldwork would have been impossible without the field assistance of Ricardo Mendez and Bruno Vaca, and logistical support by Peter van der Sleen and Estela Quintero. And last, we would like to thank Frans Bongers, Jérôme Chave, Timothy Paine, Lourens Poorter, and an anonymous reviewer for valuable comments on earlier versions of this paper. 


\section{Appendices}

Appendix 2.1: Results for the comparisons of basal area (in $\mathrm{m}^{2}$ ) among the four sampling heights in the tree: breast height (1), just below the first major branch (2), and at two heights in the crown below the first leaves ( 3 and 4; see figure on the right). For each height, the mean, minimum, maximum, and standard deviation (Stdev) of the basal area are given. A two-way ANOVA showed no interaction between species and height, and we therefore used a one-way ANOVA with TukeyHSD multiple comparisons to compare basal area at the four heights (letters indicate different groups at $\mathrm{P}<0.001)$. $\mathrm{N}$ was 43 for all comparisons.

\begin{tabular}{llllll}
\hline Height & Mean & Min & Max & Stdev & TukeyHSD* \\
\hline 1 & 0.40 & 0.13 & 1.31 & 0.24 & $\mathrm{a}$ \\
2 & 0.23 & 0.06 & 0.68 & 0.13 & $\mathrm{~b}$ \\
3 & 0.24 & 0.04 & 0.98 & 0.18 & $\mathrm{~b}$ \\
4 & 0.21 & 0.03 & 0.95 & 0.17 & $\mathrm{~b}$ \\
\hline
\end{tabular}

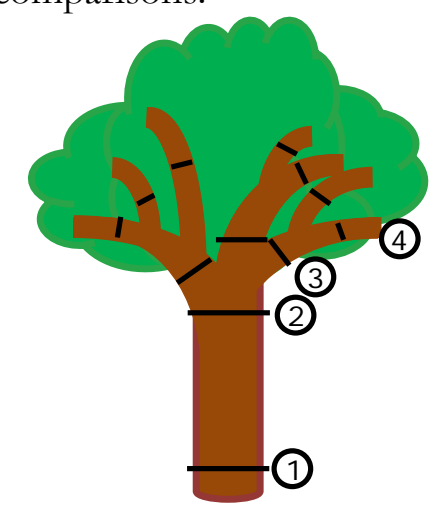

Appendix 2.2: Evaluation of the effect on the analyses when (a) including predicted sapwood area for Cariniana (as used in the manuscript) and basal area instead of tree height, and (b) when excluding Cariniana from the analyses. All subset regression analyses were used with absolute growth rate as response variable and all traits, stem basal area and species as predictor variables, and model averaging was applied over all models that differed less than 2 AIC from the 'best' model (which is considered a not significantly different fit). Empty rows indicate that the variable was excluded by the analysis. These results also show the effect of including stem basal area instead of tree height (Appendix 2.2a vs. Table 2.3). The standardized coefficient (Beta), adjusted standard error (SEadj), $z$-value and P-value are given for each predictor variable. $\mathrm{N}=43$ for the model with predicted sapwood area for Cariniana, and $\mathrm{N}=34$ for the model without Cariniana.

\begin{tabular}{lllllllll}
\hline & \multicolumn{1}{c}{ a) Including Cariniana } & \multicolumn{5}{c}{ b) Excluding Cariniana } \\
Predictor variable & Beta & SEadj & z-value & P-value & Beta & SEadj & z-value & P-value \\
\hline $\log (\mathrm{SA})$ & 0.74 & 0.15 & 4.77 & $<0.001$ & 0.91 & 0.19 & 4.71 & $<0.001$ \\
Intercept Sweetia & 0 & 0 & & & 0 & 0 & & \\
Intercept Hura & -0.28 & 0.20 & 1.44 & 0.149 & -0.54 & 0.25 & 2.19 & 0.029 \\
Intercept Schizolobium & 0.56 & 0.14 & 3.94 & $<0.001$ & 0.40 & 0.17 & 2.39 & 0.017 \\
Intercept Cariniana & 0.07 & 0.13 & 0.55 & 0.585 & & & & \\
SLA & -0.16 & 0.10 & 1.60 & 0.110 & -0.13 & 0.11 & 1.22 & 0.224 \\
Nsapw & 0.13 & 0.09 & 1.47 & 0.142 & 0.08 & 0.09 & 0.91 & 0.365 \\
sqrt(TLA) & 0.17 & 0.12 & 1.46 & 0.146 & & & & \\
Nleaf & -0.12 & 0.14 & 0.84 & 0.401 & & & & \\
\hline
\end{tabular}


Appendix 2.3: Pearson correlations among a) the predictor variables used to explain variation among trees in absolute biomass growth, and b) the predictor variables used to explain variation among trees in sapwood area.

\begin{tabular}{|c|c|c|c|c|c|c|c|}
\hline \multicolumn{5}{|l|}{ a) } & \multicolumn{3}{|l|}{ b) } \\
\hline $\log (\mathrm{SA})$ & sqrt(TLA) & SLA & $\mathbf{N}_{\text {leaf }}$ & $\mathbf{N}_{\text {sapw }}$ & $\log (\mathrm{BA})$ & $\begin{array}{l}\log \text { (sapwood } \\
\text { growth) }\end{array}$ & \\
\hline \multirow[t]{7}{*}{0.564} & 0.461 & 0.340 & 0.325 & -0.090 & & & Height \\
\hline & 0.699 & 0.474 & 0.511 & -0.204 & & & $\log (\mathrm{SA})$ \\
\hline & & 0.500 & 0.394 & 0.090 & & & sqrt(TLA) \\
\hline & & & 0.599 & 0.095 & & & SLA \\
\hline & & & & 0.007 & & & $\mathbf{N}_{\text {leaf }}$ \\
\hline & & & & & 0.314 & & $\begin{array}{l}\log \text { (sapwood } \\
\text { growth) }\end{array}$ \\
\hline & & & & & 0.478 & -0.547 & $\begin{array}{l}\text { log(sapwood } \\
\text { lifespan) }\end{array}$ \\
\hline
\end{tabular}

Appendix 2.4: Results from the linear model for basal area growth, including the same predictor variables as used for absolute biomass growth (see Table 2.3). Only the predictor variables are presented that were included in the best models (based on all subset regression analysis), and statistics are based on averaging of these 'best' models (i.e. that differed less than 2 AIC units). The standardized coefficient (Beta), standard error (SE), t-value, P-value, and relative variable importance are given for each predictor variable. The statistics for the species are based on their intercept.

\begin{tabular}{llllll}
\hline Predictor variable & Beta & SEadj & t-value & P-value & Relative importance \\
\hline $\log (\mathrm{SA})$ & 0.56 & 0.12 & 4.73 & $<0.001$ & 1 \\
Intercept Sweetia & 0 & 0 & & & $1^{*}$ \\
Intercept Hura & 0.15 & 0.17 & 0.91 & 0.362 & \\
Intercept & & & & & \\
Schizolobium & 0.79 & 0.11 & 6.91 & $<0.001$ & \\
Intercept Cariniana & 0.35 & 0.10 & 3.48 & 0.001 & \\
SLA & -0.10 & 0.08 & 1.29 & 0.199 & 0.30 \\
$\mathrm{~N}_{\text {leaf }}$ & -0.13 & 0.12 & 1.10 & 0.272 & 0.23 \\
\hline
\end{tabular}

$* \overline{\text { Relative importance was given for the variable 'species'. Therefore no importance value }}$ is shown for the intercepts of the individual species. 
Appendix 2.5: Results of the linear regression for absolute biomass growth, using stepwise exclusion of variables (based on AIC), in order to compare these with the results based on all subsets regression analysis and model averaging (Table 2.3). All traits, tree height and species were initially included as predictor variables. All continuous variables were scaled prior to analysis, by subtracting the mean and dividing by the standard deviation, to obtain standardized coefficients (Beta). Furthermore, standard error (SE), t-value and P-value are given for each predictor variable. The significance of the intercepts of the different species are relative to the intercept of Sweetia.

\begin{tabular}{lllll}
\hline Predictor variable & Beta & SE & t-value & P-value \\
\hline $\log (\mathrm{SA})$ & 0.80 & 0.14 & 5.70 & $<0.001$ \\
$\mathrm{SLA}$ & -0.15 & 0.09 & -1.63 & 0.113 \\
$\mathrm{~N}_{\text {sapw }}$ & 0.13 & 0.08 & 1.58 & 0.124 \\
Intercept Sweetia & -0.15 & 0.26 & -0.60 & 0.555 \\
Intercept Hura & -0.74 & 0.38 & -1.57 & 0.126 \\
Intercept Schizolobium & 1.13 & 0.30 & 4.21 & $<0.001$ \\
Intercept Cariniana & 0.00 & 0.29 & 0.52 & 0.607 \\
\hline
\end{tabular}

Appendix 2.6: Results of the reduced linear model for aboveground biomass growth, including only sapwood area (SA), total leaf area (TLA), tree height and species as predictor variables (without exclusion of variables). All continuous variables were scaled prior to analysis, by subtracting the mean and dividing by the standard deviation, to obtain standardized coefficients (Beta). Furthermore, standard error (SE), t-value and P-value are given for each predictor variable. The significance of the intercepts of the different species are relative to the intercept of Sweetia.

\begin{tabular}{lllll}
\hline & Beta & SE & t-value & P-value \\
\hline $\log (\mathrm{SA})$ & 0.58 & 0.19 & 3.10 & 0.004 \\
sqrt(TLA) & 0.08 & 0.11 & 0.70 & 0.486 \\
Height & 0.08 & 0.10 & 0.76 & 0.455 \\
Intercept Sweetia & -0.19 & 0.28 & -0.69 & 0.493 \\
Intercept Hura & -0.71 & 0.41 & -1.26 & 0.215 \\
Intercept Schizolobium & 1.13 & 0.32 & 4.17 & $<0.001$ \\
Intercept Cariniana & -0.02 & 0.30 & 0.57 & 0.573 \\
\hline
\end{tabular}





\section{Chapter 3}

Soil fertility and species traits, but not species diversity, drive productivity and biomass stocks in a tropical rainforest

Masha T. van der Sande, Eric J. M. M. Arets, Marielos Peña-Claros, Marcel R. Hoosbeek, Yasmani Cáceres-Siani, Peter van der Hout, Lourens Poorter

In revision

t?

a

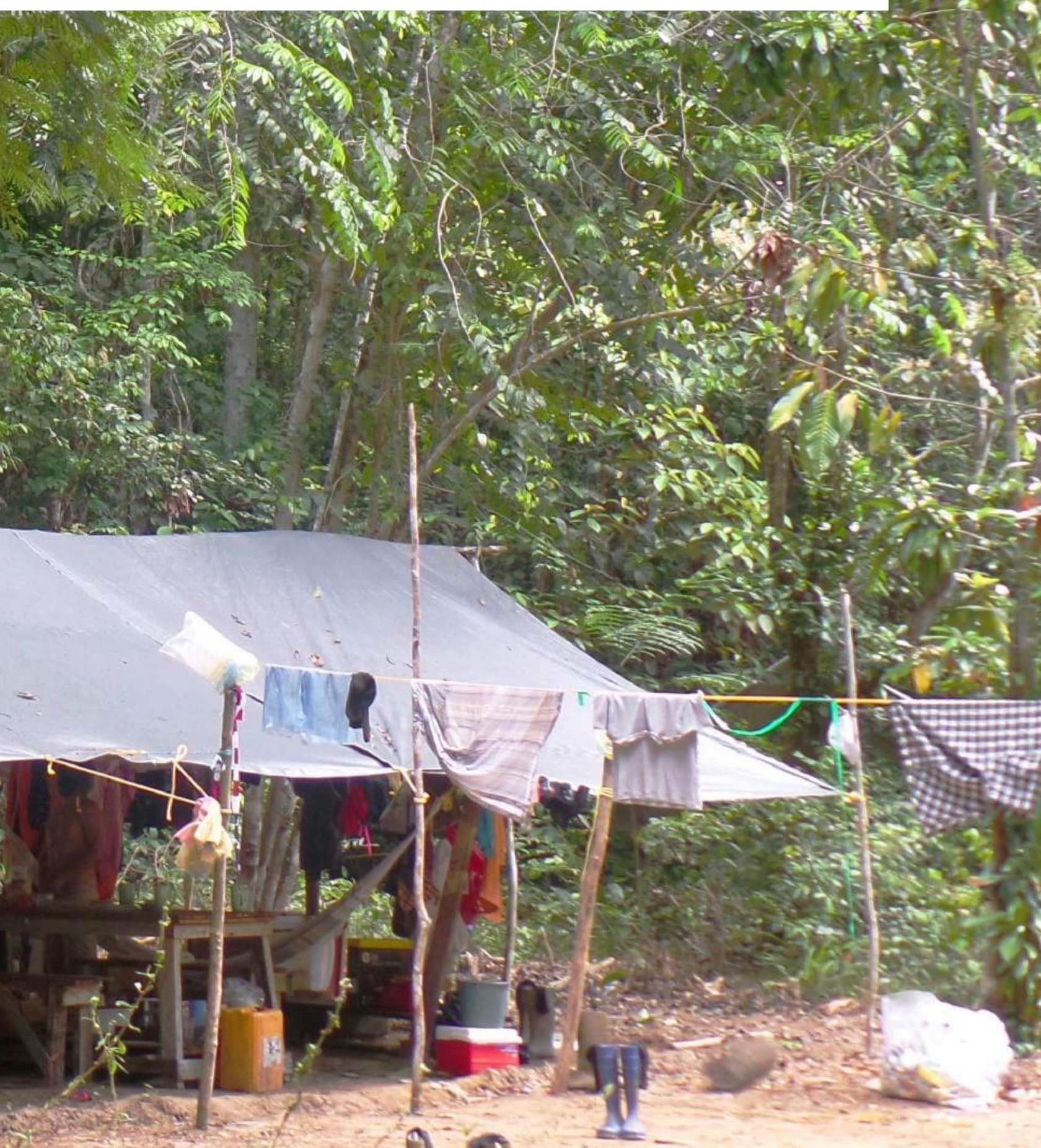




\section{Abstract}

Tropical forests store and sequester large amounts of carbon in above- and belowground plant biomass and soil organic matter (SOM), but how these are driven by abiotic and biotic factors remains poorly understood. Here, we test the effects of abiotic (soil fertility and light availability) and biotic (species richness and trait composition) factors on biomass stocks (aboveground and fine root), SOM, and productivity in a Guyanese tropical rainforest. This forest grows on nutrient poor soils and has few species that contribute most to total abundance, and we therefore expected strong effects of soil fertility and species' traits that determine resource acquisition and conservation, but not of species diversity. We evaluated 6 years of data for 30 0.4-ha plots and tested hypotheses using structural equation models. Soil phosphorus (P) increased aboveground biomass and productivity, whereas soil nitrogen $(\mathrm{N})$ increased fine root biomass, possibly because $\mathrm{N}$ is needed for $\mathrm{P}$ absorption by roots. In contrast to expectations, acquisitive trait values (e.g., high leaf $\mathrm{P}$ ) increased biomass stocks possibly because they indicate higher nutrient absorption and thus higher biomass build-up. However, under harsh conditions where biomass increase is slow, acquisitive trait values may increase respiration and vulnerability to physical and biotic hazards and therefore increase biomass loss. As expected, species richness did not increase productivity and biomass stocks. We conclude that soil fertility - especially P - strongly limits forest biomass productivity and stocks; low $\mathrm{P}$ availability may cause strong environmental filtering, which in turn results in a small set of dominant species. As a result, community trait composition but not species richness determines productivity and stocks of biomass and SOM in tropical forest on poor soils.

Keywords: biodiversity-ecosystem functioning, diversity, disturbance, fine root biomass, functional traits, mass-ratio hypothesis, niche complementarity, soil organic matter 


\section{Introduction}

Tropical forests store about 25\% of global terrestrial carbon (Bonan 2008) and account for $34 \%$ of terrestrial gross primary productivity (Beer et al. 2010), and their storage and productivity per hectare is even expected to increase with rising atmospheric $\mathrm{CO}_{2}$ and climate change (King et al. 1997). Tropical forests are thus important in the global carbon cycle and for climate change mitigation options. This carbon is divided over different stocks, such as above- and belowground living biomass, and soil organic matter (SOM) (Malhi et al. 2009, Quesada et al. 2011). However, the factors driving such carbon fluxes and pools are yet poorly understood.

Biomass stocks in living plant biomass (i.e., in roots, stems and crowns) vary greatly among tropical forests (Cairns et al. 1997, Baker et al. 2004b). Although most studies evaluate drivers of aboveground biomass stocks (e.g., Poorter et al. 2015), on average $32 \%$ of living biomass is found in the roots (Robinson 2007). Additionally, SOM represents another important stock of carbon in tropical forests, storing about half the amount of carbon (up to $1 \mathrm{~m}$ depth) as compared to all living above- and belowground plant biomass combined (Malhi et al. 1999, Robinson 2007). Here, we evaluate how abiotic and biotic factors directly and indirectly affect aboveground biomass productivity, and stocks of aboveground biomass, fine roots, and SOM for a tropical rainforest in Guyana (see the conceptual model, Fig. 3.1), to better understand underlying drivers of carbon fluxes and stocks and, hence, their role in the global carbon cycle. To our knowledge, no studies have simultaneously evaluated abiotic and biotic drivers of carbon stocks and fluxes in tropical forests.

\section{Abiotic effects on biomass productivity and stocks}

Although mature tropical forests store most biomass per hectare, forests that have suffered from human disturbances (such as logging) cover more than half of the world's tropical forest area (FAO 2010), sequester more carbon, and are therefore important in the global carbon cycle. Such disturbances directly reduce above- and belowground biomass stocks, but they may increase ecosystem productivity because of increased light levels reaching the lower tree strata (Fig. 3.1a) (PeñaClaros et al. 2008).

At large spatial scales (e.g., across different tropical forest types), climate may be a strong driver of productivity and biomass stocks (Toledo et al. 2011, Durán et al. 2015), but at smaller spatial scales (e.g., 1 ha or smaller), soil conditions instead of climate may vary more strongly (Burrough 1983). Soil fertility should positively affect biomass productivity especially in forests growing on very poor soils (Baker 
et al. 2009), such as on the old and leached soils of the Guiana shield (Quesada et al. 2011) that are very nutrient poor (van Kekem et al. 1996).

\section{Biotic effects on biomass productivity and stocks}

The richness and composition of the tree community can also be an important biotic predictor of ecosystem functions such as biomass productivity and stocks (Fig. 3.1a) (Hooper et al. 2005). For example, the presence of many different species in a system (i.e., high species richness) can increase the resource use efficiency because of niche complementarity or facilitation among species (Tilman 1999). For different systems and at different scales, positive effects (Vilà et al. 2013, Poorter et al. 2015) but also no or negative effects (Adler et al. 2011, Zhang et al. 2011) on biomass productivity and stocks have been found (Chisholm et al. 2013). We expect that niche complementarity may be weak when conditions are harsh and few species with well-adapted strategies are abundant, as in this Guyanese forest on poor soils. The abovementioned contrasting results of diversity effects may partly be explained by the fact that species richness does not provide information on the functional traits of the species.

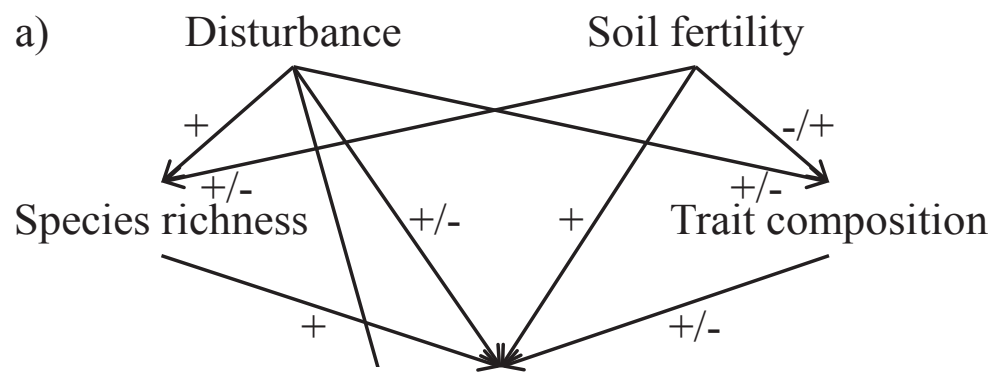

1. Aboveground biomass

2. Belowground biomass

b)

3. Productivity

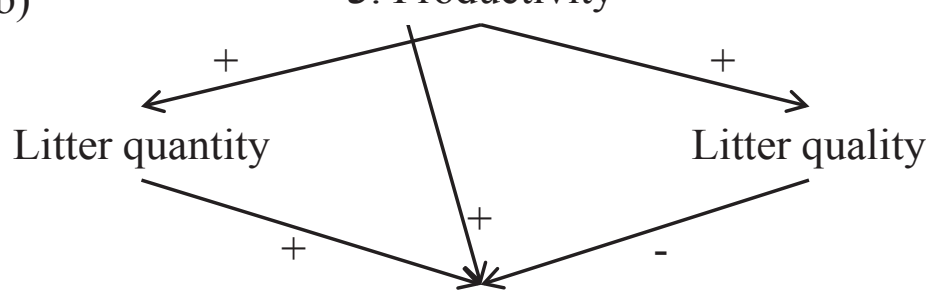

4. Soil organic matter

Figure 3.1: Expected direct and indirect effects of disturbance, soil fertility, species richness and trait composition on productivity and above- and belowground living biomass (a), and the (in)direct effects of disturbance, aboveground productivity, leaf litter quantity and leaf litter quality on soil organic matter (b). Expected positive $(+)$ and negative $(-)$ effects are given. The expected effect of disturbance is negative for above- and belowground biomass but positive for productivity. Disturbance and soil fertility favour species with acquisitive trait composition (e.g., high specific leaf area, low wood density), and an acquisitive trait composition increases productivity but decreases biomass stocks. High litter quality means that nutrient concentrations are high, which increases decomposition rates and thus decreases soil organic matter stocks. 
Species' traits are morphological or physiological plant characteristics that represent species' strategies to acquire and use resources, and thus determine their growth, reproduction and survival (Poorter and Bongers 2006, Violle et al. 2007, Baker et al. 2009). Consistent with Grime's mass-ratio hypothesis (Grime 1998), we expect that the dominant trait values in a community (i.e., the community average leaf and stem trait values weighted by species' basal area, here called the 'trait composition'), are a better predictor for biomass stocks and productivity than species richness. In temperate grasslands, the number of functional groups has a more important effect on productivity than species richness (Tilman et al. 1997), and in modelled single-species forests, wood density increases and specific leaf area decreases biomass stocks (Falster et al. 2011). However, such relations may be different in more diverse natural tropical forests where species differ in abundance and in trait values, and in forests where biomass productivity and stocks are strongly determined by abiotic factors (Ruiz-Jaen and Potvin 2011, Zhang et al. 2012, Conti and Díaz 2013).

\section{Abiotic and biotic effects on soil organic matter stocks}

Abiotic and biotic factors are thus important determinants for productivity and living biomass, but what factors would drive SOM stocks (i.e., all carbon in nonliving organic stocks, including decomposing litter)? SOM stocks are mainly balanced by input via plant litter production and output via decomposition (Amundson 2001, De Deyn et al. 2008). Plant litter production is determined by the turnover rate of living biomass and should thus relate to gross productivity of the forest. Decomposition, on the other hand, directly depends on environmental conditions, litter quality, and the decomposer community (Aerts 1997, Parton et al. 2007, Cornwell et al. 2008). High litter quality, meaning high concentrations of nutrients such as nitrogen and phosphorus that increase palatability for decomposers, increases decomposition rates (Melillo et al. 1982, Wardle et al. 2002) and should therefore reduce SOM stocks. SOM may also be affected by disturbance. Shortly after disturbance, litter input increases and so does the activity of the microbial community, and the more open vegetation leads to higher temperatures and lower humidity which may slow down decomposition and as a result increase SOM stocks (van Dam 2001). Years after disturbance, however, the activity of the microbial community should have stabilized and the canopy should have closed, resulting in weaker effects of microbial activity, temperature and moisture on SOM. Soil fertility may indirectly affect SOM, through increasing productivity and thus litter production (De Deyn et al. 2008). Hence, SOM stocks may depend on litter quantity, litter quality, environmental conditions (Fig. 3.1b), and the decomposer community. 


\section{Conceptual framework: abiotic and biotic effects on productivity and stocks of biomass and soil organic matter}

We ask two questions; First, how do abiotic factors (disturbance and soil fertility) and biotic factors (species richness and trait composition) affect aboveground productivity and aboveground and fine root biomass stocks? We expect that productivity is positively affected by disturbance, soil fertility, species richness and an acquisitive trait composition (e.g., relatively high specific leaf area and leaf nitrogen concentration). Biomass productivity tends to increase with biomass stocks within sites (Chisholm et al. 2013), and therefore aboveground and fine root biomass stocks would also increase with soil fertility. However, aboveground and fine root biomass stocks would decrease with disturbance, because of biomass removal, and with an acquisitive trait composition, because of increased tissue turnover and decreased residence time of the biomass. The Guiana shield is very nutrient poor, and thus soil fertility may be the strongest predictor. Moreover, few species account for the majority of the abundance (ter Steege and Hammond 2001), and diversity may therefore be of limited importance. Second, how do disturbance, litter quantity and litter quality affect SOM? We expect that SOM should increase with litter quantity because this represents the organic matter input, decrease with litter quality because more palatable leaves speed up decomposition and therefore decrease SOM stocks, but show little effect of disturbance because too much time has passed and microbial activity and the microclimate should already have readjusted.

\section{Methods}

\section{Research site}

This study was based on 151.96 ha permanent sample plots at Pibiri creek in Central Guyana, located $50 \mathrm{~km}$ south of Mabura Hill $\left(5^{\circ} 13^{\prime} \mathrm{N} 58^{\circ} 38^{\prime} \mathrm{W}\right)$. This site receives on average $2772 \mathrm{~mm}$ rainfall per year (van Dam 2001). The relatively dry periods are from September to November and from March to April, although monthly rainfall is always higher than $100 \mathrm{~mm}$. Mean annual temperature is $25.9{ }^{\circ} \mathrm{C}$ (ter Steege et al. 1996). The 15 plots are all positioned up to $1.5 \mathrm{~km}$ apart on brown sand ferralsols (van Kekem et al. 1996, van der Hout 1999) with very low phosphorus availability (Quesada et al. 2010). The forest is a mixed Greenheart Morabukea forest with an average canopy height of 30-40 $\mathrm{m}$ (Houter and Pons 2005) and is classified as a moist tropical forest. It has a few very dominant species, with the 8 most abundant ones accounting for $45 \%$ of the trees $(>5 \mathrm{~cm} \mathrm{DBH})$ (Arets 2005). 


\section{Permanent sample plots}

The 15 permanent sample plots were set up in 1993 as part of the Tropenbos Guyana Programme (van der Hout 1999) and are currently managed by the Guyana Forestry Commission. The plots are 1.96 ha $(140 * 140 \mathrm{~m})$ with a buffer zone of $50 \mathrm{~m}$ surrounding the plot. In the whole plot (excluding buffer zone), trees larger than $20 \mathrm{~cm}$ DBH were measured and identified. The central 1 ha $(100 * 100 \mathrm{~m})$ was subdivided into $2520 * 20 \mathrm{~m}$ subplots, and in each subplot one $10 * 10 \mathrm{~m}$ subplot was established in the South-West corner in which trees larger than $5 \mathrm{~cm} \mathrm{DBH}$ were measured and identified (composing 0.25 ha per plot).

In 1994, the plots were experimentally logged, resulting in five different treatments ( 3 repetitions per treatment): control (no logging), logging of 4 trees ha $^{-1}, 8$ trees ha ${ }^{-1}$, or 16 trees ha ${ }^{-1}$, and one silvicultural treatment with logging of 8 trees $\mathrm{ha}^{-1}$, followed by post-harvest liberation thinning (van der Hout 1999). The logging treatments caused a reduction in basal area between $1.5-35 \%$. The plots were completely re-measured in 1995, 1997 and 2000.

\section{Aboveground biomass productivity and stocks}

To determine aboveground productivity and aboveground living biomass stocks, we used the post-logging censuses of 1995 and 2000. We used this 5-year interval because over shorter time intervals productivity may be obscured by stochastic variation especially for slow growing forests, such as our study site, and because the relative effect of measurement error increases. We split each central 1-ha plot into 2 subplots of $100 * 40 \mathrm{~m}$ (i.e., 0.4 ha each) separated by a buffer zone of $100 * 20 \mathrm{~m}$. This plot size allowed us to assess the role of smaller-scale variation in soil fertility (see 'Fine root biomass, soil organic matter, soil fertility, and litter') while keeping sufficiently large plots to reliably estimate biomass and productivity (Chave et al. 2004). Because we aimed to evaluate natural processes, we excluded all trees from the dataset that died as a delayed result of logging and silviculture activities. All trees between $5-20 \mathrm{~cm} \mathrm{DBH}$, which were measured on a subsample of $1 / 4$ of the plot, were considered four times to scale this diameter group to the whole plot. Per tree and per census, we calculated living aboveground biomass (AGB) using the equation from Chave et al. (2014a):

$\mathrm{AGB}=\exp [-1.803-0.976 *(\mathrm{E})+0.976 * \ln (\mathrm{WD})+2.673 * \ln (\mathrm{DBH})-0.0299 *(\ln (\mathrm{DBH}))$

(Eq. 1)

where $\mathrm{E}$ is a measure of environmental stress of the site, which depends on temperature seasonality and water deficit and has a value of -0.1092452 at the Pibiri site (extracted from http://chave.ups-tlse.fr/pantropical_allometry/readlayers.r with the retrieve_raster function in $\mathrm{R}) . \mathrm{DBH}$ is the diameter at breast height $(\mathrm{cm})$ 
and WD is the wood density $\left(\mathrm{g} \mathrm{cm}^{-3}\right)$, which was based on local wood density if available (see explanation under Wood sampling), and otherwise on wood density data obtained from the Global Wood Density Database from DRYAD (Chave et al. 2009, Zanne et al. 2009). Biomass stock per 0.4-ha plot was calculated by summing the biomass of all live trees in 1995, summing the biomass for all live trees in 2000, and averaging these two values per plot to obtain a value that better represents the census period. Average biomass stock per 0.4 ha was multiplied by 2.5 to express per ha.

To calculate productivity $\left(\mathrm{Mg} \mathrm{ha}^{-1} \mathrm{yr}^{-1}\right)$ between 1995 and 2000, we summed the growth of all trees that were present in both censuses, and the growth of trees that were newly recruited in 2000. Growth of trees that were present in both censuses was determined by subtracting the biomass of a tree in 1995 from the biomass of the same tree in 2000. To calculate the growth of recruits between 1995 and 2000, we subtracted the biomass of that individual with a DBH of $5 \mathrm{~cm}$ from its biomass in 2000. Hence, we assumed that recruits grew from $5 \mathrm{~cm}$ at the start of the census interval until the diameter that was measured at the end of the interval. Assuming that recruits started growing from $5 \mathrm{~cm}$ DBH slightly underestimates growth, because in reality most recruits will have reached the diameter limit later. However, it still yields more accurate recruitment estimations than assuming that recruits started growing from $0 \mathrm{~cm}$ at the start of the census interval, which strongly overestimates growth (Talbot et al. 2014). All growth values per tree were summed per 0.4-ha plot, divided by the time in between the two census periods for the 0.4-ha plots (on average 5.65 years) to obtain annual productivity, and multiplied by 2.5 to obtain annual productivity per hectare. Hence, with productivity we refer to aboveground biomass growth by trees that recruit and trees that survive, and do not include mortality and belowground productivity.

\section{Fine root biomass, soil organic matter, soil fertility, and litter}

The plots are located on slightly undulating sedimentary interfluves (i.e., relatively flat surfaces in between drainage tributaries) and thus spatial variation in soil conditions should be small. For that reason, we used two sampling points per 0.4ha plot: one towards the north and one towards the south end (van Kekem et al. 1996, van der Hout 1999, Soil Survey Manual 1993). Per sampling point, soil samples were taken between $0-5 \mathrm{~cm}$ for bulk density, root biomass and concentrations of carbon, nitrogen $\left(\mathrm{N}_{\text {soil }}\right)$, phosphorus $\left(\mathrm{P}_{\text {soil }}\right)$, and the ratios between carbon and nitrogen $\left(\mathrm{C}: \mathrm{N}_{\text {soil }}\right)$ and nitrogen and phosphorus $\left(\mathrm{N}: \mathrm{P}_{\text {soil }}\right.$. Fine root biomass was additionally collected at an intermediate point in each plot (i.e., 3 sampling points per 0.4 ha plot). In addition, fine root biomass was sampled at 15$20 \mathrm{~cm}$ soil depth. Soil organic matter was averaged per plot and scaled to $\mathrm{Mg} \mathrm{ha}^{-1}$ in the $10 \mathrm{~cm}$ topsoil, and fine root biomass was scaled to $\mathrm{Mg} \mathrm{ha}^{-1}$ in the $20 \mathrm{~cm}$ topsoil 
(using an exponential function, see Appendix 3.1), in order to compare values with aboveground productivity and biomass stocks (also in $\mathrm{Mg} \mathrm{ha}{ }^{-1}$ ). At the two sampling points per plot, fragmented litter mass was determined and scaled to $\mathrm{Mg}$ $h \mathrm{~h}^{-1}$. Compared to fresh litter, nutrients in this fragmented litter may already partly have mineralized, and we may therefore expect weaker effects of litter nutrient concentrations on soil organic matter. The coefficient of variation (CV) in soil variables, based on 2 (or 3 for fine root biomass) sample points per plot, was within the range found for other published results in tropical forests (Metcalfe et al. 2008). The within-plot CV of soil organic matter was $24 \%$ (vs compared to $7-51 \%$ for other published studies), of $\mathrm{N}_{\text {soil }}$ was $19 \%$ (vs. 9-52\%), and $\mathrm{P}_{\text {soil }}$ was $49 \%$ (we found no studies to compare this with). The higher $\mathrm{CV}$ for $\mathrm{P}_{\text {soil }}$ is probably caused by the very low values that quickly result in strong relative differences. The CV of litter mass was $29 \%$ (vs. 13-60\%), of litter nutrient concentrations was between 19 and $25 \%$. We are aware that we have not sampled the full soil and litter heterogeneity, and therefore that the relations of soil and litter variables with biomass stocks and productivity may be more conservative and that the chance to find significant effects may be lower (Metcalfe et al. 2008). More details about the collection of fine root biomass, soil organic matter, soil fertility and litter can be found in Appendix 3.1. Correlations between soil variables can be found in Appendix 3.2.

\section{Disturbance}

Relative disturbance (in \%) was computed as a continuous measure per 0.4-ha subplot, based on the basal area of all trees that were logged or died during the census interval due to (post-)logging activities, divided by the total basal area of the subplot before harvesting

Logging disturbance took place in 1994 and the aboveground data (i.e., biomass productivity and stocks, species richness and trait composition) were collected in 1995-2000, but belowground data (i.e., root data, litter data, SOM, and soil fertility) could not be collected during this time and were collected in 2013. Hence, time-lag may affect some of the relations between below- and aboveground data, and between disturbance and belowground data. Shortly after disturbance (e.g., 1-5 years, which is the timeframe of the aboveground data), differences among plots may be large, whereas during later years of recovery, plots may again become more similar. Hence, we may find strong variation among plots in their aboveground variables (1995-2000), but less variation among plots in belowground variables (2013). Our relations between below- and aboveground variables and between disturbance and belowground variables may therefore be rather conservative. 


\section{Leaf and wood traits}

We used 6 leaf traits that we expected to be good predictors for productivity and above- and belowground biomass stocks (Table 3.1). Specific leaf area (SLA) increases light interception efficiency and should therefore increase productivity, but it also relates to a high turnover rate which decreases biomass retention and therefore biomass stocks (Shipley 2006). High specific force to punch (FPs; a measure for leaf toughness) increases leaf defence and should decrease productivity, but tough structures may increase biomass retention and biomass stocks (Kitajima et al. 2012). Leaf nitrogen $\left(\mathrm{N}_{\text {leaf }}\right)$ and leaf phosphorus $\left(\mathrm{P}_{\text {leaf }}\right)$ are used in photosynthesis and growth (Mercado et al. 2011), and should in this way stimulate productivity but decrease longevity and therefore biomass stocks. High ratios of leaf carbon : nitrogen $\left(\mathrm{C}: \mathrm{N}_{\text {leaf }}\right)$ and nitrogen : phosphorus $\left(\mathrm{N}: \mathrm{P}_{\text {leaf }}\right)$ decrease decomposition rate and can indicate which nutrient is relatively more limiting. Since we expect strong nutrient limitation in our forest but were not sure what element or ratio is most limiting, we used concentrations and ratios.

Leaf traits were determined for the 33 most abundant tree species, composing on average $78 \%$ of the basal area $(>5 \mathrm{~cm} \mathrm{DBH})$ in the 300.4 -ha plots over the two census years. For 5 individuals per species, between 7 and $17 \mathrm{~cm} \mathrm{DBH}$, we sampled 5 healthy and young but mature leaves growing at the outer side of the crown (thus in relatively high light conditions, but mostly in the understory).

Besides leaf traits, we measured wood density and wood dry matter content to also define the species' functional strategy in terms of their stem characteristics, as leaf and stem economics spectra can vary independently for large rainforest trees (Baraloto et al. 2010). Wood density and stem dry matter content increase wood defence and should therefore decrease productivity, but they enhance tree longevity and therefore biomass stocks (Baker et al. 2004b). We sampled wood traits for 25 species and 3 individuals per species. For more details on leaf and stem trait collection, see Appendix 3.3.

\section{Species richness and trait composition}

We calculated rarefied species richness, to account for variation in stem number among plots that could affect species richness. Rarefied richness (hereafter referred to as 'species richness') was calculated as the number of species per 100 randomly drawn stems for all live individuals per 0.4-ha plot and per census, using the rarefy function from the vegan package in $\mathrm{R}$ (Oksanen et al. 2014). We used species richness, because this measure is often used in biodiversity-ecosystem function research (e.g., Balvanera et al. 2006), and we used no other species diversity indices to limit the number of possible variables for the structural equation models. 
Table 3.1: All trait composition indices with abbreviation, description, units (the variables expressed in \% are mass-based), what it indicates, and the average (Avg), minimum value (Min) and maximum value (Max) across the 0.4-ha plots.

\begin{tabular}{|c|c|c|c|c|c|c|}
\hline Abbreviation & Variable description & Units & Indicator of: & Avg & Min & Max \\
\hline SLA & Specific leaf area & $\mathrm{cm}^{2} \mathrm{~g}^{-1}$ & $\begin{array}{l}\text { Light interception } \\
\text { efficiency }\end{array}$ & 127.55 & 119.44 & 140.07 \\
\hline $\mathrm{N}_{\text {leaf }}$ & Leaf nitrogen content & $\%$ & $\begin{array}{l}\text { Photosynthetic } \\
\text { capacity }\end{array}$ & 1.78 & 1.64 & 1.94 \\
\hline$P_{\text {leaf }}$ & $\begin{array}{l}\text { Leaf phosphorous } \\
\text { content }\end{array}$ & $\%$ & Growth capacity & 0.05 & 0.04 & 0.05 \\
\hline$C: \mathrm{N}_{\text {leaf }}$ & $\begin{array}{l}\text { Leaf carbon : nitrogen } \\
\text { ratio }\end{array}$ & & $\begin{array}{l}\text { Relative nutrient } \\
\text { limitation }\end{array}$ & 27.86 & 25.87 & 29.74 \\
\hline $\mathrm{N}: \mathrm{P}_{\text {leaf }}$ & $\begin{array}{l}\text { Leaf nitrogen : } \\
\text { phosphorous ratio }\end{array}$ & & $\begin{array}{l}\text { Relative nutrient } \\
\text { limitation }\end{array}$ & 37.74 & 34.21 & 40.58 \\
\hline FPs & Specific force to punch & $\mathrm{N} \mathrm{cm}^{-2}$ & Leaf defence & 263.37 & 243.17 & 284.42 \\
\hline WD & Wood density & $\mathrm{g} \mathrm{cm}^{-3}$ & $\begin{array}{l}\text { Volume growth, } \\
\text { wood defence }\end{array}$ & 0.89 & 0.83 & 0.95 \\
\hline WDMC & $\begin{array}{l}\text { Wood dry matter } \\
\text { content }\end{array}$ & $\mathrm{g} \mathrm{g}^{-1}$ & Wood defence & 0.74 & 0.71 & 0.76 \\
\hline
\end{tabular}

For trait composition, we calculated the community-weighted mean (CWM) for all leaf and stem traits (i.e., the trait value of an average tree in the community) per plot and per census, by multiplying each species' trait value by its relative dominance in the plot (in terms of basal area), and summing all species occurring in the subplot for which traits were measured. Hence, for each subplot at each census, we used the formula:

$$
\mathrm{CWM}=\sum_{i=1}^{S} w i * x i
$$

where $w_{i}$ is the relative basal area of species $i, x_{i}$ is the trait value of species $i$, and $\mathrm{S}$ is the total number of species. Species richness and all trait composition variables of the two censuses were averaged to obtain one value per subplot.

Note that for trait composition, we used mean trait values per species. Hence, differences in CWM trait values among plots are only due to differences in species composition, not due to intraspecific differences caused by acclimation to local environmental conditions. We did not include intraspecific trait variation, because interspecific differences generally explain most variation in trait values $(78 \%$; Rozendaal et al. 2006), and sapling traits and adult traits are strongly correlated (Poorter 2008). Moreover, we collected traits in 2013, and used species composition of 1995-2000 to calculate CWM traits values. We thus assume that species ranking in average trait values remains constant over time. Correlations between community-weighted mean leaf and stem traits can be found in Appendix 3.2. 
To evaluate the importance of environmental filtering (Keddy 1992) on functional trait diversity, we calculated functional trait dispersion (Fdis). Fdis is a multivariate trait diversity measure weighted by species basal area, and based on the mean distance in the multidimensional trait space of all individual species to the centroid of all species (Pakeman 2014). We chose this measure because other (unweighted) multivariate trait measures are more sensitive for an underestimation of diversity when traits are not sampled for all species.

\section{Statistical analyses}

Our aim was to evaluate the effects of abiotic and biotic factors on productivity and stocks of biomass and SOM (i.e., the 'response variables'), as shown in Fig. 3.1. One could think of many variables and interactions between variables to affect the response variable. However, to limit the number of possible models and the number of explanatory factors per model, we only evaluated the framework corresponding to our a priori hypotheses (see Fig. 3.1). To test this framework, we used structural equation modelling (SEM), which is based on regression analyses and allows to test multivariate and hierarchical relations (Laughlin et al. 2007). For each response variable, multiple models with different combinations of variables representing the abiotic and biotic factors were possible; we had one variable to represent species diversity and disturbance, but multiple variables for soil fertility and trait composition. From these combinations per response variable, we selected one SEM with the combination of variables that resulted in the highest explained variation $\left(\mathrm{R}^{2}\right)$ of the response variable. For details on model selection and refinement, see Appendix 3.4.

To understand the importance of environmental filtering, we evaluated effects of the four soil fertility variables $\left(\mathrm{N}_{\text {soil, }}, \mathrm{P}_{\text {soil, }}, \mathrm{C}: \mathrm{N}_{\text {soil }}\right.$, and $\left.\mathrm{N}: \mathrm{P}_{\text {soil }}\right)$ and disturbance on functional trait dispersion (Fdis), using 'all subsets regression analysis' followed by model averaging (see Appendix 3.4). All analyses were performed in R 2.15.2. Structural equation modelling was performed using the sem function of the lavaan package (Rosseel 2012). 


\section{Results}

Aboveground biomass productivity and stocks of biomass and soil organic matter (SOM) varied strongly among plots, with an average aboveground productivity of 6 $\pm 1.5 \mathrm{Mg} \mathrm{ha}^{-1} \mathrm{yr}^{-1}$ (average \pm standard error), an average aboveground biomass of $367 \pm 90 \mathrm{Mg} \mathrm{ha}^{-1}$, an average belowground fine root biomass (in the top $20 \mathrm{~cm}$ of the soil) of $17 \pm 4 \mathrm{Mg} \mathrm{ha}^{-1}$, and an average SOM (in the top $10 \mathrm{~cm}$ of the soil) of 66 $\pm 14 \mathrm{Mg} \mathrm{ha}^{-1}$ (Table 3.2).

We evaluated the effects of abiotic and biotic factors on productivity and stocks of biomass and SOM (Fig. 3.1). For results on model selection and refinement, see Appendix 3.4. For leaf and soil, we pre-selected 1 or 2 variables for each model with highest relative importance value (Appendix 3.6). For aboveground productivity, $\mathrm{P}_{\text {soil }}$ was the only selected soil variable, and SLA and $\mathrm{P}_{\text {leaf }}$ were selected as leaf variables in the SEM. $\mathrm{N}_{\text {leaf }}$ had a relative importance value slightly lower than $\mathrm{P}_{\text {leaf }}$ and was not evaluated in the SEM. The best selected SEM (with the highest $\mathrm{R}^{2}$ for productivity) showed a negative effect of SLA and positive effects of $\mathrm{P}_{\text {soil }}$ and disturbance on productivity (Fig. 3.2a, Table 3.3).

For aboveground biomass stocks, only $\mathrm{P}_{\text {soil }}$ and $\mathrm{P}_{\text {leaf }}$ were evaluated in the SEM, and showed positive effects of $\mathrm{P}_{\text {soil }}$ and $\mathrm{P}_{\text {leaf }}$ and a negative effect of disturbance on aboveground biomass stocks (Fig. 3.2b, Table 3.3).

For fine root biomass, only $\mathrm{N}_{\text {soil }}$ and C: $\mathrm{N}_{\text {leaf }}$ were evaluated in best SEM and showed a positive effect of $\mathrm{N}_{\text {soil }}$, but negative effects of disturbance, C: $\mathrm{N}_{\text {leaf }}$ and species richness on fine root biomass stocks (Fig. 3.2c, Table 3.3). All these three models showed a positive effect of soil fertility on species richness and a negative effect of disturbance on species richness. For SOM, $\mathrm{N}_{\text {litter }}$ was included in the SEM and was the only variable significantly reducing SOM (Fig. 3.2d, Table 3.3).

The test for environmental filtering on functional trait diversity showed that $\mathrm{P}_{\text {soil }}$ and $\mathrm{N}: \mathrm{P}_{\text {soil }}$ significantly positively related with Fdis (Appendix 3.5).

Table 3.2: The four response variables (aboveground productivity, aboveground biomass, fine root biomass, and soil organic matter) with description, units, mean and standard deviation (SD).

\begin{tabular}{|c|c|c|c|c|}
\hline Response variable & Description & Units & Mean & SD \\
\hline $\begin{array}{l}\text { Aboveground } \\
\text { productivity }\end{array}$ & Gross biomass increase & $\mathrm{Mg} \mathrm{ha}^{-1} \mathrm{y}^{-1}$ & 6.3 & 1.5 \\
\hline Aboveground biomass & Stem and crown biomass & $\mathrm{Mg} \mathrm{ha}^{-1}$ & 367.4 & 89.7 \\
\hline Fine root biomass & $\begin{array}{l}\text { Root biomass in top } 20 \mathrm{~cm} \text { of the } \\
\text { soil }\end{array}$ & $\mathrm{Mg} \mathrm{ha}^{-1}$ & 17.3 & 3.9 \\
\hline Soil organic matter & $\begin{array}{l}\text { Soil organic matter in top } 10 \mathrm{~cm} \text { of } \\
\text { the soil }\end{array}$ & $\mathrm{Mg} \mathrm{ha}^{-1}$ & 66.3 & 14.4 \\
\hline
\end{tabular}


Table 3.3: Results for the four structural equation models (SEMs) of aboveground productivity, aboveground biomass, fine root biomass, and soil organic matter (see also Fig. 3.2), to evaluate the effects of various abiotic and biotic factors. The regression coefficients (Coef), standardized regression coefficients (Std. coef), Z-values and P-values are given for all regressions (i.e., all arrows in Fig. 3.2), and the $\mathrm{R}^{2}$ of the endogenous variables (i.e., variables that are affected by other variables: productivity and stocks of biomass and soil organic matter, species richness, and trait composition)). All four models were accepted $\left(P=0.87,0.86,0.07,0.99\right.$, and $\chi^{2}=0.03,0.03$, 3.3, 0.30 for productivity, aboveground biomass, fine root biomass, and soil organic matter, respectively; Appendix 3.7). For trait abbreviations, see Table 3.1.

\begin{tabular}{|c|c|c|c|c|c|}
\hline SEM response variable & SEM predictor variable & Coef & Std. coef & $\mathrm{Z}$ & $\mathbf{P}$ \\
\hline \multicolumn{6}{|l|}{ Aboveground biomass productivity } \\
\hline \multirow[t]{4}{*}{ Productivity } & Disturbance & 0.01 & 0.39 & 2.70 & 0.007 \\
\hline & $\mathrm{P}_{\text {soil }}$ & 58.46 & 0.51 & 3.35 & 0.001 \\
\hline & Richness & -0.01 & -0.14 & -0.85 & 0.396 \\
\hline & SLA & -0.02 & -0.27 & -2.02 & 0.043 \\
\hline \multirow[t]{2}{*}{ Richness } & Disturbance & -0.11 & -0.34 & -2.18 & 0.029 \\
\hline & $P_{\text {soil }}$ & 714.38 & 0.42 & 2.70 & 0.007 \\
\hline \multirow[t]{2}{*}{ SLA } & Disturbance & 0.02 & 0.04 & 0.21 & 0.831 \\
\hline & $\mathrm{P}_{\text {soil }}$ & 98.79 & 0.05 & 0.25 & 0.803 \\
\hline $\mathrm{R}^{2}$ Productivity & & 0.45 & & & \\
\hline $\mathrm{R}^{2}$ Richness & & 0.29 & & & \\
\hline $\mathrm{R}^{2} \mathrm{SLA}$ & & $<0.01$ & & & \\
\hline \multicolumn{6}{|l|}{ Aboveground biomass } \\
\hline \multirow[t]{4}{*}{ Aboveground biomass } & Disturbance & -0.02 & -0.70 & -6.87 & $<0.001$ \\
\hline & $\mathrm{P}_{\text {soil }}$ & 31.81 & 0.28 & 2.61 & 0.009 \\
\hline & Richness & -0.01 & -0.14 & -1.27 & 0.203 \\
\hline & $P_{\text {leaf }}$ & 0.06 & 0.49 & 5.10 & $<0.001$ \\
\hline \multirow[t]{2}{*}{ Richness } & Disturbance & -0.11 & -0.34 & -2.18 & 0.029 \\
\hline & $\mathrm{P}_{\text {soil }}$ & 714.38 & 0.42 & 2.70 & 0.007 \\
\hline \multirow[t]{2}{*}{$P_{\text {leaf }}$} & Disturbance & 0.00 & 0.02 & 0.12 & 0.905 \\
\hline & $\mathrm{P}_{\text {soil }}$ & 66.43 & 0.07 & 0.38 & 0.701 \\
\hline $\mathrm{R}^{2}$ Aboveground biomass & & 0.73 & & & \\
\hline $\mathrm{R}^{2}$ Richness & & 0.29 & & & \\
\hline $\mathrm{R}^{2} \mathrm{P}_{\text {leaf }}$ & & 0.01 & & & \\
\hline \multicolumn{6}{|l|}{ Fine root biomass } \\
\hline \multirow[t]{4}{*}{ Fine root biomass } & Disturbance & -0.01 & -0.36 & -2.72 & 0.007 \\
\hline & $\mathrm{N}_{\text {soil }}$ & 4.09 & 0.47 & 3.38 & 0.001 \\
\hline & Richness & -0.03 & -0.44 & -2.97 & 0.003 \\
\hline & C: $\mathrm{N}_{\text {soil }}$ & -0.13 & -0.48 & -3.84 & $<0.001$ \\
\hline \multirow[t]{2}{*}{ Richness } & Disturbance & -0.10 & -0.31 & -2.11 & 0.035 \\
\hline & $\mathrm{N}_{\text {soil }}$ & 57.61 & 0.48 & 3.25 & 0.001 \\
\hline \multirow[t]{2}{*}{ C: $\mathrm{N}_{\text {soil }}$} & Disturbance & 0.03 & 0.28 & 1.63 & 0.104 \\
\hline & $\mathrm{N}_{\text {soil }}$ & -3.03 & -0.10 & -0.56 & 0.578 \\
\hline $\mathrm{R}^{2}$ Fine root biomass & & 0.57 & & & \\
\hline $\mathrm{R}^{2}$ Richness & & 0.35 & & & \\
\hline $\mathrm{R}^{2} \mathrm{C}: \mathrm{N}_{\text {leaf }}$ & & 0.09 & & & \\
\hline
\end{tabular}


Soil organic matter

Soil organic matter

$\begin{array}{lllll}\text { Disturbance } & 0.00 & 0.11 & 0.68 & 0.500 \\ \text { Litter quantity } & 0.01 & 0.13 & 0.76 & 0.450 \\ \mathrm{~N}_{\text {litter }} & -0.04 & -0.37 & -2.19 & 0.028 \\ \text { Productivity } & -0.23 & -0.17 & -0.94 & 0.346 \\ \text { Productivity } & -0.12 & -0.09 & -0.52 & 0.606 \\ & 0.16 & & & \\ & 0.03 & & & \\ & 0.01 & & & \end{array}$

a)

Litter quantity

$\mathrm{N}_{\text {litter }}$

$\mathrm{R}^{2}$ Soil organic matter

$\mathrm{R}^{2}$ Litter quantity

$\mathrm{R}^{2} \mathrm{~N}_{\text {litter }}$

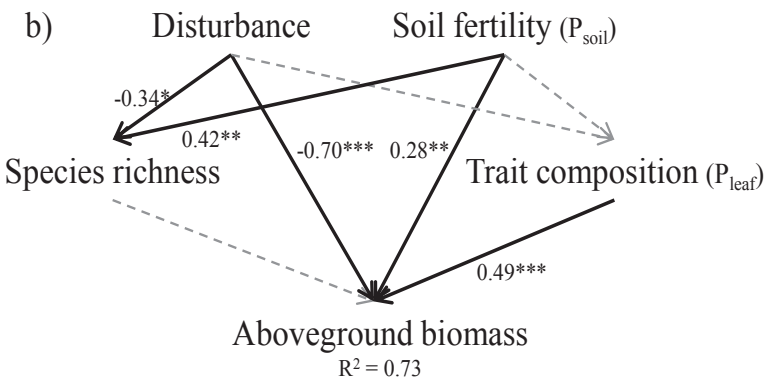

d) Aboveground biomass productivity

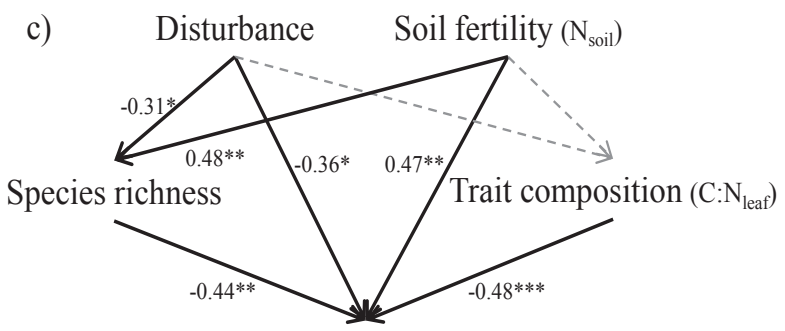

Fine root biomass $\mathrm{R}^{2}=0.57$

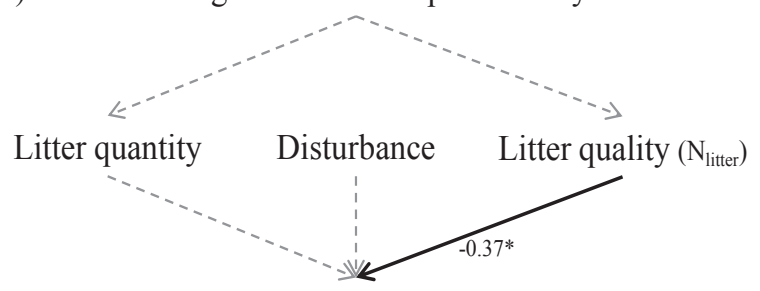

Soil organic matter

Figure 3.2: Structural equation models for aboveground biomass productivity (a), aboveground biomass (b), fine root biomass (c), and soil organic matter (d). For aboveground biomass productivity, aboveground biomass and fine root biomass, direct and indirect effects of disturbance, soil fertility, rarefied species richness (per 100 stems), and trait composition (i.e., a community-weighted mean stem or leaf trait) were evaluated. For soil organic matter, direct and indirect effects of litter quantity, litter quality, disturbance and productivity were evaluated. All four models were accepted (Appendix 3.7). For all relations that were significant (continuous black lines), the beta coefficient and significance level are given $(*=\mathrm{P}<0.05$, $* *=\mathrm{P}<0.01$, *** $=\mathrm{P}<0.001$ ), and for all non-significant relations (grey, dashed lines), no statistics are shown. $\mathrm{R}^{2}$ values show the explained variance of the ultimate response variables. The variables between brackets for soil fertility and trait composition are the variables that were selected to best predict productivity, biomass, or soil organic matter. For more statistics of the structural equation models, see Table 3.3 . 


\section{Discussion}

We evaluated the effects of abiotic and biotic factors on productivity, biomass stocks (aboveground biomass and fine root biomass), and soil organic matter (SOM) stocks. Soil P increased aboveground productivity, biomass stocks, and species richness, whereas soil N increased fine root biomass stocks. Surprisingly, species richness did not increase productivity and biomass stocks. A more acquisitive trait composition increased biomass stocks but decreased productivity, which is in contrast with current trait paradigms (Reich 2014). These results indicate that mass-ratio and soil nutrient availability, rather than niche complementarity, determine productivity and biomass in this tropical rainforest.

\section{Soil fertility - especially $\mathrm{P}$ - shapes productivity and biomass stocks}

Soils on the Guiana shield are old and leached, and as a result nutrient poor. We therefore expected that soil fertility would strongly determine productivity and biomass stocks. Soil fertility was indeed a strong predictor for productivity and biomass stocks (Fig. 3.2 and 3.3b, f, j), indicating that, as expected, this forest is limited by soil nutrients at the 0.4-ha scale. Average productivity among the undisturbed plots (4.9 Mg ha $\mathrm{yr}^{-1}$ ) was a bit lower than the average productivity of Neotropical forests (around 5.5 Mg ha $\mathrm{yr}^{-1}$; Brienen et al. 2015), which also supports the idea that in Guyana low soil fertility limits productivity. At the 1-ha scale, we found weaker effects of soil fertility on various biomass stocks in this forest (results not shown) and in a Bolivian moist forest (chapter 4), possibly because at the 1-ha scale, smaller-scale heterogeneity in soil fertility is averaged out and plots do not strongly differ anymore in their average soil fertility. However, across 0.1-ha plots in a secondary forest in Brazil, forest type also strongly determined plot basal area and species diversity (Martins et al. 2015). Soil effects on biomass productivity and stocks are thus scale-dependent and it is therefore of paramount importance to define the relevant scale for the ecological question at hand. Other studies also show positive effects of soil fertility on productivity and aboveground biomass across Neotropical forests (Malhi et al. 2004, Baraloto et al. 2011) and on fine root biomass in temperate forests (Valverde-Barrantes et al. 2015).

Both nitrogen $(\mathrm{N})$ and phosphorus $(\mathrm{P})$ are important for plant growth (Santiago et al. 2012), but the relation between these nutrients remains largely unclear. The old and leached soils in Guyana may be particularly limited in phosphorus $\left(\mathrm{P}_{\text {soil }}\right)$, as visible from the low $\mathrm{P}_{\text {soil }}$ in our forest $(0.0014 \%$ in organic and easily available forms, measured by the Bray method) and other Eastern Amazonian forests (Quesada et al. 2010). Quesada et al. (2010) argue that when $\mathrm{P}_{\text {soil }}$ 
is very low, most of the soil nitrogen $\left(\mathrm{N}_{\text {soil }}\right)$ cannot be used and gets lost, eventually resulting in a $\mathrm{N}$ deficiency. This can happen because at low $\mathrm{P}_{\text {soil }}$ availability, the lignin concentration of the litter is high and the litter decomposition rate is low (Hirobe et al. 2004), leading to a low rate at which $\mathrm{N}$ becomes available. Hence, low $\mathrm{P}_{\text {soil }}$ could lead to $\mathrm{N}$ deficiency. However, $\mathrm{N}_{2}$-fixing tree species are relatively abundant in forests of the Guianas (Roggy and Prévost 1999), and therefore $\mathrm{N}_{\text {soil }}$ may be more readily available than $\mathrm{P}_{\text {soil }}$. $\mathrm{N}_{2}$-fixing species produce more $\mathrm{N}$ requiring phosphatases, which help to mineralize organic P. Moreover, $\mathrm{N}_{2}$-fixing species can sustain larger colonies of mycorrhizal fungi that help to absorb $\mathrm{P}$ (Nasto et al. 2014). In line with this idea, Ter Steege et al. (2006) found that the proportion of trees belonging to ectomycorrhizal genera is higher in Guyana compared to the rest of the Amazon. Hence, rather than $\mathrm{P}$ determining $\mathrm{N}$ availability, it may be that $\mathrm{N}$ stimulates $\mathrm{P}$ uptake from the soil.

P-limitation rather than $\mathrm{N}$-limitation in this forest, is further supported by three findings: 1 ) the N:P of soil is higher than that of litter (718 vs. 54), indicating that relatively more $\mathrm{P}$ than $\mathrm{N}$ is taken up by plants and other organisms; 2 ) the N:P of litter is higher than that of green leaves (54 vs. 38), indicating efficient P resorption before leaf senescence (Vitousek 1984, McGroddy et al. 2004, Zhang et al. 2015); and 3) the \% plot basal area covered by Fabaceae species (of which many can fix $\mathrm{N}_{2}$ ) is strongly positively correlated with community-weighted mean (CWM) $\mathrm{P}_{\text {leaf }}(\mathrm{r}=0.65, \mathrm{P}<0.001)$ but not with $\mathrm{CWM} \mathrm{N}_{\text {leaf }}(\mathrm{r}=0.12, \mathrm{P}=0.52)$. This suggests that a higher abundance of $\mathrm{N}_{2}$-fixing species allows for more nitrogen fixation which is used to support mycorrhizae that enhance P uptake and storage in leaves. For a site close to our study area, Raaimakers (1994) also showed that P limits growth. Interestingly, $\mathrm{P}_{\text {soil }}$ was the most important soil fertility variable for aboveground productivity and aboveground biomass stocks, whereas $\mathrm{N}_{\text {soil }}$ was more important for belowground biomass stocks in fine roots (Fig. 3.2). This supports the idea that $\mathrm{P}$ is the most limiting element in this forest, but that it is $\mathrm{N}$ that stimulates root biomass which is needed for $\mathrm{P}$ absorption. Thus, in this Guyanese forest, it is most likely that $\mathrm{P}$ rather than $\mathrm{N}$ limits productivity and biomass stocks.

\section{Soil fertility increases, but disturbance decreases diversity}

At high soil fertility, the few most competitive species should outcompete other species and thus diversity would be low (Huston 1979). Contrary to this hypothesis, we found that soil fertility increased species richness. However, even the most fertile plot in this forest is still relatively nutrient poor. Possibly, an increase in soil fertility provides the opportunity for non- $\mathrm{N}_{2}$-fixing species to establish, and therefore has a positive effect on species richness. 

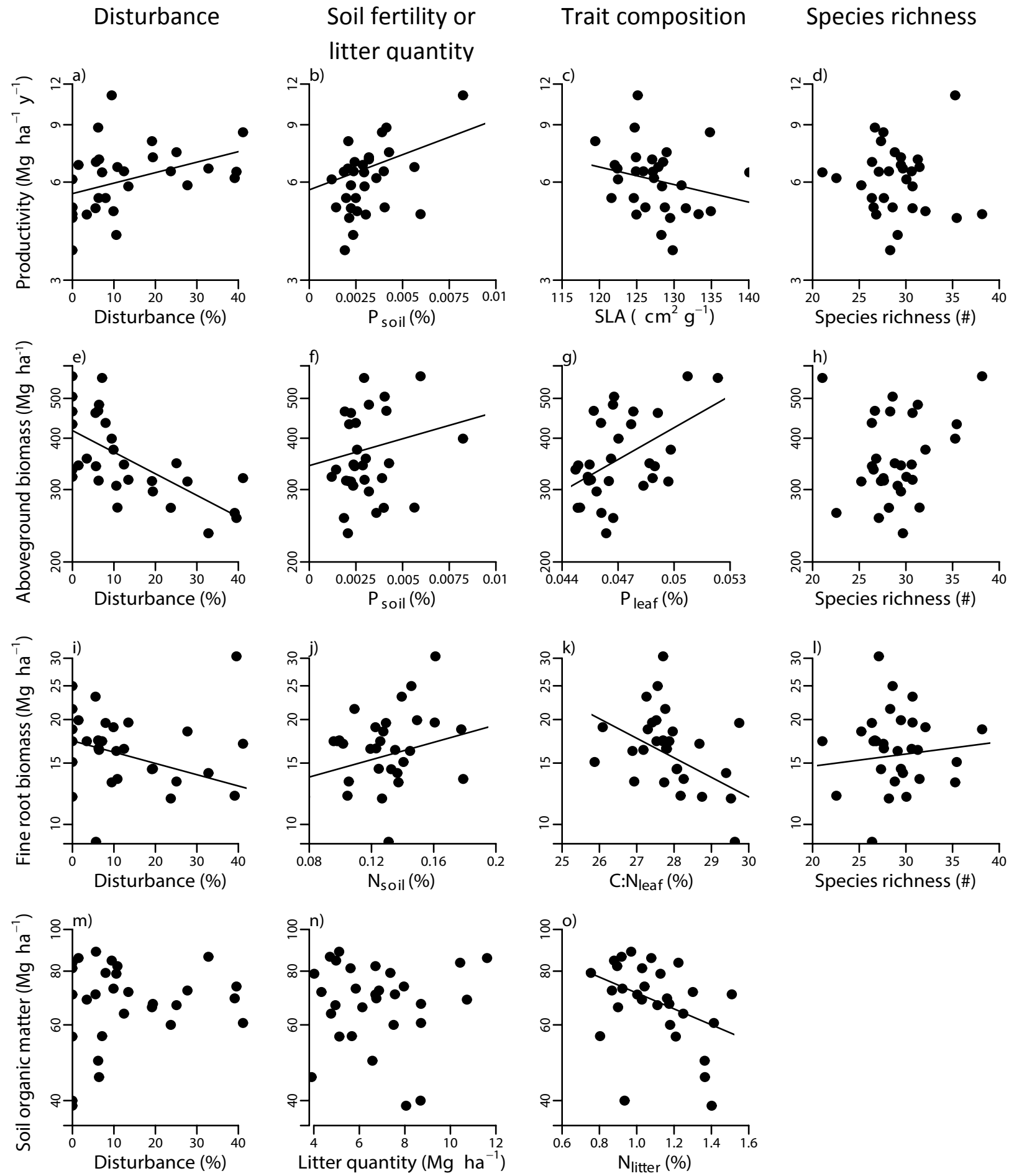

Figure 3.3: Bivariate relations of disturbance (a, e, i), soil fertility $(b, f, j)$ and litter quantity in the case of soil organic matter (n), trait composition (c, g, k, o) and rarefied species richness per 0.4 ha plot $(\mathrm{d}, \mathrm{h}, \mathrm{l})$ with aboveground biomass productivity (a-d), aboveground biomass (e-h), and fine root productivity (i-l), and soil organic matter (m-o). Each dot is one 0.4 ha plot. Regression lines are given for the relations that were significant in the structural equation models (Fig. 3.2), but are based on simple regressions and meant for illustration purposes only. SLA = specific leaf area; $\mathrm{P}_{\text {soil }}$ and $\mathrm{N}_{\text {soil }}=$ soil phosphorus and nitrogen concentration, respectively; $\mathrm{P}_{\text {leaf }}$ and $\mathrm{C}: \mathrm{N}_{\text {leaf }}=$ leaf phosphorus concentration and leaf carbon : nitrogen ratio, respectively. Note that the y-axes are in $\ln$-scale. 
The intermediate disturbance hypothesis predicts that species richness shows a hump-backed relationship with disturbance (Grime 1973, Connell 1978, Huston 1979), although this relation is generally weak for wet tropical forests (Bongers et al. 2009). We found a negative effect of disturbance on species richness, which may indicate that the disturbance intensity applied to our stands is beyond the optimum for species richness. Alternatively, the availability of more light due to disturbance should favour pioneer species that are able to make use of the extra light, but such species may be prohibited to grow well in this forest due to the low nutrient availability, or have not yet reached the $5 \mathrm{~cm}$ limit in the six years after logging.

\section{Diversity has no or sometimes negative effect on productivity and biomass stocks}

We expected that species richness would lead to facilitation and niche complementarity (or to reduced negative plant-soil feedback; Mangan et al. 2010), which would increase productivity, and hence accumulated (above- and belowground) biomass. However, this effect could be weak for this forest in Guyana, where soil fertility and growth rates are low and relatively few species are very abundant. On the other hand, an increased number of $\mathrm{N}_{2}$-fixing species, which facilitate uptake and availability of $\mathrm{P}$ for the whole community, could stimulate stand-level productivity and biomass stocks. We found that species richness did not have a significant effect on aboveground productivity and biomass stocks, and it even had a significantly negative effect on fine root biomass stocks (Fig. 3.2 and 3.3d, h, l). This contradicts with positive effects of richness on productivity for various ecosystem types (Tilman et al. 2001, Balvanera et al. 2006, Paquette and Messier 2011), a positive effect of species richness on aboveground biomass stocks across a wide range of Neotropical forests (Poorter et al. 2015), and a positive effect of phylogenetic diversity on fine root biomass stocks in temperate forests (Valverde-Barrantes et al. 2015). It could be that a positive effect of diversity is present at large spatial scales (e.g., regional and continental) where variation in species richness is stronger (Chisholm et al. 2013, Poorter et al. 2015), in systems where diversity is very low and less redundancy may occur, such as in temperate forests (Walker 1992), and in systems where growth rate is higher and diversity reduces species competing for resources.

When evaluating the single effect of species richness on fine root biomass (e.g., in a single regression analysis), its effect is not significant (see also the weak relationship in Fig. 3.31). This indicates that, to understand processes in the field where many variables are at play, a multivariate approach should be taken (see conceptual Fig. 3.1) to disentangle the contribution of species richness to ecosystem functioning. The negative effect of richness on belowground fine root biomass stocks in the structural equation model (SEM) may be caused by relatively 
few species that can cope well with low nutrient availability and produce high amounts of fine root biomass. This idea is supported by the positive effect of soil fertility on species richness, indicating that more species are able to occur when soil conditions are less limiting.

\section{Mass-ratio effects drive productivity and stocks of biomass and soil organic matter}

Trait composition, i.e. the traits of an average tree in the forest, should reflect abiotic and biotic conditions and ultimately drive the biomass stocks and growth of the forest, as predicted by the mass-ratio hypothesis (Grime 1998). Trait values representing 'acquisitive' strategies (e.g., high leaf nutrient concentrations and low wood density) increase resource use efficiency and should result in higher productivity. We indeed found that trait composition affected all four response variables (Fig. 3.2 and 3.3c, g, k, o). Surprisingly, however, productivity decreased with community-weighted mean (CWM) specific leaf area (SLA), indicating that forests with high abundance of 'conservative' (i.e., the contrast of acquisitive) species attained a higher productivity (Fig. 3.2a and 3.3c). This is contrary to our expectations, and to other studies that show positive effects of CWM SLA or other acquisitive trait values on productivity (Baker et al. 2009, Finegan et al. 2015). Compared to other Amazonian forests, this forest in Guyana is nutrient poor (Quesada et al. 2010), composed of a small number of dominant species (Arets 2005), and possesses on average very conservative trait values (ter Steege and Hammond 2001). Such conservative trait values allow trees to retain scarce (soil) resources and enhance nutrient residence time in the plants (Zhang et al. 2015). Conservative trait values (such as low $\mathrm{N}_{\text {leaf }}$ ) may result in less respiration (Poorter and Bongers 2006) and in this way enhance net carbon gain. Conservative trait values such as high wood density also protect the plants better against physical and biotic hazards, thus enhancing plant survival (van Gelder et al. 2006) and therefore also stand productivity. In a tropical dry forest, a conservative trait composition also increased productivity (Prado-Junior et al. 2016). Hence, whereas current trait paradigms (which predict that acquisitive trait values increase ecosystem process rates) may hold for most tropical forests, these relations may be contrary - with conservative traits enhancing productivity - for tropical forests growing under limiting resource availability (nutrients, water, light). Additionally, these trait paradigms might hold across large regions with very wide variation in site conditions and traits, but not within a particular site.

Acquisitive trait values are associated with short lifespan of tissues (leaves, roots), and should therefore lead to increased turnover and decreased biomass stocks (Reich 2014). Surprisingly, we found that acquisitive trait values result in 
increased biomass stocks; $\mathrm{P}_{\text {leaf }}$ had a positive effect on aboveground biomass stocks and C: $\mathrm{N}_{\text {leaf }}$ (i.e., high relative $\mathrm{C}$ content) had a negative effect on fine root biomass stocks. This finding is in agreement with some studies (Conti and Díaz 2013, Loiola et al. 2015) but in contrast with others (Falster et al. 2011). Acquisitive trait values can decrease biomass stocks due to a higher turnover, and hence, shorter residence time of the biomass (Galbraith et al. 2013), or they can increase biomass stocks due to higher potential build-up caused by a higher productivity (Chisholm et al. 2013). This last option may be relevant especially when (soil) resources are strongly limiting and acquisitive trait values (such as high nutrient concentrations) indicate an increased availability and/or uptake of nutrients, and thus an increase in the build-up of biomass. The importance of $\mathrm{P}_{\text {leaf }}$ for aboveground biomass stocks and of C: $\mathrm{N}_{\text {leaf }}$ for fine root biomass stocks is in line with the findings for the soil nutrients, and indicate that $\mathrm{P}$ may mainly limit aboveground biomass processes because it is the most limiting element, whereas $\mathrm{N}$ may limit belowground biomass processes because it is needed for $\mathrm{P}$ uptake by the roots.

\section{Environmental filtering through low soil fertility?}

The importance of mass ratio (i.e., the traits of the dominant species) for biomass stocks and productivity can indicate that there is strong environmental filtering (Keddy 1992), which means that co-occurring species share similar trait strategies because of strong environmental constraints (ter Steege and Hammond 2001). We indeed found that $\mathrm{P}_{\text {soil }}$ and $\mathrm{N}: \mathrm{P}_{\text {soil }}$ increase Fdis, suggesting that low absolute $\mathrm{P}$ and relative $\mathrm{N}$ availability select for low multivariate trait diversity. This result, combined with the low $\mathrm{P}_{\text {soil }}$ and $\mathrm{P}_{\text {leaf }}$ values, and the strong effects of soil fertility and leaf trait composition on productivity and stocks of biomass and SOM, indicate that our forest is severely constrained by $\mathrm{P}$ availability, and that this strongly limits the number and type of species that are abundant.

\section{High litter nutrient concentrations decrease soil organic matter}

Soil organic matter (SOM) stock was only explained by litter quality (Fig. 3.2d and 3.3o), which is in line with the mass-ratio hypothesis. Litter $\mathrm{N}$ had a negative effect on SOM, because litter with high $\mathrm{N}$ is easily decomposed, resulting in a reduction of litter and organic matter in the soil (Melillo et al. 1982, Wardle et al. 2002). Disturbance could have a positive effect on SOM (as disturbance can increase decomposition, which in turn increases stabilization of physically or chemically protected SOM fractions, and hence, the residence time of SOM; von Lützow et al. 2006, Hoosbeek and Scarascia-Mugnozza 2009), or a negative effect on SOM (as disturbance leads to more open forests that are drier and to a disruption of the microbial community, which would reduce decomposition and therefore increase SOM; van Dam, 2001). Instead, we found that disturbance had no effect on SOM, 
perhaps because disturbance effects are time-dependent. In our forest, disturbance happened 20 years before the collection of SOM data, and therefore the forest canopy should have closed and the microbial community should have recovered. SOM did also not depend on the litter quantity that can potentially reach the soil as SOM. Hence, SOM is determined by decomposition rates that are in turn driven by litter quality, as predicted by the mass-ratio hypothesis.

\section{Conclusions - mechanisms driving productivity and stocks of biomass and soil organic matter}

Soils on the Guiana shield are highly weathered and nutrient poor, and the forests are relatively mono-dominant with a conservative trait composition (ter Steege \& Hammond 2001). We evaluated which abiotic and biotic factors drive variation in forest productivity and stocks of biomass and SOM. $\mathrm{P}_{\text {soil }}$ strongly increased aboveground productivity and aboveground biomass stocks, whereas $\mathrm{N}_{\text {soil }}$ increased belowground biomass stocks in fine roots. This indicates that $\mathrm{P}$ is the most limiting element in this forest, and that $\mathrm{N}$ availability stimulates root biomass and $\mathrm{P}$ absorption. Moreover, an acquisitive trait composition increased biomass stocks, possibly because it indicates higher availability of soil nutrients and thus increases biomass build-up, but it decreased productivity, possibly because conservative trait values result in less respiration and biomass loss under harsh conditions. Species richness, on the other hand, did not increase productivity and biomass stocks. Hence, we found evidence for the mass-ratio hypothesis but not for the niche complementarity hypothesis. All these results indicate that this forest is severely constrained by $\mathrm{P}$ availability, which may impose strong environmental filtering and as a result limit the number and type of species that are abundant. Hence, soil fertility and species traits, but not species diversity, drive productivity and stocks of biomass and SOM in this Guyanese tropical rainforest.

\section{Acknowledgements}

The research leading to these results has received partial funding from the European Union Seventh Framework Programme (FP7/2007-2013) under grant agreement $\mathrm{n}^{\circ} 283093$ - The Role Of Biodiversity In climate change mitigatioN (ROBIN). This research is part of the strategic research program KBIV (KB-14) "Sustainable spatial development of ecosystems, landscapes, seas and regions", funded by the Dutch Ministry of Economic Affairs and carried out by Wageningen University \& Research centre (project code KB-14-003-030). We are grateful to the Guyana Forestry Commission for logistical support and support of the fieldwork, and to the Environmental Protection Agency for providing the necessary fieldwork permits. Specifically, we thank Benedict Harry for field assistance and species identification, Karlon Warde for logistical support of the fieldwork, and Rehaaz Mohamed for helping with the collection of soil, root and litter samples. We furthermore thank MSc students Jemberu Biru for help with the collection of leaf traits, and Tereza Nermcová for the collection of soil, root and litter samples. And last, we thank Eef Velthorst for doing all chemical analyses of soil, root and litter in the laboratory at Wageningen University, the Netherlands. 


\section{Appendices}

Appendix 3.1: Collection and calculation of soil organic matter, fine root biomass, nutrient concentrations, and litter variables.

The plots are located on slightly undulating sedimentary interfluves (i.e., relatively flat surfaces in between drainage tributaries), allowing two sampling points per 0.4 ha plot: one towards the north and one towards the south end (van Kekem et al. 1996, van der Hout 1999, Soil Survey Manual 1993). Root biomass was additionally collected at an intermediate point in each plot. Soil samples were collected in October-November 2013. Per sampling point, three soil samples were taken between $0-5 \mathrm{~cm}$ for root mass, bulk density, and nutrient concentrations, and also one between $15-20 \mathrm{~cm}$ for root mass, using bulk density rings of $100 \mathrm{~cm} 3$ volume $(\mathrm{r}=2.5 \mathrm{~cm}$ and $\mathrm{h}$ $=5.093 \mathrm{~cm})$. We did not collect data for the decomposer community.

The two samples for fine root mass at each sampling point (at 0-5 and 15-20 cm depth) were sieved to $1 \mathrm{~mm}$, oven-dried for 48 hours at $70{ }^{\circ} \mathrm{C}$, and weighted. Fine root mass at each sampling point was expressed in $\mathrm{g} \mathrm{cm}^{-3}$. For each 0.4-ha plot, we estimated the fine root biomass in the top $20 \mathrm{~cm}$ of the soil by applying an exponential formula to the six sampling points (two at each depth, for three points per 0.4-ha plot), and integrating that formula to calculate the root biomass between 0 and $20 \mathrm{~cm}$ depth. For this we assumed an exponential decline in root mass with depth rather than a linear decline (Gale and Grigal 1987, Jackson et al. 1996) using the formula $a * e^{b * d e p t h}$, with depth in $\mathrm{cm}$. Per plot, we estimated the unknown a and $\mathrm{b}$ using the $\mathrm{R}$ function nls and integrated the formula using the $\mathrm{R}$ function integrate, both from the stats package (R Core Team 2014). This value of root mass per plot was scaled to $\mathrm{Mg} \mathrm{ha}^{-1}$ of $20 \mathrm{~cm}$ depth $(2000 \mathrm{~m} 3)$, in order to compare values with aboveground productivity and biomass stocks that are also expressed in $\mathrm{Mg} \mathrm{ha}^{-1}$.

For soil organic matter (SOM) and soil fertility, we used two samples from the upper soil layer $(0-5 \mathrm{~cm})$ at the two sampling points per 0.4-ha plot. One soil sample per point was ovendried for 48 hours at $104{ }^{\circ} \mathrm{C}$, after which dry mass was measured. Dry mass was divided by 100 $\mathrm{cm} 3$ to obtain bulk density in $\mathrm{g} \mathrm{cm}^{-3}$. The second sample of each sampling point was stored in zip-lock bags under cool temperatures (in a small creek) before they were shipped to the soil lab at Wageningen University, the Netherlands. Here, soil samples were analysed for concentrations of total organic carbon $(\mathrm{C})$ and nitrogen $(\mathrm{N})$ and organic and easily available phosphorus $(\mathrm{P})$. For $\mathrm{C}$ and $\mathrm{N}$ analyses, sub-samples were crushed by hand and roots were removed. No carbonates were present in the soil. $\mathrm{C}$ and $\mathrm{N}$ were determined with an elemental analyzer (Interscience EA 1108) (van Lagen 1996). For P analyses, samples were first digested by addition of a seleniumsulphuric acid mixture and peroxide while heated to $330{ }^{\circ} \mathrm{C}$ (Gerhardt Kjeldatherm digestion system), after which they were diluted and $\mathrm{P}$ was determined colorimetrically (spectrophotometer Mechatronics Starrcol SC-60-S at $720 \mathrm{~nm}$ ), following the description by Novozamsky et al. (1983). This method is comparable to the Bray method for $\mathrm{P}$ analyses. Soil carbon, nitrogen and phosphorus concentrations $\left(\mathrm{C}_{\text {soil }}, \mathrm{N}_{\text {soil }}\right.$, and $\mathrm{P}_{\text {soil }}$, respectively) were expressed in $\%$ and averaged per 0.4-ha plot. We used Nsoil, Psoil, and the ratios between $\mathrm{C}_{\text {soil }}: \mathrm{N}_{\text {soil }}$ and $\mathrm{N}_{\text {soil }}: \mathrm{P}_{\text {soil }}$ as proxies for soil fertility, because total organic $\mathrm{N}_{\text {soil }}$ represents the $\mathrm{N}$ that is available for mineralization, Psoil in organic and easily available forms represents the potential pool of $\mathrm{P}$ for mineralization, and the ratios may indicate relative nutrient limitation (Koerselman and Meuleman 1996).

Bulk density $\left(\mathrm{g} \mathrm{cm}^{-3}\right)$ was first scaled to a 0-10 $\mathrm{cm}$ soil depth increment, and then scaled to represent $\mathrm{Mg} \mathrm{ha}{ }^{-1}$. This value was multiplied by the fraction of carbon in the soil to obtain $\mathrm{MgC}$ 
$\mathrm{ha}^{-1}$ in the $10 \mathrm{~cm}$ topsoil. The two sampling points per subplot were averaged and multiplied by 1.9 to scale from C content to SOM (Nelson and Sommers 1982).

At the same two sampling points per plot, also litter was collected. A circular frame with an inner diameter of $19 \mathrm{~cm}$ was placed on a representative piece of the forest floor (within a radius of $50 \mathrm{~cm}$ from the sampling point), pushed down onto the mineral soil, and litter was vertically cut to include only litter inside the frame. Recent and fragmented litter was hand-picked and stored in zip-lock bags under cool temperatures before they were shipped to the lab at Wageningen University, the Netherlands. Here, litter samples were oven-dried for 48 hours at 70 ${ }^{\circ} \mathrm{C}$, weighted, analysed for concentrations of carbon, nitrogen and phosphorus as described above. Litter dry mass was expressed in $\mathrm{Mg} \mathrm{ha}^{-1}$ and litter carbon, nitrogen and phosphorus concentrations (Clitter, Nlitter and Plitter, respectively) in \%. Due to the relatively low quantity and high spatial variability of recent litter, only fragmented litter was included in further analyses.
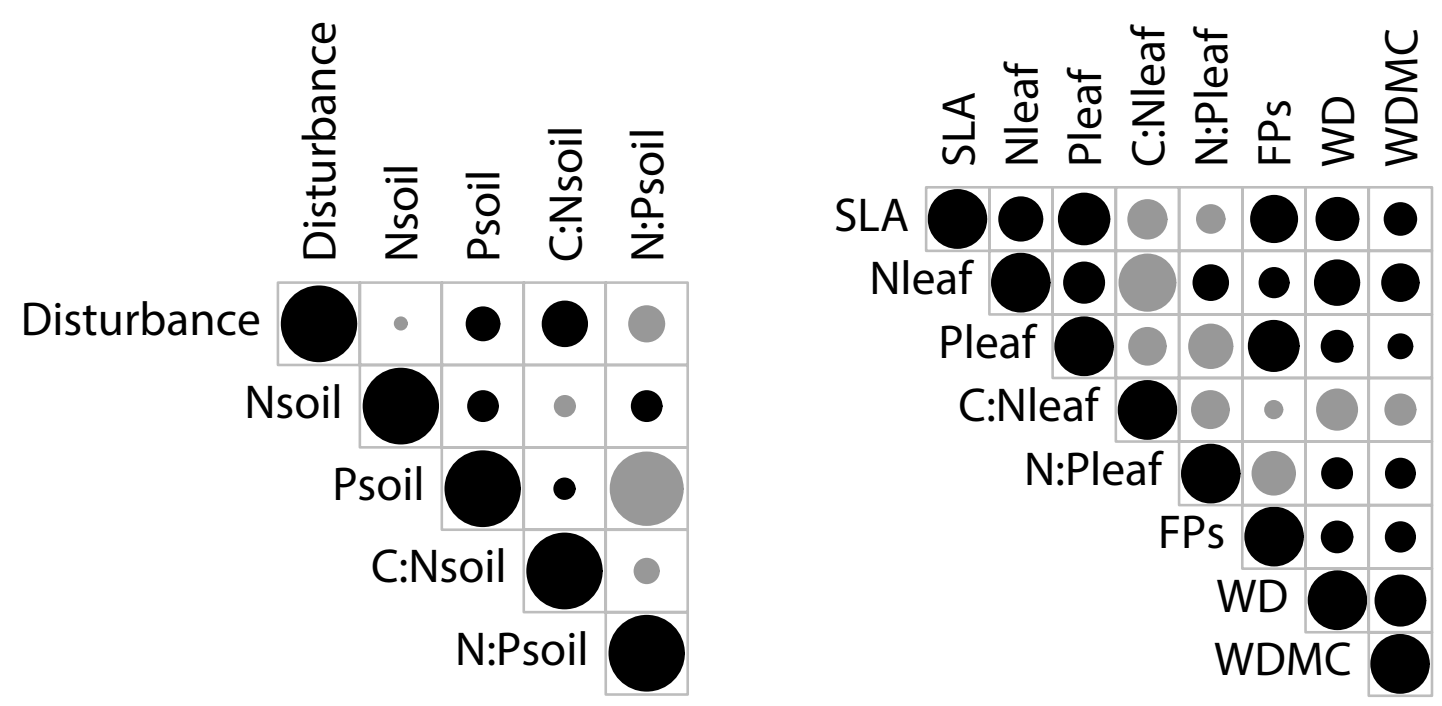

Appendix 3.2: Spearman correlations between soil variables (left graph) and communityweighted mean trait variables. Black circles indicate positive correlations and gray circles indicate negative correlations. The size of the circle indicates the strength of the correlation. For abbreviation of soil and trait variables, see Table 3.1. 
Appendix 3.3: Leaf and stem trait collection

\section{Leaf trait collection:}

We sampled leaves for the 33 most abundant tree species in terms of basal area, composing on average $78 \%$ of the basal area $(>5 \mathrm{~cm} \mathrm{DBH})$ in the 300.4 -ha plots over the two census years. For 5 individuals per species, between 7 and $17 \mathrm{~cm}$ in $\mathrm{DBH}$, we sampled 5 healthy and young but mature leaves growing at the outer side of the crown (thus in relatively high light conditions, but mostly in the understory).

Directly after collecting, we measured for each leaf the leaf area using a desktop scanner, the leaf thickness using a Mitotuyo micrometer, and the chlorophyll content using a SPAD meter (Minolta SPAD 502 Chlorophyll Meter, Spectrum Technologies Inc., Plainfield, IL, USA). For each leaf we also measured leaf toughness, which is a measure of investment in defence structures, using a penetrometer that measures the mass needed to punch the flat-ended part of a nail through the leaf (Bakker et al. 2011). The fresh mass was measured after rehydrating the leaves overnight, and the dry mass was measured after oven-drying the leaves for 48 hours at 70 ${ }^{\circ} \mathrm{C}$. Leaves were pooled per species and analysed for concentrations of carbon $\left(\mathrm{C}_{\text {leaf }}\right)$, phosphorus $\left(\mathrm{P}_{\text {leaf }}\right)$ and nitrogen $\left(\mathrm{N}_{\text {leaf }}\right)$. Sample digestion was done using the kjeldahl digestion method, and detection was done using the Nessler's reagent by UV-VIS spectrophotometer for $\mathrm{N}_{\text {leaf }}$, and the molybdenum-blue method by UV-VIS spectrophotometer for $\mathrm{P}_{\text {leaf }}$. $\mathrm{C}_{\text {leaf }}$ were determined using a Interscience elemental analyzer EA 1108.

We calculated leaf area $\left(\mathrm{cm}^{2}\right)$, including the rachis in case of compound leaves, using the software ImageJ. Specific leaf area (SLA; $\mathrm{cm}^{2} \mathrm{~g}^{-1}$ ) was then calculated by dividing the leaf area by the leaf dry mass. The values for $\mathrm{C}_{\text {leaf }}, \mathrm{N}_{\text {leaf }}$ and $\mathrm{P}_{\text {leaf }}$ (in \%) were obtained from the chemical analyses. Leaf nutrient ratios were calculated by dividing $\mathrm{C}_{\text {leaf }}$ by $\mathrm{N}_{\text {leaf }}\left(\mathrm{C}: \mathrm{N}_{\text {leaf }}\right)$ and by dividing $\mathrm{N}_{\text {leaf }}$ by $\mathrm{P}_{\text {leaf }}\left(\mathrm{N}: \mathrm{P}_{\text {leaf }}\right)$. Chlorophyll content per unit leaf area $(\mathrm{Chl})$ was calculated by translating the SPAD units into chlorophyll content per unit leaf area $(\mu \mathrm{g} \mathrm{cm}-2)$, using the formula of Coste et al. $(2010): \mathrm{Chl}=(117.1 * \mathrm{SPAD}) /(148.84-\mathrm{SPAD})$. The force needed to punch the leaf (in Newton) was calculated by multiplying the mass (in g) to punch the leaf with 0.00981 . The specific force to punch (FPs; $\mathrm{N} \mathrm{m}^{-2}$ ), was then calculated by dividing the force by the product of the circumference of the nail (in $\mathrm{m}$ ) and the thickness of the leaf (in $\mathrm{m}$ ), to correct for the fracture area on which pressure is exerted.

\section{Stem trait collection:}

We took wood samples for 25 of the 33 species using an increment borer, because wood of the remaining 8 species was too tough to sample and was therefore excluded. For three individuals per species, we took one wood core at breast height, from the outer sapwood until the pith. We aimed to select individuals that were between 20 and $40 \mathrm{~cm}$ in diameter, to include possible radial gradients in wood density, which have been found for many tropical tree species (Woodcock and Shier 2002, Plourde et al. 2014) and obtain an average wood density that more accurately describes the whole radius of the tree. However, for some species that did not grow this big or that had too tough wood at large sizes, we sampled trees of around $12-20 \mathrm{~cm}$ in diameter. Directly after collecting, the length of each core was measured and multiplied by its known radial surface to obtain fresh volume, and fresh mass was measured. The dry mass was measured after oven-drying for 48 hours at $70{ }^{\circ} \mathrm{C}$. Wood Density (WD) was calculated by dividing the dry mass of the whole core by its fresh volume $\left(\mathrm{g} \mathrm{cm}^{-3}\right)$. Species-specific WD was calculated by averaging the WD of the three individuals per species. To calculate stem dry matter content (SDMC; $\mathrm{g} \mathrm{g}^{-1}$ ), we divided the dry mass by the fresh mass per core, and averaged these per species. 
Appendix 3.4: Selection and refinement of structural equation models

\section{Procedure:}

To test our hypothesized framework (Fig. 3.1 in the main text), we used structural equation modelling (SEM). For each response variable (i.e., aboveground productivity, aboveground biomass stocks, fine root biomass stocks, or soil organic matter; SOM), multiple models with different combinations of variables representing the abiotic and biotic factors were possible. From these combinations per response variable, we finally selected one SEM with the combination of variables that resulted in the highest explained variation $\left(\mathrm{R}^{2}\right)$ of the response variable (see Fig. 3.2 in the main text). Here we describe how we selected this one model per response variable.

To limit the number of possible variables to use for the factors soil fertility and trait composition, we first made a pre-selection of 1-2 variables for each factor and per response variable. To do so, we applied 'all subsets regression analysis' (i.e., a statistical method that tests all possible combinations of predictor variables) for each of the four response variables (productivity, and stocks of aboveground biomass, belowground biomass, and SOM; Appendix 3.6). These four analyses initially included disturbance, rarefied species richness, all possible trait composition variables, and all possible soil fertility variables. $\mathrm{C}_{\text {leaf }}$ was a-priori excluded from this analysis because it differed less than 5\% among plots. We then averaged all possible models weighted by their Akaike Information Criterion. This method provides more reliable model outcomes compared to using only the single best model, because this single best model can contain other variables or variable parameters than other well-fitting models (Burnham and Anderson 2002). Based on these averaged models, for further analyses we selected the trait composition variable and soil fertility variable that had the highest importance value (which can vary between 0 and 1and which is obtained by summing the 'Akaike weights' for all models where the specific variable occurred; Barton, 2015). If the two best trait composition variables or the two best soil fertility variables differed less than 0.1 in variable importance, then we decided that their importance was rather similar and both variables were selected. For SOM, a similar analysis was performed, but using different explanatory variables: disturbance, litter quantity, and all variables for litter quality (i.e., $\mathrm{N}_{\text {litter }}, \mathrm{P}_{\text {litter }}, \mathrm{C}: \mathrm{N}_{\text {litter }}$, and $\mathrm{N}: \mathrm{P}_{\text {litter }}$ ). For litter quality, also the one or two variables with the highest relative importance were selected. The four response variables, i.e., biomass productivity and stocks of biomass and SOM, were ln-transformed to obtain equal variances and normal distribution of the residuals.

For the carbon cycle elements for which multiple trait composition and/or multiple soil fertility variables were selected, multiple SEMs were built to evaluate all selected variables. The overall fit of these models was first evaluated using a chi-square test. The models that were not rejected (i.e., with a P-value larger than 0.05), were compared based on the $\mathrm{R}^{2}$ of the response variable (Appendix 3.7), because these are our main variables of interest and we want to find the model that best explains them. We did not include other possible relations, such as an effect of soil fertility on SOM, because our sample size was too low to include more variables, and because we expected that this effect would work via productivity. All subsets regression analysis followed by model averaging was also used for the effect of soil fertility and disturbance on functional trait dispersion.

We used the $\mathrm{lm}$ function for the linear regression models, and the dredge function and the model.avg function of the MuMIn package (Barton 2015) for the all subsets regression analyses and model averaging, respectively. 


\section{Results:}

We evaluated the effects of abiotic and biotic factors on productivity and stocks of biomass and soil organic matter (SOM) (Fig. 3.1 in the main text). First, one or two variables were selected for trait composition and for soil fertility, based on relative variable importance obtained after 'all subsets regression analysis'.

For aboveground productivity, $\mathrm{P}_{\text {leaf }}$ and SLA were selected as trait composition variables and $\mathrm{P}_{\text {soil }}$ as soil fertility variable (Appendix 3.6). The two possible structural equation models (SEMs) were accepted, but the model including SLA was selected because it gave a higher $\mathrm{R}^{2}$ for productivity, which was our response variable of interest (Appendix 3.7).

For aboveground biomass stocks, $\mathrm{P}_{\text {leaf }}$ was selected as trait composition variable and $\mathrm{P}_{\text {soil }}$ as soil fertility variable (Appendix 3.6). This SEM (with disturbance, $\mathrm{P}_{\text {soil, }}$ species richness, and $\mathrm{P}_{\text {leaf }}$ ) was accepted (Appendix 3.7) and thus used as final model.

For fine root biomass stocks, C: $\mathrm{N}_{\text {leaf }}$ was selected as trait composition variable and $\mathrm{N}_{\text {soil }}$ as soil fertility variable (Appendix 3.6). The SEM was accepted (Appendix 3.7) and used as final model. For SOM, $\mathrm{N}_{\text {litter }}$ was selected as litter quality variable (Appendix 3.6), and this model was accepted (Appendix 3.7) and used as final model.

Appendix 3.5: Results of all subsets regression analysis for the effects of disturbance and soil fertility $\left(\mathrm{N}_{\text {soil }}, \mathrm{P}_{\text {soil }}, \mathrm{C}: \mathrm{N}_{\text {soil }}\right.$, and $\mathrm{N}: \mathrm{P}_{\text {soil }}$ ) on functional trait dispersion (Fdis; Pakeman (2014)), followed by model averaging of all possible models (for more explanation, see Methods). Standardized regression coefficient (Std. coeff), adjusted standard error (SEadj), z-value, P-value, and relative variable importance (Rel. imp.) are given.

\begin{tabular}{llllll}
\hline Predictor variable & Std. coeff & SEadj & z-value & P-value & Rel. imp. \\
\hline Disturbance & -0.08 & 0.19 & 0.43 & 0.667 & 0.21 \\
$\mathrm{~N}_{\text {soil }}$ & 0.16 & 0.22 & 0.72 & 0.474 & 0.26 \\
$\mathrm{P}_{\text {soil }}$ & 0.55 & 0.28 & 1.98 & 0.047 & 0.70 \\
$\mathrm{C}: \mathrm{N}_{\text {soil }}$ & -0.20 & 0.18 & 1.11 & 0.269 & 0.34 \\
$\mathrm{~N}: \mathrm{P}_{\text {soil }}$ & 0.58 & 0.28 & 2.07 & 0.039 & 0.75 \\
\hline
\end{tabular}


Appendix 3.6: Results of all subsets regression analyses for aboveground productivity, aboveground biomass, fine root biomass, and soil organic matter (i.e., the response variable), followed by averaging of all possible models. Per model, multiple indices for soil fertility and trait composition were included. The one or two soil fertility indices and trait composition indices with the highest relative variable importance (Rel. imp.), i.e., the variables in bold and italics, were selected for further analyses using structural equation modelling. Furthermore, standardized regression coefficient (Std. coeff), adjusted standard error (SEadj), z-value and P-value are given.

\begin{tabular}{|c|c|c|c|c|c|c|}
\hline Response variable & Predictor variable & Estimate & SEadj & z-value & P-value & Rel. imp. \\
\hline \multirow{15}{*}{$\begin{array}{l}\text { Aboveground } \\
\text { productivity }\end{array}$} & & & & & & \\
\hline & Disturbance & 0.41 & 0.18 & 2.32 & 0.020 & 0.86 \\
\hline & $\mathrm{N}_{\text {soil }}$ & -0.34 & 0.17 & 1.97 & 0.049 & 0.65 \\
\hline & $P_{\text {soil }}$ & 0.71 & 0.27 & 2.61 & 0.009 & 0.98 \\
\hline & $\mathrm{C}: \mathrm{N}_{\text {soil }}$ & 0.05 & 0.17 & 0.31 & 0.760 & 0.13 \\
\hline & $\mathrm{N}: \mathrm{P}_{\text {soil }}$ & 0.46 & 0.24 & 1.89 & 0.059 & 0.62 \\
\hline & Richness & 0.02 & 0.29 & 0.06 & 0.956 & 0.19 \\
\hline & SLA & -0.40 & 0.30 & 1.31 & 0.190 & 0.45 \\
\hline & $\mathrm{N}_{\text {leaf }}$ & -5.32 & 6.21 & 0.86 & 0.391 & 0.36 \\
\hline & $P_{\text {leaf }}$ & 5.50 & 6.93 & 0.79 & 0.427 & 0.37 \\
\hline & $\mathrm{C}: \mathrm{N}_{\text {leaf }}$ & 0.21 & 0.49 & 0.44 & 0.660 & 0.21 \\
\hline & $\mathrm{N}: \mathrm{P}_{\text {leaf }}$ & 6.62 & 7.07 & 0.94 & 0.349 & 0.32 \\
\hline & FPs & -0.05 & 0.24 & 0.19 & 0.851 & 0.16 \\
\hline & WD & 0.01 & 0.30 & 0.03 & 0.976 & 0.16 \\
\hline & WDMC & -0.18 & 0.22 & 0.79 & 0.430 & 0.20 \\
\hline \multicolumn{7}{|l|}{ Aboveground } \\
\hline \multirow[t]{14}{*}{ biomass } & Disturbance & -0.64 & 0.12 & 5.23 & $<0.001$ & 1.00 \\
\hline & $\mathrm{N}_{\text {soil }}$ & -0.11 & 0.13 & 0.90 & 0.370 & 0.22 \\
\hline & $P_{\text {soil }}$ & 0.23 & 0.14 & 1.70 & 0.089 & 0.52 \\
\hline & C: $\mathrm{N}_{\text {soil }}$ & -0.11 & 0.12 & 0.97 & 0.333 & 0.23 \\
\hline & $\mathrm{N}: \mathrm{P}_{\text {soil }}$ & -0.20 & 0.14 & 1.42 & 0.156 & 0.40 \\
\hline & Richness & -0.13 & 0.15 & 0.87 & 0.382 & 0.23 \\
\hline & SLA & 0.13 & 0.25 & 0.54 & 0.590 & 0.18 \\
\hline & $\mathrm{N}_{\text {leaf }}$ & 0.19 & 1.16 & 0.16 & 0.870 & 0.25 \\
\hline & $P_{\text {leaf }}$ & 0.54 & 0.65 & 0.82 & 0.412 & 0.79 \\
\hline & C: $\mathrm{N}_{\text {leaf }}$ & 0.17 & 0.52 & 0.33 & 0.739 & 0.20 \\
\hline & $\mathrm{N}: \mathrm{P}_{\text {leaf }}$ & 0.03 & 1.19 & 0.03 & 0.980 & 0.28 \\
\hline & FPs & 0.14 & 0.21 & 0.68 & 0.498 & 0.20 \\
\hline & WD & 0.09 & 0.19 & 0.48 & 0.634 & 0.21 \\
\hline & WDMC & 0.18 & 0.15 & 1.21 & 0.227 & 0.33 \\
\hline \multirow[t]{14}{*}{ Fine root biomass } & Disturbance & -0.27 & 0.23 & 1.18 & 0.238 & 0.72 \\
\hline & $N_{\text {soil }}$ & 0.28 & 0.25 & 1.10 & 0.270 & 0.69 \\
\hline & $\mathrm{P}_{\text {soil }}$ & -0.02 & 0.10 & 0.16 & 0.875 & 0.18 \\
\hline & $\mathrm{C}: \mathrm{N}_{\text {soil }}$ & -0.01 & 0.07 & 0.09 & 0.926 & 0.15 \\
\hline & $\mathrm{N}: \mathrm{P}_{\text {soil }}$ & 0.06 & 0.14 & 0.43 & 0.668 & 0.29 \\
\hline & Richness & -0.19 & 0.25 & 0.77 & 0.443 & 0.51 \\
\hline & SLA & 0.01 & 0.12 & 0.11 & 0.912 & 0.17 \\
\hline & $\mathrm{N}_{\text {leaf }}$ & -0.22 & 0.90 & 0.25 & 0.804 & 0.41 \\
\hline & $P_{\text {leaf }}$ & 0.01 & 0.65 & 0.01 & 0.989 & 0.22 \\
\hline & $C: N_{\text {leaf }}$ & -0.67 & 0.71 & 0.94 & 0.347 & 0.77 \\
\hline & $\mathrm{N}: \mathrm{P}_{\text {leaf }}$ & -0.04 & 0.68 & 0.06 & 0.949 & 0.23 \\
\hline & FPs & -0.03 & 0.13 & 0.21 & 0.838 & 0.19 \\
\hline & WD & 0.00 & 0.11 & 0.00 & 0.999 & 0.16 \\
\hline & WDMC & 0.00 & 0.10 & 0.04 & 0.968 & 0.16 \\
\hline
\end{tabular}


Soil organic matter

Disturbance
Litter quantity
$N_{\text {litter }}$
$\mathrm{P}_{\text {litter }}$
$\mathrm{C}: \mathrm{N}_{\text {litter }}$
$\mathrm{N}: \mathrm{P}_{\text {litter }}$

0.11

0.19

0.57

0.572

0.23

0.11

0.20

0.54

0.593

0.23

$-0.38$

0.26

1.45

0.146

0.61

$-0.19$

0.40

0.47

0.639

0.29

$\mathrm{N}: \mathrm{P}_{\text {litter }}$

$-0.06$

$0.22 \quad 0.14$

0.891

0.21

$\begin{array}{llll}0.42 & 0.14 & 0.893 & 0.25\end{array}$

Appendix 3.7: Statistics showing the model fit of structural equation models (SEMs) for productivity, aboveground biomass, fine root biomass, and soil organic matter. For aboveground productivity, two possible SEMs were evaluated because two trait composition variables (SLA and $\mathrm{P}_{\text {leaf }}$ ) gave comparable fit in the all subsets regression analysis (Appendix 3.6), and the SEM with highest $\mathrm{R}^{2}$ for the response variable (i.e., productivity) was selected (see Fig. 3.2). Note that other variables (i.e., disturbance and species richness) were included in all SEMs as shown in Fig. 3.2. For each model, model Chi-squared value and P-value are based on the fit of the whole model, and the $\mathrm{R}^{2}$ gives the explained variation of the response variable. A P-value $>0.05$ indicates that the model is accepted.

\begin{tabular}{|c|c|c|c|c|c|}
\hline $\begin{array}{l}\text { Response } \\
\text { variable }\end{array}$ & $\begin{array}{l}\text { Trait } \\
\text { composition } \\
\text { variable }\end{array}$ & $\begin{array}{l}\text { Soil } \\
\text { fertility } \\
\text { variable }\end{array}$ & $\begin{array}{l}\text { Model } \\
\text { Chi- } \\
\text { squared }\end{array}$ & $\begin{array}{l}\text { Model } \\
\text { P-value }\end{array}$ & $\begin{array}{l}\mathbf{R}^{2} \text { of } \\
\text { response } \\
\text { variable }\end{array}$ \\
\hline \multirow{2}{*}{$\begin{array}{l}\text { Aboveground } \\
\text { productivity }\end{array}$} & SLA & $\mathrm{P}_{\text {soil }}$ & 0.027 & 0.869 & 0.454 \\
\hline & $P_{\text {leaf }}$ & $\mathrm{P}_{\text {soil }}$ & 0.031 & 0.860 & 0.425 \\
\hline $\begin{array}{l}\text { Aboveground } \\
\text { biomass }\end{array}$ & $\mathrm{P}_{\text {leaf }}$ & $\mathrm{P}_{\text {soil }}$ & 0.031 & 0.860 & 0.730 \\
\hline Fine root biomass & $C: \mathrm{N}_{\text {leaf }}$ & $\mathrm{N}_{\text {soil }}$ & 3.296 & 0.069 & 0.574 \\
\hline Soil organic matter & - & $\mathrm{N}_{\text {litter }}$ & 0.303 & 0.990 & 0.163 \\
\hline
\end{tabular}




\section{Chapter 4}

Drivers of biomass change in a Neotropical forest: testing for niche, mass-ratio, and environmental effects

Masha T. van der Sande, Marielos Peña-Claros, Nataly Ascarrunz, Eric J. M. M. Arets, Juan Carlos Licona, Marisol Toledo and Lourens Poorter 


\section{Abstract}

Tropical forests play an important role in the global carbon cycle, but the drivers of net forest biomass change (i.e., net carbon sequestration) are poorly understood. Here, we evaluate how abiotic factors (soil conditions and disturbance) and biotic factors (forest structure, diversity and community trait composition) shape three important demographic processes (recruitment, growth, and mortality) and how these underlie net biomass change. To test this, we evaluated 9 years of biomass dynamics using 48 1-ha plots in a Bolivian tropical moist forest, and measured the most abundant species for eight functional traits that are important for plant carbon gain and loss. Demographic processes were related to the abiotic and biotic factors using structural equation models. Net biomass change was most strongly determined by stand-level mortality, but mortality itself was highly stochastic at this scale. Contrary to expectations, we found that species richness - as proxy for the niche complementarity theory - and trait composition - as indicator for the massratio theory - had little effect on the demographic processes. Biomass recruitment increased with higher resource availability (i.e., water and light) and resource use efficiency (through high species richness), whereas growth of larger, established trees increased with higher sand content (which may facilitate root growth of larger trees to deeper soil layers). Growth of larger trees also increased with plot basal area, due to the presence of more biomass that can grow. In sum, niche complementarity and mass ratio are of limited importance in this complex and species-rich forest, and demographic processes are most strongly determined by soil texture, soil water availability and forest structure. Only by simultaneously evaluating multiple abiotic and biotic drivers of demographic processes, better insights can be gained into mechanisms playing a role in the carbon sequestration potential of tropical forests and natural systems in general.

Keywords: biomass growth, Bolivia, disturbance, ecosystem functioning, functional diversity, functional traits, mortality, productivity, recruitment, soil conditions, species diversity, structural equation modelling 


\section{Introduction}

Tropical forests play an important role in global carbon storage (Saatchi et al. 2011) and sequestration (Malhi 2012), and hence, in climate change mitigation strategies (e.g., Reduced Emissions from Deforestation and forest Degradation; REDD+). Yet, it is still poorly understood what factors are driving the net forest biomass change and, thus, the net carbon sequestration (Malhi 2012). At the stand level, net biomass change is the result of three underlying demographic processes: recruitment, growth, and mortality. These demographic processes should be analysed individually to understand net biomass change, as each process may be driven by different biotic factors (e.g., the diversity and trait composition of the forest) and abiotic factors (e.g., soil properties and light availability) (see the conceptual framework in Fig. 4.1).

To explain biotic effects on demographic processes, two competing theories have been described: the niche complementarity theory (Tilman 1999) and the mass-ratio theory (Grime 1998). According to niche complementarity theory, high diversity increases the overall resource use efficiency of a community, leading to increased growth rates. A positive effect of species diversity on productivity (i.e., growth) was found for herbaceous communities (Tilman et al. 2001) and forest ecosystems (Balvanera et al. 2006, Paquette and Messier 2011). However, rather than number of species, the identity of species and their traits are thought to provide a more direct and mechanistic link with forest processes (Violle et al. 2007). Variation in plant traits positively affected productivity in grasslands (Tilman et al. 1997) and temperate forests (Butterfield and Suding 2013), but its effect may be different for diverse tropical forests where trait redundancy between species may not further enhance forest growth (Walker 1992).

Mass-ratio theory predicts that the most abundant species drive ecosystem processes (Grime 1998). This is reflected in the 'trait composition', i.e., the basal area-weighted leaf and stem trait values of the community. Few studies have simultaneously evaluated the relative importance of taxonomic diversity (i.e., species diversity), trait diversity and trait composition on demographic processes in natural communities. Mokany et al. (2008) found in temperate grasslands that trait composition is a stronger driver of productivity than taxonomic diversity. Similarly, Finegan et al. (2015) found across three tropical forests that trait composition, and not trait diversity, determined productivity, whereas Lohbeck et al. (2015) found that during secondary forest succession, neither trait composition nor trait diversity, but aboveground biomass had a positive effect on productivity. Hence, the relative importance of taxonomic and trait effects in natural systems is yet poorly understood and may depend on various factors, such as local abiotic and biotic factors. 
Abiotic factors are strong drivers of demographic processes as they determine resource availability for plant growth and survival (Fig. 4.1). For example, soil conditions are key drivers of tropical forest growth across the Amazon (Quesada et al. 2012) and locally (Paoli et al. 2005), and disturbance can increase light availability and therefore the opportunity for recruitment and growth (Peña-Claros et al. 2008). Abiotic factors can also have an indirect effect on demographic processes, via their effects on biotic factors (Fig. 4.1). For example, in African forests, sandy soils, compared to clayey soils, had a higher abundance of species with high wood density that are more drought tolerant and better survive on sandy and resourcelimited soils (Fayolle et al. 2012). In our study forest, disturbance due to logging treatments changed the trait composition of demographic groups towards more acquisitive trait values (e.g., high specific leaf area and low wood density) that are typical of pioneer species that benefit from higher light levels (Carreño-Rocabado et al. 2012). Moreover, disturbance alters the forest structure (e.g., decreases plot basal area), which may in turn result in a change in species diversity (Armesto and Pickett 1985). Consequently, forest structure can determine demographic processes directly, but also indirectly via its effects on the diversity and trait composition of different demographic groups (Vilà et al. 2013). These studies show that abiotic factors can affect the biotic factors, but they did not evaluate how the biotic factors in turn affect demographic processes (but see Vilà et al. 2013). We are not aware of studies evaluating such combined effects of abiotic and biotic factors on demographic processes that underlie net biomass change in forest systems.

We address two questions. First, how are demographic processes (recruitment, growth and mortality) driven by abiotic factors (soil conditions and disturbance) and biotic factors (forest structure, taxonomic and trait diversity, and trait composition)? We expected that i) survival and growth increase with light availability and hence, with an open forest structure and disturbance, whereas mortality is mostly a stochastic process and therefore not strongly driven by abiotic and biotic factors; ii) recruitment and growth increase with species richness (as predicted by niche theory) and with an acquisitive trait composition (as predicted by mass ratio theory); and iii) trait composition has a stronger effect on demographic processes than diversity because the bulk of these processes are determined by the dominant species. Secondly we ask: how do these demographic processes determine net biomass change? We expected that net biomass change is most strongly determined by mortality, to a lesser extent by growth of surviving trees, and least by recruitment because mortality would have highest absolute values and thus contribute most to net biomass change, followed by growth and recruitment. We tested these hypotheses using long-term data of 48 1-ha forest plots in a tropical moist forest in Bolivia, that provided strong gradients in demographic processes and abiotic and biotic factors. 


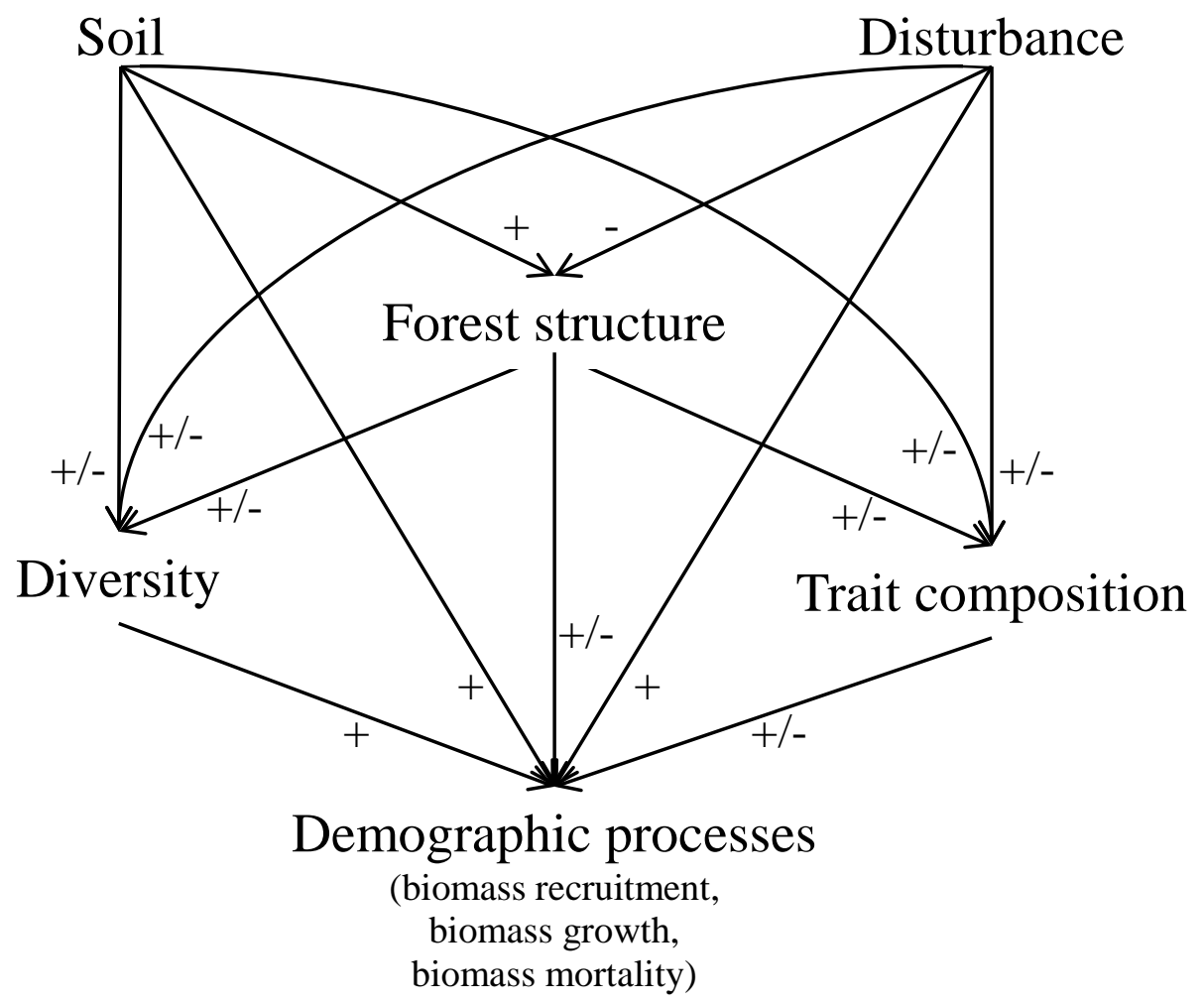

Figure 4.1: Conceptual framework showing the expected relations of abiotic factors (disturbance and soil resource availability) and biotic factors (forest structure, diversity and trait composition) on demographic processes (biomass recruitment, growth, and mortality). Forest structure (e.g., plot basal area, tree density) is based on all alive trees in the 1-ha plots, whereas diversity and trait composition are based on the individuals of that demographic group only (i.e., recruits, survivors, or trees that died). Hypothesized positive effects are indicated by + signs and hypothesized negative effects are indicated by - signs. The effect of and on trait composition depends on the trait considered; acquisitive trait values (e.g., high specific leaf area and leaf nitrogen concentration) will increase with disturbance and positively affect demographic processes, whereas conservative trait values (e.g., high leaf toughness and wood density) will decrease with disturbance and negatively affect demographic processes. Soil resource availability and disturbance can decrease diversity because of a competitive advantage of few, light-demanding species, or they can increase diversity because of the creation of more niches. Forest structure would decrease recruitment because of light-limitation but would increase growth because of more standing biomass that can grow.

\section{Methods}

\section{Research site and plots}

Research was carried out in the moist, semi-deciduous forest of La Chonta, Santa Cruz, Bolivia $\left(15^{\circ} 47^{\prime} \mathrm{S}, 62^{\circ} 55^{\prime} \mathrm{W}\right)$. Mean annual rainfall is $1580 \mathrm{~mm}$, with a dry season from April until September when precipitation is $<100 \mathrm{~mm}$, and mean annual temperature is $24.3{ }^{\circ} \mathrm{C}$. The forest is located on ultisols, with sandy-loam soils that are neutral in $\mathrm{pH}$ and rich in nutrients (Peña-Claros et al. 2012), and 
topography is homogeneous (Peña-Claros et al. 2008). On average, the forest has 367 stems (>10 cm DBH), 59 species per ha, and a canopy height of $25 \mathrm{~m}$ (PeñaClaros et al. 2012).

For this study, we used 48 one-hectare $(100 * 100 \mathrm{~m})$ permanent sample plots of the Long-Term Silvicultural Research Program (LTSRP) managed by Instituto Boliviano de Investigación Forestal (IBIF), in which all trees larger than $10 \mathrm{~cm}$ in diameter at breast height (DBH) were first recorded between September 2000 and December 2001. After the initial census, four treatments were applied, each replicated on 12 plots. The treatments varied in the intensity of logging and silvicultural practices applied, from an unlogged control treatment to an intensive silvicultural treatment with post-logging activities such as girdling to liberate trees from overtopping non-commercial trees (see Peña-Claros et al. 2008 for more details on treatments). The most recent census was done for 16 plots in 2009, for 16 plots in 2010, and for 16 plots in 2011 (each time for four plots per treatment).

\section{Demographic processes}

We calculated three demographic processes: biomass recruitment by recruiting trees, biomass growth by surviving trees, and biomass mortality by dying trees (in $\left.\mathrm{Mg} \mathrm{ha}^{-1} \mathrm{yr}^{-1}\right)$. Henceforth, these will be referred to as recruitment, growth, and mortality, respectively. We calculated demographic processes between the prelogging census and the last post-logging census. We used a long census interval of 8-10 years to reduce the effect of stochastic variation in biomass dynamics. Palms were excluded from the analyses because they do not have radial growth and thus their growth is hard to estimate, and because they have outlying trait values that would affect the relation between trait composition and demographic processes. Since we focus on natural demographic processes, we excluded all trees that were logged or that died due to logging activities (e.g., due to damage caused by logging operations or due to post-logging silvicultural treatments). We also excluded trees that died due to fire that took place in 2004 in 4 of the plots. These excluded trees were also excluded for calculations of other variables (i.e., forest structure, diversity and trait composition), but used to calculate the disturbance intensity (see 'Disturbance').

For each tree and each of the two census years, we calculated the aboveground biomass using the equation from Chave et al. (2014):

Biomass $=\exp \left(-1.803-0.976 *(E)+0.976 * \log (W D)+2.673 * \log (D B H)-0.0299 *(\log (D B H))^{\wedge} 2\right)$

where $\mathrm{DBH}$ is the diameter at breast height (in $\mathrm{cm}$ ) and WD is the wood density (in $\mathrm{g} \mathrm{cm}^{-3}$, see explanation in Appendix 4.1). $E$ is a measure of environmental stress experienced at the site, which depends on temperature seasonality and water deficit. We calculated the E-value (see Chave et al. 2014) for 26 sites across Bolivia for 
which we had accurate rainfall data (using data from Toledo 2010), and predicted the $E$-value of La Chonta based on the relation between locally available annual rainfall and the $E$-value for these surrounding Bolivian sites $\left(E_{\text {predicted }}=0.776\right.$ $0.000356 *$ precipitation; $\left.\mathrm{R}^{2}=0.79\right)$. This resulted in the $E$-value 0.25 for La Chonta.

\section{Recruitment, growth, mortality, and net biomass change}

Recruitment $\left(\mathrm{Mg} \mathrm{ha}^{-1} \mathrm{yr}^{-1}\right)$ was based on trees that recruited after the first census. Per individual, biomass recruitment was calculated as its biomass in the last census minus its biomass for a $\mathrm{DBH}$ of $10 \mathrm{~cm}$. In this way, we assumed that the recruits were $10 \mathrm{~cm} \mathrm{DBH}$ just after the initial census, and calculate growth based on the increase in diameter from $10 \mathrm{~cm}$ until its measured diameter in the last census. This may slightly underestimate biomass recruitment, as most trees may have reached the $10 \mathrm{~cm}$ limit later during the census interval, but it should yield more accurate estimations than assuming that recruits were $0 \mathrm{~cm} \mathrm{DBH}$ (which would lead to stronger overestimations of growth), and similar estimations as using the tree's growth rate during other censuses to predict when it reached the $10 \mathrm{~cm}$ limit (Talbot et al. 2014). Total annual recruitment per plot was calculated by summing the recruitment per plot and dividing this by the census length.

Growth $\left(\mathrm{Mg} \mathrm{ha}^{-1} \mathrm{yr}^{-1}\right)$ was based on the growth of trees that were present at the first census and survived until the last census. It was calculated by subtracting the biomass of a tree in the last census from the biomass of the same tree in the first census. By summing all growth values per plot and dividing it by the census length (in years), we obtained annual growth per hectare.

Mortality $\left(\mathrm{Mg} \mathrm{ha}^{-1} \mathrm{yr}^{-1}\right)$ was based on trees that died between the first and last census. It was calculated as the biomass of the tree in the initial census when it was still alive, minus its biomass for a DBH of $10 \mathrm{~cm}$, to be able to compare biomass loss (i.e., mortality) with biomass gain (i.e., recruitment and growth) (Talbot et al. 2014). Annual mortality was obtained by summing mortality per plot and dividing this by the census length. Net biomass change was calculated per plot by summing recruitment and growth, and subtracting mortality.

\section{Soil}

For each plot, soil variables were collected in 2005 from the top $30 \mathrm{~cm}$ of the soil at 20 fixed locations distributed in the plot systematically. Collection was done after logging (which occurred in 2001) but samples were taken from areas that were not affected by logging, to represent pre-logging variation in soil conditions among plots. All samples were pooled per plot and brought to the Soil Laboratory of the Centro de Investigación Agricola Tropical (CIAT), Santa Cruz, Bolivia, for analyses of the following soil nutrients and conditions: calcium, magnesium, potassium, 
sodium, cation exchange capacity as the sum of all exchangeable cations and acidity (all in $\mathrm{cmol} \mathrm{kg}^{-1}$ ), total available phosphorus $\left(\mathrm{mg} \mathrm{kg}^{-1}\right)$ using the Olson method, total nitrogen using the micro-Kjeldahl method (\%), $\mathrm{pH}$, and soil texture (sand content and clay content) (for more explanation, see Toledo 2010). Dry season soil water potential per plot $(\mathrm{MPa})$, a measure for minimum soil water availability, was obtained from L. Markesteijn (unpublished data). Soil water potential was measured during the peak of the dry season (July 2007) (Markesteijn et al. 2010). One sample per plot was taken from the first $10 \mathrm{~cm}$ of the soil, and soil water potential was determined using the filter paper method (for a more extensive description, see Markesteijn et al. 2010).

\section{Disturbance}

We developed a continuous measure for disturbance, based on the basal area of all trees that died due to fire or logging (i.e., that were logged or died due to logging and post-logging activities between the first and last census) relative to the total initial basal area of that plot, in $\%$. The disturbed plots ranged from $0.1-40.3 \%$ in basal area loss.

\section{Forest structure}

We wanted to evaluate the effect of forest structure, as a measure of biotic competition for resources and space, on the diversity and trait composition of the demographic groups and on demographic processes (Fig. 4.1). We therefore calculated several structural variables (based on trees $>10 \mathrm{~cm} \mathrm{DBH}$ ), per plot and per census (all after disturbance), that would indicate abiotic competition for light and other resources: total plot basal area $\left(\mathrm{m}^{2} \mathrm{ha}^{-1}\right)$, tree density $\left(\# \mathrm{ha}{ }^{-1}\right)$, average diameter at breast height $(\mathrm{cm})$, and the basal area of "large trees" (all trees $>60 \mathrm{~cm}$ $\left.\mathrm{DBH} ; \mathrm{m}^{2} \mathrm{ha}^{-1}\right)$. The values of the two censuses per plot were averaged to obtain one value per plot that would better represent the whole monitoring period.

\section{Diversity}

Niche complementarity theory predicts that diversity increases resource use efficiency and as a result the overall productivity of the forest stand. We used taxonomic richness and functional trait richness to evaluate diversity in functioning among species. The indices were calculated based on all trees belonging to each specific demographic group (i.e., recruitment, growth and mortality), and calculated per plot and per census. We described taxonomic richness using rarefied species richness, as the number of species found in a random sample of 50 individuals (as this number of individuals is found in all demographic groups per plot). We used rarefied richness to prevent that differences in stem number among plots would 
determine differences in species richness. Functional trait richness (Frich) was described as the amount of multivariate trait space occupied by species in the plot (Mason et al. 2005, Mouillot et al. 2005), and was based on all traits (Table 4.1). Values for taxonomic richness and trait richness of the initial and final census were averaged to obtain values that would better represent the whole census interval. Taxonomic richness was obtained using the vegan package (Oksanen et al. 2014), and trait richness using the $d b F D$ function of the FD package in $\mathrm{R}$ (Laliberté et al. 2015). Taxonomic and trait richness are hereafter collectively called 'diversity'.

Table 4.1: Overview of the leaf and stem traits that were used to calculate community-weighted mean values per plot (i.e., the trait composition), with abbreviation, variable description, units, for what function they are an indicator, and literature.

\begin{tabular}{|c|c|c|c|c|c|}
\hline $\begin{array}{l}\text { Variable } \\
\text { group }\end{array}$ & Abbreviation & $\begin{array}{l}\text { Variable } \\
\text { description }\end{array}$ & Units & Indicator for & Literature \\
\hline \multirow[t]{6}{*}{ Leaf traits } & SLA & Specific leaf area & $\mathrm{cm}^{2} \mathrm{~g}^{-1}$ & $\begin{array}{l}\text { Light interception } \\
\text { efficiency }\end{array}$ & $\begin{array}{l}\text { Poorter and } \\
\text { Remkes 1990, } \\
\text { Schieving and } \\
\text { Poorter } 1999\end{array}$ \\
\hline & $\mathrm{N}_{\text {mass }}$ & $\begin{array}{l}\text { Leaf nitrogen } \\
\text { concentration }\end{array}$ & $\%$ & $\begin{array}{l}\text { Photosynthetic } \\
\text { capacity, metabolic } \\
\text { rate }\end{array}$ & $\begin{array}{l}\text { Evans 1989, } \\
\text { Mercado et al. } 2011\end{array}$ \\
\hline & $P_{\text {mass }}$ & $\begin{array}{l}\text { Leaf phosphorus } \\
\text { concentration }\end{array}$ & $\%$ & $\begin{array}{l}\text { Photosynthetic } \\
\text { capacity, metabolic } \\
\text { rate }\end{array}$ & Mercado et al. 2011 \\
\hline & Chl & $\begin{array}{l}\text { Chlorophyll } \\
\text { content }\end{array}$ & $\mu \mathrm{g} \mathrm{cm}^{-2}$ & $\begin{array}{l}\text { Light harvesting } \\
\text { capacity }\end{array}$ & Evans 1989 \\
\hline & FPs & $\begin{array}{l}\text { Specific force to } \\
\text { punch }\end{array}$ & $\mathrm{N} \mathrm{cm}^{-2}$ & Leaf defense & $\begin{array}{l}\text { Kitajima and } \\
\text { Poorter 2010, } \\
\text { Onoda et al. } 2011\end{array}$ \\
\hline & LMFm & $\begin{array}{l}\text { Leaf mass } \\
\text { fraction of the } \\
\text { metamer }\end{array}$ & $\mathrm{g} \mathrm{g}^{-1}$ & $\begin{array}{l}\text { Light interception } \\
\text { efficiency }\end{array}$ & $\begin{array}{l}\text { Walters and Reich } \\
\text { 1999, Lusk } 2004\end{array}$ \\
\hline \multirow[t]{2}{*}{$\begin{array}{l}\text { Stem } \\
\text { traits }\end{array}$} & WD & Wood density & $\mathrm{g} \mathrm{cm}^{-3}$ & $\begin{array}{l}\text { Volume growth, stem } \\
\text { defense }\end{array}$ & $\begin{array}{l}\text { Baker et al. 2004b, } \\
\text { Chao et al. 2008, } \\
\text { Chave et al. } 2009\end{array}$ \\
\hline & $\mathrm{DBH}_{\max }$ & $\begin{array}{l}\text { Maximum stem } \\
\text { diameter at } \\
\text { breast height }\end{array}$ & $\mathrm{cm}$ & $\begin{array}{l}\text { Tree longevity and } \\
\text { life history strategy }\end{array}$ & $\begin{array}{l}\text { Kohyama et al. } \\
\text { 2003, King et al. } \\
\text { 2006a }\end{array}$ \\
\hline
\end{tabular}




\section{Trait collection}

We selected six leaf traits and two stem traits that are important components of the leaf- and stem economics spectra (Baraloto et al. 2010) and that are important for demographic processes (Table 4.1). Specific leaf area (SLA) and leaf mass fraction of the metamer $\left(\mathrm{LMF}_{\mathrm{m}}\right)$ indicate the light interception efficiency per leaf investment and metamer investment, respectively, and leaf nitrogen $\left(\mathrm{N}_{\text {mass }}\right)$ and phosphorus $\left(\mathrm{P}_{\text {mass }}\right)$ concentration and chlorophyll content $(\mathrm{Chl})$ are important for photosynthetic capacity and growth capacity. All these traits would therefore increase the rate of the demographic processes. On the other hand, high specific force to punch (FPs; a measure for leaf toughness) and wood density (WD) are part of the shade-tolerant traits that increase survival (i.e., reduce mortality) but reduce photosynthetic rates (Selaya and Anten 2010) and possibly growth. Maximum diameter $\left(\mathrm{DBH}_{\max }\right)$ is a measure for tree longevity and life-history strategy, with high values indicating species that can benefit from high light levels in the upper canopy and have the capacity to grow fast.

All traits were determined for 161 tree species that together made up on average $97.5 \%$ of the basal area across the 48 permanent sample plots in the first and last census year. The community-mean trait value weighted by species' basal area can be accurately determined if it is based on the species that together compose at least $80 \%$ of the abundance (Pakeman and Quested 2007), but a higher coverage is needed to accurately determine trait diversity (Pakeman 2014). Traits were measured on individuals between 10 and $20 \mathrm{~cm} \mathrm{DBH}$ that were exposed to direct sunlight or high lateral light levels. See Appendix 4.1 for a more detailed description of trait data collection.

\section{Trait composition indices}

Grime's (1998) mass ratio theory states that ecosystem processes are driven by the characteristics of the most dominant species in the community. We calculated the trait composition (or average trait values) of the stand as the sum of the trait values of all species multiplied by their relative basal area, which is also known as the community-weighted mean (CWM, Pla et al. 2012). We used species' basal area rather than tree abundance because basal area scales better with biomass than abundance (Poorter et al. 2015), and hence, with biomass-driven demographic processes. For these calculations only the species were used for which trait data were available, which together made up $93-100 \%$ of the basal area in the plots (averaged for the two census years). We calculated the CWM values based on the subset of trees belonging to the specific demographic group (i.e., trees that recruited, trees that survived, and trees that died), since their traits drive their biomass dynamics. The CWM values were calculated per plot and per census for 
each of the 8 traits, and values of the initial and final census per plot were averaged to represent the average trait composition of the community during the monitoring period.

\section{Statistical analyses}

We evaluated how demographic processes that underlie net biomass change were affected by abiotic factors (soil conditions, disturbance) and biotic factors (forest structure, trait composition, and diversity). We therefore developed one structural equation model (SEM) for each of the three demographic processes (Fig. 4.1). This approach allows to take the direct and indirect effects and (cor)relations among variables into account, and has the additional advantage that it can test whether the overall model is "correct" (i.e., statistically accepted) and provides an accurate description of the data.

Per demographic process, we selected one variable for each abiotic and biotic factor in Fig. 4.1, depending on which combination of variables best explained variation in the demographic process (i.e., the combination of variables providing the highest $\mathrm{R}^{2}$ ). See Appendix 4.2 for a more detailed description of model building.

The relative strengths of the effects of the three demographic processes on net biomass change were evaluated using a multiple linear regression. Recruitment and mortality were ln-transformed to meet the assumptions of equal variances and normal distribution of the residuals (also in previous analyses).

We performed all analyses in $\mathrm{R}$ 2.15.2. Linear models were evaluated using the $\mathrm{lm}$ function, and structural equation modelling was performed using the sem function of the lavaan package (Rosseel 2012).

\section{Results}

Across all plots, average net biomass change was $1.68 \mathrm{Mg} \mathrm{ha}^{-1} \mathrm{yr}^{-1} \pm 0.30$ (average \pm standard error), recruitment was $0.78 \mathrm{Mg} \mathrm{ha}^{-1} \mathrm{yr}^{-1} \pm 0.05$, growth of surviving trees was $3.78 \mathrm{Mg} \mathrm{ha}^{-1} \mathrm{yr}^{-1} \pm 0.20$, and mortality was $2.88 \mathrm{Mg} \mathrm{ha}^{-1} \mathrm{yr}^{-1} \pm 0.22$.

The structural equation model for recruitment showed a strong negative effect of plot basal area (i.e., forest structure) on biomass recruitment. Disturbance enhanced recruitment directly, and also indirectly by reducing the basal area and thus reducing the negative effect of basal area on recruitment (Fig. 4.2a, Appendix 4.3a). High taxonomic richness increased recruitment, whereas high sand content decreased recruitment (Fig. 4.3a, d, g, j, m).

The model for growth showed that plot basal area (i.e., forest structure) had a strong positive effect on growth (Fig. 4.2b, Appendix 4.3b), whereas soil water 
potential had a negative effect on growth, indicating that plots on wetter soils had slower biomass growth. Disturbance had an indirect negative effect on growth by decreasing the basal area of the growing stand (Fig. 4.2b, Fig. 4.3b, e, h, k, n). None of the abiotic and biotic variables had a significant effect on mortality (Fig. 4.2c, Fig. 4.3c, f, i, 1, o, Appendix 4.3c).

In all three SEMs, disturbance negatively affected forest structure. Other abiotic factors did not consistently relate to biotic factors. We only found a negative effect of disturbance on community-weighted mean (CWM) leaf toughness (i.e., trait composition) of recruiting trees (because disturbance may increase the abundance of light-demanding species that generally have low leaf toughness) and a negative effect of tree density (i.e., forest structure) on rarefied taxonomic richness of trees that died during the monitoring period.

All three demographic processes significantly explained net growth, with the strongest standardized coefficient for mortality (-0.72), followed by growth (0.65) and recruitment (0.18; Fig. 4.2c, Appendix 4.4, Fig. 4.4).

\section{Discussion}

We evaluated how abiotic and biotic factors drive three stand-level demographic processes, and how these underlie net biomass change. We show that mortality most strongly predicted net biomass change but was unpredictable itself. Surprisingly, niche complementarity (i.e., taxonomic and trait diversity) and mass ratio (i.e., community-average trait values) had little effect on recruitment and growth. Plot basal area (i.e., forest structure), and soil factors strongly determined recruitment and growth, indicating that vegetation quantity and abiotic factors matter most for ecosystem processes in this Amazonian tropical forest.

\section{The strongest predictor of net biomass change is unpredictable}

We hypothesized that net biomass change would be more strongly affected by growth and mortality than recruitment because of their higher absolute values. We found that all demographic processes significantly affected net growth (Fig. 4.2, 4.4, Appendix 4.4), and that natural mortality indeed had the strongest effect. This is in agreement with a modelling study, showing that mortality is a key driver of variation in aboveground biomass stocks across the Amazon (Delbart et al. 2010). This and our study indicate that mortality is a crucial process determining forest structure and dynamics, and we should therefore aim to better understand what drives stand-level mortality. We show, however, that mortality was unpredictable 


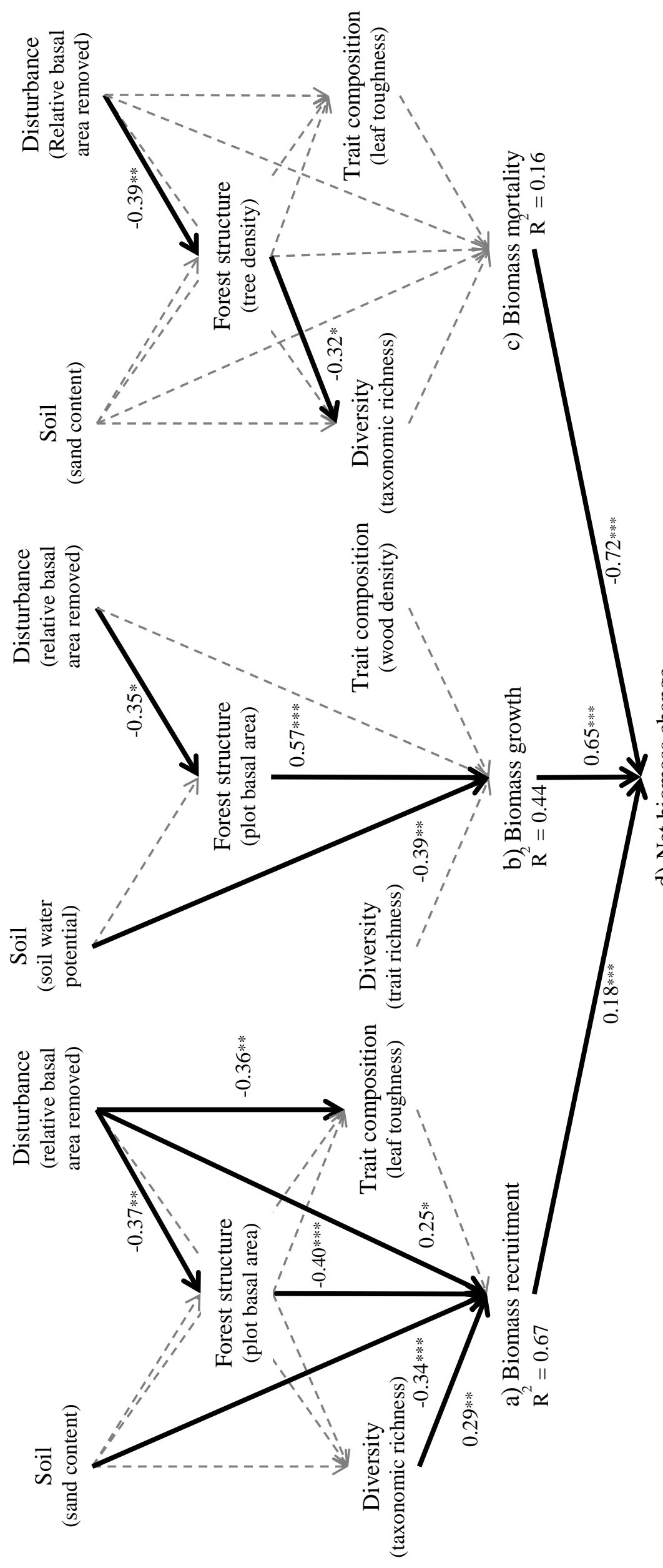

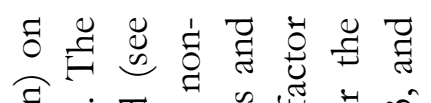

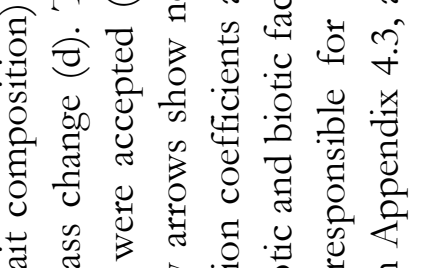

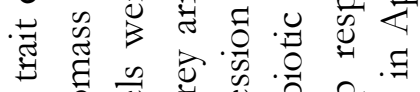
苨高

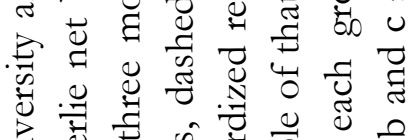

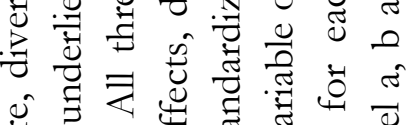

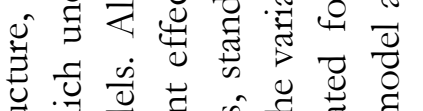

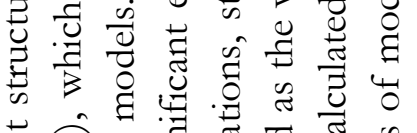

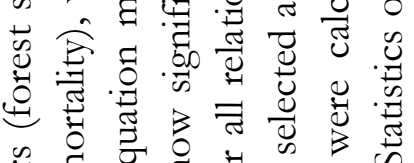

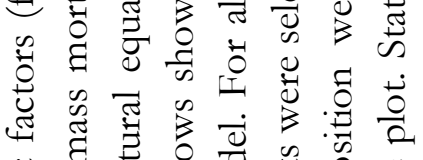

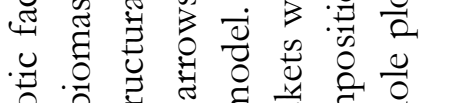
究 च च 矛

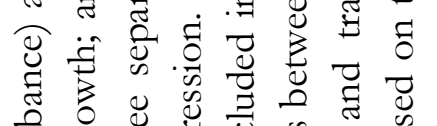

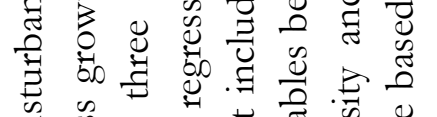

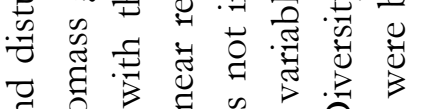

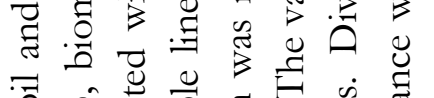

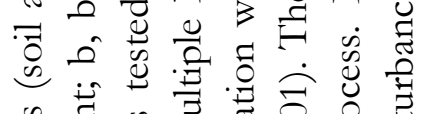

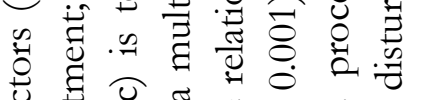

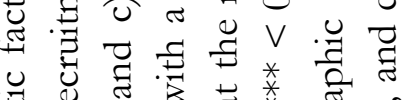
.

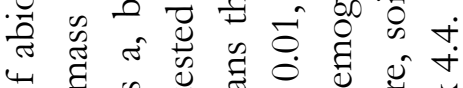

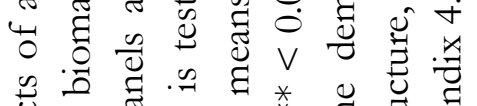
苟 है पु

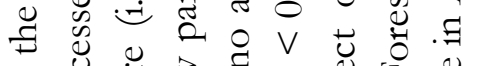

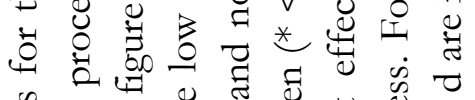

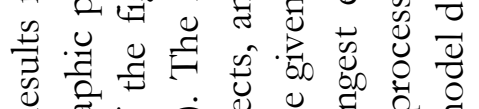

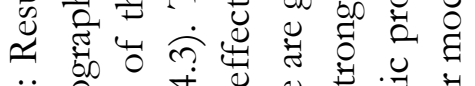

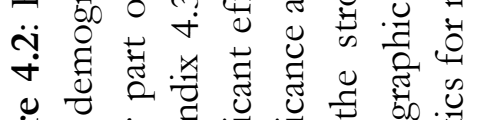
ษ

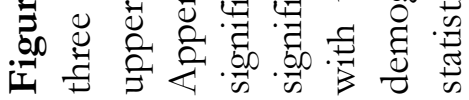


Recruitment

a)

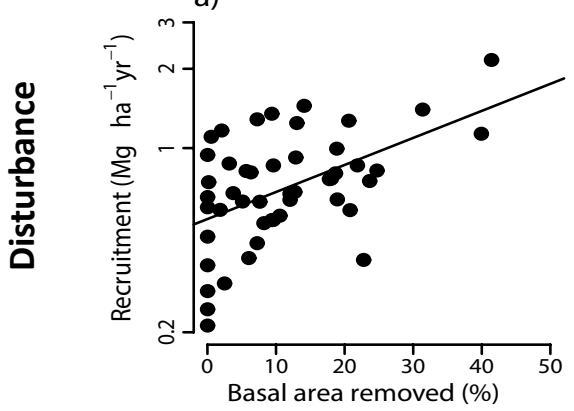

d)

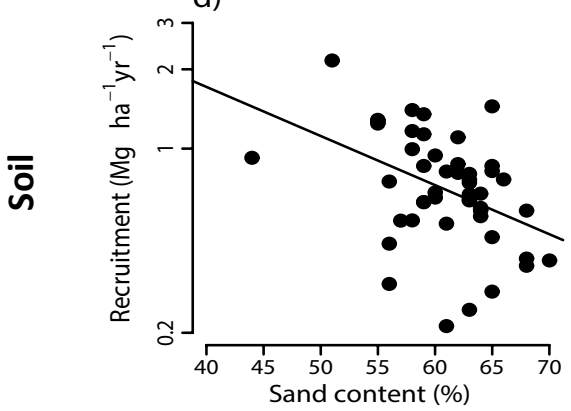

g)
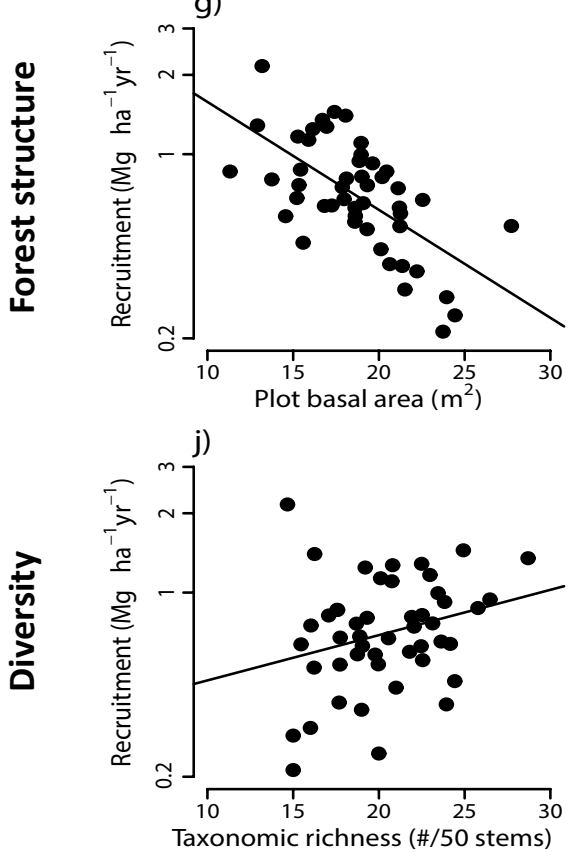

$\mathrm{m})$

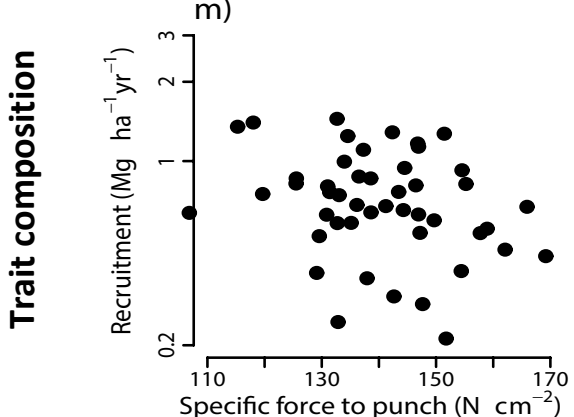

Growth

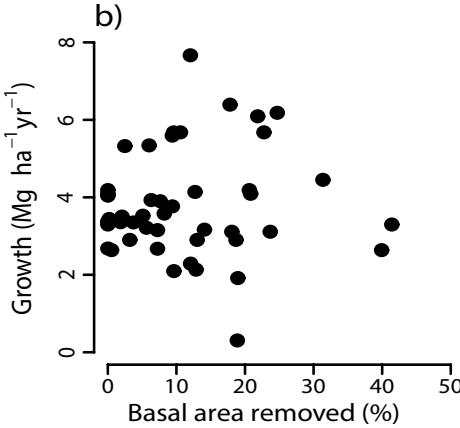

e)

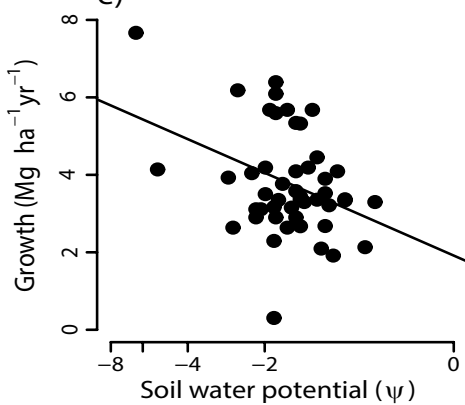

h)

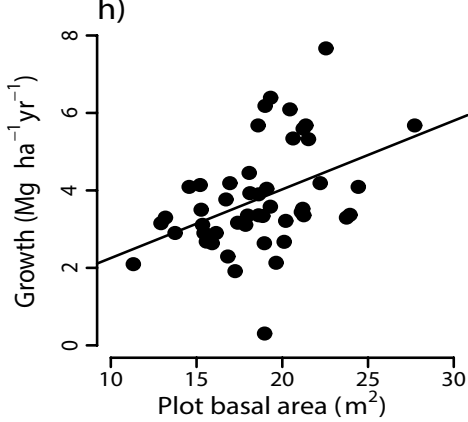

k)
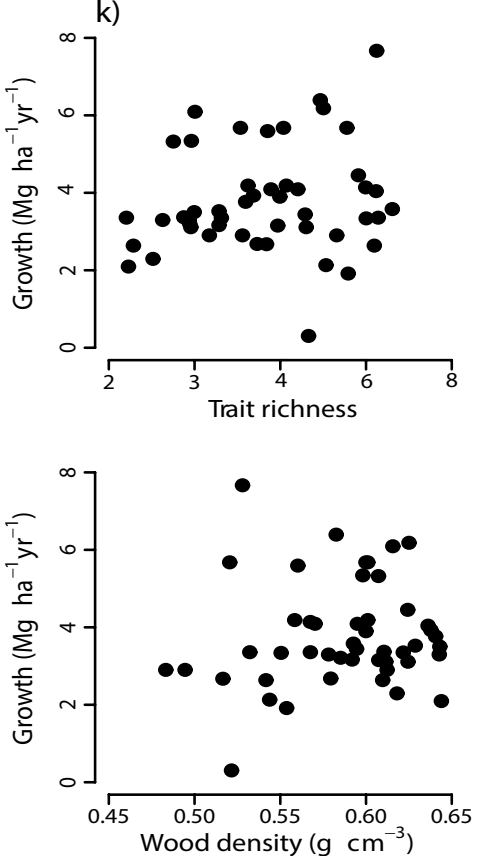

Mortality
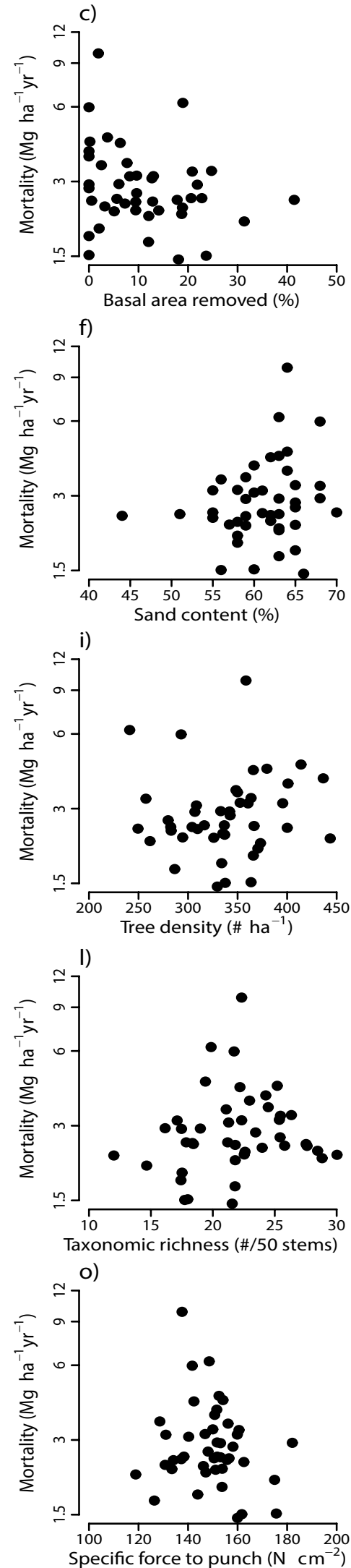

Figure 4.3: Bivariate relations of the three demographic processes (recruitment: left column, Fig. a, d, g, j, m; growth: middle column, Fig. b, e, h, k, n; and mortality: right column, Fig. c, f, I, l, o) 
with the five abiotic and biotic factors in rows (see also Fig. 4.1 and 4.2): relative basal area removed (i.e., disturbance, Fig. a-c), soil conditions (Fig d-f), forest structure (Fig g-i), diversity (Fig. j-l), and community-weighted mean trait composition (Fig. m-o) for 48 1-ha plots in the tropical moist forest of La Chonta. See Fig. 4.2 and Appendix 4.3 for results of multivariate structural equation models. Regression lines are given for the relations that were significant in the structural equation models (Fig. 4.2), but are based on simple regressions and meant for illustration purposes only. Note that the axes for recruitment (Fig. a, d, g, j, m), mortality (Fig. c, $\mathrm{f}, \mathrm{i}, \mathrm{l}, \mathrm{o}$ ), and soil water potential (e) are in ln-scale.
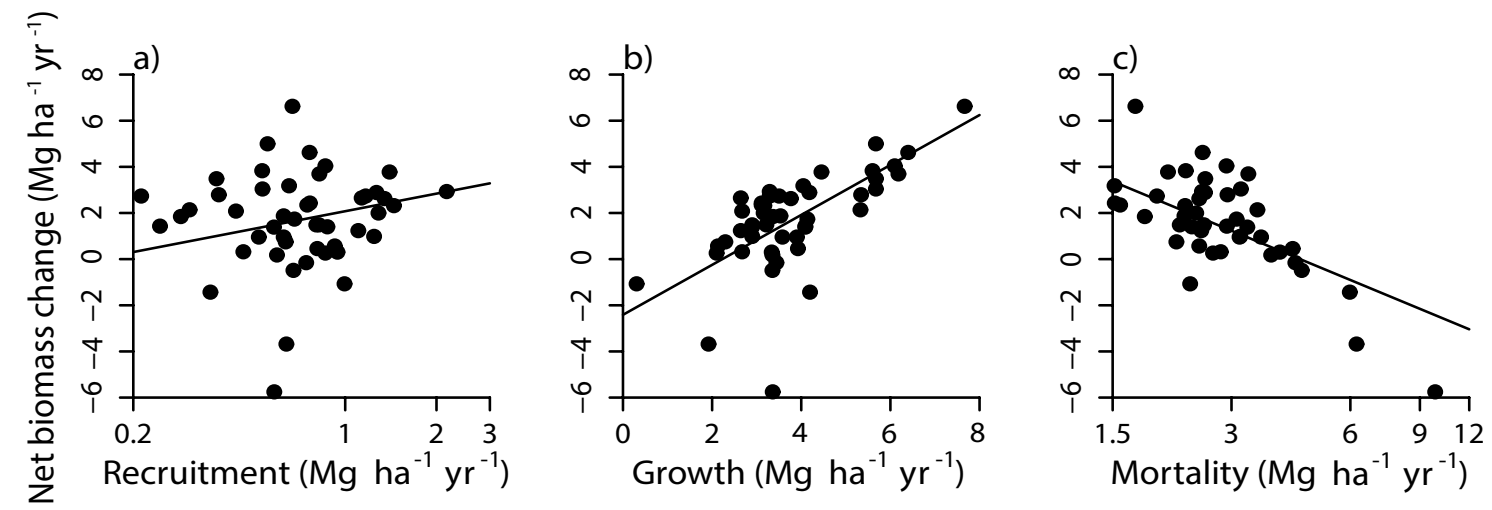

Figure 4.4: Bivariate relations of net biomass change with recruitment (a), growth (b), and mortality (c) for 48 1-ha plots in the tropical moist forest of La Chonta. Regression lines are based on the multiple regression analysis (by keeping the other predictor variables at their mean), see Appendix 4.4. Note that the axes for recruitment (a) and mortality (c) are in ln-scale.

and not explained by any of the abiotic or biotic factors included in our model (Fig. 4.2c, Fig. 4.3c, f, j, 1, o), apart from a weak positive effect of taxonomic richness (Appendix 4.3c). We did not measure direct causes of mortality, but we expected that certain trait values (e.g., high wood density) would lead to lower risk of mortality by causes such as diseases, wind storms and herbivory (Putz et al. 1983, Poorter et al. 2004). The lack of effects on mortality supports our hypothesis that mortality is a stochastic process at this scale. Although mortality may be well predictable at the individual-scale (Chao et al. 2008), species-scale (Poorter et al. 2008) and across stands at regional-scale (Quesada et al. 2012), mortality across stands at local-scale may be more stochastic as it can, for example, be strongly determined by the death of one large tree or the local effect of strong winds. Hence, the strongest predictor of net growth is unpredictable itself.

\section{Dense forests increase growth but decrease recruitment}

We hypothesized that recruitment and growth would be most strongly affected by the direct effect of disturbance. Recruits would face more light limitation than 
survivors, which would be reflected by a stronger positive effect of disturbance and a negative effect of stand basal area on recruitment growth. We indeed found that stand basal area was the most important driver for both processes, with a negative effect on recruitment and a positive effect on growth (Fig. 4.2a vs. b, Fig. 4.3g vs. h). Plot basal area is mainly composed of the basal area of surviving trees, and a higher initial basal area of surviving trees will, therefore, result in higher growth rates, especially since many of these trees are large and contribute most to growth (Stephenson et al. 2014). For recruiting trees in lower canopy layers, however, high plot basal area decreases growth probably because of low light availability (Poorter 1999). Similarly, disturbance had no effect on growth but increased recruitment due to more light availability. Canopy trees are less limited by light and do not benefit from increased light levels due to disturbance, which mostly increases light levels in lower canopy layers (IBIF, unpublished data).

\section{Water availability increases recruitment but decreases growth of larger trees}

For a wide range of ecosystems, soil fertility is an important driver of productivity and demographic processes (e.g., chapter 3), partly via its effect on species composition (Waide et al. 1999). In this forest, water availability is more important for recruitment and growth than soil fertility, and it affects these two demographic processes in a contrasting way (Fig. 4.2a vs. b, Fig. $4.3 \mathrm{~d}$ vs. e). Soil sand content had a negative effect on recruitment, indicating that a community of recruits grows slower on drier soils. In contrast, survivors grow faster on soils that are drier in the dry season (as indicated by the negative effect of, minimum soil water potential on growth). Recruits root less deeply than surviving trees, and may therefore not experience waterlogged conditions and/or the facilitating effect of sand on root growth, but rather experience the negative effect of decreased water holding capacity of the upper soil layers and thus more water stress during the dry season (Markesteijn et al. 2010).

The finding that drier soils increase growth of survivors is in contrast with studies showing that species increase their growth with increasing soil water availability (Baker et al. 2003, Sterck et al. 2011). Possibly, a high soil water potential in the dry season indicates that these microsites are waterlogged and anoxic in the wet season, thus hampering growth especially for large trees with deep roots that suffer more from waterlogged conditions (Ferry et al. 2010, AubryKientz et al. 2015). However, van der Sande et al. (2015) (chapter 2) showed for our study site that growth of large canopy trees was most strongly driven by their (water transporting) sapwood area, indicating that large trees can be strongly limited by water supply. Large trees have a high evaporative demand and probably rely on deep groundwater especially during the dry season (Nepstad et al. 1994). When we replaced soil water potential by sand content in the structural equation model, we 
found that sand content had a positive effect on growth. Possibly, sandy soils facilitate the growth of roots to deeper soil layers, thus stimulating the use of groundwater in drier periods.

Interestingly, soil conditions were important for recruitment and growth but they did not affect diversity and trait composition, as found earlier for the same site (Peña-Claros et al. 2012). This is in contrast with studies showing that soil texture affects the trait composition of African forests (Fayolle et al. 2012) and soil fertility affects trait composition across the Amazon basin (Fyllas et al. 2009), and with studies showing that soil fertility affects species richness positively in a Guyanese tropical rainforest (chapter 3) but negatively in Costa Rican forests (Huston 1980). This suggests that the effects of soil conditions and disturbance on diversity and trait composition are site-specific depend on the length of the soil gradient considered, and the amount of species turnover observed. The lack of soil effects on biotic factors could also be caused by the way we selected the structural equation models (Appendix 4.2): we used variables for soil conditions, trait composition and diversity that best explained the demographic process in which we were interested, but it could be that other soil variables had a stronger effect on our intermediate variables, trait composition and diversity.

\section{What drives recruitment and growth?: niche theory vs. mass-ratio theory}

We evaluated the role of two theories on growth and recruitment: the niche complementarity theory (Tilman 1999), which predicts that high diversity leads to facilitation and/or high resource use efficiency and increased growth and recruitment, and the mass-ratio theory (Grime 1998), which predicts that growth and recruitment are driven by the traits of an average tree in the forest. Taxonomic richness (as an indicator of the niche theory) was important for recruitment but not for growth, and trait composition was not important for any of the two processes (Appendix 4.3, Fig. 4.2a, b). Niche complementarity is thus more important than mass ratio for recruits, probably because they experience strong competition for light, and therefore higher taxonomic diversity may decrease competition and increase the growth of the recruiting community. Hence, recruitment depends strongly on light availability and light use efficiency - through high disturbance, low plot basal area, and high taxonomic diversity - and less on their own trait composition. In contrast, growth does not depend on diversity nor traits.

Several studies find a positive effect of diversity or trait composition on forest productivity (Paquette and Messier 2011, Vilà et al. 2013), but few have simultaneously evaluated the role of the two theories. The few studies that evaluated both theories for tropical forests, partly agree with our results. For a secondary forest in Mexico (Lohbeck et al. 2015), biomass instead of trait composition or trait diversity was important for growth, which is in agreement with 
our results for growth. However, in contrast with our results, across three Neotropical mature forests (Finegan et al. 2015), trait composition but not trait diversity affected growth and only biomass affected recruitment, and for a tropical rainforest in Guyana (chapter 3), trait composition but not taxonomic richness determined productivity. These studies and our study differ in various aspects, such as forest type and environmental conditions, diversity and trait composition indices used, sample size, and percentage of species for which traits were known. So far, results on the relative importance of both theories for tropical forests are not conclusive. Experimental grassland studies have advanced our knowledge on how diversity and trait composition could affect productivity and ecosystem functioning (e.g., Tilman et al. 1997), but more studies are needed in natural and more complex systems at various spatial scales, to unravel mechanisms of various processes, under varying conditions and across a spectrum of species diversity. Possibly, the effect of niche complementarity is most important for recruits because they experience strong interspecific competition for resources, at local scales (e.g., our study) where interspecific interactions take place, and in forests where environmental filtering is less important than interspecific competition. Mass-ratio effects, on the other hand, may be important at regional scales (e.g., Finegan et al. 2015) where variation in trait composition is stronger and better represents functional differences among forests, and in forests where environmental filtering and thus the selection for specific traits is strong (e.g., chapter 3).

\section{Taxonomic richness outperforms trait richness}

Taxonomic richness was selected as the best 'diversity' variable in two of the three SEMs, and it had a significantly positive effect on recruitment. Taxonomic diversity was, surprisingly, a better predictor for recruitment than trait diversity (also called functional diversity or variety, e.g. Mason et al. 2005, Finegan et al. 2015), which should be more mechanistically linked to recruitment. Taxonomic richness and trait richness were significantly positively correlated $(\mathrm{r}=0.57, \mathrm{n}=48$ plots, $\mathrm{P}<0.001$, for recruiting trees in the plot), indicating that higher taxonomic richness partly translates into higher richness in the eight traits that we measured. However, taxonomic richness better predicted recruitment, possibly because a high number of species increases the diversity of more traits or a different set of traits than we measured, such as leaf phenology or the ability to fix nitrogen. It could also be that a higher number of tree species leads to a lower concentration of species-specific soil pathogens, which allows species to maintain productivity compared to low diversity stands that suffer from pathogen attack, as has been found in temperate grasslands (Schnitzer et al. 2011, de Kroon et al. 2012). The positive effect of taxonomic richness may also be explained by only one or a few traits, and may 
therefore partly be concealed when calculating multivariate trait richness based on more but less relevant traits.

\section{Drivers of demographic processes, a matter of scale?}

The relative contribution of different drivers on demographic biomass processes may vary with the spatial and organizational scale considered (Chisholm et al. 2013). At large spatial scales, climate effects vary strongly and may overrule other effects (e.g., pantropical, Phillips et al. 2010, Banin et al. 2014), whereas at regional or local scales, soil conditions may determine demographic processes (Paoli et al. 2005, Baribault et al. 2012). We found that soil sand content and soil water potential overruled soil fertility. Possibly, soil fertility is more heterogeneous at larger spatial scales due to variation in parent material (Malhi et al. 2004, Baker et al. 2009, Toledo et al. 2011), or at smaller spatial scales such as smaller plot sizes (e.g., chapter 3) or the projection area of tree crowns, due to plant-soil feedback effects (Ehrenfeld et al. 2005, Liu et al. 2012), but is relatively homogeneous when compared among averaged samples of 1 -ha plots.

Organizational scales such as communities and species represent different units of measurements, and their demographic processes may therefore be predicted by different factors. For example, traits and forest structure may predict the mortality rate of individual trees (Chao et al. 2008) or species (King et al. 2006b, Iida et al. 2014), but for a whole stand stochastic processes, such as the death of one very large tree or the local occurrence of heavy winds, may strongly determine variation in biomass loss (Gale and Barfod 1999). Furthermore, recruitment and growth can be well explained by traits at the individual or species level (e.g., Poorter and Bongers 2006, van der Sande et al. 2015, see also chapter 2), but not by trait composition at the 1-ha stand level (this study). Species-level demographic changes in growth are a function of the species' growing strategy and average environmental conditions that the species experience, whereas community-level differences in demographic processes are a function of multiple species' strategies, species abundances, and local environmental conditions. These discrepancies between spatial and organizational scales highlight the importance for studies explicitly evaluating the drivers of demographic and other ecological processes at various scales.

\section{Conclusions}

We evaluated how three demographic processes underlying net biomass change (recruitment, growth and mortality) are determined by abiotic and biotic factors. Variation in net biomass change, and thus net carbon sequestration, was most strongly determined by stand-level mortality, implying that understanding the 
drivers of mortality is of crucial importance for the understanding of ecosystem carbon sequestration. However, we show that mortality itself is stochastic, and thus that the major part of variation in net biomass change cannot be predicted.

We expected that recruitment and growth would be driven by diversity (as predicted by the niche complementarity theory) and community-weighted mean traits (as predicted by mass-ratio theory). In contrast to what has been found for grassland experiments, both theories explained nothing or little of demographic processes in this diverse tropical forest. Biomass growth of recruits increased with soil water availability and light availability, whereas biomass growth of larger, established trees increased on dry soils (that may experience less waterlogging in the wet season) and on sandy soils that may facilitate root growth to deeper soil layers. These results highlight the importance of simultaneously testing multiple theories for demographic processes in naturally complex, species-rich systems at various (spatial and organizational) scales. This approach will yield better insights into mechanisms playing a role in the biomass dynamics, and hence in the carbon sequestration and mitigation potential of natural systems.

\section{Acknowledgements}

The research leading to these results has received partial funding from the European Union Seventh Framework Programme (FP7/2007-2013) under grant agreement no 283093 - The Role Of Biodiversity In climate change mitigatioN (ROBIN), and from the DiverSus project through Inter-American Institute for Global Change Research (IAI) CRN 2015 and SGP-CRA2015, supported by the US National Science Foundation grants GEO-0452325 and GEO-1138881. This research is part of the strategic research program KBIV (KB-14) "Sustainable spatial development of ecosystems, landscapes, seas and regions", funded by the Dutch Ministry of Economic Affairs and carried out by Wageningen University \& Research centre (project code KB-14-003-030). We thank the personnel from the Instituto Boliviano de Investigación Forestal (IBIF) for the use of database of the permanent plots, facilities and logistical support, and we thank La Chonta Forestry Concession for logistical support. We are grateful to Geovana Carreño-Rocabado, Stijn van Gils, and Danaë Rozendaal for being able to use their data on leaf traits for several species, and Lars Markesteijn for data on soil water potential. The fieldwork would have been impossible without the field assistance of Ricardo Mendez, Angel Mendez, and other field workers, who assisted in collecting leaf and stem traits and in establishing and evaluating the permanent plots for the last 10 years. 


\section{Appendices}

Appendix 4.1: Trait collection and calculation

\section{Trait collection:}

Leaf trait data were obtained from previous studies (Rozendaal et al. 2006, Carreño-Rocabado et al. 2012, van Gils 2012), and additional species were collected to obtain trait values for a larger part of the trees in the plots, using the same protocol. We collected leaves from 5 individuals between 10 and $20 \mathrm{~cm} \mathrm{DBH}$ that were exposed to direct sunlight or high lateral light levels, and from each individual we selected 5 young and healthy leaves from the outer side of the crown. By using this standardized protocol, we could compare traits across species. We sampled the whole metamer, that is, the leaf, its petiole and corresponding internode (twig section between two leaves). Wood samples were collected for 58 species on trees between 20-40 cm DBH (Poorter 2008). For three trees per species, a sample of the youngest sapwood was taken of about $2 * 2 * 2$ $\mathrm{cm}$.

\section{Trait calculations:}

Directly after the leaves were collected, we separated the leaves from their petiole and internode and measured their surface area using a desktop scanner, their chlorophyll content using a SPAD meter (Minolta SPAD 502 Chlorophyll Meter, Spectrum Technologies Inc., Plainfield, IL, USA), the leaf thickness in between the veins using a micrometer, and leaf toughness using a penetrometer that measures the force needed to punch the flat-ended side of a nail through the leaf. After rehydrating the leaves overnight, their fresh mass was determined. After oven-drying the leaves, petioles and internodes for 48 hours at $70{ }^{\circ} \mathrm{C}$, we measured the dry mass of each part separately. For a more extensive description of trait collection, see Rozendaal et al. (2006).

We then calculated SLA by dividing the leaf area by the dry mass $\left(\mathrm{cm}^{2} \mathrm{~g}^{-1}\right)$; FPs by dividing the force by the product of the circumference of the nail and the thickness of the leaf $\left(\mathrm{N} \mathrm{cm}^{-2}\right)$ to correct for the fracture area on which pressure is exerted; LMFm by dividing the leaf dry mass by the sum of the dry masses of the leaf, petiole and internode $\left(\mathrm{g} \mathrm{g}^{-1}\right)$; and Chl by translating the SPAD units into $\mu \mathrm{g} \mathrm{cm}^{-2}$ using the formula of Coste et al. (2010) for rainforest trees: Chl = (117.1*SPAD) / (148.84 - SPAD). Last, leaves (without petioles and internodes) were pooled per species and analysed for nitrogen and phosphorous concentrations ( $\%$ of dry mass) at CIAT, Santa Cruz, Bolivia. Nitrogen was analysed using the micro-Kjeldahl method, and phosphorus was analysed using digestion by HSO4 and detection using ammonium molybdate solution and a spectrophotometric reading at $882 \mathrm{~nm}$.

As stem traits, we used wood density (WD) (also known as wood specific gravity, Williamson and Wiemann 2010) and maximum stem diameter (DBHmax). For WD, the fresh volume of a sample was measured directly after collecting, using the water displacement method. After oven-drying for 48 hours at $70{ }^{\circ} \mathrm{C}$, dry mass was determined. WD could then be calculated by dividing the dry mass by the fresh volume $\left(\mathrm{g} \mathrm{cm}^{-3}\right)$. For more details on wood collection or WD calculation, see Poorter (2008). Based on the relation between WD of the youngest wood and the average WD of the whole radius of the stem that was available for 32 Bolivian tree species (WDradius $=0.0037+1.0607 *$ WDouter; $\mathrm{R}^{2}=0.90$ ), we predicted average WD of the whole radius for all species. DBHmax $(\mathrm{cm})$ per species was calculated as the $95^{\text {th }}$ percentile of all trees $>10 \mathrm{~cm} \mathrm{DBH}$ at initial and final census. 
Appendix 4.2: Building procedure and results of structural equation models

\section{Model building procedure:}

For each abiotic and biotic factor in Fig. 4.1 (except for the demographic processes, disturbance and diversity), we had more than two possible candidate variables, but could only include one at a time in the SEMs. To reduce the number of variables per abiotic and biotic factor, and thus the number of possible SEMs to choose from, we performed for each demographic process an allsubsets regression analysis for the candidate variables per factor (Burnham and Anderson 2002), and based on this pre-selected one or two variables. All subsets regression analysis evaluates all possible combinations of potential predictor variables, and provides statistics for all relations and Akaike Information Criterion (AIC) for each combination. Because our main goal was to explain variation in biomass dynamics, we used the demographic process as response variable in all cases. Hence, for each of the demographic groups this resulted in three all subsets regression models with respectively as predictors: soil variables, forest structure variables, or trait composition variables (Appendix 4.5). All subsets regression analysis was followed by averaging of the models that differed less than two AIC units from the model that was selected as 'best' (because these models are considered to not be significantly different). Based on this averaged model for each of the abiotic and biotic factors (i.e., the 3 models per demographic process), we selected two variables per factor (in case the average model was composed of more than two variables) with the highest relative variable importance (by summing the 'Akaike weights' for all models where the specific variable occurred; Barton 2012). In the case that more than two variables had the importance value 1 (i.e., the maximum), then the two variables with highest absolute regression coefficients were selected. Per demographic group, we thus had a maximum of two possible variables for three of the abiotic and biotic factors and two possible variables for diversity, which resulted in $24=$ maximum 16 possible models.

The overall fit of these 16 models was first evaluated using a chi-squared $\left(\chi^{2}\right)$ test, and the models that were not rejected (i.e., with a P-value higher than 0.05), were compared based on the R2 of the demographic process (Appendix 4.6). If needed to obtain non-rejected models, pathways that were not important (i.e., not significant and low standardized coefficient) were removed stepwise, starting with removing the pathways with the lowest P-value. We did not use AIC to compare models, because this technique is not well developed for SEM (Daniel Laughlin, personal communications). Instead, we selected the model that best explained variation in the demographic processes (i.e., with the highest $\mathrm{R}^{2}$ for the demographic process), since understanding what drives variation in demographic processes is one of the main aims of this study.

All subsets regression and model averaging were evaluated using the dredge function and the model.avg function, respectively, of the MuMIn package (Barton 2015).

\section{Model building results:}

Based on the all-subsets regression models, we developed 16 potential structural equation models (SEMs) for recruitment, and selected the SEM that was not rejected and had the highest $\mathrm{R}^{2}$ for recruitment, which was our variable of interest (Fig. 4.2a, Appendix 4.3a).

All 16 possible SEMs for growth were initially rejected. To simplify the models, we removed some pathways representing effects on biotic variables that were less important, but aimed to keep all pathways representing direct (significant and non-significant) effects on growth. First, we removed the effect of disturbance and forest structure on diversity because surviving trees were already established before disturbance took place and the forest structure was changed, 
so diversity might be minimally affected. Similarly, we removed the effects of disturbance and forest structure on trait composition because its effects were not significant. As all 16 reduced models were still rejected, soil effects on diversity and trait composition were also removed because these effects were not significant and generally the weakest (in terms of beta coefficient). From these 16 further reduced models (Appendix 4.6), one model was accepted (Fig. 4.2b, Appendix 4.3b).

The all subsets regression models yielded 8 potential SEMs for mortality (Appendix 4.6), and the selected SEM with the highest R2 for mortality is shown in Fig. 4.2c.

Appendix 4.3: Results of the three structural equation models that evaluate the effects of various abiotic and biotic factors on biomass recruitment (a), growth (b), and mortality (c). The models are also shown in Fig. 4.2a-c. The regression coefficient (Coef.), standardized coefficients (Std.Coef.), standard error (SE), Z-value and P-value are given for all regressions (i.e., all arrows in Fig. 4.2a-c), and variation explained $\left(\mathrm{R}^{2}\right)$ are given for all endogenous variables (i.e., variables that are related to predictor variables). All three models were accepted $(P=0.206,0.640$, and 0.110 for $\mathrm{a}, \mathrm{b}$ and c, respectively; Appendix 4.6). Recruitment, mortality and soil water potential (SWP) were ln-transformed. WD = community-weighted mean wood density and FPs = community-weighted mean specific force to punch (Table 4.1).

\begin{tabular}{|c|c|c|c|c|c|c|c|}
\hline & Response variable & Predictor variable & Coef. & Std.Coef. & SE & $\begin{array}{l}\mathrm{Z}- \\
\text { value }\end{array}$ & $\begin{array}{l}\text { P- } \\
\text { value }\end{array}$ \\
\hline \multicolumn{8}{|c|}{ a) } \\
\hline & \multirow{5}{*}{$\begin{array}{l}\text { Ln biomass } \\
\text { recruitment }\end{array}$} & Disturbance & 0.01 & 0.25 & 0.01 & 2.51 & 0.012 \\
\hline & & Sand content & -0.04 & -0.34 & 0.01 & -4.00 & 0.000 \\
\hline & & Plot basal area & -0.06 & -0.40 & 0.01 & -4.20 & 0.000 \\
\hline & & $\begin{array}{l}\text { Taxonomic } \\
\text { richness }\end{array}$ & 0.04 & 0.29 & 0.01 & 3.29 & 0.001 \\
\hline & & FPs & -0.01 & -0.16 & 0.00 & -1.70 & 0.090 \\
\hline & \multirow[t]{2}{*}{ Plot basal area } & Disturbance & -0.12 & -0.37 & 0.04 & -2.66 & 0.008 \\
\hline & & Sand content & 0.01 & 0.01 & 0.10 & 0.05 & 0.961 \\
\hline & \multirow[t]{3}{*}{ Taxonomic richness } & Disturbance & -0.08 & -0.24 & 0.05 & -1.55 & 0.120 \\
\hline & & Sand content & 0.02 & 0.03 & 0.10 & 0.19 & 0.847 \\
\hline & & Plot basal area & -0.27 & -0.26 & 0.15 & -1.73 & 0.084 \\
\hline & \multirow{3}{*}{ FPs } & Disturbance & -0.49 & -0.36 & 0.19 & -2.61 & 0.009 \\
\hline & & Sand content & -0.35 & -0.12 & 0.38 & -0.90 & 0.367 \\
\hline & & Plot basal area & 1.02 & 0.24 & 0.59 & 1.74 & 0.082 \\
\hline & $\begin{array}{l}\mathrm{R}^{2} \mathrm{Ln} \text { biomass } \\
\text { recruitment }\end{array}$ & & 0.667 & & & & \\
\hline & $\mathrm{R}^{2}$ Plot basal area & & 0.135 & & & & \\
\hline & $\mathrm{R}^{2}$ Taxonomic richness & & 0.080 & & & & \\
\hline & $\mathrm{R}^{2} \mathrm{FPs}$ & & 0.239 & & & & \\
\hline \multicolumn{8}{|l|}{ b) } \\
\hline & \multirow{5}{*}{ Biomass growth } & Disturbance & 0.02 & 0.15 & 0.02 & 1.27 & 0.203 \\
\hline & & SWP & 1.35 & 0.39 & 0.40 & 3.38 & 0.001 \\
\hline & & Plot basal area & 0.24 & 0.57 & 0.05 & 4.92 & 0.000 \\
\hline & & Trait richness & 0.23 & 0.14 & 0.22 & 1.09 & 0.276 \\
\hline & & WD & 0.07 & 0.20 & 0.04 & 1.63 & 0.103 \\
\hline & \multirow{2}{*}{ Plot basal area } & Disturbance & -0.11 & -0.35 & 0.04 & -2.48 & 0.013 \\
\hline & & SWP & -0.67 & -0.08 & 1.14 & -0.59 & 0.554 \\
\hline & $\mathrm{R}^{2}$ Biomass growth & & 0.444 & & & & \\
\hline & $\mathrm{R}^{2}$ Plot basal area & & 0.141 & & & & \\
\hline
\end{tabular}


c)

\begin{tabular}{ll|lllll} 
Ln biomass mortality & Disturbance & -0.01 & -0.18 & 0.01 & -1.20 & 0.230 \\
& Sand content & 0.02 & 0.19 & 0.01 & 1.38 & 0.169 \\
& Tree density & 0.12 & 0.12 & 0.15 & 0.82 & 0.413 \\
& $\begin{array}{l}\text { Taxonomic } \\
\text { richness }\end{array}$ & 0.03 & 0.27 & 0.02 & 1.92 & 0.055 \\
& & & & & & \\
FPs & 0.01 & 0.18 & 0.01 & 1.35 & 0.178 \\
Tree density & $\begin{array}{l}\text { Disturbance } \\
\text { Taxonomic richness }\end{array}$ & -0.02 & -0.39 & 0.01 & -2.84 & 0.004 \\
& Sand content & -0.01 & -0.05 & 0.01 & -0.38 & 0.705 \\
& Disturbance & 0.01 & 0.02 & 0.06 & 0.16 & 0.875 \\
FPs & Sand content & -0.01 & -0.01 & 0.12 & -0.07 & 0.942 \\
& Tree density & -2.76 & -0.32 & 1.26 & -2.19 & 0.028 \\
& Disturbance & 0.09 & 0.07 & 0.21 & 0.44 & 0.658 \\
$\mathrm{R}^{2}$ Ln biomass & Sand content & -0.39 & -0.14 & 0.42 & -0.93 & 0.354 \\
mortality & Tree density & 0.92 & 0.03 & 4.49 & 0.21 & 0.837 \\
$\mathrm{R}^{2}$ Tree density & 0.164 & & & & \\
$\mathrm{R}^{2}$ Taxonomic richness & & & & & & \\
$\mathrm{R}^{2}$ FPs & & 0.145 & & & & \\
\end{tabular}

Appendix 4.4: Results of the multiple regression model for the effects of the three demographic processes (recruitment, growth and mortality) on net biomass change. Standardized regression coefficients, standard errors (SE), t-values and P-values are given for each of the predictor variables.

\begin{tabular}{lllll}
\hline & Std. Coefficient & SE & t-value & P-value \\
\hline Recruitment & 0.18 & $<0.01$ & $5.27 * \mathrm{E}^{\wedge} 11$ & $<0.001$ \\
Growth & 0.65 & $<0.01$ & $1.94^{*} \mathrm{E}^{\wedge} 10$ & $<0.001$ \\
Mortality & -0.72 & $<0.01$ & $-2.18^{*} \mathrm{E}^{\wedge} 12$ & $<0.001$ \\
\hline
\end{tabular}


Appendix 4.5: Results of 9 all subsets regression analyses followed by averaging of all models that differed less than 2 AIC from the best fitting model. For each of the three demographic processes (recruitment, growth, and mortality), one analysis was done for three of the abiotic and biotic factors for which we had more than two candidate variables: soil variables, forest structure indices (based on all alive individuals in the plot), and trait composition indices (i.e., the community-weighted mean traits based on the specific demographic group). Each analysis contained all candidate predictor variables for the abiotic and biotic factor. Statistics are shown for the variables that were selected in the 9 averaged models. For each selected predictor variable, standardized regression coefficients ('Std. coef.'), P-values, and relative importance values ('Rel. imp.') are given. Relative importance values were calculated by summing the AIC weights for all models where the specific variable occurred (Barton 2015), and were used to select 1-2 variables per model to develop structural equation models (see variables in bold and Appendix 4.4). For abbreviations of trait composition variables, see Table 4.1.

\begin{tabular}{|c|c|c|c|c|c|c|c|c|c|c|}
\hline \multirow{2}{*}{$\begin{array}{l}\text { Abiotic } \\
\text { or biotic } \\
\text { factor }\end{array}$} & \multirow[b]{2}{*}{ Predictor variable } & \multicolumn{3}{|c|}{ Recruitment } & \multicolumn{3}{|c|}{ Growth } & \multicolumn{3}{|c|}{ Mortality } \\
\hline & & $\begin{array}{l}\text { Std. } \\
\text { coef. }\end{array}$ & $\begin{array}{l}\text { P- } \\
\text { value }\end{array}$ & $\begin{array}{l}\text { Rel. } \\
\text { imp }\end{array}$ & $\begin{array}{l}\text { Std. } \\
\text { coef. }\end{array}$ & $\begin{array}{l}\mathbf{P} \text { - } \\
\text { value }\end{array}$ & $\begin{array}{l}\text { Rel. } \\
\text { imp }\end{array}$ & $\begin{array}{l}\text { Std. } \\
\text { coef. }\end{array}$ & $\begin{array}{l}\mathrm{P}- \\
\text { value }\end{array}$ & $\begin{array}{l}\text { Rel. } \\
\text { imp }\end{array}$ \\
\hline \multirow[t]{9}{*}{ Soil } & $\mathrm{Ca}$ & -0.60 & $<0.01$ & 0.77 & 0.34 & 0.11 & 0.42 & 0.27 & 0.20 & 0.18 \\
\hline & $\mathrm{Mg}$ & & & & & & & 0.08 & 0.61 & 0.04 \\
\hline & Cation exchange capacity & -0.45 & $<0.01$ & 0.23 & 0.28 & 0.12 & 0.31 & 0.28 & 0.18 & 0.21 \\
\hline & $\mathrm{N}$ & 0.14 & 0.29 & 0.08 & 0.23 & 0.12 & 0.57 & & & \\
\hline & $\mathrm{P}$ & -0.20 & 0.25 & 0.06 & 0.14 & 0.35 & 0.03 & -0.29 & 0.17 & 0.44 \\
\hline & $\mathrm{Ph}$ & 0.29 & 0.08 & 0.40 & -0.26 & 0.16 & 0.32 & 0.16 & 0.29 & 0.16 \\
\hline & Clay $\%$ & 0.11 & 0.43 & 0.06 & & & & & & \\
\hline & Sand $\%$ & -0.36 & $<0.01$ & 1.00 & 0.18 & 0.17 & 0.43 & 0.22 & 0.14 & 0.46 \\
\hline & Soil water potential & -0.30 & 0.01 & 0.82 & -0.31 & 0.02 & 1.00 & 0.12 & 0.43 & 0.09 \\
\hline \multirow{4}{*}{$\begin{array}{l}\text { Forest } \\
\text { structure }\end{array}$} & Plot basal area & -0.63 & $<0.01$ & 0.67 & 0.48 & 0.07 & 0.57 & & & \\
\hline & Tree density & 0.21 & 0.16 & 0.43 & -0.29 & 0.18 & 0.51 & 0.11 & 0.48 & 0.30 \\
\hline & $\begin{array}{l}\text { Tree density }>60 \mathrm{~cm} \\
\text { DBH }\end{array}$ & 0.20 & 0.33 & 0.14 & -0.35 & 0.14 & 0.23 & & & \\
\hline & $\mathrm{DBH}_{\text {aver }}$ & -0.22 & 0.17 & 0.26 & 0.35 & 0.06 & 0.34 & & & \\
\hline \multirow{7}{*}{$\begin{array}{l}\text { Trait } \\
\text { compositi } \\
\text { on }\end{array}$} & SLA & -0.17 & 0.253 & 0.16 & 0.08 & 0.595 & 0.12 & & & \\
\hline & $\mathrm{N}_{\text {mass }}$ & 0.32 & 0.173 & 0.10 & & & & & & \\
\hline & $P_{\text {mass }}$ & -0.24 & 0.295 & 0.21 & & & & & & \\
\hline & Chl & & & & 0.27 & 0.176 & 0.12 & 0.08 & 0.578 & 0.22 \\
\hline & FPs & -0.36 & 0.014 & 1.00 & -0.24 & 0.189 & 0.44 & 0.08 & 0.589 & 0.21 \\
\hline & LMF & -0.21 & 0.267 & 0.25 & & & $\theta^{\circ}$ & & & \\
\hline & WD & 0.24 & 0.214 & 0.28 & 0.25 & 0.267 & 0.40 & & & \\
\hline
\end{tabular}


Appendix 4.6: Results of multiple candidate structural equation models (SEMs) per demographic process. Variables for all abiotic and biotic factors were selected based on all subsets regression analyses (Appendix 4.5), except for disturbance and diversity, for which we had respectively only one and two candidate variables. For recruitment and growth, these resulted in 16 possible variable combinations, and for mortality in 8 possible variable combinations. For recruitment and mortality, the results are based on full SEMs (as shown in Fig. 4.1), but for growth, the arrows from soil, disturbance and forest structure to diversity and trait composition were excluded in order to find accepted models (i.e., model P-value $>0.05$ ). In all cases, we had only one possible variable for disturbance, and thus this variable was included in all SEMs and therefore not shown here. For all possible combinations per demographic process, some combinations were accepted (i.e., model P-value $>0.05$ and low model $\chi^{2}$ ), from which the model with the highest $\mathrm{R}^{2}$ for the demographic process was selected and used in the manuscript (see variables and values in bold, and Fig. 4.2). Biomass recruitment and biomass mortality were ln-transformed. For abbreviations of trait composition variables, see Table 4.1.

\begin{tabular}{|c|c|c|c|c|c|c|c|}
\hline $\begin{array}{l}\text { Demographic } \\
\text { process }\end{array}$ & Soil & $\begin{array}{l}\text { Forest } \\
\text { structure }\end{array}$ & Diversity & $\begin{array}{l}\text { Trait } \\
\text { composition }\end{array}$ & Model $\chi^{2}$ & $\begin{array}{l}\text { Model } \\
\text { P-value }\end{array}$ & $\begin{array}{l}\mathrm{R}^{2} \text { of } \\
\text { demographic } \\
\text { process }\end{array}$ \\
\hline \multirow{16}{*}{$\begin{array}{l}\text { Biomass } \\
\text { recruitment }\end{array}$} & \multirow{8}{*}{$\begin{array}{l}\text { Sand } \\
\text { content }\end{array}$} & \multirow{4}{*}{$\begin{array}{l}\text { Plot basal } \\
\text { area }\end{array}$} & \multirow{4}{*}{$\begin{array}{l}\text { Taxonomic } \\
\text { richness } \\
\text { Trait richness }\end{array}$} & FPs & 1.600 & 0.206 & 0.667 \\
\hline & & & & WD & 3.483 & 0.062 & 0.663 \\
\hline & & & & FPs & 1.305 & 0.253 & 0.654 \\
\hline & & & & WD & 0.110 & 0.741 & 0.656 \\
\hline & & \multirow{4}{*}{$\begin{array}{l}\text { Tree } \\
\text { density }\end{array}$} & \multirow{4}{*}{$\begin{array}{l}\text { Taxonomic } \\
\text { richness } \\
\text { Trait richness }\end{array}$} & FPs & 12.140 & 0.016 & 0.448 \\
\hline & & & & WD & 1.866 & 0.172 & 0.568 \\
\hline & & & & FPs & 3.339 & 0.068 & 0.562 \\
\hline & & & & WD & 0.857 & 0.349 & 0.563 \\
\hline & \multirow[t]{8}{*}{ SWP } & \multirow{4}{*}{$\begin{array}{l}\text { Plot basal } \\
\text { area }\end{array}$} & \multirow{4}{*}{$\begin{array}{l}\text { Taxonomic } \\
\text { richness } \\
\text { Trait richness }\end{array}$} & FPs & 1.357 & 0.244 & 0.569 \\
\hline & & & & WD & 7.212 & 0.027 & 0.476 \\
\hline & & & & FPs & 3.538 & 0.060 & 0.592 \\
\hline & & & & WD & 1.195 & 0.274 & 0.682 \\
\hline & & \multirow{4}{*}{$\begin{array}{l}\text { Tree } \\
\text { density }\end{array}$} & \multirow{4}{*}{$\begin{array}{l}\text { Taxonomic } \\
\text { richness } \\
\text { Trait richness }\end{array}$} & FPs & 0.149 & 0.669 & 0.588 \\
\hline & & & & WD & 0.242 & 0.623 & 0.489 \\
\hline & & & & FPs & 1.938 & 0.164 & 0.498 \\
\hline & & & & WD & 3.187 & 0.074 & 0.508 \\
\hline \multirow{16}{*}{$\begin{array}{l}\text { Biomass } \\
\text { growth }\end{array}$} & \multirow[t]{8}{*}{$\mathrm{N}$} & \multirow{4}{*}{$\begin{array}{l}\text { Plot basal } \\
\text { area }\end{array}$} & \multirow{4}{*}{$\begin{array}{l}\text { Taxonomic } \\
\text { richness } \\
\text { Trait richness }\end{array}$} & FPs & 5.622 & 0.060 & 0.365 \\
\hline & & & & WD & 6.339 & 0.042 & 0.532 \\
\hline & & & & FPs & 1.517 & 0.468 & 0.309 \\
\hline & & & & WD & 0.409 & 0.815 & 0.399 \\
\hline & & \multirow{4}{*}{$\begin{array}{l}\text { Tree } \\
\text { density }\end{array}$} & \multirow{4}{*}{$\begin{array}{l}\text { Taxonomic } \\
\text { richness } \\
\text { Trait richness }\end{array}$} & FPs & 11.522 & 0.003 & 0.151 \\
\hline & & & & WD & 11.637 & 0.003 & 0.187 \\
\hline & & & & FPs & 0.947 & 0.623 & 0.157 \\
\hline & & & & WD & 4.882 & 0.087 & 0.221 \\
\hline & \multirow[t]{8}{*}{ SWP } & \multirow{4}{*}{$\begin{array}{l}\text { Plot basal } \\
\text { area }\end{array}$} & \multirow{4}{*}{$\begin{array}{l}\text { Taxonomic } \\
\text { richness } \\
\text { Trait richness }\end{array}$} & FPs & 7.587 & 0.023 & 0.558 \\
\hline & & & & WD & 2.838 & 0.242 & 0.419 \\
\hline & & & & FPs & 0.891 & 0.640 & 0.444 \\
\hline & & & & WD & 10.290 & 0.006 & 0.150 \\
\hline & & \multirow{4}{*}{$\begin{array}{l}\text { Tree } \\
\text { density }\end{array}$} & \multirow{4}{*}{$\begin{array}{l}\text { Taxonomic } \\
\text { richness } \\
\text { Trait richness }\end{array}$} & FPs & 11.020 & 0.004 & 0.165 \\
\hline & & & & WD & 0.668 & 0.716 & 0.145 \\
\hline & & & & FPs & 0.965 & 0.326 & 0.510 \\
\hline & & & & WD & 5.295 & 0.071 & 0.173 \\
\hline \multirow{3}{*}{$\begin{array}{l}\text { Biomass } \\
\text { mortality }\end{array}$} & \multirow[t]{3}{*}{$\mathrm{P}$} & \multirow{3}{*}{$\begin{array}{l}\text { Tree } \\
\text { density }\end{array}$} & \multirow{3}{*}{$\begin{array}{l}\text { Taxonomic } \\
\text { richness } \\
\text { Trait richness }\end{array}$} & Chl & 1.717 & 0.190 & 0.096 \\
\hline & & & & FPs & 3.565 & 0.059 & 0.130 \\
\hline & & & & Chl & 4.935 & 0.026 & 0.139 \\
\hline
\end{tabular}


Drivers of net biomass change in a moist tropical forest

\begin{tabular}{lllllll} 
& & & FPs & 8.614 & 0.003 & 0.241 \\
Sand & Tree & Taxonomic & Chl & 3.136 & 0.077 & 0.115 \\
content & density & richness & FPs & 2.549 & 0.110 & 0.164 \\
& & Trait richness & Chl & 4.784 & 0.029 & 0.131 \\
& & & FPs & 7.433 & 0.006 & 0.243 \\
\hline
\end{tabular}




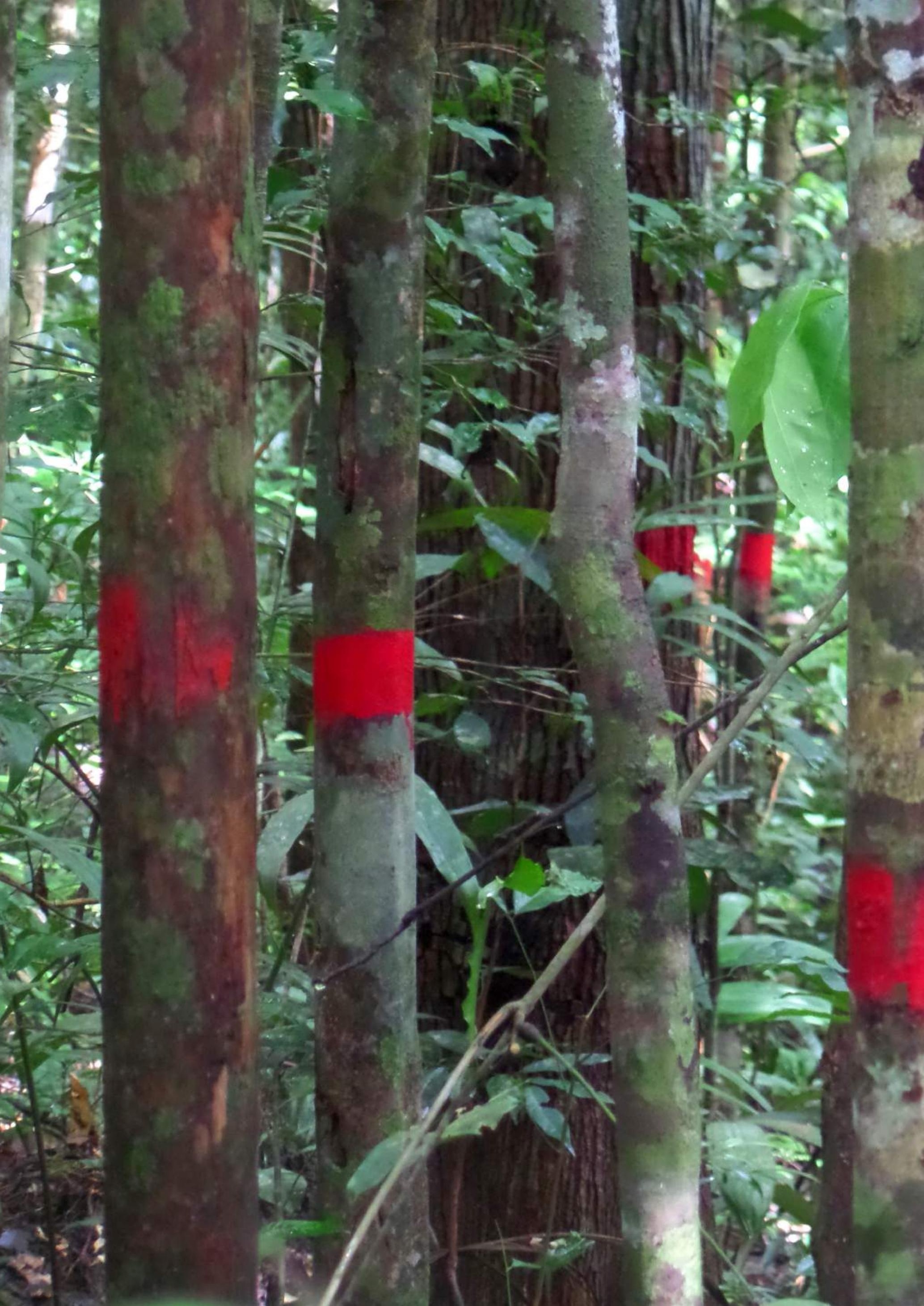




\section{Chapter 5}

\section{Biodiversity, climate and soil determine functioning of Neotropical forests}

Lourens Poorter, Masha T. van der Sande, Eric J. M. M. Arets, Nataly Ascarrunz, Brian Enquist, Bryan Finegan, Juan Carlos Licona, Miguel Martínez-Ramos, Lucas Mazzei, Jorge Meave, Rodrigo Muñoz, Christopher J. Nytch, Alexandre A. de Oliveira, Eduardo A. Pérez-García, Jamir Prado-Junior, Jorge Rodríguez-Velázques, Ademir Roberto Ruschel, Beatriz Salgado-Negret, Ivan Schiavini, Nathan G. Swenson, Elkin A. Tenorio, Jill Thompson, Marisol Toledo, Maria Uriarte, Peter van der Hout, Jess K. Zimmerman, Marielos Peña-Claros 


\section{Abstract}

Tropical forests account for $25 \%$ of the global carbon storage and $34 \%$ of the terrestrial productivity. Few studies have teased apart the relative direct and indirect importance of environmental conditions and forest attributes - including species diversity and community-mean traits - for ecosystem functioning, especially for the tropics. Here, we relate aboveground biomass (AGB), net biomass productivity, and its underlying demographic drivers (biomass recruitment, growth and mortality) to forest attributes (basal area, tree diversity, and community-weighted mean (CWM) traits) and environmental conditions (water availability, soil fertility and disturbance). We use data from $>92,000$ trees, 201 one-ha plots and 26 sites distributed across the main forest types in the lowland Neotropics. For each site we quantified water availability using annual rainfall and climatic water deficit, and soil fertility using $\mathrm{pH}$ and cation exchange capacity. For each plot we quantified the CWM of three key traits (specific leaf area, wood density, and maximum stem diameter) that we expected to be important for biomass stocks and productivity. We used structural equation models to test the hypothesis that species richness, CWM traits, basal area, and environmental conditions have independent, positive effects on biomass stocks and dynamics. We found that forest attributes were stronger drivers (significant in $73 \%$ of the relationships in the models) of biomass stocks and dynamics than environmental conditions (significant in $50 \%$ of the relationships). Increased resource availability in terms of water and soil fertility had positive effects on biomass stocks and dynamics, although they affected different components. Rarefied tree species richness had consistent positive effects on biomass stocks and dynamics, probably because of niche complementarity, but did not affect net biomass change. CWM trait values were good predictors of biomass stocks and dynamics because they reflect how species are filtered out by the environment through their response traits, and how they directly affect ecosystem processes through their effect traits. In sum, forest attributes - including species diversity and community-weighted mean traits - have independent and important effects on AGB stocks, dynamics, and ecosystem functioning, not only in relatively simple temperate ecosystems, but also in structurally complex hyper-diverse tropical forests. Furthermore, water availability has a strong positive effect on biomass stocks and productivity components, and a future predicted increase in (atmospheric) drought may therefore potentially reduce carbon storage.

Keywords: biomass, carbon stocks, carbon sequestration, functional traits, mortality, productivity, rainfall, REDD+, species richness 


\section{Introduction}

Across the globe, there are marked spatial gradients in environmental conditions that have consequences for the diversity and composition of plant communities and the functioning of ecosystems. Insights in the mechanisms underlying these relationships are crucial to understand and predict how ecosystems will respond to climate change and species loss. Most large-scale studies assume that ecosystems are under strong environmental control. Macro-ecologists have shown that largescale gradients in environmental conditions shape biodiversity (e.g., Brown et al. 1995), while ecosystem ecologists and earth system scientists have demonstrated that these environmental gradients determine ecosystem functioning (e.g., Fernández-Martínez et al. 2014). Yet, these latter studies ignore the fact that ecosystems are also under strong control of vegetation attributes, as both biodiversity (Tilman et al. 2001) and biogeography (Hoorn et al. 2010) can have strong and direct impacts on ecosystem functioning. The strong focus on environmental control has perhaps also a methodological reason; it is not only more difficult and labour intensive to quantify the biotic community (as it requires full species identification and characterization), but also the species composition in disparate ecosystems and biomes differs strongly and are therefore difficult to compare.

To facilitate comparison of disparate ecosystems and improve understanding of ecosystem functioning, plant traits have emerged as a promising tool. Such traits (or 'functional traits') allow for quantitative expression of plant form and function using the same yardstick (Westoby 1998, Violle et al. 2014). Functional traits are any measurable plant characteristic that affect the growth and survival of individuals (Violle et al. 2007), and hence, the functioning of communities and ecosystems (Garnier et al. 2004, Finegan et al. 2015). Here we evaluate the relative importance of environmental conditions and forest attributes on ecosystem functioning of 26 Neotropical forests occurring along large-scale gradients in environmental conditions. We focus 1) on biomass stocks and dynamics as key ecosystem functions, as biomass to a large extent drives local and global biogeochemical cycles in carbon, nutrients and water (Chapin et al. 2011, Lohbeck et al. 2015), and 2) on tropical forests because they play a large role in the global carbon cycle (Beer et al. 2010) but the role of forest attributes on carbon stocks and dynamics in such diverse systems remains yet largely unknown. We analyse biomass dynamics in terms of biomass growth of recruiting and surviving trees and biomass loss due to mortality)

Biomass stocks and dynamics depend on environmental conditions, in terms of resource availability (water, nutrients, and light), and on forest attributes (or biotic conditions), in terms of vegetation quantity and quality (Lohbeck et al. 2015). 
Vegetation quantity refers to the amount of photosynthetically active leaf area present (as indicated by stand basal area) and vegetation quality refers to species diversity and to the "average" traits of the community (the community-weighted mean; CWM). Disturbances may modify the vegetation quantity, by removing biomass and opening up the forest canopy, leading to an increased light availability, and hence, enhanced rates of carbon gain in the remaining forest stand (Toledo et al. 2012, Fig. 5.1). To analyse biomass stocks and dynamics, we use the conceptual framework of Poorter et al. (2015, Fig. 1a) and expand this to include effects of community-weighted mean traits and evaluate besides biomass stocks also the biomass dynamics.

Most of our knowledge on biomass dynamics of tropical forests comes from a large network of forest plots in the Amazon basin. The eastern part of the Amazon consists of extremely old and nutrient poor soils and the western part consists of young soils enriched by alluvial deposits. Biomass dynamics are strongly driven by soil fertility (e.g., phosphorus, Quesada et al. 2012) and associated variation in CWM wood density, with forests on low fertility soils being dominated by tough, long-lived tree species (Galbraith et al. 2013) with high WD (ter Steege et al. 2006), leading to a high aboveground standing biomass (Baker et al. 2004b, Malhi et al. 2006, Quesada et al. 2012). Forests on high fertility soils have, however, high biomass dynamics, which seem to be more driven by resource availability than by species traits (Baker et al. 2009). Yet, the Amazon is climatically and biogeographically a relatively homogeneous region; therefore, the question is whether different relationships emerge when the full environmental and biogeographical range of lowland Neotropical forests is considered. With larger gradients, other variables such as water availability, species richness, and different traits (e.g., specific leaf area rather than wood density) may emerge as the main drivers of biomass stocks and dynamics.

High species diversity may enhance biomass stocks and dynamics through niche complementarity because species occupy different niches or facilitate each other, leading to a more efficient resource use at the community level, resulting in higher biomass growth. This higher biomass growth may increase biomass build-up and thus increase biomass stocks (Chisholm et al. 2013). A large body of experiments has shown that species diversity indeed enhances productivity (reviewed in Cardinale et al. 2011), but the question is whether the effect is also ecologically relevant and strong enough to be observed in the field. It is difficult to empirically assess the independent effect of species diversity on biomass stocks and dynamics in the field, as both diversity and biomass stocks and dynamics can respond in a similar way to environmental conditions. Few studies have simultaneously looked at the independent effects of environmental conditions and diversity on biomass stocks and dynamics. In Canada, functional tree diversity had 
a strong positive effect on productivity in climatically harsh boreal forest, but a weaker effect in climatically more benign temperate forests (Paquette and Messier 2011). In Europe, tree diversity had a significant positive effect on biomass productivity for four out of 11 forest types (Vilà et al. 2013). For hyper-diverse tropical forests, diversity might be less relevant because of a saturation effect, but similar studies have only been done at the local-scale (chapters 3 and 4, Barrufol et al. 2013, Prado-Junior et al. 2016), for biomass stocks (Poorter et al. 2015), or have only considered some of the drivers (Finegan et al. 2015). Insights into the mechanisms underlying the effects of climate, diversity, and other forest attributes on ecosystem functioning in tropical forests are important to understand how ecosystems may respond to climate change, species loss and shifts in species composition.

Here we use dynamic data from $>92,000$ trees, 201 one-ha plots and 26 sites distributed across the main forest types in the lowland Neotropics. For each site we quantified water availability by using annual rainfall and climatic water deficit (CWD), and soil fertility by using $\mathrm{pH}$ and cation exchange capacity (CEC). For each plot we quantified the CWM of three key traits (specific leaf area, wood density, and maximum diameter) that we expected to be important for biomass stocks and dynamics (Conti and Díaz 2013).

The aim of this study is to analyse how environmental conditions and forest attributes drive biomass stocks and dynamics of Neotropical forests (Fig. 5.1). We address two questions. First, how do environmental conditions drive biomass stocks and dynamics? We hypothesize that biomass stocks and dynamics increase with water availability, soil fertility, and disturbance, and that biomass stocks and dynamics are most strongly affected by rainfall (as this is the main driver of spatial variation in biomass and diversity in the lowlands, ter Steege et al. 2003, Poorter et al. 2015), and to a lesser extent by soil fertility and disturbance. Second, how do forest attributes that are related to vegetation quality (e.g., species richness and community-weighted mean traits) and vegetation quantity (e.g., basal area) affect biomass stocks and dynamics? We hypothesize that high species diversity enhances biomass stocks and dynamics because of niche complementarity, and that communities with productive trait values (e.g., high CWM specific leaf area) have high biomass dynamics, whereas communities with conservative trait values (e.g., high CWM wood density) have longer-lived tissues and trees, and hence, large biomass stocks. 


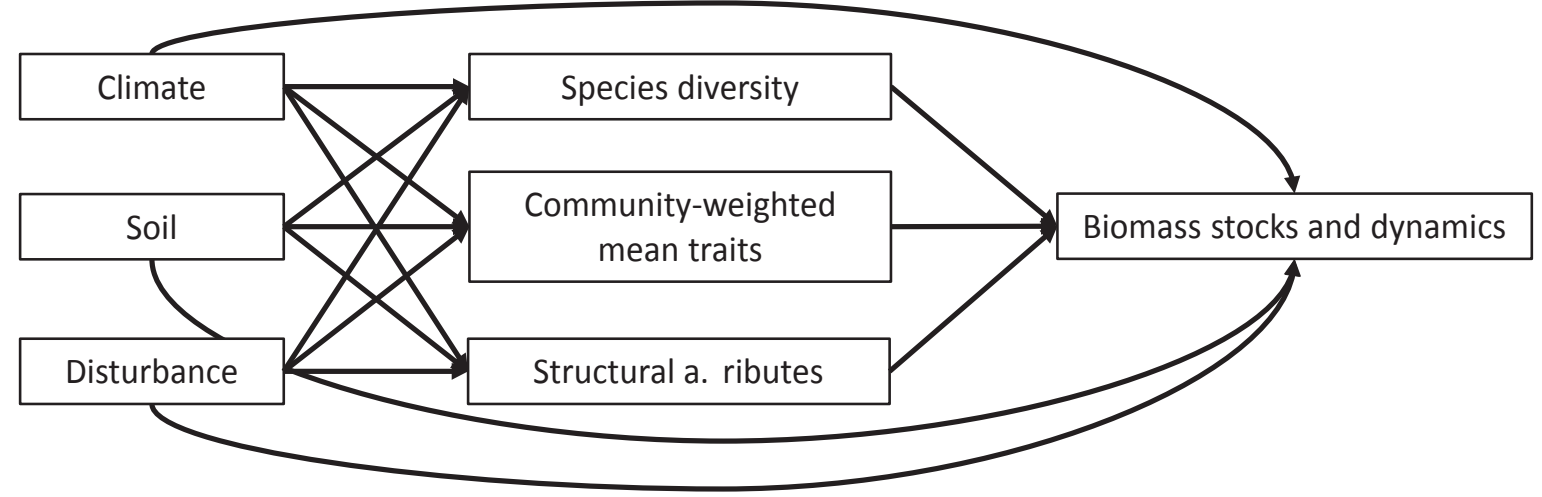

Figure 5.1: Conceptual framework linking environmental conditions and forest attributes to biomass variables (biomass stocks and dynamics).

\section{Methods}

\section{Study sites}

We used data from 201 1-ha plots in 26 sites distributed across the Neotropics, from Mexico to Bolivia (Appendix 5.1 and 5.2). Precipitation varied from 784-3991 $\mathrm{mm} \mathrm{y}^{-1}$, and the soil cation exchange capacity from 2.0-726.7 $\left(\mathrm{cmol} \mathrm{kg}^{-1}\right)$. All plots were located in mature forests, of which $47 \%$ had been subjected to timber extraction between 14-32 years ago as part of long-term experiments on the effect of logging.

\section{Plot size and measurement period}

We used plots established for different purposes; therefore, their size, shape and spatial distribution varied among sites. Most of the forest inventory plots $(66 \%$ of plots) are 1-ha and square. To standardize all other plots to this size, we combined small plots (e.g., the 50*50 m plots in Tapajós) and subdivided large ones (e.g., the 15-ha plot in Luquillo) using the same criteria as in Poorter et al. (2015).

We used data coming from two censuses to calculate biomass dynamics. The census period ranged between 4 and 11 years with an average of 7.9 years. The majority of the census periods (for 86\% of the plots) fell between 2000 and 2015 . For each plot, a list of variables was calculated representing the different boxes in the conceptual framework in Fig. 5.1.

\section{Biomass stocks and dynamics}

For each individual tree $\geq 10 \mathrm{~cm}$ stem diameter at breast height (DBH, measured at $1.3 \mathrm{~m}$ from the ground) present in the plots in one or two censuses, we calculated aboveground biomass using the allometric formula of Chave et al. (2014b). For 
each site we estimated the $\mathrm{E}$ value, which is a measure of environmental stress, using R (http://chave.ups-tlse.fr/pantropical_allometry/readlayers.r, with the retrieve_raster function from the $\mathrm{R}$ packages raster (Hijmans et al. 2015) and ncdf). Wood density (WD, $\mathrm{g} \mathrm{cm}^{-3}$ ) data came from the local sites or from the Neotropical data of the global WD database "Dryad" (Zanne et al. 2009, http://datadryad.org/handle/10255/dryad.235). For all WD estimates we used the data source (local or Dryad) that had the highest level of taxonomic resolution. When the resolution was the same, we used the local data source. When no WD information was available at the species level, we used the genus- or family-level WD values, as WD is phylogenetically strongly conserved (Chave et al. 2006). Other life forms (lianas and palms) were not considered in biomass calculations because of lack of adequate allometric equations to estimate their biomass (for palms and lianas) or because they were not consistently measured in all plots (for lianas). For multiple-stemmed trees, all stems $\geq 10 \mathrm{~cm}$ in DBH were included in the calculations of biomass. With the aboveground biomass (AGB) at individual tree level, we calculated five variables of biomass stocks and dynamics at the plot level (in $\mathrm{Mg} \mathrm{ha}{ }^{-1} \mathrm{y}^{-1}$ ):

AGB growth of survivors ( $\triangle \mathrm{AGBsurv)}$ is the annual change in biomass produced by the growth of all the stems in a plot that survived from census 1 to census 2 . Biomass growth of each stem was calculated as the difference in biomass between census 1 and 2, divided by the time interval in years between the two censuses;

AGB growth of recruits ( $\triangle \mathrm{AGBrecr}$ ) is the annual increment of biomass obtained from trees that recruited between census 1 and 2 . Biomass of each new stem $\geq 10$ $\mathrm{cm}$ DBH was calculated as the difference between the biomass when first measured in census 2 and the biomass as if the stem had a $10 \mathrm{~cm} \mathrm{DBH}$ at census one, divided by the average time between the first and second census for that specific plot. This assumes the tree recruited immediately after the first census (Talbot et al. 2014);

$A G B$ loss due to mortality ( $\triangle \mathrm{AGBmort}$ ) is the annual loss of biomass due to stems dying between census 1 and 2 . To be consistent with the calculations done for the recruits, the biomass of the each dead stem was calculated as the difference between the biomass at census 1 and the biomass of this stem as if it had a $10 \mathrm{~cm}$ $\mathrm{DBH}$, divided by the average time between the first and second census for that plot. Mortality was only based on natural tree death, not on death due to logging activities or consequences of these activities;

Net $A G B$ change $(\triangle \mathrm{AGB})$ is the annual net change in biomass between census 1 and 2. It was calculated as the difference between biomass stock in census 1 and census 2. We also calculated $\triangle \mathrm{AGB}$ as the difference between biomass growth $(\triangle \mathrm{AGBsurv}+\triangle \mathrm{AGBrecr})$ and biomass loss $(\triangle \mathrm{AGBmort})$. Both ways of calculating $\triangle \mathrm{AGB}$ were highly correlated (Pearson $\mathrm{r}=0.96, \mathrm{P}<0.001$ ). Because we did not 
have data on biomass dynamics for one of the sites (San Emilio), we further use $\triangle \mathrm{AGB}$ based on the first method;

Aboveground biomass stock (AGB) is the sum of biomass of all live trees in one census. For plots that did not receive logging disturbance, we used the average AGB of the first and second census For plots that received logging disturbance, we used a pre-logging census to calculate biomass stocks.

For each of these biomass variables, we developed a separate model as shown in Fig. 5.1, using structural equation modeling (see Appendix 5.6 for sample size used for each biomass variable). For many of the boxes of the environmental conditions and forest attributes, we had multiple possible variables to use (e.g., multiple species diversity indices).

\section{Species diversity}

For each plot, three species diversity measures were calculated for each census: species richness (number of species per plot), Shannon diversity, and rarefied species richness. Species richness is most often used, whereas Shannon diversity also incorporates information on species abundances. Species richness was calculated as the number of species per ha, based on all trees $\geq 10 \mathrm{~cm} \mathrm{DBH}$. Shannon diversity was calculated as $H^{\prime}=-\Sigma\left(p_{i} \ln \left(p_{i}\right)\right)$, where $p_{i}=$ the proportion of individuals of species $\mathrm{i}$ in the plot. Rarefied species richness is the number of species when a certain number of trees is randomly drawn from a plot, removing in this way the confounding effect of tree density on species richness. We calculated rarefied species richness as the number of species at a random draw of 200 stems, as this number of individuals was found in all plots. Multiple-stemmed individuals were counted as one individual for species diversity calculations. Calculations were done either using EstimateS 9.1.0 (Colwell 2011) or the R package vegan (Oksanen et al. 2014). Species diversity measures for the first and second census were averaged to obtain one value per plot that would better represent the species diversity experienced during the census period.

\section{Community-weighted mean traits}

It could also be that not the diversity in species but rather the most dominant species and their traits determine ecosystem functioning. The central tendency of the trait values can be described with the community-weighted Mean (CWM; the "average" trait value of individuals in the community). The CWM is a univariate trait index which was calculated for each plot, each census year, and for each of the three traits by weighing species trait values by species basal area $\left(\right.$ in $\left.\mathrm{m}^{2}\right)$ in the plot. Effects of CWM traits on ecosystem functioning are in line with the mass-ratio hypothesis of Grime (1998), which predicts that ecosystem functioning is 
determined by the trait values of the most dominant species in the community. We selected eight traits that have been found to affect productivity at the species level (Appendix 5.3). From the eight traits, only three were available for 24 (out of 26) sites: specific leaf area (SLA), wood density (WD) and maximum diameter (DBHmax). Traits were mostly measured following standardized protocols (PérezHarguindeguy et al. 2013), although site differences occurred because different sites collected traits initially for different aims. In general, traits were measured for 3-10 trees per species, for trees $>10 \mathrm{~cm} \mathrm{DBH}$. Per tree 3-20 leaves growing in the outer canopy were collected. Leaf area was measured and leaves were oven-dried at 70 ${ }^{\circ} \mathrm{C}$. SLA was calculated as leaf area divided by leaf dry mass, generally excluding the petioles. Stem samples were taken with an increment corer for 3-5 trees per species at 0.5-2 $\mathrm{m}$ height aboveground. Volume of the stem sample was measured with the water displacement method, after which they were oven-dried for 2 days at 101-104 ${ }^{\circ} \mathrm{C}$. Wood density was measured as wood mass over wood volume. To calculate maximum diameter, we first pooled for each species all trees in a site. We then calculate the $95^{\text {th }}$ percentile of stem diameter for each species.

For each plot and each census, we calculated the CWM trait values based on all species for which trait data were available. To obtain accurate estimates, CWM trait values should be calculated based on the most dominant species that contribute to at least $80 \%$ of the total basal area in the plot (Garnier et al. 2004). This criterion was met, as the average basal area covered in the plots was $89 \%$ for specific leaf area, 93\% for wood density, and almost 100\% for maximum diameter. Calculations were done with the software FDiversity (Casanoves et al. 2011, http://www.FDiversity.nucleodiversus.org/) or $\mathrm{R}$ (using the $d b F D$ function of the FD package). The CWM trait values were averaged between the two census years per plot.

\section{Structural attributes}

For each plot and census year, three structural attributes were calculated: total tree density $(\geq 10 \mathrm{~cm} \mathrm{DBH})$, density of trees $\geq 50 \mathrm{~cm} \mathrm{DBH}$, and stand basal area (in $\mathrm{m}^{2}$ ). For total tree density and density of trees $\geq 50 \mathrm{~cm} \mathrm{DBH}$, multiple-stemmed individuals counted as one individual. For multiple-stemmed trees, all stems $\geq 10$ $\mathrm{cm}$ DBH were included in the calculations of stand basal area. Also for structural attributes, the average between the two censuses was used per plot. 


\section{Environmental conditions}

For each site, mean annual rainfall was obtained from the nearest climatological station and climatic water deficit (CWD) was obtained based on the coordinates of each plot from http://chave.ups-tlse.fr/pantropical_allometry/readlayers.r (with the retrieve_raster function from the $\mathrm{R}$ packages raster and ncdf, as was done to calculate the $\mathrm{E}$ value). CWD is based on the water loss during the dry months (when evapotranspiration exceeds rainfall) and may more accurately reflect drought conditions than total annual rainfall (e.g., van Mantgem et al. 2009). CWD of 0 indicates very wet conditions, whereas large negative CWD indicates very dry conditions.

We searched for soil data per site, ideally collected at the plot level in the first $20-30 \mathrm{~cm}$ of the soil. Unfortunately, sites differed largely in the soil data available and in the extraction methods used (e.g., for phosphorus). Consequently, we decided to focus on $\mathrm{pH}$ and $\mathrm{CEC}$ as indicators of soil fertility because they were partly locally available and could otherwise be obtained from the Harmonized World Soil Database (HWSD version 1.2; Nachtergaele et al. 2010). pH is no direct measure of soil fertility, but is often positively related with concentrations of soil nutrients and cations (Quintero-Vallejo et al. 2015). We used locally available data if present, and otherwise used data from the HWSD.

\section{Disturbance}

For the logged plots ( $47 \%$ of total), logging disturbance was estimated by summing the basal area of trees that were removed from the plot due to timber extraction, or that died due to logging damage and application of additional silvicultural treatments. Disturbance was then calculated as basal area that was removed or died as a percentage of the total basal area of the plot.

\section{Statistical analyses}

To evaluate direct and indirect causal effects of environmental conditions and forest attributes on each of the biomass variables as presented in Fig. 5.1, we developed structural equation models (SEMs) (Shipley 2004, Grace 2006), with plots nested within sites. Biomass growth by recruiting trees and biomass loss due to mortality were $\log 10$-transformed to result in normally distributed residuals and equal variances. As measure for species diversity, we a-priori selected rarefied species richness because 1) this variable avoids the fact that plots with a high stem density may for this reason have a high species richness, 2) it has been shown to be strongly related to biomass stocks across Neotropical forests (Poorter et al. 2015), and 3) species richness is more widely used than Shannon diversity and thus allows for comparisons. As measure of structural attribute, we a-priori selected plot basal 
area of all trees $\geq 10 \mathrm{~cm} \mathrm{DBH}$, because this variable well represents the density and thus the competition within the plot and it was an important predictor in other single-site studies (e.g., chapter 4). For CWM trait values, we used three traits that were available for most of the plots: SLA, WD and DBHmax. For climate, we used annual rainfall because this variable was available for all sites and is often important for biomass stocks and dynamics (e.g., Poorter et al. 2015), and the climatic water deficit (CWD) at this also includes evapotranspiration. For soil conditions, we used $\mathrm{pH}$ and cation exchange capacity (CEC). The number of plots and sites in each SEM varied depending on data availability (see Appendix 5.6 for sample sizes).

Per biomass variable, we considered 12 possible structural equation models (3 possible CWM traits*2 climate variables*2 soil variables). In some cases, climate has a hump-shaped relationship with vegetation attributes or ecosystem processes. This can be solved by including a composite variable based on rainfall and rainfall ${ }^{2}$ or on CWD and $\mathrm{CWD}^{2}$. Consequently, we tested a-priori whether hump-shaped relationships needed to be included in the SEMs by relating the vegetation attributes and the five biomass variables to rainfall and rainfall ${ }^{2}$ or to CWM and $\mathrm{CWM}^{2}$ using linear mixed models with site as random variable. Only in one case we found a significant relationship (between $\mathrm{CWD}^{2}$ and rarefied species richness, Appendix 5.4), and therefore we did not include the quadratic terms in further analyses. The 12 models per biomass variable were compared based on the chisquare statistic for model fit. If the P-value of the chi-square is higher than 0.05, then the model is accepted. If several of the 12 models were accepted, then we selected the one with the highest $\mathrm{R}^{2}$ for the biomass variable because this was our main variable of interest. We also evaluated the effects of $\Delta A G B r e c r, \Delta A G B s u r v$ and $\triangle \mathrm{AGBmort}$ on $\triangle \mathrm{AGB}$ using a linear mixed model with site as random variable.

To evaluate bivariate relationships between vegetation attributes, environmental conditions, and the biomass variables, we used Spearman correlations. All analyses were performed in R 3.1.2. Correlations were evaluated using the rorr function of the Hmisc package (Harrell and Dunpot 2015), linear mixed models with the lme function of the nlme package (Pinheiro and Bates 2016), and structural equation models with the sem function of the lavaan package (Rosseel 2012). We corrected for nesting of plots within sites in the SEMs by using the svydesign function of the survey package (Lumley 2015) and the lavaan.survey function of the lavaan.survey package (Oberski 2013). 


\section{Results}

To evaluate our conceptual model (Fig. 5.1) we used structural equation modelling (SEM). We selected one model for each of the five biomass variables (Fig. 5.2, and see Appendix 5.5 for the results on model selection). The explained variation in biomass variables ranged from $31 \%$ for net biomass change to $87 \%$ for biomass stocks (Fig. 5.2).

Environmental conditions had direct and indirect effects on biomass stocks and dynamics (Fig. 5.2, 5.3, Appendix 5.6). Water availability (as indicated by rainfall or CWD) increased $\triangle \mathrm{AGBsurv}$ (standardized regression coefficient $\beta=$ 0.44, Fig. 5.2a, 5.4a), $\Delta$ AGBrecr $(\beta=0.33$, Fig. 5.2b) and AGB $(\beta=0.39$, Fig. 5.2e, 5.5b). Soil fertility (as indicated by $\mathrm{pH}$ and CEC) increased $\triangle \mathrm{AGBrecr}(\beta=0.59$, Fig. 5.2b, 5.4b), $\triangle \mathrm{AGB}(\beta=0.18$, Fig. 5.2d, Fig. 5.5a) and AGB $(\beta=0.39$, Fig. 5.2e). Soil fertility had, however, strong negative indirect effects on $\triangle A G B s u r v$ (Fig. 5.3). Additionally, disturbance increased $\Delta$ AGBsurv $(\beta=0.20)$ and $\Delta A G B r e c r$ $(\beta=0.22$, Fig. 5.2a, b). All environmental conditions had also indirect effects on all five biomass variables via forest attributes (Fig. 5.2). Figures of all bivariate relations between environmental conditions and biomass stock and dynamics included in Fig. 5.2 are shown in Appendix 5.7.

Forest attributes had generally strong and significant effects on biomass stocks and dynamics; from all 15 possible relations, 11 (73\%) were significant (Fig. 5.2). Species richness and CWM traits were important for four biomass variables and structural attributes for three biomass variables. Species richness increased $\Delta$ AGBsurv $(\beta=0.31$, Fig. 5.2a), $\Delta$ AGBrecr $(\beta=0.30$, Fig. 5.2b), $\Delta$ AGBmort $(\beta=$ 0.38, Fig. 5.2c) and AGB $(\beta=0.22$, Fig. 5.2e), while it did not affect $\triangle$ AGB (Fig. $5.2 \mathrm{~d})$. Plot basal area increased $\Delta$ AGBsurv $(\beta=0.43), \Delta$ AGBmort $(\beta=0.23)$ and AGB $(\beta=0.49)$. CWM WD had a positive effect on AGB $(\beta=0.56)$ and, surprisingly, also on $\triangle \mathrm{AGBsurv}(\beta=0.39)$. CWM DBHmax decreased $\triangle \mathrm{AGBrecr}$ $(\beta=-0.29)$, while CWM SLA increased $\triangle \mathrm{AGB}(\beta=0.51)$. Figures of all bivariate relations between forest attributes and biomass stock and dynamics included in Fig. 5.2 are shown in Appendix 5.8.

$\triangle \mathrm{AGB}$ was most strongly predicted by $\triangle \mathrm{AGBmort}(\beta=-0.97, \mathrm{P}<0.001)$, followed by $\Delta$ AGBsurv $(\beta=0.50, \mathrm{P}<0.001)$, and not by $\Delta$ AGBrecr $(\beta=0.06, \mathrm{P}=$ $0.14)$. 
a)

$0.44^{* *}$

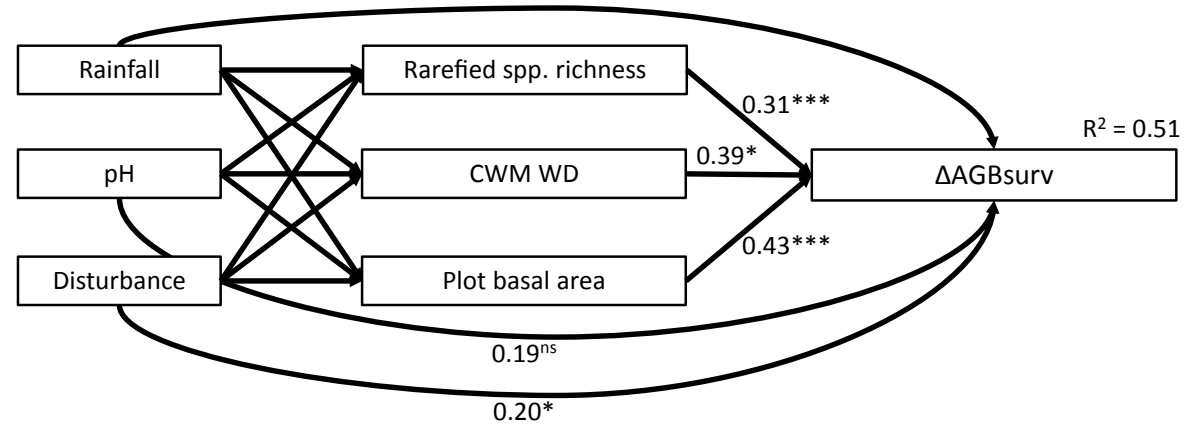

b)

$0.33^{* * *}$

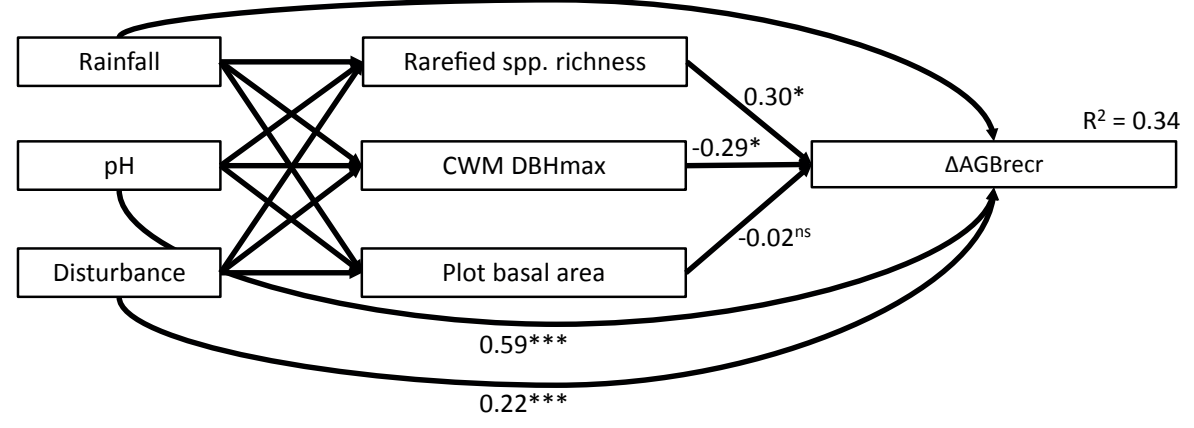

c)

$0.19^{\text {ns }}$

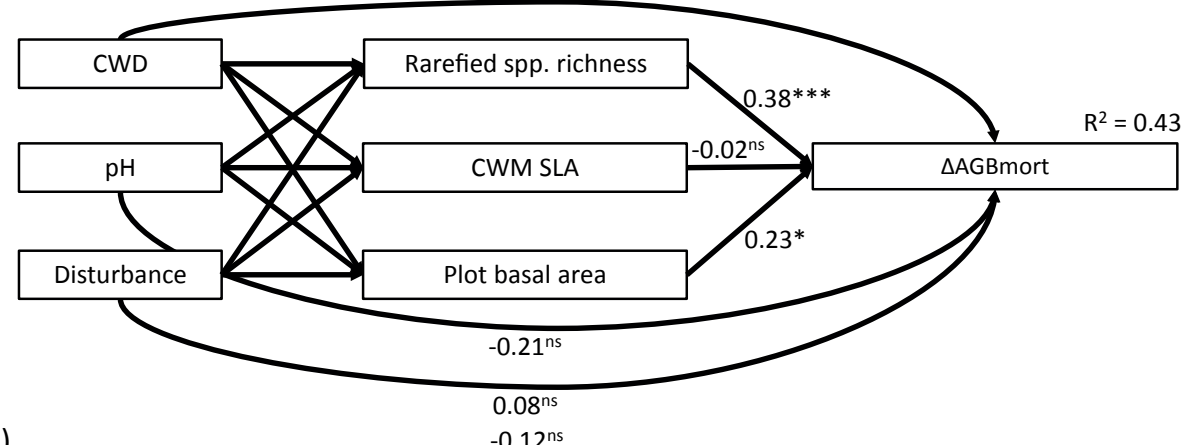

d)

$-0.12^{\text {ns }}$

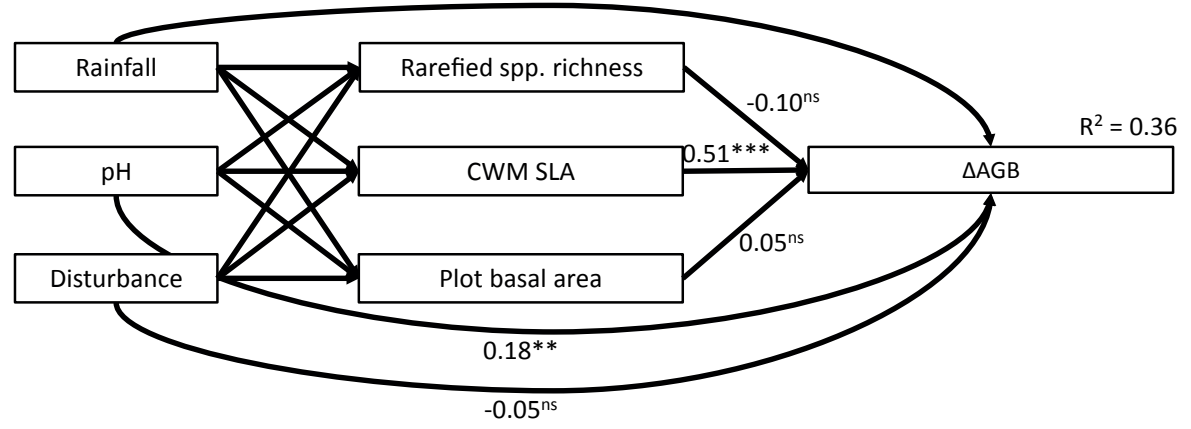

e)

$0.39^{* * *}$

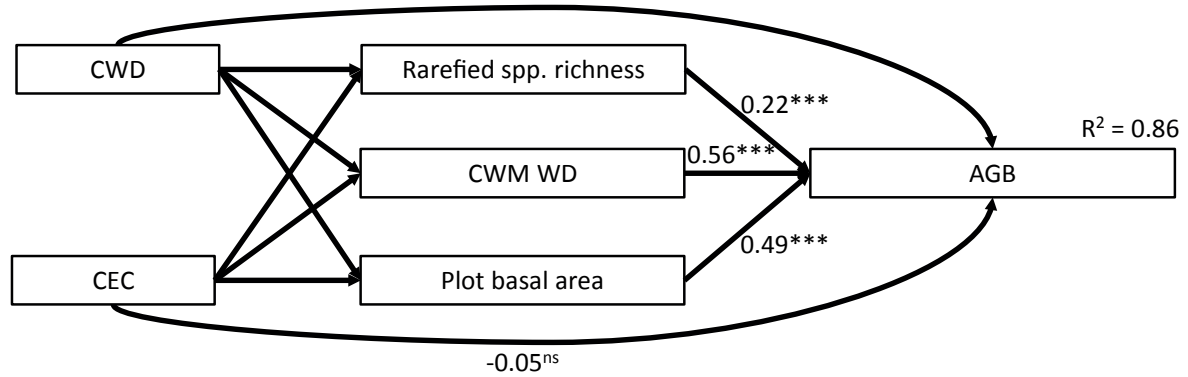

Figure 5.2: Structural equation models for the effects of the environmental conditions (climate, soil and disturbance) and forest attributes (rarefied species richness, community-weighted mean (CWM) traits, and plot basal area) on each of the five biomass variables: a) biomass growth by 
surviving trees ( $\triangle \mathrm{AGBsurv}), \mathrm{b})$ biomass growth by recruiting trees $(\triangle \mathrm{AGBrecr}), \mathrm{c}$ ) biomass loss due to mortality ( $\triangle \mathrm{AGBmort}$ ), $\mathrm{d}$ ) net biomass change $(\triangle \mathrm{AGB})$ and, $\mathrm{e})$ aboveground biomass stocks (AGB). Standardized coefficients with significance level (ns = not significant, $*<0.05, * *$ $\left.<0.01,{ }^{* * *}<0.001\right)$ are given for all direct relationships with the biomass variables. The standardized beta coefficients and significance for all other relationships can be found in Appendix 5.6. Black lines indicate significant effects, whereas dashed lines indicate nonsignificant effects. Per ecosystem process, the explained variation $\left(\mathrm{R}^{2}\right)$ is provided. For statistics of model fit, see Appendix 5.5. CWD = climatic water deficit, SLA = specific leaf area, WD = wood density.

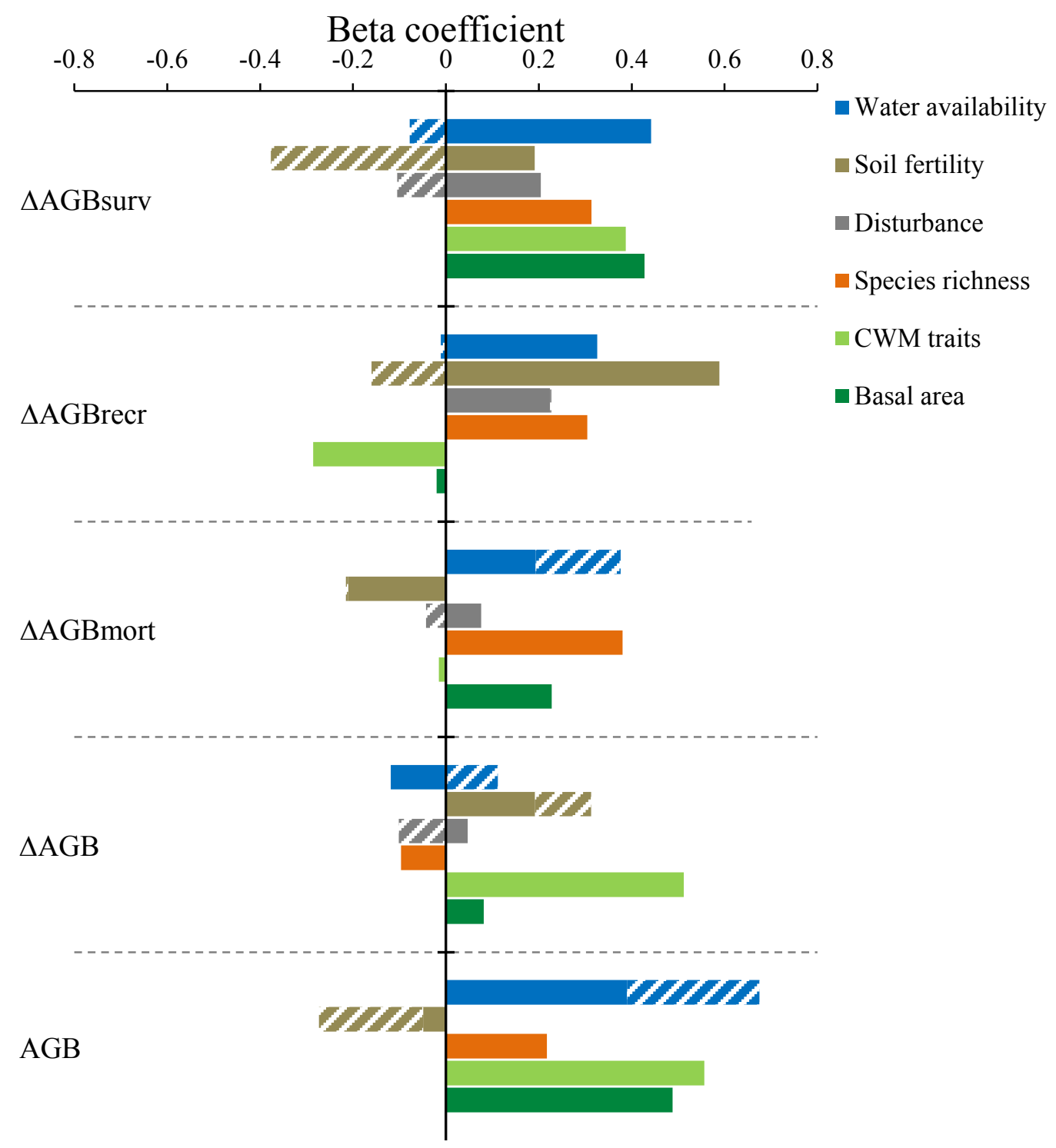

Figure 5.3: Beta coefficients of environmental conditions and forest attributes on five biomass

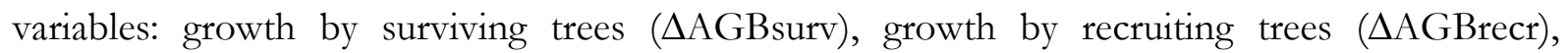
biomass mortality ( $\triangle \mathrm{AGB}$ mort), net biomass change $(\triangle \mathrm{AGB})$, and biomass stocks (AGB). The colors represent different environmental conditions or forest attributes: blue $=$ water availability (rainfall or climatic water deficit), brown $=$ soil fertility $(\mathrm{pH}$ or cation exchange capacity), grey $=$ disturbance, orange $=$ species richness, light green $=$ community-weighted mean $(C W M)$ traits, and dark green $=$ plot basal area. The filled bars show the direct effects and the dashed bars show the indirect effects of environmental conditions on biomass stocks and dynamics. 

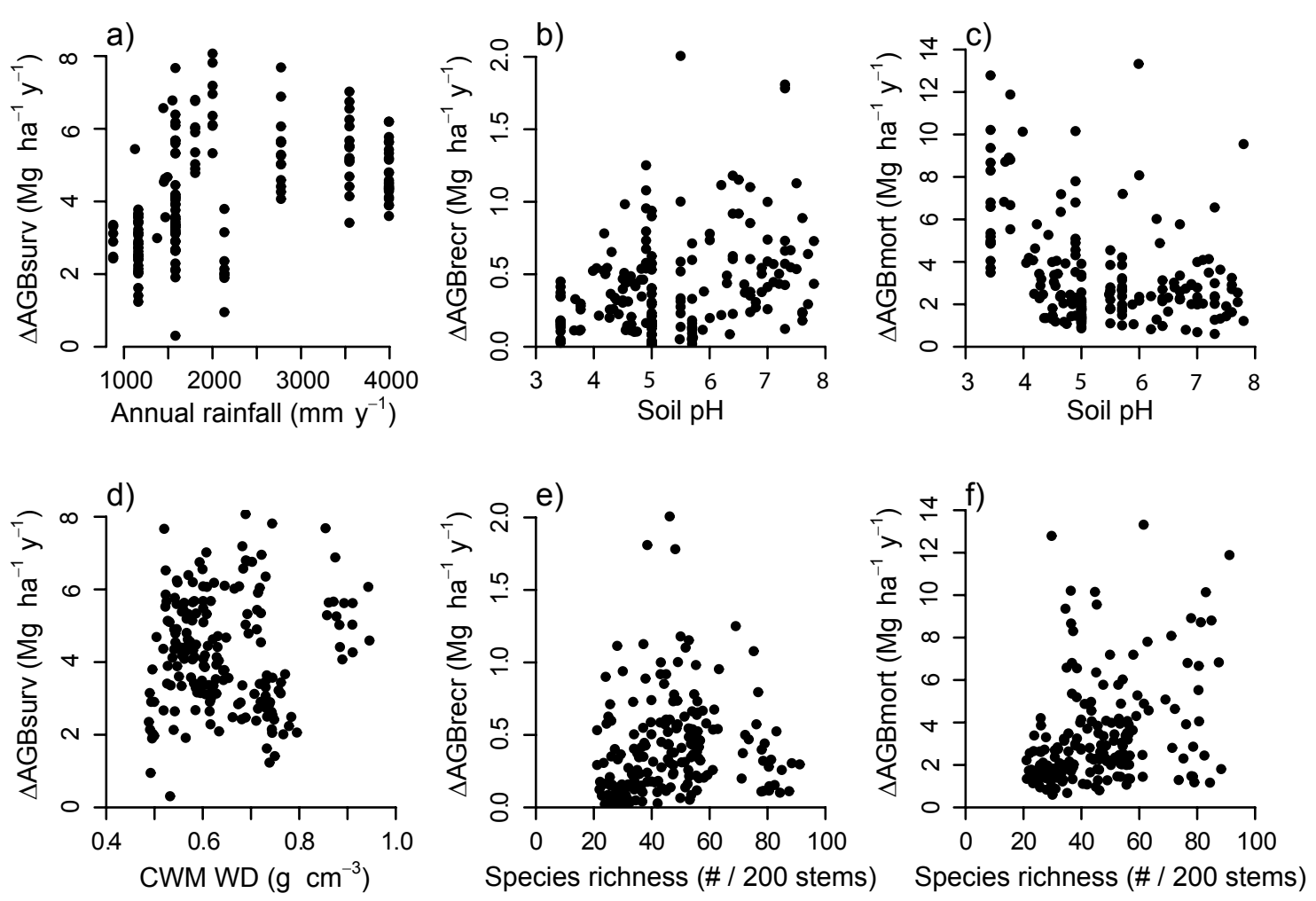

Figure 5.4: Bivariate relationships of one environmental predictor (upper row) and one 'vegetation quality' predictor (i.e., species richness or community-weighted mean (CWM) trait values; lower row) with biomass growth of surviving trees ( $\triangle$ AGBsurv; left column), biomass growth of recruiting trees ( $\triangle \mathrm{AGBrecr}$; middle column), and biomass mortality ( $\triangle \mathrm{AGBmort}$; right column). The chosen environmental and vegetation quality variables were the ones that had the strongest effect in the structural equation models (Fig. 5.2a, b, c). Each dot is a 1-ha plot. WD = wood density. Note that these bivariate relationships are for illustration purposes only and may not necessarily provide the same results as the structural equation models (Fig. 5.2a, b, c). For plots of all bivariate relationships tested in Fig. 5.2, see Appendix 5.7 and 5.8.

\section{Discussion}

We asked how environmental conditions and forest attributes (vegetation quantity and vegetation quality) drive biomass stocks and dynamics of Neotropical forests, and used structural equation models to test for their independent and causal effects. We found that 1) biomass stocks and dynamics were more strongly driven by forest attributes (significant in $73 \%$ of the relationships shown in Fig. 5.2) than by environmental conditions (significant in 50\% of the relationships), 2) where significant, water availability and soil fertility have a positive effect on biomass stocks and dynamics, 3) rarefied species richness and community-weighted mean (CWM) traits had consistent significant effects on biomass stocks and dynamics. These results suggest that large-scale environmental gradients lead to 
biogeographically and functionally distinct forest communities with cascading effects on biomass stocks and dynamics. Below we will discuss the underlying mechanisms and the implications for the conservation, management, and climate change mitigation potential of tropical forests.

$\triangle \mathrm{AGB}$

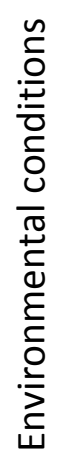

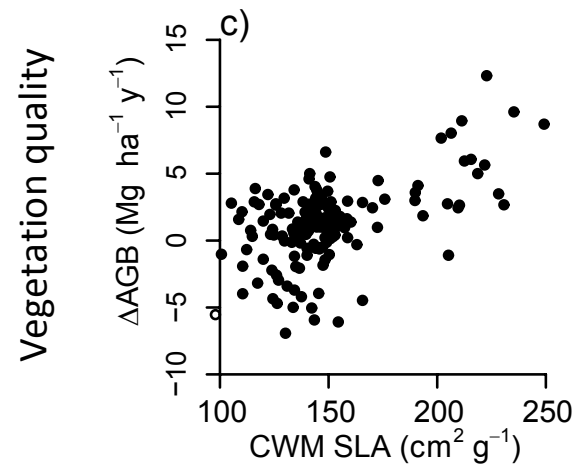

AGB
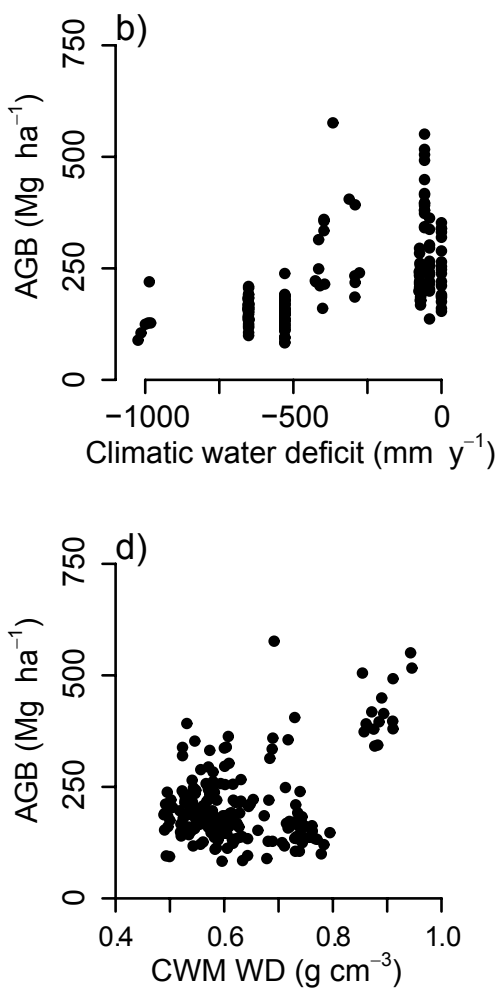

Figure 5.5: Bivariate relationships of one environmental predictor (upper row) and one 'vegetation quality' predictor (i.e., species richness or community-weighted mean (CWM) trait values; lower row) with net biomass change ( $\triangle \mathrm{AGB}$; left column) and biomass stocks (AGB; right column). The chosen environmental and vegetation quality variables were the ones that had the strongest effect in the structural equation models (Fig. 5.2d, e). Each dot is a 1-ha plot. CEC = cation exchange capacity, SLA $=$ specific leaf area, and WD $=$ wood density. Note that these bivariate relationships are for illustration purposes only and may not necessarily provide the same results as the structural equation models (Fig. 5.2d, e). For plots of all bivariate relationships tested in Fig. 5.2, see Appendix 5.7 and 5.8.

\section{Abiotic control: rainfall and soil fertility affect different demographic processes}

We hypothesized that biomass stocks and dynamics increase with resource availability (water availability, soil fertility, and increased irradiance due to disturbance), and that biomass stocks and dynamics are most strongly affected by water availability (as this is the main driver of spatial variation in biomass and diversity in lowland tropical forests, ter Steege et al. 2003, Poorter et al. 2015), and 
to a lesser extent by soil fertility. Water availability and soil fertility indeed generally increased biomass dynamics. In contrast to our hypothesis, they had similarly large effect sizes and affected partly different biomass variables: water availability increased growth of surviving trees ( $\triangle \mathrm{AGBsurv}$ ), growth of recruiting trees $(\triangle \mathrm{AGBrecr})$ and $\mathrm{AGB}$, whereas soil fertility increased $\triangle \mathrm{AGBrecr}$ and net biomass change $(\Delta \mathrm{AGB})$.

High water availability year round increases the length of the growing season and growth of individual trees, and therefore also the growth of whole stands (Toledo et al. 2012, cf. Fig. 5.2a). This higher growth rate results, in turn, in the build-up and maintenance of a larger standing biomass over time, leading to the well-known increase in forest stature, structural complexity (Beard 1955) and biomass (Fig. 5.2e, cf. Poorter et al. 2015) with an increase in rainfall. Several climate change scenarios predict an increase in the intensity and frequency of droughts. Field studies indicate that such droughts may lead to increased mortality and reduced biomass in the short term (Phillips et al. 2010, Lewis et al. 2011). The effect of water availability in our SEMs indicates that such droughts may also lead to reductions in forest biomass stocks and biomass dynamics in the long-term (Fig. $5.2)$.

Current paradigms on the environmental drivers of biomass dynamics are mostly based on results from the Amazon, and they show that highly fertile sites have higher productivity because of a combination of higher nutrient availability and selection for fast-growing pioneer species. At the same time, high nutrient availability may speed up the life cycle of plants which, in combination with an inherently short lifespan of pioneers, leads to high biomass mortality and lower standing biomass stocks (Baker et al. 2009). Our results only partly support this hypothesis. We indeed found that soil fertility increases $\triangle A G B r e c r$ but, surprisingly, soil fertility did not directly affect $\triangle A G B s u r v$, and even had a strong indirect negative effect (through its negative effect on CWM WD) on $\triangle \mathrm{AGBsurv}$ (Fig. 5.3). Soil fertility indeed tended to decrease AGB, although this was not because of higher mortality, as $\triangle \mathrm{AGBmort}$ actually tended to be lower on fertile soils (Fig. 5.2c). Discrepancies between the results from our study and the Amazonian studies can be attributed to various causes. First, our results may be different because we consider a wider range of soil and rainfall conditions. Especially dry forest (with rainfall between 750 and $1500 \mathrm{~mm} \mathrm{yr}^{-1}$ and climatic water deficit between -1000 and $-600 \mathrm{~mm} \mathrm{yr}^{-1}$ ) show a strong increase in biomass dynamics with water availability, after which it tends to levels off (e.g., Fig 5.4a). Hence, these dry forests drive most of the Neotropics-wide patterns but they are systematically excluded from comparative Amazonian rainforest studies. Second, we have explicitly assessed the independent effects of soil and rainfall, whereas most of the other studies did not. Third, for many of our sites soil fertility was 
obtained from a global database rather than measured in situ. Fourth, we used only proxies for soil fertility (CEC and $\mathrm{pH}$ ) and we did not measure phosphorus or nitrogen availability, which are often the main limiting factors for productivity on old and weathered tropical soils (chapter 3, Quesada et al. 2012). Hence, we may underestimate the role of soil fertility.

Water availability and soil fertility affect partly different processes. Water availability is especially important for large growing trees (van der Sande et al. 2015, see chapter 2); with their exposed crowns in the forest canopy (Peña-Claros et al. 2008) they face higher radiation loads and vapor pressure deficits which, in combination with longer hydraulic path lengths, lead to increased drought stress (Koch et al. 2004, Poorter et al. 2010b, Bennett et al. 2015). Water availability and soil fertility may be important for small recruiting trees because they face more drought and nutrient limitation due to their small root system. Soil fertility also increased net biomass change (cf. Quesada et al. 2012).

Logging disturbance opens up the canopy, leading to increased light levels in the lower forest strata (cf. Peña-Claros et al. 2008). Therefore, we hypothesized and found that logging disturbance increased $\triangle A G B$ surv and $\triangle A G B r e c r$ (Fig. 5.2a, b). This is in line with the observation that light is a limiting factor for tree growth, not only in wet forests (Kitajima and Poorter 2008, Rüger et al. 2012) but also in dry tropical forests (Villegas et al. 2009, Prado-Junior et al. 2016).

\section{Biotic control: how does species diversity affect biomass stocks and dynamics?}

We hypothesized that high species diversity enhances biomass stocks and dynamics through niche complementarity, which would lead to a more efficient overall resource use and higher biomass stocks and dynamics. Additionally, species diversity may enhance biomass stocks and dynamics through the selection effect, meaning that at high diversity there is a higher chance of including productive species with traits that dominate and drive the system (Loreau and Hector 2001), and through the insurance effect, meaning that species with different trait values may buffer biomass stocks and dynamics against temporal variation in environmental conditions (Yachi and Loreau 1999, Isbell et al. 2011). Our measure of species diversity (rarefied species richness) had a significant, independent and positive effect on biomass stocks and dynamics, but did not affect net biomass change. Also other measures of species diversity had a similarly strong correlation with biomass stocks and dynamics (Appendix 5.9). Clearly, diversity enhances the overall carbon stocks and productivity of the forests, leading to higher biomass dynamics, but also to higher biomass loss due to mortality, and hence no net effect on net biomass change. 
To our knowledge, this is the first large-scale study analysing the relationships between biomass dynamics in tropical forests and its multiple underlying drivers, and the first to demonstrate that species diversity has a positive and independent effect on dynamics. Most large-scale studies that looked at diversity effects ignored confounding effects of environment (e.g., Asase et al. 2012, Chisholm et al. 2013) or forest structure (e.g., Baker et al. 2009, Finegan et al. 2015). Poorter et al. (2015) used a similar approach as we did, and found a positive effect of tree species diversity on AGB across 59 Neotropical forest sites. A few single-site studies carried out for tropical forests did find a positive and independent effect of species diversity on productivity (Barrufol et al. 2013, during succession) whereas other studies did not (chapter 3, Prado-Junior et al. 2016), perhaps because within forest sites the range in diversity is smaller. Our large-scale study shows that the importance of diversity for ecosystem functioning found by experimental studies (Tilman et al. 2001, van Ruijven and Berendse 2005) and relatively more simple temperate systems (Gamfeldt et al. 2013) can also be extended to hyper-diverse tropical forests.

\section{Biotic control: how do community-mean traits affect biomass stocks and dynamics?}

Most studies assume that trait-rate relationships observed at the species level should also apply at the community level. We hypothesized, therefore, in line with the mass-ratio hypothesis (Grime 1998), that communities dominated by productive trait values (e.g., high CWM SLA, low WD) would realize a high $\triangle \mathrm{AGBsurv}, \triangle \mathrm{AGBrecr}$ and $\triangle \mathrm{AGB}$, whereas communities dominated by conservative trait values (e.g., high CWM WD) would realize large AGB. We indeed found that CWM SLA increased light capture, and hence $\triangle A G B$ (Fig. 5.2d, 5.5c, cf. Reich 2014, and Finegan et al. 2015 for $\Delta A G B s u r v$ and $\Delta A G B r e c r)$. We also found that CWM WD increased AGB, either directly because high WD implies more stem biomass per volume, or indirectly because WD enhances stem longevity and thus biomass build-up. Other comparative studies also found that regional variation in WD and especially stem survival have strong positive effects on AGB (Johnson et al. in press, Baker et al. 2009). Surprisingly, high CWM WD increased the biomass growth of surviving trees, which contrasts sharply with studies carried out at the species level, where high WD decreased the stem diameter growth of trees (Poorter et al. 2008, Rüger et al. 2012). Although high WD implies less volumetric growth, this does not mean that it should also lead to less biomass growth, as high WD contributes directly to higher biomass. Finally, an increase in CWM DBHmax decreased $\triangle \mathrm{AGBrecr}$, probably because communities dominated 
by potentially large trees cast a deeper shade, leading to less recruitment in the understory.

Other studies also found that current paradigms on trait-rate relationships at the species level can play out differently at the community level, especially when resources become limiting. For example, in tropical dry forests in Brazil (PradoJunior et al. 2016) or on nutrient poor soils in Guyana (chapter 3), conservative CWM trait values (i.e., a low SLA) rather than acquisitive trait values increase productivity, and acquisitive CWM trait values (i.e., a higher leaf phosphorus concentration) rather than conservative trait values increase biomass stocks in Guyana. The authors argue that communities dominated by trees with conservative trait values (e.g., thick, dense, and long-lived leaves) reduce transpiration and enhance the residence time of nutrients in plants. As a result, these communities are more efficient in their water and nutrient use, which enhances their productivity under limiting resource conditions.

Overall, we found that CWM trait values are good predictors of biomass stocks and dynamics because they significantly affect four of the five biomass variables evaluated, and have similar effect sizes as the other drivers (Fig. 5.2, 5.3). CWM traits are good predictors for three reasons. First, these traits have a direct and mechanistic impact on forest functioning (i.e., 'effect traits', Lavorel \& Garnier, 2002). Second, the CWM reflects the traits of the dominant species in the community, and especially these dominant species have a large impact on ecosystem productivity and fluxes, simply because they account for most of the community biomass (cf. Fauset et al. 2015). Third, these traits are not only effect traits, but also response traits, as they reflect how species are filtered out by the environment (see the arrows from abiotic conditions to CWM traits in Fig. 5.2). Therefore, they also account for the indirect effects of abiotic conditions on biomass stocks and dynamics (chapters 3 and 4).

\section{Biomass mortality is the strongest predictor of net biomass change, but unpredictable itself}

To understand net biomass change, and thus carbon sequestration potential, we need to look at the underlying demographic processes. Interestingly, $\triangle \mathrm{AGB}$ was the biomass variable that was least explained by our SEM models $\left(\mathrm{R}^{2}=0.31\right.$, compared to $0.35-0.54$ for the other variables of carbon dynamics). $\triangle A G B$ was most strongly driven by $\triangle \mathrm{AGBmort}(\beta=-0.97)$, followed by $\triangle \mathrm{AGBsurv}(\beta=0.50)$, and not significantly by $\triangle A G B r e c r$. The question then becomes: what drives biomass mortality? In small 1-ha plots, biomass mortality is partly a stochastic process, as it for example depends whether a large storm hits the stand during the monitoring period. Recent studies also show that mortality is the main driver of net 
biomass change in Bolivia (chapter 4) and of stand biomass across the Amazon (Johnson et al. in press). Global vegetation models cannot accurately predict standing biomass because they cannot accurately simulate mortality. Interestingly, in our study biomass loss due to mortality did not depend on environmental conditions or on CWM trait values (Fig. 5.2c), which makes it more difficult to model mortality in a mechanistic way. Mortality is an absolute flux rate, and it therefore increased with the biomass of the vegetation (as reflected in the basal area), but also with the species richness because species-rich forests have high AGB. Johnson et al. (in press) showed that aboveground forest biomass is more strongly driven by tree mortality than by biomass mortality. This suggests that tree mortality shapes the size class distribution of tropical forests (cf. Farrior et al. 2016) which, in turn, dictates how many trees attain large sizes. As large trees contribute disproportionally to forest biomass (Slik et al. 2013), this then ultimately determines total above-ground biomass.

\section{Demographic processes are shaped by different drivers}

To understand net biomass change we need to look at the underlying demographic processes, especially mortality. Demographic processes are driven by trees of different sizes that experience different limiting resources and environmental hazards. For example, small trees that regenerate in the understory or treefall gaps mainly drive $\triangle A G B r e c r$, whereas tall canopy trees mainly drive $\triangle A G B s u r v$ and $\triangle A G B m o r t$. From the understory to the canopy, irradiance, temperature, wind exposure and atmospheric water stress increase (Yoda 1974). Hence, the $\triangle A G B r e c r$ of understory trees may be more limited by light (in our case reflected by disturbance and CWM DBHmax, Fig. 5.2b) or by nutrient availability (because of their small root systems), whereas the $\triangle \mathrm{AGBsurv}$ of exposed canopy trees may be more limited by water availability (i.e., rainfall, Fig. 5.2a). Additionally, $\triangle \mathrm{AGBmort}$ of large trees canopy may be mainly driven by strong episodic droughts (Bennett et al. 2015) and stochastic wind disturbances, and hence, cannot be predicted by the environmental and forest variables that we considered. If we had analysed only net biomass change, then we would have found a somewhat different suite of variables to be important, and all these size and process dependent drivers would have been concealed.

\section{Conclusions and implications}

We demonstrate that biomass stocks and dynamics of Neotropical forests are under strong control of environmental conditions and especially forest attributes. Water availability exerts a strong effect on forest biomass and dynamics, which indicates that forest functioning is sensitive to climate change. Increasing 
(atmospheric) drought may especially reduce biomass growth by large trees and ultimately carbon stocks.

Tree species diversity had strong positive effects on biomass stocks, biomass growth and biomass turnover (i.e., mortality). As a result, diversity had no effect on net biomass change. Given our relatively small plot size (1 ha) and census period (5-10 y) we are not sure whether patterns in net biomass change are just the result of stochastic mortality during the monitoring period, or whether they really reflect long-term trends. If the latter is true, then the conclusion is that diversity enhances carbon storage and components of productivity but that it does not affect the net carbon sequestration potential. However, biodiversity is more than only species richness as it encompasses forest attributes in general. We show that forest attributes - including species diversity and community-weighted mean trait values are very strong drivers of biomass stocks and dynamics, indicating that the biodiversity of the vegetation strongly shapes ecosystem functioning. Additionally, high tree diversity makes tropical forests more resilient to climate change (Sakschewski et al. in revision). Biodiversity conservation in the broader sense including functional attributes - should therefore be an integral component for global strategies such as REDD+ and the Convention of Biological Diversity.

\section{Acknowledgements}

We gratefully thank all the people that have established and measured the plots, and the institutions and funding agencies that have supported this work over the years. Luquillo forest Dynamics plot was supported by Luquillo Long Term Ecological Research program (LTER), USA National Science Foundation and others. The Nizanda project was funded by CONACYTSEMARNAT Grant CB-1281326 and PAPIIT-UNAM Grants IN216007-3 and IN218416.The San Emilio plot was funded by an NSF CAREER and a Fulbright Fellowship award to BJE. This study was partly funded by the European Union's Seventh Framework Programme ([FP7/20072013]) under grant agreement $n^{\circ} 283093$; Role Of Biodiversity In climate change mitigatioN (ROBIN), with co-funding for MvdS and EA from the Dutch Ministry of Economic Affairs (KB-14-003-030). We thank Ben Turner for providing the soil data for the Luquillo plots, and Jennifer Powers for providing soil data for the San Emilio plots. MMR was supported by PAPIIT-DGAPA (UNAM) grants IN227210 and IN213714, and we thank Gilberto Jamangape García for his fieldwork support, and the Chajul Station and Natura Mexicana for their logistic facilities provided. 


\section{Appendices}

Appendix 5.1: Overview of sites included in the study. Main characteristics of each site, number of plots. $\mathrm{CWD}=$ climatic water deficit, $\mathrm{CEC}=$ cation exchange capacity .

\begin{tabular}{|c|c|c|c|c|c|c|c|c|c|}
\hline Site name & Country & Latitude & Longitude & Nr. plots & $\begin{array}{l}\text { Nr. Plots } \\
\text { logged }\end{array}$ & $\begin{array}{l}\text { Rainfall } \\
\mathrm{mm} \mathrm{y}^{-1}\end{array}$ & $\begin{array}{l}\text { CWD } \\
\mathrm{mm} \mathrm{y}^{-1}\end{array}$ & $\mathrm{pH}$ & CEC \\
\hline $\mathrm{AGU}$ & Brazil & -18.50 & -48.39 & 1 & 0 & 1375 & -424 & 6.35 & 13.82 \\
\hline Chajul_FLOODED & Mexico & 16.12 & -90.94 & 1 & 0 & 2844 & -293 & 6.00 & 18.00 \\
\hline Chajul-ALLUVIAL & Mexico & 16.11 & -90.94 & 1 & 0 & 2844 & -291 & 5.99 & 18.00 \\
\hline Chajul-KARST & Mexico & 16.11 & -90.99 & 1 & 0 & 2844 & -277 & 6.61 & 18.00 \\
\hline Chajul-LOWHILL1 & Mexico & 16.12 & -90.95 & 1 & 0 & 2844 & -291 & 5.00 & 18.00 \\
\hline Chajul-LOWILL2 & Mexico & 16.12 & -90.94 & 1 & 0 & 2844 & -293 & 4.20 & 18.00 \\
\hline Corinto & Costa Rica & 10.20 & -83.87 & 9 & 6 & 3900 & 0 & 4.90 & 23.00 \\
\hline GLO & Brazil & -18.95 & -48.20 & 1 & 0 & 1491 & -411 & 4.90 & 10.33 \\
\hline Ilha do Cardoso & Brazil & -25.08 & -47.93 & 9 & 0 & 2134 & 0 & 5.50 & 2.00 \\
\hline INPA_1 & Bolivia & -16.12 & -61.72 & 16 & 12 & 1160 & -651 & 5.00 & 2.00 \\
\hline INPA_2 & Bolivia & -16.12 & -61.72 & 16 & 12 & 1160 & -651 & 5.00 & 2.00 \\
\hline IRA & Brazil & -19.15 & -48.15 & 1 & 0 & 1465 & -395 & 4.74 & 2.87 \\
\hline La Chonta_12 & Bolivia & -15.78 & -62.92 & 32 & 28 & 1580 & -529 & 6.96 & 8.61 \\
\hline La Chonta_3 & Bolivia & -15.78 & -62.92 & 16 & 12 & 1580 & -529 & 6.92 & 10.93 \\
\hline La Planada & Colombia & 1.15 & -77.99 & 25 & 0 & 3991 & -73 & 4.46 & 48.00 \\
\hline Luquillo & Puerto Rico & 18.32 & -65.82 & 15 & 0 & 3548 & -40 & 5.70 & 7.00 \\
\hline MON & Brazil & -18.75 & -47.51 & 1 & 0 & 1124 & -414 & 5.48 & 6.01 \\
\hline Nizanda & México & 16.66 & -95.02 & 6 & 0 & 878 & -1000 & 6.90 & 30.62 \\
\hline PAN & Brazil & -19.17 & -48.39 & 1 & 0 & 1450 & -402 & 5.88 & 8.40 \\
\hline Paragominas & Brazil & -3.52 & -48.79 & 9 & 6 & 1805 & -397 & 4.56 & 8.65 \\
\hline PER & Brazil & -18.93 & -48.06 & 1 & 0 & 1469 & -426 & 4.65 & 2.13 \\
\hline Pibiri & Guyana & 5.22 & -58.63 & 15 & 12 & 2772 & -57 & 3.43 & 3.00 \\
\hline San Emilio & Costa Rica & 10.81 & -85.61 & 12 & 0 & 1740 & -652 & 6.08 & 21.66 \\
\hline SÃO & Brazil & -18.86 & -48.23 & 1 & 0 & 1445 & -415 & 4.64 & 3.38 \\
\hline Tapajós & Brazil & -3.32 & -54.95 & 8 & 7 & 2000 & -312 & 3.77 & 9.17 \\
\hline UBE & Brazil & -19.68 & -48.03 & 1 & 0 & 1547 & -366 & 5.71 & 5.67 \\
\hline
\end{tabular}


Appendix 5.2: Map of South and Mesoamerica, with the locations of the 26 study sites. Note that the location of some sites that were very close together are shown as one plot (i.e., for INPA and La Chonta, Bolivia, and the Chajul-sites in Mexico).

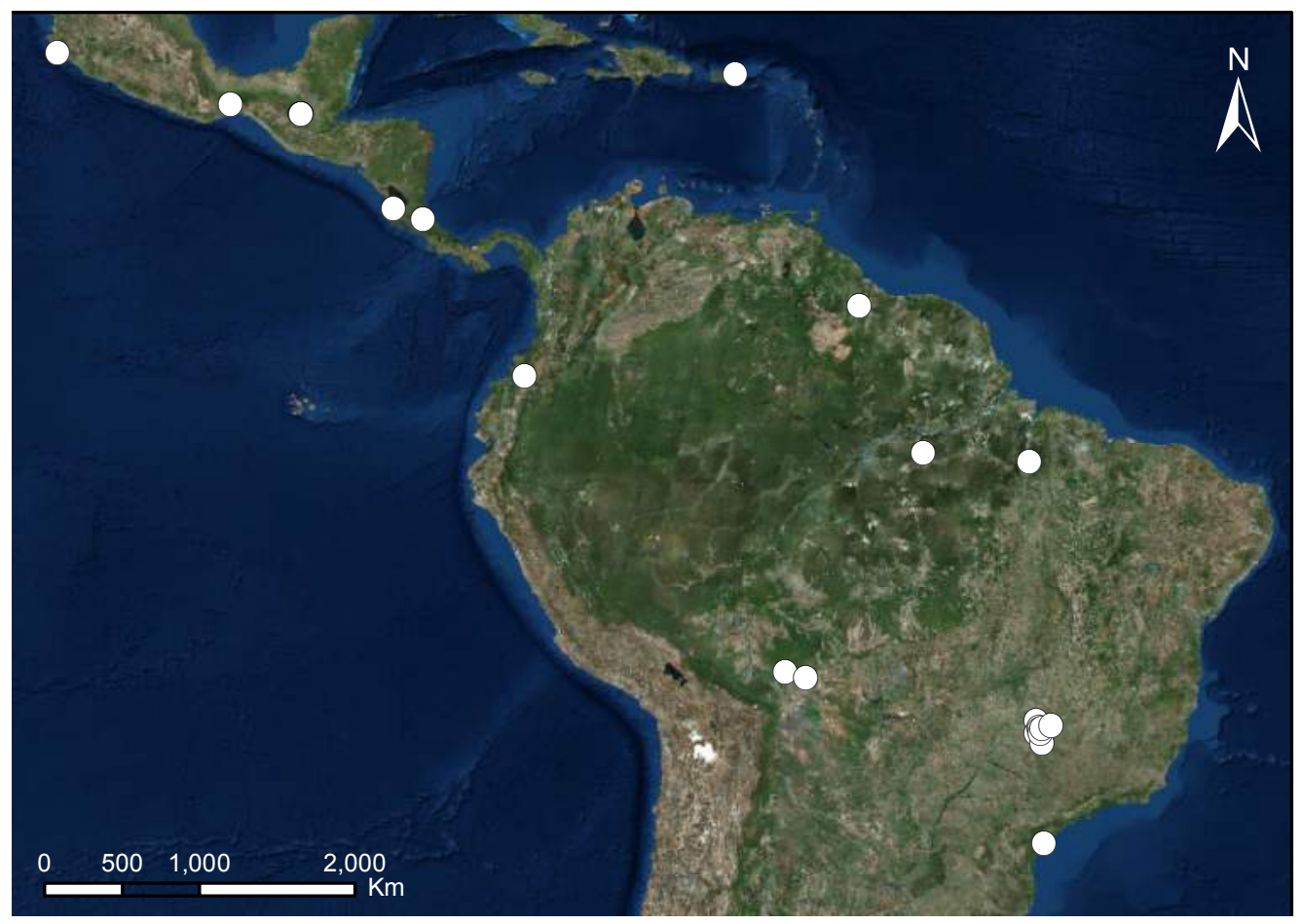

Appendix 5.3: List of eight traits considered in this study because of their hypothesized relationship $(+=$ positive, $0=$ no or unknown relationship, $-=$ negative) with biomass stocks and dynamics. These traits are indicators of different plant processes. $\mathrm{DBH}=$ diameter at $1.30 \mathrm{~m}$ aboveground.

\begin{tabular}{|c|c|c|}
\hline Trait & Indicator of & $\begin{array}{l}\text { Productivity } \\
\left(\mathrm{Mg} \mathrm{ha}^{-1} \mathrm{y}^{-1}\right)\end{array}$ \\
\hline $\begin{array}{l}\text { Specific leaf area (leaf area per unit leaf dry } \\
\text { mass, } \mathrm{cm}^{2} \mathrm{~g}^{-1} \text { ) }\end{array}$ & $\begin{array}{l}\text { Efficiency of leaf deployment for light } \\
\text { capture }\end{array}$ & + \\
\hline $\begin{array}{l}\text { Leaf nitrogen concentration } \\
\text { (nitrogen mass per leaf dry mass, } \mathrm{mg} \mathrm{g}^{-1} \text { ) }\end{array}$ & $\begin{array}{l}\text { Photosynthetic capacity, } \mathrm{CO}_{2} \\
\text { assimilation }\end{array}$ & + \\
\hline Leaf phosphorous concentration $\left(\mathrm{mg} \mathrm{g}^{-1}\right)$ & $\begin{array}{l}\text { Photosynthetic capacity, metabolic } \\
\text { activity (ATP), } \mathrm{CO}_{2} \text { assimilation }\end{array}$ & + \\
\hline Leaf $\mathrm{C}: \mathrm{N}$ ratio & Leaf longevity & - \\
\hline $\begin{array}{l}\text { Leaf dry matter content (leaf dry mass } \\
\text { divided by leaf fresh mass, } \mathrm{g} \mathrm{g}^{-1} \text { ) }\end{array}$ & $\begin{array}{l}\text { Leaf defense, leaf longevity (i.e., } \\
\text { lifespan) }\end{array}$ & - \\
\hline $\begin{array}{l}\text { Wood density (wood dry mass per wood } \\
\text { volume, } \mathrm{g} \mathrm{cm}^{-3} \text { ) }\end{array}$ & $\begin{array}{l}\text { Wood construction cost, hydraulic } \\
\text { efficiency, longevity of carbon stock }\end{array}$ & - \\
\hline $\begin{array}{l}\text { Adult stature }\left(95^{\text {th }} \text { quantile of maximum }\right. \\
\text { DBH of species, } \mathrm{cm})\end{array}$ & $\begin{array}{l}\text { Proxy for height and therefore light } \\
\text { capture }\end{array}$ & 0 \\
\hline
\end{tabular}


Appendix 5.4: Results from the linear mixed regression analyses (with site as random factor) of rainfall and rainfall squared (rainfall ${ }^{2}$ ) and of climatic water deficit (CWD) and CWM squared $\left(\mathrm{CWD}^{2}\right)$ with all variables that were included as 'endogenous' variables in the SEMs (i.e., that had an arrow pointing towards them). Here, only the results of the squared variable are shown. $\mathrm{AGB}=$ aboveground biomass, $\triangle \mathrm{AGB}$ urv $=$ biomass growth from surviving trees, $\triangle \mathrm{AGBrecr}=$ biomass growth from recruiting trees, $\triangle \mathrm{AGBmort}=$ biomass lost due to mortality, $\triangle \mathrm{AGB}=$ net change biomass, rarSPR $=$ rarefied species richness $($ at 200 randomly pulled stems), CWM = community-weighted mean, WD = wood density, SLA = specific leaf area, $\mathrm{DBHmax}=$ maximum diameter. Only for the rarefied species richness, the CWD ${ }^{2}$ had a significant effect.

\begin{tabular}{llllll}
\hline Response variable & Coef & SE & df & t-value & P-value \\
\hline Rainfall $^{2}$ & & & & & \\
$\Delta$ AGBsurv & $<0.01$ & $<0.01$ & 16 & -1.08 & 0.295 \\
$\Delta$ AGBrecr & $<0.01$ & $<0.01$ & 16 & 0.09 & 0.926 \\
$\Delta$ AGBmort & $<0.01$ & $<0.01$ & 16 & -1.77 & 0.096 \\
AGB & -0.07 & 0.04 & 17 & -1.85 & 0.081 \\
$\Delta$ AGB & $<0.01$ & $<0.01$ & 16 & 0.71 & 0.485 \\
rarSPR & $<0.01$ & $<0.01$ & 17 & -1.62 & 0.123 \\
CWM WD & $<0.01$ & $<0.01$ & 17 & -0.04 & 0.970 \\
CWM SLA & $<0.01$ & $<0.01$ & 15 & 0.57 & 0.576 \\
CWM DBHmax & $<0.01$ & $<0.01$ & 17 & -1.79 & 0.091 \\
Basal area & $<0.01$ & $<0.01$ & 17 & -0.58 & 0.572 \\
rarSPR pre-logging & $<0.01$ & $<0.01$ & 17 & -1.57 & 0.134 \\
CWM WD pre-logging & $<0.01$ & $<0.01$ & 17 & -0.20 & 0.847 \\
CWM SLA pre-logging & $<0.01$ & $<0.01$ & 15 & 0.61 & 0.552 \\
CWM DBHmax pre- & & & & & \\
logging & $<0.01$ & $<0.01$ & 17 & -1.56 & 0.138 \\
Basal area pre-logging & $<0.01$ & $<0.01$ & 17 & -0.57 & 0.579 \\
CWD ${ }^{2}$ & & & & & \\
$\Delta$ AGBsurv & $<0.01$ & $<0.01$ & 162 & -1.34 & 0.184 \\
$\Delta$ AGBrecr & $<0.01$ & $<0.01$ & 162 & 1.44 & 0.153 \\
$\Delta$ AGBmort & $<0.01$ & $<0.01$ & 162 & -0.93 & 0.355 \\
AGB & 0.3 & 0.91 & 117 & 0.29 & 0.774 \\
$\Delta$ AGB & $<0.01$ & $<0.01$ & 173 & 0.35 & 0.725 \\
rarSPR & $<0.01$ & $<0.01$ & 173 & -2.10 & 0.037 \\
CWM WD & $<0.01$ & $<0.01$ & 173 & -0.16 & 0.875 \\
CWM SLA & $<0.01$ & $<0.01$ & 141 & -0.13 & 0.899 \\
CWM DBHmax & $<0.01$ & $<0.01$ & 173 & -1.52 & 0.129 \\
Basal area & $<0.01$ & $<0.01$ & 173 & -0.32 & 0.752 \\
rarSPR pre-logging & $<0.01$ & $<0.01$ & 173 & -1.89 & 0.060 \\
CWM WD pre-logging & $<0.01$ & $<0.01$ & 173 & -0.97 & 0.332 \\
CWM SLA pre-logging & $<0.01$ & $<0.01$ & 141 & -0.14 & 0.893 \\
CWM DBHmax pre- & & & & & \\
logging & $<0.01$ & $<0.01$ & 173 & -1.07 & 0.285 \\
Basal area pre-logging & $<0.01$ & $<0.01$ & 173 & -0.44 & 0.659 \\
\hline & & & & &
\end{tabular}


Appendix 5.5: Results from the 12 structural equation models (SEMs) for biomass stocks and each component of biomass dynamics. Each SEM (i.e., each row) is a combination of one of the two climate variables (rainfall and climatic water deficit, CWD), one of the two soil variables ( $\mathrm{pH}$ and cation exchange capacity, CEC), and one of the three community-weighted mean (CWM) traits (wood density, WD; specific leaf area, SLA; maximum diameter, DBHmax). Rarefied species richness and plot basal area are included in all models, and relative disturbance is included in all models except the ones for aboveground biomass. Per SEM, model fit (chi-square and accompanying $\mathrm{P}$-value) and $\mathrm{R}^{2}$ of the ecosystem process are given. The SEMs in bold and italics were chosen as the 'best' model per ecosystem process. For other abbreviations, see legend of Appendix 5.4.

\begin{tabular}{|c|c|c|c|c|c|c|}
\hline Response variable & Climate & Soil & CWM trait & Chi-square & P-value & $\mathbf{R}^{2}$ \\
\hline$\triangle A G B$ surv & Rainfall & $p H$ & $W D$ & 6.51 & 0.09 & 0.51 \\
\hline$\Delta$ AGBsurv & Rainfall & $\mathrm{pH}$ & SLA & 5.50 & 0.14 & 0.45 \\
\hline$\Delta \mathrm{AGBsurv}$ & Rainfall & $\mathrm{pH}$ & DBHmax & 5.34 & 0.15 & 0.44 \\
\hline$\Delta \mathrm{AGBsurv}$ & Rainfall & CEC & WD & 3.89 & 0.27 & 0.47 \\
\hline$\Delta \mathrm{AGBsurv}$ & Rainfall & CEC & SLA & 4.17 & 0.24 & 0.44 \\
\hline$\Delta \mathrm{AGBsurv}$ & Rainfall & CEC & DBHmax & 4.63 & 0.20 & 0.42 \\
\hline$\Delta \mathrm{AGBsurv}$ & CWD & $\mathrm{pH}$ & WD & 5.77 & 0.12 & 0.45 \\
\hline$\Delta \mathrm{AGBsurv}$ & CWD & $\mathrm{pH}$ & SLA & 3.76 & 0.29 & 0.43 \\
\hline$\Delta \mathrm{AGBsurv}$ & CWD & $\mathrm{pH}$ & DBHmax & 5.11 & 0.16 & 0.42 \\
\hline$\Delta$ AGBsurv & CWD & CEC & WD & 2.85 & 0.42 & 0.45 \\
\hline$\Delta$ AGBsurv & CWD & CEC & SLA & 2.17 & 0.54 & 0.44 \\
\hline$\Delta$ AGBsurv & CWD & CEC & DBHmax & 3.77 & 0.29 & 0.40 \\
\hline $\log (\Delta$ AGBrecr $)$ & Rainfall & $\mathrm{pH}$ & WD & 6.51 & 0.09 & 0.29 \\
\hline $\log (\Delta A G B r e c r)$ & Rainfall & $\mathrm{pH}$ & SLA & 5.50 & 0.14 & 0.31 \\
\hline $\log (\triangle A G B r e c r)$ & Rainfall & $p H$ & $D B H \max$ & 5.34 & 0.15 & 0.35 \\
\hline $\log (\Delta \mathrm{AGBrecr})$ & Rainfall & CEC & WD & 3.89 & 0.27 & 0.29 \\
\hline $\log (\Delta \mathrm{AGBrecr})$ & Rainfall & CEC & SLA & 4.17 & 0.24 & 0.27 \\
\hline $\log (\Delta \mathrm{AGBrecr})$ & Rainfall & CEC & DBHmax & 4.63 & 0.20 & 0.21 \\
\hline $\log (\Delta \mathrm{AGBrecr})$ & CWD & $\mathrm{pH}$ & WD & 5.77 & 0.12 & 0.27 \\
\hline $\log (\Delta \mathrm{AGBrecr})$ & CWD & $\mathrm{pH}$ & SLA & 3.76 & 0.29 & 0.30 \\
\hline $\log (\Delta$ AGBrecr $)$ & CWD & $\mathrm{pH}$ & DBHmax & 5.11 & 0.16 & 0.33 \\
\hline $\log (\Delta \mathrm{AGBrecr})$ & CWD & CEC & WD & 2.85 & 0.42 & 0.29 \\
\hline $\log (\Delta \mathrm{AGBrecr})$ & CWD & CEC & SLA & 2.17 & 0.54 & 0.27 \\
\hline $\log (\Delta$ AGBrecr $)$ & CWD & CEC & DBHmax & 3.77 & 0.29 & 0.21 \\
\hline $\log (\triangle \mathrm{AGBmort})$ & Rainfall & $\mathrm{pH}$ & WD & 6.51 & 0.09 & 0.33 \\
\hline $\log (\Delta \mathrm{AGBmort})$ & Rainfall & $\mathrm{pH}$ & SLA & 5.50 & 0.14 & 0.42 \\
\hline $\log (\Delta \mathrm{AGBmort})$ & Rainfall & $\mathrm{pH}$ & DBHmax & 5.34 & 0.15 & 0.25 \\
\hline $\log (\Delta \mathrm{AGBmort})$ & Rainfall & CEC & WD & 3.89 & 0.27 & 0.31 \\
\hline $\log (\Delta$ AGBmort $)$ & Rainfall & CEC & SLA & 4.17 & 0.24 & 0.38 \\
\hline $\log (\Delta \mathrm{AGBmort})$ & Rainfall & CEC & DBHmax & 4.63 & 0.20 & 0.27 \\
\hline $\log (\Delta \mathrm{AGBmort})$ & CWD & $\mathrm{pH}$ & WD & 5.77 & 0.12 & 0.36 \\
\hline $\log (\triangle A G B m o r t)$ & $C W D$ & $p H$ & $S L A$ & 3.76 & 0.29 & 0.43 \\
\hline $\log (\triangle \mathrm{AGBmort})$ & CWD & $\mathrm{pH}$ & DBHmax & 5.11 & 0.16 & 0.28 \\
\hline $\log (\Delta \mathrm{AGBmort})$ & CWD & CEC & WD & 2.85 & 0.42 & 0.33 \\
\hline $\log (\Delta \mathrm{AGBmort})$ & CWD & CEC & SLA & 2.17 & 0.54 & 0.40 \\
\hline $\log (\Delta$ AGBmort $)$ & CWD & CEC & DBHmax & 3.77 & 0.29 & 0.28 \\
\hline$\Delta \mathrm{AGB}$ & Rainfall & $\mathrm{pH}$ & WD & 6.42 & 0.09 & 0.15 \\
\hline$\triangle A G B$ & Rainfall & $p H$ & $S L A$ & 3.61 & 0.31 & 0.36 \\
\hline$\triangle \mathrm{AGB}$ & Rainfall & $\mathrm{pH}$ & DBHmax & 5.43 & 0.14 & 0.16 \\
\hline$\triangle \mathrm{AGB}$ & Rainfall & CEC & WD & 3.58 & 0.31 & 0.17 \\
\hline$\triangle \mathrm{AGB}$ & Rainfall & CEC & SLA & 4.13 & 0.25 & 0.32 \\
\hline$\Delta \mathrm{AGB}$ & Rainfall & CEC & DBHmax & 4.77 & 0.19 & 0.28 \\
\hline
\end{tabular}




\begin{tabular}{lllllll}
$\Delta$ AGB & CWD & pH & WD & 5.97 & 0.11 & 0.20 \\
$\Delta$ AGB & CWD & pH & SLA & 2.59 & 0.46 & 0.35 \\
$\Delta$ AGB & CWD & pH & DBHmax & 5.48 & 0.14 & 0.18 \\
$\Delta$ AGB & CWD & CEC & WD & 2.76 & 0.43 & 0.20 \\
$\Delta$ AGB & CWD & CEC & SLA & 2.25 & 0.52 & 0.32 \\
$\Delta$ AGB & CWD & CEC & DBHmax & 4.44 & 0.22 & 0.29 \\
\hline AGB & Rainfall & pH & WD & 4.68 & 0.20 & 0.83 \\
AGB & Rainfall & pH & SLA & 4.02 & 0.26 & 0.71 \\
AGB & Rainfall & pH & DBHmax & 2.40 & 0.49 & 0.68 \\
AGB & Rainfall & CEC & WD & 2.89 & 0.41 & 0.86 \\
AGB & Rainfall & CEC & SLA & 7.36 & 0.06 & 0.59 \\
AGB & Rainfall & CEC & DBHmax & 2.71 & 0.44 & 0.61 \\
AGB & CWD & pH & WD & 7.53 & 0.06 & 0.86 \\
AGB & CWD & pH & SLA & 0.69 & 0.88 & 0.72 \\
AGB & CWD & pH & DBHmax & 2.40 & 0.49 & 0.69 \\
$A G B$ & $C W D$ & $C E C$ & WD & 2.74 & 0.43 & 0.86 \\
AGB & CWD & CEC & SLA & 4.75 & 0.19 & 0.59 \\
AGB & CWD & CEC & DBHmax & 3.12 & 0.37 & 0.60 \\
\hline
\end{tabular}


Appendix 5.6: Results from the structural equation models for biomass growth by surviving trees ( $\triangle \mathrm{AGBsurv}$ ), biomass growth by recruiting trees $(\triangle \mathrm{AGBrecr})$, biomass loss due to mortality ( $\triangle \mathrm{AGBmort})$, net biomass change $(\triangle \mathrm{AGB})$, and aboveground biomass stocks (AGB). Each row indicates one relationship (i.e., one arrow) in Fig. 5.2. Per relationship, the coefficient (Coeff), standardized coefficient (Std. Coeff), standard error (SE), Z-value and P-value are given. Number of sites and number of plots included per model are provided (number sites; number plots). $\mathrm{CWM}=$ community-weighted mean, $\mathrm{WD}=$ wood density, SLA $=$ specific leaf area, DBHmax $=$ maximum diameter, CEC = cation exchange capacity. For other abbreviations, see legend of Appendix 5.4.

\begin{tabular}{|c|c|c|c|c|c|c|c|}
\hline $\begin{array}{l}\text { Biomass } \\
\text { variable }\end{array}$ & $\begin{array}{l}\text { Response } \\
\text { variable }\end{array}$ & Predictor variable & Coeff & $\begin{array}{l}\text { Std. } \\
\text { Coeff }\end{array}$ & SE & $\mathrm{Z}$ & $\mathbf{P}$ \\
\hline$\Delta \mathrm{AGBsurv}$ & $\Delta \mathrm{AGBsurv}$ & $\begin{array}{l}\text { Rarefied spp. } \\
\text { richness }\end{array}$ & 0.03 & 0.31 & 0.01 & 3.97 & $<0.001$ \\
\hline (25 sites; & & Basal area & 0.13 & 0.43 & 0.03 & 4.88 & $<0.001$ \\
\hline \multirow[t]{13}{*}{188 plots) } & & CWM WD & 0.06 & 0.39 & 0.03 & 2.35 & 0.019 \\
\hline & & Rainfall & 0.07 & 0.44 & 0.02 & 3.16 & 0.002 \\
\hline & & Disturbance & 0.04 & 0.20 & 0.01 & 2.45 & 0.014 \\
\hline & & $\mathrm{pH}$ & 0.26 & 0.19 & 0.21 & 1.22 & 0.224 \\
\hline & \multirow{3}{*}{$\begin{array}{l}\text { Rarefied spp. } \\
\text { richness }\end{array}$} & Disturbance & 0.10 & 0.06 & 0.18 & 0.57 & 0.568 \\
\hline & & Rainfall & 0.35 & 0.22 & 0.43 & 0.82 & 0.412 \\
\hline & & $\mathrm{pH}$ & -1.64 & -0.12 & 3.76 & -0.44 & 0.664 \\
\hline & \multirow[t]{3}{*}{ Basal area } & Disturbance & -0.21 & -0.36 & 0.06 & -3.73 & $<0.001$ \\
\hline & & Rainfall & 0.09 & 0.18 & 0.12 & 0.79 & 0.428 \\
\hline & & $\mathrm{pH}$ & -0.65 & -0.14 & 0.79 & -0.83 & 0.407 \\
\hline & \multirow[t]{3}{*}{ CWM WD } & Disturbance & 0.09 & 0.08 & 0.11 & 0.80 & 0.422 \\
\hline & & Rainfall & -0.58 & -0.57 & 0.10 & -5.75 & $<0.001$ \\
\hline & & $\mathrm{pH}$ & -6.46 & -0.72 & 1.86 & -3.48 & 0.001 \\
\hline$\Delta \mathrm{AGBrecr}$ & $\log (\Delta \mathrm{AGBrecr})$ & $\begin{array}{l}\text { Rarefied spp. } \\
\text { richness }\end{array}$ & 0.08 & 0.30 & 0.03 & 2.41 & 0.016 \\
\hline \multirow{14}{*}{$\begin{array}{l}\text { (25 sites; } \\
188 \text { plots) }\end{array}$} & & Basal area & -0.02 & -0.02 & 0.08 & -0.20 & 0.844 \\
\hline & & CWM DBHmax & -0.08 & -0.29 & 0.03 & -2.69 & 0.007 \\
\hline & & Disturbance & 0.10 & 0.22 & 0.02 & 4.29 & $<0.001$ \\
\hline & & Rainfall & 0.13 & 0.33 & 0.04 & 3.01 & 0.003 \\
\hline & & $\mathrm{pH}$ & 2.12 & 0.59 & 0.40 & 5.34 & $<0.001$ \\
\hline & \multirow{3}{*}{$\begin{array}{l}\text { Rarefied spp. } \\
\text { richness }\end{array}$} & Disturbance & 0.10 & 0.06 & 0.18 & 0.57 & 0.568 \\
\hline & & Rainfall & 0.35 & 0.22 & 0.43 & 0.82 & 0.412 \\
\hline & & $\mathrm{pH}$ & -1.64 & -0.12 & 3.76 & -0.43 & 0.664 \\
\hline & \multirow[t]{3}{*}{ Basal area } & Disturbance & -0.21 & -0.36 & 0.06 & -3.73 & $<0.001$ \\
\hline & & Rainfall & 0.09 & 0.18 & 0.12 & 0.79 & 0.428 \\
\hline & & $\mathrm{pH}$ & -0.65 & -0.14 & 0.79 & -0.83 & 0.407 \\
\hline & \multirow[t]{3}{*}{ CWM DBHmax } & Disturbance & 0.12 & 0.08 & 0.17 & 0.72 & 0.470 \\
\hline & & Rainfall & 0.37 & 0.26 & 0.42 & 0.88 & 0.380 \\
\hline & & $\mathrm{pH}$ & 5.55 & 0.45 & 2.10 & 2.64 & 0.008 \\
\hline
\end{tabular}




\begin{tabular}{|c|c|c|c|c|c|c|c|}
\hline$\Delta \mathrm{AGBmort}$ & $\log (\Delta \mathrm{AGBmort})$ & $\begin{array}{l}\text { Rarefied spp. } \\
\text { richness }\end{array}$ & 0.07 & 0.38 & 0.02 & 4.56 & $<0.001$ \\
\hline \multirow{13}{*}{$\begin{array}{l}\text { (23 sites; } \\
155 \text { plots) }\end{array}$} & & Basal area & 0.11 & 0.23 & 0.05 & 2.23 & 0.026 \\
\hline & & CWM SLA & 0.00 & -0.02 & 0.01 & -0.17 & 0.867 \\
\hline & & Disturbance & 0.02 & 0.08 & 0.03 & 0.72 & 0.474 \\
\hline & & CWD & 0.00 & 0.19 & 0.00 & 1.44 & 0.149 \\
\hline & & $\mathrm{pH}$ & -0.49 & -0.21 & 0.30 & -1.65 & 0.098 \\
\hline & \multirow{3}{*}{$\begin{array}{l}\text { Rarefied spp. } \\
\text { richness }\end{array}$} & Disturbance & 0.21 & 0.13 & 0.18 & 1.15 & 0.250 \\
\hline & & CWD & 0.02 & 0.28 & 0.02 & 1.09 & 0.275 \\
\hline & & $\mathrm{pH}$ & 0.75 & 0.06 & 3.59 & 0.21 & 0.834 \\
\hline & \multirow[t]{3}{*}{ Basal area } & Disturbance & -0.24 & -0.41 & 0.06 & -3.94 & $<0.001$ \\
\hline & & CWD & 0.01 & 0.34 & 0.00 & 1.99 & 0.046 \\
\hline & & $\mathrm{pH}$ & -0.50 & -0.10 & 0.79 & -0.63 & 0.532 \\
\hline & \multirow[t]{2}{*}{ CWM SLA } & Disturbance & 0.04 & 0.02 & 0.28 & 0.14 & 0.886 \\
\hline & & CWD & 0.01 & 0.11 & 0.03 & 0.30 & 0.764 \\
\hline$\triangle \mathrm{AGB}$ & $\Delta \mathrm{AGB}$ & $\begin{array}{l}\text { Rarefied spp. } \\
\text { richness }\end{array}$ & -0.02 & -0.10 & 0.02 & -1.20 & 0.232 \\
\hline \multirow{14}{*}{$\begin{array}{l}\text { (24 sites; } \\
167 \text { plots) }\end{array}$} & & Basal area & 0.04 & 0.08 & 0.06 & 0.73 & 0.464 \\
\hline & & CWM SLA & 0.05 & 0.51 & 0.01 & 4.52 & $<0.001$ \\
\hline & & Disturbance & 0.01 & 0.05 & 0.03 & 0.48 & 0.632 \\
\hline & & Rainfall & -0.40 & -0.12 & 0.28 & -1.45 & 0.147 \\
\hline & & $\mathrm{pH}$ & 0.47 & 0.19 & 0.18 & 2.67 & 0.008 \\
\hline & \multirow{3}{*}{$\begin{array}{l}\text { Rarefied spp. } \\
\text { richness }\end{array}$} & Disturbance & 0.17 & 0.11 & 0.17 & 1.04 & 0.298 \\
\hline & & Rainfall & 4.17 & 0.24 & 5.30 & 0.79 & 0.431 \\
\hline & & $\mathrm{pH}$ & 0.18 & 0.01 & 3.48 & 0.05 & 0.958 \\
\hline & \multirow[t]{3}{*}{ Basal area } & Disturbance & -0.24 & -0.41 & 0.05 & -4.99 & $<0.001$ \\
\hline & & Rainfall & 2.51 & 0.38 & 0.62 & 4.03 & $<0.001$ \\
\hline & & $\mathrm{pH}$ & -0.62 & -0.13 & 0.76 & -0.82 & 0.412 \\
\hline & \multirow[t]{3}{*}{ CWM SLA } & Disturbance & -0.32 & -0.11 & 0.47 & -0.69 & 0.493 \\
\hline & & Rainfall & 6.54 & 0.20 & 6.08 & 1.08 & 0.282 \\
\hline & & $\mathrm{pH}$ & 6.00 & 0.26 & 3.21 & 1.87 & 0.062 \\
\hline AGB & AGB & $\begin{array}{l}\text { Rarefied spp. } \\
\text { richness }\end{array}$ & 0.16 & 0.22 & 0.03 & 5.32 & $<0.001$ \\
\hline \multirow{10}{*}{$\begin{array}{l}\text { (26 sites; } \\
188 \text { plots) }\end{array}$} & & Basal area & 0.86 & 0.49 & 0.10 & 8.32 & $<0.001$ \\
\hline & & CWM WD & 5.08 & 0.56 & 0.87 & 5.82 & $<0.001$ \\
\hline & & CWD & 0.01 & 0.39 & 0.00 & 4.47 & $<0.001$ \\
\hline & & CEC & -0.03 & -0.05 & 0.04 & -0.83 & 0.407 \\
\hline & \multirow{2}{*}{$\begin{array}{l}\text { Rarefied spp. } \\
\text { richness }\end{array}$} & CWD & 0.01 & 0.18 & 0.01 & 1.05 & 0.294 \\
\hline & & CEC & 0.38 & 0.42 & 0.13 & 2.91 & 0.004 \\
\hline & \multirow[t]{2}{*}{ Basal area } & CWD & 0.01 & 0.40 & 0.00 & 1.94 & 0.052 \\
\hline & & CEC & 0.00 & -0.01 & 0.07 & -0.03 & 0.974 \\
\hline & \multirow[t]{2}{*}{ CWM WD } & CWD & 0.00 & 0.01 & 0.00 & 0.03 & 0.979 \\
\hline & & CEC & -0.03 & -0.40 & 0.02 & -1.56 & 0.119 \\
\hline
\end{tabular}



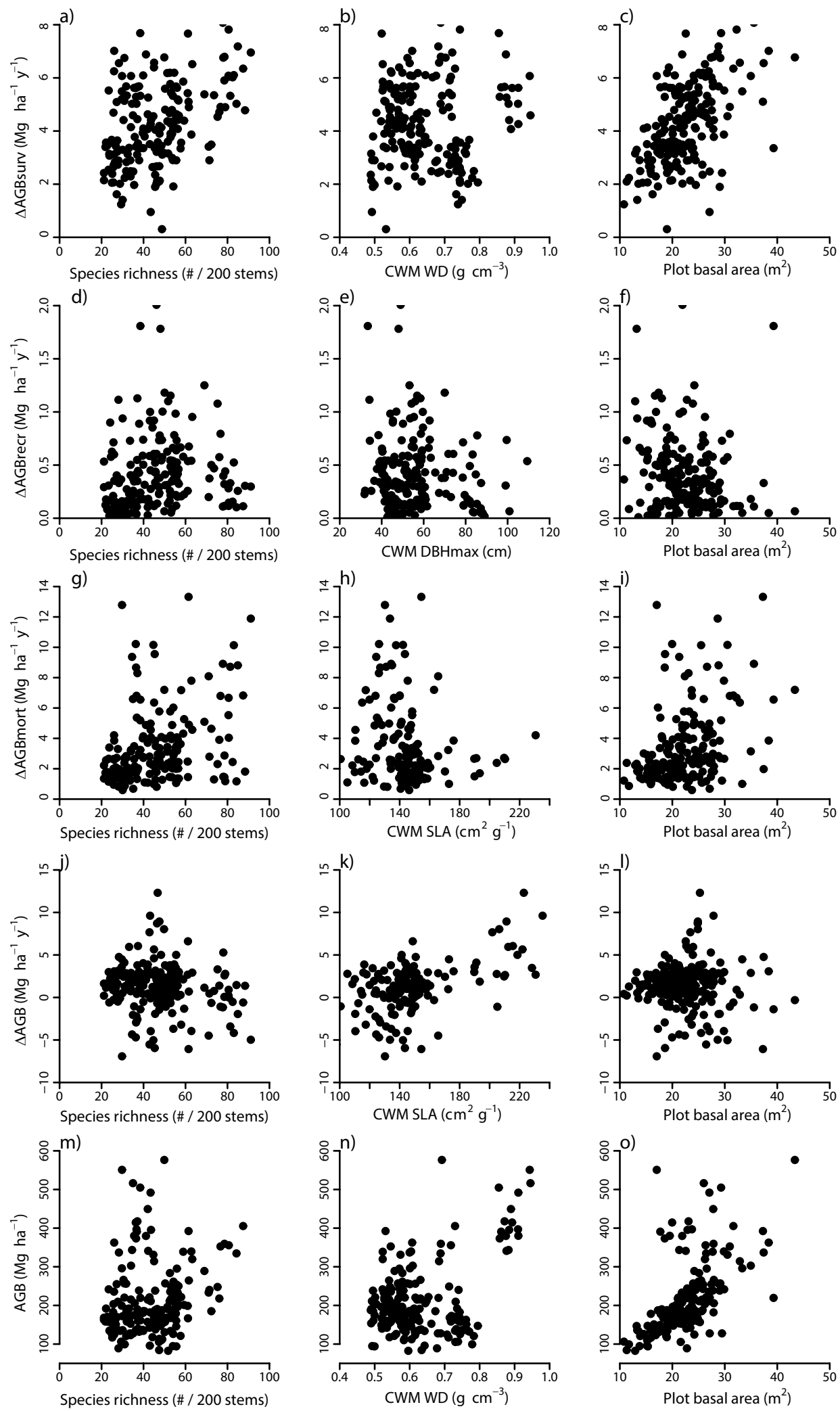

Appendix 5.7: Bivariate relationships for growth by surviving trees ( $\triangle \mathrm{AGBsurv;}$ a-c), growth by recruiting trees $(\Delta A G B r e c r ; d-f)$, biomass mortality ( $\Delta$ AGBmort; $g-i)$, net biomass change $(\triangle \mathrm{AGB} ; \mathrm{j}-\mathrm{l})$ and aboveground biomass stocks (AGB; $\mathrm{m}-\mathrm{n})$ in relation to three groups of environmental conditions: annual rainfall or climatic water deficit (first column), soil $\mathrm{pH}$ or soil cation exchange capacity (CEC) (second column), and disturbance measured by basal area removed (third column). Each dot is a 1-ha plot. Please note that these bivariate relationships are for illustration purposes only and may not necessarily provide the same results as in the structural equation models (Fig. 5.2). 

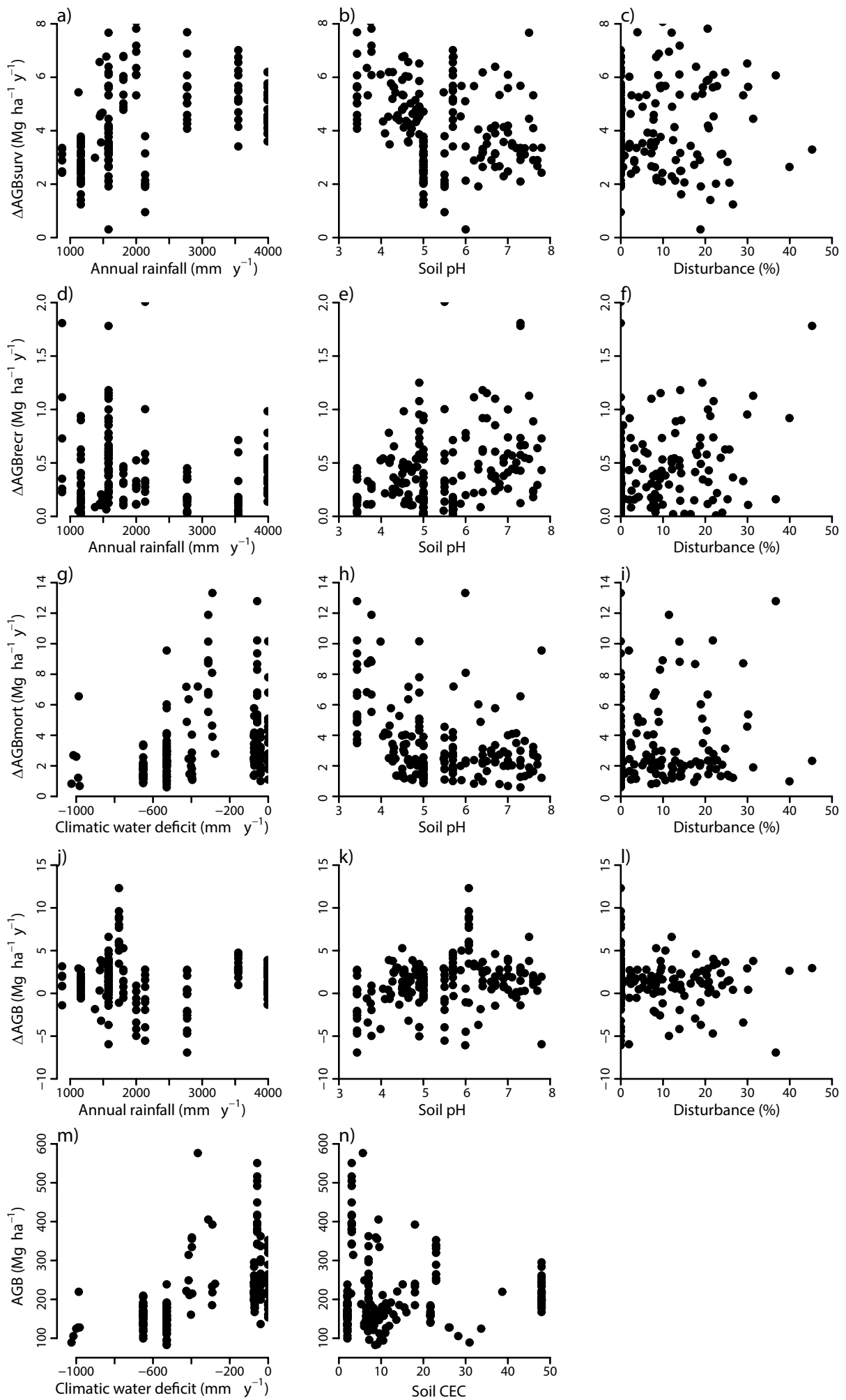

Appendix 5.8: Bivariate relationships for growth by surviving trees ( $\triangle A G B s u r v ; ~ a-c)$, growth by recruiting trees ( $\triangle \mathrm{AGBrecr}$; $\mathrm{d}-\mathrm{f}$ ) biomass mortality ( $\triangle \mathrm{AGBmort;} \mathrm{g}-\mathrm{i})$, net biomass change $(\triangle \mathrm{AGB} ; \mathrm{j}-\mathrm{l})$ and aboveground biomass stocks (AGB; $\mathrm{m}-\mathrm{o})$ in relation to three groups of forest attributes: species richness (first column), community-weighted mean (CWM) specific leaf area (SLA), wood density (WD), or maximum diameter (DBHmax) (second column), and plot basal area (third column). Each dot is a 1-ha plot. Please note that these bivariate relationships are for illustration purposes only and may not necessarily provide the same results as in the structural equation models (Fig. 5.2). 


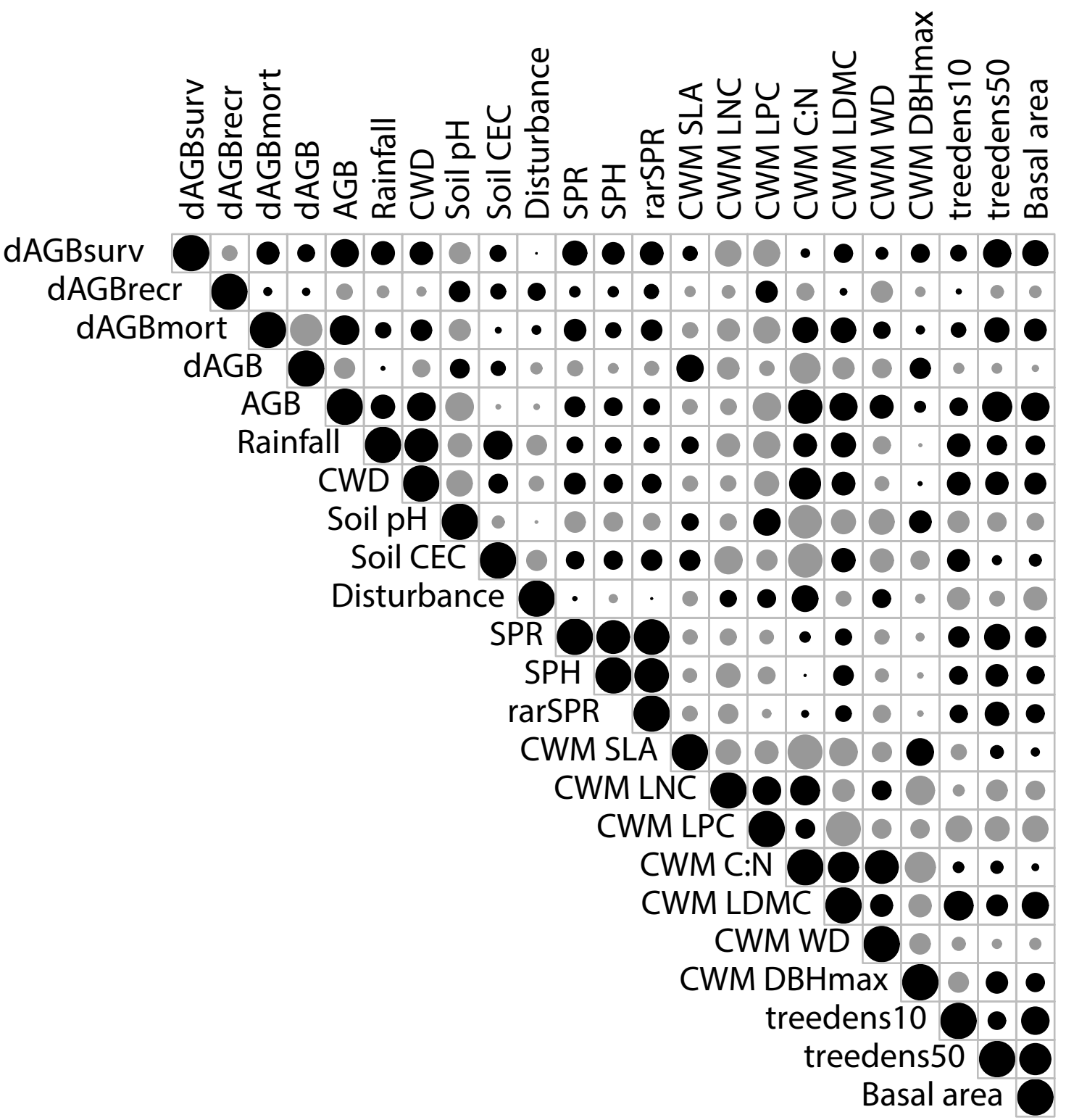

Appendix 5.9: Spearman's correlations between all variables used in the manuscript. Black circles indicate positive correlations and gray circles indicate negative correlations. The size of the circle indicates the strength of the correlation. dAGBsurv = biomass growth from surviving trees, $\mathrm{dAGBrecr}=$ biomass growth from recruiting trees, $\mathrm{dAGBmort}=$ biomass lost due to mortality, $\mathrm{dAGB}=$ net change biomass, $\mathrm{SPR}=$ species richeness, $\mathrm{SPH}=$ Shannon index, $\mathrm{LNC}=$ leaf nitrogen content, LPC = leaf phosphorous content, $\mathrm{CN}=$ leaf $\mathrm{C}: \mathrm{N}$ ratio, treedens $10=$ density of trees $>10 \mathrm{~cm}$ in $\mathrm{DBH}$, treedens $50=$ density of trees $>50 \mathrm{~cm}$ in DBH. For other abbreviations see legend of Appendix 5.4. 


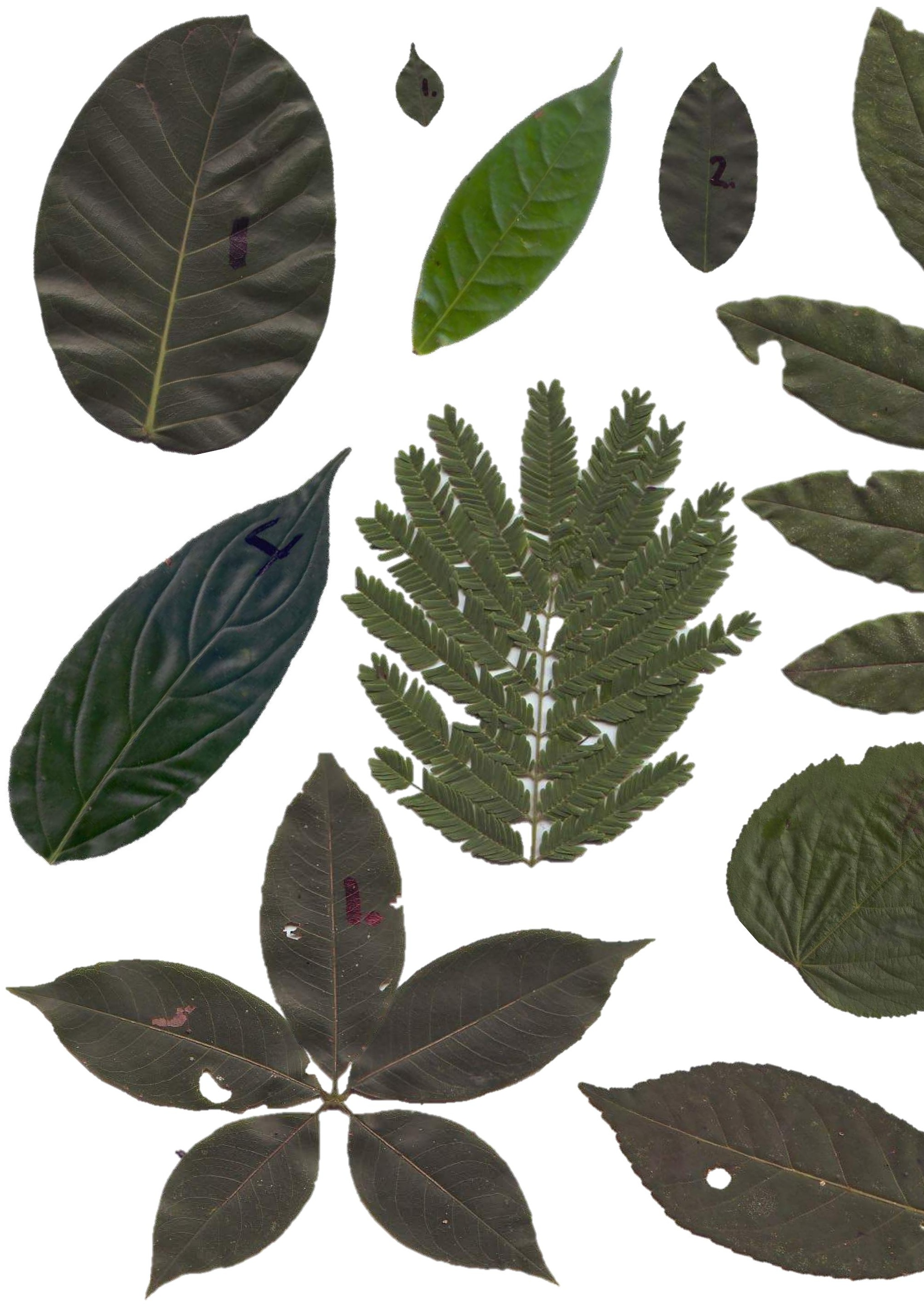




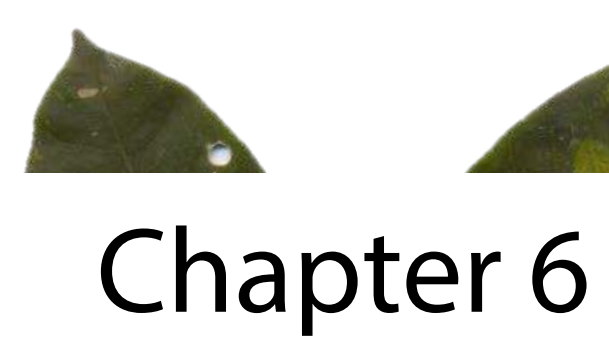

Old-growth Neotropical forests are shifting in species and trait composition

Masha T. van der Sande, Eric J. M. M. Arets, Marielos Peña-Claros, Angela L. de Avila, Anand Roopsind, Lucas Mazzei, Nataly Ascarrunz, Bryan Finegan, Alfredo Alarcón, Yasmani Cáceres-Siani, Juan Carlos Licona, Ademir Ruschel, Marisol Toledo, Lourens Poorter

Ecological Monographs (2016) 86: 228-243

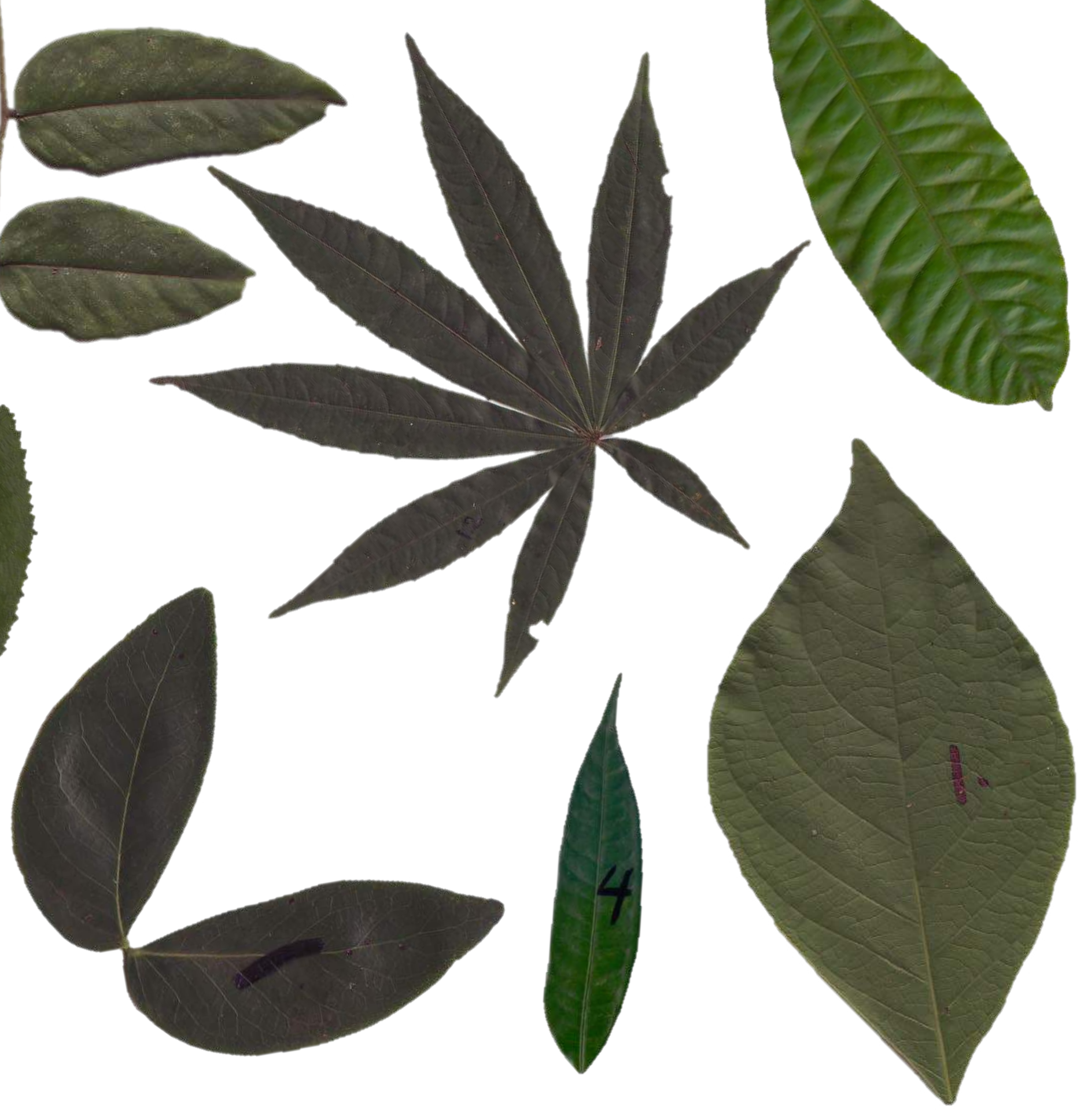




\section{Abstract}

Tropical forests have long been thought to be in stable state, but recent insights indicate that global change is leading to shifts in forest dynamics and species composition. These shifts may be driven by environmental changes such as increased resource availability, increased drought stress, and/or recovery from past disturbances. The relative importance of these drivers can be inferred from analysing changes in trait values of tree communities. Here, we evaluate a decade of change in species and trait composition across five old-growth Neotropical forests in Bolivia, Brazil, Guyana and Costa Rica that cover large gradients in rainfall and soil fertility. To identify the drivers of compositional change, we used data from 29 permanent sample plots and measurements of 15 leaf, stem and whole-plant traits that are important for plant performance and should respond to global change drivers.

We found that forests differ strongly in their community-mean trait values, resulting from differences in soil fertility and annual rainfall seasonality. The abundance of deciduous species with high specific leaf area increases from wet to dry forests. The community-mean wood density is high in the driest forests to protect xylem vessels against drought cavitation, and is high in nutrient poor forests to increase wood longevity and enhance nutrient residence time in the plant. The species composition changed over time in three of the forests, and the communitymean wood density increased and the specific leaf area decreased in all forests, indicating that these forests are changing towards later successional stages dominated by slow-growing, shade-tolerant species. We did not see changes in other traits that could reflect responses to increased drought stress, such as increased drought deciduousness or decreased maximum adult size, or that could reflect increased resource availability $\left(\mathrm{CO}_{2}\right.$, rainfall or nitrogen). Changes in species and trait composition in these forests are, therefore, most likely caused by recovery from past disturbances. These compositional changes may also lead to shifts in ecosystem processes, such as a lower carbon sequestration and "slower" forest dynamics.

Keywords: disturbance, drought, environmental gradients, forest dynamics, functional traits, global change, rainfall, resource availability, soil fertility 


\section{Introduction}

Tropical forests are of global importance for maintaining biodiversity, storing and sequestering carbon, and regulating the world's climate (Bonan 2008, Alkama and Cescatti 2016). Evidence continues to grow, however, that these forests are not in stable state (Heckenberger et al. 2003) but are undergoing large-scale changes in species composition and dynamics (Brienen et al. 2015), which may be attributed to various global change drivers (Wright 2005). To predict the future of old-growth forests, a better understanding is needed of the direction of forest change and its underlying drivers. One way to achieve this is by evaluating community-level changes in functional traits. Here, we evaluate changes in species composition and 15 leaf, stem and whole-plant traits among five Neotropical forests and infer the underlying global drivers by analysing whether and how traits change.

\section{Spatial variation in species and trait composition}

Species distributions are amongst others determined by species' responses to climate (Engelbrecht et al. 2007) and soil conditions (Clark and Palmer 1999, Toledo et al. 2012). Such species-specific responses in distribution are associated with species' traits, which ultimately determine species' strategies to acquire and use resources (Violle et al. 2007). Analysis of shifts in traits in relation to environmental conditions (also referred to as 'response traits'; Suding et al. 2008) are therefore expected to provide mechanistic insights into the underlying drivers of change. Many studies have addressed the effect of environmental conditions on species composition and community-level trait values for grasslands (Pakeman 2004) and individual forests (Feeley et al. 2011, Fauset et al. 2012). These studies generally find that the values of community-level traits respond to environmental gradients. However, environmental conditions vary more at larger spatial scales (e.g., across the Neotropics), leading to strong species turnover. As a result, the composition of species, and thus the composition of traits, should differ more strongly at large than at local scales. Few studies have addressed community-level changes across large-scale environmental gradients, and studies that do exist tend to focus only on a few traits (e.g., Baker et al. 2004b, Wright et al. 2004). Here, we evaluate changes in 15 traits for five forests spanning large environmental gradients from Bolivia to Costa Rica to test the hypothesis that differences in community-mean trait values among forests are a result of gradients in environmental conditions. 


\section{Temporal variation in species and trait composition}

Old-growth tropical forests are not in stable state. Natural or anthropogenic disturbances can set back a forest to an earlier successional state, causing community reassembly (Chazdon 2003). Moreover, global change, such as increased atmospheric $\mathrm{CO}_{2}$ concentrations or increased drought stress can alter species composition, eventually pushing the forest to an alternative stable state. Several studies have demonstrated changes in species composition over the last decades, although results and hypothesized drivers are contradictory, which could be caused by differences among sites in changing environmental conditions. Some studies find an increase in the abundance of drought-tolerant and deciduous species possibly due to increasing (atmospheric) drought stress as caused by decreased rainfall and/or increased temperature (Enquist and Enquist 2011, Feeley et al. 2011, Fauset et al. 2012, Zhou et al. 2014). Other studies find an increase in the abundance of emergent and canopy species due to increased resource availability such as $\mathrm{CO}_{2}$ (Laurance et al. 2004) or recovery from recent disturbances (Nelson 2005), and again others find an increased abundance of slow-growing species with high wood density, indicating that the forest is recovering from more historical disturbances and/or facing a reduction in resource availability (Chave et al. 2008). We aim to obtain a better understanding of possible underlying causes of compositional change by evaluating temporal changes in the community-weighted mean trait values of functional leaf, stem and whole-plant traits across tropical forests.

\section{Questions and hypotheses}

We address two questions. First, how do community-weighted mean trait values differ across five Neotropical forests? We expect that an increase in soil nutrient availability would increase the abundance of species with acquisitive trait values (e.g., high specific leaf area and leaf nutrient concentrations) that acquire more resources and grow faster. Trait responses along the precipitation gradient should be determined by drought adaptations at low rainfall, for example by droughtdeciduousness, and by shade adaptations at high rainfall. Drought-deciduous species at low rainfall may compensate for their short leaf lifespan with more acquisitive trait values that lead to faster growth in the short growing season, whereas evergreen species at high rainfall may have conservative trait values to increase leaf lifespan. Wood traits will be most conservative (e.g., high wood density) at dry sites or at sites with low nutrient availability to reduce drought cavitation and increase wood resistance to pathogens (Muller-Landau 2004, Romero and Bolker 2008, Markesteijn et al. 2011b). 
For the second question, we ask how species composition and communityweighted mean trait values change over time. We identify three important environmental change drivers that should favour species with certain trait values more than others, leading to changes in the community-mean trait values (Table 6.1, 6.2):

a) Increased resource availability (e.g., $\mathrm{CO}_{2}$ and nutrient deposition; Laurance et al. 2004, Hietz et al. 2011) would increase the abundance of i) species with acquisitive trait values that can make use of the increased availability of resources, ii) species with a tall adult stature that are better competitors for aboveground resources (i.e., light) in a denser forest canopy, and iii), in the case of nutrient deposition, reduce the Fabaceae abundance because of reduced advantage from $\mathrm{N}_{2}$-fixation. Such changes in community-weighted mean trait values could also be observed in response to recent disturbances, such as wind storms, which open up the canopy and favour the establishment of acquisitive species.

b) Increased drought stress (through decreased rainfall and/or increased temperature) would increase the abundance of i) drought-avoiding, deciduous species that generally have high specific leaf area (Enquist and Enquist 2011), ii) physiologically drought-tolerant species with high wood density that are cavitation resistant (Markesteijn et al. 2011b), iii) species with a small adult stature that suffer less from water transport limitations (Bennett et al. 2015), and iv) species with small leaves that allow for a better convective heat cooling.

c) Recovery from past disturbances should cause a shift from early-successional species with acquisitive trait values towards late-successional species with more 'conservative' trait values and tall adult stature, whereas Fabaceae should become less abundant due to decreased N limitation in older forests (Batterman et al. 2013, Sullivan et al. 2014).

\section{Methods}

\section{Sites}

We used data from permanent sample plots in five Neotropical forests, spanning a large latitudinal gradient in the Neotropics (from $16^{\circ} 07^{\prime} \mathrm{S}$ in Bolivia to $10^{\circ} 12^{\prime} \mathrm{N}$ in Costa Rica, see the map in Appendix 6.1), and broad gradients in rainfall (1160 $3900 \mathrm{~mm} \mathrm{y}^{-1}$ ) and soil conditions (Table 6.3). From low to high annual precipitation, we used two forest sites in Bolivia (INPA and La Chonta), one in Brazil (Tapajós), one in Guyana (Pibiri), and one in Costa Rica (Corinto). These forests also differ in soil fertility, from young and fertile soils in La Chonta to old and poor soils in Pibiri. Hereafter, these forest sites will be referred to as dry 
deciduous (DD; INPA), moist semi-deciduous (MSD; La Chonta), moist evergreen (ME; Tapajós and Pibiri), and wet evergreen (WE; Corinto).

Table 6.1: Traits with abbreviations, descriptions, units, and an explanation of what it indicates.

\begin{tabular}{|c|c|c|c|}
\hline Abbreviation & Variable description & Units & Indicator of \\
\hline SLA & Specific leaf area & $\mathrm{cm}^{2} \mathrm{~g}^{-1}$ & Light interception efficiency \\
\hline LA & Ln-transformed leaf area & $\mathrm{cm}^{2}$ & $\begin{array}{l}\text { Light interception, heat } \\
\text { balance }\end{array}$ \\
\hline $\mathrm{N}_{\text {leaf }}$ & Leaf nitrogen concentration & $\%$ & Photosynthetic capacity \\
\hline$P_{\text {leaf }}$ & Leaf phosphorus concentration & $\%$ & $\begin{array}{l}\text { Growth and photosynthetic } \\
\text { capacity }\end{array}$ \\
\hline $\mathrm{N}: \mathrm{P}_{\text {leaf }}$ & Leaf nitrogen : phosphorus ratio & & Relative nutrient limitation \\
\hline Chl & Leaf chlorophyll content & $\mu \mathrm{g} \mathrm{cm}^{-2}$ & Light harvesting capacity \\
\hline LDMC & Leaf dry matter content & $\mathrm{g} \mathrm{g}^{-1}$ & Leaf defense \\
\hline $\mathrm{FP}_{\mathrm{s}}$ & Specific force to punch & $\mathrm{N} \mathrm{cm}^{-2}$ & Leaf defense \\
\hline $\mathrm{LMF}_{\mathrm{m}}$ & Leaf mass fraction of the metamer & $\mathrm{g} \mathrm{g}^{-1}$ & Light interception efficiency \\
\hline WD & Wood density & $\mathrm{g} \mathrm{cm}^{-3}$ & $\begin{array}{l}\text { Stem defense, drought } \\
\text { tolerance }\end{array}$ \\
\hline $\mathrm{DBH}_{\max }$ & $\begin{array}{l}95 \% \text { quantile of stem diameter for all } \\
\text { individuals per species }\end{array}$ & $\mathrm{cm}$ & $\begin{array}{l}\text { Tree longevity and life history } \\
\text { strategy }\end{array}$ \\
\hline $\mathrm{CE}_{\max }$ & $\begin{array}{l}95 \% \text { quantile of crown exposure index } \\
\text { for all individuals per species }\end{array}$ & \# (1-5) & $\begin{array}{l}\text { Tree longevity and life history } \\
\text { strategy }\end{array}$ \\
\hline$\% \mathrm{Fab}$ & $\begin{array}{l}\text { Percentage of individuals from } \\
\text { Fabaceae }\end{array}$ & $\%$ & $\mathrm{~N}$ fixing capacity \\
\hline$\%$ compound & $\begin{array}{l}\text { Percentage of individuals with } \\
\text { compound leaves }\end{array}$ & $\%$ & Heat balance \\
\hline$\%$ deciduous & $\begin{array}{l}\text { Percentage of individuals that is } \\
\text { deciduous }\end{array}$ & $\%$ & Drought avoidance \\
\hline
\end{tabular}

\section{Plot design}

We used permanent plots in old-growth forests that were not disturbed by human activities or fire during the time of monitoring. To facilitate comparisons across sites, we used a similar time window for all sites (2000-2013), a plot size of 1 ha (if available), and included all trees $\geq 10 \mathrm{~cm} \mathrm{DBH}$. The plots in the dry deciduous site (INPA) were established and all trees $\geq 10 \mathrm{~cm} \mathrm{DBH}$ were identified and measured by Instituto Boliviano de Investigación Forestal (IBIF). The plots in the moist semi-deciduous site (La Chonta) were also established and measured by IBIF. The plots in the moist evergreen forest of Tapajós were established and all trees $\geq 5 \mathrm{~cm}$ DBH were identified and measured by Empresa Brasileira de Pesquisa Agropecuária (EMBRAPA). To use the same diameter limit as for the other sites, we used only trees $\geq 10 \mathrm{~cm} \mathrm{DBH}$. Besides the time window of about 10 years, we included an analysis of longer-term changes ( 29 years) for Tapajós. The plots in the moist evergreen forest of Pibiri were established and measured by Tropenbos. All trees $\geq 20 \mathrm{~cm} \mathrm{DBH}$ were measured in the whole plot, and trees $\geq 5 \mathrm{~cm}$ were 
measured in 25 subplots that in total covered an area of 0.25 ha per plot. We considered the trees between 10 and $20 \mathrm{~cm} \mathrm{DBH}$, which were measured on 0.25 ha per 1-ha plot, four times (to scale to $1 \mathrm{ha}$ ). The plots in the evergreen wet forest (Corinto) were established and all trees $\geq 10 \mathrm{~cm}$ in DBH were measured by Centro Agronómico Tropical de Investigación y Enseñanza (CATIE).

Table 6.2: Hypothesized temporal changes in 15 community-weighted mean traits in response to three potential drivers of environmental change: a) increased resource availability, b) increased drought stress, and c) recovery from past disturbances. The traits used, are: specific leaf are (SLA), leaf area (LA), leaf nitrogen concentration $\left(\mathrm{N}_{\text {leaf }}\right)$, leaf phosphorus concentration $\left(\mathrm{P}_{\text {leaf }}\right)$, leaf $\mathrm{N}: P$ ratio $\left(\mathrm{N}: \mathrm{P}_{\text {leaf }}\right)$, leaf chlorophyll content $(\mathrm{Chl})$, leaf dry matter content (LDMC), specific force to punch $\left(\mathrm{FP}_{\mathrm{s}}\right.$, a measure for leaf toughness), leaf mass fraction $\left(\mathrm{LMF}_{\mathrm{m}}\right)$, wood density (WD), species-specific maximum diameter $\left(\mathrm{DBH}_{\max }\right)$, species-specific maximum crown exposure index $\left(\mathrm{CE}_{\max }\right)$, percentage of individuals belonging to Fabaceae (\% Fab), \% of individuals with compound leaves ( $\%$ compound), and percentage of individuals that is deciduous ( $\%$ deciduous) (see Table 6.1 for more details). In the first three rows, hypothesized positive changes are shown by a ' $\uparrow$ ', hypothesized negative changes by a ' $\downarrow$ ', and no hypothesized changes by a '-'. The last row shows the observed changes in CWM traits across the five forest sites, with ' $\uparrow$ ' indicating a consistent increase over sites, ' $\downarrow$ ' a consistent decrease over sites, arrows in between brackets an increase or decrease for part of the sites, and '-' no significant temporal changes for any of the sites.

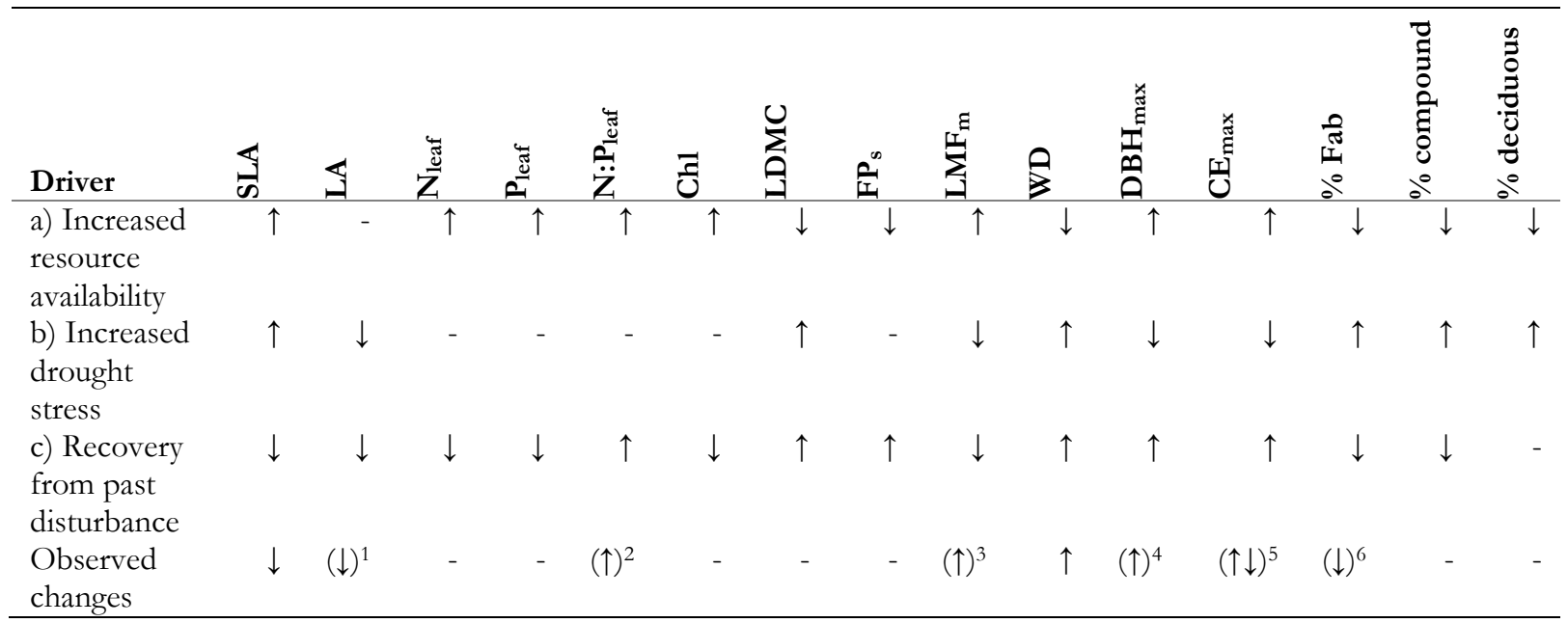

${ }^{1}$ Decrease for the moist evergreen forest (Tapajós) over 30 years.

2 Increase for the dry deciduous forest.

3 Increase for the moist semi-deciduous forest.

${ }^{4}$ Increase for the moist evergreen forest (Pibiri).

${ }^{5}$ Increase for the moist semi-deciduous forest and decrease for the moist evergreen forest (Pibiri).

${ }^{6}$ Decrease for the moist evergreen forest (Tapajós). 
Table 6.3: Details of the five forest sites used in this study: INPA, La Chonta, Tapajós, Pibiri, and Corinto. Information on the setup of the plots, climate, soil, and relevant references are given. SPEI $=$ Standardized Precipitation and Evapotranspiration Index, with high values indicating wet conditions. n.a. $=$ not available, n.s. $=$ not significant, $\uparrow=$ a significant temporal increase, and $\downarrow=$ a significant temporal decrease.

\begin{tabular}{|c|c|c|c|c|c|}
\hline & INPA & La Chonta & Tapajós & Pibiri & Corinto \\
\hline Coordinates & $\begin{array}{l}16^{\circ} 07^{\prime} \mathrm{S}, \\
61^{\circ} 43^{\prime} \mathrm{W}\end{array}$ & $\begin{array}{l}15^{\circ} 47^{\prime} \mathrm{S}, \\
62^{\circ} 55^{\prime} \mathrm{W}\end{array}$ & $\begin{array}{l}3^{\circ} 19^{\prime} \mathrm{S}, \\
54^{\circ} 57^{\prime} \mathrm{W}\end{array}$ & $\begin{array}{l}5^{\circ} 13^{\prime} \mathrm{N}, \\
58^{\circ} 38^{\prime} \mathrm{W}\end{array}$ & $\begin{array}{l}10^{\circ} 12^{\prime} \mathrm{N}, \\
83^{\circ} 52^{\prime} \mathrm{W}\end{array}$ \\
\hline Country & Bolivia & Bolivia & Brazil & Guyana & Costa Rica \\
\hline Forest type & $\begin{array}{l}\text { Dry deciduous } \\
\text { (DD) }\end{array}$ & $\begin{array}{l}\text { Moist semi- } \\
\text { deciduous } \\
\text { (MSD) }\end{array}$ & $\begin{array}{l}\text { Moist } \\
\text { evergreen } \\
\text { (MEtap) }\end{array}$ & $\begin{array}{l}\text { Moist } \\
\text { evergreen } \\
\text { (MEpib) }\end{array}$ & $\begin{array}{l}\text { Wet evergreen } \\
\text { (WE) }\end{array}$ \\
\hline $\begin{array}{l}\text { Number of control } \\
\text { plots }\end{array}$ & 8 & 9 & 6 & 3 & 3 \\
\hline Size of plots (ha) & $1(100 * 100 \mathrm{~m})$ & $1(100 * 100 \mathrm{~m})$ & $0.25(50 * 50 \mathrm{~m})$ & $1(100 * 100 \mathrm{~m})$ & $1(100 * 100 \mathrm{~m})$ \\
\hline First census & $2002-2003$ & 2000-2001 & $\begin{array}{l}2003 \text { (and } \\
1983 \text { ) }\end{array}$ & 2000 & 2000 \\
\hline Last census & 2012-2013 & 2009-2011 & 2012 & 2013 & 2010 \\
\hline Timespan (y) & 10 & 8 & 9 (and 29) & 13 & 10 \\
\hline Rainfall $\left(\mathrm{mm} \mathrm{y}^{-1}\right)$ & 1160 & 1580 & 2110 & 2772 & 3900 \\
\hline $\begin{array}{l}\text { Number of dry months } \\
<100 \mathrm{~mm} \text { rainfall }\end{array}$ & 7 & 6 & 3 & 0 & 0 \\
\hline $\begin{array}{l}\text { Average annual } \\
\text { temperature }\left({ }^{\circ} \mathrm{C}\right)\end{array}$ & 24.3 & 24.3 & 25 & 25.9 & 23.7 \\
\hline $\begin{array}{l}\text { Temporal change in } \\
\text { annual rainfall (1900- } \\
\text { 2013) }\end{array}$ & n.s. & n.s. & n.s. & $\uparrow$ & $\uparrow$ \\
\hline $\begin{array}{l}\text { Temporal change in } \\
\text { SPEI (1900-2013) }\end{array}$ & $\downarrow$ & n.s. & n.s. & $\uparrow$ & $\uparrow$ \\
\hline Soil type & Oxisols & Ultisols & Oxisols & Ferralsols & Inceptisols \\
\hline $\begin{array}{l}\text { Soil fertility from } \\
\text { highest (1) to lowest } \\
\text { (5), based on Fig. } 2 \text { of } \\
\text { Quesada et al. (2010). }\end{array}$ & $\begin{array}{l}2 \text { (middle- } \\
\text { high) }\end{array}$ & 1 (high) & 3 (middle-low) & 4 (low) & n.a. \\
\hline References & $\begin{array}{l}\text { Peña-Claros et } \\
\text { al. } 2012\end{array}$ & $\begin{array}{l}\text { Peña-Claros et } \\
\text { al. } 2012\end{array}$ & $\begin{array}{l}\text { de Carvalh, } \\
\text { 1992, Silva et } \\
\text { al. 1995, } \\
\text { Aragão et al. } \\
2009\end{array}$ & $\begin{array}{l}\text { van Kekem et } \\
\text { al. 1996, van } \\
\text { der Hout 1999, } \\
\text { van Dam } 2001\end{array}$ & $\begin{array}{l}\text { Sesnie et al. } \\
\text { 2009, Finegan } \\
\text { et al. } 2015\end{array}$ \\
\hline $\begin{array}{l}\text { Number of species } \\
\text { with leaf and stem trait } \\
\text { data }\end{array}$ & 98 & 158 & 68 & 33 & 72 \\
\hline $\begin{array}{l}\% \text { plot abundance } \\
\text { covered with traits, } \\
\text { averaged per site }\end{array}$ & 96.7 & 82.9 & 72.5 & 78.6 & 85.1 \\
\hline $\begin{array}{l}\text { Reference for more } \\
\text { details on trait } \\
\text { collection }\end{array}$ & $\begin{array}{l}\text { Markesteijn et } \\
\text { al. 2011b }\end{array}$ & $\begin{array}{l}\text { Chapter 4, } \\
\text { Rozendaal et } \\
\text { al. 2006, } \\
\text { Poorter 2008, } \\
\text { Carreño- } \\
\text { Rocabado et } \\
\text { al. 2012, van } \\
\text { Gils 2012 }\end{array}$ & $\begin{array}{l}\text { de Avila et al. } \\
\text { in prep. }\end{array}$ & Chapter 3 & \\
\hline
\end{tabular}




\section{Trait collection}

Here, we provide a short description of the collection of traits (see Table 6.3 for references providing more detailed information). All traits were expressed at the plot level and in general, traits were measured according to standard protocols (Pérez-Harguindeguy et al. 2013). We measured traits that are important for the carbon-, water-, nutrient- and heat-balance of the plant (Table 6.1), and hence, should respond to global change drivers. We used specific leaf area (SLA), leaf area (LA), leaf nitrogen $\left(\mathrm{N}_{\text {leaf }}\right)$ and phosphorus concentration $\left(\mathrm{P}_{\text {leaf }}\right)$, leaf $\mathrm{N}: \mathrm{P}$ ratio $(\mathrm{N}$ : $\mathrm{P}_{\text {leaf }}$ ), leaf chlorophyll content $(\mathrm{Chl})$, leaf dry matter content (LDMC), specific force to punch $\left(\mathrm{FP}_{\mathrm{s}}\right)$, leaf mass fraction of the metamer $\left(\mathrm{LMF}_{\mathrm{m}}\right)$, wood density (WD), maximum stem diameter $\left(\mathrm{DBH}_{\max }\right)$, maximum crown exposure index $\left(\mathrm{CE}_{\max }\right)$, percentage of individuals from Fabaceae, percentage of individuals with compound leaves, and percentage of individuals from deciduous species (Table 6.1).

For each site, we measured leaf and stem traits for the most abundant tree species (on average representing $84 \%$ of all individuals in the plots). All leaf traits were measured on about 5 (range 1-10) individuals per species and 4-5 leaves per individual. To have comparable measurements among species and sites, individuals were selected that were growing in relatively open conditions and that had a DBH of $8-20 \mathrm{~cm}$. Trees in this size class are well-established and their leaves are still accessible with a pruner on an extension pole. Leaves harvested were healthy and exposed to high-light conditions. LA was measured on fresh leaves without the petiole, and ln-transformed for a normal distribution. SLA was calculated as the fresh leaf area divided by the dry mass $\left(\mathrm{cm}^{2} \mathrm{~g}^{-1}\right)$, and was based on the whole leaf (including rachis for compound leaves). Chlorophyll content was defined as mass per unit leaf area $\left(\mu \mathrm{g} \mathrm{cm}^{-2}\right)$ using a SPAD-meter (Minolta SPAD 502 Chlorophyll Meter, Spectrum Technologies Inc., Plainfield, IL, USA), $\mathrm{N}_{\text {leaf }}$ and $\mathrm{P}_{\text {leaf }}$ (in \%) as concentrations of dry mass, and $\mathrm{N}_{\text {leaf }}: \mathrm{P}_{\text {leaf }}$ provided the $\mathrm{N}: \mathrm{P}_{\text {leaf }}$ ratio. LDMC was calculated by dividing the leaf dry mass by the leaf fresh mass $\left(\mathrm{g} \mathrm{g}^{-1}\right) \cdot \mathrm{FP}_{\mathrm{s}}$ was measured using a penetrometer, which measures the force needed to punch the flat-ended side of a nail through the leaf. $\mathrm{FP}_{\mathrm{s}}$ was then calculated by dividing the force needed to punch the leaf by the product of the circumference of the nail and the thickness of the leaf $\left(\mathrm{N} \mathrm{cm}^{-2}\right)$, to correct for the fracture area on which pressure is exerted. $\mathrm{LMF}_{\mathrm{m}}$ was calculated by dividing the leaf dry mass by the sum of the biomass of the whole metamer, i.e., the dry masses of the leaf, petiole and internode $\left(\mathrm{g} \mathrm{g}^{-1}\right)$.

To take into consideration the possible radial variation in wood density (Hietz et al. 2013), WD ( $\mathrm{g} \mathrm{cm}-3)$ was based on the average of the whole stem radius of a tree. Per species, a wood core was taken from about 3 individuals of $20-40 \mathrm{~cm}$ $\mathrm{DBH}$. WD was calculated by dividing the oven-dried mass (for 48 hours at $70{ }^{\circ} \mathrm{C}$ ) by the fresh volume. For most species of the moist semi-deciduous site (La 
Chonta), wood was collected from the outer sapwood of the tree. These WD values were converted to WD values for the whole radius, based on the relation between WD of the youngest sapwood and WD of the whole radius for 32 Bolivian species $\left(\mathrm{WD}_{\text {radius }}=0.0037+1.0607 * \mathrm{WD}_{\text {outer }} ; \mathrm{R}^{2}=0.90\right.$; see chapter 4$)$. For Corinto, WD was only measured on the outer sapwood of the tree. $\mathrm{DBH}_{\max }$ (cm) per species was based on the 95\% quantile of diameters for all individuals in a site that were larger than $0.1 *$ maximum diameter found for that species (King et al. 2006a), and $\mathrm{CE}_{\max }$ was calculated for each species as the $95 \%$ quantile of crown exposure values (between 1 and 5; Dawkins \& Field, 1978) for all individuals in a site. See Appendix 6.2 for alternative ways to calculate $\mathrm{DBH}_{\max }$. The moist evergreen site (Tapajós) was excluded for $\mathrm{CE}_{\max }$ because it could not be calculated in the same way.

\section{Community-weighted mean trait composition}

To evaluate differences in community-level traits among sites and between census years, we calculated the abundance-weighted mean trait values, also known as the community-weighted mean (CWM, Pla et al. 2012), for the 15 leaf, stem and wholeplant traits. We weighted by species abundance rather than by species basal area, to give equal weight to recruiting and dying trees and in this way increase the effect of small, newly recruited trees on changes in mean trait values. A test with basal areaweighted mean trait values showed similar trends in community-weighted mean trait values (Appendix 6.3). Hereafter, we therefore only report analyses based on abundance-weighted trait values.

Per plot, these CWM trait values were calculated based on all live individuals (for which trait data were available) in the first census and all live individuals in the final census. Hence, these resulted in 29 plots $* 2$ censuses $=58 \mathrm{CWM}$ values per trait. Additionally, we calculated CWM trait values for Tapajós in an earlier census (1983), to evaluate longer-term changes (29 years). Note that we used mean trait values per species. Therefore, we only evaluate changes in CWM trait values due to changes in species composition, not due to plastic changes in species' trait values over time. Although many species show plastic phenotypic responses within and across individuals to environmental conditions (Poorter et al. 2010a), in general the variation explained by intraspecific trait differences is small (12\%) compared to interspecific differences ( $72 \%$, Rozendaal et al. 2006). Sites differed in the number of species with trait data (Table 6.3). Leaf and stem traits were available for species representing $73-97 \%$ (average $84 \%$ ) of all individual trees per plot.

We also calculated the percentage of individuals of Fabaceae per plot as an indicator of the nitrogen fixing potential (as different subfamilies of Fabaceae have 62\% (Papilionoideae), 54\% (Mimosoideae) and 5\% (Caesalpinoideae) of $\mathrm{N}_{2}$-fixing genera; Hedin et al. 2009). Furthermore, for each plot and census we calculated the 
percentage of individuals with compound leaves, and the percentage of individuals that belonged to deciduous species. A species was categorized as deciduous when some (or all) of its individuals possess a yearly leafless period. $\mathrm{DBH}_{\max }, \mathrm{CE}_{\max }$, and Fabaceae abundance were obtained for all species in the plots.

\section{Environmental drivers}

Globally, the concentration of atmospheric $\mathrm{CO}_{2}$ has increased from about $320 \mathrm{ppm}$ in 1960 to almost $400 \mathrm{ppm}$ in 2013 (Appendix 6.4). Annual rainfall between 1900 and 2013 significantly increased for the two wettest sites (Corinto and Pibiri) and did not change for the three driest sites (INPA, La Chonta and Tapajós) (Table 6.3, Appendix 6.5). The Standardized Precipitation and Evapotranspiration Index (SPEI) is a measure for dryness, with positive values indicating humid conditions and negative values indicating dry conditions. Over the period 1900-2013, SPEI significantly decreased for the dry deciduous site (i.e., it became drier), significantly increased for the two wettest sites (i.e., it became more humid), and did not significantly change for the two intermediate sites (Table 6.3, Appendix 6.6). Also over the period 1991-2013 (i.e., the time period in which the data were collected), SPEI values significantly decreased for the dry deciduous site $(\mathrm{P}<0.001)$, significantly increased for the wet evergreen site $(\mathrm{P}<0.001$, both only for the 12 month timescale, see Appendix 6.6), and did not change for the intermediate sites. Hence, the sites do not show consistent increases or decreases in drought. However, extreme drought events (the lowest peaks in Appendix 6.6) occur repeatedly (with a monthly SPEI value $<-2$ occurring every 3-8 years, based on a 12 -month timescale), and may therefore still cause changes in species and trait composition.

\section{Statistical analyses}

To evaluate how trait composition (i.e., the multivariate CWM trait space) and single CWM trait values differ amongst sites and change over time (between the censuses) we performed several analyses. Differences in the multivariate CWM trait composition among the five sites and between the first and final census were tested using a redundancy analysis, using the 10 traits that were collected at all sites. Site and census were included as constrained axes, to test for differences in multivariate CWM trait composition. The significance of the constrained axes was tested using a permuted ANOVA, by allowing permutations within plots (Oksanen 2011). To evaluate whether annual precipitation and soil fertility could explain differences between sites (because we do not have variation among plots and between census years), we repeated the analysis twice: on time to include annual precipitation and one time to include soil fertility as constrained axis instead of site. Soil fertility was 
based on the ranking from low to high soil fertility between sites (Table 6.3). The wet evergreen forest was given the highest soil fertility, because this forest is growing on volcanic soils (Finegan et al. 2015). Both soil fertility and annual precipitation were included as continuous variables.

For each univariate trait, differences in CWM values among the five sites and between censuses were evaluated using a linear mixed model, with site, census and their interaction as fixed factors, and plot as random factor (to account for census as repeated measures per plot). In case of significant effects of site and/or the interaction of site and year, Tukey's post-hoc test was used for multiple comparisons. To test whether the observed changes over a decade were also found for a longer time period, we evaluated temporal changes in CWM trait values in Tapajós between 1983 and 2012, using a linear mixed model with census as explanatory variable and plot as random factor. To evaluate associations among CWM trait values, we used a principal component analysis on the centered (with a mean of 0 ) and standardized (by dividing the centered trait values by their standard deviations) trait values.

To evaluate whether the first and last census differed in species composition, we applied a redundancy analysis on the species abundance data, with census as the constrained axis and permutations within plots. This analysis was done for each site separately, since species composition was too different to be able to combine sites. For Tapajós, a change in species composition was also tested between the years 1983 and 2012.

All analyses were performed in $\mathrm{R}$ version 3.1.2. Linear mixed models were performed with the lme function of the nmle package (Pinheiro and Bates 2016), and multiple comparisons with the glht function of the multcomp package (Hothorn et al. 2014). Redundancy and principal component analyses were performed with the rda function, and the ANOVA to test for constrained axes with the anova.cca function, both of the vegan package (Oksanen et al. 2014).

\section{Results}

The multivariate composition of $10 \mathrm{CWM}$ traits (that were collected at all sites) differed significantly among sites $\left(\mathrm{F}_{4,51}=78.1, \mathrm{P}=0.018\right.$; Fig. 6.1a), and also with annual precipitation $\left(\mathrm{F}_{1,54}=20.7, \mathrm{P}=0.012\right.$; not shown in Fig. 6.1) and soil fertility $\left(\mathrm{F}_{1,54}=16.3, \mathrm{P}=0.012\right)$. All individual CWM traits differed significantly among sites, except for $\mathrm{CE}_{\max }$ (Table. 6.4, Appendix 6.7). In general, SLA, $\mathrm{N}_{\text {leaf }}, \mathrm{P}_{\text {leaf }}$ and percentage of deciduous species increased towards drier forests (except for the wettest forest where SLA and leaf nutrients were high, Fig. 6.2, Appendix 6.8). In contrast, LDMC, $\mathrm{FP}_{\mathrm{s}}$ and leaf area increased towards wetter forests. In 
combination, this indicates that leaf trait values tend to be more acquisitive in dry forests. Chl, $\mathrm{N}: \mathrm{P}_{\text {leaf }}$ and $\mathrm{LMF}_{\mathrm{m}}$ showed an optimum with rainfall, whereas the other traits did not show a clear pattern with rainfall.

Species composition of the three driest forests (INPA, La Chonta and Tapajós) changed significantly over time (Table 6.5, Fig. 6.3). The shift in species composition towards the centre of Fig. 6.3 indicates a directional convergence of plots over time. Multivariate trait composition did not change significantly over time $\left(\mathrm{F}_{1,51}=0.35, \mathrm{P}=0.609\right.$; Fig. 6.1a), but individual traits did (Table 6.4). Across all sites, specific leaf area decreased and wood density increased over time (Fig. 6.2). Five traits $\left(\mathrm{DBH}_{\max }, \mathrm{CE}_{\max }, \mathrm{N}: \mathrm{P}_{\text {leaf }}, \mathrm{LMF}_{\mathrm{m}}\right.$, and \% individuals of Fabaceae) changed over time for one or two sites only (Fig. 6.2, Appendix 6.9).

For the moist evergreen forest of Tapajós we could evaluate longer-term (29 years) changes. We found significant changes over time in species composition (Table 6.5), marginal changes in multivariate trait composition $\left(\mathrm{P}=0.093, \mathrm{~F}_{1,9}=\right.$ 0.665), a significant increase in $\mathrm{DBH}_{\max }$ and $\mathrm{WD}$, and a decrease in LA and the percentage individuals of Fabaceae and with compound leaves (Appendix 6.10). Hence, on both the short-term (10 years) and longer-term (29 years), WD and $\mathrm{DBH}_{\max }$ increased, and Fabaceae abundance decreased for this forest.

Table 6.4: Significance values (P-values) of ANOVAs for each community-weighted mean (CWM) trait, with census year (first census around 2000 vs. last census around 2010, Table 6.3), site, and the interaction between census and site as explanatory variables. See Appendix 6.8 for multiple comparisons among sites for the CWM traits that had no significant interaction between census and site, and see Appendix 6.9 for multiple comparisons for the CWM traits that had a significant interaction between census and site. LA was ln-transformed. Significant P-values are shown in bold.

\begin{tabular}{llll}
\hline CWM trait & Site & Census & Census*Site \\
\hline $\mathrm{SLA}$ & $<\mathbf{0 . 0 0 1}$ & $<\mathbf{0 . 0 0 1}$ & 0.052 \\
$\mathrm{LA}$ & $<\mathbf{0 . 0 0 1}$ & 0.560 & 0.151 \\
$\mathrm{~N}_{\text {leaf }}$ & $<\mathbf{0 . 0 0 1}$ & 0.597 & 0.567 \\
$\mathrm{Pl}_{\text {eaf }}$ & $<\mathbf{0 . 0 0 1}$ & 0.129 & 0.325 \\
$\mathrm{~N}_{\text {leaf }}$ & $<\mathbf{0 . 0 0 1}$ & 0.056 & $\mathbf{0 . 0 0 4}$ \\
$\mathrm{Chl}$ & $<\mathbf{0 . 0 0 1}$ & 0.152 & 0.345 \\
$\mathrm{LDMC}$ & $<\mathbf{0 . 0 0 1}$ & 0.493 & 0.408 \\
$\mathrm{FP}_{\mathrm{s}}$ & $<\mathbf{0 . 0 0 1}$ & 0.086 & 0.065 \\
$\mathrm{LMF}_{\mathrm{m}}$ & $<\mathbf{0 . 0 0 1}$ & $\mathbf{0 . 0 0 4}$ & $\mathbf{0 . 0 0 1}$ \\
$\mathrm{WD}_{\mathrm{DBH}}$ & $<\mathbf{0 . 0 0 1}$ & $\mathbf{0 . 0 0 1}$ & 0.214 \\
$\mathrm{CE} \mathrm{m}_{\max }$ & $\mathbf{0 . 0 4 0}$ & 0.143 & $<\mathbf{0 . 0 0 1}$ \\
$\%$ Fab & 0.232 & 0.885 & $<\mathbf{0 . 0 0 1}$ \\
\% compound & $<\mathbf{0 . 0 0 1}$ & 0.947 & $<\mathbf{0 . 0 0 1}$ \\
\% deciduous & $<\mathbf{0 . 0 0 1}$ & 0.928 & 0.227 \\
\hline
\end{tabular}



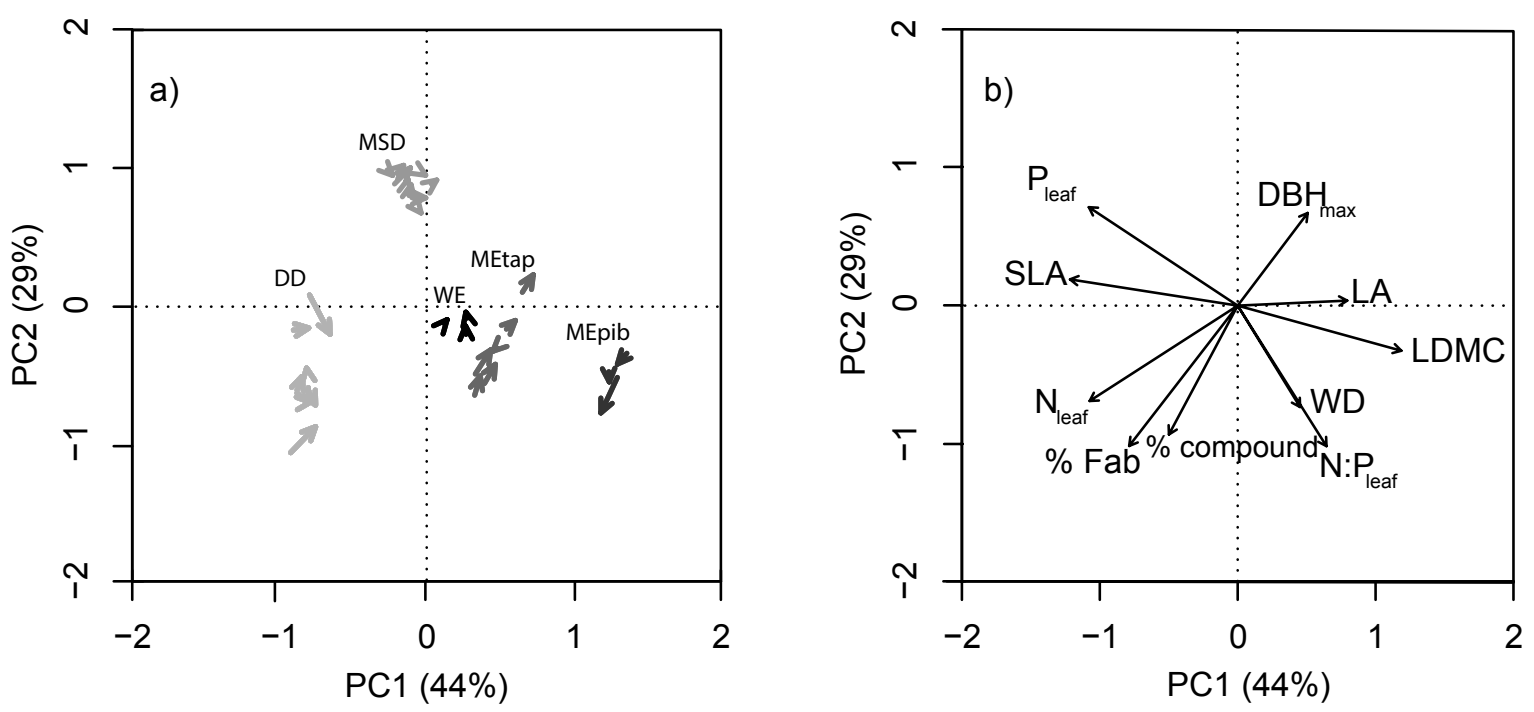

Figure 6.1: Multivariate trait composition for plots in two census years in the five sites, based on 10 community-weighted mean traits (a), and associations among community-weighted mean traits (b). The arrows in a) show the change in multivariate trait composition over the $\sim 10$ years per plot. The different shades of gray indicate the sites, ordered from dry deciduous (lightest grey) to wet evergreen (black): dry deciduous (DD; INPA), moist semi-deciduous (MSD; La Chonta), moist evergreen (MEtap; Tapajós and MEpib; Pibiri), and wet evergreen (WE; Corinto) (Table 6.3). For trait abbreviations of Figure $b$, see Table 6.1. Sites differed significantly in multivariate trait composition $\left(\mathrm{F}_{4,51}=78.1, \mathrm{P}=0.018\right)$, but census did not $\left(\mathrm{F}_{1,51}=0.3, \mathrm{P}=0.609\right)$. Chl, $\mathrm{FP}_{\mathrm{s}}$, $\mathrm{LMF}_{\mathrm{m}}, \mathrm{CE}_{\text {max }}$, and \% deciduous were left out of these analyses because of missing values for some sites. Percentages behind the axes is the variation explained by the principal component axes.

Table 6.5: The effect of census year (as the constrained axis of the redundancy analysis) on species composition, tested using a permuted ANOVA per site (La Chonta, INPA, Tapajós, Pibiri and Corinto) (Oksanen 2011). For each site, the variance (Var), F-value (F) with degrees of freedom in subscript, and P-value (P) are given. 'Tapajós 29 years' compares the species composition over a 29-year time interval. Significant P-values are shown in bold.

\begin{tabular}{llll}
\hline Site & Var & F & $\mathbf{P}$ \\
\hline Dry deciduous (INPA) & 59.40 & $0.22_{(1,13)}$ & $\mathbf{0 . 0 3 0}$ \\
Moist semi-deciduous( La Chonta) & 26.27 & $0.22_{(1,15)}$ & $\mathbf{0 . 0 0 6}$ \\
Moist evergreen (Tapajós 10 years) & 6.46 & $0.35_{(1,9)}$ & $\mathbf{0 . 0 1 6}$ \\
Moist evergreen (Pibiri) & 34.70 & $0.03_{(1,3)}$ & 1.000 \\
Wet evergreen (Corinto) & 41.50 & $0.08_{(1,3)}$ & 0.625 \\
Moist evergreen (Tapajós 29 years) & 14.14 & $0.70_{(1,9)}$ & $\mathbf{0 . 0 1 6}$ \\
\hline
\end{tabular}




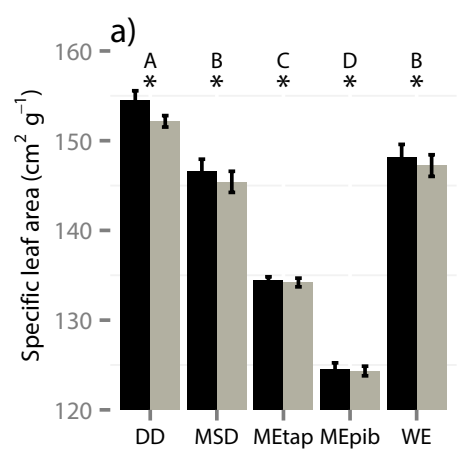

b)

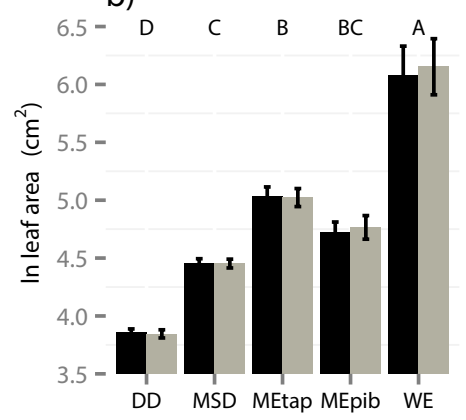

d)

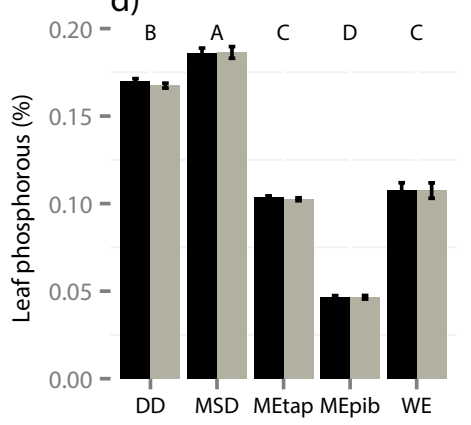

g)

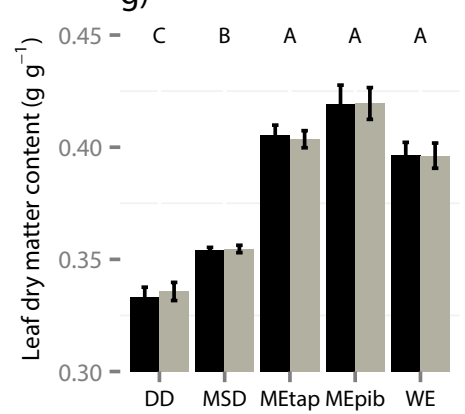

j)
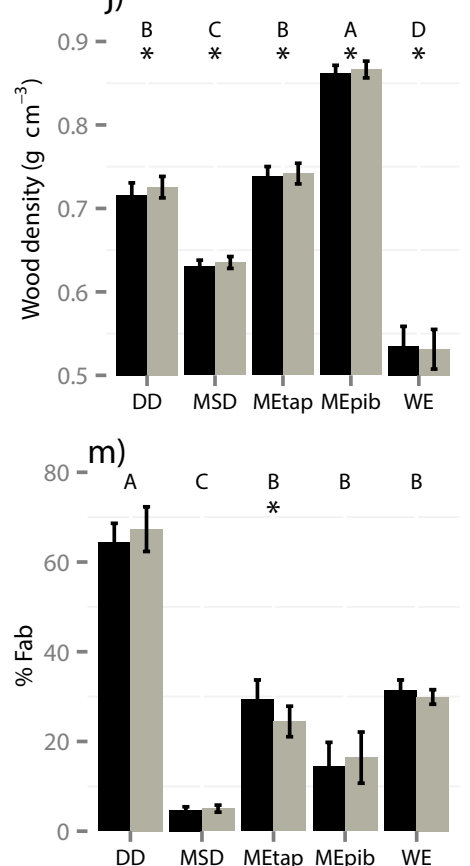

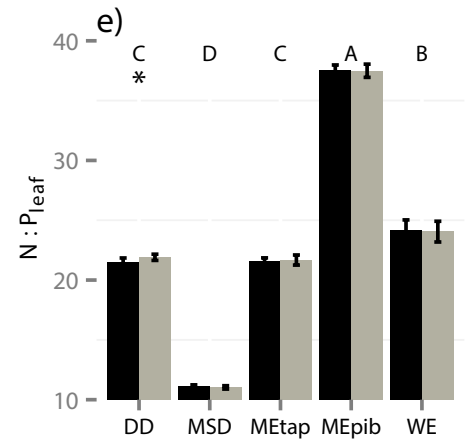

h)

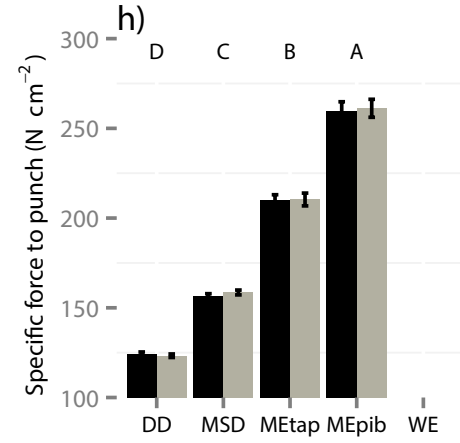

$50-k)$

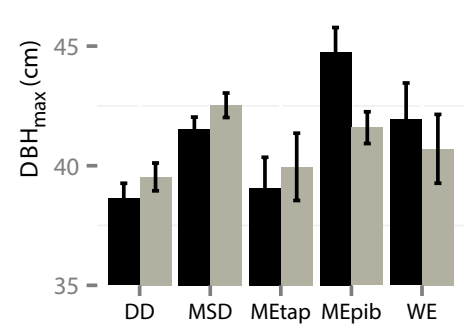

n)

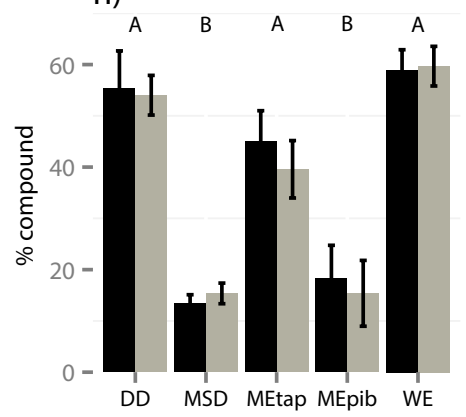

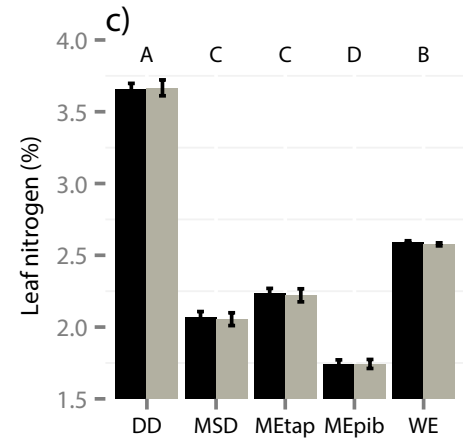
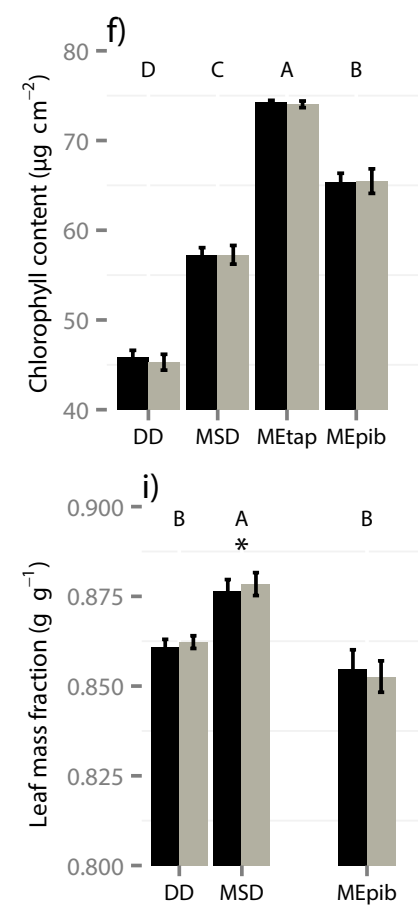

$\begin{array}{ccc}\text { I) } & & \\ & \text { A } & \text { A } \\ & * & *\end{array}$

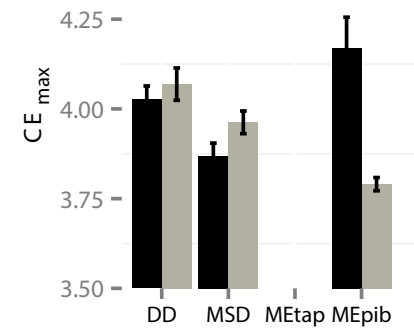

O)

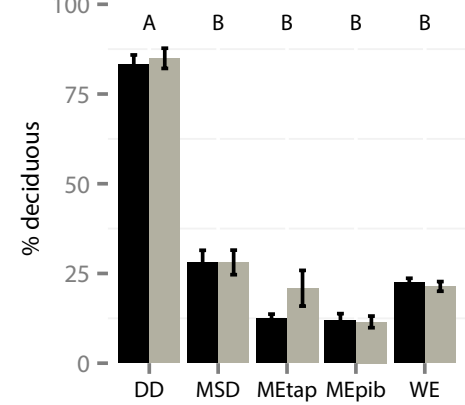

Figure 6.2: Average community-weighted mean (CWM) leaf, stem and whole-plant traits for different sites and two census years (black: first year, grey: last year). The sites are ordered 
according to increasing rainfall; dry deciduous (DD; INPA), moist semi-deciduous (MSD; La Chonta), moist evergreen (MEtap; Tapajós and MEpib; Pibiri), and wet evergreen (WE; Corinto) (Table 6.3). 15 traits were analysed: a) specific leaf area, b) ln-transformed leaf area, c) leaf nitrogen concentration, d) leaf phosphorus concentration $\left(\mathrm{P}_{\text {leaf }}\right)$, e) leaf $\mathrm{N}: \mathrm{P}$ ratio $\left(\mathrm{N}: \mathrm{P}_{\text {leaf }}\right)$, f) leaf chlorophyll content, g) leaf dry matter content, h) specific force to punch (i.e., leaf toughness), i) leaf mass fraction of the metamer, j) wood density, $\mathrm{k})$ maximum diameter $\left.\left(\mathrm{DBH}_{\max }\right), \mathrm{l}\right)$ maximum crown exposure index $\left.\left(\mathrm{CE}_{\text {max }}\right), \mathrm{m}\right)$ the percentage of individuals belonging to the Fabaceae family $(\% \mathrm{Fab}), \mathrm{n})$ the percentage of individuals with compound leaves (\% compound), and o) the percentage of deciduous individuals (\% deciduous) (Table 6.1). Means and standard errors are givens. Capital letters above the bar graphs indicate significant differences between sites, and an asterisk ( $\left({ }^{\prime}\right)$ indicates significant differences between the censuses within a site. For $\mathrm{N}: \mathrm{P}_{\text {leaf }}$, $\mathrm{LMF}_{\mathrm{m}}, \mathrm{DBH}_{\max }, \mathrm{CE}_{\max }$ and \% Fab, an interaction between site and census was found, and hence census was not significant across all sites. Sites were considered significantly different when both census years were significantly different (Appendix 6.9). Note that $\mathrm{DBH}_{\max }$ differed across sites in the ANOVA (Table 6.4), but not in the post-hoc test and this figure. For statistics on effects of site, census, and the interaction between site and census, see Table 6.4 and Appendices 6.3 and 6.4.
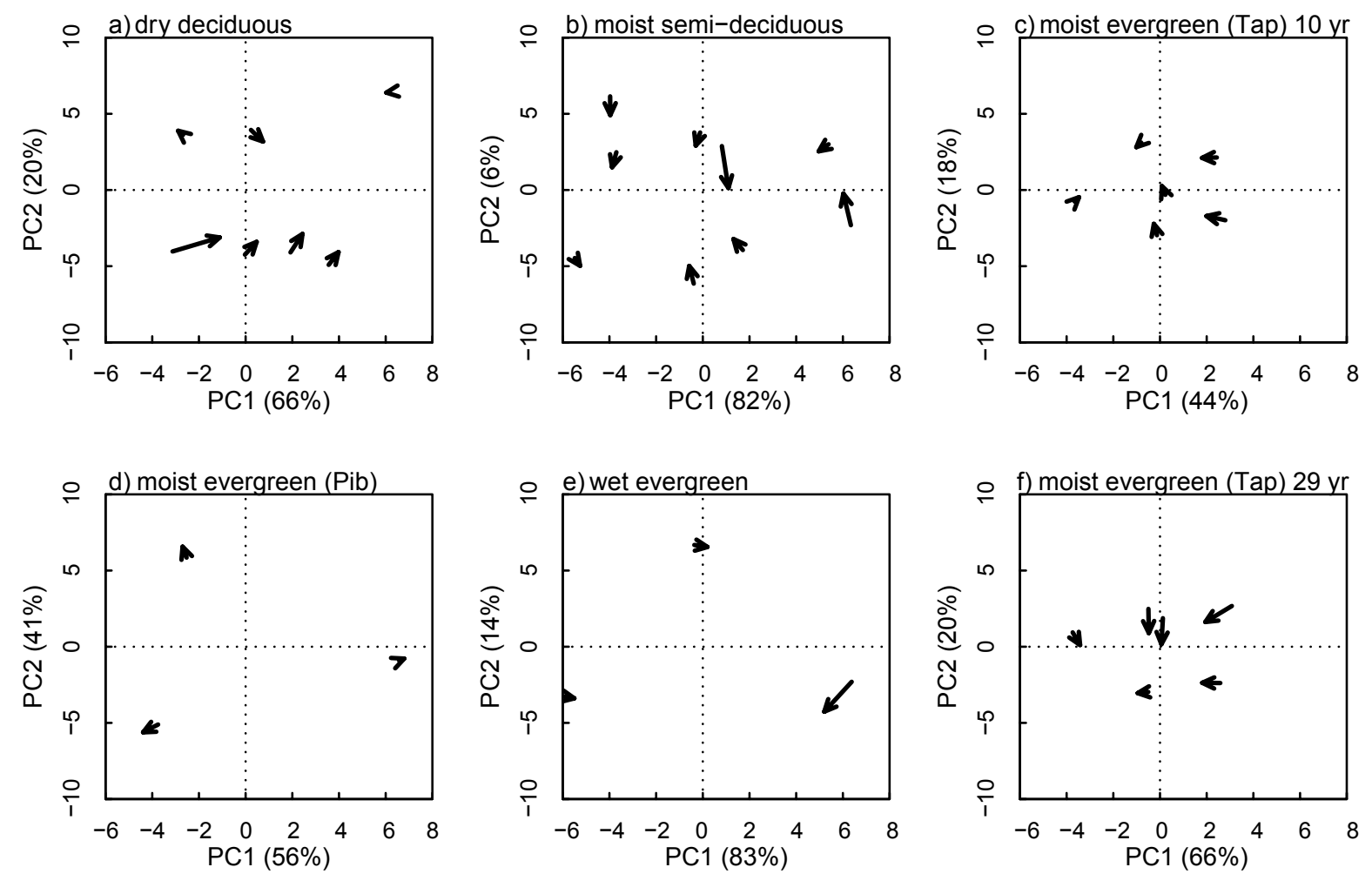

Figure 6.3: The temporal change in species composition over $\sim 10$ years for the five sites: a) dry deciduous, b) moist semi-deciduous, c) and d) moist evergreen (Tapajós and Pibiri), e) wet evergreen (Table 6.2), and f) over 29 years for the moist evergreen forest (Tapajós). The arrows show the unconstrained positioning of plots in the first census (the start of the arrow) and last census (the tip of the arrow) along the first and second principal component axes. Percentages behind the axes is the variation explained by the principal component axes. See Table 6.5 for statistics on temporal changes in species composition. 


\section{Discussion}

We evaluated how old-growth tropical forests vary in their community-weighted mean (CWM) trait composition, and whether their species and trait composition changed over time. Multivariate CWM trait composition and individual CWM traits differed strongly among the five Neotropical sites. Species composition changed over time for the three driest sites, and several CWM key traits changed significantly over time for all sites.

\section{Strong differences in trait composition among Neotropical forests}

The five sites differ strongly in rainfall and soil fertility (Table 6.3, Quesada et al. 2010), and we therefore expected that they would differ strongly in their multivariate trait composition (i.e., the multivariate trait space) and CWM values of individual traits (Fyllas et al. 2009, Patiño et al. 2012). Traits related to drought resistance, such as wood density, should be higher in drier sites, and traits related to nutrient acquisition and use, such as $\mathrm{P}_{\text {leaf }}$ and $\mathrm{N}_{\text {leaf }}$, should be higher in fertile sites. We indeed found differences across sites using a multivariate analysis including the traits collected at all sites (Fig. 6.1b), and for most CWM traits individually (Table 6.4, Fig. 6.2). These site differences suggest that both drought and soil fertility determine CWM trait values.

The drought effect is most evident for the increase in abundance of deciduous individuals with acquisitive leaf trait values (higher SLA, lower LDMC and $\mathrm{FP}_{\mathrm{s}}$ ) and the decrease in leaf area towards drier sites (Fig. 6.2). At drier sites, many species follow a drought-avoiding strategy; by being drought-deciduous, they reduce water loss in the dry season, and by having small leaves, they increase heat exchange and reduce their water requirements for transpirational heat loss (Poorter and Rozendaal 2008). Drought-deciduous species have relatively short-lived leaves, and therefore invest less in structural components (e.g., low LDMC and $\mathrm{FP}_{\mathrm{s}}$ ) that protect the leaves against physical damage, and more in acquisitive trait values (high SLA and $\mathrm{N}_{\text {leaf }}$ ) to attain fast growth rates during the short growing season (Poorter 2009). High $\mathrm{N}_{\text {leaf }}$ may also decrease water loss in dry forests, as a high concentration of photosynthetic enzymes (that are rich in $\mathrm{N}$ ) allows for a larger drawdown of internal $\mathrm{CO}_{2}$ concentration in the leaf, and thus for lower stomatal conductance and water loss (Wright et al. 2001). At the wettest end of the gradient (3900 mm annual rainfall), however, SLA values strongly increase. These acquisitive trait values may be needed for efficient light capture and use in a dense forest where light is limiting tree growth and survival. Alternatively, acquisitive trait values that increase growth rates may be allowed because of the higher soil fertility at this site compared to the two moist evergreen sites. 
Drought and, hence, deciduousness should affect leaf nutrient concentrations to a lesser extent than other leaf traits, as leaf nutrients can be translocated prior to leaf abscission and reused to produce new leaves (Aerts 1996, Zhang et al. 2015). Instead, soil fertility, especially phosphorus, might be a stronger driver of leaf nutrient concentrations; higher phosphorus availability (e.g., in the dry deciduous and moist semi-deciduous site) may increase the uptake of nutrients and the nutrient concentrations in the leaves (Maire et al. 2015), and may increase the abundance of species that can make use of high nutrient availability. The remarkably high $\mathrm{N}_{\text {leaf }}$ in the dry deciduous forest (INPA) is not solely explained by high soil fertility (Table 6.3), but also by high abundance of Fabaceae (Fig. 6.2m). Fabaceae species are very abundant in dry forests (Vargas et al. 2015) and have on average higher leaf nitrogen concentrations $(2.79 \%$ in our dataset) than other families $(2.32 \%$, Appendix 6.11), because of their nitrogen fixing potential. Similarly, the relatively high $\mathrm{N}_{\text {leaf }}$ in the wet evergreen forest (Corinto) may be explained by the high dominance of the Fabaceae Pentaclethra macroloba. The almost four-fold differences among sites in leaf N:P ratio (Fig. 6.2e) show similar ranking among sites as $\mathrm{P}_{\text {leaf }}$. The lowest $\mathrm{N}: \mathrm{P}_{\text {leaf }}$ values are found at the richest site (La Chonta) that contains tracts of anthropogenic enriched 'terra preta' soils with high P values (Quintero-Vallejo 2015). In contrast, the highest $\mathrm{N}: \mathrm{P}_{\text {leaf }}$ values, indicating a relative P shortage, are found for the poorest site (Pibiri, chapter 3) that is located on the very old and highly weathered Guiana shield (Quesada et al. 2011).

The differences between wood traits among sites indicate an effect of both rainfall and soil fertility, since sites with high WD are either low in rainfall and high in soil fertility (INPA) or high in rainfall and relatively low in soil fertility (Tapajós and Pibiri). High WD entails higher cavitation resistance, and hence continued hydraulic functioning during drought in dry forest (Markesteijn et al. 2011b). Moreover, high WD increases pathogen resistance and stem longevity (Romero and Bolker 2008), which enhances nutrient conservation on very nutrient poor soils (e.g., Pibiri) (Baraloto et al. 2011, Gourlet-Fleury et al. 2011).

We cannot fully disentangle the effect of rainfall and soil fertility, as rainfall increases and fertility decreases from South-West to North-East Amazon (Quesada et al. 2010). However, the various CWM traits seem to be affected differently, which allows us to infer the effects of multiple environmental drivers. Using this approach, we find that rainfall most likely shapes CWM values of leaf traits associated with drought avoidance and deciduousness (e.g., SLA, $\mathrm{FP}_{\mathrm{s}}$ ), soil fertility mainly shapes leaf nutrient concentrations, the two drivers combined shape wood density, and none of the two environmental drivers determines adult stature $\left(\mathrm{DBH}_{\max }\right.$ and $\left.\mathrm{CE}_{\max }\right)$. 


\section{Old-growth forests are changing in species and trait composition}

Old-growth forests are exposed to changing environmental conditions, and we therefore expected that their species composition and trait composition would change over time (cf., Enquist and Enquist 2011, Feeley et al. 2011). We indeed found significant changes in species composition over the short term (10 years) for the three driest sites (INPA, La Chonta and Tapajós; Fig. 6.3, Table 6.5), and over the long term (29 years) for the site for which long-term data were available (Tapajós) (Appendix 6.10). The species composition seems to shift towards the centre of Fig. 6.3, which indicates a directional convergence of plots in terms of species composition. We did not find changes in species composition for the two wettest sites (Pibiri and Corinto), possibly because wetter forests are less sensitive to changes in environmental conditions than drier forests, or simply because the number of plots in these sites ( 3 plots per site) was too low to detect significant changes in composition. Despite the changes in species composition for most sites, we did not find significant temporal changes in multivariate trait composition (Fig. 6.1a). Instead, we found significant temporal changes for individual CWM traits (Fig. 6.2, Table 6.4). Apparently, directional changes in species composition are reflected by a limited set of traits, and not by the multivariate set of traits (cf. Butterfield and Suding 2013). Focusing on multivariate strategies alone can therefore conceal important species responses to environmental change.

\section{What drives temporal changes in trait composition?}

We expected that old-growth tropical forests are affected by current changes in resource availability, drought stress, or by (historical) disturbances, and that this would cause temporal changes in CWM trait values (Table 6.2). We found that WD consistently increased and SLA consistently decreased over time across all sites (Fig. 6.2, Table 6.4). Hence, both leaf and stem traits change towards a higher abundance of conservative trait values. But what is driving these changes?

Resource availability - We expected that increased availability of resources, such as $\mathrm{CO}_{2}$ (Appendix 6.4) and rainfall, would result in more acquisitive trait values rather than the more conservative trait values that we observed. Increased resource availability is therefore most likely not driving the changes in our forests. Similarly, we found no changes in nutrient concentrations and Fabaceae abundance over time (except for an increase in $\mathrm{N}: \mathrm{P}_{\text {leaf }}$ in the driest site and a decrease in Fabaceae abundance in Tapajós). Therefore, increased nitrogen deposition (cf. Hietz et al. 2011) is not a likely driver of the changes we observed.

Drought - The increase in conservative trait values could be the result of increased (atmospheric) drought and/or temperature stress (Enquist and Enquist 2011, Feeley et al. 2011). We did not observe a consistent decrease in annual 
rainfall or increase in rainfall seasonality (Table 6.3, Appendix 6.5) or in drought (Appendix 6.6) in our sites, but atmospheric drought stress also depends on changes in factors such as temperature and drought events, and drought events have occurred repeatedly since 1900 (Appendix 6.6). With increasing temperature, we would expect a decrease in leaf area and also a decrease in the abundance of species with compound leaves, as small leaves or leaflets facilitate heat exchange (Poorter and Rozendaal 2008), but we did not find such changes (Fig. 6.2b and n). With an increase in drought, we would expect an increase in the abundance of drought-avoiding deciduous species, which we did not find (Fig. 6.20). Moreover, we would expect a reduction in potential adult stature (indicated by $\mathrm{DBH}_{\max }$ ), as tall species have more exposed crowns and longer hydraulic path lengths, which makes them more prone to hydraulic failure under drier conditions (Phillips et al. 2010, Bennett et al. 2015). We indeed found a tendency for a significant temporal decrease in $\mathrm{DBH}_{\max }$ for the two wettest sites, but a tendency of $\mathrm{DBH}_{\max }$ to increase in the three driest sites. Possibly, wet forests suffer more from drought than dry forests, although the safety margins to cavitation are rather similar for wet and dry forest trees (Choat et al. 2012), and our wettest forests have experienced increasing rather than decreasing rainfall patterns (Table 6.3, Appendix 6.6). Furthermore, the reduction in percentage of Fabaceae trees in moist evergreen forest (Tapajós), on the short term (Fig. 6.2) and long term (Appendix 6.10), indicates no increased drought stress, as Fabaceae species are generally more drought-tolerant and more abundant in dry forests (Adams et al. 2010, Vargas et al. 2015). Alternatively, drought is affecting other aspects that we did not measure, such as rooting depth. Nevertheless, a lack of trend in the \% deciduous, an increase in DBHmax in the driest sites, and no increase in Fabaceae abundance, suggest that compositional changes are not due to increased drought stress. Hence, although we cannot fully exclude an increased drought stress on a longer timescale, it seems not to be the main driver of changes in species and trait composition in our forests.

Disturbances - It is most likely that these forests are undergoing a successional change from early-successional, light-demanding species with high SLA and low WD towards a higher abundance of late-successional, shade-tolerant species with lower SLA and higher WD (Poorter et al. 2006, van Gelder et al. 2006). Most observed trait changes are in line with what we expected when forests recover after disturbances (Table 6.2). The decrease in percentage of Fabaceae individuals in moist evergreen forest (Tapajós) suggests, for example, a successional change towards older forests, which are generally less N limited (Batterman et al. 2013, Sullivan et al. 2014). This decrease in $\mathrm{N}$ limitation is further supported by an increase in the $\mathrm{N}: \mathrm{P}_{\text {leaf }}$ for dry deciduous forest (INPA) (Fig 6.2e). Possibly, the forests are still recovering from past disturbances. After disturbance, forest structure and species richness recover relatively fast (e.g., de Avila et al. 2015, 
Poorter et al. 2016), but many tropical tree species can live for hundreds of years (Chambers et al. 1998), and therefore the recovery of species composition, and hence functional trait composition, can take more than a century for temperate forests (Vellend et al. 2006), and probably even longer for some tropical forests (Chazdon 2003). We found no relation between changes in trait composition and changes in biomass (Appendix 6.12), which suggests that the successional changes in trait composition in our sites do not result in a change in forest structure, possibly because forest structure recovers faster than trait composition (Martin et al. 2013).

Many recent studies show that old-growth Neotropical forests are not pristine, but disturbed by pre-Columbian (Heckenberger et al. 2003, Clement et al. 2015) or more recent human occupation (Redford 1992, van Gemerden et al. 2003). For example, for one of our sites (La Chonta), the presence of terra preta soils suggest that it had been occupied by indigenous people a long time ago (Quintero-Vallejo et al. 2015). Recovering from other disturbances is also possible, such as intense (El Niño) drought events (as opposed to a long-term increase in atmospheric droughtstress), large-scale and intense fires, and wind storms (Nelson et al. 1994), which are all frequently observed across the Amazon (Nelson 2005). Disturbance events lead to canopy tree dieback and more light availability in the understory (Nepstad et al. 2007, Phillips et al. 2010). During initial recovering from such events, we would expect to see an increase in the abundance of light-demanding species with low WD and high SLA (Carreño-Rocabado et al. 2012, Karfakis and Andrade 2013), but during later phases of recovery when light availability reduces, the abundance of shade-tolerant species with high WD and low SLA should increase. Such patterns of successional change could also be observed as an artefact due to small plot size and distribution of plots (Fisher et al. 2008). When natural disturbances (e.g., tree-fall events) are of a similar size as the plots, then the chance is high that these events will not occur during the census period. Instead, it is then more likely to sample plots that were disturbed before the census period, and that are thus undergoing successional change. However, most natural disturbance events occur on small spatial scales (<0.1 ha) (Jans et al. 1993, Espírito-Santo et al. 2014), and we therefore expect that our plots of $0.25-1$ ha well represent the heterogeneity in forest dynamics and structure (Chave et al. 2004), and thus that this possible artefact cannot explain the successional changes in species and trait composition across our forests.

The type of disturbance responsible for the observed changes in species and trait composition should have a relatively low intensity and/or have occurred many decades to centuries ago, as these old-growth forests seem to be in late phases of recovery. Given the consistent changes in composition across the five forests, recovery from disturbance events that occur regularly across the Neotropics, such 
as El Niño droughts, are more likely to explain the observed changes in trait composition than local-scale disturbances that do not occur across the Neotropics, such as wind storms and fire. All sites have experienced frequent drought events since 1900 at different moments in time (Appendix 6.5 and 6.6), and some of these may have caused considerable disturbance to the forest. Hunting pressure could also change the species and trait composition, but this would decrease the dispersal of large seeds and the abundance of large-seeded and late-successional species (Foster and Janson 1985, Galetti et al. 2013), and can thus not explain our results. An alternative explanation for the observed successional patterns is that in the past, anthropogenic disturbances by rural people were more widespread. With a recent migration of rural people to urban areas, this pressure has been released, leading to forest recovery (Wright 2005).

For the longer-term temporal changes (29 years) in a moist evergreen forest (Tapajós), we found an increase in $\mathrm{DBH}_{\text {max }}$ and WD (Appendix 6.10), supporting the successional change that we found across all sites for a shorter time period. Although SLA did not change, we found a decrease in LA, possibly because late successional species have on average small or intermediate-sized leaves (Poorter and Rozendaal 2008). Moreover, the abundance of individuals of Fabaceae decreases over this long-term period, which supports our hypothesis that a gradual increase in drought stress is likely not the main driver of change.

\section{Conclusions}

Even over relatively short timescales (10 and 29 year), we find consistent changes in species and trait composition. The shifts in functional composition across the sites suggest that not only the species and trait composition, but also the ecosystem processes are changing, with lower SLA and higher WD leading to slower carbon sequestration, longer-term carbon storage and "slower" forests (Finegan et al. 2015). A recent analysis of three decades of carbon dynamics in Amazonian forest plots also shows that these forests are slowing down in carbon sequestration (Brienen et al. 2015). The authors suggested that this slowing down of carbon sequestration is caused by higher $\mathrm{CO}_{2}$ concentrations leading to a speeding up of the life cycle of trees, and a faster tree turnover. For our old-growth forests, however, we find that the slowing down of the forest is most likely explained by successional forest recovery from disturbances that occur regularly across the Neotropics (e.g., El Niño droughts). 


\section{Acknowledgements}

The research leading to these results has received partial funding from the European Union Seventh Framework Programme (FP7/2007-2013) under grant agreement n 283093 - The Role Of Biodiversity In climate change mitigatioN (ROBIN). This research is part of the strategic research program KBIV (KB-14) "Sustainable spatial development of ecosystems, landscapes, seas and regions", funded by the Dutch Ministry of Economic Affairs and carried out by Wageningen University \& Research centre (project code KB-14-003-030). We are grateful to personnel from the Guyana Forestry Commission, the Instituto Boliviano de Investigación Forestal (IBIF), and Empresa Brasileira de Pesquisa Agropecuária (EMBRAPA) for logistical support and support of the fieldwork and laboratory work. Furthermore, we thank the Environmental Protection Agency in Guyana for providing the necessary fieldwork permits, the University of Guyana for the use of lab facilities, and the La Chonta Forestry Concession in Bolivia for logistical support. We are grateful to Geovana Carreño-Rocabado, Stijn van Gils, Danaë Rozendaal, and Lars Markesteijn for being able to use their data on leaf traits for several species in Bolivia. The fieldwork would have been impossible without the help of many field assistants. MS was financially sponsored for fieldwork by Stichting Het Kronendak, Alberta Mennega Stichting, and the Treub Maatschappij. 


\section{Appendices}

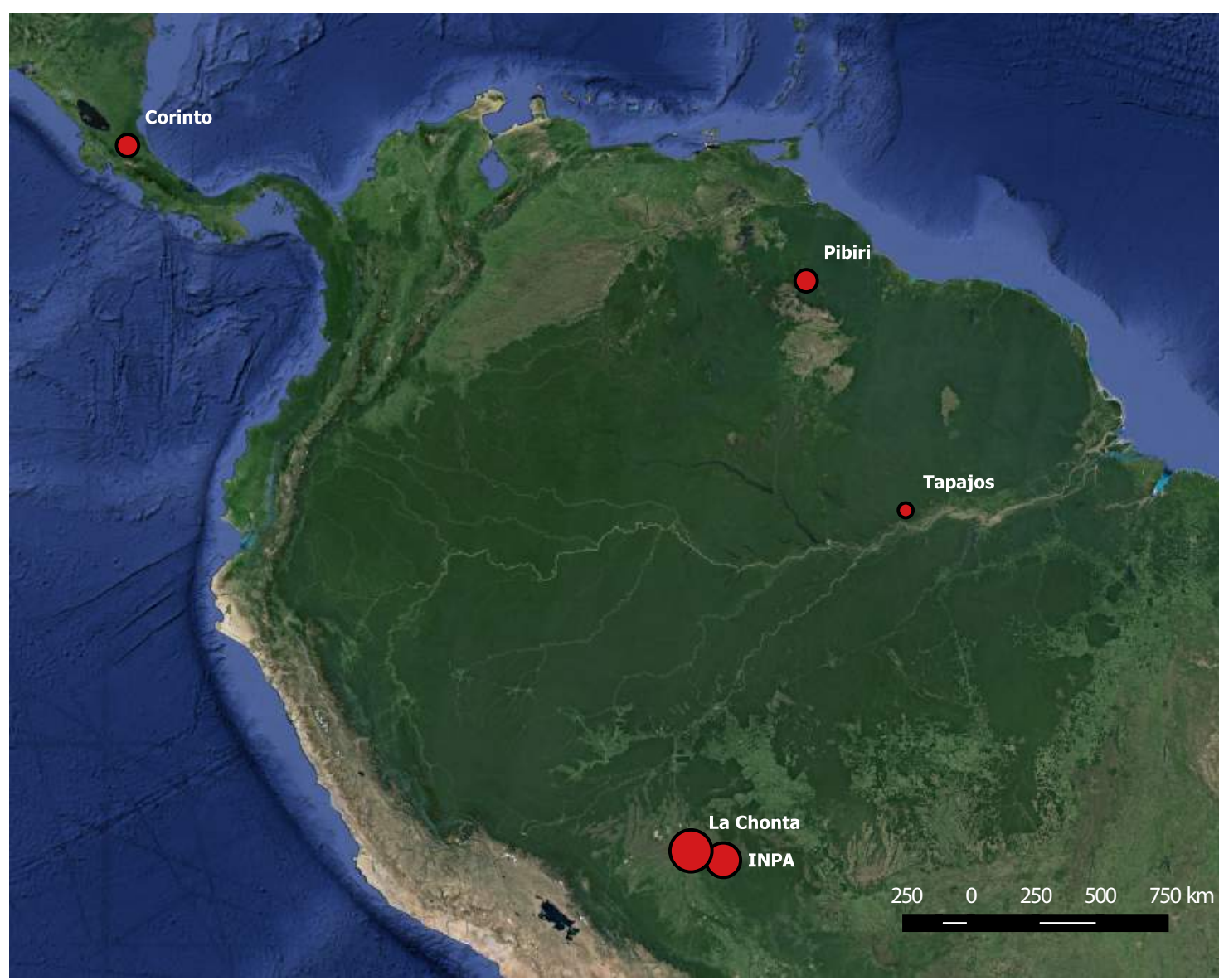

Appendix 6.1: Map showing the location of the five Neotropical forest sites, with a SouthNorth gradient in annual rainfall: INPA (dry deciduous), La Chonta (moist semi-deciduous), Tapajós (moist evergreen), Pibiri (moist evergreen), and Corinto (wet evergreen). The size of the point represents the total plot area used (see Table 6.3).

Appendix 6.2: Calculation of species-specific maximum diameter $\left(\mathrm{DBH}_{\text {max }}\right)$ by using only species with at least 10 individuals.

$\mathrm{DBH}_{\text {max }}$ may be underestimated for species with few individuals, and therefore CWM DBH $\mathrm{Dax}_{\text {max }}$ may be underestimated. We also calculated $\mathrm{CWM} \mathrm{DBH}_{\max }$ by using only the species that had at least 10 individuals in the whole site. Using these values, results were similar (see Appendix 6.2.1), except for higher values of moist semi-deciduous forest (La Chonta) compared to all other forests. The correlation between the two estimates of $\mathrm{CWM} \mathrm{DBH}_{\max }$ is good for dry deciduous (INPA) and moist evergreen (Tapajós and Pibiri) forest, but $\mathrm{CWM} \mathrm{DBH}_{\max }$ based on all species provides much lower values than $\mathrm{CWM} \mathrm{DBH}_{\max }$ based on species with at least 10 individuals for moist semi-deciduous and wet evergreen forest (see Appendix 6.2.2). These sites have few dominant, large-sized species and many rare small-sized species, and when excluding the rare species, the $\mathrm{CWM} \mathrm{DBH}_{\text {max }}$ shifts considerably towards higher $\mathrm{DBH}_{\max }$. 


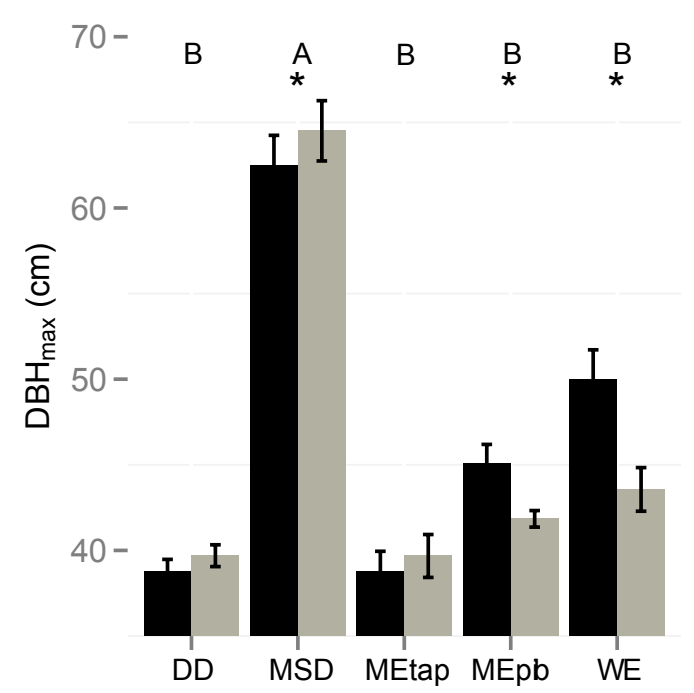

Appendix 6.2.1: Community-weighted mean maximum diameter $\left(\mathrm{DBH}_{\text {max }}\right)$ based on all species that had at least 10 individuals in the whole site, for five sites and two census years (black bar = first census, grey bar $=$ second census). The sites are ordered according to increasing rainfall; dry deciduous (DD; INPA), moist semi-deciduous (MSD; La Chonta), moist evergreen (MEtap; Tapajós and MEpib; Pibiri), and wet evergreen (WE; Corinto) (Table 6.3). Letters above the bars indicate significant differences between sites, and asterisks above the bars indicate significant differences between the two censuses.

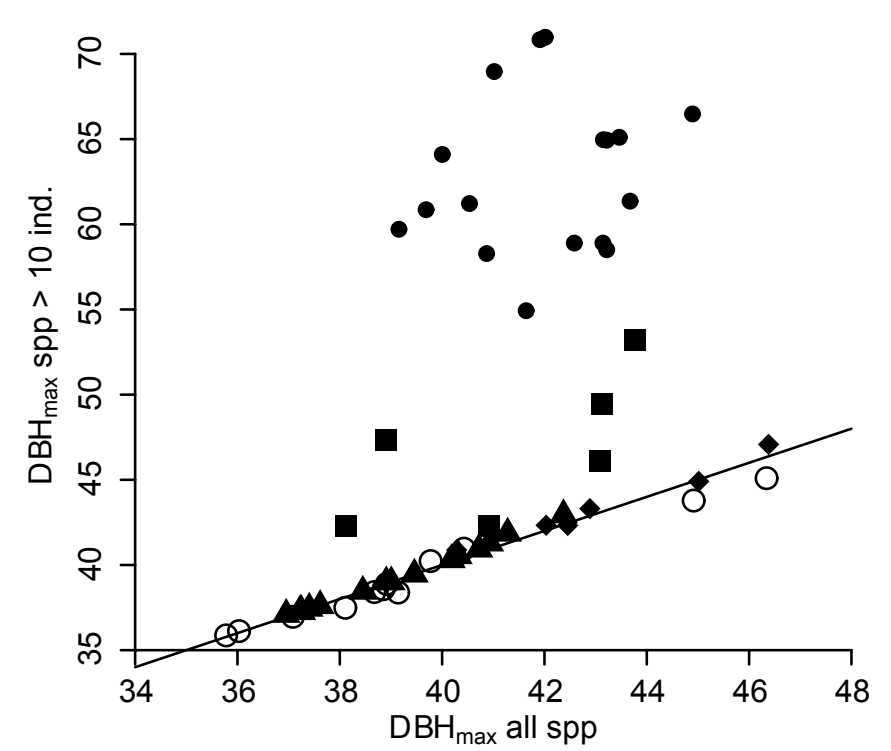

Appendix 6.2.2: Relation between community-weighted mean maximum diameter $\left(\mathrm{DBH}_{\max }\right)$ values estimated in two ways: based on an estimate of $\mathrm{DBH}_{\max }$ for all species (x-axis) and based on an estimate for species that have at least 10 individuals in the whole site (y-axis). Symbols indicate sites; triangles: dry deciduous (DD; INPA), closed circles: moist semi-deciduous (MSD; La Chonta), open circles: moist evergreen (MEtap; Tapajós), diamonds: moist evergreen (MEpib; Pibiri), and squares: wet evergreen (WE; Corinto). 
a)

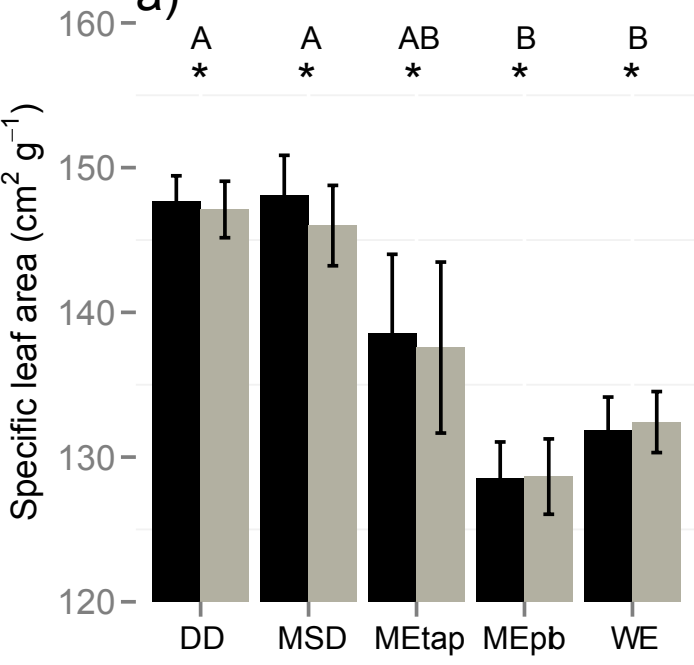

b)

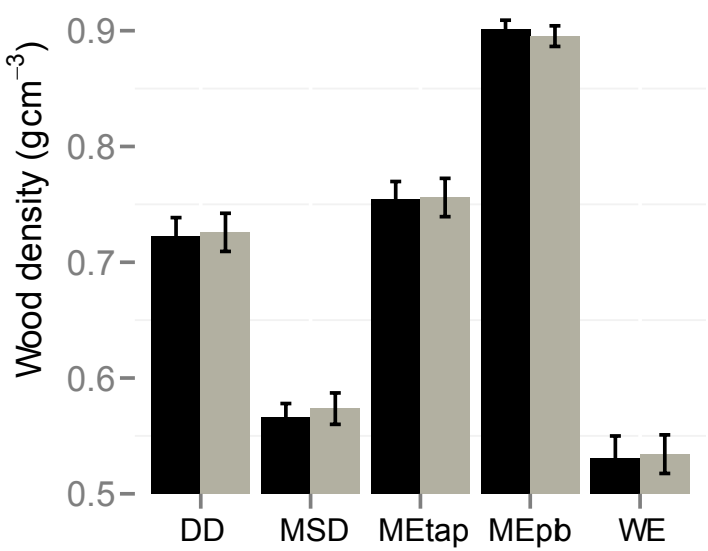

Appendix 6.3: Average basal area-weighted mean specific leaf area (a) and wood density (b) for different sites and two census years (black: first year, grey: last year). The sites are ordered according to increasing rainfall; dry deciduous (DD; INPA), moist semi-deciduous (MSD; La Chonta), moist evergreen (MEtap; Tapajós and MEpib; Pibiri), and wet evergreen (WE; Corinto) (Table 6.3). Means and standard errors are givens. Capital letters above the bar graphs indicate significant differences between sites, and an asterisk ${ }^{(*)}$ indicates significant differences between the censuses within a site.

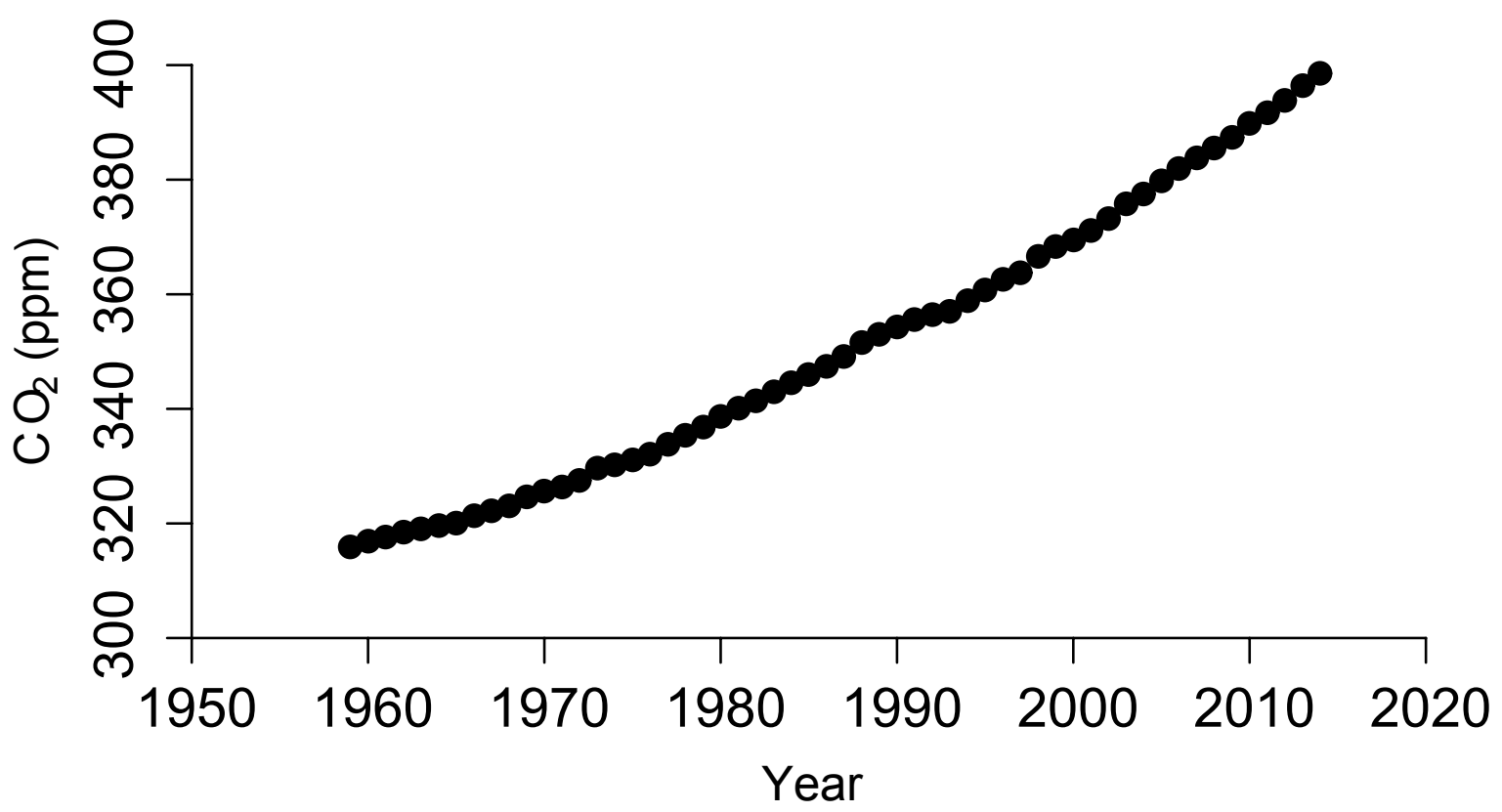

Appendix 6.4: Temporal changes in annual atmospheric $\mathrm{CO}_{2}$ concentration between 1958 and 2013. Data were obtained from the Earth System Research Laboratory (http://www.esrl.noaa.gov/gmd/ccgg/trends/\#mlo_full) and measured in Mauna Loa, Hawaii. 


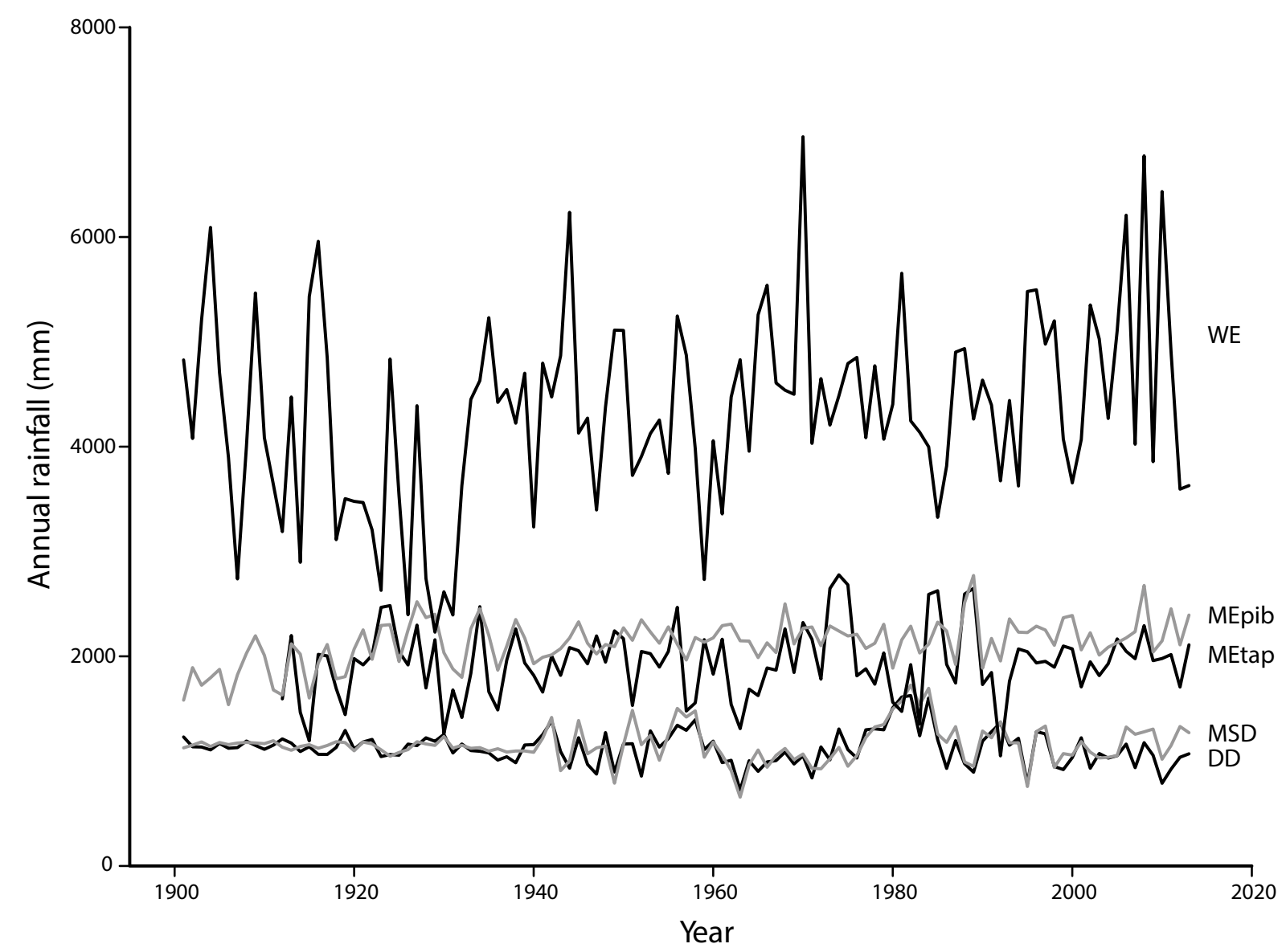

Appendix 6.5: Temporal changes in annual rainfall between 1900 and 2015 for the five sites. Lower black line: dry deciduous (DD; INPA); lower gray line: moist semi-deciduous (MSD; La Chonta); middle black line: moist evergreen (MEtap; Tapajós); upper gray line: moist evergreen (MEpib; Pibiri); and upper black line: wet evergreen (WE; Corinto) (Table 6.3). Data were obtained from Climate Explorer (http://climexp.knmi.nl/start.cgi?id=someone@somewhere) and are interpolated data based on the site coordinates. Note that the interpolated values do not match the locally measured values. For example, the difference in rainfall between dry deciduous (INPA) and moist semi-deciduous (La Chonta) forests is small because the spatial interpolation results in very similar values. Nevertheless, the data represent well the site ranking in average annual rainfall. Annual rainfall of the two wettest sites (black and green lines) increased significantly over time $(\mathrm{P}=0.018$ for the MEpib and $\mathrm{P}<0.001$ for the WE forest), whereas annual rainfall of the three driest sites did not change over time. 


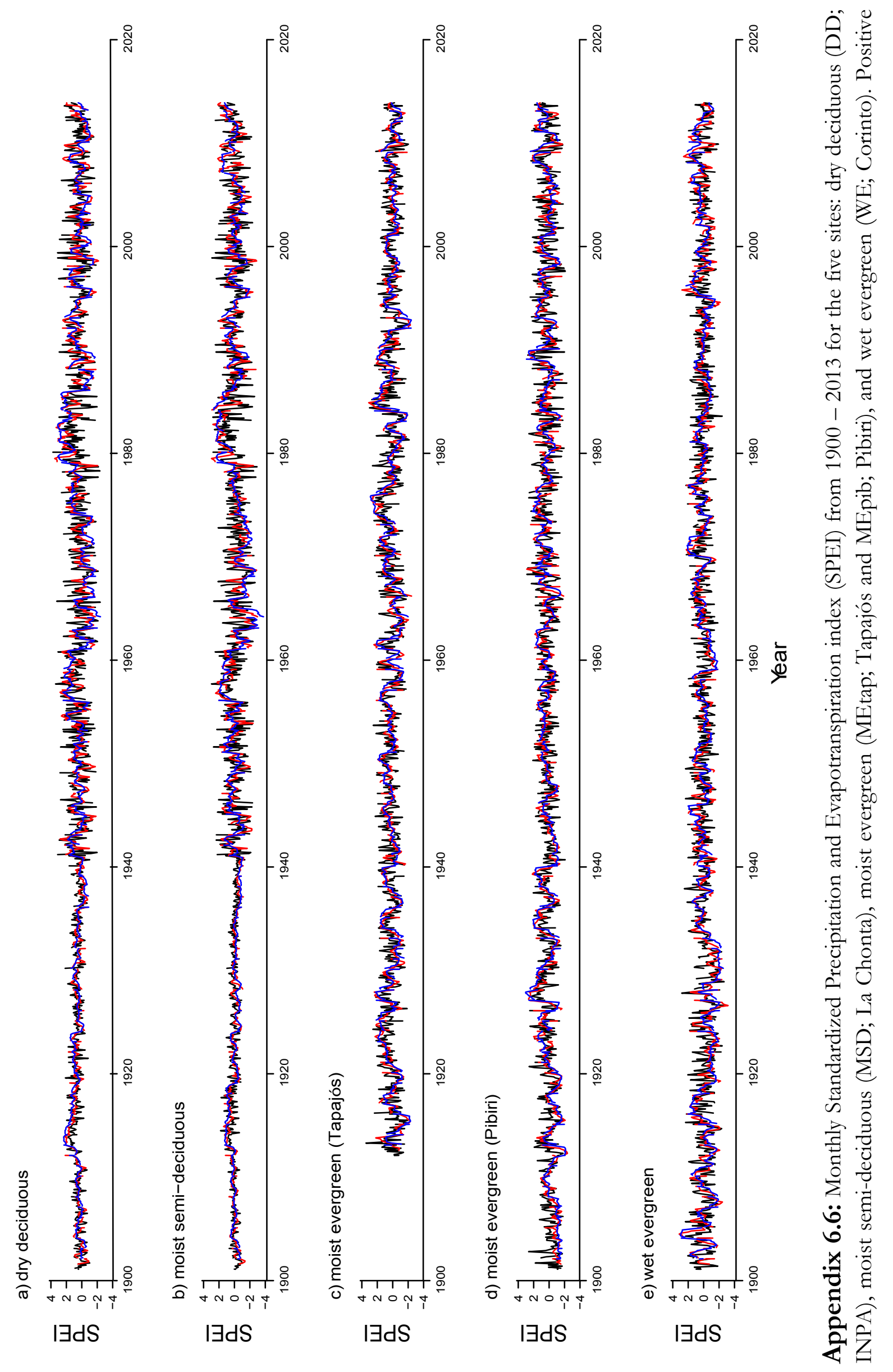


.

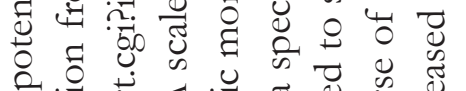

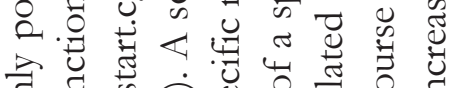

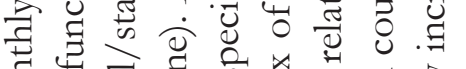

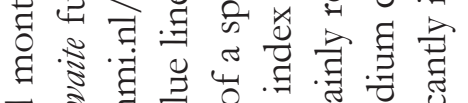

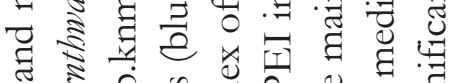
光

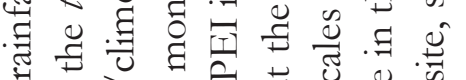

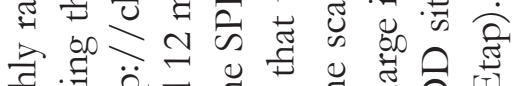

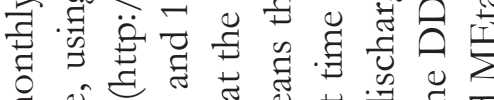

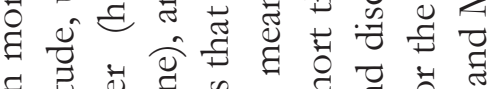

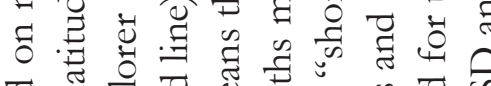

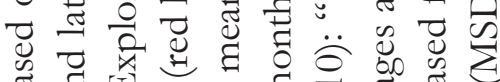

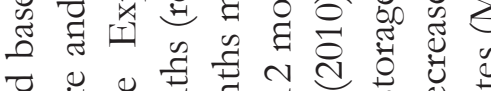

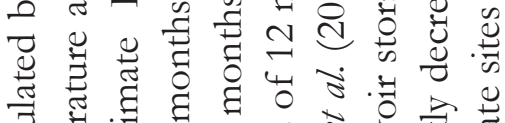

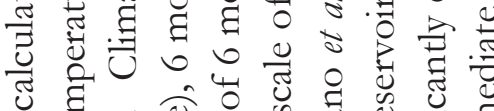

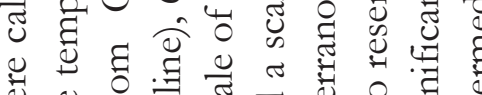

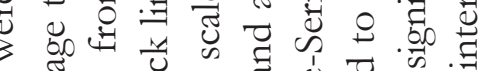

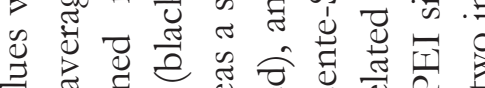

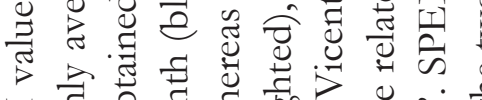

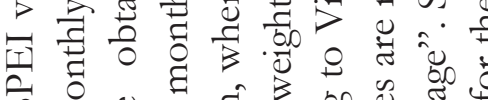

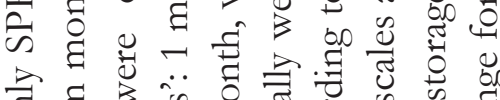

司范药

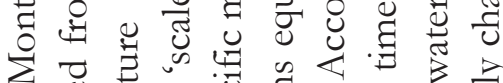

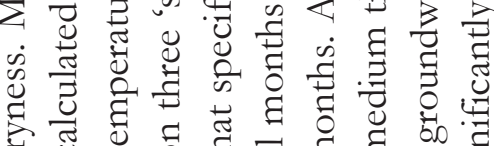

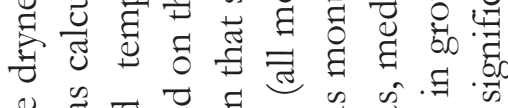

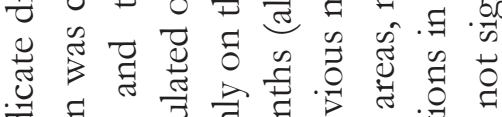

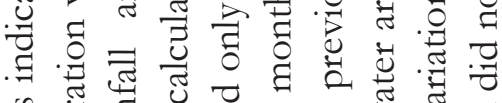

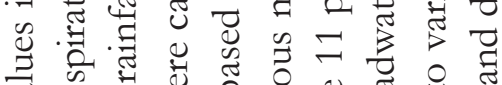

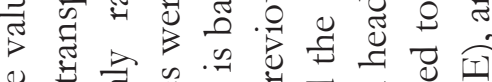

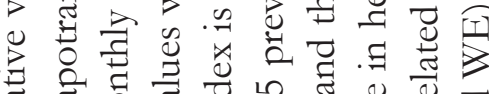

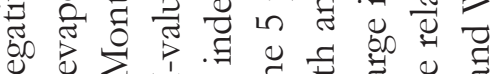

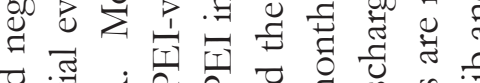

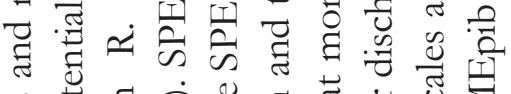

क

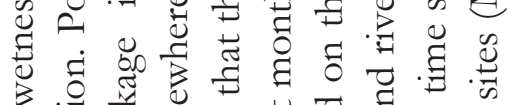

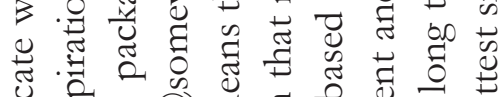

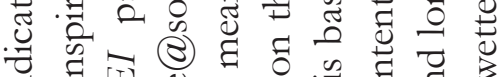

.

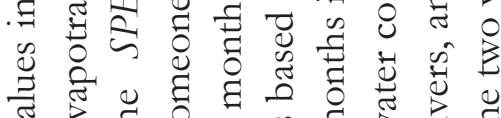

$\because \bar{c}$

$\stackrel{\mathscr{J}}{.}$

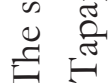

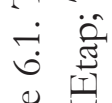

तै

. :

ఫ્త

. 苗

ती

苞

도

I

¿

를

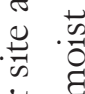

ปे

岂 学

.

$\exists=$

苛

总 总

.00

둥

昰密焉

हृ

$\infty$ 屯.

苞. 䒕

$>0$

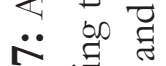

ช்

雚

पे 
Appendix 6.8: Multiple comparisons using Tukey's post-hoc test for all community-weighted mean (CWM) traits that differed between sites but showed no interaction between site and census year (Table 6.5). The two columns with 'Site' show the two sites that are compared (DD: dry deciduous, INPA; MSD: moist semi-deciduous, La Chonta; MEtap: moist evergreen, Tapajós; MEpib: moist evergreen, Pibiri; WE: wet evergreen, Corinto). For each comparison, the estimate, standard error, Z-value ( $\mathrm{Z})$ and $\mathrm{P}$-value $(\mathrm{P})$ are given. LA was $\ln$-transformed.

\begin{tabular}{|c|c|c|c|c|c|c|}
\hline CWM trait & Site & Site & Est. & SE & $\mathrm{Z}$ & $\mathbf{P}$ \\
\hline \multirow[t]{10}{*}{ SLA } & $\mathrm{DD}$ & WE & 6.33 & 2.14 & 2.96 & 0.025 \\
\hline & MSD & WE & -1.57 & 2.12 & -0.74 & 0.945 \\
\hline & MEpib & WE & -23.61 & 2.47 & -9.55 & $<0.001$ \\
\hline & MEtap & WE & -13.67 & 2.21 & -6.19 & $<0.001$ \\
\hline & MSD & DD & -7.91 & 1.71 & -4.61 & $<0.001$ \\
\hline & MEpib & DD & -29.95 & 2.14 & -14.00 & $<0.001$ \\
\hline & MEtap & DD & -20.00 & 1.83 & -10.94 & $<0.001$ \\
\hline & MEpib & MSD & -22.04 & 2.12 & -10.42 & $<0.001$ \\
\hline & MEtap & MSD & -12.10 & 1.80 & -6.72 & $<0.001$ \\
\hline & MEtap & MEpib & 9.94 & 2.21 & 4.50 & $<0.001$ \\
\hline \multirow[t]{10}{*}{ LA } & $\mathrm{DD}$ & WE & -2.22 & 0.13 & -16.95 & $<0.001$ \\
\hline & MSD & WE & -1.62 & 0.13 & -12.57 & $<0.001$ \\
\hline & MEpib & WE & -1.35 & 0.15 & -8.74 & $<0.001$ \\
\hline & MEtap & WE & -1.04 & 0.14 & -7.68 & $<0.001$ \\
\hline & MSD & DD & 0.60 & 0.10 & 6.00 & $<0.001$ \\
\hline & MEpib & DD & 0.87 & 0.13 & 6.61 & $<0.001$ \\
\hline & MEtap & DD & 1.17 & 0.11 & 10.88 & $<0.001$ \\
\hline & MEpib & MSD & 0.27 & 0.13 & 2.09 & 0.218 \\
\hline & MEtap & MSD & 0.58 & 0.11 & 5.47 & $<0.001$ \\
\hline & MEtap & MEpib & 0.31 & 0.14 & 2.27 & 0.150 \\
\hline \multirow[t]{10}{*}{$\mathrm{N}_{\text {leaf }}$} & DD & WE & 1.06 & 0.08 & 12.51 & 0.001 \\
\hline & MSD & WE & -0.53 & 0.08 & -6.30 & 0.001 \\
\hline & MEpib & WE & -0.85 & 0.10 & -8.45 & 0.001 \\
\hline & MEtap & WE & -0.36 & 0.09 & -4.09 & 0.001 \\
\hline & MSD & DD & -1.59 & 0.06 & -24.89 & 0.001 \\
\hline & MEpib & DD & -1.91 & 0.08 & -22.53 & 0.001 \\
\hline & MEtap & DD & -1.42 & 0.07 & -20.41 & 0.001 \\
\hline & MEpib & MSD & -0.32 & 0.08 & -3.86 & 0.001 \\
\hline & MEtap & MSD & 0.17 & 0.07 & 2.44 & 0.103 \\
\hline & MEtap & MEpib & 0.49 & 0.09 & 5.55 & 0.001 \\
\hline \multirow[t]{10}{*}{$P_{\text {leaf }}$} & $\mathrm{DD}$ & WE & 0.06 & 0.00 & 12.77 & $<0.001$ \\
\hline & MSD & WE & 0.08 & 0.00 & 16.24 & $<0.001$ \\
\hline & MEpib & WE & -0.06 & 0.01 & -10.84 & $<0.001$ \\
\hline & MEtap & WE & 0.00 & 0.01 & -0.81 & 0.925 \\
\hline & MSD & DD & 0.02 & 0.00 & 4.13 & $<0.001$ \\
\hline & MEpib & DD & -0.12 & 0.00 & -25.35 & $<0.001$ \\
\hline & MEtap & DD & -0.07 & 0.00 & -16.03 & $<0.001$ \\
\hline & MEpib & MSD & -0.14 & 0.00 & -28.97 & $<0.001$ \\
\hline & MEtap & MSD & -0.08 & 0.00 & -20.21 & $<0.001$ \\
\hline & MEtap & MEpib & 0.06 & 0.01 & 11.35 & $<0.001$ \\
\hline \multirow[t]{3}{*}{ Chl } & MSD & DD & 11.41 & 1.22 & 9.36 & $<0.001$ \\
\hline & MEpib & DD & 19.53 & 1.62 & 12.03 & $<0.001$ \\
\hline & MEtap & $\mathrm{DD}$ & 28.42 & 1.33 & 21.33 & $<0.001$ \\
\hline
\end{tabular}




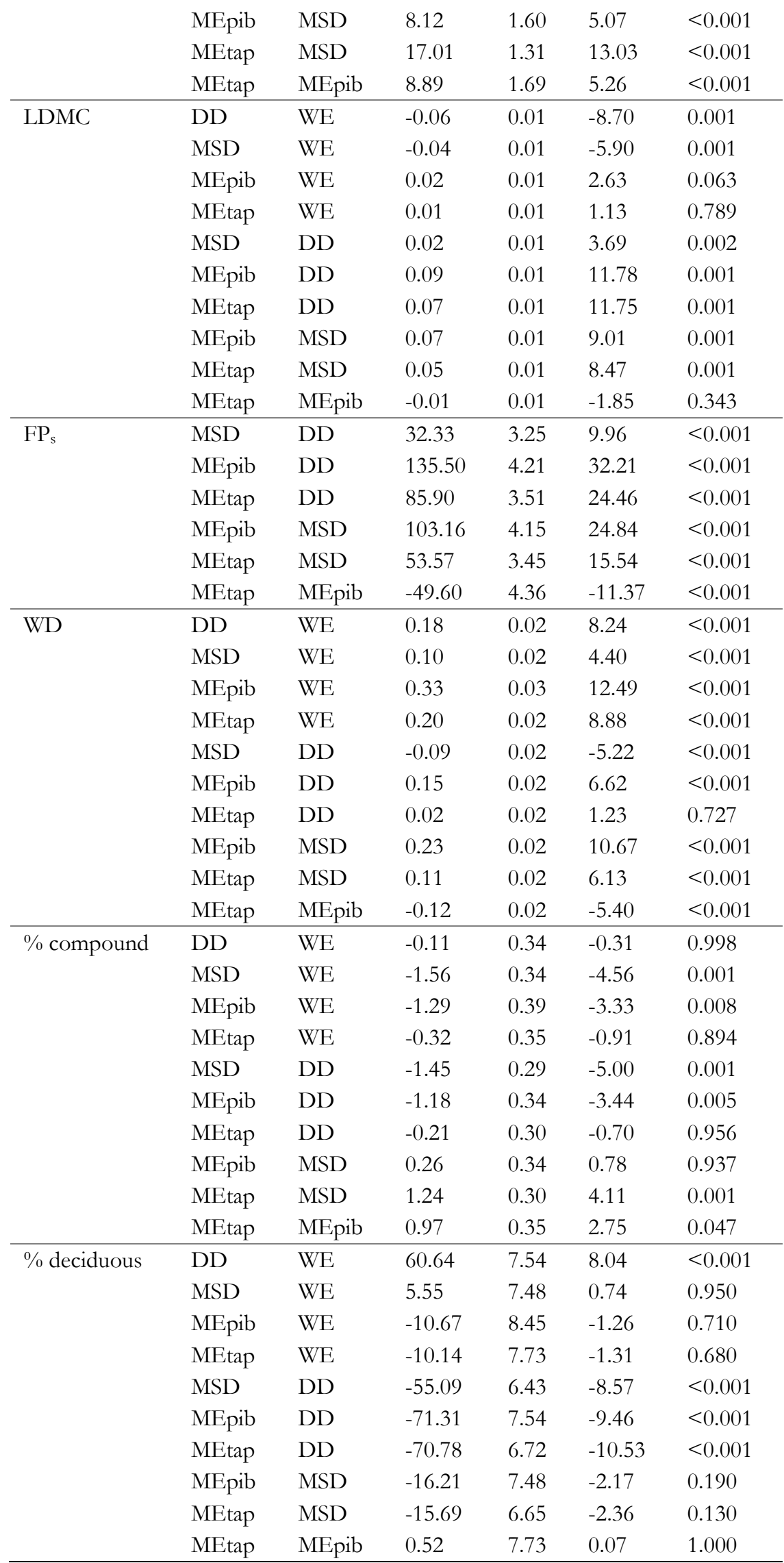


Appendix 6.9: Multiple comparisons using Tukey's post-hoc test for all community-weighted mean (CWM) traits that showed an interaction between site and census year (Table 6.5). The two columns with 'Site.Year' show which site in which census is being compared (DD: dry deciduous, INPA; MSD: moist semi-deciduous, La Chonta; MEtap: moist evergreen, Tapajós; MEpib: moist evergreen, Pibiri; WE: wet evergreen, Corinto; 1: first census; 2: last census). For each comparison, the estimate, standard error, $\mathrm{Z}$-value $(\mathrm{Z})$ and $\mathrm{P}$-value $(\mathrm{P})$ are given.

\begin{tabular}{|c|c|c|c|c|c|c|}
\hline CWM Trait & Site.Year & Site.Year & Est. & SE & $\mathrm{Z}$ & $\mathbf{P}$ \\
\hline \multirow[t]{25}{*}{$\mathrm{N}: \mathrm{P}_{\text {Leaf }}$} & DD.1 & WE.1 & -2.63 & 0.61 & -4.33 & 0.001 \\
\hline & MSD.1 & WE.1 & -13.03 & 0.60 & -21.74 & 0.001 \\
\hline & MEpib.1 & WE.1 & 13.42 & 0.72 & 18.71 & 0.001 \\
\hline & MEtap.1 & WE.1 & -2.60 & 0.63 & -4.12 & 0.001 \\
\hline & WE.2 & WE.1 & -0.09 & 0.16 & -0.54 & 1.000 \\
\hline & MSD.1 & DD.1 & -10.40 & 0.46 & -22.53 & 0.001 \\
\hline & MEpib.1 & DD.1 & 16.05 & 0.61 & 26.43 & 0.001 \\
\hline & MEtap.1 & DD.1 & 0.03 & 0.50 & 0.06 & 1.000 \\
\hline & DD.2 & DD.1 & 0.40 & 0.10 & 4.05 & 0.001 \\
\hline & MEpib.1 & MSD.1 & 26.45 & 0.60 & 44.14 & 0.001 \\
\hline & MEtap.1 & MSD.1 & 10.43 & 0.49 & 21.19 & 0.001 \\
\hline & MSD.2 & MSD.1 & -0.08 & 0.09 & -0.84 & 0.995 \\
\hline & MEtap.1 & MEPIB.1 & -16.02 & 0.63 & -25.40 & 0.001 \\
\hline & MEpib.2 & MEpib.1 & -0.06 & 0.16 & -0.38 & 1.000 \\
\hline & MEtap.2 & MEtap.1 & 0.14 & 0.11 & 1.22 & 0.943 \\
\hline & DD.2 & WE.2 & -2.15 & 0.61 & -3.53 & 0.008 \\
\hline & MSD.2 & WE.2 & -13.02 & 0.60 & -21.73 & 0.001 \\
\hline & MEpib.2 & WE.2 & 13.45 & 0.72 & 18.74 & 0.001 \\
\hline & MEtap.2 & WE.2 & -2.37 & 0.63 & -3.76 & 0.003 \\
\hline & MSD.2 & DD.2 & -10.87 & 0.46 & -23.56 & 0.001 \\
\hline & MEpib.2 & DD.2 & 15.59 & 0.61 & 25.68 & 0.001 \\
\hline & MEtap.2 & DD.2 & -0.23 & 0.50 & -0.46 & 1.000 \\
\hline & MEpib.2 & MSD.2 & 26.47 & 0.60 & 44.17 & 0.001 \\
\hline & MEtap.2 & MSD.2 & 10.65 & 0.49 & 21.63 & 0.001 \\
\hline & MEtap.2 & MEpib.2 & -15.82 & 0.63 & -25.08 & 0.001 \\
\hline \multirow[t]{9}{*}{$\mathrm{LMF}_{\mathrm{m}}$} & DD.2 & DD.1 & 0.00 & 0.00 & 2.30 & 0.129 \\
\hline & MSD.1 & DD.1 & 0.02 & 0.00 & 3.77 & 0.001 \\
\hline & MEpib.1 & DD.1 & -0.01 & 0.01 & -1.08 & 0.836 \\
\hline & MSD.2 & DD.2 & 0.02 & 0.00 & 3.91 & 0.001 \\
\hline & MEpib.2 & DD.2 & -0.01 & 0.01 & -1.74 & 0.400 \\
\hline & MSD.2 & MSD.1 & 0.00 & 0.00 & 3.47 & 0.004 \\
\hline & MEpib.1 & MSD.1 & -0.02 & 0.01 & -3.91 & 0.001 \\
\hline & MEpib.2 & MSD.2 & -0.03 & 0.01 & -4.68 & $<0.001$ \\
\hline & MEpib.2 & MEpib.1 & 0.00 & 0.00 & -2.26 & 0.143 \\
\hline \multirow[t]{6}{*}{$\mathrm{DBH}_{\max }$} & DD.1 & WE.1 & -3.30 & 1.68 & -1.97 & 0.515 \\
\hline & MSD.1 & WE.1 & -0.40 & 1.66 & -0.24 & 1.000 \\
\hline & MEpib.1 & WE.1 & 2.83 & 1.95 & 1.45 & 0.858 \\
\hline & MEtap.1 & WE.1 & -2.88 & 1.74 & -1.66 & 0.738 \\
\hline & WE.2 & WE.1 & -1.23 & 0.61 & -2.01 & 0.481 \\
\hline & MSD.1 & DD.1 & 2.90 & 1.34 & 2.17 & 0.370 \\
\hline
\end{tabular}




\begin{tabular}{|c|c|c|c|c|c|c|}
\hline & MEpib.1 & DD.1 & 6.13 & 1.68 & 3.65 & 0.006 \\
\hline & MEtap.1 & DD.1 & 0.42 & 1.43 & 0.30 & 1.000 \\
\hline & DD. 2 & DD.1 & 0.90 & 0.37 & 2.42 & 0.231 \\
\hline & MEpib.1 & MSD.1 & 3.23 & 1.66 & 1.95 & 0.531 \\
\hline & MEtap.1 & MSD.1 & -2.48 & 1.41 & -1.76 & 0.665 \\
\hline & MSD.2 & MSD.1 & 0.99 & 0.35 & 2.82 & 0.086 \\
\hline & MEtap.1 & MEpib.1 & -5.71 & 1.74 & -3.29 & 0.021 \\
\hline & MEpib.2 & MEpib.1 & -3.17 & 0.61 & -5.20 & 0.001 \\
\hline & MEtap. 2 & MEtap.1 & 0.90 & 0.43 & 2.08 & 0.433 \\
\hline & DD. 2 & WE.2 & -1.17 & 1.68 & -0.70 & 0.999 \\
\hline & MSD.2 & WE.2 & 1.82 & 1.66 & 1.10 & 0.973 \\
\hline & MEpib.2 & WE.2 & 0.88 & 1.95 & 0.45 & 1.000 \\
\hline & MEtap.2 & WE.2 & -0.75 & 1.74 & -0.43 & 1.000 \\
\hline & MSD.2 & DD.2 & 2.99 & 1.34 & 2.24 & 0.327 \\
\hline & MEpib.2 & DD. 2 & 2.06 & 1.68 & 1.23 & 0.945 \\
\hline & MEtap. 2 & DD.2 & 0.42 & 1.43 & 0.29 & 1.000 \\
\hline & MEpib.2 & MSD.2 & -0.94 & 1.66 & -0.56 & 1.000 \\
\hline & MEtap. 2 & MSD.2 & -2.57 & 1.41 & -1.83 & 0.616 \\
\hline & MEtap.2 & MEpib.2 & -1.64 & 1.74 & -0.94 & 0.990 \\
\hline & MEpib.2 & MEpib.1 & -0.38 & 0.04 & -9.19 & 0.001 \\
\hline & MEtap.1 & MEpib.1 & 0.32 & 0.09 & 3.70 & 0.004 \\
\hline & MEtap.2 & MEpib.2 & 0.70 & 0.09 & 8.07 & 0.001 \\
\hline & MEtap.2 & MEtap.1 & 0.00 & 0.03 & -0.07 & 1.000 \\
\hline $\mathrm{CE}_{\max }$ & MSD.1 & DD.1 & -0.16 & 0.08 & -1.98 & 0.298 \\
\hline & MEpib.1 & DD.1 & 0.14 & 0.09 & 1.51 & 0.598 \\
\hline & DD. 2 & DD.1 & 0.04 & 0.03 & 1.50 & 0.606 \\
\hline & MEpib.1 & MSD.1 & 0.30 & 0.09 & 3.20 & 0.013 \\
\hline & MSD.2 & MSD.1 & 0.09 & 0.03 & 3.47 & 0.005 \\
\hline & MEpib.2 & MEpib.1 & -0.38 & 0.05 & -8.08 & $<0.001$ \\
\hline & MSD.2 & DD. 2 & -0.11 & 0.08 & -1.34 & 0.714 \\
\hline & MEpib.2 & DD. 2 & -0.28 & 0.09 & -2.94 & 0.029 \\
\hline & MEpib.2 & MSD.2 & -0.17 & 0.09 & -1.83 & 0.381 \\
\hline \% Fab & DD.1 & WE.1 & 33.01 & 6.46 & 5.11 & 0.001 \\
\hline & MSD.1 & WE.1 & -26.63 & 6.38 & -4.18 & 0.001 \\
\hline & MEpib.1 & WE.1 & -16.87 & 7.64 & -2.21 & 0.329 \\
\hline & MEtap.1 & WE.1 & -1.94 & 6.72 & -0.29 & 1.000 \\
\hline & WE.2 & WE.1 & -1.41 & 1.68 & -0.84 & 0.995 \\
\hline & MSD.1 & DD.1 & -59.64 & 4.90 & -12.17 & 0.001 \\
\hline & MEpib.1 & DD.1 & -49.87 & 6.46 & -7.72 & 0.001 \\
\hline & MEtap.1 & DD.1 & -34.95 & 5.33 & -6.55 & 0.001 \\
\hline & DD.2 & DD.1 & 3.00 & 1.03 & 2.92 & 0.059 \\
\hline & MEpib.1 & MSD.1 & 9.76 & 6.38 & 1.53 & 0.803 \\
\hline & MEtap.1 & MSD.1 & 24.69 & 5.23 & 4.72 & 0.001 \\
\hline & MSD.2 & MSD.1 & 0.33 & 0.97 & 0.34 & 1.000 \\
\hline & MEtap.1 & MEpib.1 & 14.93 & 6.72 & 2.22 & 0.319 \\
\hline & MEpib.2 & MEpib.1 & 1.94 & 1.68 & 1.16 & 0.957 \\
\hline & MEtap. 2 & MEtap.1 & -4.93 & 1.18 & -4.16 & 0.001 \\
\hline & DD.2 & WE.2 & 37.41 & 6.46 & 5.79 & 0.001 \\
\hline
\end{tabular}




\begin{tabular}{llllll} 
MSD.2 & WE.2 & -24.89 & 6.38 & -3.90 & 0.002 \\
MEpib.2 & WE.2 & -13.52 & 7.64 & -1.77 & 0.642 \\
MEtap.2 & WE.2 & -5.46 & 6.72 & -0.81 & 0.996 \\
MSD.2 & DD.2 & -62.30 & 4.90 & -12.71 & 0.001 \\
MEpib.2 & DD.2 & -50.93 & 6.46 & -7.88 & 0.001 \\
MEtap.2 & DD.2 & -42.87 & 5.33 & -8.04 & 0.001 \\
MEpib.2 & MSD.2 & 11.37 & 6.38 & 1.78 & 0.631 \\
MEtap.2 & MSD.2 & 19.43 & 5.23 & 3.72 & 0.004 \\
MEtap.2 & MEpib.2 & 8.06 & 6.72 & 1.20 & 0.947 \\
\hline
\end{tabular}

Appendix 6.10: Averages of all community-weighted mean (CWM) trait values for a moist evergreen forest (Tapajós) in 1983 and 2012, and the P-value of the difference in CWM trait values between these census years (P). For description of traits, see Table 6.1. LA was lntransformed.

\begin{tabular}{llll}
\hline Trait & $\mathbf{1 9 8 3}$ & $\mathbf{2 0 1 2}$ & P of difference \\
\hline $\mathrm{SLA}$ & 134.24 & 134.19 & 0.928 \\
$\mathrm{LA}$ & 5.08 & 5.02 & 0.010 \\
$\mathrm{~N}_{\text {leaf }}$ & 2.23 & 2.22 & 0.625 \\
$\mathrm{P}_{\text {leaf }}$ & 0.103 & 0.102 & 0.247 \\
$\mathrm{~N}_{\text {leaf }}$ & 21.59 & 21.67 & 0.766 \\
$\mathrm{Chl}$ & 74.38 & 74.04 & 0.193 \\
$\mathrm{LDMC}$ & 0.404 & 0.404 & 0.799 \\
$\mathrm{FP}_{\mathrm{s}}$ & 208.91 & 210.35 & 0.151 \\
$\mathrm{WD}_{\mathrm{WDMC}}$ & 0.731 & 0.742 & 0.039 \\
$\mathrm{DBH}$ & 0.622 & 0.625 & 0.160 \\
\% Fab & 38.07 & 39.95 & 0.001 \\
\% compound & 33.96 & 24.46 & 0.002 \\
\hline
\end{tabular}




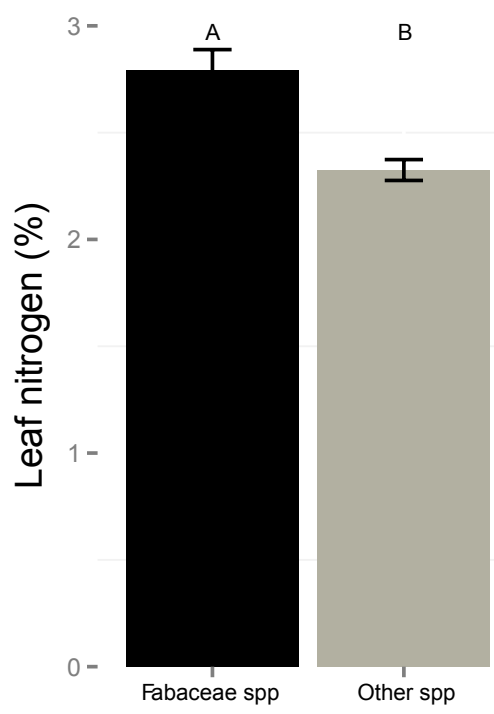

Appendix 6.11: Leaf nitrogen concentration for species from the Fabaceae family (Fabaceae spp) versus species from non-Fabaceae families (Other spp). Average and standard error are shown. $\mathrm{t}=4.25, \mathrm{P}<0.001, \mathrm{~N}=274$ species, of which 52 from the Fabaceae family. No interaction with site was found.
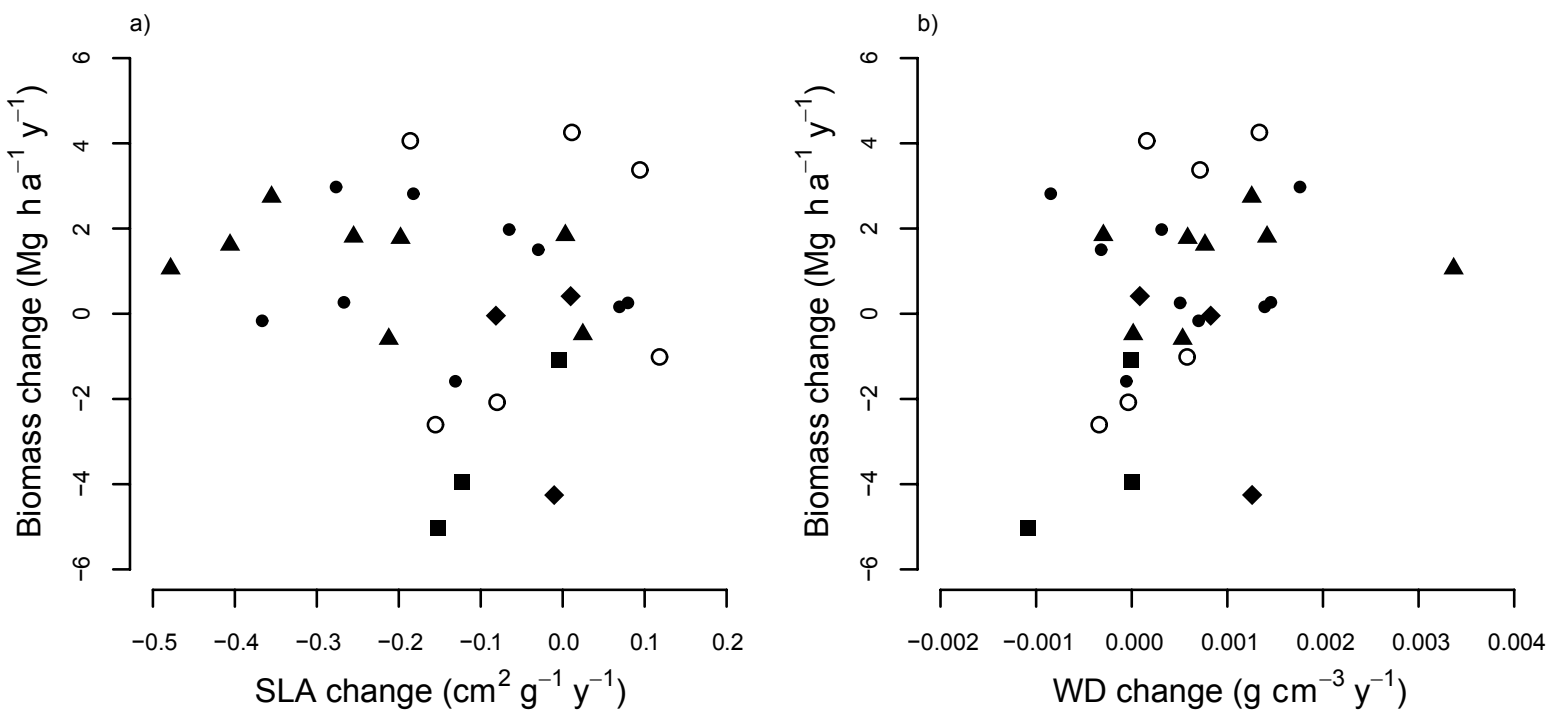

Appendix 6.12: Relationship of temporal change in a) specific leaf area (SLA) and b) wood density (WD) with temporal change in biomass. Symbols indicate sites; triangles: dry deciduous (DD; INPA), closed circles: moist semi-deciduous (MSD; La Chonta), open circles: moist evergreen (MEtap; Tapajós), diamonds: moist evergreen (MEpib; Pibiri), and squares: wet evergreen (WE; Corinto). For both graphs, the effect of change in trait value, site and their interaction were evaluated. In both cases, only sites were significantly different (for SLA: P = $0.04, \mathrm{~F}=3.1$, and for WD: $\mathrm{P}=0.02, \mathrm{~F}=3.7$ ). However, multiple comparisons using Tukey's post-hoc test did not show any significant differences among sites. 


\section{Chapter 7}

\section{Biodiversity enhances climate change mitigation by tropical forests}

Masha T. van der Sande, Lourens Poorter, Lammert Kooistra, Patricia Balvanera, Kirsten Thonicke, Jill Thompson, Eric J. M. M. Arets, Alice Boit, Loïc P. Dutrieux, Julian Equihua, Nashieli Garcia Alaniz, France Gerard, Martin Herold, Laurence Jones, Melanie Kolb, Francisco Mora, Tuyeni H. Mwampamba, Terry Parr, Boris Sakschewski, Margareth Simões, Marielos Peña-Claros

In revision 


\section{Abstract}

Rapidly increasing rates of climate change require society to urgently develop ways to reduce carbon dioxide concentrations in the atmosphere. Tropical forests present an important opportunity, as they store and sequester large amounts of carbon. It is often suggested that high biodiversity forests have high carbon uptake and stocks. Evidence is, however, scattered across geographic areas, scales and approaches, and it remains unclear whether biodiversity is just a co-benefit or also a requirement for the maintenance of carbon stocks and dynamics. Here, we review relationships between biodiversity attributes and carbon stocks and dynamics in tropical forests, focusing on empirical, remote sensing, and modelling approaches. Our results convincingly show that biodiversity is not only a co-benefit, but also a requirement for short- and long-term enhancement and maintenance of carbon stocks and uptake. This indicates that biodiversity should be included as an integral component of climate mitigation policies.

Keywords: carbon dynamics, carbon stocks, empirical studies, functional traits, modelling, remote sensing 


\section{Introduction}

The global increase in emissions of greenhouse gases such as $\mathrm{CO}_{2}$ has led to rapid changes in climate, at unprecedented rates over the last 1300 years (IPCC 2007). Simultaneously, anthropogenic disturbances have resulted in a loss of species diversity, with the current rate of extinctions being at least 1000 times higher than natural extinction rates (De Vos et al. 2015). These changes have raised international concern and stimulated the emergence of initiatives such as the Kyoto protocol (to reduce emissions and combat climate change) and the Convention on Biological Diversity (CBD, for the conservation and sustainable use of biodiversity). A policy initiative arising from the United Nations Framework Convention on Climate Change (UNFCCC) Conference of the Parties in 2007 is the Reduced Emissions from Deforestation and forest Degradation (REDD), which explicitly focuses on conserving the carbon stored in tropical forests.

Tropical forests are particularly relevant for these initiatives because they are hotspots of both carbon storage and biodiversity; they host around 47,000 tree species (Slik et al. 2015), store $25 \%$ of global terrestrial carbon in plant biomass (Bonan 2008) and account for 34\% of gross primary productivity (Beer et al. 2010), which helps mitigate climate change. In the last years, REDD+ has also recognized the importance of conserving biodiversity as a co-benefit of conserving carbon. The question remains, however, whether biodiversity also directly contributes to, and is thus a requirement for, maintaining carbon stocks and carbon dynamics (hereafter termed CSD, see Table 7.1; Balvanera et al. 2006; Díaz et al. 2009).

Ecological theories predict that a higher diversity of species results in greater resource use efficiency (Tilman 1999) and therefore higher CSD. Evidence for a positive relationship between species diversity and CSD has been provided by small-scale experiments and empirical field studies carried out mainly in temperate grasslands or other relatively simple ecosystems (Tilman et al. 2001, Balvanera et al. 2006, Paquette and Messier 2011, Fraser et al. 2015). Yet, evidence for highly diverse and structurally complex tropical forests has only recently become available and is still fragmented (e.g., Bunker et al. 2005; Poorter et al. 2015). The application of this evidence is therefore insufficient to inform the design and implementation of REDD+. Moreover, 'biodiversity' is more than species diversity, as it also comprises variation in ecosystems and other ecosystem properties such as plant functional traits and vegetation structure (Table 7.1, 7.2). Several reviews have been carried out to evaluate the role of biodiversity on CSD, but they were dominated by results from temperate grasslands (e.g., Hooper et al. 2012) or focused on forests in general (e.g., Díaz et al. 2009). Furthermore, these reviews have not explicitly evaluated how different study approaches - empirical (field or experimental), 
remote sensing, and modelling - contribute and complement each other in the understanding of this relationship.

Field studies measure directly on the ground and are useful for evaluating ecological mechanisms underlying the biodiversity-CSD relationship. Remote sensing studies are able to scale up to cover and monitor large spatial gradients. Finally, simulation models can be used to disentangle mechanisms underlying the biodiversity-CSD relationship or to forecast changes in this relationship under different scenarios of global change. Hence, while either of these approaches can provide useful insights, only through their combined use may we be able to obtain a more complete understanding of how biodiversity affects CSD (Bustamante et al. 2015).

Here, we review results from these three complementary research approaches (empirical, remote sensing, and modelling) to evaluate the biodiversity-carbon stocks and dynamics (CSD) relationship in tropical forests. We focus on different attributes of biodiversity (taxonomic diversity, functional trait diversity, trait mean, and structural attributes; Table 7.2) related to community-level vegetation properties - representing average as well as variation in vegetation properties. For the empirical evidence, the large body of information available allows a more detailed testing of additional hypotheses on the role of scale, forest management and analytical approach on biodiversity-CSD relationships. Next, we synthesize this information to evaluate under what conditions biodiversity is important for CSD, which ecological theories can explain this, and we identify the main knowledge gaps and potential solutions to fill these gaps. Finally, we provide recommendations on the policy implications of our findings.

\section{Empirical studies}

\section{Relevance}

Biodiversity attributes (Table 7.2) can be related with CSD. That is, biodiversity can be a co-benefit of the REDD+ mechanism, or biodiversity can directly influence CSD and thus is a requirement. The niche complementarity theory (Tilman 1999) predicts that diversity in the number and functioning (i.e., trait diversity) of species should increase resource use efficiency and therefore lead to higher carbon dynamics, and hence, higher carbon accumulation over time and larger carbon stocks per area of forest (Chisholm et al. 2013). In addition, the mass-ratio theory (Grime 1998) predicts that the most dominant species and their characteristics, rather than the diversity of species, determine ecosystem processes. Apart from effects of number and type of species, it could be that the structural attributes of the vegetation or the environmental conditions most strongly determine CSD. To 
evaluate effects of biodiversity attributes and environmental conditions (such as soil fertility and rainfall) on CSD, long-term sampling plots have been set up in many tropical forests. These data provide an important basis for testing the mechanisms underlying the relationships between biodiversity attributes and CSD.

Table 7.1: Glossary

\begin{tabular}{|c|c|}
\hline Biodiversity & $\begin{array}{l}\text { "The variability among living organisms from all sources including terrestrial, } \\
\text { marine, and other aquatic ecosystems and the ecological complexes of which } \\
\text { they are part; this includes diversity within species, among species, and of } \\
\text { ecosystems" (Convention on Biological Diversity). }\end{array}$ \\
\hline Biodiversity attributes & $\begin{array}{l}\text { Taxonomic diversity, trait diversity, community-mean trait values, and/or } \\
\text { structural attributes (see also Table 7.2). }\end{array}$ \\
\hline Carbon dynamics & $\begin{array}{l}\text { The fluxes in carbon per unit area per unit time. Examples of positive fluxes } \\
\text { (i.e. carbon uptake) are: aboveground biomass increase, tree growth, seedling } \\
\text { recruitment, or litter production. Tree mortality is a negative flux, but was } \\
\text { incorporated in some studies to evaluate the net carbon flux (net uptake). In } \\
\text { this review, carbon dynamics are mostly based on positive fluxes. Carbon } \\
\text { dynamics can be independent from carbon stocks. }\end{array}$ \\
\hline Carbon stocks & $\begin{array}{l}\text { The amount of carbon (or biomass) per unit area. This carbon can be based } \\
\text { on aboveground living biomass, (fine) root biomass, or soil organic matter. }\end{array}$ \\
\hline $\begin{array}{l}\text { Community-mean } \\
\text { traits }\end{array}$ & $\begin{array}{l}\text { Community average trait values, such as specific leaf area, wood density and } \\
\text { leaf nitrogen concentration, often weighted by species' basal area or } \\
\text { abundance. }\end{array}$ \\
\hline Functional trait & $\begin{array}{l}\text { Any measurable characteristic of an individual that is expected to have an } \\
\text { effect on one or multiple specific ecosystem processes and is affected by } \\
\text { environmental conditions. }\end{array}$ \\
\hline Insurance theory & $\begin{array}{l}\text { Species respond differently to environmental changes and in this way the } \\
\text { community insures long-term ecosystem functioning under environmental } \\
\text { change (Yachi and Loreau 1999). }\end{array}$ \\
\hline Mass-ratio theory & $\begin{array}{l}\text { The most dominant species and their traits mostly determine ecosystem } \\
\text { processes (Grime 1998). That is, the community-weighted mean (e.g., of trait } \\
\text { values) more strongly determines ecosystem processes than diversity (in } \\
\text { species or trait values) in the community. }\end{array}$ \\
\hline $\begin{array}{l}\text { Niche } \\
\text { complementarity } \\
\text { theory }\end{array}$ & $\begin{array}{l}\text { Species are complementary in their resource acquisition and use. Therefore, } \\
\text { high diversity (of species or traits) results in efficient acquisition and use at } \\
\text { the community level, and thus in high carbon stocks and dynamics (Tilman } \\
\text { 1999). }\end{array}$ \\
\hline Remote sensing & $\begin{array}{l}\text { Information on biodiversity and CSD obtained from a distance, e.g. by using } \\
\text { aircrafts or satellites. }\end{array}$ \\
\hline Resilience & $\begin{array}{l}\text { The capacity of an ecosystem to return to the pre-condition state following a } \\
\text { perturbation, including maintaining its essential characteristics taxonomic } \\
\text { composition, structures, ecosystem functions, and process rates (Holling } \\
\text { 1973). }\end{array}$ \\
\hline Structural attributes & $\begin{array}{l}\text { Community-average or community-total values of structural components of } \\
\text { the community, such as plot basal area and average stem diameter. }\end{array}$ \\
\hline Taxonomic div & Variation in species (e.g., the number or diversity) within a community. \\
\hline Trait diversity & $\begin{array}{l}\text { Variation in trait values within a community. This can be based both on } \\
\text { multivariate trait diversity as well as on the variation in single traits (Table } \\
7.2 \text {. }\end{array}$ \\
\hline
\end{tabular}




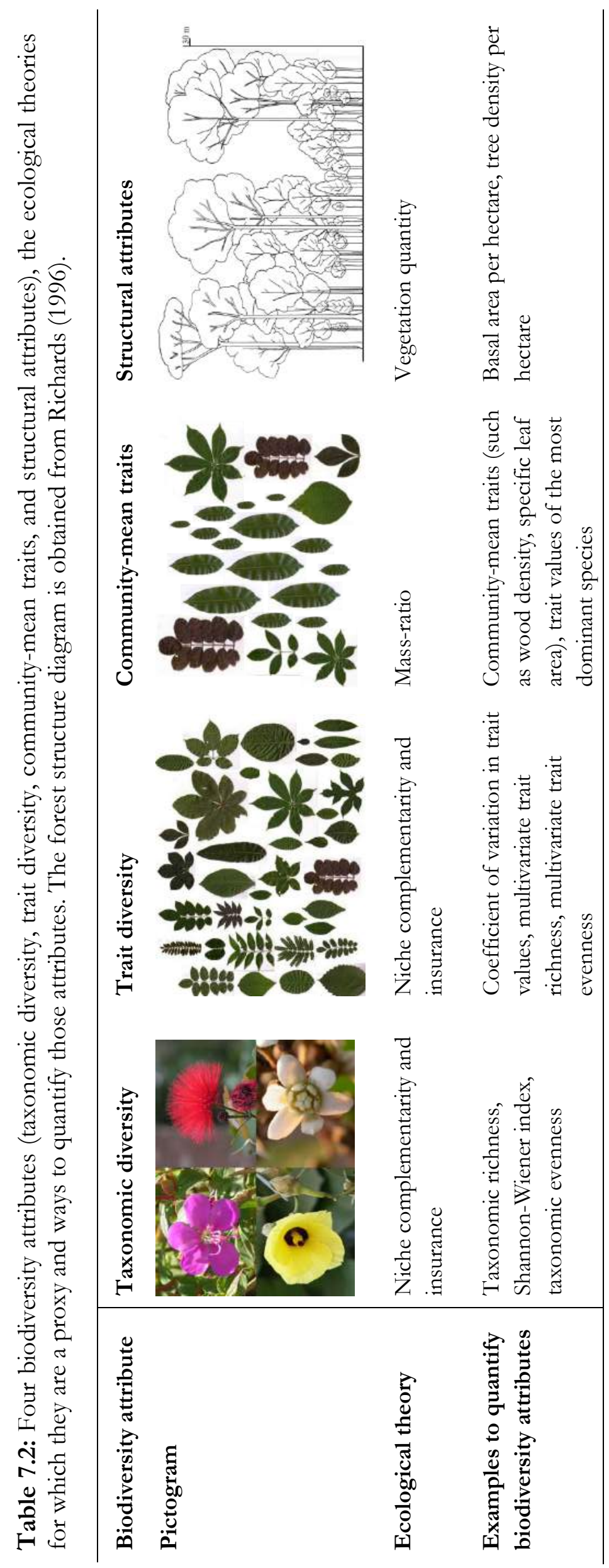




\section{Evidence}

We summarized 38 empirical studies that describe 165 relationships between one or more of the four biodiversity attributes and CSD in tropical forests (Table 7.2, and Appendix 7.1 for details on study selection and analyses and Appendices 7.2 and 7.3 for details about the studies). We evaluated whether biodiversity effects on CSD were positive, negative, both positive and negative (which can happen when multiple measures of the same biodiversity attribute are tested) or not significant. For trait mean effects, we did not distinguish between positive and negative, because the relevance of the direction depends on the trait considered.

Carbon stocks were significantly and positively related to taxonomic diversity (in $42 \%$ of the relationships), supporting the niche complementarity theory (Fig. 7.1). The diversity of trait values had a positive effect (17\%) or both positive and negative effects $(33 \%)$ on carbon stocks. Trait diversity is a complex measure that is constructed using a variety of traits of which only a subset may be important for CSD. Carbon stocks were also significantly related to community-mean trait values (in 100\% of the relationships; Fig. 7.1), providing support for the mass-ratio theory. Structural attributes, generally indicating forest density (Table 7.2), were positively related to carbon stocks in $78 \%$ of the relationships. Forest density was positively related to carbon stocks because denser forests have more stems, and since most carbon is held in stems, this directly increases carbon stocks.

In comparison with carbon stocks, carbon dynamics were more often significantly and positively related to taxonomic diversity (53\% for dynamics vs. $42 \%$ for stocks), but less often significantly related to community-mean traits $(47 \%$ for dynamics vs. $100 \%$ for stocks). These results suggest that carbon stocks are more frequently related to the average traits of the community, whereas carbon dynamics are lightly more frequently related to the species diversity. Structural attributes were positively related to carbon dynamics in $44 \%$ of the relationships and negatively in $33 \%$ of the relationships, in contrast to the always-positive effect of structural attributes on carbon stocks. On the one hand, a large quantity of leafy vegetation could lead to high productivity because many leaves are available to assimilate carbon. On the other hand, large plants and dense vegetation that compete for resources and space can reduce stand-level carbon dynamics because less light, water and nutrients are available for growth of other individuals. Environmental variables were often reported to have a significant effect on both carbon stocks ( $82 \%$ of the relationships) and dynamics (79\%), indicating that environmental conditions may be at least as important as biodiversity attributes in explaining CSD.

Effects of biodiversity on CSD depend on various factors related to scale, site properties, and the analytical approach used. We therefore evaluated how the 
biodiversity-CSD relationship differs with spatial scale, management intensity, and the analytical framework.

Biodiversity-CSD relationship at different spatial scales - Ecological processes operate at different spatial scales (McGill 2010). At small spatial scales (e.g., within one plot or study site), species-specific interactions are important, whereas at larger spatial scales with strong variation in environmental conditions environmental filtering may be more important (Laliberté et al. 2009). Therefore, the importance of biodiversity attributes and environmental conditions for CSD may vary with spatial scale. We found that for both carbon stocks and dynamics the effects of taxonomic diversity, vegetation structure and environmental conditions were more often important at large scales (i.e., all scales larger than local site-studies) than at local scales (Appendix 7.4). In contrast, trait diversity effects were more often important for stocks and dynamics at local than at large scales. Furthermore, trait mean effects on carbon dynamics were more often important at large than local scales, and trait mean effects on carbon stocks were not sensitive to scale.

Biodiversity-CSD relationship at different management intensities - We hypothesized that biodiversity effects on CSD may be more important in disturbed forests and plantations than in mature forests, because overall diversity is lower and less functional redundancy may occur than in mature forests without recent anthropogenic disturbance (see also Hooper et al. 2005). We found, however, that biodiversity was more often significantly related to CSD in mature forests than in plantations and disturbed forests, especially for carbon dynamics (Appendix 7.4). Possibly, the strong light differences among plots in disturbed forests and plantations may be so important for CSD that it overwhelms the effect of taxonomic diversity, or the lower diversity and structural complexity lead to less complementarity. Strong diversity effects on CSD have been widely documented by theoretical, experimental, and observational studies mainly in temperate grasslands (Tilman et al. 2014). Here we show that this relationship also applies to tropical plantations and to more diverse and complex managed and mature tropical forests.

Biodiversity-CSD relationship evaluated by different analytical approaches The studies included in this review used a range of analytical approaches that are likely to affect the observed biodiversity-CSD relationship. For example, independent effects of biodiversity on CSD can only be evaluated when controlling for possible confounding factors, such as variation in environmental conditions. In the studies reviewed here, especially for carbon dynamics, biodiversity attributes more frequently significantly affected CSD when analysed separately, compared to when analysed together with environmental variables (e.g., in a multiple regression; Appendix 7.4). This suggests that some of the biodiversity-CSD relationships are explained by environmental variables that drive both biodiversity and CSD. In other words, some of the biodiversity-CSD relationships are associations rather 
than causal relationships, although a large part of the relationships still shows independent effects of biodiversity attributes on CSD (on average $83 \%$ for stocks and $41 \%$ for dynamics). Hence, for a full understanding of underlying drivers and independent biodiversity effects on CSD, a more complete and mechanistic framework should be used that includes multiple biodiversity and environmental drivers and their mutual relationships.

\section{Outlook}

Strong evidence for an independent effect of biodiversity on CSD in tropical forests is emerging, but yet remains in its infancy, especially compared to wellstudied temperate (experimental) grassland systems (e.g., Tilman et al. 2001, van Ruijven and Berendse 2005). To obtain a better understanding of biodiversity effects on CSD in tropical forests in the face of global change, long-term data should be collected covering a range of spatial scales, environmental conditions and land-use intensities. To separate effects of biodiversity attributes and environmental conditions on CSD, more comprehensive and mechanistic analytical frameworks should be used.
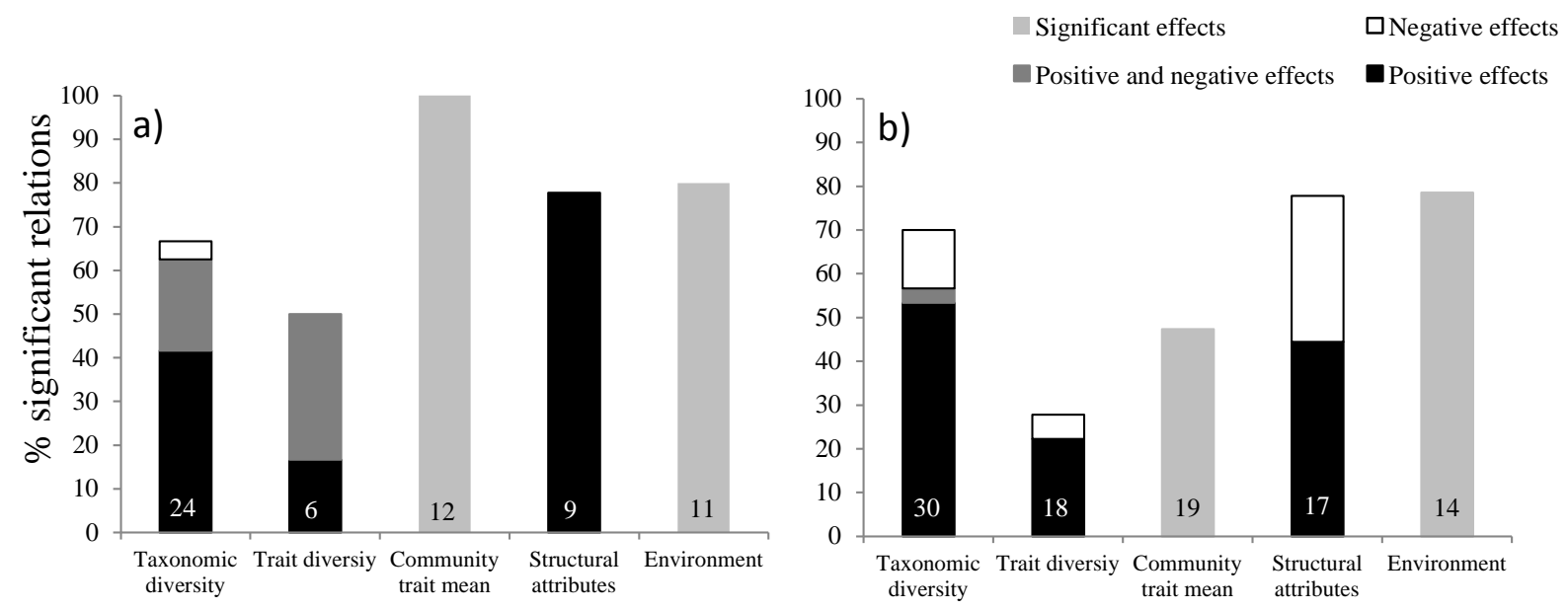

Figure 7.1: Percentage significant relationships (in the 38 reviewed studies) of biodiversity attributes on (a) carbon stocks and (b) carbon dynamics (see Glossary for definition and Appendix 7.1 for more information). The bars represent the four biodiversity attributes (taxonomic diversity, trait diversity, community trait mean, and forest structural attributes) and environment, and the colours show the \% relationships reporting a positive effect (black), negative effect (white), or both positive and negative (dark grey) effects. For community trait mean and environment, significant effects were not separated into positive and negative (because these are only meaningful when elaborating on the meaning of the variable used), and therefore only the total percentage of significant relations are shown (light grey). The numbers in the bars represent the total number of relationships evaluated. 


\section{Remote sensing studies}

\section{Relevance}

Remote sensing provides spatial information that can extend our view of biodiversity attributes and CSD in tropical forests to spatial and temporal scales that are intractable on the ground. Therefore, remote sensing can provide the means to test the scale-dependence of the biodiversity-CSD relationship and identify synergies between them. Several recent studies have reviewed the potential and limitations of remote sensing based methods for measuring and monitoring carbon (De Sy et al. 2012) and biodiversity (Duro et al. 2007, Kuenzer et al. 2014) of tropical forests. For forest carbon, wall-to-wall pan-tropical benchmark maps based on different techniques and resolutions have been developed (Saatchi et al. 2011, Baccini et al. 2012, Avitabile et al. 2015). However, remote sensing based maps of biodiversity are still rare (Asner 2015), and as a result the number of studies evaluating biodiversity-CSD relationships is limited and mainly focused at local scales.

\section{Evidence}

We identified 10 studies that used remote sensing techniques to evaluate biodiversity-CSD relationships (Appendix 7.5a, c), and conducted a qualitative assessment (see full description in Appendix 7.5b). Nine of the ten studies show a positive relationship between biodiversity and carbon stocks (no studies evaluated carbon dynamics), for different biodiversity indicators: plant species diversity ( 7 studies), fauna species diversity (2) and trait diversity (1).

The strength of the biodiversity-CSD relationship varies considerably among studies $(r=-0.01-0.83)$ but seems to be scale-independent. For example, both the strongest and the weakest correlations were found at the local scale (Appendix 7.5a). At least three possible reasons may explain why the correlation between biodiversity and carbon varies in strength. First, differences in environmental conditions may determine the correlation strength. Fig. 7.2 shows an example of spatial variation in correlation strength, which is significantly and positively related to rainfall seasonality and predicted species richness (Appendix 7.7), indicating that the positive effect of species richness on carbon stocks increases towards drier and more diverse forests. Second, the strength may depend on the method used to derive biodiversity and carbon variables. When biodiversity attributes and carbon stocks are derived using the same method (e.g., LiDAR), then they are not independent and may show a stronger correlation compared to when the variables are obtained from independent sources. Third, the strength may depend on the prediction accuracy of remote sensing indicators for biodiversity and CSD. In 
remote sensing, a range of methods is used to estimate carbon stocks by relating remote sensing indicators to field observations (Appendix 7.5b), but yet no agreement has been reached on which remote sensing methods should be adopted for tracking biodiversity (Skidmore et al. 2015). Although the small number of studies does not allow formal testing of the biodiversity-CSD relationship and the approach used in remote sensing studies (i.e., correlations) cannot differentiate whether biodiversity is a co-benefit or a requirement for CSD, the studies convincingly show that hotspots for carbon storage are also hotspots for biodiversity. Therefore, simultaneous and optimal conservation of biodiversity and carbon can be achieved by focusing on such areas.

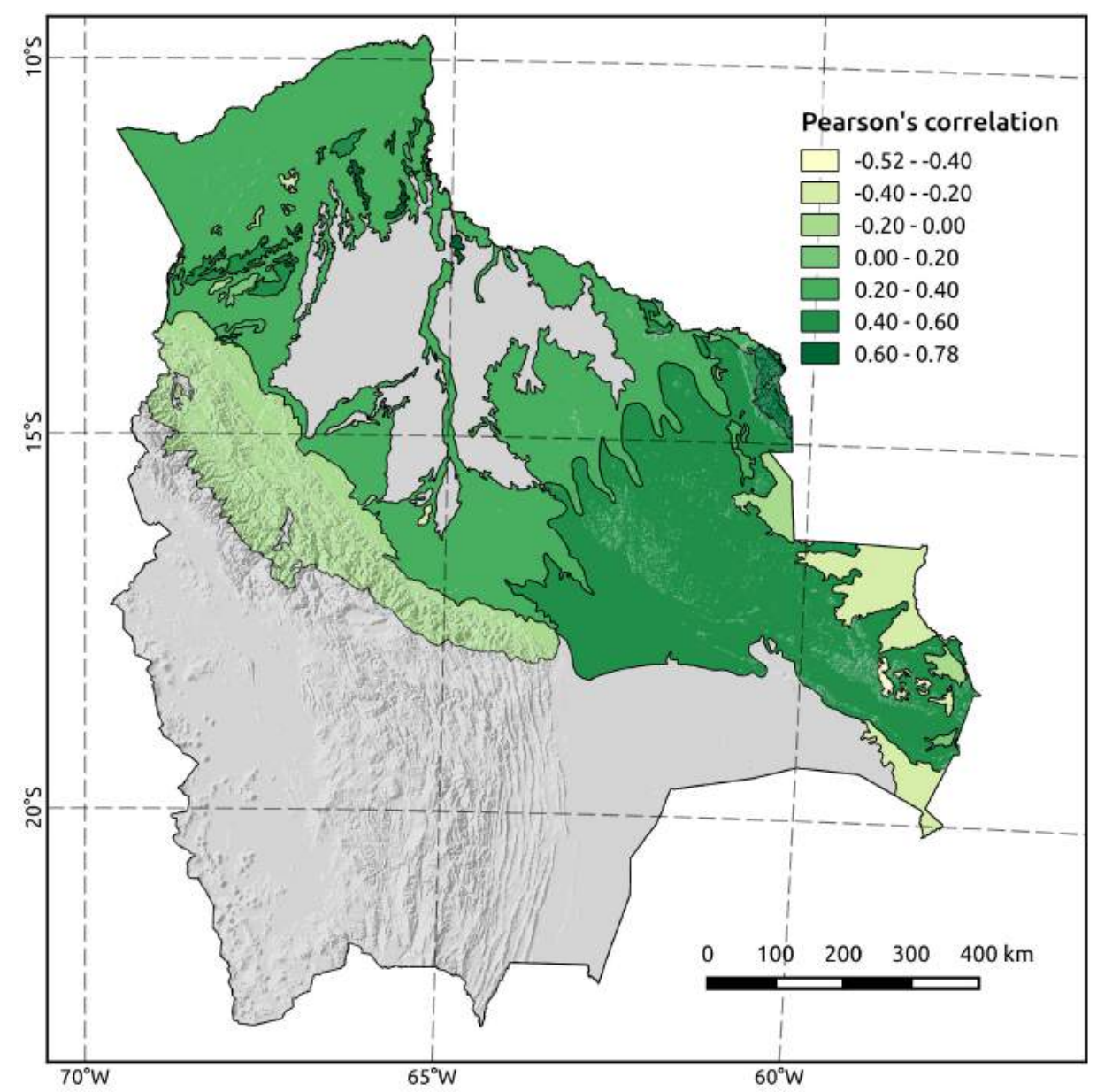

Figure 7.2: Spatial correlation between remote sensing-derived tree species richness and aboveground biomass for tropical forest in different biogeographic zones in lowland Bolivia (see Appendix 7.7 for a description of methods). The correlation strength increased with rainfall seasonality (i.e., the coefficient of variation of monthly rainfall; $\mathrm{P}<0.001, \mathrm{t}=4.3, \mathrm{~N}=53$ ) and with predicted species richness $(\mathrm{P}<0.001, \mathrm{t}=5.4, \mathrm{~N}=53)$. In both regression analyses, we included the size of the area as a variable to correct for possible effect of differences in pixel number on which the correlation coefficient was based. Rainfall seasonality and predicted species richness were not significantly correlated $(\mathrm{r}=0.20, \mathrm{P}=0.12, \mathrm{t}=1.55)$. Data were obtained from Kooistra et al. (2015), based on which the map was prepared by L. Dutrieux. 


\section{Outlook}

Over the past decade, data quality (i.e., temporal and spectral resolution using veryhigh resolution remote sensing datasets from space borne or (unmanned) airborne sensors), data availability, and the ability to link remote sensing derived variables with field observations have improved considerably (see Appendix 7.6 for more details). This will allow further testing of biodiversity-CSD relationships. Future developments are likely to link field observations to these high quality images and to upscale point observations to continuous maps of biodiversity attributes (Feret and Asner 2014) or of specific plant traits (Asner et al. 2015). These in turn could be linked to carbon stocks and to carbon dynamics.

\section{Modelling studies}

\section{Relevance}

Numerical ecosystem models are complementary to empirical and remote sensing approaches in their ability to test hypotheses related to biodiversity and ecosystem function in an experimental way and to develop scenarios. Models that quantify the influence of biodiversity on CSD in tropical forests are only starting to emerge, but they may nevertheless provide valuable information on the current and potential role of biodiversity for CSD.

\section{Evidence}

We found two models that have been used to study biodiversity-CSD relationships (see Appendix 7.8). The first is a dynamic plant functional trait model that was applied to Australian forests (Pichancourt et al. 2014). This study found that, with modest climate change, plant trait diversity increased carbon sequestration in lowland forests, but this effect decreases with strong climate change (under SRES A1FI scenario). In a second modelling study, species diversity weakly increased forest productivity in northern India (simulated by the remote-sensing based Carnegie-Ames-Stanford Approach (CASA) model) under current climate conditions (Chitale et al. 2012).

Another potentially useful ecosystem modelling approach consists of dynamic global vegetation models (DGVMs). Initially, DGVMs had a very simplified representation of biodiversity, using several plant functional types (PFTs) (e.g., Sitch et al. 2008), which could not be used to evaluate the biodiversity-CSD relationship. Improvements to more realistically model biodiversity using DGVMs were done by including variation of a selected number of plant traits (JSBACH by Verheijen et al. 2013), as implemented using adaptive functional traits (aDGVM2 by Scheiter et al. 2013), multiple trait ranges (JeDi by Pavlick et al. 2012, and TFS 
by Fyllas et al. 2014), and the leaf and stem economics spectrum (LPJmL-FIT by Sakschewski et al. 2015) (see Appendix 7.9 for more details on the models). These new trait-based DGVMs with partly adaptive features of functional biodiversity represent promising approaches to test the biodiversity-CSD relationship at the landscape to regional scale. Research of the next few years will show whether these next-generation DGVMs will live up to the expectations.

\section{Outlook}

Only two numerical modelling studies have looked into the biodiversity-CSD relationship. To improve our understanding of this relationship and underlying mechanisms that play a role under changing climate conditions and at large spatiotemporal scales, many existing modelling approaches should be (further) developed. Trait-based modelling approaches represent a suitable tool to explore how biodiversity influences CSD, especially under climate change conditions because changes in the relationship between individual traits and CSD as well as forest composition can be quantified. Incorporating more elements or processes would further improve model predictions, such as adaptive responses in trait values (within individuals and over generations) and a better representation of belowground processes.

Given the diverse nature of modelling approaches used, it is yet too early to draw a consistent model-based conclusion on the biodiversity-CSD relationship. Nevertheless, the few modelling attempts available so far show that biodiversity has a positive effect on long-term CSD in tropical forests.

\section{Synthesis}

How important is biodiversity for CSD? We assessed the biodiversity-CSD relationship using three complementary approaches, and found a significant positive relationship between biodiversity and carbon stocks or dynamics in $75 \%$ of the empirical studies and $90 \%$ of the remote sensing studies. Modellers have only recently started to include biodiversity in a more realistic way in their ecosystem models, and found that biodiversity has a weak positive effect on long-term CSD. These results extend the findings from experimental studies and temperate systems that biodiversity matters for ecosystem functioning, even in bighly diverse tropical forests.

What biodiversity attributes matter for CSD and under what conditions? Empirical studies indicate that not only species diversity, but a suite of biodiversity attributes (taxonomic and trait diversity, community-mean trait values, and structural attributes, Table 7.2) is important for CSD. They also indicate that the biodiversity-CSD relationship is stronger at larger spatial scales, possibly because of 
stronger variation in species diversity and other biodiversity attributes across these larger environmental gradients. In contrast, remote sensing studies found that the strength of the biodiversity-CSD relationship did not vary with scale, perhaps because of the indirect way in which they assess both carbon and biodiversity. Empirical studies also found that the biodiversity-CSD relationship was strongest in mature forests, possibly because of higher diversity and structural complexity leading to more complementarity. In sum, the biodiversity-CSD relationship tends to be weaker in disturbed forest and at local scales, and stronger in old-growth forest and across larger spatial scales.

Which ecological theories explain biodiversity effects on CSD? We evaluated several ecological theories on how biodiversity can affect CSD (Table 7.2), and found that not only the quantity (i.e., structural attributes) but also the quality (i.e., taxonomic diversity, trait diversity, or community-mean traits) of the vegetation is important for CSD. The traits of the dominant species (reflecting the mass-ratio theory) were most important for carbon stocks, whereas taxonomic diversity (reflecting the niche complementarity theory) was most important for carbon dynamics. We also expect that over the long term, biodiversity enhances ecosystem resilience in the face of environmental change (the insurance theory), which assures long-term stability of CSD (e.g., Loreau et al. 2003, Isbell et al. 2015). Only with sufficient variation of species and ecological strategies in the plant community, the community has the potential to adapt to environmental change, in which the currently marginal species become the dominant species in the future and maintain ecosystem functioning (Yachi and Loreau 1999). Hence, strong evidence suggests that three mechanistic reasons (niche complementarity, mass-ratio, and the insurance effect) explain why biodiversity matters for CSD.

How can different research approaches inform us about the biodiversity-CSD relationship? Empirical studies and controlled experiments in the field can provide insight into underlying mechanisms of the biodiversity-CSD relationship, identify what aspect of biodiversity matters most, and provide evidence whether this relationship is strong enough to have a significant effect on the functioning of natural systems. Empirical studies have the disadvantage that site-specific factors may modify this relationship and that they cover small areas. Remote sensing allows to assess the biodiversity-CSD relationship at continuous and larger spatial scales that are relevant to policy development. Remote sensing can also monitor changes in CSD and biodiversity over time, which is important for the measurement, verification and reporting of REDD+. Remote sensing has the disadvantage that it remains an indirect proxy for what is happening on the ground, and needs to deal with co-varying site conditions. Modelling studies can take an experimental approach to test the independent effects of biodiversity for CSD and allow sensitivity analyses of complex ecological systems. They also allow 
assessments of the biodiversity-CSD relationship at large temporal scales, which are needed to inform us about how forest systems may respond to climate change, and to develop scenario analyses on the impact of policy interventions. Modelling studies have the disadvantage that they are a simplification of the real world and their representation of multiple interacting processes is difficult to validate. In sum, field studies, remote sensing, and modelling are three complementary research approaches that differ in ecological realism, spatial and temporal scale, and that can provide complementary information on the biodiversity-CSD relationship and its (policy) implications.

\section{Policy implications}

The findings in this review indicate that biodiversity is a requirement for the longterm conservation of carbon stocks and for enhancing the uptake of carbon from the atmosphere. These findings have implications for policies related to biodiversity and carbon conservation in tropical forests. We consider three broad policy issues that are particularly relevant.

Diverse, carbon rich and productive mature forests should be given priority under the REDD + framework when threatened by degradation or land-use change. Data on carbon stocks and biodiversity attributes can be used to identify strategic targets across space, allowing alignment of global and national strategies aimed at maximizing biodiversity and carbon conservation (Phelps et al. 2012a). At the national scale, it would allow to prioritise protection of forests rich in carbon and biodiversity in their Nationally Appropriate Mitigation Actions (NAMAs, http://unfccc.int/focus /mitigation/items/7172.php). At the local scale, a range of context-dependent interventions including community involvement, strengthening ownership, and the development of pro-conservation local governance would be needed to enhance the cost-effectiveness and long-term impact of biodiversity and carbon conservation initiatives (Gardner et al. 2012).

Besides existing and mature forests, efforts to increased forest cover (through natural regeneration, restoration, afforestation, and land use systems that enhance tree cover) under REDD+, the New York challenge, and other national and local initiatives should recognize and incorporate biodiversity as a requirement to obtain carbon-rich and resilient systems. Carbon stocks and uptake could be maximized through the selection of a large variety of species with specific desirable traits at a range of spatial scales. Care should be given to the fact that biodiversity attributes that increase carbon uptake are not necessarily the same as the ones increasing carbon stocks, as these are the result of different processes.

A suite of complementary approaches can best address the needs for data generation for improving carbon and biodiversity conservation in the context of performance-based incentive 
regimes (such as $R E D D+$ ). Remote sensing could not only identify target areas with the highest carbon stocks and dynamics, but also inform on different attributes of biodiversity (Skidmore et al. 2015) to be targeted by Monitoring Reporting and Verification initiatives. Refined models at multiple scales could assess the impacts of alternative policies, management interventions, and future climate change scenarios (Ay et al. 2014). The long-term scientific monitoring of the dynamics of old-growth and disturbed forests will be necessary to enhance the realism of models and targeting exercises, and will provide relevant information on carbon and biodiversity change (Gardner et al. 2012). Additionally, community monitoring of forest carbon and biodiversity could generate adequate data and increase ownership and negotiation power in carbon markets (e.g., Butt et al. 2015).

Consideration of these policy issues is necessary to realise the full potential of tropical forests to mitigate climate change through optimizing biodiversity.

\section{Acknowledgements}

The research leading to these results has received partial funding from the European Union Seventh Framework Programme (FP7/2007-2013) under grant agreement n 283093 - The Role Of Biodiversity In climate change mitigatioN (ROBIN). MS and EA received partial funding through the strategic research program KBIV (KB-14) "Sustainable spatial development of ecosystems, landscapes, seas and regions", funded by the Dutch Ministry of Economic Affairs and carried out by Wageningen University \& Research centre (project code KB-14-003-030). The modelling part of this study has been supported by the TRY initiative on plant traits (http://www.try-db.org). We thank Nataly Ascarrunz, Marcel Hoosbeek, Octavio Perez Maqueo, Mauro Garcia-Esteban, Rodrigo Ferraz, and Andrei Olak for input on earlier versions of the manuscript. 


\section{Appendices}

Appendix 7.1: Details on empirical studies selection

\section{Selection of studies:}

We selected studies that explicitly look at the relation between biodiversity and carbon stocks and dynamics (CSD) in tropical forests. That is, studies should evaluate the effects of at least one of the biodiversity attributes (taxonomic diversity, trait diversity, community-mean trait, and vegetation structure) on at least one variable of biomass (or carbon) stocks and dynamics (or vice versa). We sought these studies on Web of Science and Scopus, using different combinations of the following keywords: 'biodiversity', 'diversity', 'species richness', 'species diversity', 'tropical forests', 'carbon', 'carbon stocks', 'carbon dynamics', 'biomass', 'biomass stocks', 'biomass dynamics', 'productivity', 'community-weighted mean trait', 'functional trait', 'functional diversity', 'forest structure'. We also found studies through scanning references of already found studies. All studies were found before November 13, 2015. Additionally, some still unpublished studies were included that were produced as part of the ROBIN-project (http://robinproject.info/).

\section{Evaluation of studies:}

In total, we gathered 38 studies, which used different components indicating carbon stocks (e.g., biomass, soil organic matter) or dynamics (e.g., litter or biomass productivity, mortality; see Appendix 7.2 and 7.3). For each study and each CSD component (70 in total), we evaluated the effect of four biodiversity attributes: taxonomic diversity, trait diversity, trait mean, and vegetation structure (Table 7.2). We also considered the effect of environment if reported in the study. As our unit of replication, we used each reported relationship between a biodiversity attribute group or environmental attribute and CSD. Hence, if a study tested the effect of one biodiversity attribute on one component of CSD, then this study yielded one relationship, but if the study tested for multiple (2,3 or 4) biodiversity attributes and/or multiple CSD components, then this study yielded multiple relationships. In total, we included 165 relationships (between 1 and 15 relationships per study) (Appendix 7.4). To increase the number of relationships assessed per group and better understand the role of biodiversity attributes in carbon stocks and the dynamics of carbon, we grouped CSD components into 'carbon stocks' (including above- and belowground carbon or biomass stocks, and soil organic matter or soil carbon) and 'carbon dynamics' (including (litter) productivity, biomass or carbon (net) growth, and biomass or carbon loss through tree mortality; see Appendix 7.2 for the original CSD components reported in the studies and the grouping into 'stocks' and 'dynamics').

For all these 165 relationships (64 for stocks and 101 for dynamics), we evaluated whether the effect was positive $(+)$, negative $(-)$, both negative and positive $(+/-$, which can happen when multiple variables within the same biodiversity attribute group show contrasting results), or not significant (0). In many cases, studies used multiple variables within the same biodiversity attribute group to predict CSD (for example, taxonomic richness and Shannon diversity). In these cases, we summarized the multiple variables within one biodiversity attribute group as one relationship in the following way: in case both positive and not significant effects were found, then we gave the relation a + . Similarly, in case both negative and not significant effects were found, then the relation was given a -. Finally, when it showed both positive and negative (and non-significant) relationships, it was given a $+/$-. We neglected the non-significant effects in 
these cases because the absolute amount of variables in each biodiversity attribute group may not be representative, as authors will pre-select some variables and/or not report variables that do not show a significant effect. The relationship between a biodiversity attribute group and a CSD component was attributed a 0 when all tested effects were not significant.

\section{Geographical range, forest type and spatial scale of the studies used:}

Studies incorporated in the review were carried out mainly in the Neotropics (68\%), but also in Africa (8\%), Asia (5\%) and across multiple continents (18\%). We included mature forests (45\%) as well as disturbed forests $(29 \%)$ and plantations $(26 \%)$, and at local scales $(63 \%)$ and large (i.e., anything larger than local site-studies) scales (37\%) (see Appendix 7.2). From all studies, 18\% received partial financial support by the ROBIN project. 


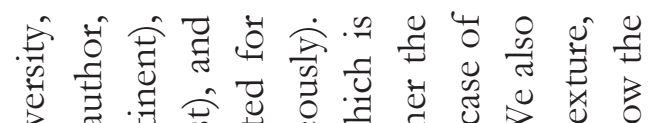

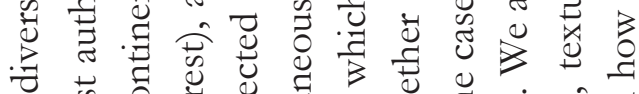

.

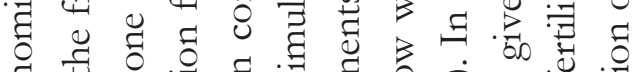

व

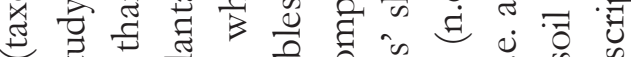

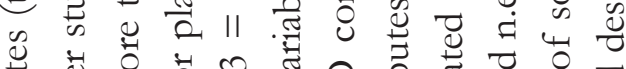

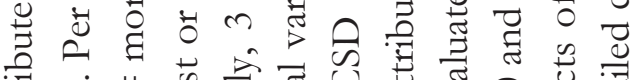

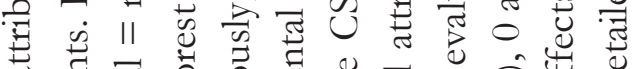

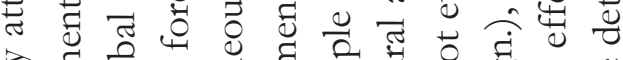

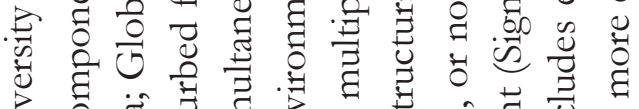

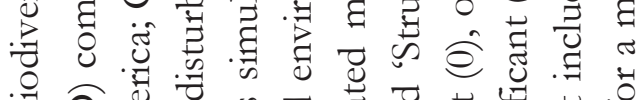

ลิ)

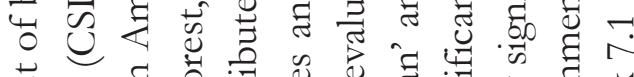

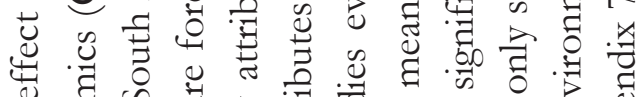

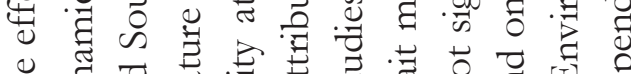

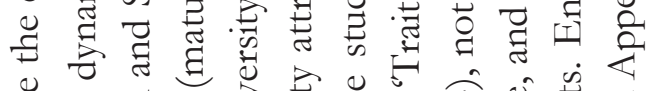

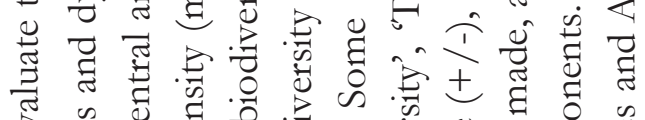

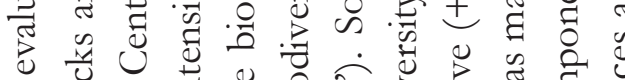

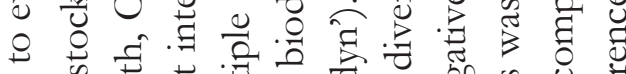

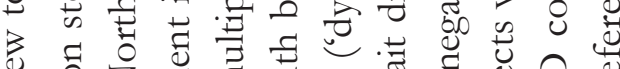

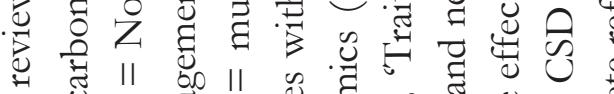

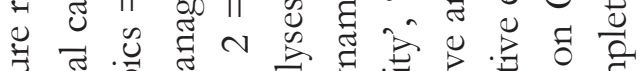

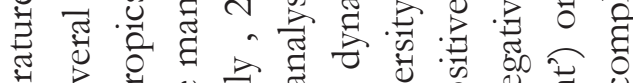

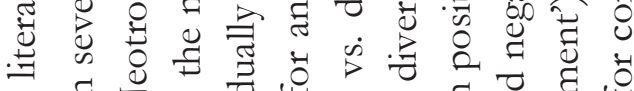

छ

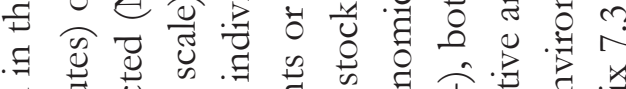

च

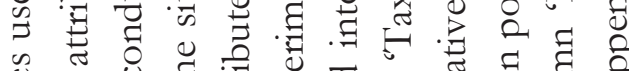

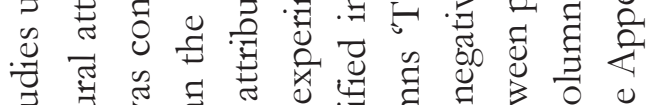

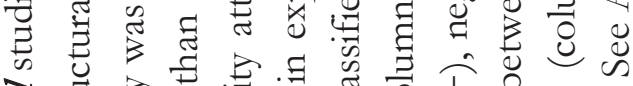

तु

:

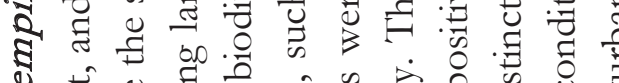

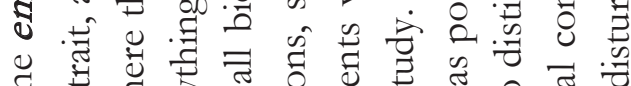

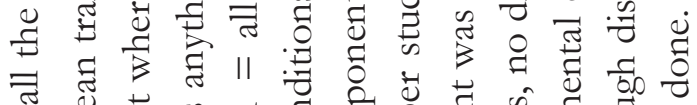

స

虫

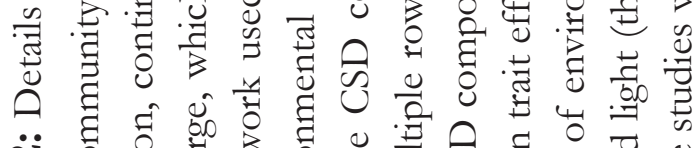

ஸ்

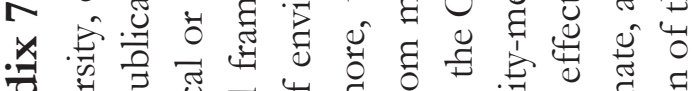

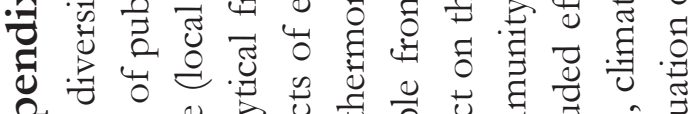

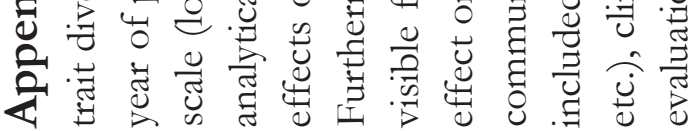

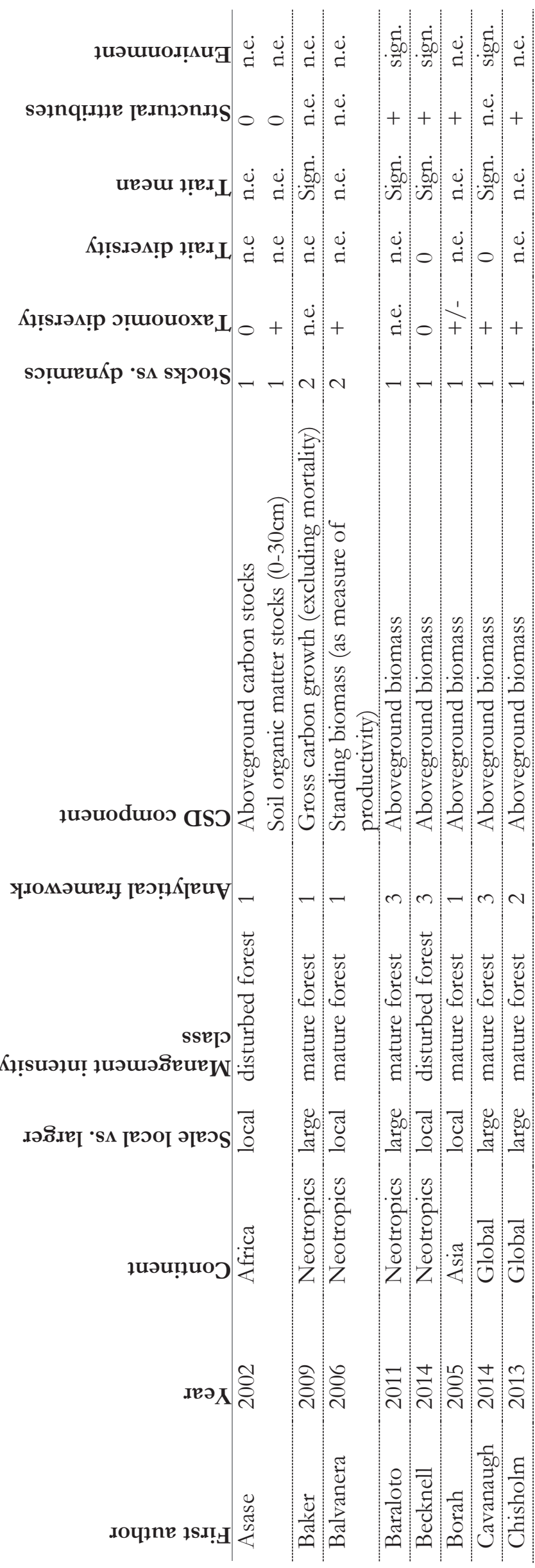




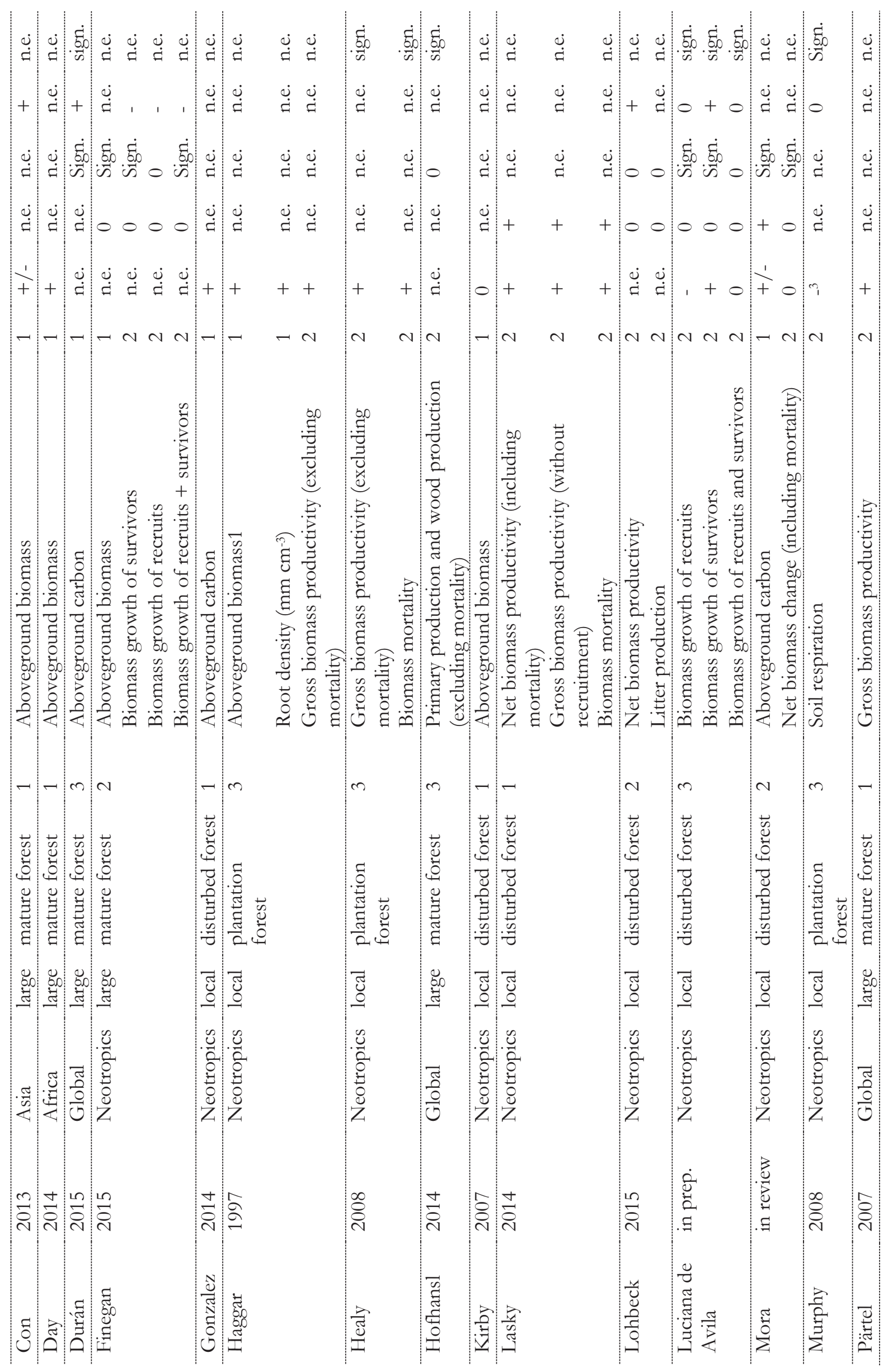




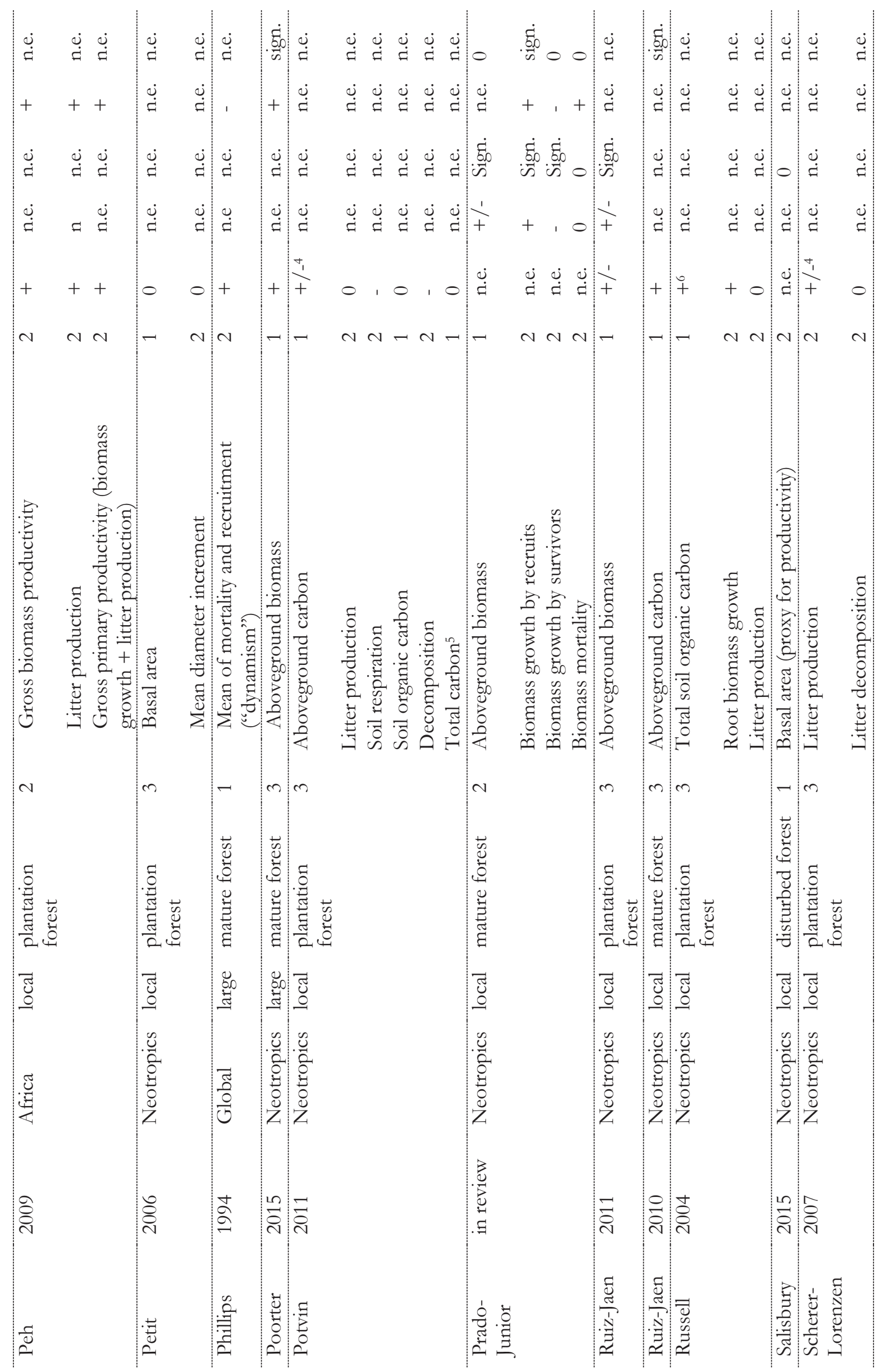


Chapter 7

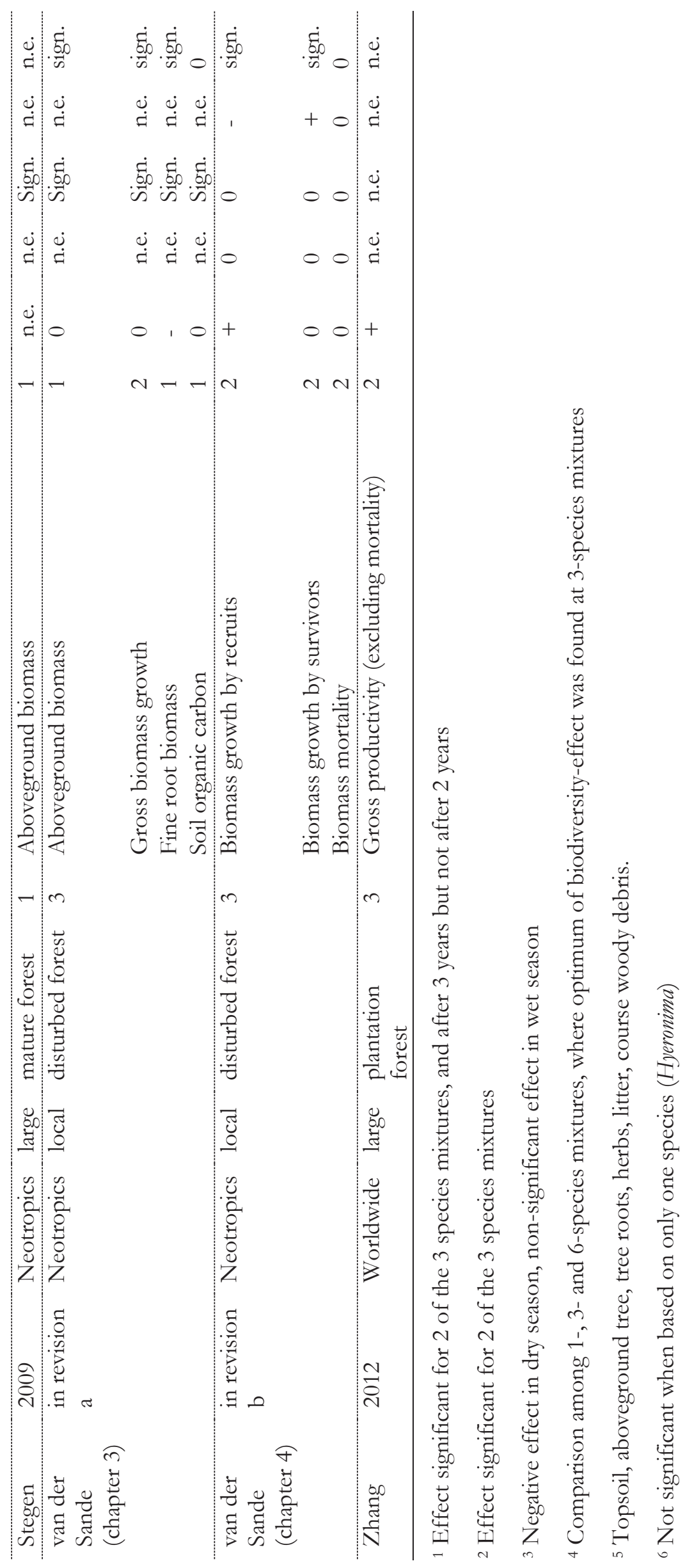


Appendix 7.3: References of all empirical studies used in the literature review to evaluate the effect of biodiversity attributes (taxonomic diversity, trait diversity, community-mean trait, and structural attributes) on carbon stocks and dynamics. See Appendix 7.2 for more details about the studies.

Asase A, Asitoakor BK, and Ekpe PK. 2012. Linkages between tree diversity and carbon stocks in unlogged and logged West African tropical forests. Int J Biodivers Sci Ecosyst Serv Manag 8: 217-30.

Baker TR, Phillips OL, Laurance WF, et al. 2009. Do species traits determine patterns of wood production in Amazonian forests? Biogeosciences 6: 297-307.

Balvanera P and Aguirre E. 2006. Tree diversity, environmental heterogeneity, and productivity in a Mexican tropical dry forest. Biotropica 38: 479-91.

Baraloto C, Rabaud S, Molto Q, et al. 2011. Disentangling stand and environmental correlates of aboveground biomass in Amazonian forests. Glob Chang Biol 17: 2677-88.

Becknell JM and Powers JS. 2014. Stand age and soils as drivers of plant functional traits and aboveground biomass in secondary tropical dry forest. Can J For Res 613: 604-13.

Borah M, Das D, Kalita J, et al. 2015. Tree species composition, biomass and carbon stocks in two tropical forest of Assam. Biomass and Bioenergy 78: 25-35.

Cavanaugh KC, Gosnell JS, Davis SL, et al. 2014. Carbon storage in tropical forests correlates with taxonomic diversity and functional dominance on a global scale. Glob Ecol Biogeogr 23: 563-73.

Chisholm RA, Muller-Landau HC, Abdul Rahman K, et al. 2013. Scale-dependent relationships between tree species richness and ecosystem function in forests. J Ecol 101: 1214-24.

Con T Van, Thang NT, Ha DTT, et al. 2013. Relationship between aboveground biomass and measures of structure and species diversity in tropical forests of Vietnam. For Ecol Manage 310: 213-8.

Day M, Baldauf C, Rutishauser E, and Sunderland TCH. 2013. Relationships between tree species diversity and above-ground biomass in Central African rainforests: implications for REDD. Environ Conserv 41: 64-72.

Durán SM, Sánchez-azofeifa GA, Rios RS, and Gianoli E. 2015. The relative importance of climate, stand variables and liana abundance for carbon storage in tropical forests. Glob Ecol Biogeogr.

Finegan B, Peña-Claros M, Oliveira A de, et al. 2015. Does functional trait diversity predict above-ground biomass and productivity of tropical forests? Testing three alternative hypotheses (C Canham, Ed). J Ecol 103: 191-201.

Gonzalez P, Kroll B, and Vargas CR. 2014. Tropical rainforest biodiversity and aboveground carbon changes and uncertainties in the Selva Central, Peru. For Ecol Manage 312: 78-91.

Haggar JP and Ewel JJ. 1997. Primary productivity and resource partitioning in model tropical ecosystems. Ecology 78: 1211-21.

Healy C, Gotelli NJ, and Potvin C. 2008. Partitioning the effects of biodiversity and environmental heterogeneity for productivity and mortality in a tropical tree plantation. J Ecol 96: 903-13.

Hofhansl F, Schnecker J, Singer G, and Wanek W. 2014. New insights into mechanisms driving carbon allocation in tropical forests. New Phytol.

Kirby KR and Potvin C. 2007. Variation in carbon storage among tree species: Implications for the management of a small-scale carbon sink project. For Ecol Manage 246: 208-21.

Lasky JR, Uriarte M, Boukili VK, et al. 2014. The relationship between tree biodiversity and biomass dynamics changes with tropical forest succession. Ecol Lett.

Lohbeck M, Poorter L, Martínez-Ramos M, and Bongers F. 2015. Biomass is the main driver of changes in ecosystem process rates during tropical forest succession. Ecology 96: 1242-52.

Luciana de Avila A, Sande MT van der, Dormann CF, et al. Effects of management intensity, residual diversity and trait composition on biomass recovery of a tropical rain forest. In prep.

Mora F. Changes in carbon stocks along tropical dry forest succession: Explanatory and predictive ability of forest age, tree diversity and chronosequence models. Glob Chang Biol.

Murphy M, Balser T, Buchmann N, et al. 2008. Linking tree biodiversity to belowground process in a young tropical plantation: Impacts on soil CO2 flux. For Ecol Manage 255: 2577-88. 
Pärtel M, Laanisto L, and Zobel M. 2007. Contrasting plant productivity-diversity relationships across latitude: The role of evolutionary history. Ecology 88: 1091-7.

Peh KS. 2009. The relationship between species diversity and ecosystem function in low- and highdiversity gropical African forests.

Petit B and Montagnini F. 2006. Growth in pure and mixed plantations of tree species used in reforesting rural areas of the humid region of Costa Rica, Central America. For Ecol Manage 233: 338-43.

Phillips OL, Hall P, Gentry a H, et al. 1994. Dynamics and species richness of tropical rain forests. Proc Natl Acad Sci U S A 91: 2805-9.

Poorter L, Sande MT van der, Thompson J, et al. 2015. Diversity enhances carbon storage in tropical forests. Glob Ecol Biogeogr.

Potvin C, Mancilla, Lady, Buchmann N, et al. 2011. An ecosystem approach to biodiversity effects: Carbon pools in a tropical tree plantation. For Ecol Manage 261: 1614-24.

Prado-Junior JA, Schiavini I, Vale VS, et al. Conservative species drive biomass productivity in tropical dry forests. Rev.

Ruiz-Jaen MC and Potvin C. 2010. Tree diversity explains variation in ecosystem function in a neotropical forest in Panama. Biotropica 42: 638-46.

Ruiz-Jaen MC and Potvin C. 2011. Can we predict carbon stocks in tropical ecosystems from tree diversity? Comparing species and functional diversity in a plantation and a natural forest. New Phytol 189: 978-87.

Russell AE, Cambardella C a., Ewel JJ, and Parkin TB. 2004. Species, Rotation, and Life-Form Diversity Effects on Soil Carbon in Experimental Tropical Ecosystems. Ecol Appl 14: 47-60.

Salisbury CL and Potvin C. 2015. Does tree species composition affect productivity in a tropical planted forest? Biotropica 47: 559-68.

Sande MT van der, Arets EJMM, Peña-Claros M, et al. Soil fertility and species traits, but not species diversity, drive productivity and biomass stocks in a tropical rainforest. In revision, a. (chapter 3)

Sande MT van der, Peña-Claros M, Ascarrunz N, et al. Drivers of biomass change in a tropical forest: testing for effects of diversity, traits, and environment. In revision, b. (chapter 4)

Scherer-Lorenzen M, Bonilla JL, and Potvin C. 2007. Tree species richness affects litter production and decomposition rates in a tropical biodiversity experiment. Oikos 116: 2108-24.

Stegen JC, Swenson NG, Valencia R, et al. 2009. Above-ground forest biomass is not consistently related to wood density in tropical forests. Glob Ecol Biogeogr 18: 617-25.

Zhang Y, Chen HYH, and Reich PB. 2012. Forest productivity increases with evenness, species richness and trait variation: a global meta-analysis. J Ecol 100: 742-9. 
Carbon stocks

a)

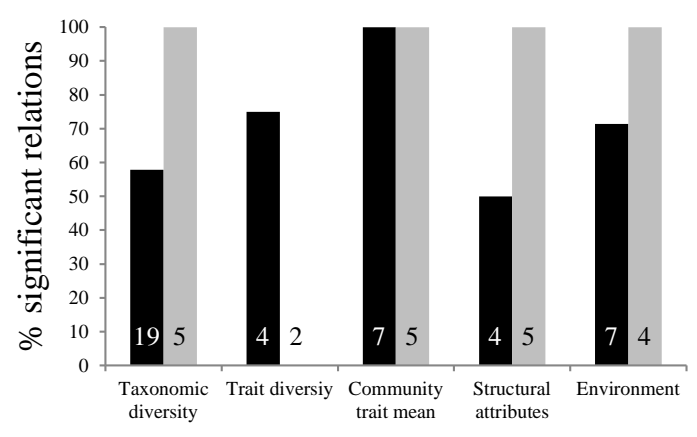

c)

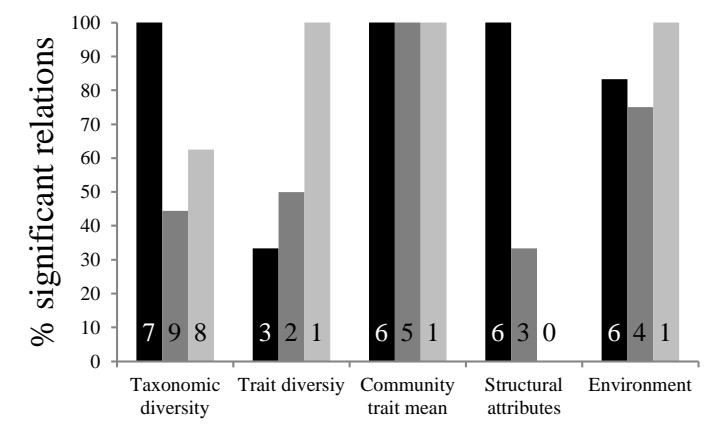

e)

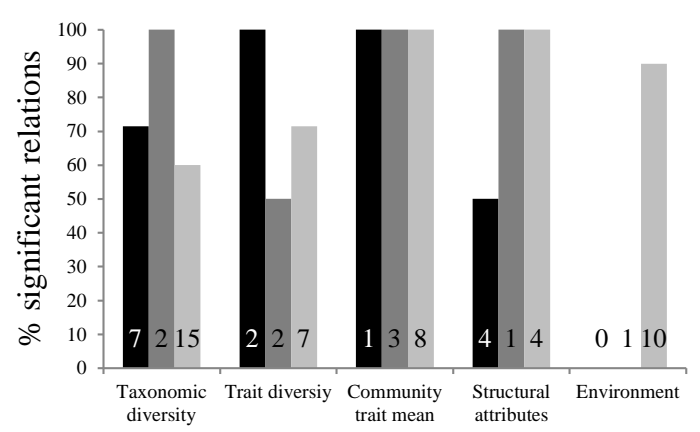

b) Carbon dynamics

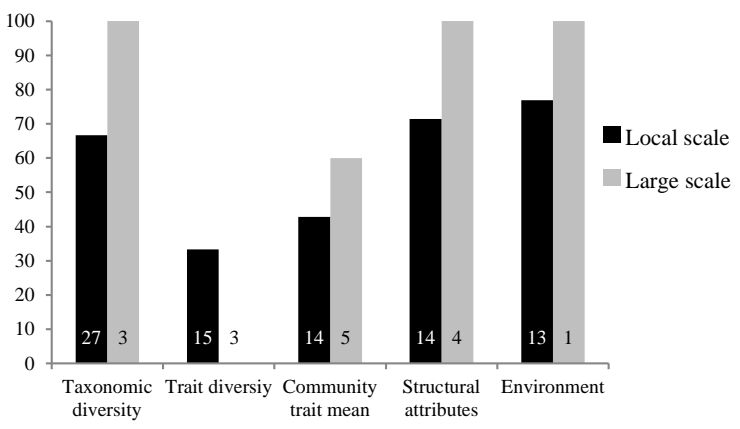

d)

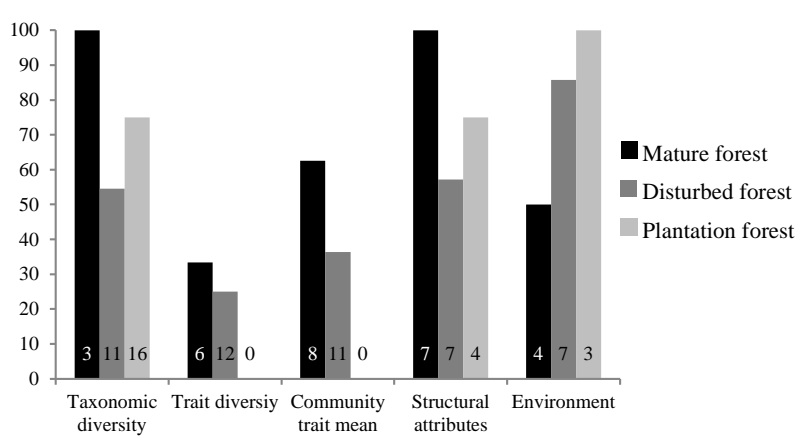

f)

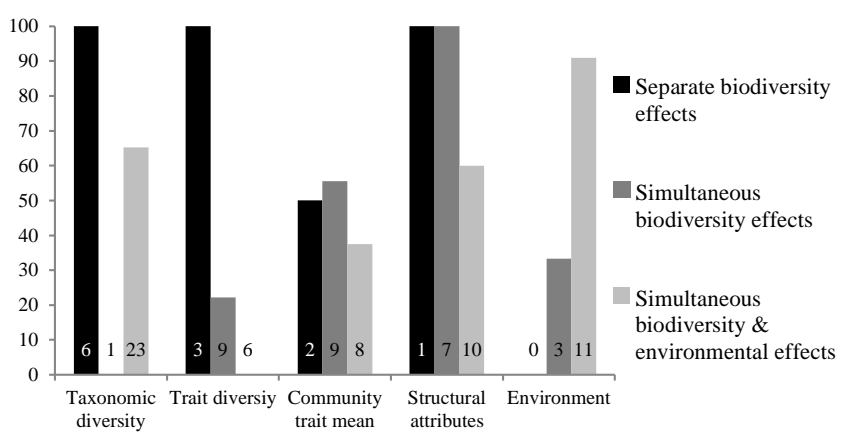

Appendix 7.4: Percentage of relationships showing a significant effect of four biodiversity attribute groups (taxonomic diversity, trait diversity, community trait mean and structural attributes) and environment on carbon stocks (all left panels) and carbon dynamics (all right panels) in empirical studies. Each pair of graphs shows a different grouping of studies: (a and b) by scale, comparing local vs. large spatial scale; ( $c$ and $d$ ) by management intensity, comparing plantation forests, disturbed forests and mature forests; and (e and f) by analytical framework used in the studies: all biodiversity attributes individually ('Separate biodiversity effects'), for multiple biodiversity attributes simultaneously ('Simultaneous biodiversity effects'), and for multiple biodiversity attributes and environmental variables simultaneously ('Simultaneous biodiversity \& environmental effects'). The numbers in the bars indicate the number of relationships that was evaluated. 


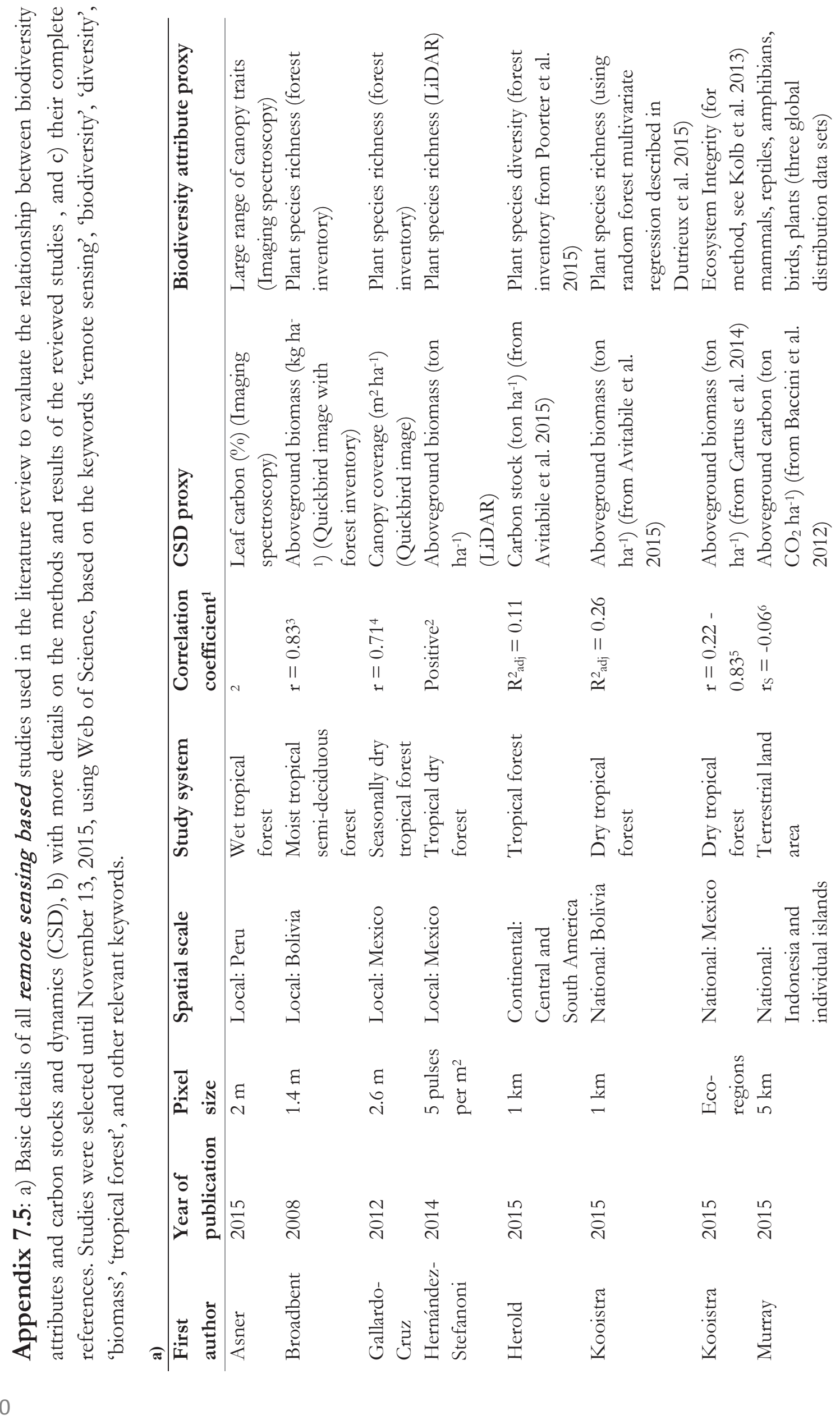




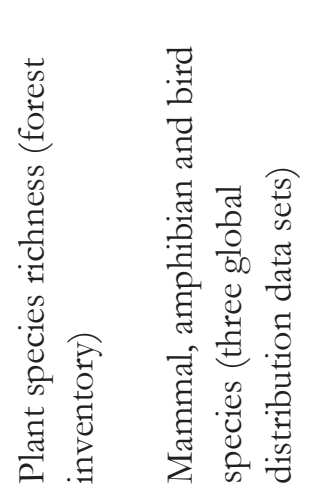

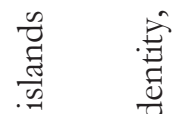

赵密苞

苟 $\quad \frac{0}{0}$

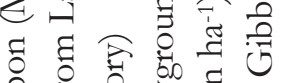

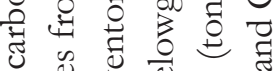

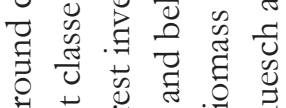

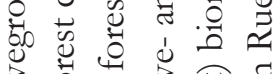

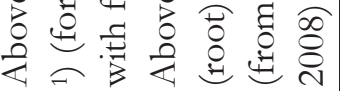

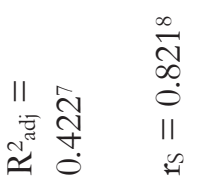

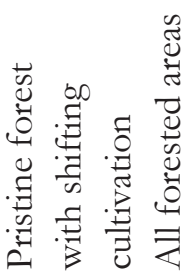

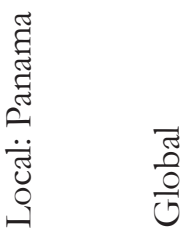

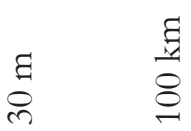

곡

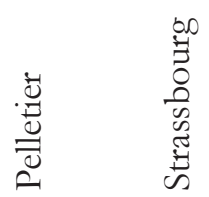

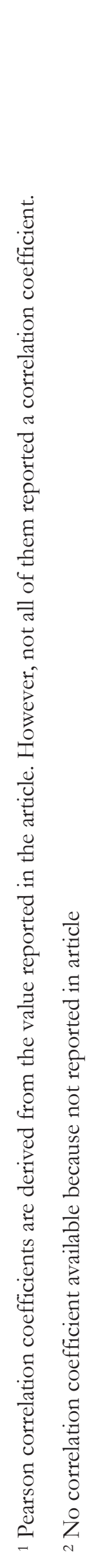

学 蔍

荧

趈 $\quad 0$

䒿 壱

章

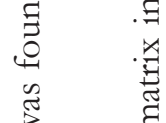

๙ 0

ป .

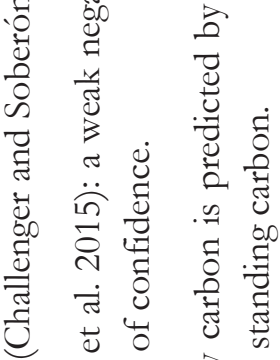

8 逮命

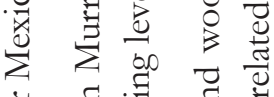

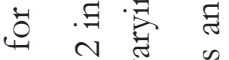

च

毒 त्ञ

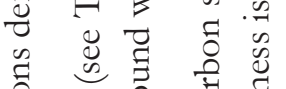

.5.

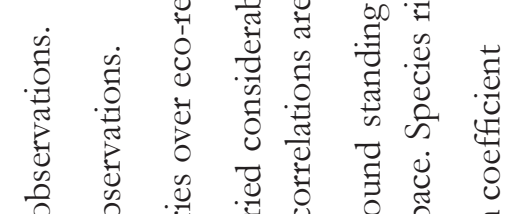

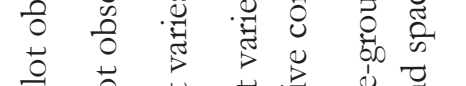

至 $\frac{0}{2}$ 苞

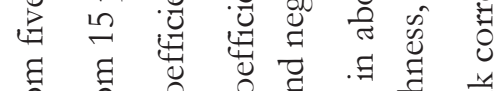

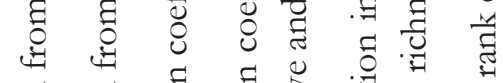

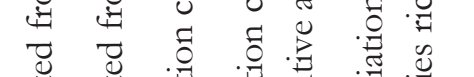

离

Uี

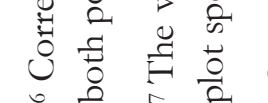




\section{b) Details on methods and results of reviewed studies, ordered from global to local scale.}

On a global level, Strassburg et al. (2010) used the best available global data sets on terrestrial biodiversity and carbon storage to map and investigate potential synergies between biodiversity and carbon across 124 regions. These regions had unique carbon stock values based on the IPCC Tier-1 method for estimating vegetation carbon stocks using the globally consistent default values provided for aboveground biomass. Belowground biomass (root) carbon stocks were added using the IPCC root to shoot ratios for each vegetation type (Ruesch and Gibbs 2008). A strong positive correlation $\left(r_{S}=0.82\right)$ between carbon stocks and species richness suggests that such synergies would be high, but resulting spatial maps also show an uneven distribution in correlation strength.

Pelletier et al. (2012) used remote sensing to distinguish for a forest reserve in Panama four forest classes differing in forest-use intensity and time-since-intervention (i.e., since deforestation). Their results show that $61.4 \%$ of the variation in aboveground standing carbon stocks and woody carbon is predicted by an explanatory matrix including land use, dominant species identity, plot species richness and space $\left(R^{2}\right.$ adjusted $\left.=0.42\right)$. Species richness showed a positive relation with standing carbon and was the explanatory variable most closely related to it.

Broadbent et al. (2008) linked field observations on the spatial distribution of biomass and tree species diversity to high-resolution Quickbird satellite imagery for a Bolivian lowland moist forest. They show that trees with crowns visible to nadir (i.e. observing straight down from sensor) remote sensing instruments compromise $86 \%$ of all tree species $>20 \mathrm{~cm}$ stem diameter as a structural forest trait. Hence, canopy biodiversity can be estimated well using remote sensing observations.

Gallardo-Cruz et al. (2012) examined in a dry forest in Mexico whether the structure and diversity of forest ecosystems can be estimated using the texture (the spatial variation of the image elements) of very high-resolution satellite imagery (pixel size $=2.6 \mathrm{~m}$ ). Basal area $\left(\mathrm{R}^{2}=\right.$ $0.93)$, vegetation height and cover (0.89), species richness (0.87), and stand age (0.85) were the best-described attributes by a two-variable regression model. Such image-texture analysis can reliably estimate basal area and fallow-age, thus allowing for the assessment of carbon sequestration and biodiversity loss rates.

Hernández-Stefanoni et al. (2014) used LiDAR to assess simultaneously species diversity and biomass for the Yucatan Peninsula in Mexico. They found that species richness was mainly explained by habitat heterogeneity $(27 \%-42 \%$; standard deviation values of LiDAR metrics in the plots), whereas biomass was mainly explained by vegetation structure (16-20\%; mean values of LiDAR metrics in the plots), and thereafter by habitat heterogeneity $(5-12 \%)$. Additionally, the study shows that plot size and plot spatial arrangement strongly influence the accuracy for the estimates of AGB and species richness obtained from LiDAR.

In a recent study, Asner et al. (2015) adopted airborne laser-guided imaging spectroscopy to develop maps of 16 forest canopy traits and in this way provided spatial distributions of plant functional traits within and across landscapes. Expanding this type of spectroscopic mapping of tropical forest landscapes can reveal the inter-connections between biological diversity, biogeochemical processes and carbon stocks and dynamics for tropical forest ecosystems.

Finally, as part of the ROBIN project (Role Of Biodiversity In climate change mitigatioN), three examples of remote sensing based assessment of the relationship between tree diversity and carbon stocks have been elaborated (Kooistra et al. 2015). First, at the continental level including tropical forest in Central and South America, Herold et al. (2015) found a weak positive relation $\left(\mathrm{R}_{\text {adj }}^{2}=0.11\right)$ between species richness and carbon stocks. Second, across ecoregions in Mexico, a 
positive relation between the biodiversity indicator Ecosystem Integrity (EI) and aboveground biomass (AGB) was found across ecoregions in Mexico (Challenger and Soberón, 2008). In this context, ecosystem integrity is constructed as a composite indicator composed of separate components related to structural diversity, functional diversity, taxonomic diversity and landscape level characteristics (Kolb et al. 2013). Third, for a case in Bolivian forest (Kooistra et al. 2015), a positive relation was observed between species richness (Dutrieux et al. in review) and aboveground biomass (Avitabile et al. 2015) both independently derived from remote sensing based data sources. For this study, a large part of the variation can be explained by the dry-wet gradient observed in the country (Fig. 7.2).

\section{c) Complete references of studies reviewed}

Asner GP, Anderson CB, Martin RE, et al. 2015. Landscape biogeochemistry reflected in shifting distributions of chemical traits in the Amazon forest canopy. Nat Geosci 8: 567-73.

Broadbent EN, Asner GP, Peña-Claros M, et al. 2008. Spatial partitioning of biomass and diversity in a lowland Bolivian forest: Linking field and remote sensing measurements. For Ecol Manage 255: 2602-16.

Gallardo-Cruz JA, Meave JA, González EJ, et al. 2012. Predicting tropical dry forest successional attributes from space: is the key hidden in image texture? PLoS One 7: e30506.

Hernández-Stefanoni JL, Dupuy JM, Johnson KD, et al. 2014. Improving species diversity and biomass estimates of tropical dry forests using airborne LiDAR. Remote Sens 6: 4741-63.

Herold M, Garcia-Esteban M, Lau Sarmiento A, et al. 2015. Effects of land use changes on ecosystem processes, carbon storage and climate change mitigation. Report ROBIN project D123.

Kooistra L, Dutrieux L, Equihua J, et al. 2015. Current contributions of biodiversity and ecosystems to climate change mitigation - an analysis using remote sensing datasets. Report ROBIN project D113.

Murray JP, Grenyer R, Wunder S, et al. 2015. Spatial patterns of carbon, biodiversity, deforestation threat, and REDD+ projects in Indonesia. Conservation Biology 29: 1434-1445.

Pelletier J, Codjia C, and Potvin C. 2012. Traditional shifting agriculture: tracking forest carbon stock and biodiversity through time in western Panama. Glob Chang Biol 18: 3581-95.

Strassburg BBN, Kelly A, Balmford A, et al. 2010. Global congruence of carbon storage and biodiversity in terrestrial ecosystems. Conserv Lett 3: 98-105. 
Appendix 7.6: Explanation of relevant changes in remote sensing during the last decade

The field of remote sensing has strongly changed over the last decade. First, the availability of remote sensing based data sources has increased substantially. This increase includes both image sources derived from satellite-based platforms (Kuenzer et al. 2014) manned airborne planes (Asner and Martin 2009) and Unmanned Aerial Vehicles (UAV) or drones (Getzin et al. 2014), which are increasingly used to map and monitor tropical forests states and processes at a detailed local scale (Asner et al. 2015).

Second, the increased temporal resolution of remote sensing observations has opened a whole new field of research in earth system monitoring (Wulder et al. 2012). For example, in 2008 Landsat provided a 40 year archive of $30 \mathrm{~m}$ resolution images. This development has led to a surge in high temporal resolution change detection methods, several of them specifically designed for monitoring forest cover (Hansen et al. 2013), biomass and diversity (DeVries et al. 2015).

Third, the improved spectral resolution, either through more spectral bands or narrower spectral bands, requires the development of alternative analysis methods like advanced multivariate statistical techniques or machine learning techniques, from which relations between a large number of spectral variables and ecological target variables can be explored (Dutrieux et al. in review). The next step is for these quantitative relations to be established and then adopted for scaling from point observations to a continuous map of specific plant traits (Asner and Martin 2009, Asner et al. 2015) and related diversity (Feret and Asner 2014).

Finally, the increasing availability of very-high resolution remote sensing datasets from space borne or sensors on (un)manned aerial platforms will allow an increased understanding between ground-based observation and the structural properties of tropical forest canopies (Broadbent et al. 2008). In that case the pixel is not the unit of analysis but instead an individual tree, tree gap or agricultural field is characterized as the object, allowing to take structural variables into account both spatially and through time.

\section{Appendix 7.7: Description of methods of Fig. 7.2}

Data were derived from Kooistra et al. (2015), based on which the figure was prepared by L. Dutrieux. Remote sensing-derived species richness was obtained from Dutrieux et al. (in review) and remote sensing derived aboveground biomass from Avitabile et al. (2015). Tree species richness is determined as the number of tree species from a list of 100 focal species that occur in a 1-ha permanent plots distributed across the lowlands of Bolivia. The correlation is derived and displayed for biogeographic zones defined from the map from Olson et al. (2001). Biogeographic zones with a forested area (defined by a tree cover $>40 \%$ ) lower than $20 \%$ of the total zone area were excluded from the analysis. Climatic variables (annual rainfall, temperature and rainfall seasonality) to explain spatial variation in correlation strength were obtained from WorldClim (Hijmans et al. 2005), and climatic water deficit was obtained from Chave et al. (2014b). In the description of Fig. 7.2, only the climatic variables that significantly affected the correlation strength are shown. 
Biodiversity effects on climate change mitigation

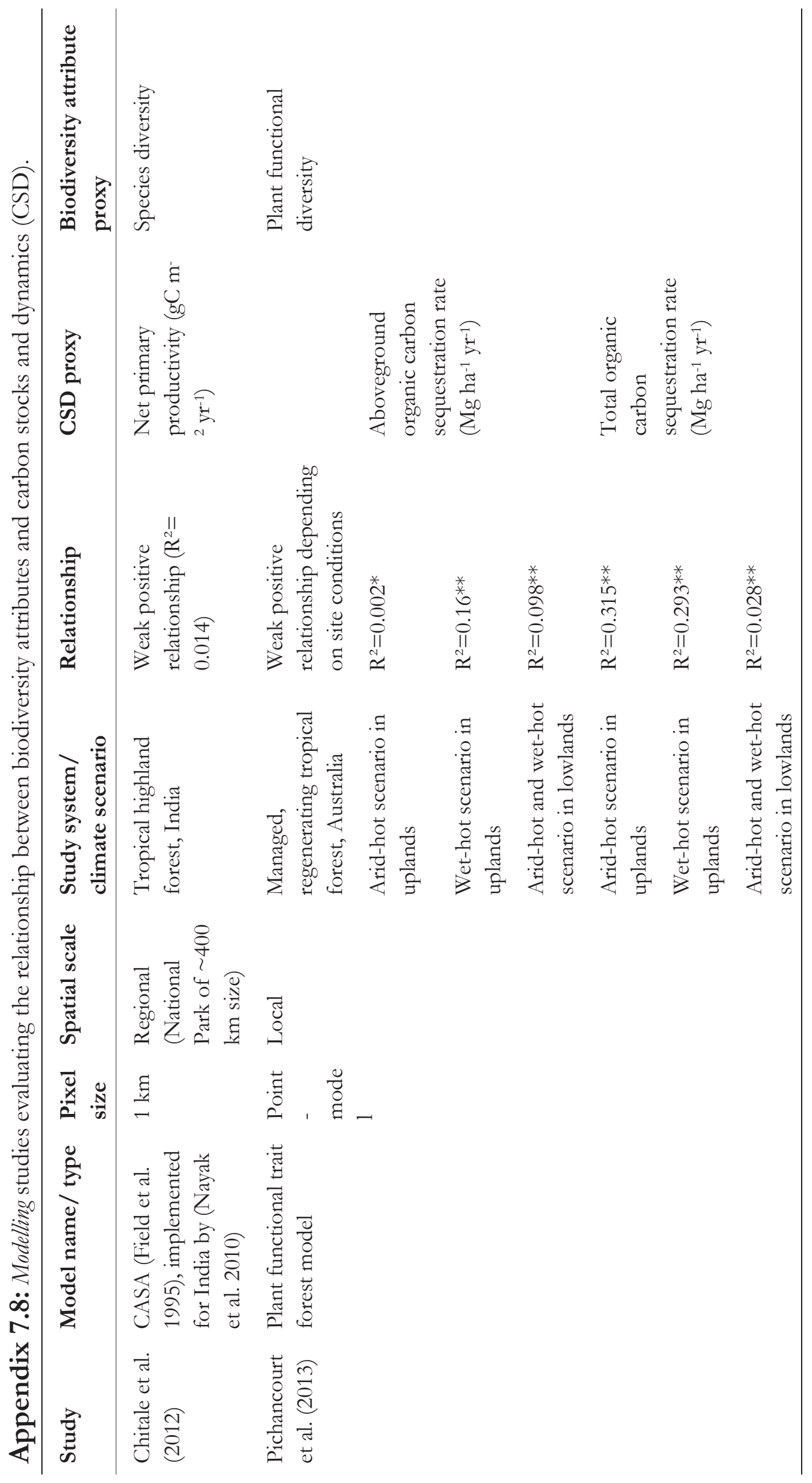


Appendix 7.9: Detailed information on Global Dynamic Vegetation Models

Given their power to represent physiology and carbon dynamics at regional to global scales and also under climate change conditions, DGVMs with embedded flexible individual traits provide an adequate framework to quantify biodiversity-CSD relationships, but need to diversify the influence of functional and morphological plant traits on carbon dynamics and the water cycle (e.g., McMahon et al. 2011; Van Bodegom et al. 2012). The LPJmL-FIT model, as an example for the next-generation variable-trait DGVMs, describes the interaction between the leaf and the stem economics spectrum for individual trees and CSD, water fluxes and plant competition in tropical forests. The importance to not only include trait ranges but to consider their trade-offs has been shown as essential to reproduce observed trait ranges for specific leaf area and wood density (Sakschewski et al. 2015). DGVMs can contribute to the investigation of potential longterm changes in the relationship between biodiversity and CSD by conducting experiments at the regional and continental scale, whereas dynamic plant functional trait models (Pichancourt et al. 2014) could conduct similar tests for specific sites. 



\section{Introduction - functioning forests across space and time}

Tropical forests are major contributors to globally important biogeochemical cycles, such as removal of $\mathrm{CO}_{2}$ from the atmosphere and water recycling (Bonan 2008, Alkama and Cescatti 2016). The magnitude of these processes, however, varies across space and time. For example, Poorter et al. (2015) have shown that there is strong spatial variation in aboveground biomass stocks across Neotropical forests, and Brienen et al. (2015) have shown there is temporal variation in $\mathrm{CO}_{2}$ sequestration capacity of tropical forests and that over time this capacity decreases. Spatial and temporal variation in these processes may be due to variation in abiotic conditions (such as climate and soil) and biotic conditions (i.e. properties of the vegetation: Box 1.1) (Mayle et al. 2004, Malhi et al. 2015). Global change may therefore strongly alter ecosystem processes by affecting abiotic and biotic conditions.

Besides changes in abiotic conditions, one of the main drivers of changes in ecosystem processes is expected to be biodiversity loss (an indicator of biotic conditions) caused by deforestation, fragmentation, environmental change, and hunting (Wright 2005, Betts et al. 2008). Field studies on temperate grasslands and theoretical studies provide important insights into the importance of species diversity for ecosystem processes and have mainly focused on biomass productivity (e.g. Loreau et al. 2001, Tilman et al. 2001, van Ruijven and Berendse 2010). Generally, these studies support the hypothesis that diversity increases ecosystem processes because of niche complementarity among species.

Tropical forests, however, are more diverse, structurally complex, and are composed of longer-lived plants, and the effects of their biodiversity on ecosystem processes may differ from the effects of simpler ecosystems. Recent studies on tropical forests found positive (Chisholm et al. 2013, Poorter et al. 2015), nonsignificant (e.g. Russell et al. 2004) or even negative (e.g. Potvin et al. 2011) relationships between species diversity and ecosystem processes. Hence, the role of species diversity on ecosystem processes of tropical forests remains debated. These contradictory results may be partly explained by the fact that species diversity measures do not provide information on the functional differences among species. The idea of niche complementarity implies that the functional traits of the species that determine the functional diversity of an ecosystem should be more important for ecosystem processes than species diversity. For this reason, research on 'biodiversity and ecosystem functioning' has undergone a shift from species-based towards using a trait-based approach in order to better understand and underpin the effects of species and of biodiversity on ecosystem processes.

The role of functional traits on performance at the species level is fairly well understood (Wright et al. 2004, 2010, Poorter et al. 2008). However, the aspects of 
tropical forests that provide globally important benefits are mainly due to community-level processes (e.g. biomass productivity of forest stands). We therefore need to understand how species diversity, trait diversity, and the mean trait value of the community (i.e. the community-weighted mean (CWM) traits) determine community-level processes. Henceforth, I will group species and trait diversity, CWM traits, and other vegetation attributes under the term 'biotic conditions' (see Box 1.1).

A complication in testing effects of biotic conditions on ecosystem processes of tropical forests is that it is difficult to tease apart the true and individual effect of biotic conditions from the effects of other co-varying variables. For example, species richness can be positively related to biomass stocks across Neotropical forests, but this relationship is at least partly driven by annual rainfall, which increases both species richness and biomass stocks (Poorter et al. 2015). Various studies have evaluated the effects of abiotic and biotic conditions separately (Baker et al. 2009, Finegan et al. 2015), but this does not reveal the independent role of biotic conditions on ecosystem processes. To avoid this problem, I tested for biotic effects while correcting for possible confounding abiotic variables, using a structural equation modelling approach.

The importance of mechanisms underlying ecosystem processes may in addition depend on spatial and temporal scale. I therefore assessed how spatial and temporal scales determine the relationships between abiotic and biotic conditions and ecosystem processes. As measures for ecosystem processes, I focused on biomass stocks and biomass dynamics (see Box 1.1). Biomass stock is a state variable rather than a process, but for simplicity, in this chapter I consider it as part of 'ecosystem processes'.

Following from these challenges, the main questions of this thesis (see also Figs. 1.1 and 1.2) were:

1. What are the independent relationships between abiotic conditions, biotic conditions, and biomass stocks and dynamics in tropical forests (chapters 2-5 and 7)?

2. How does spatial scale influence these relationships (chapters 2-5, 7 and additional analyses in this chapter)?

3. How does temporal scale influence these relationships?

a. How do biotic conditions respond to short-term temporal changes in abiotic conditions (chapter 6)?

b. How do biotic conditions determine the long-term stability of biomass stocks and dynamics (chapter 7 and this chapter)? 
In this final chapter (chapter 8), I synthesize the results of this thesis and other studies in order to answer my research questions. I will also discuss the role of science and its research priorities for safeguarding the functioning of tropical forests, and elaborate on the role that policy and society should have in assuring diverse and functioning tropical forests for the long term.

\section{Abiotic and biotic effects on ecosystem processes}

In this thesis I refer to biotic conditions as properties of the vegetation, such as species or trait diversity, CWM trait values, and forest structure. Biotic conditions could determine ecosystem processes in three main ways. First, diversity in species and traits would result in greater spatial and temporal complementarity in resource use among species and in faster process rates or larger stocks (i.e. the niche complementarity theory, Tilman 1999), and, in the face of an unstable or changing climate, in more stable processes (i.e. the insurance theory, Yachi and Loreau 1999). Second, the dominant species and their traits, also called the community-weighted mean trait values, would determine the processes of the community (i.e. the mass-ratio theory, Grime 1998). Third, the vegetation structure and quantity (e.g. the stem number or total basal area) determine ecosystem processes (Lohbeck et al. 2015). In this discussion chapter, I will mainly focus on the niche complementarity and mass-ratio theories, because these were evaluated in most of the chapters.

Abiotic conditions such as soil fertility, annual rainfall, and light availability can determine ecosystem processes directly, and indirectly by steering biotic conditions (e.g. through environmental filtering, Keddy 1992). In several chapters (2-5 and 7) I tested these hypotheses and the independent relationships between abiotic conditions, biotic conditions, and biomass stocks and dynamics. The vegetation can also affect the abiotic conditions (e.g. Wardle et al. 2004), but I have not evaluated these effects in this thesis.

\section{The findings from the four test cases}

I first evaluated the individual-tree level (chapter 2), then scaled up to the local community level (chapters 3 and 4) and to communities across the Neotropics (chapter 5), and finally reviewed the literature (chapter 7). In chapter 2, we ${ }^{1}$ evaluated how biotic conditions (functional traits) determine the biomass growth of individual canopy trees in a Bolivian moist semi-deciduous tropical forest. We found that the single most important driver of growth is the sapwood area of the

\footnotetext{
1 "we" is used when referring to research chapters in which co-authors are involved, and "I" for information or discussion that is new or related to this thesis in general.
} 
stem. A large sapwood area increases water transport and storage and may therefore be important for the growth of these large individuals that have long hydraulic paths and exposed crowns that face high evaporative demands, especially during dry periods. We found no effects of soil texture and fertility (as biotic conditions) on canopy tree growth (results not shown in this thesis). Unfortunately, because of the great extent of their root system (Jones et al. 2011) it is practically impossible to obtain a good measure for the soil conditions that large trees experience, which may partly explain why we found no effect of soil conditions. In addition, the soil in this forest is relatively fertile (Quintero-Vallejo et al. 2015) and thus nutrient availability may not be the main limiting factor for tree growth (see also chapter 4). Since all trees studied were emergent, they received equal and high amounts of light and therefore it is also unlikely that light availability is a factor limiting biomass growth. Instead, the importance of water transport and storage by sapwood area suggests that an important determinant of the growth of canopy trees in this semi-deciduous forest is water availability.

In chapters 3 and 4 we scaled up from individual tree growth to communitylevel biomass dynamics and tested for effects of abiotic and biotic conditions. For this we used data from two forests at the extremes of environmental and floristic (i.e. species composition) gradients found in the Amazon: Guyana and Bolivia (Quesada et al. 2010, ter Steege et al. 2013). In both chapters we used structural equation modelling, which allows for causal hypothesis testing (Shipley 2004, Grace 2006) and enables the separate effects of abiotic and biotic conditions on biomass stocks and dynamics to be discerned.

For a tropical wet forest in Guyana on very nutrient-poor soils (van Kekem et al. 1996, Quesada et al. 2011), we tested how abiotic and biotic conditions drive the productivity and stocks of aboveground biomass, fine root biomass, and soil organic matter in plots of 0.4 ha (chapter 3). Soil fertility and CWM leaf traits had strong effects on biomass stocks and productivity, whereas species richness had no effect on aboveground biomass, soil organic matter or productivity, and even affected fine root biomass negatively. These results indicate that soil fertility especially phosphorus concentration - is strongly limiting in this forest, and therefore determines biomass stocks and productivity not only directly, but also indirectly by allowing only a small set of species with the appropriate traits to become dominant. Hence, due to the exceptionally poor soils in this forest, environmental filtering (Keddy 1992) is strong, and therefore we found important effects of the CWM trait values, but not of species richness, on biomass stocks and productivity.

For a moist semi-deciduous forest on very fertile soils in Bolivia, we evaluated at the 1-ha scale how abiotic and biotic conditions drive three demographic processes that underlie net biomass change: biomass growth by trees that recruit 
(referred to as 'biomass recruitment'), biomass growth by trees that survive ('biomass growth'), and biomass loss due to tree mortality ('biomass mortality'; chapter 4). Net biomass change was most directly related to globally important ecosystem functions such as net $\mathrm{CO}_{2}$ sequestration, but we may only be able to understand net biomass change through evaluating its underlying demographic processes. We found that net biomass change was most strongly determined by biomass mortality, but that mortality itself was not related to any of the abiotic and biotic variables. Diversity and CWM trait values predicted biomass recruitment and biomass growth poorly or not at all. Instead, biomass recruitment decreased with plot basal area (a measure of forest structure) because of low light availability in dense stands, and it increased with soil water availability. Biomass growth increased with plot basal area because more biomass was available to contribute to growth. In addition, biomass growth increased on sandy soils, possibly because here the roots could more easily penetrate to greater depths, thereby increasing their access to water. Hence, for a wet forest on poor soils (chapter 3), biomass stocks and dynamics are driven by soil fertility and CWM traits, whereas for a moist semideciduous forest on fertile soils (chapter 4), biomass dynamics are driven by soil water availability and forest structure.

The relationships between abiotic conditions, biotic conditions, and biomass stocks and dynamics thus seem to be strongly site-dependent. But how would abiotic and biotic conditions drive biomass stocks and dynamics across multiple sites with a wide range of environmental conditions? In chapter 5 we evaluated this question across 26 Neotropical forests, using an approach similar to that described in chapters 3 and 4. We evaluated demographic processes (biomass recruitment, growth, and mortality), net biomass change, and biomass stocks. In contrast to the two site studies, we found very strong effects of biotic conditions especially species diversity and CWM trait values - on ecosystem processes; species richness increased all demographic processes and biomass stocks (except for net biomass change), CWM trait values significantly determined biomass stocks and all demographic processes (except for biomass mortality), while plot basal area increased biomass growth, mortality, and stocks. Species richness may increase biomass mortality because it leads to inherently more dynamic forests. Effects of species richness and CWM trait values on ecosystem processes may be strong because vegetation properties (i.e. biotic conditions) determine vegetation dynamics most directly and because they also reflect variation in abiotic conditions that leads to different vegetation types.

Water availability increased biomass growth, recruitment, and stocks, while soil fertility increased biomass recruitment and net biomass change. It seems counterintuitive that across large abiotic gradients, abiotic conditions are less important than biotic conditions. It could be that most of the abiotic effects are 
manifested through their effect on biotic conditions, and thus that abiotic conditions have stronger indirect effects at the expense of their direct effects (Figs. 5.2 and 5.3).

Net biomass change was most strongly determined by biomass mortality (chapters 4 and 5). Mortality itself, however, was only explained by few abiotic and biotic conditions, indicating that the strongest driver of net biomass change is largely unpredictable (see also chapter 4).

\section{Generalizing biotic effects on ecosystem processes}

The four chapters discussed so far (2-5) present one tree-level study, two community-level case studies in contrasting forest types, and one study on 26 sites across the Neotropics. Other studies have also evaluated the effects of biotic conditions on biomass stocks and dynamics. However, these studies differ in the indices used for biotic variables, measures of biomass stocks and dynamics, forest type, spatial scale, site conditions, and in their analytical approach. All these differences in variables and approaches limit the possibilities to integrate studies, and thereby limit our understanding of the role of biotic conditions on biomass stocks and dynamics. To nevertheless be able to evaluate the relationship between community-level biotic conditions (i.e. species diversity, trait diversity, CWM traits, and vegetation quantity) and community-level stocks and dynamics of biomass (or 'carbon') in tropical forests, in chapter 7 we reviewed the literature, focusing on studies that use empirical data, remote sensing data, or modelling data. This review included 38 empirical studies that evaluated relationships of biotic conditions (or 'biodiversity attributes', Box 1.1) on biomass stocks and dynamics. We found that most $(74 \%)$ of the studies report significant effects of one or multiple biotic conditions on biomass stocks and dynamics. For the studies that also took abiotic conditions into account and that evaluated the independent effect of biotic conditions on biomass stocks and dynamics, the percentage of studies reporting significant biotic effects was slightly lower $(71 \%)$. Hence, even when accounting for co-varying abiotic conditions, the biotic effects on biomass stocks and dynamics remained very important. This importance of biotic conditions had also been reported by the few remote sensing and modelling studies that we found. This confirms my earlier findings that biotic conditions have an important effect on biomass stocks and dynamics that is independent of effects of abiotic conditions. 


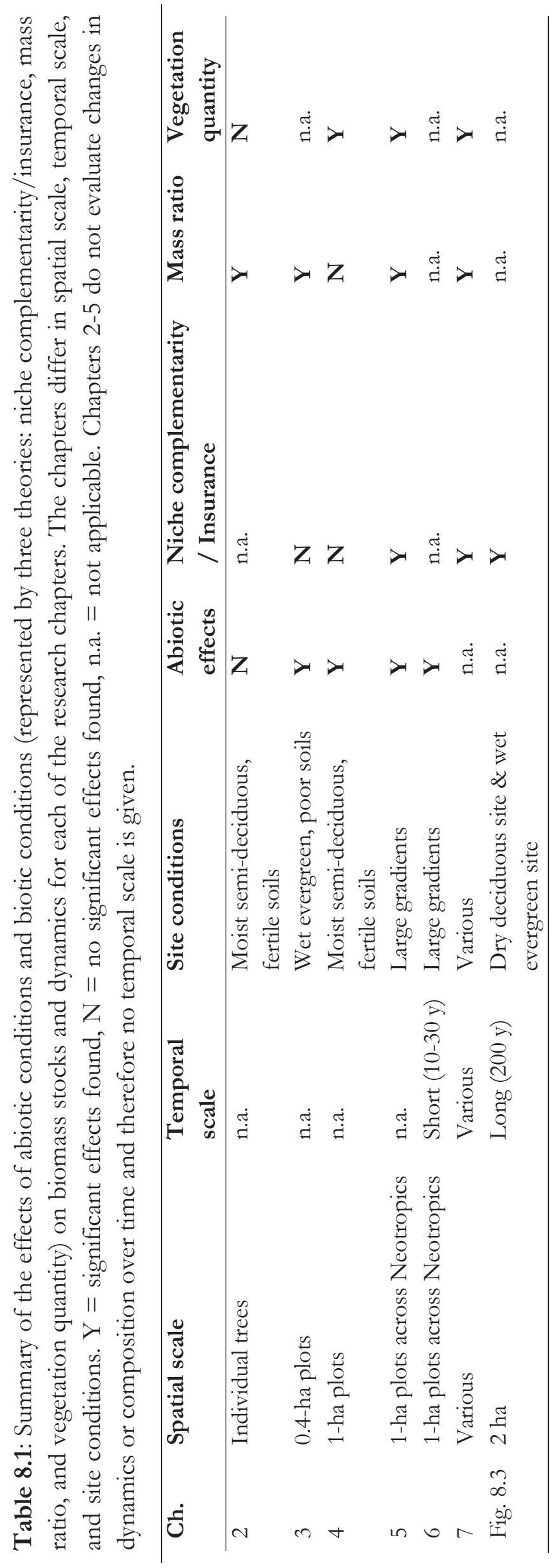




\section{Mechanisms underlying abiotic and biotic effects on ecosystem processes}

The results presented in chapter 7 thus show that in most of the studies, biotic conditions were important for biomass stocks and dynamics. But can we also understand which mechanisms underlie this relationship? In chapters 2-5 and 7 we evaluated the relative importance of niche complementarity, mass ratio, and vegetation quantity, and found that overall the mass ratio (represented by CWM trait values) and vegetation quantity were more important than niche complementarity (represented by species diversity and functional trait diversity) in explaining biomass stocks and dynamics at local scales (Table 8.1). However, across Neotropical forests (chapter 5), niche complementarity and mass ratio were most important. This indicates that at a given moment in time and at a local scale, niche complementarity through species and trait diversity contributes to biomass stocks and dynamics to a limited extent only. Possibly, the effect of diversity on ecosystem processes weakens at the high diversity found in these forests (Balvanera et al. 2005, Reich et al. 2012), or maybe high diversity becomes important only when evaluating multiple ecosystem processes simultaneously (Hector and Bagchi 2007, Isbell et al. 2011, but see Lohbeck et al. under review), across longer timescales (Isbell et al. 2015), or across larger spatial scales (chapter 5, see also '2. A matter of spatial scale?').

Contrary to the results across Neotropical forests, the major drivers of smallscale and short-term biomass stocks and dynamics are the average type of species in the forest (i.e. mass ratio; Finegan et al. 2015) and the quantity of vegetation in the forest (Lohbeck et al. 2015). The importance of these mechanisms, however, also depends on the ecosystem process considered. Mass ratio was important for biomass stocks in $100 \%$ of the cases evaluated and was important for biomass dynamics in $47 \%$ of the cases (chapter 7). In contrast, niche complementarity was important for biomass dynamics in $18-30 \%$ of the cases $30 \%$ for species diversity and $18 \%$ for trait diversity) and for biomass stocks in $6-24 \%$ of the cases (24\% for species and $6 \%$ for trait diversity). This indicates that the traits of the dominant species (i.e. mass ratio) determine the amount of biomass that can be stored per area, whereas the species diversity (i.e. niche complementarity) mainly determines the rates of biomass change.

How do abiotic site conditions affect the relationship between biotic conditions and biomass stocks and dynamics? We tested the hypothesis that resource limitation strongly determines the species type and diversity in the forest, and thus that biotic conditions would be more important for biomass stocks and dynamics in resource-poor sites than in resource-rich sites. This thesis and other studies do indeed show that CWM trait values are more important in forests experiencing strongly limiting resource availability, such as on the nutrient-poor soils in Guyana (chapter 3) and in dry forests in Brazil (Prado-Junior et al. 2016), 
than they are in forests with greater resource availability, such as those on the fertile soils in Bolivia (chapter 4). This indicates that strong environmental filtering determines the type of species that become dominant and hence determines the CWM trait values, which in turn strongly affect ecosystem processes. It could also be that in sites with extremely high availability of water and soil nutrients, competition becomes so strong that only the most competitive species become abundant and drive ecosystem processes, and thus that mass ratio is also important at the extremely resource-rich end of the spectrum. Hence, limiting abiotic conditions strongly shape the type of species present in the community (i.e. strong mass-ratio effect).

How do abiotic conditions determine biomass stocks and dynamics? In most of the chapters in this thesis we demonstrated that abiotic conditions have direct effects on ecosystem processes. Other studies have also shown strong effects of abiotic conditions on ecosystem processes (e.g. Malhi et al. 2004, Aragão et al. 2009, Baraloto et al. 2011, Durán et al. 2015). As expected, my findings show that the relevant abiotic variables are context-dependent: the most limiting resources in a forest are also those that most strongly determine biomass stocks and dynamics. For example, what is most important for ecosystem processes on old and leached soils of the Guyana Shield is nutrient availability (chapter 3), whereas in moist forests at the southern fringe of the Amazon basin it is soil water availability (chapter 4), and across large spatial scales both soil fertility and water availability (through rainfall) are important because these scales include forests where soil fertility is limiting and forests where water availability limits the biomass stocks and dynamics (chapter 5, but see Poorter et al. 2015). Thus, abiotic conditions are of major importance for biomass stocks and dynamics. At local scales abiotic effects are strongly context-dependent, whereas at larger spatial scales all abiotic conditions play a role because they are limiting in at least some forests.

\section{Which diversity and trait indices are most relevant for ecosystem processes?}

Multiple indices can be used to scale from individual trees to community properties, and can serve as proxies for niche complementarity, mass ratio, and vegetation quantity. For niche complementarity, I expected that variation in species' strategies and thus trait diversity would be a better indicator for ecosystem processes than species diversity. Surprisingly, species diversity indices rather than multivariate trait diversity indices more often explained biomass stocks and dynamics (chapters 4 and 7). In chapter 4, we argued that the weaker effect of trait diversity could be due either to important traits that were not included in the 
multivariate trait diversity indices, such as physiological traits and dispersal traits, or to including similar traits that result in a relatively small multivariate space. An alternative explanation is that the diversity in only one or a few traits is important but their effect is diminished when less important traits are included in multivariate trait indices.

For the mass-ratio theory, I expected that the CWM traits that were hypothesized to be most relevant for ecosystem processes would show the strongest effect on biomass stocks and dynamics. However, we found that the most important CWM traits seem to depend mainly on the limiting abiotic variable. For example, leaf nutrient concentrations are important when soil fertility is low (chapter 3), and sapwood area is important when water availability is limiting (for canopy trees in chapter 2). Hence, only in the tight context can traits be called 'functional'.

Of the studies reviewed in chapter 7, most used only wood density (WD) (13 out of 16 studies) and specific leaf area (SLA) (9 out of 16) to predict biomass stocks and dynamics. WD showed significant effects on biomass stocks and dynamics in $46 \%$ of the 13 studies and SLA did so in $44 \%$ of the 9 studies. Both WD and SLA are used as proxy for multiple processes or 'hard' traits (see Table 3.1, 4.1, 6.1, Appendix 5.3), which are traits that are more directly linked to performance but are also more time-consuming to measure (Hodgson et al. 1999, Larjavaara and Muller-Landau 2010). These multiple roles of 'soft' traits such as WD and SLA may make it difficult to understand trait effects on one process. For example, high SLA values increase light interception efficiency and are generally related to an acquisitive growing strategy (Poorter and Bongers 2006), but SLA values are also high in dry forests because of a short leaf lifespan, and may thus also indicate a conservative growing strategy (see discussion in chapter 6). WD is often related to mechanical strength and drought tolerance (because narrow and tough vessels increase resistance to drought-induced cavitation: Poorter and Markesteijn 2008), but various wood tissues contribute to WD, with vessel properties mainly determining drought tolerance (Cochard and Tyree 1990). Traits such as SLA and WD can thus be indicators of multiple processes or ecological strategies, depending on where the plant is growing. None of the studies reviewed used hard traits such as photosynthetic capacity or hydraulic conductivity to predict biomass stocks and dynamics. Moreover, many studies may overlook the traits that are most directly influenced by the limiting abiotic conditions, and therefore they may underestimate the importance of mass ratio for ecosystem processes. In sum, species diversity better explains biomass stocks and dynamics than trait diversity, the most relevant CWM traits depend on the locally limiting abiotic conditions, and a shift from soft to hard traits may be needed to better understand ecosystem processes. 


\section{A matter of spatial scale?}

\section{Scale effects in this thesis}

The importance of different mechanisms underlying biomass stocks and dynamics may also depend on the spatial scale considered (Chisholm et al. 2013, Poorter et al. 2015). Across large spatial scales, such as the Neotropics, variation in abiotic conditions is strong and may therefore strongly determine variation in biomass stocks and dynamics (Fig. 1.2). At smaller spatial scales, such as within one forest type, variation in abiotic conditions is smaller and biotic interactions may be more important (McGill 2010, but see Messier et al. 2010). Biotic mechanisms such as those defined in the niche complementarity and mass-ratio theories may therefore most strongly determine biomass stocks and dynamics at small spatial scales.

We tested the relationships between abiotic and biotic conditions and biomass stocks and dynamics at various spatial scales: individual trees (chapter 2), 0.4-ha and 1-ha communities within one forest type (chapters 3 and 4), and 1-ha communities across Neotropical forests (chapter 5). We found strong effects of traits on growth of individual trees, support for the mass ratio for biomass stocks and dynamics in 0.4-ha communities within one forest type, and support for niche complementarity and mass ratio across Neotropical forests, but no support for niche complementarity or mass ratio in 1-ha communities within one forest type (Table 8.1). Abiotic conditions were important for the 0.4-ha plots, 1-ha plots, and across Neotropical forests, but not important for individual canopy trees. Hence, these results show that biotic conditions were most influential at the smallest and largest spatial scales (cf. Messier et al. 2010), and that abiotic conditions were very influential at all spatial scales except for the individual-tree scale.

The review in chapter 7 also shows that biotic conditions are generally more important at scales larger than local (e.g. across Neotropical forests, as in chapter 5). Possibly, the biotic conditions at small community-level spatial scales vary only modestly and therefore less strongly determine differences in biomass stocks and dynamics. In contrast, at very large spatial scales, abiotic conditions vary strongly and lead to strong differences in biotic conditions (e.g. the CWM traits) which, in turn, affect biomass stocks and dynamics (e.g. Fig. 5.2).

\section{Explicit tests for scale effects}

The observed differences between scales in abiotic and biotic effects on biomass stocks and dynamics might be attributable to measurements of abiotic conditions at the smallest and largest spatial scales being less accurate than at the intermediate spatial scale. In addition, the chapters in this thesis and the external studies reviewed in chapter 7 differ not only in spatial scale, but also in site conditions and 
variables used. It is therefore impossible to fully tease apart the effect of spatial scale from other differences among the studies.

To explicitly test how spatial scale determines the importance of biotic conditions for biomass productivity, I here perform additional analyses at various spatial scales but covering the same region of La Chonta, Bolivia: across individuals of the same species, across 0.1 -ha plots, across 0.5 -ha plots, across 1 -ha plots, and finally, across Neotropical forests. At each scale, I calculated the coefficient of variation (CV) of specific leaf area (SLA) and leaf nitrogen concentration $\left(\mathrm{N}_{\text {leaf }}\right)$. A high $\mathrm{CV}$ at a given scale may indicate that differences in abiotic conditions are strong enough to result in large variation in (CWM) trait values, and could result in a potentially strong effect of the trait on biomass stocks and dynamics. I used SLA and $\mathrm{N}_{\text {leaf }}$ because they are 1) closely related to photosynthetic capacity and thus to productivity, 2) often-used and easy-to-measure traits, 3) easy to scale up from individual trees to communities, and 4) available for all five spatial scales.

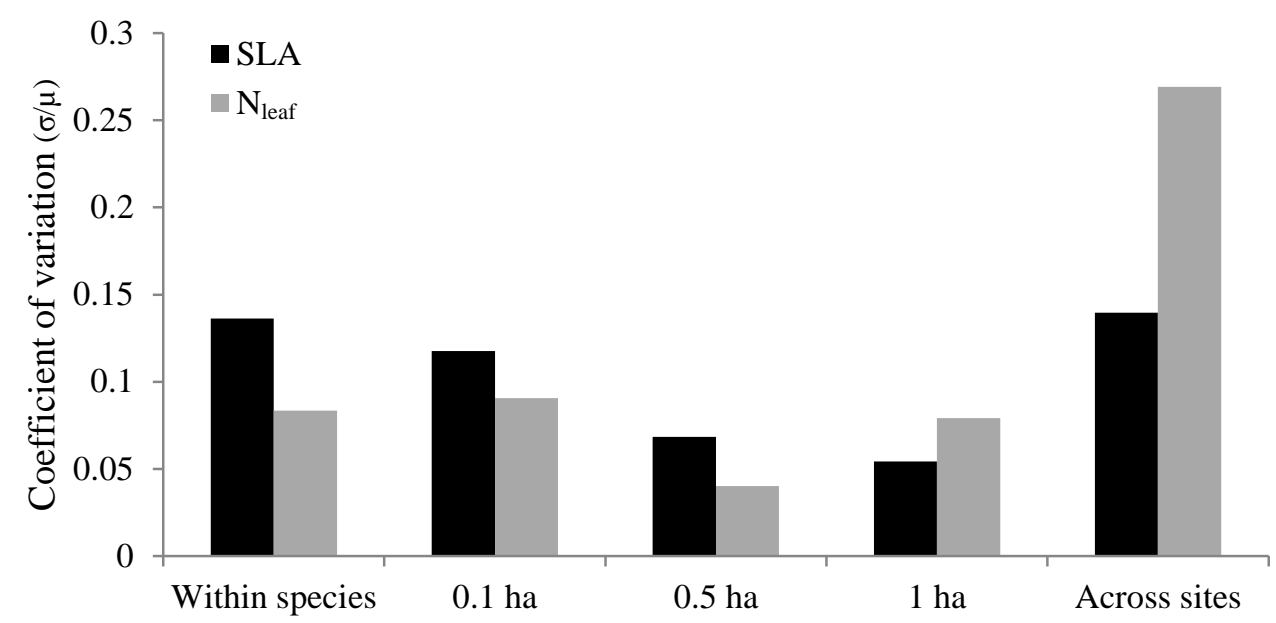

Figure 8.1: Coefficient of variation (calculated as the standard deviation divided by the mean) of specific leaf area (SLA, black bars) and leaf nitrogen concentration $\left(\mathrm{N}_{\text {leaf }}\right.$, grey bars) at various spatial scales: among individuals of the same species, among 0.1-ha plots, among 0.5-ha plots, among 1-ha plots, and for 1-ha plots across 23 Neotropical forest sites. Within species, SLA and $\mathrm{N}_{\text {leaf }}$ were based on average trait values of individual canopy trees (from chapter 2). Coefficient of variation within species was first calculated per species ( 4 species and 43 trees in total), and then averaged across the species. For all the other scales, community-weighted mean (CWM) trait values were calculated weighted by species' basal area, based on undisturbed plots. The 0.1-, 0.5-, and 1-ha scales were based on 9 1-ha plots in La Chonta, Bolivia. For the 0.1-ha scale, the central $32 * 32 \mathrm{~m}$ of each plot was used, and for the 0.5 -ha scale, the central $70 * 70 \mathrm{~m}$ of each plot was used. Data across the 23 sites were obtained from chapter 5 (Appendix 5.1 and 5.2). For sites with multiple plots, the average CWM SLA and CWM $\mathrm{N}_{\text {leaf }}$ were used. $\mathrm{N}=23$ for SLA and $\mathrm{N}=$ 8 for $\mathrm{N}_{\text {leaf }}$. 
SLA and $\mathrm{N}_{\text {leaf }}$ showed the highest $\mathrm{CV}$ at smallest and largest scales, and lowest $\mathrm{CV}$ at intermediate scales (Fig. 8.1). Possibly, small-scale heterogeneity in environmental conditions (such as soil) drives variation in individual-tree trait values or CWM trait values, while large-scale gradients in climatic conditions drive large-scale variation in species composition and thus in CWM trait values. At intermediate scales, however, small-scale soil heterogeneity is averaged out and variation in climatic conditions is still weak, so therefore the variation in CWM trait values is small. This is in line with our findings that biotic conditions most strongly determine biomass stocks and dynamics at smallest (chapters 2 and 3) and largest spatial scales (chapter 5). The next question then is whether these differences in the $\mathrm{CV}$ of traits between spatial scales do indeed lead to different relationships between traits and productivity (as hypothesized in Fig. 1.2). Surprisingly, I found that the effect of both SLA and $\mathrm{N}_{\text {leaf }}$ on aboveground biomass growth did not differ between spatial scales (Fig. 8.2), except for a significant effect of $\mathrm{N}_{\text {leaf }}$ for all community-level scales, but that there was no effect of $\mathrm{N}_{\text {leaf }}$ for individual trees (Fig. 8.2b vs. d). These results of CWM trait values do not agree with the results of studies evaluating species diversity effects on productivity at the community level, which report stronger effects at smaller spatial scales (i.e. 0.04-0.1 ha) than at intermediate spatial scales (0.25-1 ha: Chisholm et al. 2013, Poorter et al. 2015). CWM trait values are indicators for mass-ratio effects, whereas species and trait diversity are indicators for niche complementarity, and these mechanisms may differ in scale dependence. It could be that spatial scale is important for the effect of niche complementarity, since at small spatial scales species interact and less redundancy may occur among species. In contrast, scale is less important for the effect of mass ratio, which may mainly depend on environmental conditions. Alternatively, it could be that scale effects are only visible when using plots that have been established over a larger area (as done in Chisholm et al. 2013, Poorter et al. 2015). Instead, other factors than scale may determine the differences in results among the chapters of this thesis, such as abiotic site conditions or variables used. I only evaluated this scale effect for one site, two traits, and plot sizes up to 1 ha; nevertheless, these results provide a first indication that although the variation in (CWM) traits is scale-dependent, their effect on ecosystem processes is not strongly scale-dependent. 

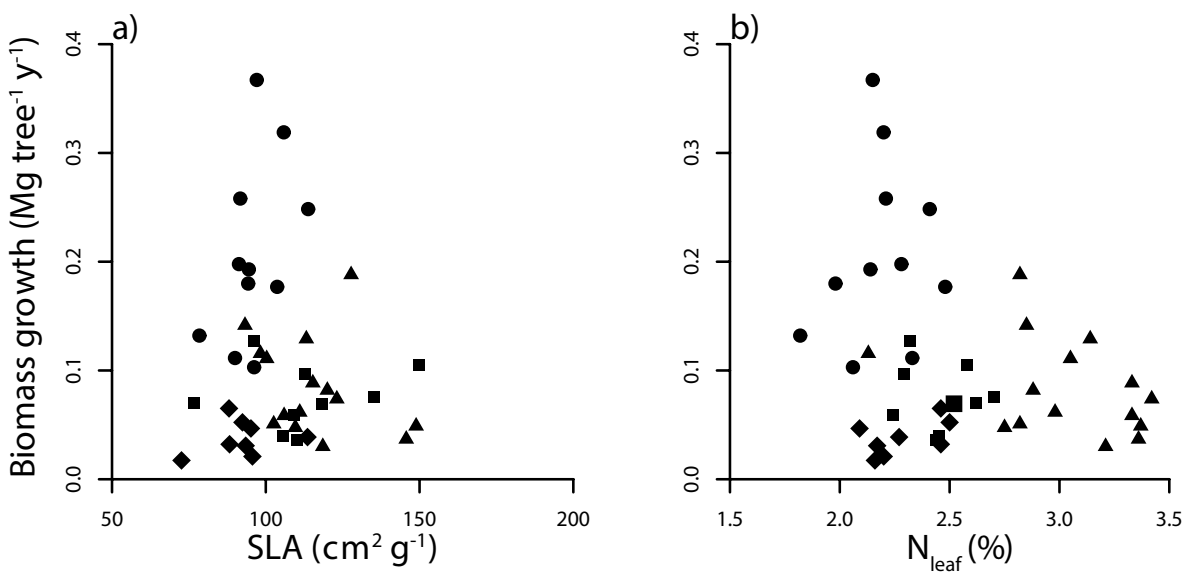

- Cariniana

- Hura

- Schizolobium

- Sweetia
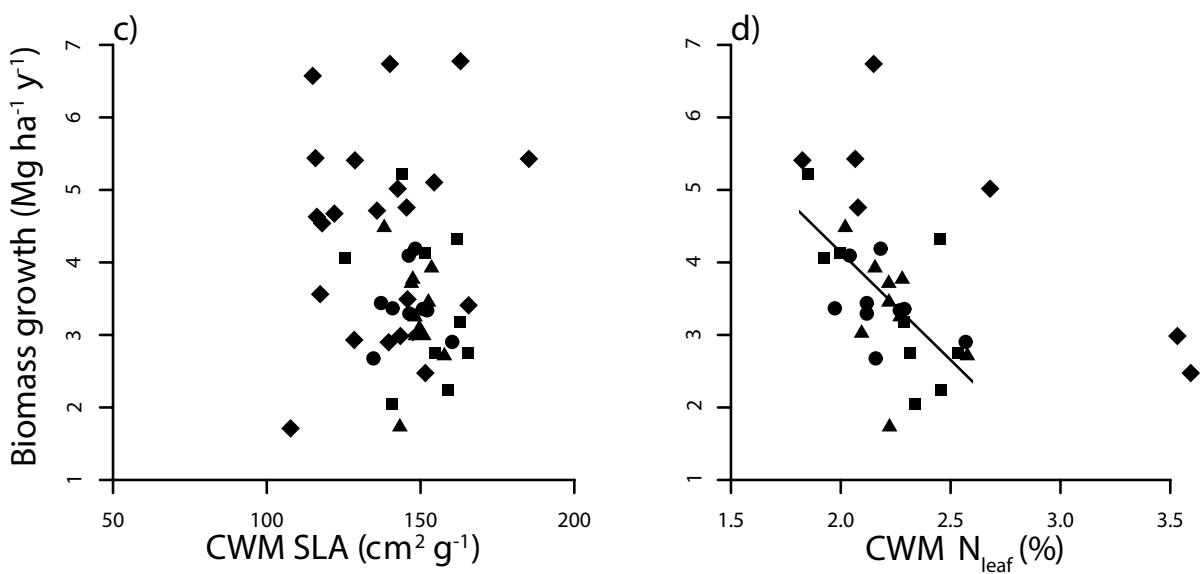

Figure 8.2: Relationship of specific leaf area (SLA) and leaf nitrogen concentration per unit leaf mass $\left(\mathrm{N}_{\text {leaf }}\right)$ with biomass growth at different spatial scales in the La Chonta forest, Bolivia: (a and b) among individual canopy trees (from chapter 2) and (c and d) at the community level. The canopy trees in panels a and b belong to four species: Cariniana ianeirensis (black dots), Hura crepitans (red dots), Schizolobium parabyba (blue dots), and Sweetia fruticose (grey dots). For the community level, we used four spatial scales: 0.1-ha plots (black dots), 0.5-ha plots (red dots) and 1-ha plots within La Chonta (blue dots), and 1-ha plots across Neotropical forest sites (grey dots). Biomass growth for individual canopy trees was based on the 5 -year average biomass growth (for more details see chapter 2), and the biomass growth at the community level was based on growth of surviving trees (for more details see chapter 4) because these trees are tall and would thus be best comparable with growth of the individual canopy trees. At the community level, the community-weighted mean (CWM) SLA and $\mathrm{N}_{\text {leaf }}$ were weighted by species' basal area. For details on plot selection and sample size, see the description of Fig. 8.1. Relationships were tested using linear models with an interaction between trait and species in panel a and b, and an interaction between trait and spatial scale in panels $\mathrm{c}$ and d. SLA did not affect biomass growth of canopy trees (panel a; $\mathrm{F}=1.42, \mathrm{P}=0.24$ ) and did not interact with species $(\mathrm{F}=2.19, \mathrm{P}=0.11$ ). Nor did SLA affect biomass growth at the community level (panel c; $\mathrm{F}=0.70, \mathrm{P}=0.41$ ) or interact with spatial scale $(F=0.28, P=0.84)$. $\mathrm{N}_{\text {leaf }}$ did not affect biomass growth of canopy trees (panel b; $\mathrm{F}=0.34, \mathrm{P}=0.56$ ) and did not interact with species $\left(\mathrm{F}=0.74, \mathrm{P}=0.53\right.$ ), but $\mathrm{N}_{\text {leaf }}$ increased biomass growth at the community level (panel d; $\mathrm{F}=22.33, \mathrm{P}<0.001$ ) independent of spatial scale $(\mathrm{F}=0.55, \mathrm{P}=0.65)$. 


\section{Tropical forest functioning across temporal scales}

The first part of this thesis demonstrates that ecosystem processes depend on spatial variation in abiotic and biotic conditions. Besides this spatial aspect, global change causes temporal changes in abiotic conditions, which may lead to changes in biotic conditions and ecosystem process rates. It is becoming clear that tropical forests are not in a stable state but are responding to changes in abiotic conditions (e.g. Bush et al. 2011, Enquist and Enquist 2011, Feeley et al. 2011). Questions remain, however, of how tropical forests change over time, what drives these changes, and how diversity contributes to the long-term stability of tropical forests. If tropical forests are in a stable state, then the species composition and CWM trait values of old-growth forests should remain constant over time. However, if they are not in a stable state but are affected by changing abiotic conditions, then the direction of changes in CWM trait values should demonstrate the underlying driver(s) of change. In chapter 6 we evaluated how species composition and CWM trait values (or 'trait composition' as we call it in chapter 6) changed over 10-30 years, using $15 \mathrm{CWM}$ traits for 29 old-growth forest plots across five Neotropical forests. We expected that distinct changes in CWM trait values would be driven by distinct drivers. First, we expected that increasing resource availability such as $\mathrm{CO}_{2}$ and rainfall would increase the abundance of species with acquisitive trait values such as high SLA and high leaf nutrient concentrations (Wright et al. 2004). Second, we expected that increasing drought stress caused by a reduction in rainfall and/or an increase in temperature that leads to increased vapour pressure deficit and atmospheric drought would result in more drought-tolerant traits such as high WD (Markesteijn et al. 2011a) and in drought-avoiding traits such as deciduousness. And third, we expected that recovery from past disturbances would result in an increasing abundance of late-successional traits such as low SLA (Lohbeck et al. 2013).

\section{3a. Short-term temporal changes in biotic conditions}

Across the five forests, we found a consistent increase in CWM WD and a consistent decrease in CWM SLA over time. These changes may have been caused by increased drought stress, because high WD is associated with high drought tolerance (Markesteijn et al. 2011b), or they may be attributable to recovery from disturbances, because high WD and low SLA are typical for late-successional species (van Gelder et al. 2006, Carreño-Rocabado et al. 2012, Lohbeck et al. 2013). We also found that in some forests there was an increase in species-specific maximum stem diameter, which can be seen as another indicator of increased abundance of late-successional species. Since we found no changes in other 
drought-tolerance or -avoidance traits, such as the leaf dry matter content and the abundance of deciduous species, it is most likely that the observed changes reflect successional recovery from past disturbances, and not increased drought stress. The consistent changes in CWM WD and SLA across our forests indicate that they are most probably caused by disturbances that frequently occur throughout the Neotropics, such as El Niño droughts; other possible causes are wind storms, fire, or human occupation in the far past.

It is remarkable that old-growth tropical forests undergo significant changes in trait composition during only 10-30 years. This could indicate that tropical forests are flexible and able to respond to changes in abiotic conditions (here caused by successional recovery). The increasing abundance of species with high WD would also help forests to respond to possible increases in drought stress (for example atmospheric). Although it remains uncertain how rainfall patterns will change, drought stress is expected to increase because it is predicted that extreme drought and wet events will become more common and that temperatures and, hence, vapour pressure deficit will increase (Phillips et al. 2009, Wright et al. 2009).

If tropical forests are undergoing successional development, then how would this affect ecosystem processes? The Amazon is a net carbon sink, yet its sequestration capacity is diminishing (Brienen et al. 2015), suggesting that the buffering effect of anthropogenic $\mathrm{CO}_{2}$ emissions by tropical forests may come to a halt or even reverse. The results presented in chapter 6 show a similar trend: species with high WD and low SLA generally have low growth and mortality rates (King et al. 2006b, Poorter et al. 2008, Rüger et al. 2012), and thus an increase in their abundance may result in less dynamic forests with lower carbon sequestration rates. Nevertheless, the positive message from these same results is that tropical forests seem flexible to respond to changes in abiotic conditions over a relatively short timescale. This, however, does not provide information on the extent to which forests are able to adapt to long-term climate change, or on the consequences of this for biomass stocks and dynamics.

\section{3b. Long-term diversity effects on stability of forest functioning}

Several studies on temperate grasslands have provided strong evidence for the importance of species or trait diversity on the long-term stability of ecosystems (e.g. Tilman et al. 2006, Hector et al. 2010, Isbell et al. 2015). These results are in line with the insurance theory (Yachi and Loreau 1999), which postulates that a high diversity of species and their traits would buffer the ecosystem against environmental changes and result in greater stability of ecosystem processes. But would this theory also hold for tropical forests that are more diverse and structurally complex? We addressed this question by using the global dynamic vegetation model LPJmL-FIT (Lund-Potsdam-Jena managed Land with Flexible 
Individual Traits) to simulate the effect of trait diversity on the long-term stability of biomass stocks in response to inter-annual climatic fluctuations (van der Sande, Sakschewski et al. in prep.). We tested this for a dry tropical forest and a wet tropical forest because, as shown earlier in this chapter, abiotic site conditions can strongly determine the importance of diversity.

We found that for both forest types, diversity in SLA values within a community increased long-term (200 y) biomass stocks and the stability of biomass stocks (Fig. 8.3) due to greater asynchrony in species' responses to inter-annual climatic fluctuations. These results indicate that trait diversity does indeed increase ecosystem process rates through niche complementarity, and increases the stability of ecosystem processes through the insurance effect. High functional trait diversity of tropical forests is also crucially important for a forest's resilience to severe and directional changes in climate, because some of the species present will be well adapted to cope with the changed climatic conditions (Sakschewski 2015). Hence, for diverse tropical forests too, diversity ensures the long-term stability of ecosystem processes and their resilience to external pressures.
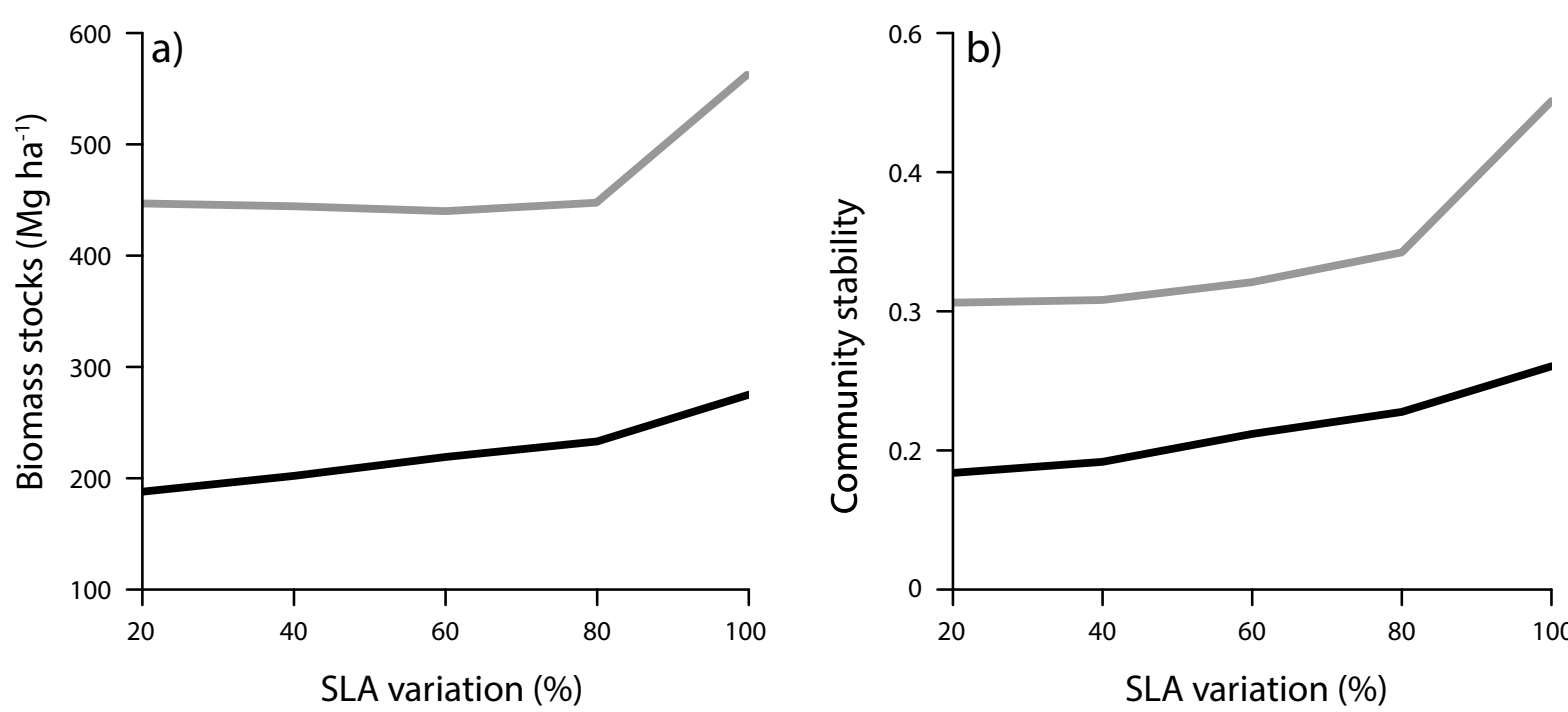

Figure 8.3: Relationship between variation in specific leaf area (SLA) - as measure for functional trait diversity - and the long-term average (a) biomass stocks and (b) stability of biomass stocks (calculated as the mean divided by the standard deviation of biomass stocks). The relationships are given for a simulated wet tropical forest receiving $2772 \mathrm{~mm}$ annual rainfall (gray line) and a dry tropical forest receiving $1270 \mathrm{~mm}$ annual rainfall (black line). Results were obtained from simulations done using the LPJmL-FIT model (Sakschewski et al. 2015). Five model simulations were run: at $20 \%, 40 \%, 60 \%, 80 \%$, and $100 \%$ SLA variation. The $100 \%$ SLA variation refers to the full range of simulated SLA values that can naturally establish in the site. The $100 \%$ SLA variation was reduced in four subsequent simulations, by each time excluding $10 \%$ of the cumulative biomass-weighted trait variation at both extremes of the SLA distribution (leading to a $20 \%$ reduction in each subsequent simulation). 


\section{Wrapping up: what determines forest functioning?}

In this thesis, I focus on effects of biotic conditions - especially species and trait diversity and community-weighted mean traits - and (changing or static) abiotic conditions on ecosystem processes. The relative importance of relationships between abiotic conditions, biotic conditions, and ecosystem processes depend mainly on spatial scale and/or site conditions, and on temporal scale (Fig. 8.4). At small spatial scales at the community level (chapters 3,4 and 7), abiotic conditions always have an important effect on ecosystem processes, whereas biotic effects seem to depend on site conditions (i.e. strong for forests on poor soils and weak for forests on fertile soils). At larger spatial scales (chapters 5, 7, Poorter et al. 2015), biotic conditions have stronger effects than abiotic conditions, possibly because abiotic effects work partly via biotic conditions. At relatively short temporal scales (10$30 \mathrm{y}$; chapter 6), biotic conditions respond to temporal changes in abiotic conditions, possibly as a result of disturbances in the far past. This indicates that old-growth tropical forests are dynamic and flexible. At long temporal scales $>200$ years; chapter 7 and Fig. 8.3), modelling studies show that biotic conditions are important for the long-term stability of ecosystem processes. With fluctuations or directional changes in climate, high species and trait diversity increase the likelihood of the presence of species that are well adapted to the new conditions (Sakschewski 2015). These species may now seem redundant or too rare to provide an important contribution to ecosystem processes, but may become important and dominant in the future (Walker et al. 1999).

\section{Outlook: the way forward in tropical forest ecology}

The functioning of individually growing plants is fairly well understood, but we have limited understanding of the functioning of diverse plant communities. Diverse ecosystems that consist of long-lived organisms, such as tropical forests, are more complex because many factors are at play that cannot be easily controlled for, and because most studies are of short duration whereas the time lag between changes and effects is long. As a result, many studies at the community level are able to describe patterns but are unable to identify the underlying mechanisms. Understanding such mechanisms is crucial in order to be able to interpret and apply the results beyond the specific case study, and to provide information and advice for decision makers and stakeholders on how to maintain and achieve tropical forests that provide multiple important functions such as carbon sequestration, nutrient retention, and water cycling. 

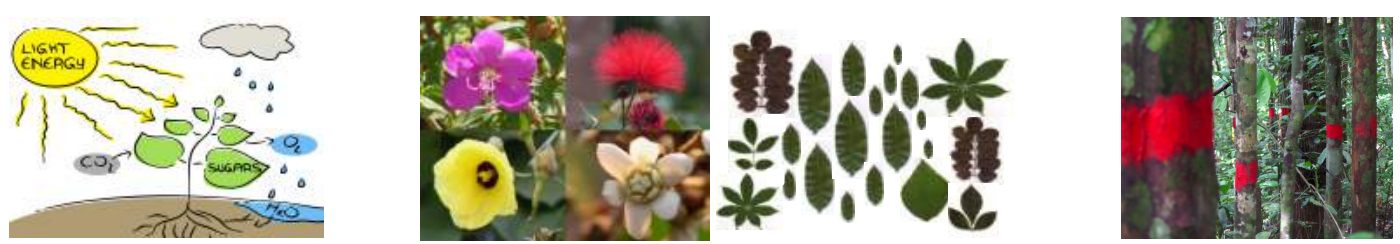

Small spatial scale

(ch. 3, 4 \& 7)

Abiotic conditions

Biotic conditions EPs

Large spatial scale

(ch. 5 \& 7)

Abiotic conditions

Biotic conditions

EPs
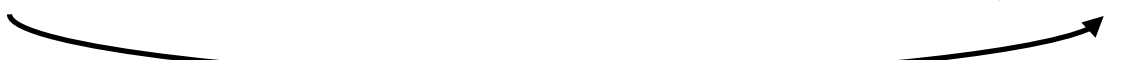

Short temporal scale

(ch. 6)

Abiotic conditions

Biotic conditions - - - - - $\rightarrow$ EPs

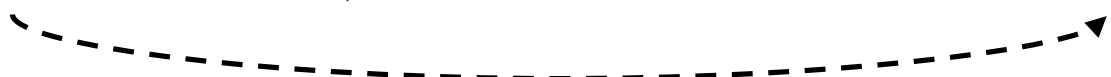

Long temporal scale

(ch. 7 \& Fig. 8.3)

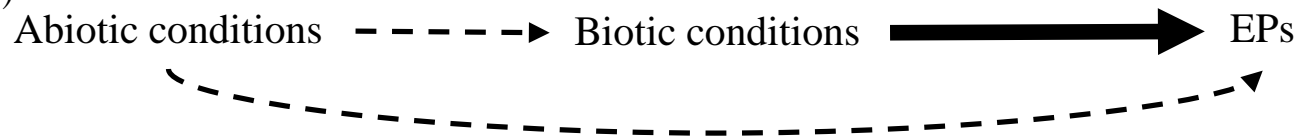

Figure 8.4: Relationships between abiotic conditions, biotic conditions, and ecosystem processes (EPs) as found in this thesis for four different scales at the community level: small spatial scale (chapters 3, 4 and 7), large spatial scale (chapters 5 and 7), short temporal scale (chapter 6), and long temporal scale (chapter 7 and Fig. 8.3). Biotic conditions are based on species and trait diversity and on community-weighted mean trait, not on vegetation quantity, because that was evaluated in fewer chapters. Note that chapter 2 (the individual-tree scale) is also not included in this framework. The thick black arrows indicate the generally strongest relationship at that scale, thin black arrows indicate evaluated relationships that were generally less important, and dashed arrows indicate relationships that were not evaluated in this thesis.

The mechanisms predicted by the niche complementarity, insurance and mass-ratio theories are generally relevant for ecosystem processes (chapters 2-5 and 7). Nevertheless, the complex nature of tropical forests makes it difficult to understand how these relationships change with abiotic site conditions, with spatial and temporal scales, and for different ecosystem processes. To improve such understanding, future research will need to focus on 1) long-term monitoring, 2) the use of mechanistic approaches, and 3) the combination of different research approaches such as empirical, remote sensing, and modelling studies. 


\section{Long-term monitoring}

Long-term monitoring (i.e. decades to centuries) is necessary to detect patterns and underlying mechanisms, as this is the timescale that corresponds to the life cycle of most tree species and during which environmental change, acclimation, and natural large-scale disturbances may take place (Zuidema et al. 2013). In this thesis, I mostly use time periods between 5-30 y, which is sufficient to address questions related to spatial variation in abiotic and biotic conditions and ecosystem processes (such as in chapters 2-5) but is rather short for addressing questions related to global change (such as in chapter 6). We found strong temporal changes in CWM traits across 5 Neotropical forests over 10-30 y, which we attributed to recovery from large-scale disturbances. It would be important, however, to monitor these sites for at least several decades more, to ascertain whether the recovery that we observed is due to directional abiotic changes after disturbance or is part of a longterm periodic cycle of disturbance and recovery. The latter case may mean that these forests will likely never reach a 'stable state'. Furthermore, long-term monitoring would allow the evaluation of how forests respond to future environmental changes, which may be more extreme than the changes that have occurred during recent decades. Besides long-term monitoring in the future, longterm time series of past vegetation development (i.e. obtained from pollen records) may also yield important insights into changes in vegetation composition and underlying drivers of change (Mayle et al. 2000, Gosling et al. 2009).

\section{Mechanistic approaches}

The use of mechanistic approaches will be crucial in developing an understanding of mechanisms underlying ecosystem processes and functions. These approaches range from analytical approaches that allow the testing for cause-effect relationships, such as path modelling or structural equation modelling (SEM; Grace and Pugesek 1997, Shipley 2004, Grace 2006), to mechanistic models based on simulations (e.g. Bunker et al. 2005) or process-based dynamic global vegetation models (DGVMs). Data analytical approaches such as SEM can go far in teasing apart various abiotic and biotic effects, but can never fully account for variables inherent to empirical field data that confound the observed relationships. Ecosystem models such as DGVMs are a strong tool for creating an experimental setting and testing hypotheses and scenarios, but their results rely on model assumptions and are difficult to verify, especially without long-term monitoring.

Several ecosystem models have recently been made more realistic by including higher levels of functional diversity, such as LPJmL-FIT (Sakschewski et al. 2015) and TFS (Fyllas et al. 2014) (see also chapter 7). The processes or relationships in these models are based on a small number of still poorly understood empirical 
relationships between abiotic conditions, traits, and performance. Many of these relationships have been well documented under optimal conditions at the species level (e.g. Wright et al. 2004, Poorter et al. 2008), but this does not mean that they would hold at the community level. At the community level such relationships may mainly reflect the local abiotic conditions rather than species' strategies under optimal conditions, which may result in different trait effects on performance (Kunstler et al. 2016). For example, SLA generally increases growth rate at the species level (Poorter and Bongers 2006), but CWM SLA decreases aboveground biomass productivity in a Guyanese wet forest (chapter 3) and in a Brazilian dry forest (Prado-Junior et al. 2016). Similarly, Fig. 8.2b shows an unexpected negative relationship between CWM leaf nitrogen concentration and biomass growth. Conclusions based on this thesis provide a first important step in scaling up, but many uncertainties remain. A more systematic testing of such relationships is thus urgently needed, in order to understand the mechanisms underlying communitylevel processes in the field and to improve model predictions at large spatial and temporal scales.

An alternative strong, mechanistic approach that does not rely on model assumptions is the use of manipulative field experiments. In temperate grasslands, field experiments that are fully manipulated (e.g. van Ruijven and Berendse 2005, Hector et al. 2010) or semi-manipulated (e.g. Hautier et al. 2014) have provided strong evidence for the importance of species and trait diversity and CWM traits on the amplitude and stability of community processes. Several biodiversity experiments have also been carried out in plantation forests (e.g. Ruiz-Jaen and Potvin 2011, Bruelheide et al. 2014), but these do not represent natural tropical forests, which are structurally more complex, composed of larger individuals, and have numerous species. Species-removal experiments could provide a way to test for biodiversity effects in natural forests. Although these experiments may be difficult, costly, and time-consuming to implement, they could be a robust way of evaluating the relationships between biodiversity and ecosystem functioning.

\section{Combining empirical, remote sensing, and modelling approaches}

Empirical (including experimental), remote sensing, and modelling approaches each have their advantages and disadvantages, as discussed in chapter 7. Empirical studies present direct measurements of field data but are limited in their spatial and temporal scale. Remote sensing approaches can easily scale up to large spatial areas but can lack detail. Modelling approaches are a powerful tool for going beyond measured data and exploring timescales, hypotheses, and scenarios that are impossible to evaluate by empirical or remote sensing data, but they rely on assumptions that are crucial for data generation. These approaches are important on their own, but can also potentially overcome each other's limitations. Such a 
combined approach may result in a whole that is greater than the sum of its parts. For example, remote sensing data need to be linked to empirical data in order to demonstrate that their images provide realistic information (Dutrieux et al. in review, Spasojevic et al. 2015, Jetz et al. 2016). Furthermore, empirical data should be used as input and validation for vegetation models (such as DGVMs or other Earth System Models) in order to increase the realism of their concepts, assumptions, and results (Sakschewski 2016). Remote sensing data verified by or linked to empirical measurements can also be used to develop land-use change scenarios, for example with the CLUE model (Conversion of Land Use and its Effects) (e.g. Verburg et al. 2006). Output from such models and remote sensing, backed up by field data, will then provide very valuable information for decision makers.

\section{Outlook: towards long-term resilient systems and a safe climate}

The global atmospheric $\mathrm{CO}_{2}$ concentration is increasing at unprecedented rates, and this has created global concern about rising temperatures and associated problems. During the $21^{\text {st }}$ session of the Conference of the Parties (COP) of the United Nations Framework Convention on Climate Change (UNFCCC) in 2015 in Paris, all countries agreed on 'holding the increase in the global average temperature to well below $2{ }^{\circ} \mathrm{C}$ above pre-industrial levels' (United Nations 2015). This means that emissions of $\mathrm{CO}_{2}$ and other greenhouse gases need to be reduced and $\mathrm{CO}_{2}$ uptake from the atmosphere enhanced. Concern about climate change is growing in parallel with concern about other environmental problems, such as the rate of species extinction, which is currently at least 1000 times greater than background extinction rates (De Vos et al. 2015). Species extinction rates have also received political attention, as illustrated by the Convention on Biological Diversity (CBD) for the conservation and sustainable use of biodiversity (or biotic conditions').

Tropical forests are particularly relevant for climate change mitigation and biodiversity conservation. They are important for climate mitigation because deforestation accounts for $10-12 \%$ of the total global greenhouse gas emissions (Pan et al. 2011, Grace et al. 2014, Houghton et al. 2015), growing tropical forests have a high potential to remove $\mathrm{CO}_{2}$ from the atmosphere (Baker et al. 2004a, Poorter et al. 2016), and because high evaporation in tropical forests leads to cooling and cloud cover, which increases sunlight reflection and in this way leads to further cooling and thus reduction of global warming (Bonan 2008, Canadell and Raupach 2008). Although the carbon sequestration capacity of old-growth tropical 
forests seems to be declining, (Brienen et al. 2015, chapter 6), they may remain a net sink for decades, and it is imperative that the large amounts of carbon stored in forests are conserved. Moreover, their high biodiversity (Slik et al. 2015) makes tropical forests highly relevant for biodiversity conservation (ter Steege et al. 2015). Several approaches have been proposed to increase the climate change mitigation potential of tropical forests and/or maintain their high levels of biodiversity. Here, I will discuss those that are potentially successful and for which the result of this thesis may have implications: 1) reducing carbon emissions from deforestation and forest degradation, 2) sustainable forest (or land) management for timber and nontimber forest products, and 3) increasing forest cover by forest landscape restoration.

\section{Reducing emissions from deforestation and forest degradation}

Land for carbon stocks and sequestration is, and will increasingly be, in competition with land for production of food, bioenergy, and urban development (Agrawal et al. 2011, Canadell and Schulze 2014). For example, given the current trends in technological development, crop and pasture area will need to grow from $44 \%$ to about $50 \%$ of ice-free land to feed 9 billion people (Canadell and Schulze 2014). This will undoubtedly increase the pressure of deforestation. Therefore, during the UNFCCC COP in 2007, the Reduced Emissions from Deforestation and forest Degradation (REDD) mechanism was developed, which is a financial incentives-based strategy for conserving the carbon stored in tropical forests (Agrawal et al. 2011). During the COP in 2008, forest conservation, sustainable forest management, and enhancements of forest carbon stocks were included, and REDD was changed into REDD+. In the recent Paris Agreement, the importance of forests in reducing emissions and increasing uptake is also explicitly mentioned, although no concrete REDD+ payment framework has yet been adopted. Potentially, REDD+ can be a powerful mechanism for increasing carbon stocks in tropical forests while improving conservation and sustainable forest management.

The concern has been raised, however, that REDD+ may favour carbon-rich forests at the expense of forests that are important for other ecosystem functions or biodiversity (also called 'leakage') (Venter et al. 2009, Di Marco et al. 2015, Murray et al. 2015). We showed that species diversity increases carbon storage and uptake by tropical forests across large spatial scales (chapters 5, 7, and Poorter et al. 2015), but not in all local case studies (chapters 2, 3, and 7). To avoid REDD+ from driving a change from carbon-poor and species-rich forests to carbon-rich and species-poor plantations, biodiversity conservation should be included as an integral component of REDD+ (Díaz et al. 2009, Phelps et al. 2012 and, for biodiversity safeguards, UNFCCC 2014). Forest conservation that focuses on both carbon and biodiversity has been shown to be effective (Venter et al. 2009). 
Moreover, although high biodiversity does not always result in increased carbon storage and sequestration locally, forest with high biodiversity will likely prove more resilient and therefore able to cope with climate change (chapter 7, Fig. 8.3) and provide multiple ecosystem functions (e.g. Hector and Bagchi 2007). Biodiversity should thus be considered a prerequisite for ensuring the longterm carbon storage and sequestration, and not as only a co-benefit of REDD+.

\section{Sustainable forest management}

Compared with disturbed forests, old-growth tropical forests store more carbon and host more species per unit terrestrial area (Gibson et al. 2011). Nevertheless, $24 \%$ of tropical forest area is used for timber harvesting (Blaser et al. 2011), and given the increasing demand for wood, this area is likely to expand. It is therefore important to sustainably manage the forest to reduce $\mathrm{CO}_{2}$ emissions, guarantee ecological functioning and biodiversity, and sustain local livelihoods that depend on timber and non-timber forest products (Arets and Veeneklaas 2014, Edwards et al. 2014). Sustainable forest management (SFM) is an attractive solution to assure forest functioning, though only $1.8 \%$ of the total tropical forest area is managed sustainably (Blaser et al. 2011).

Two major challenges need to be tackled in order for SFM to become more widespread and successful. First, SFM is less profitable than unrestricted logging (Rice et al. 1997), especially by comparison with other land-use options, so there is an economic incentive to extract all valuable timber as quickly as possible and convert the land for agricultural purposes or other uses (Zimmerman and Kormos 2012). To make SFM financially more attractive, REDD+ could offer a solution, and SFM in turn could provide benefits for REDD+; financial compensation by REDD+ can make SFM more competitive with unrestricted logging or land conversion, and SFM reduces carbon emissions and therefore increases the benefits from REDD+. For example, only a small fraction of tropical forests with a management plan is managed sustainably (Blaser et al. 2011). Unsustainable management leads to depletion of the most valuable timber species within three harvest cycles (Zimmerman and Kormos 2012) and to additional forest degradation because it increases the occurrence of other disturbances, such as fire and hunting (Asner et al. 2006, Ghazoul and Sheil 2010). Reduced impact logging techniques (Putz et al. 2008a, 2008b) and silvicultural treatments (Dauber et al. 2005, PeñaClaros et al. 2008) can help to increase timber recovery. The reduced harvest yield that results from these solutions could then be offset by a REDD+ payment scheme. This coupling of REDD+ and SFM could assure that ecological functioning and biodiversity are maintained by managed forests that are 
economically viable (Putz et al. 2012), provided that appropriate governance is in place.

A second challenge is that there can be trade-offs in optimizing the recovery of timber species, carbon stocks, and biodiversity (Putz et al. 2012). For example, applying silvicultural treatments such as liana cutting and girdling of noncommercial species increases the growth rate of the commercial species (PeñaClaros et al. 2008), but may decrease species diversity. Nevertheless, win-win situations also exist, for example through the positive effect of biodiversity on the recovery of timber and carbon. To optimize SFM, such win-win situations should be identified at a local level, and implemented in national sustainable forest management programmes to avoid leakage (Agrawal et al. 2011). Hence, truly sustainable forest management has yet to be implemented at larger spatial scales, but with the positive effect of biodiversity on forest recovery, financial compensation by REDD+, and appropriate governance, sustainable forest management can provide a way to assure long-term economically viable and ecologically functioning forests.

\section{Forest landscape restoration}

Worldwide, more than 2 billion hectares of deforested and degraded land have the potential to be restored (FAO 2014), either by active restoration or passive recovery. This would benefit carbon sequestration and storage, biodiversity conservation, and other forest functions. For example, if degraded (or secondary) forests are left to recover passively, they can sequester about $3.05 \mathrm{Mg}$ carbon $\mathrm{ha}^{-1}$ $\mathrm{y}^{-1}$ (Poorter et al. 2016). Countries could greatly benefit from including forest recovery and restoration in their $\mathrm{CO}_{2}$ emission reporting and in their policies and actions to reduce greenhouse gas emissions, as specified in their Nationally Appropriate Mitigation Actions. Furthermore, it would contribute to the New York challenge to have reforested 350 million hectares of degraded land by 2030, and to the Aichi Biodiversity Targets adopted by the Parties to the CBD to reduce biodiversity loss and enhance benefits from biodiversity. Degraded forests have huge potential for biodiversity conservation because of their vast area and their importance for increasing the connectivity between patches of old-growth forests (Wright and Muller-landau 2006). Restoring biodiversity along with restoring vegetation biomass will be important for the resilience of naturally recovering tropical forests (e.g. de Avila et al. in prep.), for active forest restoration projects such as plantations (e.g. chapter 7), and hence for the resilience of multifunctional landscapes. 


\section{Concluding remarks - understanding ecosystem processes to assure long-term functioning tropical forests}

Many of our daily activities and necessities benefit directly or indirectly from the presence of vast areas of tropical forest. In this thesis, I show and argue that the existence and persistence of important ecosystem processes in tropical forests in the near and far future depend on abiotic and biotic conditions and on human actions and decisions. Abiotic and biotic conditions - including diversity and species traits - determine the rate of ecosystem processes today and their stability in an unstable and insecure future. Future research efforts should aim to further clarify how mechanisms underlying ecosystem processes depend on spatial and temporal scale and site conditions, and how sensitive they are to global change. This should be done at local levels in order to improve local forest management and safeguard sustainable ecosystems and livelihoods, and at the global level in order to influence national and international policies that tackle global problems and provide a framework for local-level sustainability. By combining ecological and socio-economic research with appropriate political and technological developments, we can shift the focus from short-term profitable and simplified systems towards a focus on long-term profitable and ecologically functioning forests that benefit local as well as global players. Conserving tropical forests for their carbon, timber, and diversity is and will be a challenge, but a challenge that must be overcome for the benefit of all organisms on Earth - including humans. 
References 
Adams, M. A., J. Simon, and S. Pfautsch. 2010. Woody legumes: a (re)view from the South. Tree Physiology 30:1072-1082.

Adler, P. B., E. W. Seabloom, E. T. Borer, H. Hillebrand, Y. Hautier, A. Hector, W. S. Harpole, L. R. O’Halloran, et al. 2011. Productivity is a poor predictor of plant species richness. Science 333:1750-1753.

Aerts, R. 1997. Climate, leaf litter chemistry and leaf litter decomposition in terrestrial ecosystems: a triangular relationship. Oikos 79:439-449.

Aerts, R. 1996. Nutrient resorption from senescing leaves of perennials: are there general patterns? Journal of Ecology 84:597-608.

Agrawal, A., D. Nepstad, and A. Chhatre. 2011. Reducing emissions from deforestation and forest degradation. Annual Review of Environment and Resources 36:373-396.

Alkama, R., and A. Cescatti. 2016. Biophysical climate impacts of recent changes in global forest cover. Science 351:600-604.

Amundson, R. 2001. The carbon budget in soils. Annual Review of Earth and Planetary Sciences 29:535-562.

Anten, N. P. R., and T. Hirose. 1999. Interspecific differences in above-ground growth patterns result in spatial and temporal partitioning of light among species in a tall-grass meadow. Journal of Ecology 87:583-597.

Aragão, L. E. O. C., Y. Malhi, D. B. Metcalfe, J. E. Silva-Espejo, E. Jiménez, D. Navarrete, S. Almeida, A. C. L. Costa, et al. 2009. Above- and below-ground net primary productivity across ten Amazonian forests on contrasting soils. Biogeosciences 6:2759-2778.

Arets, E. J. M. M. 2005. Long-term responses of populations and communities of trees to selective logging in tropical rain forests in Guyana. PhD thesis, Utrecht University, Utrecht.

Arets, E. J. M. M., and F. R. Veeneklaas. 2014. Costs and benefits of a more sustainable production of tropical timber. Technical Report 10. Wettelijke Onderzoekstaken Natuur en Milieu, Wageningen UR, Wageningen, the Netherlands.

Armesto, J. J., and S. T. A. Pickett. 1985. Experiments on disturbance in old-field plant communities: impact on species richness and abundance. Ecology 66:230-240.

Asase, A., B. K. Asitoakor, and P. K. Ekpe. 2012. Linkages between tree diversity and carbon stocks in unlogged and logged West African tropical forests. International Journal of Biodiversity Science, Ecosystem Services \& Management 8:217-230.

Asner, G. P., E. N. Broadbent, P. J. C. Oliveira, M. Keller, D. E. Knapp, and J. N. M. Silva. 2006. Condition and fate of logged forests in the Brazilian Amazon. Proceedings of the National Academy of Sciences of the United States of America 103:12947-12950.

Asner, G. P., and R. E. Martin. 2009. Airborne spectranomics: mapping canopy chemical and taxonomic diversity in tropical forests. Frontiers in Ecology and the Environment 7:269-276.

Asner, G. P. 2015. Organismic remote sensing for tropical forest ecology and conservation. Annals of the Missouri Botanical Garden 100:127-140.

Asner, G. P., C. B. Anderson, R. E. Martin, R. Tupayachi, D. E. Knapp, and F. Sinca. 2015. Landscape biogeochemistry reflected in shifting distributions of chemical traits in the Amazon forest canopy. Nature Geoscience 8:567-573.

Aubry-Kientz, M., V. Rossi, F. Wagner, and B. Hérault. 2015. Identifying climatic drivers of tropical forest dynamics. Biogeosciences Discussions 12:3145-3176.

de Avila, A. L., M. T. van der Sande, C. F. Dormann, L. Mazzei, A. R. Ruschel, J. O. Pereira de Carvalho, J. N. Macedo Silva, L. Poorter, et al. Effects of management intensity, residual diversity and trait composition on biomass recovery of a tropical rain forest. In review.

de Avila, A. L., A. R. Ruschel, J. O. P. de Carvalho, L. Mazzei, J. N. M. Silva, J. D. C. Lopes, M. M. Araujo, C. F. Dormann, et al. 2015. Medium-term dynamics of tree species composition in response to silvicultural intervention intensities in a tropical rain forest. Biological Conservation 191:577-586.

Avitabile, V., M. Herold, G. B. M. Heuvelink, S. L. Lewis, O. L. Phillips, G. P. Asner, J. Armston, P. Asthon, et al. 2015. An integrated pan-tropical biomass map using multiple reference datasets. Global Change Biology 22:14061420.

Baccini, A., S. J. Goetz, W. S. Walker, N. T. Laporte, M. Sun, D. Sulla-Menashe, J. Hackler, P. S. A. Beck, et al. 2012. Estimated carbon dioxide emissions from tropical deforestation improved by carbon-density maps. Nature Climate Change 2:182-185.

Baker, T. R., O. L. Phillips, W. F. Laurance, N. C. A. Pitman, S. Almeida, L. Arroyo, A. Difiore, T. Erwin, et al. 2009. Do species traits determine patterns of wood production in Amazonian forests? Biogeosciences 6:297-307.

Baker, T. R., O. L. Phillips, Y. Malhi, S. Almeida, L. Arroyo, A. Di Fiore, T. Erwin, N. Higuchi, et al. 2004 a. Increasing biomass in Amazonian forest plots. Philosophical Transactions of the Royal Society B: Biological Sciences 359:353-365.

Baker, T. R., O. L. Phillips, Y. R. Mahli, S. Almeida, L. Arroyo, A. Di Fiore, T. Erwin, T. J. . Killeen, et al. 2004b. Variation in wood density determines spatial patterns in Amazonian forest biomass. Global Change Biology 10:545-562.

Baker, T. R., D. F. R. P. Burslem, and M. D. Swaine. 2003. Associations between tree growth, soil fertility and water 
availability at local and regional scales in Ghanaian tropical rain forest. Journal of Tropical Ecology 19:109-125.

Bakker, M. A., G. Carreño-Rocabado, and L. Poorter. 2011. Leaf economics traits predict litter decomposition of tropical plants and differ among land use types. Functional Ecology 25:473-483.

Balvanera, P., C. Kremen, and M. Martínez-ramos. 2005. Applying community structure analysis to ecosystem function: examples from pollination and carbon storage. Ecological Applications 15:360-375.

Balvanera, P., A. B. Pfisterer, N. Buchmann, J.-S. He, T. Nakashizuka, D. Raffaelli, and B. Schmid. 2006. Quantifying the evidence for biodiversity effects on ecosystem functioning and services. Ecology letters 9:1146-56.

Banin, L., S. L. Lewis, G. Lopez-Gonzalez, T. R. Baker, C. A. Quesada, K.-J. Chao, D. F. R. P. Burslem, R. Nilus, et al. 2014. Tropical forest wood production: a cross-continental comparison. Journal of Ecology 102:1025-1037.

Baraloto, C., S. Rabaud, Q. Molto, L. Blanc, C. Fortunel, B. Hérault, N. Dávila, I. Mesones, et al. 2011. Disentangling stand and environmental correlates of aboveground biomass in Amazonian forests. Global Change Biology 17:2677-2688.

Baraloto, C., C. E. Timothy Paine, L. Poorter, J. Beauchene, D. Bonal, A.-M. Domenach, B. Hérault, S. Patiño, et al. 2010. Decoupled leaf and stem economics in rain forest trees. Ecology letters 13:1338-47.

Baribault, T. W., R. K. Kobe, and A. O. Finley. 2012. Tropical tree growth is correlated with soil phosphorus, potassium, and calcium, though not for legumes. Ecological Monographs 82:189-203.

Barrufol, M., B. Schmid, H. Bruelheide, X. Chi, A. Hector, K. Ma, S. Michalski, Z. Tang, et al. 2013. Biodiversity promotes tree growth during succession in subtropical forest. PLoS ONE 8:1-9.

Barton, K. 2015. MuMIn: Multi-model inference. R package version 1.13.4. http://CRAN.Rproject.org/package $=$ MuMIn.

Batterman, S. A., L. O. Hedin, M. van Breugel, J. Ransijn, D. J. Craven, and J. S. Hall. 2013. Key role of symbiotic dinitrogen fixation in tropical forest secondary succession. Nature 502:224-227.

Beard, J. S. 1955. The classification of tropical American vegetation-types. Ecology 36:89-100.

Beer, C., M. Reichstein, E. Tomelleri, P. Ciais, M. Jung, N. Carvalhais, C. Rödenbeck, M. A. Arain, et al. 2010. Terrestrial gross carbon dioxide uptake: global distribution and covariation with climate. Science 329:834-838.

Bennett, A. C., N. G. McDowell, C. D. Allen, and K. J. Anderson-Teixeira. 2015. Larger trees suffer most during drought in forests worldwide. Nature Plants 1:1-5.

Binkley, D., J. L. Stape, W. L. Bauerle, and M. G. Ryan. 2010. Explaining growth of individual trees: Light interception and efficiency of light use by Eucalyptus at four sites in Brazil. Forest Ecology and Management 259:1704-1713.

Blaser, J., A. Sarre, D. Poore, and S. Johnson. 2011. Status of tropical forest management 2011. Technical series 38. International Tropical Timber Organization, Yokohama, Japan.

Van Bodegom, P. M., J. C. Douma, J. P. M. Witte, J. C. Ordoñez, R. P. Bartholomeus, and R. Aerts. 2012. Going beyond limitations of plant functional types when predicting global ecosystem-atmosphere fluxes: Exploring the merits of traits-based approaches. Global Ecology and Biogeography 21:625-636.

Bolnick, D. I., R. Svanbäck, J. a Fordyce, L. H. Yang, J. M. Davis, C. D. Hulsey, and M. L. Forister. 2003. The ecology of individuals: incidence and implications of individual specialization. The American naturalist 161:1-28.

Bonan, G. B. 2008. Forests and climate change: forcings, feedbacks, and the climate benefits of forests. Science 320:1444-1449.

Bongers, F., L. Poorter, W. D. Hawthorne, and D. Sheil. 2009. The intermediate disturbance hypothesis applies to tropical forests, but disturbance contributes little to tree diversity. Ecology Letters 12:798-805.

Brienen, R. J. W., and P. A. Zuidema. 2006. Lifetime growth patterns and ages of Bolivian rain forest trees obtained by tree ring analysis. Journal of Ecology 94:481-493.

Brienen, R. J. W., O. L. Phillips, T. R. Feldpausch, E. Gloor, T. R. Baker, J. Lloyd, G. Lopez-Gonzalez, A. Moneagudo-Mendoza, et al. 2015. Long-term decline of the Amazon carbon sink. Nature 519:344-348.

Broadbent, E. N., G. P. Asner, M. Peña-Claros, M. Palace, and M. Soriano. 2008. Spatial partitioning of biomass and diversity in a lowland Bolivian forest: Linking field and remote sensing measurements. Forest Ecology and Management 255:2602-2616.

Brown, I. F., L. A. Martinellli, W. W. Thomas, M. Z. Moreira, C. A. C. Ferreira, and R. A. Victoria. 1995. Uncertainty in the biomass of Amazonian forests: An example from Rondonia, Brazil. Forest Ecology and Management 75:175-189.

Bruelheide, H., K. Nadrowski, T. Assmann, J. Bauhus, S. Both, F. Buscot, X. Y. Chen, B. Ding, et al. 2014. Designing forest biodiversity experiments: General considerations illustrated by a new large experiment in subtropical China. Methods in Ecology and Evolution 5:74-89.

Bunker, D. E., F. Declerck, J. C. Bradford, R. K. Colwell, I. Perfecto, O. L. Phillips, M. Sankaran, and S. Naeem. 2005. Species loss and aboveground carbon storage in a tropical forest. Science 310:1029-31.

Burnham, K. P., and D. R. Anderson. 2002. Formal inference from more than one model: multimodel inference (MMI). Pages 149-205 in K. P. Burnham and D. R. Anderson, editors. Model selection and multimodell inference. A practical information-theoretic approach. Second edi. Springer, New York.

Burrough, P. A. 1983. Multiscale sources of spatial variation in soil. I. The application of fractal concepts to nested levels of soil variation. Journal of Soil Science 34:577-597. 
Bush, M. B., W. D. Gosling, and P. A. Colinvaux. 2011. Climate and vegetation change in the lowlands of the Amazon Basin. Pages 1-24 in M. B. Bush, J. R. Flenley, and W. D. Gosling, editors. Tropical rainforest fesponses to climatic change. Second. Springer-Verlag Berlin Heidelberg.

Bustamante, M. M. C., I. Roitman, T. M. Aide, A. Alencar, L. Anderson, L. Aragão, G. P. Asner, J. Barlow, et al. 2015. Towards an integrated monitoring framework to assess the effects of tropical forest. Global Change Biology 22:92-109.

Butterfield, B. J., and K. N. Suding. 2013. Single-trait functional indices outperform multi-trait indices in linking environmental gradients and ecosystem services in a complex landscape. Journal of Ecology 101:9-17.

Cairns, M. A., S. Brown, E. H. Helmer, and G. A. Baumgardner. 1997. Root biomass allocation in the world's upland forests. Oecologia 111:1-11.

Canadell, J. G., and E. D. Schulze. 2014. Global potential of biospheric caron management for climate mitigation. Nature communications 5:5282.

Canadell, J. G., and M. R. Raupach. 2008. Managing forests for climate change mitigation. Science 320:320-1456.

Cardinale, B. J., K. L. Matulich, D. U. Hooper, J. E. Byrnes, E. Duffy, L. Gamfeldt, P. Balvanera, M. I. O’Connor, et al. 2011. The functional role of producer diversity in ecosystems. American journal of botany 98:572-92.

Carreño-Rocabado, G., M. Peña-claros, F. Bongers, J. Licona, and L. Poorter. 2012. Effects of disturbance intensity on species and functional diversity in a tropical forest. Journal of Ecology 100:1453-1463.

de Carvalho, J. O. P. 1992. Structure and dynamics of a logged over Brazilian Amazonian rainforest. PhD Thesis. University of Oxford, Oxford, UK.

Chambers, J. Q., N. Higuchi, and J. P. Schimel. 1998. Ancient trees in Amazonia. Nature 391:135-6.

Chao, K.-J., O. L. Phillips, E. Gloor, A. Monteagudo, A. Torres-Lezama, and R. V. Martínez. 2008. Growth and wood density predict tree mortality in Amazon forests. Journal of Ecology 96:281-292.

Chapin, F. S. I., P. A. Matson, and H. A. Mooney. 2011. Principles of terrestrial ecosystem ecology. Principles of terrestrial ecosystem ecology. Springer New York Dordrecht Heidelberg Londen.

Chave, J., R. Condit, S. Aguilar, A. Hernandez, S. Lao, and R. Perez. 2004. Error propagation and scaling for tropical forest biomass estimates. Philosophical transactions of the Royal Society of London. Series B, Biological sciences 359:409-20.

Chave, J., R. Condit, H. C. Muller-Landau, S. C. Thomas, P. S. Ashton, S. Bunyavejchewin, L. L. Co, H. S. Dattaraja, et al. 2008. Assessing evidence for a pervasive alteration in tropical tree communities. PLoS Biology 6:0455-0462.

Chave, J., D. Coomes, S. Jansen, S. L. Lewis, N. G. Swenson, and A. E. Zanne. 2009. Towards a worldwide wood economics spectrum. Ecology letters 12:351-66.

Chave, J., H. C. Muller-Landau, T. R. Baker, T. A. Easdale, H. ter Steege, and C. O. Webb. 2006. Regional and phylogenetic variation of wood density across 2456 Neotropical tree species. Ecological applications 16:2356-67.

Chave, J., M. Réjou-Méchain, A. Búrquez, E. Chidumayo, M. S. Colgan, W. B. C. Delitti, A. Duque, T. Eid, et al. 2014a. Improved allometric models to estimate the aboveground biomass of tropical trees. Global Change Biology 20:3177-3190.

Chazdon, R. L. 2003. Tropical forest recovery: legacies of human impact and natural disturbances. Perspectives in Plant Ecology, Evolution and Systematics 6:51-71.

Chisholm, R. A., H. C. Muller-Landau, K. Abdul Rahman, D. P. Bebber, Y. Bin, S. A. Bohlman, N. A. Bourg, J. Brinks, et al. 2013. Scale-dependent relationships between tree species richness and ecosystem function in forests. Journal of Ecology 101:1214-1224.

Chitale, V. S., P. Tripathi, M. D. Behera, S. K. Behera, and R. Tuli. 2012. On the relationships among diversity, productivity and climate from an Indian tropical ecosystem: A preliminary investigation. Biodiversity and Conservation 21:1177-1197.

Choat, B., S. Jansen, T. J. Brodribb, H. Cochard, S. Delzon, R. Bhaskar, S. J. Bucci, T. S. Feild, et al. 2012. Global convergence in the vulnerability of forests to drought. Nature 491:752-755.

Clark, D. B., and M. W. Palmer. 1999. Edaphic factors and the landscape-scale distributions of tropical rain forest trees. Ecology 80:2662-2675.

Clark, D. B., and D. A. Clark. 1996. Abundance, growth and mortality of very large trees in neotropical lowland rain forest. Forest Ecology and Management 80:235-244.

Clark, D. A., and D. B. Clark. 1999. Assessing the growth of tropical rain forest trees: issues for forest modeling and management. Ecological Applications 9:981-997.

Clark, J. S. 2010. Individuals and the variation needed for high species diversity in forest trees. Science 327:1129-32.

Clark, J. S., D. M. Bell, M. H. Hersh, M. C. Kwit, E. Moran, C. Salk, A. Stine, D. Valle, et al. 2011. Individual-scale variation, species-scale differences: inference needed to understand diversity. Ecology letters 14:1273-87.

Clement, C. R., W. M. Denevan, M. J. Heckenberger, A. B. Junqueira, E. G. Neves, W. G. Teixeira, and W. I. Woods. 2015. The domestication of Amazonia before European conquest. Proceedings of the Royal Society B: Biological Sciences 282:20150813.

Cochard, H., and M. T. Tyree. 1990. Xylem dysfunction in Quercus: vessel sizes, tyloses, cavitation and seasonal changes in embolism. Tree physiology 6:393-407.

Colwell, R. K. 2011. EstimateS: Statistical Estimation of Species Richness and Shared Species from Samples. Version 
9. 2011. User's Guide and application published at http://purl.oclc.org/estimates.

Connell, J. H. 1978. Diversity in tropical rain forests and coral reefs. Science 199:1302-1310.

Conti, G., and S. Díaz. 2013. Plant functional diversity and carbon storage - an empirical test in semi-arid forest ecosystems. Journal of Ecology 101:18-28.

Cornwell, W. K., J. H. C. Cornelissen, K. Amatangelo, E. Dorrepaal, V. T. Eviner, O. Godoy, S. E. Hobbie, B. Hoorens, et al. 2008. Plant species traits are the predominant control on litter decomposition rates within biomes worldwide. Ecology letters 11:1065-71.

Coste, S., C. Baraloto, C. Leroy, É. Marcon, A. Renoud, D. Richardson, Andrew, J. Roggy, H. Schimann, et al. 2010. Assessing foliar chlorophyll contents with the SPAD-502 chlorophyll meter: a calibration test with thirteen tree species of tropical rainforest in French Guiana. Annals of Forest Science 67:1-5.

Cox, P. M., R. a Betts, C. D. Jones, S. a Spall, and I. J. Totterdell. 2000. Acceleration of global warming due to carbon-cycle feedbacks in a coupled climate model. Nature 408:184-7.

Crutzen, P. J. 2002. Geology of mankind. Nature 415:2002.

van Dam, O. 2001. Forest filled with gaps. Effects of gap size on water and nutrient cycling in tropoical rain forest. A study in Guyana. PhD thesis, Utrecht University, Utrecht.

Dauber, E., T. S. Fredericksen, and M. Peña. 2005. Sustainability of timber harvesting in Bolivian tropical forests. Forest Ecology and Management 214:294-304.

Dawkins, H. C., and D. R. B. Field. 1978. A long-term surveillance system for British woodland vegetation. Occassional Papers No. 1. Department of Forestry, Oxford University, Oxford.

Delbart, N., P. Ciais, J. Chave, N. Viovy, Y. Malhi, and T. Le Toan. 2010. Mortality as a key driver of the spatial distribution of aboveground biomass in Amazonian forest: Results from a dynamic vegetation model.

Biogeosciences 7:3027-3039.

DeVries, B., J. Verbesselt, L. Kooistra, and M. Herold. 2015. Robust monitoring of small-scale forest disturbances in a tropical montane forest using Landsat time series. Remote Sensing of Environment 161:107-121.

De Deyn, G. B., J. H. C. Cornelissen, and R. D. Bardgett. 2008. Plant functional traits and soil carbon sequestration in contrasting biomes. Ecology letters 11:516-31.

Díaz, S., A. Hector, and D. A. Wardle. 2009. Biodiversity in forest carbon sequestration initiatives: not just a side benefit. Current Opinion in Environmental Sustainability 1:55-60.

Dirzo, R., and P. H. Raven. 2003. Global state of biodiversity loss. Annual Review of Environment and Resources 28:137-167.

Durán, S. M., G. A. Sánchez-azofeifa, R. S. Rios, and E. Gianoli. 2015. The relative importance of climate, stand variables and liana abundance for carbon storage in tropical forests. Global Ecology and Biogeography 24:939-949.

Duro, D. C., N. C. Coops, M. A. Wulder, and T. Han. 2007. Development of a large area biodiversity monitoring system driven by remote sensing. Progress in Physical Geography 31:235-260.

Dutrieux, L. P., L. Poorter, J. Equihua, N. Ascarrunz, M. Herold, M. Penã-Claros, G. Roerink, M. Toledo, et al. Country wide mapping of forest diversity and structure by combining forest inventories with remote sensing.In review.

Dutrieux, L. P., J. Verbesselt, L. Kooistra, and M. Herold. 2015. Monitoring forest cover loss using multiple data streams, a case study of a tropical dry forest in Bolivia. ISPRS Journal of Photogrammetry and Remote Sensing 107:112-125.

Edwards, D. P., J. A. Tobias, D. Sheil, E. Meijaard, and W. F. Laurance. 2014. Maintaining ecosystem function and services in logged tropical forests. Trends in Ecology \& Evolution 29:511-520.

Ehrenfeld, J. G., B. Ravit, and K. Elgersma. 2005. Feedback in the plant-soil system. Annual Review of Environment and Resources 30:75-115.

Engelbrecht, B. M. J., L. S. Comita, R. Condit, T. A. Kursar, M. T. Tyree, B. L. Turner, and S. P. Hubbell. 2007. Drought sensitivity shapes species distribution patterns in tropical forests. Nature 447:80-2.

Engelbrecht, B. M. J., and T. A. Kursar. 2003. Comparative drought-resistance of seedlings of 28 species of cooccurring tropical woody plants. Oecologia 136:383-393.

Enquist, B. J., and C. A. F. Enquist. 2011. Long-term change within a Neotropical forest: assessing differential functional and floristic responses to disturbance and drought. Global Change Biology 17:1408-1424.

Espírito-Santo, F. D. B., M. Gloor, M. Keller, Y. Malhi, S. Saatchi, B. Nelson, R. C. O. Junior, C. Pereira, et al. 2014. Size and frequency of natural forest disturbances and the Amazon forest carbon balance. Nature communications 5:3434.

Evans, J. R. 1989. Photosynthesis and nitrogen relationship in leaves of C3 plants. Oecologia 78:9-19.

Falster, D. S., Å. Brännström, U. Dieckmann, and M. Westoby. 2011. Influence of four major plant traits on average height, leaf-area cover, net primary productivity, and biomass density in single-species forests: a theoretical investigation. Journal of Ecology 99:148-164.

FAO. 2010. FAO statistical yearbook 2010. URL:http://www. Fao.Org/economic/ess/ess-publications/essyearbook/ess-yearbook 2010/yearbook2010-consumption/en/.

FAO. 2014. Forest and landscape restoration mechanism. Rome, Italy.

FAO. 2016. Forests and poverty reduction. Retrieved March 14, 2016. http://www.fao.org/forestry/livelihoods/. 
http://www.fao.org/forestry/livelihoods/en/.

Farrior, C. E., S. A. Bohlman, S. Hubbell, and S. W. Pacala. 2016. Dominance of the suppressed: power-law size structure in tropical forests. Science 351:155-157.

Fauset, S., T. R. Baker, S. L. Lewis, T. R. Feldpausch, K. Affum-Baffoe, E. G. Foli, K. C. Hamer, and M. D. Swaine. 2012. Drought-induced shifts in the floristic and functional composition of tropical forests in Ghana. Ecology letters 15:1120-9.

Fauset, S., M. O. Johnson, M. Gloor, T. R. Baker, A. Monteagudo M., R. J. W. Brienen, T. R. Feldpausch, G. LopezGonzalez, et al. 2015. Hyperdominance in Amazonian forest carbon cycling. Nature Communications 6:6857.

Fayolle, A., B. Engelbrecht, V. Freycon, F. Mortier, M. Swaine, M. Réjou-Méchain, J.-L. Doucet, N. Fauvet, et al. 2012. Geological substrates shape tree species and trait distributions in African moist forests. PloS ONE 7:e42381.

Feeley, K. J., S. J. Davies, R. Perez, S. P. Hubbell, and R. B. Foster. 2011. Directional changes in the species composition of a tropical forest. Ecology 92:871-82.

Feret, J.-B., and G. P. Asner. 2014. Mapping tropical forest canopy diversity using high-fidelity imaging spectroscopy. Ecological Applications 24:1289-1296.

Fernández-Martínez, M., S. Vicca, I. A. Janssens, J. Sardans, S. Luyssaert, M. Campioli, F. S. Chapin III, P. Ciais, et al. 2014. Nutrient availability as the key regulator of global forest carbon balance. Nature Climate Change 4:471476.

Ferry, B., F. Morneau, J. D. Bontemps, L. Blanc, and V. Freycon. 2010. Higher treefall rates on slopes and waterlogged soils result in lower stand biomass and productivity in a tropical rain forest. Journal of Ecology 98:106-116.

Finegan, B., M. Peña-Claros, A. de Oliveira, N. Ascarrunz, M. S. Bret-Harte, G. Carreño-Rocabado, F. Casanoves, S. Díaz, et al. 2015. Does functional trait diversity predict above-ground biomass and productivity of tropical forests? Testing three alternative hypotheses. Journal of Ecology 103:191-201.

Fisher, J. I., G. C. Hurtt, R. Q. Thomas, and J. Q. Chambers. 2008. Clustered disturbances lead to bias in large-scale estimates based on forest sample plots. Ecology Letters 11:554-563.

Foster, S., and C. H. Janson. 1985. The relationship between seed size and establishment conditions in tropical woody plants. Ecology 66:773-780.

Fraser, L. H., J. Pither, A. Jentsch, M. Sternberg, M. Zobel, D. Askarizadeh, S. Bartha, C. Beierkuhnlein, et al. 2015. Worldwide evidence of a unimodal relationship between productivity and plant species richness. Science 349:302306.

Fyllas, N. M., S. Patiño, T. R. Baker, G. Bielefeld Nardoto, L. A. Martinelli, C. A. Quesada, R. Paiva, M. Schwarz, et al. 2009. Basin-wide variations in foliar properties of Amazonian forest: phylogeny, soils and climate. Biogeosciences 6:2677-2708.

Fyllas, N. M., E. Gloor, L. M. Mercado, S. Sitch, C. A. Quesada, T. F. Domingues, D. R. Galbraith, A. TorreLezama, et al. 2014. Analysing Amazonian forest productivity using a new individual and trait-based model (TFS v.1). Geoscientific Model Development Discussions 7:1413-1452.

Galbraith, D., Y. Malhi, K. Affum-Baffoe, A. D. A. Castanho, C. E. Doughty, R. A. Fisher, S. L. Lewis, K. S.-H. Peh, et al. 2013. Residence times of woody biomass in tropical forests. Plant Ecology \& Diversity 6:139-157.

Gale, M. R., and D. F. Grigal. 1987. Vertical root distributions of northern tree species in relation to successional status. Canadian Journal of Forest Research 17:829-834.

Gale, N., and A. S. Barfod. 1999. Canopy tree mode of death in a western Ecuadorian rain forest. Journal of Tropical Ecology 15:415-436.

Galetti, M., R. Guevara, M. C. Côrtes, R. Fadini, S. Von Matter, A. B. Leite, F. Labecca, T. Ribeiro, et al. 2013. Functional extinction of birds drives rapid evolutionary changes in seed size. Science 340:1086-1090.

Gallardo-Cruz, J. A., J. A. Meave, E. J. González, E. E. Lebrija-Trejos, M. A. Romero-Romero, E. A. Pérez-García, R. Gallardo-Cruz, J. L. Hernández-Stefanoni, et al. 2012. Predicting tropical dry forest successional attributes from space: is the key hidden in image texture? PloS one 7:e30506.

Galván, J. D., J. J. Camarero, G. Sangüesa-Barreda, A. Q. Alla, and E. Gutiérrez. 2012. Sapwood area drives growth in mountain conifer forests. Journal of Ecology 100:1233-1244.

Gamfeldt, L., T. Snäll, R. Bagchi, M. Jonsson, L. Gustafsson, P. Kjellander, M. C. Ruiz-Jaen, M. Fröberg, et al. 2013. Higher levels of multiple ecosystem services are found in forests with more tree species. Nature communications 4:1340.

Gardner, T. A., N. D. Burgess, N. Aguilar-Amuchastegui, J. Barlow, E. Berenguer, T. Clements, F. Danielsen, J. Ferreira, et al. 2012. A framework for integrating biodiversity concerns into national REDD+ programmes. Biological Conservation 154:61-71.

Garnier, E., J. Cortez, G. Billès, M.-L. Navas, C. Roumet, M. Debussche, G. Laurent, A. Blanchard, et al. 2004. Plant functional markers capture ecosystem properties during secondary succession. Ecology 85:2630-2637.

van Gelder, H. A., L. Poorter, and F. J. Sterck. 2006. Wood mechanics, allometry, and life-history variation in a tropical rain forest tree community. New Phytologist 171:367-378.

van Gemerden, B. S., H. Olff, M. P. E. Parren, and F. Bongers. 2003. The pristine rain forest? Remnants of historical human impacts on current tree species composition and diversity. Journal of Biogeography 30:1381-1390. 
Getzin, S., R. S. Nuske, and K. Wiegand. 2014. Using Unmanned Aerial Vehicles (UAV) to Quantify Spatial Gap Patterns in Forests. Remote Sensing 6:6988-7004.

Ghazoul, J., and D. Sheil. 2010. Tropoical rain forest ecology, diversity, and conservation. Oxford University Press Inc., New York.

Gibson, L., T. M. Lee, L. P. Koh, B. W. Brook, T. A. Gardner, J. Barlow, C. A. Peres, C. J. A. Bradshaw, et al. 2011. Primary forests are irreplaceable for sustaining tropical biodiversity. Nature 478:378-381.

van Gils, S. 2012. Changes in functional properties in a moist tropical forest during secondary succession. MSc thesis, Wageningen University, Wageningen.

Goldstein, G., J. L. Andrade, F. C. Meinzer, N. M. Holbrook, J. Cavelier, P. Jackson, and A. Celis. 1998. Stem water storage and diurnal patterns of water use in tropical forest canopy trees. Plant, Cell and Environment 21:397-406.

Goodman, R. C., and M. Herold. 2014. Why maintaining tropical forests is essential and urgent for a stable climate. CGD climate and forest paper series \#11.

Gosling, W. D., J. A. Hanselman, C. Knox, B. G. Valencia, and M. B. Bush. 2009. Long term drivers of change in Polylepis woodland distribution in the central Andes. Journal of Vegetation Science 20:1041-1052.

Gourlet-Fleury, S., V. Rossi, M. Rejou-Mechain, V. Freycon, A. Fayolle, L. Saint-André, G. Cornu, J. Gérard, et al. 2011. Environmental filtering of dense-wooded species controls above-ground biomass stored in African moist forests. Journal of Ecology 99:981-990.

Grace, J. B., and B. H. Pugesek. 1997. A structural equation model of plant species richness and its application to a coastal wetland. The American Naturalist 149:436-460.

Grace, J. B. 2006. Structural equation modeling and natural systems. Cambridge University Press, Cambridge.

Grace, J., E. Mitchard, and E. Gloor. 2014. Perturbations in the carbon budget of the tropics. Global change biology 20:3238-55.

Grime, J. P. 1998. Benefits of plant diversity to ecosystems: immediate, filter and founder effects. Journal of Ecology 86:902-910.

Grime, J. P. 1973. Competitive exclusion in herbaceous vegetation. Nature 242:344-347.

Hall, J. B., and M. D. Swaine. 1976. Classification and ecology of closed-canopy forest in Ghana. Journal of Ecology 64:913-951.

Hansen, M. C., P. V Potapov, R. Moore, M. Hancher, S. A. Turubanova, A. Tyukavina, D. Thau, S. V Stehman, et al. 2013. High-resolution global maps of 21st-century forest cover change. Science 342:850-3.

Harrell, F. E., and C. Dunpot. 2015. Package "Hmisc": Harrell miscellaneous. http://biostat.mc.vanderbilt.edu/Hmisc, https://github.com/harrelfe/Hmisc.

Hautier, Y., E. W. Seabloom, E. T. Borer, P. B. Adler, W. S. Harpole, H. Hillebrand, E. M. Lind, A. S. MacDougall, et al. 2014. Eutrophication weakens stabilizing effects of diversity in natural grasslands. Nature 508:521-5.

Heckenberger, M. J., A. Kuikuro, U. T. Kuikuro, J. C. Russell, M. Schmidt, C. Fausto, and B. Franchetto. 2003. Amazonia 1492: pristine forest or cultural parkland? Science 301:1710-1714.

Hector, A., Y. Hautier, P. Saner, L. Wacker, R. Bagchi, J. Joshi, M. Scherer-Lorenzen, E. M. Spehn, et al. 2010. General stabilizing effects of plant diversity on grassland productivity through population asynchrony and overyielding. Ecology 91:2213-2220.

Hector, A., and R. Bagchi. 2007. Biodiversity and ecosystem multifunctionality. Nature 448:188-190.

Hedin, L. O., E. N. J. Brookshire, D. N. L. Menge, and A. R. Barron. 2009. The nitrogen paradox in tropical forest ecosystems. Annual Review of Ecology, Evolution, and Systematics 40:613-635.

Hérault, B., B. Bachelot, L. Poorter, V. Rossi, F. Bongers, J. Chave, C. E. T. Paine, F. Wagner, et al. 2011. Functional traits shape ontogenetic growth trajectories of rain forest tree species. Journal of Ecology 99:1431-1440.

Hernández-Stefanoni, J. L., J. M. Dupuy, K. D. Johnson, R. Birdsey, F. Tun-Dzul, A. Peduzzi, J. P. Caamal-Sosa, G. Sánchez-Santos, et al. 2014. Improving species diversity and biomass estimates of tropical dry forests using airborne LiDAR. Remote Sensing 6:4741-4763.

Hietz, P., B. L. Turner, W. Wanek, A. Richter, C. A. Nock, and S. J. Wright. 2011. Long-term change in the nitrogen cycle of tropical forests. Science 334:664-666.

Hietz, P., R. Valencia, and S. J. Wright. 2013. Strong radial variation in wood density follows a uniform pattern in two neotropical rain forests. Functional Ecology 27:684-692.

Hijmans, R. J., S. E. Cameron, J. L. Parra, P. G. Jones, and A. Jarvis. 2005. Very high resolution interpolated climate surfaces for global land areas. International Journal of Climatology 25:1965-1978.

Hijmans, R. J., J. van Etten, J. Cheng, M. Mattiuzzi, M. Sumner, J. A. Greenberg, A. Bevan, and A. Shortridge. 2015. Package "raster": geographic data analysis and modeling. http://cran.r-project.org/package=raster.

Hirobe, M., J. Sabang, B. K. Bhatta, and H. Takeda. 2004. Leaf-litter decomposition of 15 tree species in a lowland tropical rain forest in Sarawak: Decomposition rates and initial litter chemistry. Journal of Forest Research 9:341346.

Hodgson, J. G. G., P. J. J. Wilson, R. Hunt, J. P. P. Grime, and K. Thompson. 1999. Allocating C-S-R plant functional types: a soft approach to a hard problem. Oikos 85:282-294.

Holling, C. S. 1973. Resilience and stability of ecological systems. Annual Review of Ecology and Systematics 4:1-23. Hooper, D. U., F. S. Chapin III, J. J. Ewel, A. Hector, P. Inchausti, S. Lavorel, J. H. Lawton, D. M. Lodge, et al. 
2005. Effects of biodiversity on ecosystem functioning: a consensus of current knowledge. Ecological Monographs 75:3-35.

Hooper, D. U., E. C. Adair, B. J. Cardinale, J. E. K. Byrnes, B. A. Hungate, K. L. Matulich, A. Gonzalez, J. E. Duffy, et al. 2012. A global synthesis reveals biodiversity loss as a major driver of ecosystem change. Nature 486:105-108.

Hoorn, C., F. P. Wesselingh, H. ter Steege, M. A. Bermudez, A. Mora, J. Sevink, I. Sanmartín, A. Sanchez-Meseguer, et al. 2010. Amazonia through time: Andean uplift, climate change, landscape evolution, and biodiversity. Science 330:927-931.

Hoosbeek, M. R., and G. E. Scarascia-Mugnozza. 2009. Increased litter build up and soil organic matter stabilization in a poplar plantation after 6 years of atmospheric CO2 enrichment (FACE): Final results of POP-EuroFACE compared to other forest FACE experiments. Ecosystems 12:220-239.

Hothorn, T., F. Bretz, and P. Westfall. 2014. Package multcomp: simultaneous inference in general parametric models. R package version 1.3-8. http://multcomp.R-forge.R-project.org.

Houghton, R. A. 2005. Aboveground forest biomass and the global carbon balance. Global Change Biology 11:945958.

Houghton, R. A., B. Byers, and A. A. Nassikas. 2015. A role for tropical forests in stabilizing atmospheric CO2. Nature Climate Change 5:1022-1023.

van der Hout, P. 1999. Reduced impact logging in the tropical rain forest of Guyana: ecological, economical and silvicultural consequences. PhD thesis, Utrecht University, Utrecht.

Houter, N. C., and T. L. Pons. 2005. Gap size effects on photoinhibition in understorey saplings in tropical rainforest. Plant Ecology 179:43-51.

Huston, M. 1979. A general hypothesis of species diversity. The American Naturalist 130:81-101.

Huston, M. 1980. Soil nutrients and tree species richness in Costa Rican forests. Journal of Biogeography 7:147-157.

Iida, Y., L. Poorter, F. Sterck, A. R. Kassim, M. D. Potts, T. Kubo, and T. S. Kohyama. 2014. Linking sizedependent growth and mortality with architectural traits across 145 co-occurring tropical tree species. Ecology 95:353-363.

IPCC. 2007. Climate change 2007 - The physical science basis. Contribution of Working Group I to the Fourth Assessment Report of the Intergovernmental Panel on Climate Change (eds S. Solomon., D. Qin, M. Manning, Z. Chen, M. Marquis, K.B. Averyt, M. Tignor \& H.L. Mil.

Isbell, F., V. Calcagno, A. Hector, J. Connolly, W. S. Harpole, P. B. Reich, M. Scherer-Lorenzen, B. Schmid, et al. 2011. High plant diversity is needed to maintain ecosystem services. Nature 477:199-202.

Isbell, F., D. Craven, J. Connolly, M. Loreau, B. Schmid, C. Beierkuhnlein, T. M. Bezemer, C. Bonin, et al. 2015. Biodiversity increases the resistance of ecosystem productivity to climate extremes. Nature 526:574-577.

Jackson, R. B., J. Canadell, J. R. Ehleringer, H. A. Mooney, O. E. Sala, and E. D. Schulze. 1996. A global analysis of root distributions for terrestrial biomes. Oecologia 108:389-411.

Jans, L., L. Poorter, R. S. A. R. van Rompaey, and F. Bongers. 1993. Gaps and forest zones in tropical moist forest in Ivory Coast. Biotropica 25:258-269.

Jetz, W., J. Cavender-Bares, R. Pavlick, D. Schimel, F. W. Davis, G. P. Asner, R. Guralnick, J. Kattge, et al. 2016. Monitoring plant functional diversity from space. Nature Plants 2:16024.

Johnson, J. B., and K. S. Omland. 2004. Model selection in ecology and evolution. Trends in ecology \& evolution 19:101-8.

Johnson, M. O., D. Galbraith, E. Gloor, and E. Al. 2016. Variation in stem mortality rates determines patterns of aboveground biomass in Amazonian forests: implications for dynamic global vegetation models. Global Change Biology.

Jones, F. A., D. L. Erickson, M. A. Bernal, E. Bermingham, W. J. Kress, E. A. Herre, H. C. Muller-Landau, and B. L. Turner. 2011. The roots of diversity: below ground species richness and rooting distributions in a tropical forest revealed by DNA barcodes and inverse modeling. PloS one 6:e24506.

Jucker, T., O. Bouriaud, D. Avacaritei, and D. A. Coomes. 2014. Stabilizing effects of diversity on aboveground wood production in forest ecosystems: linking patterns and processes. Ecology letters 17:1560-1569.

Karfakis, T. N. S., and A. Andrade. 2013. Dynamics of functional composition of a Brazilian tropical forest in response to drought stress. World Academy of Science, Engineering and Technolgy 7:693-697.

Keddy, P. A. 1992. Assembly and response rules: two goals for predictive community ecology. Journal of Vegetation Science 3:157-164.

van Kekem, A. J., J. H. . Pulles, and Z. Khan. 1996. Soils of the rainforest in central Guyana. Tropenbos Guyana Series Vol. 2. Georgetown, Guyana.

King, A. W., W. M. Post, and S. D. Wullschleger. 1997. The potential response of terrestrial carbon storage to changes in climate and atmospheric CO2. Climatic Change 35:199-227.

King, D. A., S. J. Davies, and N. S. M. Noor. 2006a. Growth and mortality are related to adult tree size in a Malaysian mixed dipterocarp forest. Forest Ecology and Management 223:152-158.

King, D. A., S. J. Davies, S. Tan, and N. S. M. Noor. 2006b. The role of wood density and stem support costs in the growth and mortality of tropical trees. Journal of Ecology 94:670-680.

Kitajima, K., and L. Poorter. 2008. Functional basis for resource niche partitioning by tropical trees. Pages 160-181 
in W. P. Carson and S. A. Schnitzer, editors. Tropical forest community ecology. Blackwell Science.

Kitajima, K., A.-M. Llorens, C. Stefanescu, M. V. Timchenko, P. W. Lucas, and S. J. Wright. 2012. How cellulosebased leaf toughness and lamina density contribute to long leaf lifespans of shade-tolerant species. New Phytologist 195:640-52.

Kitajima, K., and L. Poorter. 2010. Tissue-level leaf toughness, but not lamina thickness, predicts sapling leaf lifespan and shade tolerance of tropical tree species. New Phytologist 186:708-21.

Koch, G. W., S. C. Sillett, G. M. Jennings, and S. D. Davis. 2004. The limits to tree height. Nature 428:851-854.

Koerselman, W., and A. Meuleman. 1996. The vegetation N: P ratio: a new tool to detect the nature of nutrient limitation. Journal of Applied Ecology 33:1441-1450.

Kohyama, T., E. Suzuki, T. Partomihardjo, T. Yamada, and T. Kubo. 2003. Tree species differentiation in growth, recruitment and allometry in relation to maximum height in a Bornean mixed dipterocarp forest. Journal of Ecology 91:797-806.

Kooistra, L., L. Dutrieux, J. Equihua, M. Kolb, G. Roerink, F. Gerard, and M. Peña Claros. 2015. Current contributions of biodiversity and ecosystems to climate change mitigation - an analysis using remote sensing datasets. Report ROBIN project D113.

de Kroon, H., M. Hendriks, J. van Ruijven, J. Ravenek, F. M. Padilla, E. Jongejans, E. J. W. Visser, and L. Mommer. 2012. Root responses to nutrients and soil biota: drivers of species coexistence and ecosystem productivity. Journal of Ecology 100:6-15.

Kuenzer, C., M. Ottinger, M. Wegmann, H. Guo, C. Wang, J. Zhang, S. Dech, and M. Wikelski. 2014. Earth observation satellite sensors for biodiversity monitoring: potentials and bottlenecks. International Journal of Remote Sensing 35:6599-6647.

Kunstler, G., D. Falster, D. A. Coomes, F. Hui, M. Kooyman, Robert, D. C. Laughlin, L. Poorter, M. Vanderwel, et al. 2016. Plant functional traits have globally consistent effects on competition. Nature 529:204-207.

van Lagen, B. 1996. Soil analyses. Page 314 in P. Buurman, B. van Lagen, and E. J. Velthorst, editors. Manual for soil and water analyses. Backhuys Publishers, Leiden, the Netherlands.

Laliberté, A. E., P. Legendre, and B. Shipley. 2015. Package FD: Measuring functional diversity (FD) from multiple traits, and other tools for functional ecology. R package version 1.0-12. http://cran.rproject.org/web/packages/FD/.

Laliberté, E., A. Paquette, P. Legendre, and A. Bouchard. 2009. Assessing the scale-specific importance of niches and other spatial processes on beta diversity: a case study from a temperate forest. Oecologia 159:377-88.

Larjavaara, M., and H. C. Muller-Landau. 2010. Rethinking the value of high wood density. Functional Ecology 24:701-705.

Laughlin, D. C., S. R. Abella, W. W. Covington, and J. B. Grace. 2007. Species richness and soil properties in Pinus ponderosa forests: A structural equation modeling analysis. Journal of Vegetation Science 18:231-242.

Laurance, W. F. 1999. Effects on the tropical deforestation crisis. Biological conservation 91:109-117.

Laurance, W. F., P. M. Fearnside, S. G. Laurance, P. Delamonica, T. E. Lovejoy, J. M. Rankin-de Merona, J. Q. Chambers, and C. Gascon. 1999. Relationship between soils and Amazon forest biomass: a landscape-scale study. Forest Ecology and Management 118:127-138.

Laurance, W. F., A. A. Oliveira, S. G. Laurance, R. Condit, H. E. M. Nascimento, A. C. Sanchez-Thorin, T. E. Lovejoy, A. Andrade, et al. 2004. Pervasive alteration of tree communities in undisturbed Amazonian forests. Nature 428:171-174.

Lavorel, S., and E. Garnier. 2002. Predicting changes in community composition and ecosystem functioning from plant traits: revisiting the Holy Grail. Functional Ecology 16:545-556.

Lewis, S. L., O. L. Phillips, T. R. Baker, J. Lloyd, Y. Malhi, S. Almeida, N. Higuchi, W. F. Laurance, et al. 2004. Concerted changes in tropical forest structure and dynamics: evidence from 50 South American long-term plots. Philosophical transactions of the Royal Society of London. Series B, Biological sciences 359:421-436.

Lewis, S. L., P. M. Brando, O. L. Phillips, G. M. F. van der Heijden, and D. Nepstad. 2011. The 2010 Amazon drought. Science 331:554.

Lewis, S. L., G. Lopez-Gonzalez, B. Sonké, K. Affum-baffoe, T. R. Baker, S. L. Lewis, G. Lopez-gonzalez, B. Sonke, et al. 2009. Increasing carbon storage in intact African tropical forests. Nature 457:1003-1006.

Lindenmayer, D. B., W. F. Laurance, and J. F. Franklin. 2012. Ecology. Global decline in large old trees. Science 338:1305-6.

Liu, X., N. G. Swenson, S. J. Wright, L. Zhang, K. Song, Y. Du, J. Zhang, X. Mi, et al. 2012. Covariation in plant functional traits and soil fertility within two species-rich forests. PloS ONE 7:e34767.

Lohbeck, M. 2014. Functional ecology of tropical forest recovery. PhD thesis, Wageningen University, Wageningen.

Lohbeck, M., L. Poorter, E. Lebrija-Trejos, M. Martínez-Ramos, J. A. Meave, H. Paz, E. A. Pérerz-García, I. E. Romero-Pérez, et al. 2013. Successional changes in functional composition contrast for dry and wet tropical forest. Ecology 94:1211-1216.

Lohbeck, M., L. Poorter, M. Martínez-Ramos, and F. Bongers. 2015. Biomass is the main driver of changes in ecosystem process rates during tropical forest succession. Ecology 96:1242-1252.

Loiola, P. P., M. Scherer-lorenzen, and M. Antônio. 2015. The role of environmental filters and functional traits in 
predicting the root biomass and productivity in savannas and tropical seasonal forests. Forest Ecology and Management 342:49-55.

Lopez, L., R. Villalba, and M. Peña-Claros. 2012. Determining the annual periodicity of growth rings in seven tree species of a tropical moist forest in Santa Cruz, Bolivia. Forest Systems 21:508-514.

Loreau, M. 2000. Biodiversity and ecosystem functioning: recent theoretical advances. Oikos 91:3-17.

Loreau, M., and A. Hector. 2001. Partitioning selection and complementarity in biodiversity experiments. Nature 412:72-76.

Loreau, M., S. Naeem, P. Inchausti, J. Bengtsson, J. P. Grime, A. Hector, D. U. Hooper, M. A. Huston, et al. 2001. Biodiversity and ecosystem functioning: current knowledge and future challenges. Science 294:804-809.

Loreau, M., N. Mouquet, and A. Gonzalez. 2003. Biodiversity as spatial insurance in heterogeneous landscapes. Proceedings of the National Academy of Sciences 100:12765-12770.

Lumley, T. 2015. Package "survey": Analysis of complex survey samples. http://r-survey.r-forge.rproject.org/survey/. Version 3.30-3.

Lusk, C. H. 2004. Leaf area and growth of juvenile temperate evergreens in low light: species of contrasting shade tolerance change rank during ontogeny. Functional Ecology 18:820-828.

von Lützow, M., I. Kögel-Knabner, K. Ekschmitt, E. Matzner, G. Guggenberger, B. Marschner, and H. Flessa. 2006. Stabilization of organic matter in temperate soils: Mechanisms and their relevance under different soil conditions A review. European Journal of Soil Science 57:426-445.

Maire, V., I. J. Wright, I. C. Prentice, N. H. Batjes, R. Bhaskar, P. M. van Bodegom, W. K. Cornwell, D. Ellsworth, et al. 2015. Global effects of soil and climate on leaf photosynthetic traits and rates. Global Ecology and Biogeography 24:706-717.

Malhi, Y., D. D. Baldocchi, and P. G. Jarvis. 1999. The carbon balance of tropical, temperate and boreal forests. Plant, Cell and Environment 22:715-740.

Malhi, Y. 2012. The productivity, metabolism and carbon cycle of tropical forest vegetation. Journal of Ecology 100:65-75.

Malhi, Y., L. E. O. C. Aragão, D. B. Metcalfe, R. Paiva, C. A. Quesada, S. Almeida, L. Anderson, P. Brando, et al. 2009. Comprehensive assessment of carbon productivity, allocation and storage in three Amazonian forests. Global Change Biology 15:1255-1274.

Malhi, Y., T. R. Baker, O. L. Phillips, S. Almeida, E. Alvarez, L. Arroyo, J. Chave, C. I. Czimczik, et al. 2004. The above-ground coarse wood productivity of 104 Neotropical forest plots. Global Change Biology 10:563-591.

Malhi, Y., C. E. Doughty, G. R. Goldsmith, D. B. Metcalfe, C. A. J. Girardin, T. R. Marthews, J. del Aguila-Pasquel, L. E. O. C. Aragão, et al. 2015. The linkages between photosynthesis, productivity, growth and biomass in lowland Amazonian forests. Global Change Biology 21:2283-2295.

Malhi, Y., T. A. Gardner, G. R. Goldsmith, M. R. Silman, and P. Zelazowski. 2014. Tropical forests in the Anthropocene. Annual Review of Environment and Resources 39:125-159.

Malhi, Y., J. T. Roberts, R. A. Betts, T. J. Killeen, W. Li, and C. A. Nobre. 2008. Climate change, deforestation and the fate of the Amazon. Science 319:169-172.

Malhi, Y., D. Wood, T. R. Baker, J. Wright, O. L. Phillips, T. Cochrane, P. Meir, J. Chave, et al. 2006. The regional variation of aboveground live biomass in old-growth Amazonian forests. Global Change Biology 12:1107-1138.

Mangan, S. A., S. A. Schnitzer, E. A. Herre, K. M. L. Mack, M. C. Valencia, E. I. Sanchez, and J. D. Bever. 2010. Negative plant-soil feedback predicts tree-species relative abundance in a tropical forest. Nature 466:752-5.

van Mantgem, P. J., N. L. N. Stephenson, J. C. Byrne, L. D. Daniels, J. F. Franklin, P. Z. Fulé, M. E. Harmon, A. J. Larson, et al. 2009. Widespread increase of tree mortality rates in the western United States. Science 323:521-524.

Di Marco, M., S. H. M. Butchart, P. Visconti, G. M. Buchanan, G. F. Ficetola, and C. Rondinini. 2015. Synergies and trade-offs in achieving global biodiversity targets. Conservation Biology 30:189-195.

Markesteijn, L., J. Iraipi, F. Bongers, and L. Poorter. 2010. Seasonal variation in soil and plant water potentials in a Bolivian tropical moist and dry forest. Journal of Tropical Ecology 26:497-508.

Markesteijn, L., L. Poorter, F. Bongers, H. Paz, and L. Sack. 2011a. Hydraulics and life history of tropical dry forest tree species: coordination of species' drought and shade tolerance. New phytologist 191:480-95.

Markesteijn, L., L. Poorter, H. Paz, L. Sack, and F. Bongers. 2011b. Ecological differentiation in xylem cavitation resistance is associated with stem and leaf structural traits. Plant, Cell and Environment 34:137-148.

Martin, P. A., A. C. Newton, and J. M. Bullock. 2013. Carbon pools recover more quickly than plant biodiversity in tropical secondary forests. Proceedings of the Royal Society 280:20132236.

Martins, K. G., M. C. M. Marques, E. dos Santos, and R. Marques. 2015. Effects of soil conditions on the diversity of tropical forests across a successional gradient. Forest Ecology and Management 349:4-11.

Mason, N. W. H., D. Mouillot, W. G. Lee, and J. B. Wilson. 2005. Functional richness, functional evenness and functional divergence: the primary components of functional diversity. Oikos 1:112-118.

Mayle, F. E., R. Burbridge, and T. J. Killeen. 2000. Millennial-scale dynamics of southern Amazonian rain forests. Science 290:2291-4.

Mayle, F. E., D. J. Beerling, W. D. Gosling, and M. B. Bush. 2004. Responses of Amazonian ecosystems to climatic and atmospheric carbon dioxide changes since the last glacial maximum. Philosophical transactions of the Royal 
Society of London. Series B, Biological sciences 359:499-514.

McDowell, N., H. Barnard, B. Bond, T. Hinckley, R. Hubbard, H. Ishii, B. Köstner, F. Magnani, et al. 2002. The relationship between tree height and leaf area: sapwood area ratio. Oecologia 132:12-20.

McGill, B. J. 2010. Matters of scale. Science 328:575-6.

McGill, B. J., B. J. Enquist, E. Weiher, and M. Westoby. 2006. Rebuilding community ecology from functional traits. Trends in ecology \& evolution 21:178-85.

McGroddy, M. E., T. Daufresne, and L. O. Hedin. 2004. Scaling of C : N : P stoichiometry in forests worldwide : implications of terrestrial Redfield-type ratios. Ecology 85:2390-2401.

McMahon, S. M., C. J. E. Metcalf, and C. W. Woodall. 2011. High-dimensional coexistence of temperate tree species: functional traits, demographic rates, life-history stages, and their physical context. PloS one 6:e16253.

McMurtrie, R. E., R. J. Norby, B. E. Medlyn, R. C. Dewar, D. A. Pepper, P. B. Reich, and C. V. M. Barton. 2008. Why is plant-growth response to elevated CO2 amplified when water is limiting, but reduced when nitrogen is limiting? A growth-optimisation hypothesis. Functional Plant Biology 35:521-534.

Meinzer, F. C., G. Goldstein, and J. L. Andrade. 2001. Regulation of water flux through tropical forest canopy trees: do universal rules apply? Tree physiology 21:19-26.

Meinzer, F. C., P. I. Campanello, J.-C. Domec, M. Genoveva Gatti, G. Goldstein, R. Villalobos-Vega, and D. R. Woodruff. 2008. Constraints on physiological function associated with branch architecture and wood density in tropical forest trees. Tree physiology 28:1609-17.

Meir, P., and J. Grace. 2002. Scaling relationships for woody tissue respiration in two tropical rain forests. Plant, Cell and Environment 25:963-973.

Melillo, J. M., J. D. Aber, and J. F. Muratore. 1982. Nitrogen and lignin control of hardwood leaf litter decomposition dynamics. Ecology 63:621-626.

Mercado, L. M., S. Patiño, T. F. Domingues, N. M. Fyllas, G. P. Weedon, S. Sitch, C. A. Quesada, O. L. Phillips, et al. 2011. Variations in Amazon forest productivity correlated with foliar nutrients and modelled rates of photosynthetic carbon supply. Philosophical transactions of the Royal Society of London. Series B, Biological sciences 366:3316-29.

Messier, J., B. J. McGill, and M. J. Lechowicz. 2010. How do traits vary across ecological scales? A case for traitbased ecology. Ecology letters 13:838-48.

Metcalfe, D., P. Meir, L. E. O. C. Aragão, A. da Costa, S. Almeida, A. Braga, P. Gonçalves, J. Athaydes, et al. 2008. Sample sizes for estimating key ecosystem characteristics in a tropical terra firme rainforest. Forest Ecology and Management 255:558-566.

Midgley, G. F. 2012. Biodiversity and ecosystem function. Science 335:174-175.

Mokany, K., J. Ash, and S. Roxburgh. 2008. Functional identity is more important than diversity in influencing ecosystem processes in a temperate native grassland. Journal of Ecology 96:884-893.

Morin, X., L. Fahse, C. de Mazancourt, M. Scherer-Lorenzen, and H. Bugmann. 2014. Temporal stability in forest productivity increases with tree diversity due to asynchrony in species dynamics. Ecology Letters 17:1526-1535.

Morin, X., L. Fahse, M. Scherer-Lorenzen, and H. Bugmann. 2011. Tree species richness promotes productivity in temperate forests through strong complementarity between species. Ecology Letters 14:1211-1219.

Mouillot, D., W. H. N. Mason, O. Dumay, and J. B. Wilson. 2005. Functional regularity: a neglected aspect of functional diversity. Oecologia 142:353-9.

Muller-Landau, H. C. 2004. Interspecific and inter-site variation in wood specific gravity of tropical trees. Biotropica 36:20-32.

Murray, J. P., R. Grenyer, S. Wunder, N. Raes, and J. P. G. Jones. 2015. Spatial patterns of carbon, biodiversity, deforestation threat, and REDD+ projects in Indonesia. Conservation Biology 29:1434-1445.

Nachtergaele, F., H. van Velthuizen, and L. Verelst. 2010. The harmonized world soil database. Version 1.2. FAO and IIASA.

Nasto, M. K., S. Alvarez-Clare, Y. Lekberg, B. W. Sullivan, A. R. Townsend, and C. C. Cleveland. 2014. Interactions among nitrogen fixation and soil phosphorus acquisition strategies in lowland tropical rain forests. Ecology Letters 17:1282-1289.

Nelson, B. W., V. Kapos, J. B. Adams, W. J. Oliveira, O. P. G. Braun, and I. L. do Amaral. 1994. Forest disturbance by large blowdowns in the Brazilian Amazon. Ecology 75:853-858.

Nelson, B. W. 2005. Pervasive alteration of tree communities in undisturbed Amazonian forests. Biotropica 37:158159.

Nelson, D. W., and L. E. Sommers. 1982. Total carbon, organic carbon, and organic matter. Pages 539-579 in A. L. Page, editor. Methods of soil analysis. Part 2. Chemical and microbiological properties. American Society of Agronomy, Inc., Soil Science Society of America.

Nepstad, D. C., C. R. Carvalho, E. A. Davidson, P. H. Jipp, P. A. Lefebvre, G. H. Negreiros, E. D. da Silva, T. A. Stone, et al. 1994. The role of deep roots in the hydrological and carbon cycles of Amazonian forests and pastures. Nature 372:666-669.

Nepstad, D. C., I. M. Tohver, D. Ray, P. Moutinho, and G. Cardinot. 2007. Mortality of large trees and lianas following experimental drought in an Amazon forest. Ecology 88:2259-69. 
Novozamsky, I., V. J. G. Houba, R. van Eck, and W. van Vark. 1983. A novel digestion technique for multi-element plant analysis. Communications in Soil Science and Plant Analysis 14:239-248.

Oberski, D. 2013. lavaan. survey: An R package for complex survey analysis of structural equation models. Journal of Statistical Software 57:1-27.

Oksanen, J. 2011. Multivariate analysis of ecological communities in R: vegan tutorial.

Oksanen, J., F. G. Blanchet, R. Kindt, P. Legendre, P. R. Minchin, R. B. O’Hara, G. L. Simpson, P. Solymos, et al. 2014. Package vegan: Community ecology package. R package version 2.2-0. http://cran.rproject.org/web/packages/vegan/.

Olson, D. M., E. Dinerstein, E. D. Wikramanayake, N. D. Burgess, G. V. N. Powell, E. C. Underwood, J. A.

D'amico, I. Itoua, et al. 2001. Terrestrial ecoregions of the world: a new map of life on Earth. BioScience 51:933.

Onoda, Y., M. Westoby, P. B. Adler, A. M. F. Choong, F. J. Clissold, J. H. C. Cornelissen, S. Díaz, N. J. Dominy, et al. 2011. Global patterns of leaf mechanical properties. Ecology letters 14:301-12.

Ordoñez, J. C., P. M. van Bodegom, J.-P. M. Witte, I. J. Wright, P. B. Reich, and R. Aerts. 2009. A global study of relationships between leaf traits, climate and soil measures of nutrient fertility. Global Ecology and Biogeography 18:137-149.

Paine, C. E. T., C. Baraloto, J. Chave, and B. Hérault. 2011. Functional traits of individual trees reveal ecological constraints on community assembly in tropical rain forests. Oikos 120:720-727.

Pakeman, R. J. 2014. Functional trait metrics are sensitive to the completeness of the species' trait data? Methods in Ecology and Evolution 5:9-15.

Pakeman, R. J. 2004. Consistency of plant species and trait responses to grazing along a productivity gradient: a multi-site analysis. Journal of Ecology 92:893-905.

Pakeman, R. J., and H. M. Quested. 2007. Sampling plant functional traits: What proportion of the species need to be measured? Applied Vegetation Science 10:91-96.

Pan, Y., R. A. Birdsey, J. Fang, R. Houghton, P. E. Kauppi, W. A. Kurz, O. L. Phillips, A. Shvidenko, et al. 2011. A large and persistent carbon sink in the World's forests. Science 333:988-93.

Paoli, G. D., L. M. Curran, and D. R. Zak. 2005. Phosphorus efficiency of Bornean rain forest productivity: Evidence against the unimodal efficiency hypothesis. Ecology 86:1548-1561.

Paquette, A., and C. Messier. 2011. The effect of biodiversity on tree productivity: from temperate to boreal forests. Global Ecology and Biogeography 20:170-180.

Parton, W., W. L. Silver, I. C. Burke, L. Grassens, M. E. Harmon, W. S. Currie, J. Y. King, E. C. Adair, et al. 2007. Global-scale similarities in nitrogen release patterns during long-term decomposition. Science 315:361-364.

Patiño, S., N. M. Fyllas, T. R. Baker, R. Paiva, C. A. Quesada, A. J. B. Santos, M. Schwarz, H. ter Steege, et al. 2012. Coordination of physiological and structural traits in Amazon forest trees. Biogeosciences 9:775-801.

Pavlick, R., D. T. Drewry, K. Bohn, B. Reu, and A. Kleidon. 2012. The Jena Diversity-Dynamic Global Vegetation Model (JeDi-DGVM): a diverse approach to representing terrestrial biogeography and biogeochemistry based on plant functional trade-offs. Biogeosciences Discussions 9:4627-4726.

Peet, R. K. 1974. The measurement of species diversity. Annual Review of Ecology and Systematics 5:285-307.

Pelletier, J., C. Codjia, and C. Potvin. 2012. Traditional shifting agriculture: tracking forest carbon stock and biodiversity through time in western Panama. Global Change Biology 18:3581-3595.

Peña-Claros, M., T. S. Fredericksen, A. Alarcón, G. M. Blate, U. Choque, C. Leaño, J. C. Licona, B. Mostacedo, et al. 2008. Beyond reduced-impact logging: Silvicultural treatments to increase growth rates of tropical trees. Forest Ecology and Management 256:1458-1467.

Peña-Claros, M., L. Poorter, A. Alarcón, G. Blate, U. Choque, T. S. Fredericksen, M. J. Justiniano, C. Leaño, et al. 2012. Soil effects on forest structure and diversity in a moist and a dry tropical forest. Biotropica 44:276-283.

Pérez-Harguindeguy, N., S. Díaz, E. Garnier, S. Lavorel, H. Poorter, P. Jaureguiberry, W. K. Cornwell, J. M. Craine, et al. 2013. New handbook for standardised measurement of plant functional traits worldwide. Australian Joural of Botany 61:167-234.

Phelps, J., D. A. Friess, and E. L. Webb. 2012a. Win-win REDD+ approaches belie carbon-biodiversity trade-offs. Biological Conservation 154:53-60.

Phelps, J., E. L. Webb, and W. M. Adams. 2012b. Biodiversity co-benefits of policies to reduce forest-carbon emissions. Nature Climate Change 2:497-503.

Phillips, N. G., M. G. Ryan, B. J. Bond, N. G. McDowell, T. M. Hinckley, and J. Cermák. 2003. Reliance on stored water increases with tree size in three species in the Pacific Northwest. Tree physiology 23:237-45.

Phillips, O. L., L. E. O. C. Aragão, S. L. Lewis, J. B. Fisher, J. Lloyd, G. López-González, Y. Malhi, A. Monteagudo, et al. 2009. Drought sensitivity of the Amazon rainforest. Science 323:1344-1347.

Phillips, O. L., G. Van Der Heijden, S. L. Lewis, G. López-gonzález, L. E. O. C. Aragão, J. Lloyd, Y. Malhi, A. Monteagudo, et al. 2010. Drought-mortality relationships for tropical trees. New Phytologist 187:631-646.

Pichancourt, J.-B., J. Firn, I. Chadès, and T. G. Martin. 2014. Growing biodiverse carbon-rich forests. Global change biology 20:382-93.

Pinheiro, J., and D. Bates. 2016. Package "nlme": Fit and compare Gaussian linear and nonlinear mixed-effects models. Version 3.1-124. 
Pla, L., F. Casanoves, and J. Di Rienzo. 2012. Quantifying functional biodiversity. Pages 27-51 in L. Pla, F. Casanoves, and J. Di Rienzo, editors. Quantifying Functional Biodiversity. Springer Netherlands, Dordrecht.

Plourde, B. T., V. K. Boukili, and R. L. Chazdon. 2014. Radial changes in wood specific gravity of tropical trees: inter- and intraspecific variation during secondary succession. Functional Ecology 29:111-120.

Poorter, H., Ü. Niinemets, A. Walter, F. Fiorani, and U. Schurr. 2010a. A method to construct dose-response curves for a wide range of environmental factors and plant traits by means of a meta-analysis of phenotypic data. Journal of Experimental Botany 61:2043-2055.

Poorter, H., and C. Remkes. 1990. Leaf area ratio and net assimilation rate of 24 wild species differing in relative growth rate. Oecologia 83:553-559.

Poorter, L., S. J. Wright, H. Paz, D. D. Ackerly, R. Condit, G. Ibarra-Manríquez, K. E. Harms, J. C. Licona, et al. 2008. Are functional traits good predictors of demographic rates? Evidence from five neotropical forests. Ecology 89:1908-20.

Poorter, L. 1999. Growth responses of 15 rain-forest tree species to a light gradient: the relative importance of morphological and physiological traits. Functional Ecology 13:396-410.

Poorter, L., M. Plassche, S. Willems, and R. G. A. Boot. 2004. Leaf traits and herbivory rates of tropical tree species differing in successional status. Plant Biology 6:746-754.

Poorter, L., M. T. van der Sande, J. Thompson, E. J. M. M. Arets, A. Alarcón, J. Álvarez-Sánchez, N. Ascarrunz, P. Balvanera, et al. 2015. Diversity enhances carbon storage in tropical forests. Global Ecology and Biogeography 24:1314-1328.

Poorter, L. 2008. The relationships of wood-, gas- and water fractions of tree stems to performance and life history variation in tropical trees. Annals of botany 102:367-75.

Poorter, L. 2009. Leaf traits show different relationships with shade tolerance in moist versus dry tropical forests. New Phytologist 181:890-900.

Poorter, L., and F. Bongers. 2006. Leaf traits are good predictors of plant performance across 53 rain forest species. Ecology 87:1733-1743.

Poorter, L., F. Bongers, T. M. Aide, A. M. Almeyda Zambrano, P. Balvanera, J. M. Becknell, V. Boukili, P. H. S. Brancalion, et al. 2016. Biomass resilience of Neotropical secondary forests. Nature 530:211-214.

Poorter, L., L. Bongers, and F. Bongers. 2006. Architecture of 54 moist-forest tree species: traits, trade-offs, and functional groups. Ecology 87:1289-301.

Poorter, L., and L. Markesteijn. 2008. Seedling traits determine drought tolerance of tropical tree species. Biotropica 40:321-331.

Poorter, L., I. McDonald, A. Alarcón, E. Fichtler, J.-C. Licona, M. Peña-Claros, F. Sterck, Z. Villegas, et al. 2010 b. The importance of wood traits and hydraulic conductance for the performance and life history strategies of 42 rainforest tree species. The New phytologist 185:481-92.

Poorter, L., and D. M. A. Rozendaal. 2008. Leaf size and leaf display of thirty-eight tropical tree species. Oecologia 158:35-46.

Potvin, C., Lady Mancilla, N. Buchmann, J. Monteza, T. Moore, M. Murphy, Y. Oelmann, M. Scherer-Lorenzen, et al. 2011. An ecosystem approach to biodiversity effects: Carbon pools in a tropical tree plantation. Forest Ecology and Management 261:1614-1624.

Prado-Junior, J. A., I. Schiavini, V. S. Vale, C. S. Arantes, M. T. van der Sande, M. Lohbeck, and L. Poorter. 2016. Conservative species drive biomass productivity in tropical dry forests. Journal of Ecology.

Putz, F. E., P. Sist, T. Fredericksen, and D. Dykstra. 2008a. Reduced-impact logging: Challenges and opportunities. Forest Ecology and Management 256:1427-1433.

Putz, F. E., P. D. Coley, K. Lu, A. Montalvo, and A. Aiello. 1983. Uprooting and snapping of trees: structural determinants and ecological consequences. Canadian Journal of Forest Research 13:1011-1020.

Putz, F. E., P. A. Zuidema, M. A. Pinard, R. G. A. Boot, J. A. Sayer, D. Sheil, P. Sist, and J. K. Vanclay. 2008 b. Improved tropical forest management for carbon retention. PLoS biology 6:e166.

Putz, F. E., P. A. Zuidema, T. Synnott, M. Peña-Claros, M. A. Pinard, D. Sheil, J. K. Vanclay, P. Sist, et al. 2012. Sustaining conservation values in selectively logged tropical forests: the attained and the attainable. Conservation Letters 5:296-303.

Quesada, C. A., J. Lloyd, L. O. Anderson, N. M. Fyllas, M. Schwarz, and C. I. Czimczik. 2011. Soils of Amazonia with particular reference to the RAINFOR sites. Biogeosciences 8:1415-1440.

Quesada, C. A., J. Lloyd, M. Schwarz, S. Patiño, T. R. Baker, C. Czimczik, N. M. Fyllas, L. Martinelli, et al. 2010. Variations in chemical and physical properties of Amazon forest soils in relation to their genesis. Biogeosciences 7:1515-1541.

Quesada, C. A., O. L. Phillips, M. Schwarz, C. I. Czimczik, T. R. Baker, S. Patiño, N. M. Fyllas, M. G. Hodnett, et al. 2012. Basin-wide variations in Amazon forest structure and function are mediated by both soils and climate. Biogeosciences 9:2203-2246.

Quintero-Vallejo, E., Y. Klomberg, F. Bongers, L. Poorter, M. Toledo, and M. Peña-Claros. 2015. Amazonian dark earth shapes the understory plant community in a Bolivian forest. Biotropica 47:152-161.

Quintero-Vallejo, E. M. 2015. Legacies of Amazonian dark earths on forest composition, structure and dynamics. 
$\mathrm{PhD}$ thesis, Wageningen University, Wageningen.

R Core Team. 2014. R: A language and environment for statistical computing. R Foundation for Statistical Computing, Vienna, Austria. URL http://www.R-project.org/.

Raaimakers, D. 1994. Growth of tropical rain forest trees as dependent on phosphorus supply. Tree saplings differing in regeneration strategy and their adaptations to a low phosphorus environment in Guyana. University Utrecht.

Redford, K. H. 1992. The empty forest. BioScience 42:412-422.

Reich, P. B., D. Tilman, F. Isbell, K. Mueller, S. E. Hobbie, D. F. B. Flynn, and N. Eisenhauer. 2012. Impacts of biodiversity loss escalate through time as redundancy fades. Science 336:589-592.

Reich, P. B. 2012. Key canopy traits drive forest productivity. Proceedings of the royal society Biological Sciences 279:2128-2134.

Reich, P. B. 2014. The world-wide "fast - slow" plant economics spectrum: a traits manifesto. Journal of Ecology 102:275-301.

Reiss, J., J. R. Bridle, J. M. Montoya, and G. Woodward. 2009. Emerging horizons in biodiversity and ecosystem functioning research. Trends in Ecology and Evolution 24:505-514.

Rice, R. E., R. E. Gullison, and J. W. Reid. 1997. Can sustainable management save tropical forests? Scientific American:44-49.

Richards, P. W. 1996. The tropical rain forest. Second edi. University Press, Cambridge, Cambridge.

Robinson, D. 2007. Implications of a large global root biomass for carbon sink estimates and for soil carbon dynamics. Proceedings of the Royal Society B: Biological Sciences 274:2753-2759.

Rockström, J., W. Steffen, K. Noone, A. Persson, F. S. Chapin, E. F. Lambin, T. M. Lenton, M. Scheffer, et al. 2009. A safe operating space for humanity. Nature 461:472-475.

Roggy, J. C., and M. F. Prévost. 1999. Nitrogen-fixing legumes and silvigenesis in a rain forest in French Guiana: a taxonomic and ecological approach. New Phytologist 144:283-294.

Romero, C., and B. M. Bolker. 2008. Effects of stem anatomical and structural traits on responses to stem damage: an experimental study in the Bolivian Amazon. Canadian Journal of Forest Research 38:611-618.

Rosseel, Y. 2012. lavaan: an R package for structural equation modeling. Journal of Statistical Software 48:1-36, URL http://www.jstatsoft.org/v48/i02/.

Roy, S., and J. S. Singh. 1994. Consequences of habitat heterogeneity for availability of nutrients in a dry tropical forest. Journal of Ecology 82:503-509.

Rozendaal, D. M. A., V. H. Hurtado, and L. Poorter. 2006. Plasticity in leaf traits of 38 tropical tree species in response to light; relationships with light demand and adult stature. Functional Ecology 20:207-216.

Rozendaal, D. M. A., and P. A. Zuidema. 2011. Dendroecology in the tropics: a review. Trees 25:3-16.

Ruesch, A., and H. K. Gibbs. 2008. New IPCC Tier-1 global biomass carbon map for the year 2000. Available online from the Carbon Dioxide Information Analysis Center [http://cdiac.ornl.gov], Oak Ridge National Laboratory, Oak Ridge, Tennessee.

Rüger, N., C. Wirth, S. J. Wright, and R. Condit. 2012. Functional traits explain light and size response of growth rates in tropical tree species. Ecology 93:2626-36.

van Ruijven, J., and F. Berendse. 2005. Diversity-productivity relationships: Initial effects, long-term patterns, and underlying mechanisms. Proceedings of the National Academy of Sciences of the United States of America 102:695-700.

van Ruijven, J., and F. Berendse. 2010. Diversity enhances community recovery, but not resistance, after drought. Journal of Ecology 98:81-86.

Ruiz-Jaen, M. C., and C. Potvin. 2011. Can we predict carbon stocks in tropical ecosystems from tree diversity? Comparing species and functional diversity in a plantation and a natural forest. The New phytologist 189:978-87.

Russell, A. E., C. A. Cambardella, J. J. Ewel, and T. B. Parkin. 2004. Species, rotation, and life-lorm diversity effects on soil carbon in experimental tropical ecosystems. Ecological Applications 14:47-60.

Ryan, M. G., R. M. Hubbard, D. A. Clark, and R. L. Sanford. 1994. Woody-tissue respiration for Simarouba amara and Minquartia guianensis, two tropical wet forest trees with different growth habits. Oecologia 100:213-220.

Saatchi, S. S., N. L. Harris, S. Brown, M. Lefsky, E. T. A. Mitchard, W. Salas, B. R. Zutta, W. Buermann, et al. 2011. Benchmark map of forest carbon stocks in tropical regions across three continents. Proceedings of the National Academy of Sciences of the United States of America 108:9899-904.

Sakschewski, B. 2015. Impacts of major anthropogenic pressures on the terrestrial biosphere and its resilience to global change. University of Potsdam, Potsdam.

Sakschewski, B., W. von Bloh, A. Boit, A. Rammig, J. Kattge, L. Poorter, J. Peñuelas, and K. Thonicke. 2015. Leaf and stem economics spectra drive diversity of functional plant traits in a dynamic global vegetation model. Global Change Biology 24:2711-2725.

van der Sande, M. T., E. J. M. M. Arets, M. Peña-Claros, M. R. Hoosbeek, Y. Cáceres-Siani, P. van der Hout, and L. Poorter. Soil fertility and species traits, but not species diversity, drive productivity and biomass stocks in a tropical rainforest. In review.

van der Sande, M., B. Sakschewski, E. Arets, W. von Bloh, A. Boit, M. Peña-Claros, K. Thonicke, and L. Poorter. 
Does trait diversity insure the long-term stability of biomass in tropical dry and wet forest? In prep.

van der Sande, M. T., M. Peña-Claros, N. Ascarrunz, E. J. M. M. Arets, J. C. Licona, M. Toledo, and L. Poorter.

Drivers of biomass change in a tropical forest: testing for effects of diversity, traits, and environment. In review.

van der Sande, M. T., P. A. Zuidema, and F. Sterck. 2015. Explaining biomass growth of tropical canopy trees: the importance of sapwood. Oecologia 117:1145-1155.

Santiago, L. S., S. J. Wright, K. E. Harms, J. B. Yavitt, C. Korine, M. N. Garcia, and B. L. Turner. 2012. Tropical tree seedling growth responses to nitrogen, phosphorus and potassium addition. Journal of Ecology 100:309-316.

Scheiter, S., L. Langan, and S. I. Higgins. 2013. Next-generation dynamic global vegetation models: learning from community ecology. The New phytologist 198:957-69.

Schieving, F., and H. Poorter. 1999. Carbon gain in a multispecies canopy: the role of specific leaf area and photosynthetic nitrogen-use e ffi ciency in the tragedy of the commons. New Phytologist 143:201-211.

Schnitzer, S. A., J. N. Klironomos, J. HilleRisLambers, L. L. Kinkel, P. B. Reich, K. Xiao, M. C. Rillig, B. A. Sikes, et al. 2011. Soil microbes drive the classic plant diversity-productivity pattern. Ecology 92:296-303.

Scholz, F. G., S. J. Bucci, G. Goldstein, F. C. Meinzer, A. C. Franco, and F. Miralles-Wilhelm. 2007. Biophysical properties and functional significance of stem water storage tissues in Neotropical savanna trees. Plant, cell \& environment 30:236-48.

Selaya, N. G., and N. P. R. Anten. 2010. Leaves of pioneer and later-successional trees have similar lifetime carbon gain in tropical secondary forest. Ecology 91:1102-1113.

Sesnie, S. E., B. Finegan, P. E. Gessler, and Z. Ramos. 2009. Landscape-scale environmental and floristic variation in Costa Rican old-growth rain forest remnants. Biotropica 41:16-26.

Shipley, B. 2006. Net assimilation rate, specific leaf area and leaf mass ratio: which is most closely correlated with relative growth rate? A meta-analysis. Functional Ecology 20:565-574.

Shipley, B. 2004. Cause and correlation in biology. A user's guide to path analysis, structural equations and causal inference. Cambridge University Press, Cambridge.

Silva, J. N. M., J. O. P. de Carvalho, J. C. A. Lopes, B. F. de Almeida, D. H. M. Costa, L. C. de Oliveira, J. K. Vanclay, and J. P. Skovsgaard. 1995. Growth and yield of a tropical rain forest in the Brazilian Amazon 13 years after logging. Forest Ecology and Management 71:267-274.

Sitch, S., C. Huntingford, N. Gedney, P. E. Levy, M. Lomas, S. L. Piao, R. Betts, P. Ciais, et al. 2008. Evaluation of the terrestrial carbon cycle, future plant geography and climate-carbon cycle feedbacks using five Dynamic Global Vegetation Models (DGVMs). Global Change Biology 14:2015-2039,.

Skidmore, A. K., N. Pettorelli, N. C. Coops, G. N. Geller, M. Hansen, R. Lucas, C. A. Mücher, B. O’Connor, et al. 2015. Agree on biodiversity metrics to track from space. Nature 523:403-405.

Slik, J. W. F., V. Arroyo-Rodríguez, S.-I. Aiba, P. Alvarez-Loayza, L. F. Alves, P. Ashton, P. Balvanera, M. L. Bastian, et al. 2015. An estimate of the number of tropical tree species. Proceedings of the National Academy of Sciences 111:7472-7477.

Slik, J. W. F., G. Paoli, K. McGuire, I. Amaral, J. Barroso, M. Bastian, L. Blanc, F. Bongers, et al. 2013. Large trees drive forest aboveground biomass variation in moist lowland forests across the tropics. Global Ecology and Biogeography 22:1261-1271.

Spasojevic, M. J., C. A. Bahlai, B. A. Bradley, B. J. Butterfield, M.-N. Tuanmu, S. Sistla, R. Wiederholt, and K. N. Suding. 2015. Scaling up the diversity-resilience relationship with trait databases and remote sensing data: the recovery of productivity after wildfire. Global Change Biology 22:1421-1432.

Spicer, R., and N. M. Holbrook. 2007. Parenchyma cell respiration and survival in secondary xylem: does metabolic activity decline with cell age? Plant, cell \& environment 30:934-43.

Spicer, R., and B. L. Gartner. 2001. The effects of cambial age and position within the stem on specific conductivity in Douglas-fir ( Pseudotsuga menziesii) sapwood. Trees 15:222-229.

Staudhammer, C. L., L. H. O. Wadt, and K. A. Kainer. 2013. Tradeoffs in basal area growth and reproduction shift over the lifetime of a long-lived tropical species. Oecologia 173:45-57.

ter Steege, H., and D. S. Hammond. 2001. Character convergence, diversity, and disturbance in tropical rain forest in Guyana. Ecology 82:3197-3212.

ter Steege, H., N. C. A. Pitman, T. J. Killeen, W. F. Laurance, C. A. Peres, J. E. Guevara, R. P. Salomão, C. V Castilho, et al. 2015. Estimating the global conservation status of more than 15,000 Amazonian tree species. Science Advances 1:e1500936.

ter Steege, H., N. C. A. Pitman, O. L. Phillips, J. Chave, D. Sabatier, A. Duque, J.-F. Molino, M.-F. Prévost, et al. 2006. Continental-scale patterns of canopy tree composition and function across Amazonia. Nature 443:444-7.

ter Steege, H., N. C. A. Pitman, D. Sabatier, C. Baraloto, R. P. Salomão, J. E. Guevara, O. L. Phillips, C. V Castilho, et al. 2013. Hyperdominance in the Amazonian tree flora. Science 342:1243092.

ter Steege, H., N. Pitman, D. Sabatier, H. Castellanos, P. van der Hout, C. Douglas, M. Silveira, O. Phillips, et al. 2003. A spatial model of tree $\alpha$-diversity and tree density for the Amazon. Biodiversity and Conservation 12:22552277.

Steffen, W., J. Grinevald, P. Crutzen, and J. McNeill. 2011. The Anthropocene: conceptual and historical. Philosophical Transactions of the Royal Society A 369:842-867. 
Stephenson, N. L., A. J. Das, R. Condit, S. E. Russo, P. J. Baker, N. G. Beckman, D. A. Coomes, E. R. Lines, et al. 2014. Rate of tree carbon accumulation increases continuously with tree size. Nature 507:90-93.

Sterck, F., L. Markesteijn, M. Toledo, F. Schieving, and L. Poorter. 2014. Sapling performance along resource gradients drives tree species distributions within and across tropical forests. Ecology 95:2514-2525.

Sterck, F. J., M. Martinéz-Ramos, G. Dyer-Leal, J. Rodríguez-Velazquez, and L. Poorter. 2003. The consequences of crown traits for the growth and survival of tree saplings in a Mexican lowland rainforest. Functional Ecology 17:194-200.

Sterck, F. J., L. Poorter, and F. Schieving. 2006. Leaf traits determine the growth-survival trade-off across rain forest tree species. The American naturalist 167:758-765.

Sterck, F. J., R. Zweifel, U. Sass-Klaassen, and Q. Chowdhury. 2008. Persisting soil drought reduces leaf specific conductivity in Scots pine (Pinus sylvestris) and pubescent oak (Quercus pubescens). Tree physiology 28:529-36.

Sterck, F., L. Markesteijn, F. Schieving, and L. Poorter. 2011. Functional traits determine trade-offs and niches in a tropical forest community. Proceedings of the National Academy of Sciences of the United States of America 108:20627-32.

Sterck, F., and F. Schieving. 2011. Modelling functional trait acclimation for trees of different height in a forest light gradient: emergent patterns driven by carbon gain maximization. Tree physiology 31:1024-37.

Strassburg, B. B. N., A. Kelly, A. Balmford, R. G. Davies, H. K. Gibbs, A. Lovett, L. Miles, C. D. L. Orme, et al. 2010. Global congruence of carbon storage and biodiversity in terrestrial ecosystems. Conservation Letters 3:98105.

Suding, K. N., S. Lavorel, F. S. Chapin, J. H. C. Cornelissen, S. Díaz, E. Garnier, D. Goldberg, D. U. Hooper, et al. 2008. Scaling environmental change through the community-level: a trait-based response-and-effect framework for plants. Global Change Biology 14:1125-1140.

Sullivan, B. W., W. K. Smith, A. R. Townsend, M. K. Nasto, S. C. Reed, R. L. Chazdon, and C. C. Cleveland. 2014. Spatially robust estimates of biological nitrogen (N) fixation imply substantial human alteration of the tropical $\mathrm{N}$ cycle. Proceedings of the National Academy of Sciences of the United States of America 111:8101-6.

De Sy, V., M. Herold, F. Achard, G. P. Asner, A. Held, J. Kellndorfer, and J. Verbesselt. 2012. Synergies of multiple remote sensing data sources for REDD+ monitoring. Current Opinion in Environmental Sustainability 4:696-706.

Talbot, J., S. L. Lewis, G. Lopez-Gonzalez, R. J. W. Brienen, A. Monteagudo, T. R. Baker, T. R. Feldpausch, Y. Malhi, et al. 2014. Methods to estimate aboveground wood productivity from long-term forest inventory plots. Forest Ecology and Management 320:30-38.

Telles, E. C. C., P. Barbosa de Camargo, L. A. Martinelli, S. E. Trumbore, E. Salazar da Costa, J. Santos, N. Higuichi, and R. Cosme Oliveira Jr. 2003. Influence of soil texture on carbon dynamics and storage potential in tropical forest soils of Amazonia. Global Biogeochemical Cycles 17:1-12.

Thomas, R. Q., J. R. Kellner, D. B. Clark, and D. R. Peart. 2013. Low mortality in tall tropical trees. Ecology 94:920929.

Tilman, D., P. B. Reich, J. Knops, D. Wedin, T. Mielke, and C. Lehman. 2001. Diversity and productivity in a longterm grassland experiment. Science 294:843-5.

Tilman, D., J. Knops, D. Wedin, P. Reich, M. Ritchie, and E. Siemann. 1997. The influence of functional diversity and composition on ecosystem processes. Science 277:1300-1302.

Tilman, D. 1999. The ecological consequences of changes in biodiversity: a search for general principles. Ecology 80:1455-1474.

Tilman, D., F. Isbell, and J. M. Cowles. 2014. Biodiversity and ecosystem functioning. Annual Review of Ecology and Systematics 45:471-493.

Tilman, D., P. B. Reich, and J. M. H. Knops. 2006. Biodiversity and ecosystem stability in a decade-long grassland experiment. Nature 441:629-632.

Toledo, M. 2010. Neotropical lowland forests along environmental gradients. PhD thesis, Wageningen University, Wageningen.

Toledo, M., M. Peña-Claros, F. Bongers, A. Alarcón, J. Balcázar, J. Chuviña, C. Leaño, J. C. Licona, et al. 2012. Distribution patterns of tropical woody species in response to climatic and edaphic gradients. Journal of Ecology 100:253-263.

Toledo, M., L. Poorter, M. Peña-Claros, A. Alarcón, J. Balcázar, C. Leaño, J. C. Licona, O. Llanque, et al. 2011. Climate is a stronger driver of tree and forest growth rates than soil and disturbance. Journal of Ecology 99:254 264.

UNFCCC. 2014. Report of the Conference of the Parties on its nineteenth session, held in Warsaw from 11 to 23 November 2013. FCCC/CP/2013/10/Add.1.

United Nations. 2015. Adoption of the Paris agreement. FCCC/CP/2015/L.9/Rev.1.

Valverde-Barrantes, O. J., K. A. Smemo, L. M. Feinstein, M. W. Kershner, and C. B. Blackwood. 2015. Aggregated and complementary: symmetric proliferation, overyielding, and mass effects explain fine-root biomass in soil patches in a diverse temperate deciduous forest landscape. New Phytologist 205:731-742.

Vargas, G. G., L. K. Werden, and J. S. Powers. 2015. Explaining legume success in tropical dry forests based on seed germination niches : a new hypothesis. Biotropica 47:277-280. 
Vellend, M., K. Verheyen, H. Jacquemyn, A. Kolb, H. Van Calster, G. Peterken, and M. Hermy. 2006. Extinction debt of forest plants persists for more than a century following habitat fragmentation. Ecology 87:542-548.

Venter, O., W. F. Laurance, T. Iwamura, K. A. Wilson, R. A. Fuller, and H. P. Possingham. 2009. Harnessing carbon payments to protect biodiversity. Science 326:1368.

Verburg, P. H., K. P. Overmars, M. G. A. Huigen, W. T. de Groot, and A. Veldkamp. 2006. Analysis of the effects of land use change on protected areas in the Philippines. Applied Geography 26:153-173.

Verheijen, L. M., V. Brovkin, R. Aerts, G. Bonisch, J. H. C. Cornelissen, J. Kattge, P. B. Reich, I. J. Wright, et al. 2013. Impacts of trait variation through observed trait-climate relationships on performance of an Earth system model: a conceptual analysis. Biogeosciences 10:5497-5515.

Vertessy, R. A., R. G. Benyon, S. K. O’Sullivan, and P. R. Gribben. 1995. Relationships between stem diameter, sapwood area, leaf area and transpiration in a young mountain ash forest. Tree physiology 15:559-67.

Vilà, M., A. Carrillo-Gavilán, J. Vayreda, H. Bugmann, J. Fridman, W. Grodzki, J. Haase, G. Kunstler, et al. 2013. Disentangling biodiversity and climatic determinants of wood production. PLoS ONE 8:e53530.

Villegas, Z., M. Peña-Claros, B. Mostacedo, A. Alarcón, J.C.Licona, C. Leaño, W. Pariona, and U. Choque. 2009. Silvicultural treatments enhance growth rates of future crop trees in a tropical dry forest. Forest Ecology and Management 258:971-977.

Violle, C., M.-L. Navas, D. Vile, E. Kazakou, C. Fortunel, I. Hummel, and E. Garnier. 2007. Let the concept of trait be functional! Oikos 116:882-892.

Violle, C., P. B. Reich, S. W. Pacala, B. J. Enquist, and J. Kattge. 2014. The emergence and promise of functional biogeography. Proceedings of the National Academy of Sciences 111:13690-13696.

Vitousek, P. M. 1984. Litterfall, nutrient cycling, and nutrient limitation in tropical torests. Ecology 65:285-298.

De Vos, J. M., L. N. Joppa, J. L. Gittleman, P. R. Stephens, and S. L. Pimm. 2015. Estimating the normal background rate of species extinction. Conservation Biology 29:452-462.

Waide, R. B., M. R. Willig, C. F. Steiner, G. Mittelbach, L. Gough, S. I. Dodson, G. P. Juday, and R. Parmenter. 1999. The relationship between productivity and species richness. Annual Review of Ecology and Systematics 30:257-300.

Walker, B. H. 1992. Biodiversity and ecological redundancy. Biological Conservation 6:18-23.

Walker, B., A. Kinzig, and J. Langridge. 1999. Plant attribute diversity, resilience, and ecosystem function: the nature and significance of dominant and minor species. Ecosystems 2:95-113.

Walters, M. B., and P. B. Reich. 1999. Low-light carbon balance and shade tolerance in the seedlings of woody plants: do winter deciduous and broad-leaved evergreen species differ? New Phytologist 143:143-154.

Wardle, D. A., K. I. Bonner, and G. M. Barker. 2002. Linkages between plant litter decomposition, litter quality, and vegetation responses to herbivory. Functional Ecology 16:585-595.

Wardle, D. A., R. D. Bardgett, J. N. Klironomos, H. Setälä, W. H. van der Putten, and D. H. Wall. 2004. Ecological linkages between aboveground and belowground biota. Science 304:1629-1633.

Westoby, M. 1998. A leaf-height-seed (LHS) plant ecology strategy scheme. Plant and Soil 199:213-227.

Whitehead, D., W. R. N. Edwards, and P. G. Jarvis. 1984. Conducting sapwood area, foliage area, and permeability in mature trees of Picea sitchensis and Pinus contorta. Canadian Journal of Forest Research 14:940-947.

Williamson, G. B., and M. C. Wiemann. 2010. Measuring wood specific gravity...Correctly. American journal of botany 97:519-24.

Woodcock, D. W., and A. D. Shier. 2002. Wood specific gravity and its radial variations: the many ways to make a tree. Trees 16:437-443.

Wright, I. J., P. B. Reich, and M. Westoby. 2001. Strategy-shifts in leaf physiology, structure and nutrient content between species of high and low rainfall, and high and low nutrient habitats. Functional Ecology 15:423-434.

Wright, I. J., P. B. Reich, J. H. C. Cornelissen, D. S. Falster, E. Garnier, K. Hikosaka, B. B. Lamont, W. Lee, et al. 2005. Assessing the generality of global leaf trait relationships. The New phytologist 166:485-96.

Wright, I. J., P. B. Reich, M. Westoby, D. D. Ackerly, Z. Baruch, F. Bongers, J. Cavender-Bares, T. Chapin, et al. 2004. The worldwide leaf economics spectrum. Nature 428:821-7.

Wright, S. J., K. Kitajima, N. J. B. Kraft, P. B. Reich, I. J. Wright, D. E. Bunker, R. Condit, J. W. Dalling, et al. 2010. Functional traits and the growth-mortality trade-off in tropical trees. Ecology 91:3664-74.

Wright, S. J., and H. C. Muller-landau. 2006. The uncertain future of tropical forest species. Biotropica 38:443-445.

Wright, S. J. 2005. Tropical forests in a changing environment. Trends in Ecology and Evolution 20:553-560.

Wright, S. J., H. C. Muller-Landau, and J. Schipper. 2009. The future of tropical species on a warmer planet. Conservation Biology 23:1418-1426.

Wulder, M. A., J. G. Masek, W. B. Cohen, T. R. Loveland, and C. E. Woodcock. 2012. Opening the archive: How free data has enabled the science and monitoring promise of Landsat. Remote Sensing of Environment 112:2-10.

Wullschleger, S. D., F. C. Meinzer, and R. A. Vertessy. 1998. A review of whole-plant water use studies in trees. Tree Physiology 18:499-512.

Yachi, S., and M. Loreau. 1999. Biodiversity and ecosystem productivity in a fluctuating environment: the insurance hypothesis. Proceedings of the National Academy of Sciences of the United States of America 96:1463-1468.

Zanne, A. E., G. Lopez-Gonzalez, D. A. Coomes, J. Ilic, S. Jansen, S. L. Lewis, R. B. Miller, N. G. Swenson, et al. 
2009. Data from: Towards a worldwide wood economics spectrum. Dryad Digital Repository.

http://dx.doi.org/10.5061/dryad.234. http://dx.doi.org/10.5061/dryad.234.

Zhang, J.-L., S.-B. Zhang, Y.-J. Chen, Y.-P. Zhang, and L. Poorter. 2015. Nutrient resorption is associated with leaf vein density and growth performance of dipterocarp tree species. Journal of Ecology 103:541-549.

Zhang, Y., H. Y. H. Chen, and P. B. Reich. 2012. Forest productivity increases with evenness, species richness and trait variation: a global meta-analysis. Journal of Ecology 100:742-749.

Zhang, Y., B. Duan, J. Xian, H. Korpelainen, and C. Li. 2011. Links between plant diversity, carbon stocks and environmental factors along a successional gradient in a subalpine coniferous forest in Southwest China. Forest Ecology and Management 262:361-369.

Zhou, G., B. Z. Houlton, W. Wang, W. Huang, Y. Xiao, Q. Zhang, S. Liu, M. Cao, et al. 2014. Substantial reorganization of China's tropical and subtropical forests: Based on the permanent plots. Global Change Biology 20:240-250.

Zimmerman, B. L., and C. F. Kormos. 2012. Prospects for Sustainable Logging in Tropical Forests. BioScience 62:479-487.

Zuidema, P. a, P. J. Baker, P. Groenendijk, P. Schippers, P. van der Sleen, M. Vlam, and F. Sterck. 2013. Tropical forests and global change: filling knowledge gaps. Trends in plant science 18:1-7.

Zuidema, P. A., R. J. W. Brienen, H. J. During, and B. Güneralp. 2009. Do persistently fast-growing juveniles contribute disproportionately to population growth? A new analysis tool for matrix models and its application to rainforest trees. The American naturalist 174:709-19. 
Summary 
Tropical forests are critically important for the global carbon cycle. They remove carbon dioxide from the atmosphere through photosynthesis and store this carbon as biomass. Tropical forests contain $25 \%$ of all carbon that is stored in the terrestrial biosphere and annually remove about $24 \%$ of our greenhouse gas emissions, thereby helping to mitigate climate change. For this reason the potential of tropical forests for climate change mitigation is increasingly acknowledged by international policies, such as in the agreements reached during the climate negotiations of the United Nations Framework Convention on Climate Change (UNFCCC), in December 2015 in Paris.

On top of their importance for climate change mitigation, tropical forests are the most diverse terrestrial ecosystems, hosting an estimated 47,000 tree species. This high diversity makes them particularly relevant for biodiversity conservation, especially given the threat of deforestation, forest degradation, hunting and climate change on the survival of many species. Research in less complex ecosystems such as grasslands or temperate forests has shown that high biodiversity is not only nice to have, but it also results in more productive ecosystems that store more carbon. These results are in line with the ecological niche complementarity bypothesis, which predicts that species have different strategies to acquire and use resources (such as water and nutrients), and therefore a very diverse community of plant species can more efficiently use all resources and result in higher overall productivity. The question remains, however, whether this would also be true for tropical forests, where species numbers are much higher and most plants live much longer. It could also be that instead of the diversity in species, the species that are most abundant mainly determine the storage and uptake of carbon in the forest, which is in line with the ecological mass-ratio hypothesis.

In this thesis, I evaluate the relationship between biodiversity and the capacity of tropical forests to store and take up carbon. As measure for carbon storage I use biomass stock, i.e. the standing biomass per area, and as measure for carbon uptake I use biomass dynamics, i.e. the fluxes in biomass over time such as biomass growth and biomass mortality. Biodiversity is mostly seen as the number of species per area. Just counting species, however, does not provide information on the functional characteristics (or 'traits') of the species, which is implicitly assumed to be important in the niche complementarity and mass-ratio hypotheses; biomass stocks and dynamics of the plant community would increase with the functional trait diversity within the community (in line with the niche complementarity hypothesis), and would depend on the average trait values of the community (in line with the mass-ratio hypothesis). For that reason, this thesis has a strong focus on plant functional traits (such as leaf nutrient concentrations and wood density) that should provide a functional understanding of biomass stocks and dynamics. 'Biodiversity' in this thesis can thus refer to species and trait diversity (reflecting 
niche complementarity), but also to the average traits of a community of trees (or 'community-mean traits', reflecting mass ratio).

The effects of biodiversity on biomass stocks and dynamics can depend on various variables, such as environmental conditions (e.g. soil fertility and rainfall) and the spatial and temporal scale considered. The main objectives of my thesis therefore were: 1) to understand the effect of biodiversity and environmental conditions on biomass stocks and dynamics in tropical forests, 2) to evaluate how these relationships depend on the spatial scale considered, and 3) to assess how these relationships depend on the temporal scale considered. To reach these objectives, I collaborated with research institutions in Bolivia, Brazil and Guyana that manage and monitor many hectares of tropical forest. In these forests, I collected data on leaf and stem traits for the most abundant plant species. By combining these two datasets, we had data for different measures of biodiversity, environmental conditions, and biomass stocks and dynamics.

Large trees are responsible for most of the carbon uptake by tropical forests. It is therefore important to understand what determines variation in biomass growth among large trees. In chapter 2 we looked at the effects of different traits on the biomass growth of large trees in a moist tropical forest in Bolivia. We found that biomass growth strongly increased with the size of the sapwood area, which is the living part of the wood that is responsible for water storage and transport from the roots to the leaves. Having a high capacity to transport and store water may especially be important for such large trees because they are tall, receive a lot of sunlight and experience high temperatures, and therefore they transpire a lot of water. Thus, the biomass growth of large tropical trees seems mainly limited by their high demand for water.

We then scaled from individual trees to whole communities of trees to evaluate the relationships of biodiversity and environmental conditions with community-level biomass stocks and dynamics. In chapter 3 we evaluated these relationships for a tropical wet forest growing on very nutrient-poor soils in Guyana. We found no effects of niche complementarity (i.e. of species diversity) but a strong effect of mass ratio (i.e. of community-mean trait values) and soil fertility on biomass stocks and dynamics. This means that on these poor soils, only a small set of species with the appropriate set of traits can survive well, grow fast, and become large and abundant.

In chapter 4 we evaluated the same relationships for a completely different forest: a moist tropical forest on very nutrient rich soils in Bolivia (the same forest as in chapter 2) that experiences 6 months of dry season. In this case we found that neither niche complementarity nor mass ratio were important for biomass dynamics. Instead, the soil water availability determined the biomass dynamics in 
this more seasonal and drier forest, which is in agreement with the importance of water availability for growth of large trees in chapter 2 .

The Neotropics (i.e, the tropical regions in South and Mesoamerica) have many more forest types and more variation in environmental conditions than included in chapters 3 and 4 . In chapter 5 we therefore considered the whole region of the Neotropics, using data from 201 1-ha plots and 26 forest sites, and assessed how biodiversity and environmental conditions determine biomass stocks and dynamics. In contrast with the previous two chapters, we found that both niche complementarity and mass ratio were very important for biomass stocks and dynamics. It appears that the strong differences among forests in soil conditions and climate result in strong differences in biodiversity, which in turn lead to strong differences in biomass stocks and dynamics at this very large spatial scale.

Tropical forests are dynamic systems with plants recruiting and dying all the time. We may therefore expect that tropical forests can respond to changes in environmental conditions, for example due to climate change. Depending on which environmental conditions change, some species with the right set of traits will grow and survive better than others and therefore become more abundant over time. In chapter 6 we assessed how five old-growth tropical forests (i.e. mature forests that are full-grown and undisturbed for at least the past decades) are changing in community mean trait values over time, and evaluated which causes are most likely underlying these changes. We found that over time the abundance increases of species that usually occur most in very old forests, whereas the abundance decreases of species that usually occur most in younger forests. This means that these forests seem to be in a recovering process, possibly caused by disturbances that happened long ago, such as intense drought events or disturbances by (preColumbian) human occupation.

The above chapters show different effects of biodiversity on biomass stocks and dynamics in tropical forests. To search for generalities in this relationship, we performed a literature review in chapter 7 . To do so, we focused not only on results from empirical studies (such as the other chapters of this thesis), but also on results from studies using remote sensing techniques and ecosystem computer models. From the 38 empirical studies, $74 \%$ showed that biodiversity was important for biomass stocks and carbon uptake in tropical forests. Remote sensing can be used to easily scale to large areas, and 9 out of 10 studies reported important effects of biodiversity on biomass stocks. Ecosystem models can be useful to evaluate future scenarios of, for example, biodiversity loss or climate change on biomass stocks. Currently only few ecosystem models can simulate higher levels of biodiversity, but these all show potentially strong positive effects of biodiversity on carbon uptake. Results of model simulations indicate that biodiversity is particularly important for assuring stable carbon uptake in the face of climate change. 
Consequently, we argue that biodiversity conservation is not only a side benefit of climate change mitigation efforts, but instead should be regarded as a requirement for improving and securing long-term carbon storage and uptake.

In sum, I found that biodiversity - including species diversity and communitymean traits - is important for the functioning of tropical forests, but that its precise effect depends on the environmental conditions, spatial scale, and temporal scale considered. Niche complementarity was most important at large spatial scales possibly because of strong differences in biodiversity between forests (chapter 5), whereas mass ratio was important at large spatial scales and in forests with harsh environmental conditions (e.g. the nutrient-poor soils in Guyana, chapter 3). Furthermore, tropical forests are not in a stable state, but changing environmental conditions lead to changes in biodiversity (measured as community-mean traits, chapter 6). This in turn may lead to changes in the future biomass stocks and carbon uptake of the forest, as biodiversity strongly determines the functioning of the forest in the long term (chapters 7, 8).

Tropical forests provide many local and global benefits to society such as climate change mitigation, water recycling, and the production of timber and nontimber forest products. In this thesis I show and discuss that the existence and persistence of these forests partly depend on their biodiversity. Ecological research should combine more forces with socio-economic research and appropriate political and technological developments to move towards long-term ecologically sustainable and profitable tropical forest landscapes. The conservation of tropical forests will be a challenge, but a challenge that must be overcome for the benefit of all organisms on Earth - including humans. 
Samenvatting 
Tropische bossen zijn van groot belang voor de wereldwijde koolstof cyclus. Ze nemen koolstofdioxide op uit de atmosfeer door middel van fotosynthese en slaan deze koolstof op als biomassa. Tropische bossen bevatten $25 \%$ van alle koolstof dat opgeslagen zit in natuur op land en nemen jaarlijks ongeveer $24 \%$ van onze broeikasgasemissies op. Hiermee leveren tropische bossen een belangrijke bijdrage aan het tegengaan van klimaatsverandering, ook wel klimaatmitigatie genoemd. Om deze reden wordt de potentie van tropische bossen in klimaatmitigatie steeds meer erkend in de internationale politieke agenda. Dit blijkt bijvoorbeeld uit de overeenstemmingen die bereikt zijn tijdens de klimaatonderhandelingen van de United Nations Framework Convention on Climate Change (UNFCCC) in december 2015 in Parijs.

Met een geschatte 47.000 verschillende boomsoorten zijn tropische bossen, naast hun rol die ze hebben in klimaatmitigatie, ook de meest diverse terrestrische ecosystemen. Door deze hoge soortdiversiteit zijn ze relevant voor biodiversiteitsbescherming, vooral vanwege ontbossing, bosdegradatie, jacht en klimaatsverandering die de overleving van veel soorten bedreigen. Onderzoek in minder complexe ecosystemen zoals graslanden en gematigde bossen hebben aangetoond dat een hoge biodiversiteit niet alleen mooi is, maar ook zorgt voor productievere ecosystemen die meer koolstof opslaan. Deze resultaten komen overeen met de niche complementarity (niche complementariteit) hypothese die voorspelt dat soorten verschillende strategieën hebben om hulpbronnen (zoals water en nutriënten) te verkrijgen en te gebruiken. Hierdoor kan een diverse plantengemeenschap efficiënter de aanwezige hulpbronnen gebruiken en in zijn geheel een hogere productiviteit bereiken. De vraag blijft echter of dit ook het geval zal zijn voor tropische bossen, waar veel meer soorten voorkomen en de meeste planten langlevend zijn. Het zou namelijkook kunnen dat de koolstofopslag en opname in plaats van door de hoeveelheid soorten, bepaald wordt door de meest veelvoorkomende soorten, wat overeenstemt met de ecologische mass-ratio (massaverhouding) hypothese.

In dit proefschrift evalueer ik de relatie tussen biodiversiteit en de capaciteit van tropische bossen om koolstof op te nemen en op te slaan. Als maat voor koolstofopslag gebruik ik de hoeveelheid plantbiomassa per eenheid grondoppervlak, ofwel de biomassavoorraad. Als maat voor koolstofopname gebruik ik de biomassadynamiek, gemeten als de verandering van biomassa in de tijd zoals groei en mortaliteit. Biodiversiteit wordt meestal gezien als het aantal soorten per eenheid grondoppervlak. Enkel het tellen van soorten geeft echter geen informatie over de functionele eigenschappen (zoals bladnutriëntenconcentraties en houtdichtheid) van de soorten, wat impliciet belangrijk is in de niche complementarity en de mass-ratio hypotheses. De niche complementarity hypothese voorspelt dat de biomassavoorraad en -dynamiek van de plantengemeenschap toenemen door een 
hoge diversiteit in functionele eigenschappen van de soorten. De mass-ratio hypothese, daarentegen, voorspelt dat biomassavoorraad en -dynamiek afhangen van de eigenschappen van de meest veelvoorkomende soorten. Functionele planteigenschappen moeten dus een beter functioneel begrip geven van biomassavoorraden en -dynamiek. Om die reden heeft dit proefschrift een sterke focus op de functionele eigenschappen van planten. 'Biodiversiteit' in dit proefschrift kan daarom verwijzen naar de diversiteit in soorten en hun eigenschappen, als indicatie voor niche complementarity, maar ook naar de eigenschappen van een 'gemiddelde' boom in de gemeenschap, als indicatie voor mass-ratio.

De effecten van biodiversiteit op biomassavoorraden en -dynamiek kunnen afhangen van verschillende variabelen, zoals milieuomstandigheden (bijvoorbeeld bodemvruchtbaarheid en regenval) en de ruimtelijke schaal en tijdsschaal waarop het onderzoek is uitgevoerd. Een kleine ruimtelijke schaal richt zich bijvoorbeeld op één hectare bos, terwijl een grote ruimtelijke schaal zicht richt op grotere gebieden zoals de hele Amazone. De belangrijkste doelstellingen van dit proefschrift zijn daarom: 1) het effect begrijpen van biodiversiteit en omgevingscondities op biomassavoorraden en -dynamiek in tropische bossen, 2) evalueren hoe deze relaties afhangen van de ruimtelijke schaal waarop het onderzoek is uitgevoerd en 3) evalueren hoe deze relaties afhangen van de tijdschaal die beschouwd is in het onderzoek. Om deze doelstellingen te bereiken heb ik samengewerkt met onderzoeksinstellingen in Bolivia, Brazilië en Guyana die vele hectares tropisch bos beheren en monitoren. In deze bossen heb ik gegevens verzameld over blad- en houteigenschappen van de meest voorkomende boomsoorten. Deze dataset kon ik combineren met gegevens van de lokale onderzoeksinstellingen om verschillende maten van biodiversiteit, milieuomstandigheden en biomassavoorraden en -dynamiek te kunnen berekenen.

Grote bomen zijn verantwoordelijk voor het grootste deel van de opname van koolstof in tropische bossen. Daarom is het belangrijk om te begrijpen waardoor variatie in biomassagroei bij grote bomen bepaald wordt. In hoofdstuk 2 kijken we naar de effecten van verschillende boomeigenschappen op de biomassagroei van grote bomen in een vochtig tropisch bos in Bolivia. We zien dat de biomassagroei sterk toeneemt met de grootte van het spinthout. Spinthout is het levende deel van het hout wat verantwoordelijk is voor wateropslag en -transport van de wortels naar de bladeren. De grote capaciteit om water op te slaan en te transporteren kan vooral belangrijk zijn voor grote bomen, omdat deze hoog zijn en veel zonlicht en hoge temperaturen te verdragen krijgen en daardoor veel water transpireren. Het lijkt er dus op dat de biomassagroei van grote tropische bomen vooral gelimiteerd is door hun hoge vraag naar water. 
Vervolgens schalen we op van individuele bomen naar hele gemeenschappen van bomen om de effecten van biodiversiteit en milieuomstandigheden op totale biomassavoorraden en -dynamiek te evalueren. In hoofdstuk 3 onderzoeken we deze relaties voor een tropisch regenwoud groeiend op zeer voedselarme bodems in Guyana. We vinden geen effecten van niche complementarity (gemeten als soortenrijkdom), maar wel een sterk effect van mass-ratio (gemeten als de gemiddelde boomeigenschappen) en de bodemvruchtbaarheid op biomassavoorraden en -dynamiek. Dit betekent dat op deze arme gronden slechts een klein aantal soorten die de juiste set van eigenschappen heeft goed kan overleven, snel kan groeien en groot en dominant kan worden.

In hoofdstuk 4 onderzoeken we dezelfde relaties voor een heel ander bostype: een vochtig tropisch bos op zeer voedselrijke bodems in Bolivia die 6 maanden droogseizoen ervaart (hetzelfde bos als in hoofdstuk 2). Voor dit bos zien we dat noch niche complementarity noch mass-ratio belangrijk zijn voor de biomassa dynamiek. In plaats daarvan bepaalt de bodemwaterbeschikbaarheid de biomassa dynamiek in dit meer seizoensgebonden en droger bos. Dit is in overeenstemming met hoofdstuk 2 waar we het belang van waterbeschikbaarheid voor de groei van grote bomen aantonen.

De Neotropen (de tropische gebieden in Zuid- en Meso-Amerika) omvatten veel meer bostypes en variatie in milieuomstandigheden dan de bossen gebruikt in hoofdstukken 3 en 4. In hoofdstuk 5 beschouwen we daarom de hele regio van de Neotropen door gegevens te gebruiken van 201 één-ha percelen in 26 bosgebieden. Hiermee evalueren we hoe de biodiversiteit en milieuomstandigheden de biomassavoorraden en -dynamiek bepalen. In tegenstelling tot de vorige twee hoofdstukken vinden we dat zowel niche compementarity als mass-ratio zeer belangrijk zijn voor biomassavoorraad en -dynamiek. Het lijkt erop dat de sterke verschillen tussen de bossen in bodemcondities en klimaat leiden tot grote verschillen in biodiversiteit, die op hun beurt leiden tot grote verschillen in biomassavoorraden en -dynamiek op deze grote ruimtelijke schaal.

Tropische bossen zijn dynamische systemen waarin continu nieuwe planten opkomen en andere sterven. We kunnen daarom verwachten dat tropische bossen in staat zijn te reageren op veranderingen in de omgeving, bijvoorbeeld als gevolg van de klimaatverandering. Afhankelijk van welke milieuomstandigheden veranderen, zal een aantal soorten met de juiste set van eigenschappen beter groeien en overleven dan andere soorten en daarom met de tijd steeds meer voorkomen. In hoofdstuk $\boldsymbol{\sigma}$ onderzoeken we hoe vijf tropische oerbossen (dat wil zeggen: oude bossen die volgroeid zijn en onverstoord voor tenminste enkele decennia) veranderen over tijd in termen van hun gemiddelde boomeigenschappen. Vervolgens evalueren we welke factoren de meest waarschijnlijke oorzaken zijn van deze veranderingen. We zien dat na verloop van tijd de hoeveelheid individuen 
toeneemt van soorten die normaal veel voorkomen in zeer oude bossen, terwijl de hoeveelheid afneemt van soorten die normaal veel voorkomen in jongere bossen. Dit betekent dat deze bossen zich in een herstellend proces bevinden, mogelijk veroorzaakt door verstoringen die lang geleden hebben plaats gevonden, zoals intense droogte of verstoringen door (precolumbiaanse) menselijke invloeden.

De bovenstaande hoofdstukken tonen verschillende effecten aan van biodiversiteit op biomassavoorraden en -dynamiek in tropische bossen. Om een beter algemeen beeld te krijgen van deze relaties voeren we een literatuurstudie uit in hoofdstuk 7. Hiervoor richten we ons niet alleen op resultaten van empirische studies (gebaseerd op gemeten data, zoals de andere hoofdstukken van dit proefschrift), maar ook op resultaten van studies die gebruik maken van satelliettechnieken en ecosysteem computermodellen. Van de 38 empirische studies laat $74 \%$ zien dat biodiversiteit belangrijk is voor biomassavoorraden en de opname van koolstof in tropische bossen. Satellietbeelden kunnen worden gebruikt om eenvoudig op te schalen naar grote gebieden. Bij 9 uit 10 studies met satellietbeelden zien we belangrijke effecten van biodiversiteit op biomassavoorraden. Ecosysteem modellen kunnen nuttig zijn voor het evalueren van toekomstige scenario's, zoals het gevolg van klimaatverandering en verlies van biodiversiteit op biomassavoorraden. Op dit moment zijn er slechts enkele ecosysteem modellen die een hoge biodiversiteit kunnen simuleren, maar deze wijzen op potentiele sterke en positieve effecten van biodiversiteit op de opname van koolstof. De resultaten van modelsimulaties tonen aan dat biodiversiteit met name belangrijk is om bossen weerbaar en veerkrachtig te maken voor bijvoorbeeld klimaatverandering (omdat er bij hoge biodiversiteit altijd genoeg soorten zijn die goed aangepast zijn aan de nieuwe klimaatcondities). Op die manier zorgt biodiversiteit voor een stabiele koolstofvoorraad en -opname. Daarom pleiten wij ervoor dat het behoud van biodiversiteit niet alleen een bijkomend voordeel is van bosbescherming voor klimaat mitigatie, maar dat biodiversiteit beschouwd moet worden als een voorwaarde om hoge en stabiele koolstofopslag en -opname, en dus klimaatmitigatie, te waarborgen op de lange termijn.

Kortom, in dit proefschrift toon ik aan dat biodiversiteit - waaronder de gemiddelde en diversiteit in boomeigenschappen - belangrijk is voor het functioneren van tropische bossen, maar dat het precieze effect afhankelijk is van de milieuomstandigheden, ruimtelijke schaal en tijdsschaal. Niche complementarity is het meest belangrijk op grote ruimtelijke schaal mogelijk als gevolg van grote verschillen in de biodiversiteit tussen bossen (hoofdstuk 5), terwijl mass-ratio belangrijk is op grote ruimtelijke schaal en in bossen met suboptimale milieuomstandigheden (bijvoorbeeld de voedselarme bodems in Guyana, hoofdstuk 3). Bovendien zijn tropische bossen niet stabiel, maar veranderen ze in hun biodiversiteit (in gemiddelde boomeigenschappen, hoofdstuk 6) als gevolg van 
veranderende milieuomstandigheden (waarschijnlijk veroorzaakt door verstoringen). Omdat biodiversiteit sterk bepalend is voor het functioneren van het bos op de lange termijn, zullen die veranderingen in biodiversiteit kunnen leiden tot veranderingen in de toekomstige biomassavoorraden en koolstof opname van het bos (hoofdstukken 7, 8).

Tropische bossen hebben vele lokale en mondiale voordelen, zoals het tegengaan van klimaatverandering, water recycling en de productie van hout- en andere bosproducten. In dit proefschrift laat ik zien en bediscussieer ik dat het bestaan en voortbestaan van deze bossen mede afhankelijk is van hun biodiversiteit. Ecologisch onderzoek zal zijn krachten moeten bundelen met sociaaleconomisch onderzoek en met passende politieke en technologische ontwikkelingen. Dit is nodig om te garanderen dat tropische boslandschappen ecologisch duurzaam en winstgevend zijn en zullen blijven. De bescherming en het behoud van tropische bossen zal een uitdaging zijn. Een uitdaging die overwonnen moet worden in het belang van alle organismen op aarde - inclusief de mens. 
Resumen 
Los bosques tropicales son muy importantes para el ciclo global del carbono. Eliminan el dióxido de carbono de la atmósfera a través de la fotosíntesis, secuestrándolo en forma de biomasa. Los bosques tropicales contienen el 25\% de todo el carbono almacenado en la biosfera terrestre y anualmente eliminan alrededor del $24 \%$ de las emisiones de gases de efecto invernadero, lo cual contribuye a mitigar el cambio climático. Por esta razón, las políticas internacionales tales como los acuerdos alcanzados durante las negociaciones sobre el clima de la United Nations Framework Convention on Climate Change (UNFCCC), en diciembre 2015 en París, reconocen cada vez más el potencial de los bosques tropicales para ayudar en la mitigación del cambio climático.

Además de su importancia para la mitigación del cambio climático, los bosques tropicales son los ecosistemas terrestres más diversos, albergando cerca de 47.000 especies de árboles. Esta alta diversidad hace que los bosques tropicales sean particularmente relevantes para la conservación de la biodiversidad, especialmente dada la amenaza por deforestación, degradación de los bosques, caza y cambio climático a la sobrevivencia de muchas especies. Investigaciones en ecosistemas menos complejos, tales como praderas o bosques templados, han demostrado que no solo es bueno tener alta biodiversidad, sino que también podría resultar en ecosistemas más productivos al almacenar y secuestrar más carbono. Estos resultados son afines con la hipótesis de complementariedad de nichos ecológicos, que predice que las especies tienen diferentes estrategias para obtener y utilizar recursos (tales como agua y nutrientes), y por tanto, una comunidad muy diversa de especies de plantas puede utilizar los recursos de una manera más eficiente y resultar en una mayor productividad total. Sin embargo, continuamos preguntándonos si esta hipótesis también se cumple para los bosques tropicales, donde el número de especies es mucho más alto y la mayoría de las plantas viven por mucho más tiempo. También podría ser que en vez de la diversidad de especies, las especies más abundantes son las que principalmente determinan la cantidad de carbono almacenado y absorbido por el bosque, lo cual concuerda con la hipótesis ecológica de peso proporcional ("mass-ratio" en Inglés).

En esta tesis, investigo la relación entre la biodiversidad y la capacidad de los bosques tropicales para almacenar y capturar carbono. Como medidas del almacenamiento de carbono utilizo las 'reservas de biomasa', tales como la biomasa en pie por unidad de superficie. Como medida de la captación de carbono utilizo 'dinámica de la biomasa', tales como los flujos de biomasa a través del tiempo: crecimiento y reducción de biomasa. Usualmente, a la biodiversidad se la conoce como el número de especies por área. No obstante, el sólo hecho de contar el número de especies no provee ninguna información sobre las características (o "rasgos") funcionales de las especies, los cuales son implícitamente importantes para las hipótesis de complementariedad de nichos ecológicos y peso proporcional. 
En otras palabras, donde las reservas y la dinámica de biomasa de una comunidad de plantas debería aumentar con la diversidad de rasgos funcionales (según la hipótesis de complementariedad de nichos ecológicos), y debería depender de los valores promedios de rasgos funcionales (según la hipótesis de peso proporcional). Por esa razón, esta tesis tiene un enfoque mayor en rasgos funcionales de plantas (tales como la concentración de nutrientes en la hoja y la densidad de la madera) que deberían proveer una mejor comprensión del funcionamiento de las reservas y dinámica de biomasa. Por lo tanto, en esta tesis se entiende por 'biodiversidad' a la diversidad de especies y sus rasgos (que refleja la complementariedad de nichos), pero también a los rasgos promedios de una comunidad de árboles (que refleja el peso proporcional).

Los efectos de la biodiversidad en las reservas y dinámica de biomasa pueden depender de varias variables, tales como las condiciones ambientales (por ejemplo, la fertilidad del suelo y precipitación), la escala espacial, y la escala temporal en consideración. Por tanto, los objetivos principales de mi tesis fueron los siguientes:

1) entender el efecto de la biodiversidad y el medio ambiente en las reservas y dinámica de biomasa de los bosques tropicales,

2) evaluar cómo estas relaciones dependen de la escala espacial considerada,

3) evaluar cómo estas relaciones dependen de la escala temporal considerada.

Para alcanzar estos objetivos, colaboré con instituciones de investigación en Bolivia, Brasil y Guyana que manejan e investigan una cantidad considerable de hectáreas de bosques tropicales. En estos bosques, colecté datos de los rasgos de las hojas y tallos de las especies de árboles más abundantes. Mediante la combinación de estas dos bases de datos, obtuvimos datos para las diferentes medidas de biodiversidad, de condiciones ambientales, y de reservas y dinámica de biomasa.

Los árboles grandes son responsables de la mayor parte del carbono absorbido por los bosques tropicales. Por tanto, es importante entender lo que determina la variación en el crecimiento de la biomasa entre los árboles grandes. En el capítulo 2 investigamos los efectos de las diferentes características de las plantas en el crecimiento de la biomasa de árboles grandes en un bosque húmedo tropical en Bolivia. Encontramos que el crecimiento de biomasa aumentó fuertemente con el tamaño de la albura del tronco, que es la parte viva de la madera responsable del almacenamiento y del transporte de agua desde las raíces hasta las hojas. El hecho de tener una alta capacidad para transportar y almacenar agua puede ser importante especialmente para estos árboles grandes. Por ser altos, reciben una gran cantidad de luz solar y están expuestos a temperaturas altas, y por lo tanto transpiran una mayor cantidad de agua. De esta manera, el crecimiento de la biomasa de árboles 
grandes tropicales parece estar limitado principalmente por su alta demanda de agua.

Seguidamente, ampliamos nuestro estudio de árboles individuales a comunidades enteras de árboles para evaluar las relaciones entre la biodiversidad y las condiciones ambientales con las reservas y dinámica de biomasa. En el capítulo 3 evaluamos estas relaciones para un bosque tropical húmedo que crece en suelos muy pobres en nutrientes de Guyana. No encontramos efectos de la complementariedad de nichos (medido a través de la diversidad de especies), pero un efecto importante del peso proporcional (medido a través de los rasgos promedios) y la fertilidad del suelo en las reservas y dinámica de biomasa. Este significa que en estos suelos pobres, sólo un pequeño grupo de especies con un conjunto de rasgos apropiados pueden sobrevivir más, crecer rápidamente, y llegar a ser grande y abundante.

En el capítulo 4 investigamos los mismos efectos para un bosque completamente diferente: un bosque tropical húmedo en suelos muy ricos en nutrientes en Bolivia (el mismo bosque del capítulo 2) que experimenta 6 meses de época seca al año. En este caso, encontramos que ni la complementariedad de nichos ni el peso proporcional son importantes para la dinámica de la biomasa. Más bien, la disponibilidad de agua en el suelo determinó la dinámica de biomasa en este bosque más estacional y más seco, lo que concuerda con la importancia de la disponibilidad de agua para el crecimiento de árboles grandes del capítulo 2 .

El Neotrópico (es decir, las regiones tropicales de Sudamérica y Mesoamérica) comprende mucho más tipos de bosques y mayor variabilidad en cuanto a condiciones ambientales de los que incluimos en los capítulos 3 y 4 . Por lo tanto, en el capítulo 5 consideramos toda la región del Neotrópico, utilizando datos de 201 parcelas de una hectárea en 26 sitios de bosques, y evaluamos cómo la biodiversidad y las condiciones ambientales determinan las reservas y la dinámica de biomasa. En contraste con los dos capítulos anteriores, encontramos que ambos complementariedad de nichos y peso proporcional fueron muy importantes para las reservas y la dinámica de biomasa. Parece que las grandes diferencias en las condiciones del suelo y clima entre los bosques ocasionan grandes diferencias en la diversidad biológica, que a su vez conllevan a grandes diferencias en las reservas y la dinámica de biomasa a una escala espacial mayor.

Los bosques tropicales son ecosistemas dinámicos con plantas que nacen y mueren continuamente. Por esta razón podemos esperar que los bosques tropicales pueden responder a los cambios en las condiciones ambientales, por ejemplo debido al cambio climático. Dependiendo de cómo cambian las condiciones ambientales, algunas especies con los rasgos adecuados crecerán y sobrevivirán mejor que otros, y por lo tanto serán más abundantes con el tiempo. En el capítulo 6 evaluamos como cinco bosques tropicales primarios (es decir, bosques 
maduros que no han experimentado disturbios mayores durante las últimas décadas) están transformando sus rasgos promedios con el tiempo, y evaluamos las causas más probables de estos cambios. Encontramos que la abundancia de especies que normalmente son comunes en los bosques primarios aumentó con el tiempo, mientras que la abundancia de especies que normalmente son comunes en los bosques más jóvenes disminuyó. Esto significa que estos bosques parecen estar en un proceso de recuperación, posiblemente a causa de disturbios que sucedieron hace mucho tiempo, como sequías intensas u ocupación humana precolombina.

Los capítulos anteriores muestran diferentes efectos de la biodiversidad en las reservas y la dinámica de biomasa de los bosques tropicales. Para poder generalizar mejor, hemos realizado una revisión de la literatura en el capítulo 7. Para esto, nos enfocamos no sólo en los resultados de estudios empíricos (como los otros capítulos de esta tesis), sino también en los resultados de estudios que utilizan técnicas de teledetección y modelos informáticos para ecosistemas. De los 38 estudios empíricos, el 74\% mostró que la biodiversidad es importante para las reservas de biomasa y la captura de carbono en los bosques tropicales. La teledetección se puede utilizar para medir fácilmente grandes áreas. 9 de 10 estudios que utilizan técnicas de teledetección reportaron efectos importantes de la biodiversidad en las reservas de biomasa. Los modelos de ecosistemas pueden ser útiles para evaluar escenarios futuros, por ejemplo para evaluar el efecto de la pérdida de biodiversidad o del cambio climático en las reservas de biomasa. Actualmente, sólo unos pocos modelos de ecosistemas pueden simular niveles de biodiversidad más altos, pero todos ellos mostraron efectos potencialmente fuertes y positivos de la biodiversidad en la absorción de carbono. Los resultados de las simulaciones indican que la biodiversidad es especialmente importante para asegurar una absorción estable de carbono frente al cambio climático. En consecuencia, abogamos que la conservación de biodiversidad no es solamente un beneficio adicional de los bosques para la mitigación del cambio climático, más bien, se debería considerar a la biodiversidad como un requisito previo para mejorar y garantizar la reserva y absorción alta y estable de carbono a largo plazo.

En resumen, en esta tesis encontré que la biodiversidad - incluyendo la diversidad de especies y los rasgos funcionales promedios - es importante para el funcionamiento de los bosques tropicales, pero que su efecto depende de las condiciones ambientales, y la escala espacial y temporal en consideración. La complementariedad de nichos fue más importante a mayor escala espacial, posiblemente a causa de los fuertes diferencias en diversidad entre los bosques (capítulo 5), mientras que el peso proporcional fue importante a mayor escala espacial y en bosques con condiciones ambientales duras (por ejemplo, los suelos pobres en nutrientes en Guyana, capítulo 3). Además, los bosques tropicales no se encuentran en un estado estable, sino que las condiciones ambientales cambiantes conllevan a 
cambios en biodiversidad (expresado en los rasgos promedios, capítulo 6). En consecuencia, esto podría conllevar a cambios en las reservas futuras de biomasa y absorción de carbono de los bosques, ya que la biodiversidad es muy importante para el funcionamiento de los bosques a largo plazo (capítulos 7, 8).

Los bosques tropicales proveen muchos beneficios locales y globales para la sociedad, tales como la mitigación del cambio climático, reciclaje del agua, y la producción de productos forestales maderables y no maderables. En esta tesis demuestro y argumento que la existencia y persistencia de estos bosques dependen en parte de su biodiversidad. La investigación ecológica debería combinarse más con investigación socio-económica y con el desarrollo de políticas y tecnología apropiados para lograr paisajes forestales tropicales que sean ecológicamente sostenibles y rentables a largo plazo. La conservación de los bosques tropicales será un desafío, pero un desafío que debe ser superada para el beneficio de todos los organismos de la Tierra - incluyendo los seres humanos. 
Acknowledgements 
The cover of this book mentions my name only. This agrees with the criteria of Wageningen University but does not agree with the procedure behind the development of this thesis. The years of my $\mathrm{PhD}$ have been among the most inspiring, challenging and fun of my life, in which I grew both personally and as a researcher. For all of this I am grateful to a great team of people. If the rules of Wageningen University had not been that strict, I would have added many names on the cover.

Most obviously, I would have added dear Prof. dr Poorter. Lourens, I certainly owe most to you in having reached the status of independent scientist. Back in 2010, you started investing time and energy in supervising me as an MSc student. You motivated me to join the Research Master in Ecology - you seemed to know that I liked research even before I knew myself. When you had a PhD position available, you provided me the opportunity, something that I have not regretted ever since. During my $\mathrm{PhD}$ you have always helped me to get the most out of it. The speed with which you provide comments is just astonishing. Your humour, pragmatic solutions, and positive energy are often exactly what is necessary. Besides being a great researcher and supervisor, you have certainly become my scientific father!

Marielos, also your name would be in the list! You are a wonderful woman: strong, critical and diplomatic, but simultaneously very warm and loving. Thank you for your guidance in all facets of research, and for your always-open door. People often say that the gender imbalance is partly because women lack a role model. I don't think that is true - you are a great role model for me and many others!

Eric, I don't know how you combined an Alterra-job with supervising a $\mathrm{PhD}$, but you did! Thank you for spending the time that you actually did not have on supervising me. Your critical input, humour, and all the preparations for fieldwork and life in Guyana also make you an essential part of the list. Thank you for all of this!

Lourens, Marielos and Eric, you have spent an enormous amount of time, energy, enthusiasm and humour in supervising $\mathrm{me}$ - in prosperous and challenging times. Words will fail in expressing my gratitude. It almost breaks my heart knowing that you won't be my supervisors anymore. I just hope the collaboration continues.

Someone else's name also appears in some of the chapters: Yasmani. There is no doubt that this thesis owes part of its existence to your mental and physical support. The picture on page 46-47 shows the camp that we would not have had without you, many graphs show data that we would not have had without you, and 
this whole thesis hopefully shows some passion that it would not have had without you. Muchisimas gracias!!

Once I heard someone saying: "If you don't like your friends, you choose others. If you don't like your colleagues, you change jobs. But if you don't like your family, you're stuck". I am blessed with a wonderful family. On top of that list of course are Adorien and Kees. Mum and dad, I will be grateful my lifelong for your unconditional support with everything I do, every step I make, every plane I take. Your unconditional love and care are among the most important in my life. I hope you feel that this thesis is also partly yours, because it certainly is!

Since I was one year old I was surrounded by one, and later two, brothers: Joren and Wessel. Bros, you are responsible for many moments of fighting, laughing, talking, crying, playing and traveling, which all contributed to the relationship we have now - one of trust, support and fun! And of course: Joren, thanks for the help on the wonderful cover; and Wessel, thanks for your input on the general discussion! En ook bedankt aan de rest van de familie voor jullie interesse, steun en gezelligheid, vooral aan mijn oma's!

"And she is off again...". Those were the words of one of my best friends, just after I had left for fieldwork. But unconditional friendship has no limits in time or space. Karlien and Laura, thanks for being the most wonderful friends I could wish! And thanks to all other unconditional friends who make my life so enjoyable: Anne, Anne, Dagmar, Danielle, Eva, Gerben, Joanne, Joost, Kyle, Lysanne, Maarten, Nicolas, Stijn.

Everyone who once visited the Forest Ecology and Forest Management (FEM) group will admit that this is the most amazing, inspiring and warm research group that they have ever seen. We discuss, help, party, laugh, dance, sport, eat, etc. It is therefore not strange that this group of people is also known as the "FEMfamily". I am grateful and proud to be part of this! Thanks to ALL of you, and in particular to my wonderful paranymphs Marlene and Monique, to my close colleagues Madelon and Cata ("Para") who let me be their paranymph, to my office mates Merel and Meike, to Frank (with Harry-Potter scar) and Pieter for supervision during my MSc, to Joke for making sure that FEM keeps functioning, to Frits for allowing me to be part of this group, and to all other FEMily members: Addisalem, Alan, Alejandra, André, Arildo, Bernardo, Canisius, Carol, Ellen, Estela, Federico, Frans, Geovana, Gert-Jan, Jamir, Jan, Jose, Juan-Ignacio, Kathelyn, Koen, Kwame, Lan, Leo, Linar, Lu, Lucy, Mart, Paul, the "Peters", Surya, Tieb, Ute, and Vency! 
Not only was I part of FEM, but also of the Vegetation, Forest and Landscape Ecology team (VBL) of Alterra. It was great and interesting to be part of this group, where people work really hard but still manage to enjoy, and which functions so differently from chair groups at the university. Some of the highlights of course were the team outings and the Friday-noon-squash! Thank you all for the experiences!

During my PhD I had the fortune to visit the most wonderful forests and to work with a very diverse group of people. I want to thank all 100+ ROBIN-colleagues for great opportunities to collaborate and learn from other disciplines, including the PIK team (Kirsten, Alice and Boris), the remote sensing crew (Lammert, Loïc), and Terry for bringing ROBIN to life, keeping it alive, and making it thrive.

I also want to thank Insituto Boliviano de Investigación Forestal (IBIF) in Santa Cruz (Bolivia), Empresa Brasileira de Pesquisa Agropecuária (EMBRAPA) in Belém (Brazil), and the Guyana Forestry Commission (GFC) in Georgetown (Guyana). These institutes hosted me, collaborated and helped me wherever needed, provided me the data that I needed, and made me feel welcome! Specifically, I want to thank Nataly from IBIF for her collaboration on various of my thesis chapters and co-organizing an amazing course on functional traits. Furthermore, Lucas, you have always been so incredibly helpful and made us feel very welcome - thanks for that! And last but not least, I owe many thanks for all their energy and enthusiasm in the field to: Ricardo, Bruno, Wicho, Harry, Miguel, Edenaldo, and Marcio!

Also thanks to my PE\&RC-day buddies, Chantal, Claudius, and everyone else involved in the $\mathrm{PhD}$ council of the PE\&RC graduate school and the Wageningen $\mathrm{PhD}$ Council for providing many great moments of fun and opportunities to grow!

Indeed, that is a long list of people. Just to conclude: thanks mum and dad for your unconditional support, thanks Lourens, Marielos and Eric for doing everything possible to get the best out of my $\mathrm{PhD}$, and thanks Yasmani for your physical en mental support and, above all, your love. 


\section{Short biography}

Masha Tamara van der Sande was born on the 24th of May 1989 in Rotterdam, the Netherlands. At the age of three her family moved to Dordrecht, where Masha attended Geert Grootte primary school. When she was 13 , the family moved to Sevenum, a small village in the east of the country, where she finished high school at Dendron college in Horst.

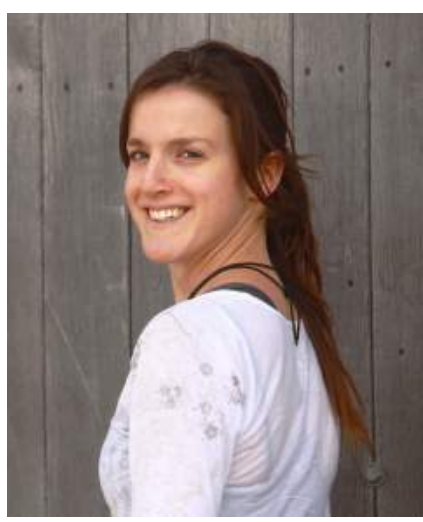

In 2007, Masha moved to Utrecht to start a BSc study in Biology at Utrecht University, which she finished cum laude in 2010. During this time, she started traveling to places such as Romania, India, and Curaçao. From Utrecht she moved to Wageningen to start an MSc in Biology at Wageningen University. As part of her studies, and to meet her wishes to discover the complex, dynamic and diverse world of tropical forests, she completed two MSc thesis projects in tropical forest ecology. Her first thesis was entitled "The trade-off between cavitation-resistance and conductivity differs between tropical tree and liana species", for which she travelled to Panama to collect data in collaboration with the Smithsonian Tropical Research Institute. Her second thesis was entitled "Using traits to explain growth of tropical canopy trees: quantity overrules quality", for which she travelled to Bolivia to collect data and collaborate with the Instituto Boliviano de Investigación Forestal (IBIF). These two thesis projects motivated Masha for research and stimulated her interest in tropical forest ecology. Therefore, parallel with the second year of her MSc in Biology, she started a one-year Research Master in Ecology at Wageningen University.

In August 2012 Masha finished her MSc Biology cum laude, and in September 2012 she started a $\mathrm{PhD}$ at Wageningen University. During her $\mathrm{PhD}$, she was privileged to work with a variety of people and in a variety of places; she was part of the international and interdisciplinary project on the Role Of Biodiversity In climate change mitigatioN (ROBIN), and did fieldwork in two forests in Bolivia in collaboration with IBIF, one forest in Guyana in collaboration with the Guyana Forestry Commission, and one forest in Brazil in collaboration with EMBRAPA. She was also involved in activities from the PE\&RC PhD Council (2013-2016) and became chair of the Wageningen PhD Council (2015-2016). These opportunities and almost four years of work (Sept 2012-July 2016) resulted in the dissertation "Biodiversity and the functioning of tropical forests" that you are now holding in your hands.

Now, having finished her PhD thesis, Masha is ready to move on in science. By doing so she aims and hopes to contribute to the understanding of (tropical) forest dynamics and complexity, which is crucially important to assure the existence and persistence of these ecosystems and thereby the quality of life of many organisms - including humans. 


\section{Publications}

\section{Published articles:}

van der Sande, M.T., Arets, E.J.M.M., Peña-Claros, M., Luciana de Avila, A., Roopsind, A., Mazzei, L., Ascarrunz, N., Finegan, B., Alarcón, A., Cáceres-Siani, Y., Licona, J.C., Ruschel, A., Toledo, M. \& Poorter, L. (2016) Old-growth Neotropical forests are shifting in species and trait composition. Ecological Monographs, 86, 228-243.

Prado-Junior, J.A., Schiavini, I., Vale, V.S., Arantes, C.S., van der Sande, M.T., Lohbeck, M. \& Poorter, L. (2016) Conservative species drive biomass productivity in tropical dry forests. Journal of Ecology, 104, 817-827.

Poorter, L., van der Sande, M.T., Thompson, J., Arets, E.J.M.M., Alarcón, A., Álvarez-Sánchez, J., Ascarrunz, N., Balvanera, P., Barajas-Guzmán, G., Boit, A., Bongers, F., Carvalho, F.A., Casanoves, F., Cornejo-Tenorio, G., Costa, F.R.C., de Castilho, C. V., Duivenvoorden, J.F., Dutrieux, L.P., Enquist, B.J., Fernández-Méndez, F., Finegan, B., Gormley, L.H.L., Healey, J.R., Hoosbeek, M.R., Ibarra-Manríquez, G., Junquira, A.B., Levis, C., Licona, J.C., Lisboa, L.S., Magnusson, W.E., Martínez-Ramos, M., Martínez-Yrizar, A., Martorano, G., Makell, L.C., Mazzei, L., Meave, J.A., Mora, F., Muñoz, R., Nytch, C., Pansonato, M.P., Parr, T.W., Paz, H., Simoes Penello, M., Pérez-García, E.A., Rentería, L.Y., Rodríguez-Velazquez, J., Rozendaal, D.M.A., Ruschel, A.R., Sakschewski, B., Salgado Negret, B., Schietti, J., Sinclair, F.L., Souza, P.F., Souza, F.C., Stropp, J., ter Steege, H., Swenson, N.G., Thonicke, K., Toledo, M., Uriarte, M., van der Hout, P., Walker, P., Zamora, N. \& Peña-Claros, M. (2015) Diversity enhances carbon storage in tropical forests. Global Ecology and Biogeography, 24, 1314 1328.

van der Sande, M.T., Zuidema, P.A., Sterck, F. (2015) Explaining biomass growth of tropical canopy trees: the importance of sapwood. Oecologia, 117, 1145-1155.

van der Sande, M.T., Poorter, L., Schnitzer, S.A. \& Markesteijn, L. (2013) Are lianas more drought-tolerant than trees? A test for the role of hydraulic architecture and other stem and leaf traits. Oecologia, 172, 961-72.

Immers, A.K., van der Sande, M.T., van der Zande, R.M., Geurts, J.J.M., Donk, E. \& Bakker, E.S. (2013) Iron addition as a shallow lake restoration measure: impacts on charophyte growth. Hydrobiologia, 710, 241-251.

\section{Submitted articles:}

van der Sande, M.T., Peña-Claros, M., Ascarrunz, N., Arets, E.J.M.M., Licona, J.C., Toledo, M. \& Poorter, L. Drivers of biomass change in a tropical forest: testing for effects of diversity, traits, and environment.

van der Sande, M.T., Poorter, L., Kooistra, L., Balvanera, P., Thonicke, K., Thompson, J., Arets, E.J.M.M., Garcia Alaniz, N., Jones, L., Mora, F., Mwampamba, T.H., Parr, T. \& PeñaClaros, M. Biodiversity enhances climate change mitigation by tropical forests.

van der Sande, M.T., Arets, E.J.M.M., Peña-Claros, M., Hoosbeek, M.R., Cáceres-Siani, Y., van der Hout, P. \& Poorter, L. Soil fertility and species traits, but not species diversity, drive productivity and biomass stocks in a tropical rainforest. 
van der Sande, M.T., Poorter, L., Kooistra, L., Balvanera, P., Thonicke, K., Boit, A., Dutrieux, L.P., Equihua, J., Garcia Alaniz, N., Gerard, F., Herold, M., Kolb, M., Sakschewski, B., Simões, M. \& Peña-Claros, M. Integrating empirical, remote sensing and modelling approaches to evaluate biodiversity effects on climate mitigation in tropical forests.

Poorter, L., van der Sande, M.T., Arets, E.J.M.M., Ascarrunz, N., Enquist, B., Finegan, B., Licona, J.C., Martínez-Ramos, M., Mazzei, L., Meave, J.A., Muñoz, R., Nytch, C., de Oliveira, A.A., Pérez-García, E.A., Powers, J.S., Prado-Junior, J., Rodríguez-Velázques, J., Ruschel, A., Salgado-Negret, B., Schiavini, I., Swenson, N., Tenorio, E.A., Thompson, J., Toledo, M., Uriarte, M., van der Hout, P., Zimmerman, J. K. \& Peña-Claros, M. Biodiversity, climate, and soil determine functioning of Neotropical forests.

de Avila, A.L., van der Sande, M.T., Dormann, C.F., Peña-Claros, M., Poorter, L., Mazzei, L., Ruschel, A.R., Macedo Silva, J.N., Pereira de Carvalho, J.O. \& Bauhus, J. What drives biomass resilience in a managed tropical forest: disturbance intensity or remaining forest composition? 


\section{PE\&RC Training and Education Statement}

With the training and education activities listed below the $\mathrm{PhD}$ candidate has complied with the requirements set by the C.T. de Wit Graduate School for Production Ecology and Resource Conservation (PE\&RC) which comprises of a minimum total of 32 ECTS (= 22 weeks of activities).

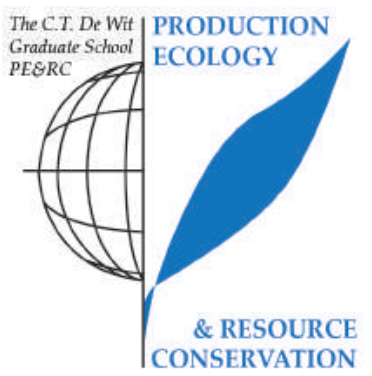

\section{Review of literature (4.5 ECTS)}

- The role of (functional) diversity in climate change mitigation in tropical forests (2012, 2013, 2015)

\section{Writing of project proposal (3 ECTS)}

- Explaining growth with traits and environment; scaling from leaf to regional tropical forests $(2012,2013)$

\section{Post-graduate courses (12 ECTS)}

- Bayesian statistics; PE\&RC (2012)

- Photosynthesis, climate and change; PE\&RC (2013)

- Linear models; PE\&RC (2013)

- Summer School: an interdisciplinary perspective on biodiversity and ecosystem services; Peyresq, France; ALTER-Net (2013)

- Multivariate analysis; PE\&RC (2014)

- Generalized linear models; PE\&RC (2015)

- Structural equation modelling; PE\&RC (2015)

- Thematic School: functional ecology of tropical rainforests in the context of climate changes: from real observations to simulations; French Guiana; UMR Ecofog, Kourou and UMR EEF, Nancy (2016)

\section{Laboratory training and working visits (4.5 ECTS)}

- Diversity, traits and environmental effects on biomass dynamics of a tropical forest in Bolivia; Instituto Boliviano de Investigación Forestal (2014)

\section{Invited review of (unpublished) journal manuscript (2 ECTS)}

- Biotropica, Forest Ecology and Management (2), Frontiers in Plant Science, Functional Ecology, Global Ecology and Biogeography, Journal of Ecology, Journal of Plant Ecology, New Phytologist, Plant and Soil (2), Plant Ecology (2013-2016). 


\section{Deficiency, refresh, brush-up courses (0.3 ECTS)}

- Lectures on sustainable forest management of the course advanced forest ecology and forest management (2013)

\section{Competence strengthening / skills courses (3.45 ECTS)}

- Competence assessment; WGS (2012)

- Reviewing a scientific paper; PE\&RC (2013)

- Workshop gender and diversity awareness; WUR (2015)

- Writing grant proposals; WGS (2015)

- The last stretch of the PhD programme; WGS (2015)

- PhD Coaching; Environmental Science Group (2015-2016)

\section{PE\&RC Annual meetings, seminars and the PE\&RC weekend (2.7 ECTS)}

- PE\&RC Day $(2011,2014,2015)$

- PE\&RC First year PhD weekend (2012)

- Current themes in ecology: biodiversity on the crossroads (2014)

- PE\&RC Last year PhD weekend (2015)

\section{Discussion groups / local seminars / other scientific meetings (6.9 ECTS)}

- Ecology and application discussion group (2012-2016)

- FEM Journal club discussion group (2013-2016)

- Netherlands Annual Ecology Meeting; oral presentation (2013)

- R Users meeting discussion group (2013)

- Wageningen evolution and ecology seminars (2013)

- Netherlands Annual Ecology Meeting; poster presentation (2014)

- Vegetation-Soil interactions: from Rhizosphere to Ecosystem; organization (2014)

- Empresa Brasileira de Pesquisa Agropecuária; oral presentation; Brazil (2014)

- Instituto Boliviano de Investigación Forestal; oral presentation; Bolivia (2014)

- Terra Preta symposium (2015)

- Netherlands Annual Ecology Meeting; oral presentation (2016)

\section{International symposia, workshops and conferences (7.8 ECTS)}

- Meeting of the ROBIN-project; Santa Cruz, Bolivia (2012)

- Annual meeting of the GTÖ; oral presentation; Zürich, Switzerland (2015)

- Annual meeting of the ATBC; oral presentation; Hawaii, USA (2015)

- Workshop on functional traits along environmental gradients, with the group of Prof. Dr. Yadvinder Malhi; oral presentation; Hawaii, USA (2015)

- Meeting of the ROBIN-project; oral presentation; Brussels, Belgium (2015) 
- Annual meeting of the GTÖ; oral presentation and session convener; Göttingen, Germany (2016)

- Annual meeting of the ATBC; oral presentation; Montpellier, France (2016)

\section{Lecturing / supervision of practicals / Tutorials (3.15 ECTS)}

- Tropical ecology; UvA (2012)

- Rasgos funcionales (functional traits); Santa Cruz, Bolivia (2014)

- Resource use and sustainable utilization; WU (2015)

- Ecology I; WU (2016)

\section{Supervision of MSc students (3 ECTS)}

- The role of functional traits and disturbance intensity on carbon stocks in a tropical forest in Guyana

- Soil carbon and fine root biomass in a tropical forest in Guyana 

The research described in this thesis was financially supported by the European Union Seventh Framework Programme (FP7/2007-2013) under grant agreement $\mathrm{n}^{\circ} 283093$ - The Role Of Biodiversity In climate change mitigatioN (ROBIN). This research was also part of the strategic research program KBIV (KB-14) "Sustainable spatial development of ecosystems, landscapes, seas and regions", funded by the Dutch Ministry of Economic Affairs and carried out by Wageningen University \& Research centre (project code KB-14-003-030).

Financial support from Wageningen University for printing this thesis is gratefully acknowledged

Cover design:

Joren van der Sande

jorenvdsande@gmail.com

Printed by:

Digiforce / Proefschriftmaken.nl, Vianen 



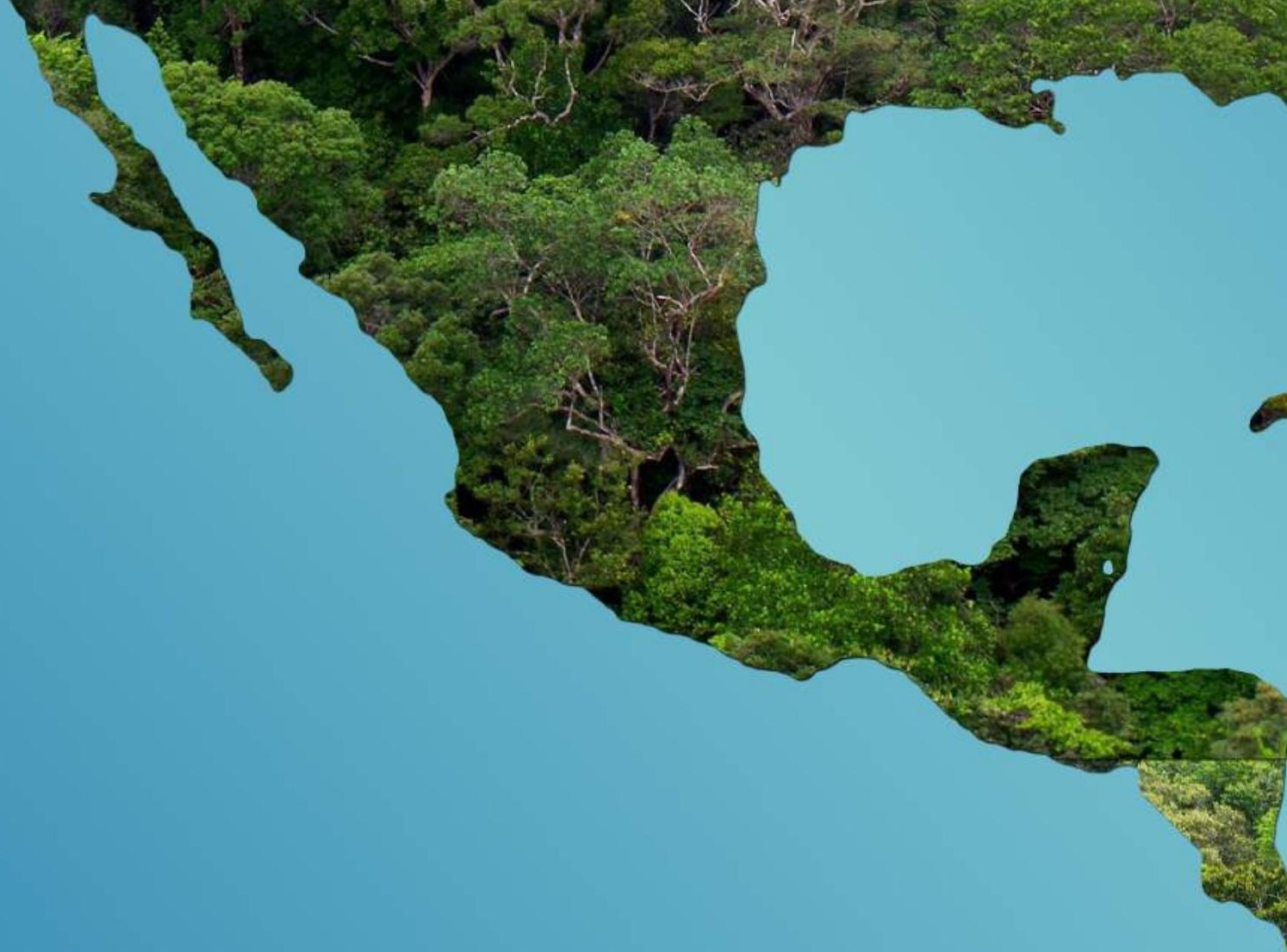

